Prepared for the U.S. Department of Energy under Contract DE-AC05-76RL01830

\title{
Assessment of the Group 3-4 (HV-S1, HV-S2, IHLW-S1) Stack Sampling Probe Locations for Compliance with ANSI/HPS \\ N13.1-1999
}

JA Glissmeyer

JE Flaherty

EJ Antonio

January 2013

Pacific Northwest

NATIONAL LABORATORY

Proudly Operated by Battelle Since 1965 


\title{
DISCLAIMER
}

This report was prepared as an account of work sponsored by an agency of the United States Government. Neither the United States Government nor any agency thereof, nor Battelle Memorial Institute, nor any of their employees, makes any warranty, express or implied, or assumes any legal liability or responsibility for the accuracy, completeness, or usefulness of any information, apparatus, product, or process disclosed, or represents that its use would not inf ringe privately owned rights. Reference herein to any specific commercial product, process, or service by trade name, trademark, manufacturer, or otherwise does not necessarily constitute or imply its endorsement, recommendation, or favoring by the United States Government or any agency thereof, or Battelle Memorial Institute. The views and opinions of authors expressed herein do not necessarily state or reflect those of the United States Government or any agency thereof.

\author{
PACIFIC NORTHWEST NATIONAL LABORATORY \\ operated by \\ BATTELLE \\ for the \\ UNITED STATES DEPARTMENT OF ENERGY \\ under Contract DE-AC05-76RL01830
}

Printed in the United States of America

Available to DOE and DOE contractors from the

Office of Scientific and Technical Information,

P.O. Box 62, Oak Ridge, TN 37831-0062;

ph: (865) 576-8401

fax: $(865) 576-5728$

email: reports@adonis.osti.gov

Available to the public from the National Technical Information Service

5301 Shawnee Rd., Alexandria, VA 22312

ph: (800) 553-NTIS (6847)

email: orders@antis.gov <http://www.ntis.gov/about/form.aspx>

Online ordering: http://www.ntis.gov

This document was printed on recycled paper.

$(8 / 2010)$ 


\section{Assessment of the Group 3-4 (HV-S1, HV-S2, IHLW-S1) Stack Sampling Probe Locations for Compliance with ANSI/HPS N13.1-1999}

JA Glissmeyer

JE Flaherty

EJ Antonio

January 2013

Test Specification:

Statement of Work

Work Authorization:

Test Plan:

Test Exceptions:

Test Scoping Statement(s):

QA Technology Level:
$\mathrm{N} / \mathrm{A}$

24590-QL-SRA-W000-00101

WA\# 009

TP-WTPSP-032, Rev 0.0

N/A

NA

Developmental Research

Prepared for

the U.S. Department of Energy

under Contract DE-AC05-76RL01830 and

Subcontract Number MOA: 24590-QL-HC9-WA49-00001

Pacific Northwest National Laboratory

Richland, Washington 99352 


\section{Completeness of Testing}

This report describes the results of work and testing specified by test plan TP-WTPSP-032. The work and any associated testing followed the quality assurance requirements outlined in the test plan. The descriptions provided in this test report are an accurate account of both the conduct of the work and the data collected. Test plan results are reported. Also reported are any unusual or anomalous occurrences that are different from expected results. The test results and this report have been reviewed and verified.

\section{Approved:}

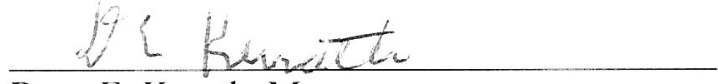

Dean E. Kurath, Manager

WTP Support Project

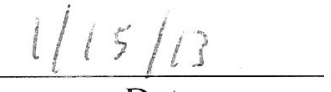

Date 


\section{Summary}

This document reports on a series of tests conducted to assess the proposed air sampling locations for the Hanford Tank Waste Treatment and Immobilization Plant (WTP) Group 3-4 exhaust stacks with respect to the applicable criteria regarding the placement of an air sampling probe. The HV-S1, HV-S2, and IHLW-S1 exhaust stacks were tested together as a group (Test Group 3-4) because they share a geometric attribute: the common factor in their design is that the last significant flow disturbance upstream of the air sampling probe is a jog (i.e., two conjoined bends of equal and opposite curvature resulting in a change in elevation of the duct). Federal regulations ${ }^{1}$ require that a sampling probe be located in the exhaust stack according to criteria established by the American National Standards Institute/Health Physics Society (ANSI/HPS) N13.1-1999, Sampling and Monitoring Releases of Airborne Radioactive Substances from the Stack and Ducts of Nuclear Facilities. These criteria address the capability of the sampling probe to extract a sample that represents the effluent stream.

The testing on scale models of the stacks conducted for this project was part of the River Protection Project-Waste Treatment Plant Support Program under Contract No. DE-AC05-76RL01830 according to the statement of work issued by Bechtel National, Inc. (BNI, 24590-QL-SRA-W000-00101, N13.1-1999 Stack Monitor Scale Model Testing and Qualification, Revision 1, 9/12/2007) and Work Authorization 09 of Memorandum of Agreement 24590-QL-HC9-WA49-00001. The internal Pacific Northwest National Laboratory (PNNL) project for this task is 53024, Work for Hanford Contractors Stack Monitoring. The testing described in this document was further guided by the Test Plan Air Sampling Probe Location Tests for Waste Treatment Plant HV-S1, HV-S2 and IHLW-S1 (Group 3-4) Air Exhaust Systems (TP-WTPSP-032).

The tests conducted by PNNL during 2012 on the Group 3-4 scale model systems are described in this report. The series of tests consists of various measurements taken over a grid of points in the duct cross section at the designed sampling probe locations. The ANSI/HPS N13.1-1999 qualification criteria concern the following properties of the air flowing through the ducts where the air sampling probes are to be located:

1. Uniform Air Velocity-The gas momentum across the stack cross section where the sample is extracted should be well mixed or uniform. The uniformity is expressed as the variability of the measurements about the mean, expressed as the percent coefficient of variance $(\% \mathrm{COV})$. It is calculated as the standard deviation divided by the mean and expressed as a percentage - the lower the $\% \mathrm{COV}$ value, the more uniform the velocity.

2. Angular Flow-The purpose of this test is to determine whether the air velocity vector is aligned with the sampling nozzle.

3. Uniform Concentration of Tracer Gases-A uniform contaminant concentration in the sampling plane enables the extraction of samples that represent the true concentration.

\footnotetext{
${ }^{1}$ Title 40 of the Code of Federal Regulations (CFR), Part 61, National Emissions Standards for Hazardous Air Pollutants (NESHAP), Subpart H, National Emission Standard For Emissions of Radionuclides other than Radon from Department of Energy Facilities.
} 
4. Uniform Concentration of Tracer Particles - Uniformity in contaminant concentration at the sampling probe was further demonstrated using tracer particles large enough to exhibit inertial effects.

Particles of $10-\mu \mathrm{m}$ aerodynamic diameter were used.

The scale model test results for the proposed sampling probe locations are summarized below.

Table S.1. Summary of Preferred Sampling Probe Location Results for the Group 3-4 Scale Model Stacks

\begin{tabular}{llllll}
\hline & Acceptance Criteria & Units & HV-S1 & HV-S2 & IHLW-S1 \\
\hline $\begin{array}{l}\text { Velocity } \\
\text { Uniformity }\end{array}$ & $\leq 20$ & \%COV & $2.8-6.6$ & $2.5-7.8$ & $4.2-10$ \\
\hline Flow Angle & $\leq 20$ & Degrees & $3.1-4.7$ & $3.9-12.5$ & $1.3-9.3$ \\
\hline $\begin{array}{l}\text { Gas Tracer } \\
\text { Uniformity }\end{array}$ & $\leq 20$ & \%COV & $1.1-4.7$ & $1.8-7.2$ & $0.9-5.8$ \\
\cline { 2 - 6 } & $\leq 30$ & $\begin{array}{l}\text { Maximum \% } \\
\text { Deviation from Mean }\end{array}$ & $2.6-7.9$ & $4.3-14.2$ & $1.8-11.2$ \\
\hline $\begin{array}{l}\text { Particle Tracer } \\
\text { Uniformity }\end{array}$ & $\leq 20$ & Normalized \%COV & $4.4-11.7$ & $5.3-13$ & $3.3-15.2$ \\
\hline
\end{tabular}

For the HV-S1 and HV-S2 stacks alternate test ports also showed satisfactory results and make allowance for limited construction variance in the probe locations.

Based on these scale model tests, the locations proposed for the air sampling probes in each of the three Group 3-4 stacks meet the requirements of the ANSI/HPS N13.1-1999 standard. Additional velocity uniformity and flow angle tests on the actual stacks will be necessary during cold-startup to confirm the validity of the scale model results in representing the actual stacks. In particular, the velocity uniformity test results for the actual stacks must be within 5\%COV of the range of results listed for the scale model so that scale model results can be said to be representative of the stack. If the velocity uniformity results on the actual stack fall within these bounds and flow angle test results fall within qualification criteria (mean angle $\leq 20^{\circ}$ ), the scale model results may be used as a substitute for the actual stack.

\section{Quality Assurance}

The PNNL quality assurance (QA) program is based on the requirements defined in the U.S. Department of Energy Order 414.1D, Quality Assurance, and 10 CFR 830, Energy/Nuclear Safety Management, and Subpart A-Quality Assurance Requirements (a.k.a., the Quality Rule). PNNL has chosen to implement the following consensus standards in a graded approach:

- ASME NQA-1-2000, Quality Assurance Requirements for Nuclear Facility Applications, Part I, "Requirements for Quality Assurance Programs for Nuclear Facilities".

- ASME NQA-1-2000, Part II, Subpart 2.7, Quality Assurance Requirements for Computer Software for Nuclear Facility Applications.

- ASME NQA-1-2000, Part IV, Subpart 4.2, Graded Approach Application of Quality Assurance Requirements for Research and Development. 
The procedures necessary to implement the requirements are documented through PNNL's "How Do I...?" (HDI), which is a system for managing the delivery of laboratory-level policies, requirements, and procedures.

The Waste Treatment Plant Support Program (WTPSP) implements an NQA-1-2000 QA program, using a graded approach presented in NQA-1-2000, Part IV, Subpart 4.2. The WTPSP Quality Assurance manual (QA-WTPSP-002) describes the technology life cycle stages under the WTPSP QA plan (QA-WTPSP-0001). The technology life cycle includes the progression of technology development, commercialization, and retirement in process phases of basic and applied research and development $(R \& D)$, engineering and production, and operation until process completion. The life cycle is characterized by flexible and informal QA activities in basic research, which becomes more structured and formalized through the applied R\&D stages. The work described in this report has been completed under the QA Technology level of Developmental Work as the data will be used for applying air discharge permits. Developmental Work is described below:

- DEVELOPMENTAL WORK-Developmental work consists of research tasks moving toward technology commercialization. These tasks still require a degree of flexibility, and there is still a degree of uncertainty that exists in many cases. The role of quality on development work is to make sure that adequate controls exist to support movement into commercialization.

WTPSP addresses internal verification and validation activities by conducting an Independent Technical Review of the final data report in accordance with WTPSP's procedure QA-WTPSP-601, Document Preparation and Change. This review verifies that the reported results are traceable, that inferences and conclusions are soundly based, and the reported work satisfies the test plan objectives. Appendix E lists the reviewed test plan, test instructions, and calculation packages used for the tests documented in this report. 



\title{
Acronyms
}

\author{
acfm \\ actual cubic feet per minute \\ afpm \\ $\mathrm{AD}$ \\ actual feet per minute \\ ANSI \\ aerodynamic diameter \\ ASME \\ American National Standards Institute \\ BNI \\ American Society of Mechanical Engineers \\ CCP \\ Bechtel National, Inc. \\ CFR \\ computer-assisted calculation package \\ DOE \\ Code of Federal Regulations \\ DV \\ U.S. Department of Energy \\ EPA \\ hydraulic diameter $\times$ mean velocity \\ FA \\ U.S. Environmental Protection Agency \\ GT \\ flow angle test \\ HDI \\ gaseous tracer test \\ HEPA \\ "How Do I...?" \\ HPS \\ high-efficiency particulate air (filter) \\ $\mathrm{HV}-\mathrm{S} 1$ \\ Health Physics Society \\ $\mathrm{HV}-\mathrm{S} 2$ \\ WTP High-Level Waste C3 (non-process) ventilation system exhaust stack \\ IHLW-S1 \\ NESHAP \\ WTP High-Level Waste C5 (process) ventilation system exhaust stack \\ WTP High-Level Waste C3 (canister) ventilation system exhaust stack \\ National Emissions Standards for Hazardous Air Pollutants \\ $\mathrm{OPC}$ \\ optical particle counter \\ $\% \mathrm{COV}$ \\ percent coefficient of variation \\ PNNL \\ Pacific Northwest National Laboratory \\ PT \\ particulate tracer test \\ QA \\ quality assurance \\ RMS \\ root mean square \\ scfm \\ standard cubic feet per minute \\ sfpm \\ standard feet per minute \\ $\mathrm{SF}_{6}$ \\ TI \\ sulfur hexafluoride \\ test instruction \\ VT \\ velocity uniformity test \\ WTP \\ Hanford Tank Waste Treatment and Immobilization Plant \\ WTPSP \\ Waste Treatment Plant Support Program
}





\section{Acknowledgments}

Preparing, executing, and post-processing these scale model measurements involved a number of Pacific Northwest National Laboratory staff. We would like to particularly acknowledge the support of our quality engineer, Kirsten Meier, and the administrative support from Andrea Boehler, Chrissy Charron, and Mona Champion. We would also like to express our appreciation to scientific staff members Carmen Arimescu, Yin-Fong Su, and Xiao-Ying Yu who conducted measurements under a variety of weather conditions. Additionally, Matthew Barnett, Carmen Arimescu, Rosanne Aaberg, Elizabeth Golovich, and Susan Sande provided technical reviews. Cary Counts and Megan Peters provided editorial support for this report.

Funding for this effort was provided by the Department of Energy's Waste Treatment and Immobilization Plant Project through a subcontract with Bechtel National, Inc. Pacific Northwest National Laboratory is operated for the U.S. Department of Energy by Battelle under Contract DE-AC05-76RL01830. 



\section{Contents}

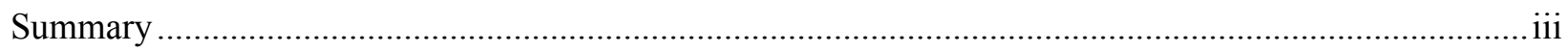

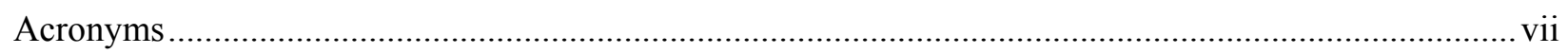

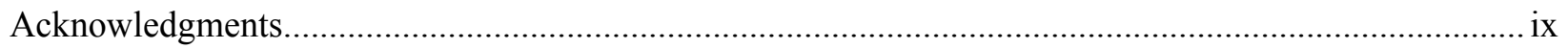

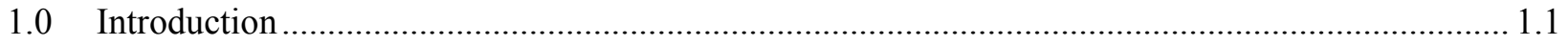

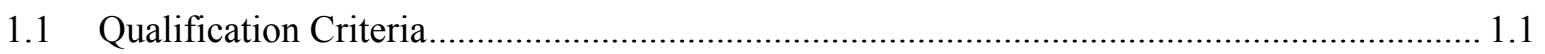

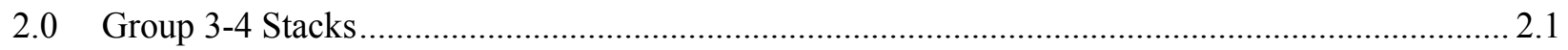

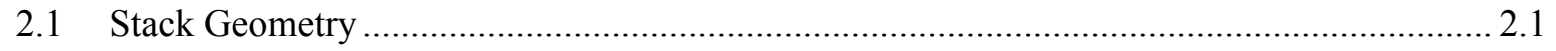

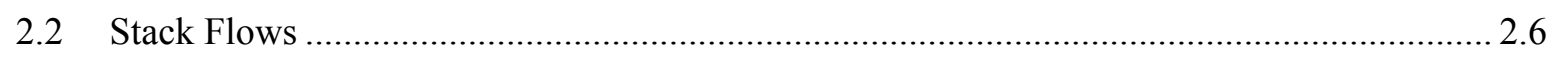

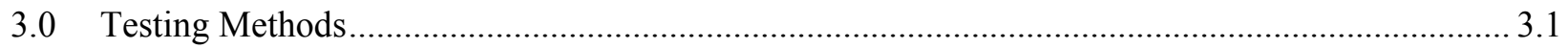

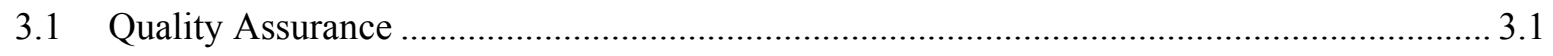

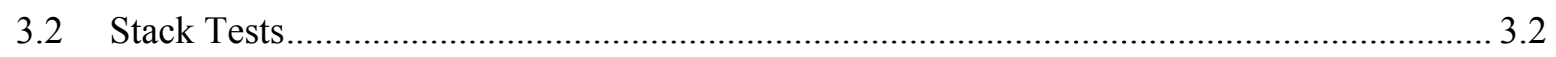

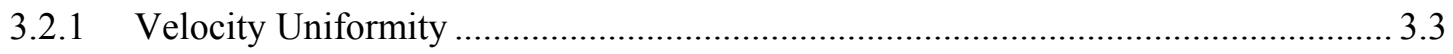

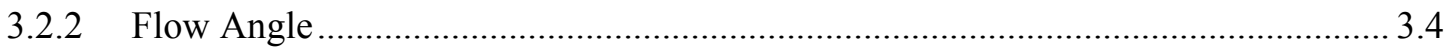

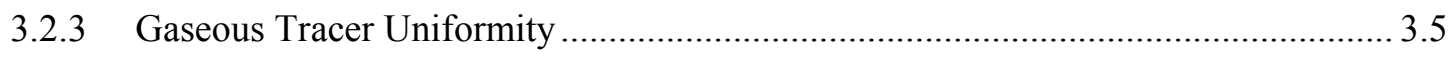

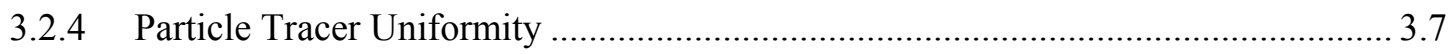

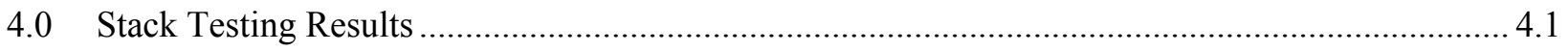

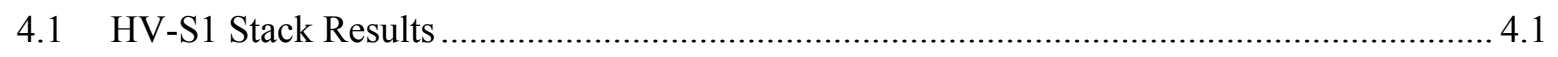

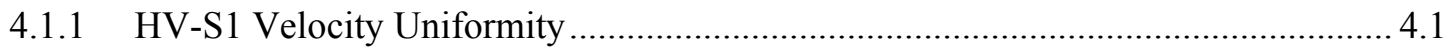

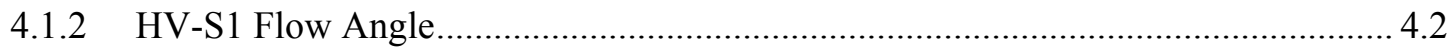

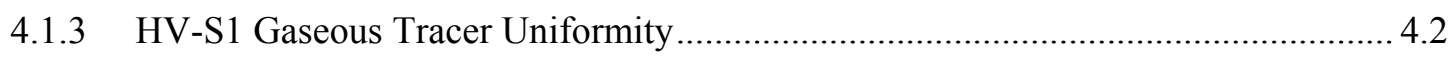

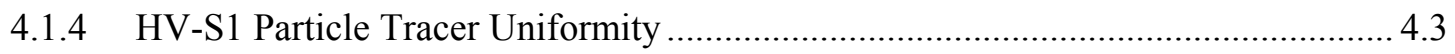

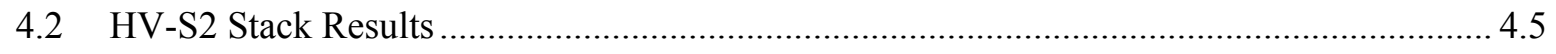

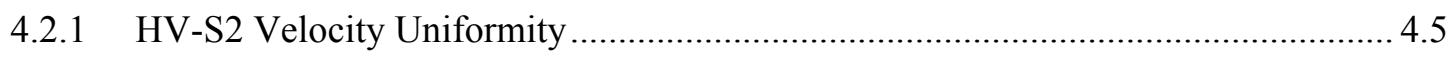

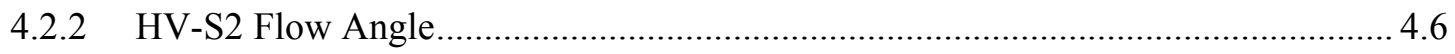

4.2.3 HV-S2 Gaseous Tracer Uniformity ……............................................................ 4.6

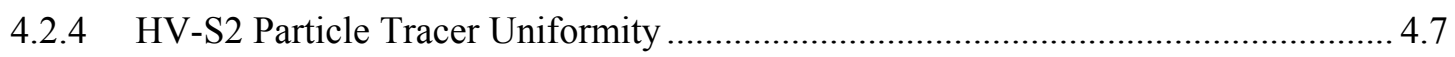

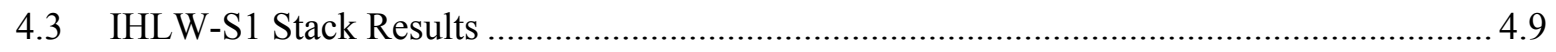

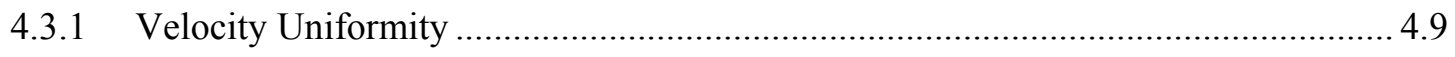

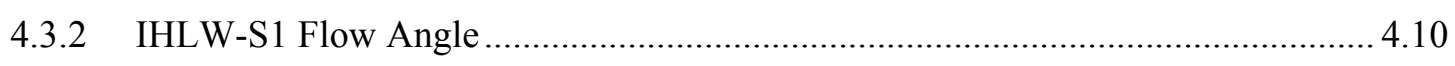

4.3.3 IHLW-S1 Gaseous Tracer Uniformity ............................................................ 4.10

4.3.4 IHLW-S1 Particle Tracer Uniformity ….......................................................... 4.11

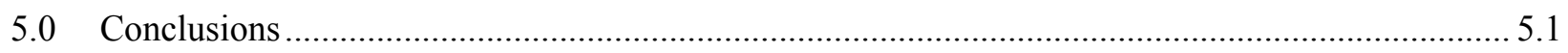

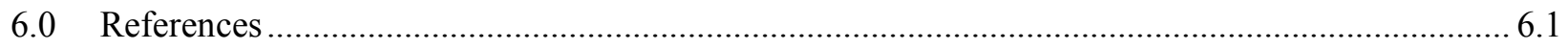

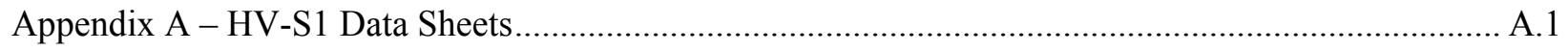

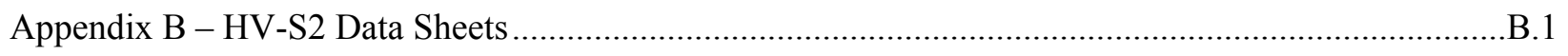

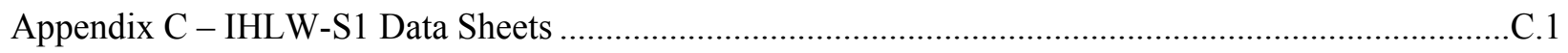




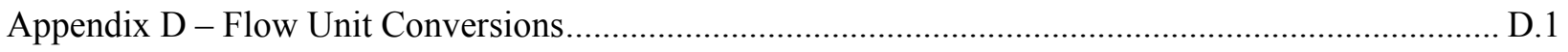

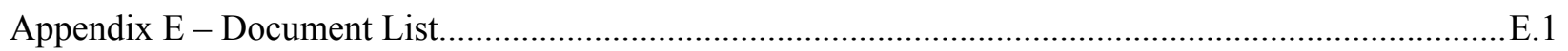

\section{Figures}

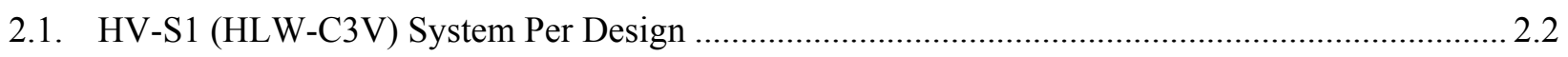

2.2. HV-S2 (HLW-C5V) System Per Design ................................................................................ 2.2

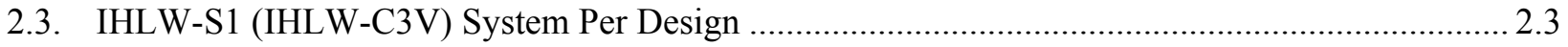

2.4. Scale Model Layout of the HV-S1 (HLW-C3V) Model............................................................. 2.3

2.5. Scale Model Layout of the HV-S2 (HLW-C5V) Model................................................................ 2.4

2.6. Scale Model Layout of the IHLW-S1 (IHLW-C3V-Canister) Model ............................................ 2.4

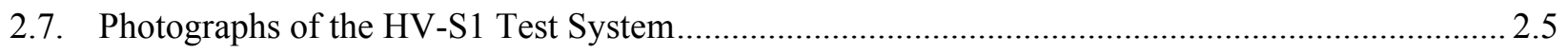

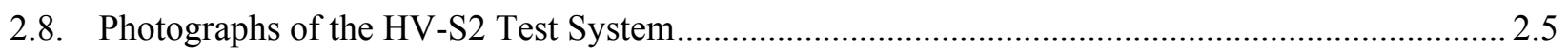

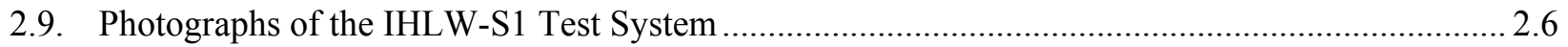

3.1. Cross Section of the Duct at the Testing Ports with Measurement Points ..................................... 3.3

3.2. Equipment Used for the Velocity Uniformity Test .................................................................... 3.4

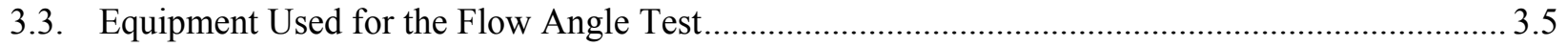

3.4. Equipment Used for the Gaseous Tracer Injection ................................................................ 3.6

3.5. Illustration of Five Injection Points in a Circular Duct ................................................................ 3.6

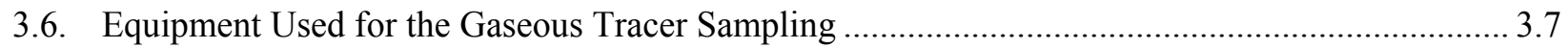

3.7. Equipment Used for Particle Injection (IHLW-S1) ................................................................ 3.8

3.8. Particle Counters Used for the Particle Sampling ........................................................................ 3.9

3.9. Probes Used for the Particle Sampling................................................................................. 3.10

4.1. Measurement and Reference Particle Test Data from PT-3 on the HV-S1 Stack .......................... 4.4

4.2. Measurement and Reference Particle Test Data from PT-6 on the HV-S1 Stack.......................... 4.5

4.3. Measurement and Reference Particle Tracer Test Data from PT-1 of HV-S2 ............................ 4.8

4.4. Measurement and Reference Particle Tracer Test Data from PT-7 of HV-S2 ............................. 4.9

4.5. Measurement and Reference Particle Tracer Test Data from PT-3 of IHLW-S1 ....................... 4.12

\section{Tables}

S.1. Summary of Preferred Sampling Probe Location Results for the Group 3-4 Scale Model Stacks ..... iv

2.1. Scaling Factor for 12-in.-Diameter Scale Model Stack ............................................................ 2.6

2.2. Summary of Flow Parameters for Scale Model Stacks ............................................................... 2.7

2.3. Summary of DV Values for Scale Model Stacks .......................................................................... 2.8 
4.1. Summary of HV-S1 Velocity Uniformity Tests ......................................................................... 4.2

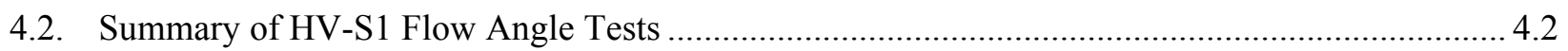

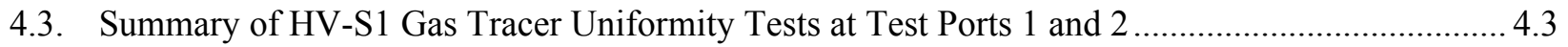

4.4. Summary of HV-S1 Particle Tracer Uniformity Tests.................................................................... 4.4

4.5. Summary of HV-S2 Velocity Uniformity Tests ........................................................................ 4.6

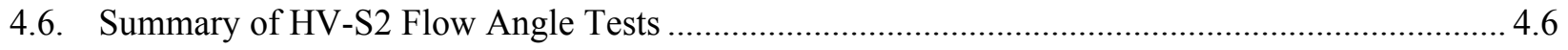

4.7. Summary of HV-S2 Gas Tracer Uniformity Tests at Test Port 2 .............................................. 4.7

4.8. Summary of HV-S2 Particle Tracer Uniformity Tests................................................................ 4.8

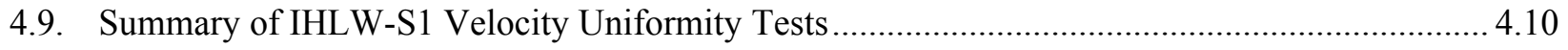

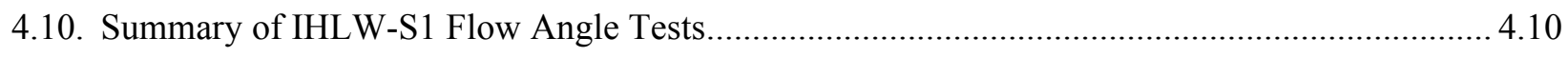

4.11. Summary of IHLW-S1 Gas Tracer Uniformity at Test Ports 1 and 2 ....................................... 4.11

4.12. Summary of IHLW-S1 Particle Tracer Uniformity Tests .......................................................... 4.12

5.1. Summary of Test Port 2 Results for the Group 3-4 Scale Model Stacks ...................................... 5.1

5.2. Summary of Test Port 1 Results for the Group 3-4 Scale Model Stacks ...................................... 5.1 



\subsection{Introduction}

The purpose of this series of scale model tests is to document the extent to which the current Bechtel National, Inc. (BNI) designs for the HV-S1, HV-S2, and IHLW-S1 air exhaust stacks in the Hanford Tank Waste Treatment and Immobilization Plant (WTP) meet the applicable regulatory criteria governing effluent monitoring systems. The emissions from these high-level waste facility air exhaust stacks may exceed the 0.1-millirem per year threshold limit given in Title 40 of the Code of Federal Regulations (CFR), Part 61, National Emissions Standards for Hazardous Air Pollutants (NESHAP), Subpart H, National Emission Standard For Emissions of Radionuclides other than Radon from Department of Energy Facilities. The NESHAP rule requires that a sampling probe be located in the exhaust stack according to criteria established by the American National Standards Institute/Health Physics Society (ANSI/HPS) N13.1-1999, Sampling and Monitoring Releases of Airborne Radioactive Substances from the Stack and Ducts of Nuclear Facilities. ${ }^{1}$ The capability of the sampling probe locations to meet this standard has been demonstrated with a series of tests on scale models. These data will be used by BNI as input to the air discharge permitting process. These three stacks were tested together as a group (Test Group 3-4) because they share a geometric attribute: the common factor in their design is that the last significant flow disturbance upstream of the air sampling probe is a jog (i.e., two conjoined bends of equal and opposite curvature resulting in a change in elevation of the duct).

This work is performed as part of the River Protection Project-Waste Treatment Plant Support Program under Contract No. DE-AC05-76RL01830 according to the statement of work issued by BNI, 24590-QL-SRA-W000-00101, N13.1-1999 Stack Monitor Scale Model Testing and Qualification, Revision 1, 09/12/2007 and Work Authorization 09 of Memorandum of Agreement 24590-QL-HC9-WA49-00001. The internal Pacific Northwest National Laboratory (PNNL) project for this task is 53024, Work for Hanford Contractors Stack Monitoring.

PNNL personnel conducted scale model tests during 2012. No BNI personnel were directly involved in the tests. The BNI WTP point of contact and facility engineers provided the most current engineering input to support PNNL's tests. BNI retains responsibility for the technical design of the stack discharge and air monitoring systems.

\subsection{Qualification Criteria}

The qualification criteria for the location of a stack air monitoring probe are taken from ANSI/HPS N13.1-1999, section 5.2.2 and are paraphrased as follows:

1. Uniform Air Velocity - It is important that the gas velocity be fairly uniform across the stack cross section where the sample is extracted. Consequently, the velocity is measured at several discrete points in the duct cross section at the proposed location of the sampling nozzle. The uniformity is expressed as the variability of the measurements about the mean. This is expressed using the percent coefficient of variation $(\% \mathrm{COV}),{ }^{2}$ which is the standard deviation divided by the mean and expressed as a percentage - the lower the \% $\mathrm{COV}$ value, the more uniform the velocity. The qualification

\footnotetext{
${ }^{1}$ Health Physics Society, McLean, Virginia. The standard has been reaffirmed in 2011 and is identical to the 1999 version. The regulations have not been updated yet, so the 1999 version is still referenced.

${ }^{2}$ Coefficient of variation is considered "dated" terminology. The modern terminology is percent relative standard deviation. However, because the standard uses the older terminology, it will likewise be used here.
} 
criterion is that the $\% \mathrm{COV}$ of the air velocity must be $\leq 20 \%$ in the center two-thirds of the duct cross section where the sampling probe is to be located.

2. Angular Flow-Sampling nozzles are typically aligned with the axis of the stack. If the air travels through the stack in cyclonic fashion, the air velocity vector approaching a sampling nozzle could be sufficiently misaligned with the nozzle to impair extraction of particles. Consequently, the flow angle is measured in the duct at the proposed location of the sampling probe. The average of the flow angle measurements (made at the same grid of points as the velocity measurements) should not exceed $20^{\circ}$ relative to the sampling nozzle axis.

3. Uniform Concentration of Tracer Gases-A uniform contaminant concentration in the sampling plane enables the extraction of samples that represent the true concentration within the duct. The uniformity of the concentration is first tested with a tracer gas to represent gaseous effluents. The fan is a good mixer, so injecting the tracer downstream of the fan provides worst-case results. The qualification criteria are that 1 ) the $\% \mathrm{COV}$ of the measured tracer gas concentration is $\leq 20 \%$ across the center two-thirds of the duct cross section at the sampling location, and 2) the concentrations at all the measurement points cannot deviate from the mean by $>30 \%$.

4. Uniform Concentration of Tracer Particles - The second set of tests addressing contaminant concentration uniformity at the sampling position uses tracer particles large enough to exhibit inertial effects. Tracer particles of $10-\mu \mathrm{m}$ aerodynamic diameter (AD) are used by default unless it is known that larger contaminant particles will be present in the airstream. The qualification criterion is that the $\% \mathrm{COV}$ of particle concentration is $\leq 20 \%$ across the center two-thirds of the duct at sampling location.

Tests to determine if Criteria 1 through 4 were met were conducted on the three scale models of the Group 3-4 stacks (HV-S1, HV-S2, and IHLW-S1) at the proposed sampling location along the exhaust duct. By conducting tests on scale models of the exhaust systems, the designed air sampling locations can be qualified before cold commissioning, and compensatory measures could be made in the design if testing results were not satisfactory. All of the tracer concentration, velocity, and flow angle measurements were made using the same grid of points in a given cross section of the duct. The ANSI/HPS N13.1-1999 standard sets additional qualification criteria for the use of a scale model as a substitute for the actual stack.

- The scale model and its sampling location must be geometrically similar to the actual stack.

- The product of the hydraulic diameter and the mean velocity (DV) for the scale model must be within a factor of six of the DV for the actual stack.

- The Reynolds number for the actual and model stacks must be $>10,000$.

- The scale model results are considered valid if it is further shown that:

- The velocity profile in the actual stack meets the uniformity criterion ( $\% \mathrm{COV} \leq 20 \%)$.

- The velocity uniformity COV values for the actual and model stacks agree within 5\%COV.

- The flow angle criterion (with a mean value less than or equal to $20^{\circ}$ ) is met.

The tests to determine the validity of the scale model testing will be performed during cold startup testing on the actual WTP stacks under separate test plans. The scale model testing conducted, as well as the results of these tests, is described in subsequent sections of this report. 


\subsection{Group 3-4 Stacks}

\subsection{Stack Geometry}

Group 3-4 consists of three different stacks: HV-S1, HV-S2, and IHLW-S1. These three stacks were tested together as a group (Test Group 3-4) because they share a geometric attribute in their design in that the last significant flow disturbance upstream of the air sampling probe is a jog (i.e., two conjoined bends of equal and opposite curvature resulting in a change in elevation of the duct).

In these three stacks, the designed sampling probes will be located in horizontal sections of duct. Figure 2.1 through Figure 2.3 show the layout for each of the three stack designs, from the fan outlet to the base of the vertical duct. Figure 2.4 through Figure 2.6 show the scale model layout for each of the three stack designs. The simplified models are based on assumptions about the necessary simulation detail. These assumptions are listed below.

- Geometric simulation of the components upstream of the backdraft damper was ignored. Backdraft damper blades do not usually open fully. The partially open blades direct the air velocity vector toward one side of the duct resulting in considerable disruption to the air flow. Consequently, it was assumed that the air velocity and tracer uniformity downstream of the dampers would not be greatly influenced by equipment upstream of the dampers. This assumption has not been tested; however, this assumption had the benefit of reducing the cost of the models by using a single fan/filter/heater arrangement and the elimination of the control damper.

Components several duct diameters downstream of the sampling point are not modeled. It was assumed that the only effect of any components downstream of the sampling probe location would be to slightly change the pressure at the sampling port. While this assumption was not tested, components generally do not influence flow patterns upstream. Figure 2.7. through Figure 2.9 show photos of the scale models for each of the three stack designs.

The same fans were used for each of the three scale models. The fans were connected to a flexible duct that was connected to the backdraft damper. The backdraft damper was subsequently connected to each of the scale models for testing.

For each of the scale model stacks, Test Port 2 represents the planned location for operational stack sampling according to the current WTP BNI designs. The distance from the end of the duct jog to the center of Test Port 2 was approximately 20, 20, and 8 duct diameters for the HV-S1, IHLW-S1, and HV-S2 models, respectively. On the HV-S1 and IHLW-S1 systems, Test Port 1 is located approximately five duct diameters downstream of Test Port 2 to allow some flexibility in testing. For the HV-S2 system, Test Port 1 is located only two duct diameters downstream of Test Port 2.

The ratio of the prototype dimensions to the scale model dimensions varies with each system. Each scale model was constructed with a primary duct diameter of $12 \mathrm{in}$. for convenience and to maintain the ability to re-use the duct sections for subsequent stack designs. Table 2.1 lists the final diameter of the actual stack with the scaling factor for the 12-in. scale model diameter. The calculations of the key scale model dimensions were performed in spreadsheets and then verified and validated in accordance with appropriate quality assurance (QA) procedures. ANSI/HPS N13.1-1999 requires that the models be geometrically similar to the actual stacks. Acceptable deviations in key dimensions of the scale model 
arising from scaling and fabrication errors are within about $\pm 5 \%$ for cross-sectional dimensions and about $25 \%$ of a duct diameter in overall length between the sampling point and the flow disturbances. These deviations would have less impact on the test results than the normal standard deviation of repeat tests. The key scale model dimensions for the as-built scale models were measured and recorded by testing staff.

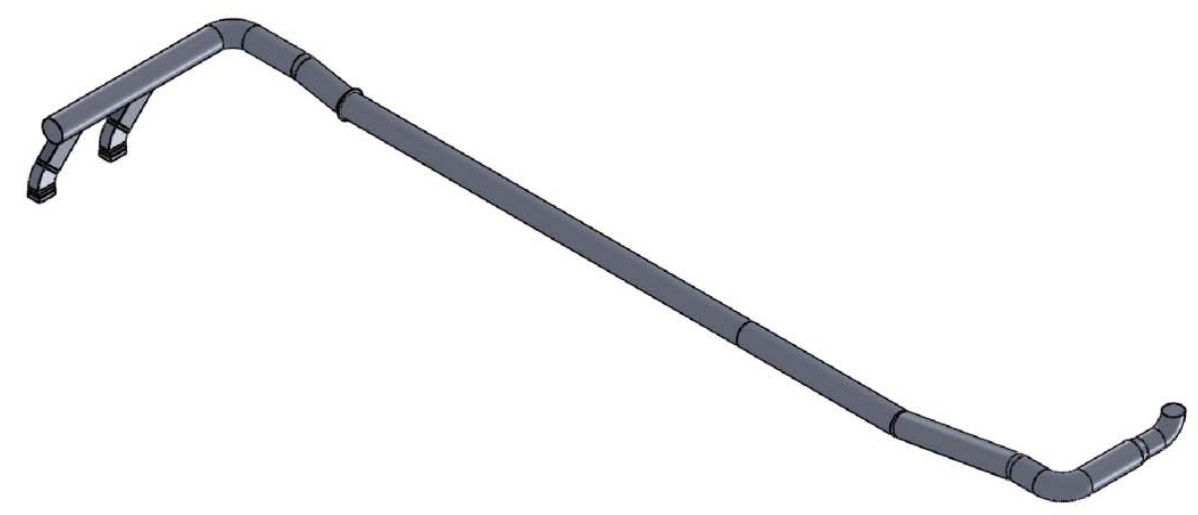

Figure 2.1. HV-S1 (HLW-C3V) System Per Design

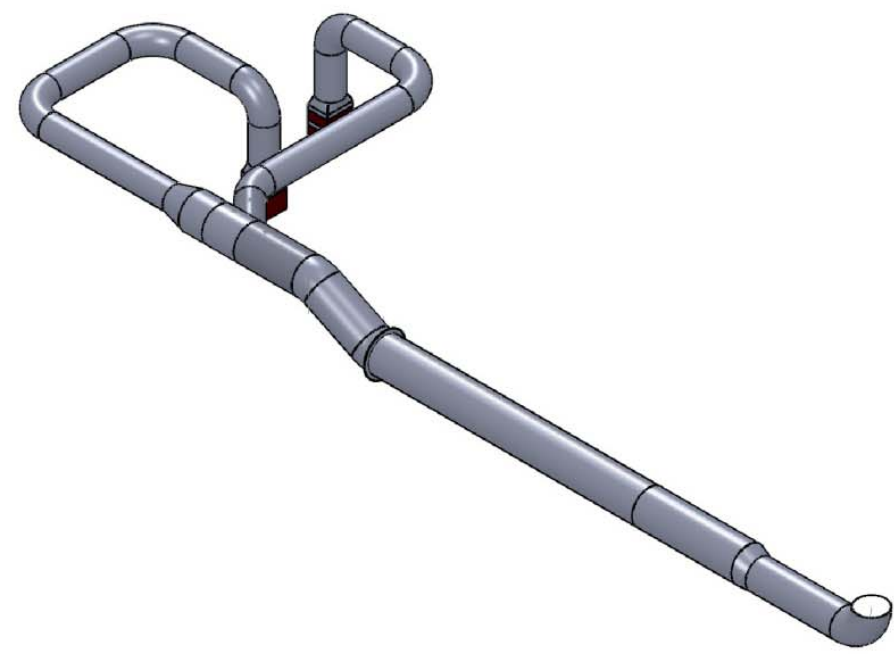

Figure 2.2. HV-S2 (HLW-C5V) System Per Design 


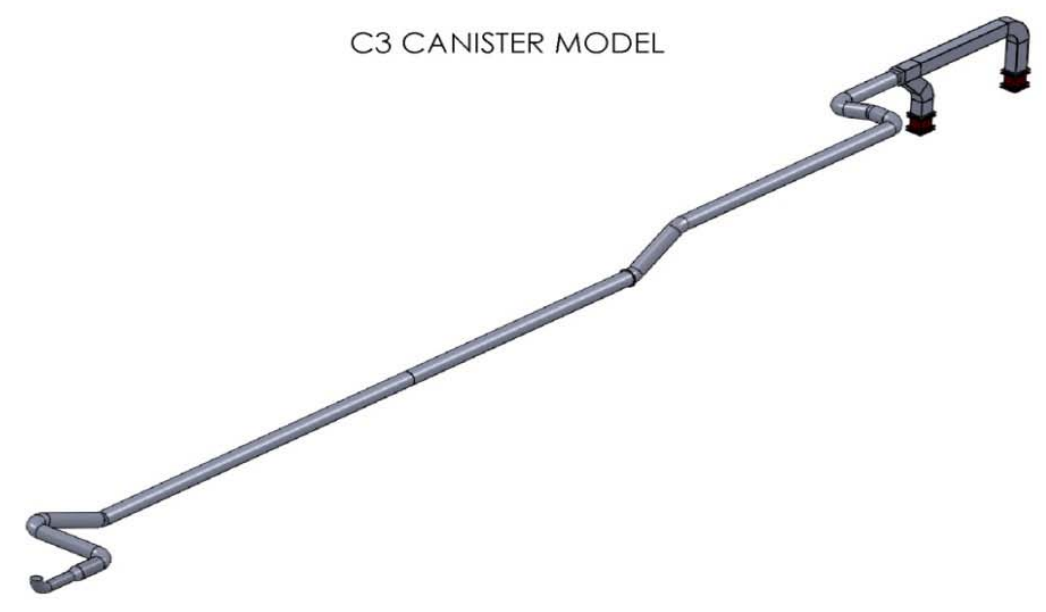

Figure 2.3. IHLW-S1 (IHLW-C3V) System Per Design

\section{C3V TEST LAYOUT}

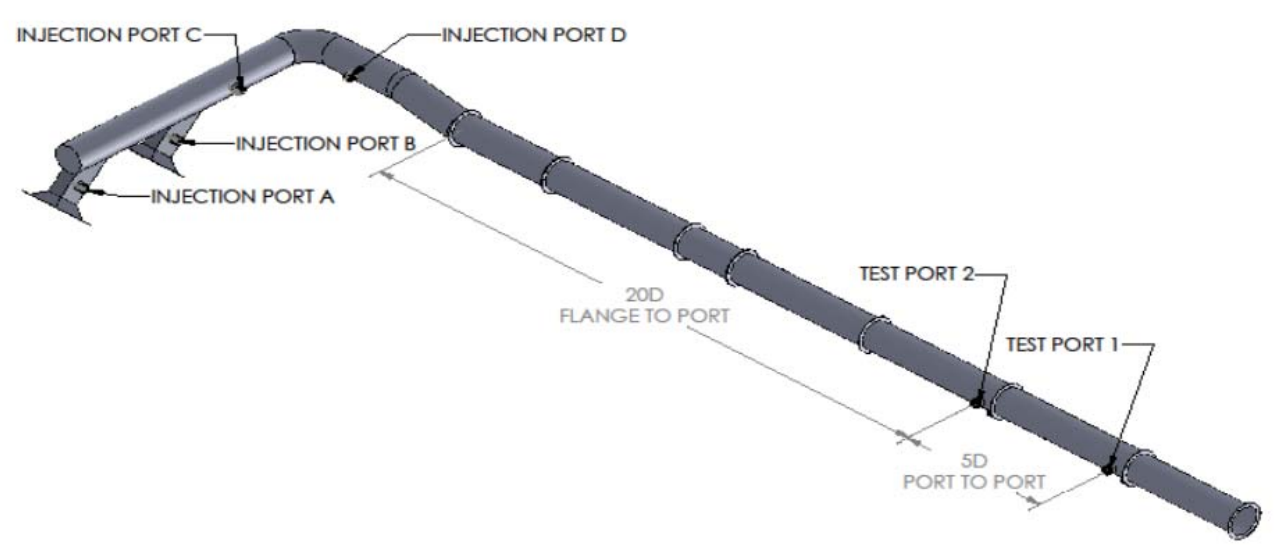

Figure 2.4. Scale Model Layout of the HV-S1 (HLW-C3V) Model 


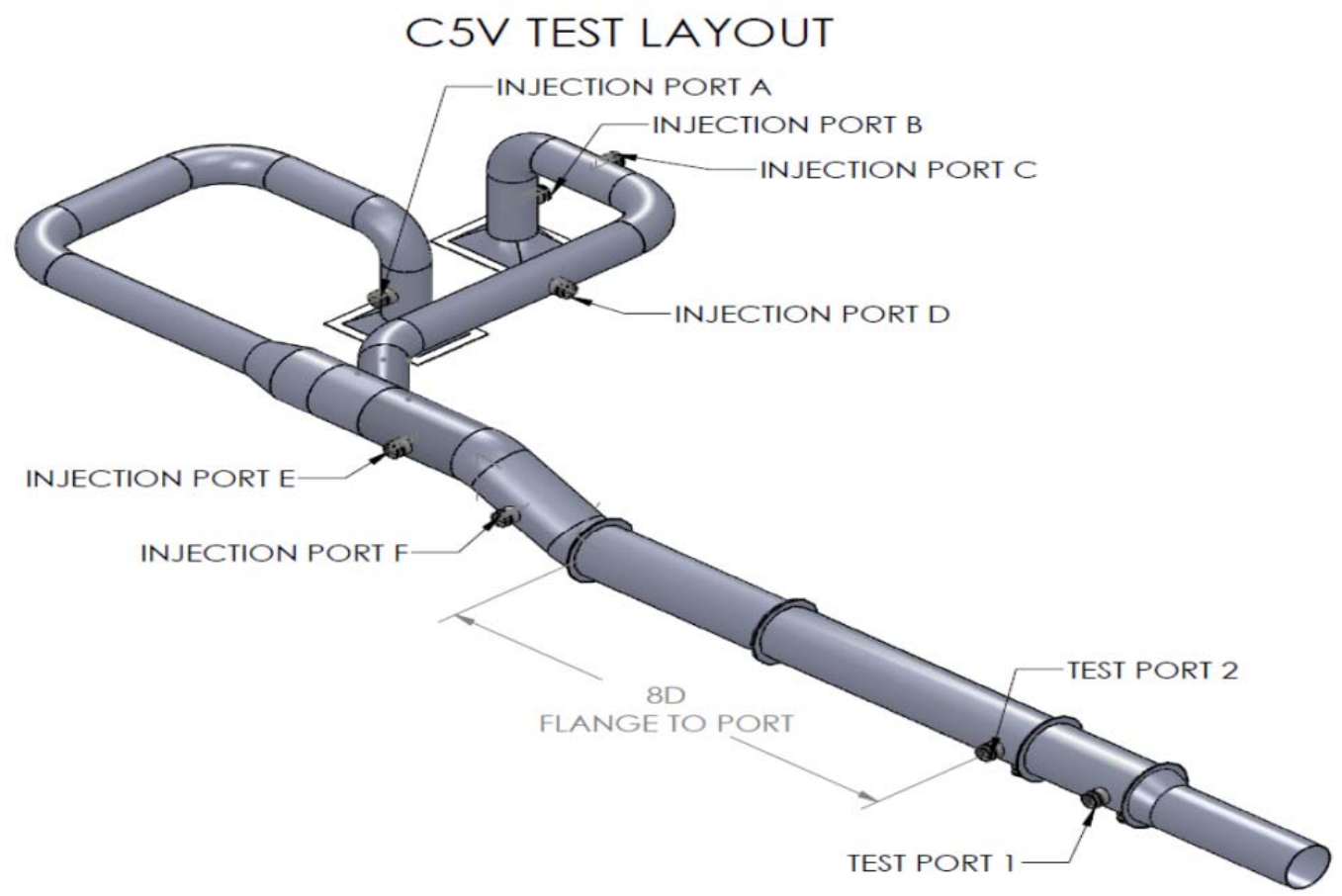

Figure 2.5. Scale Model Layout of the HV-S2 (HLW-C5V) Model

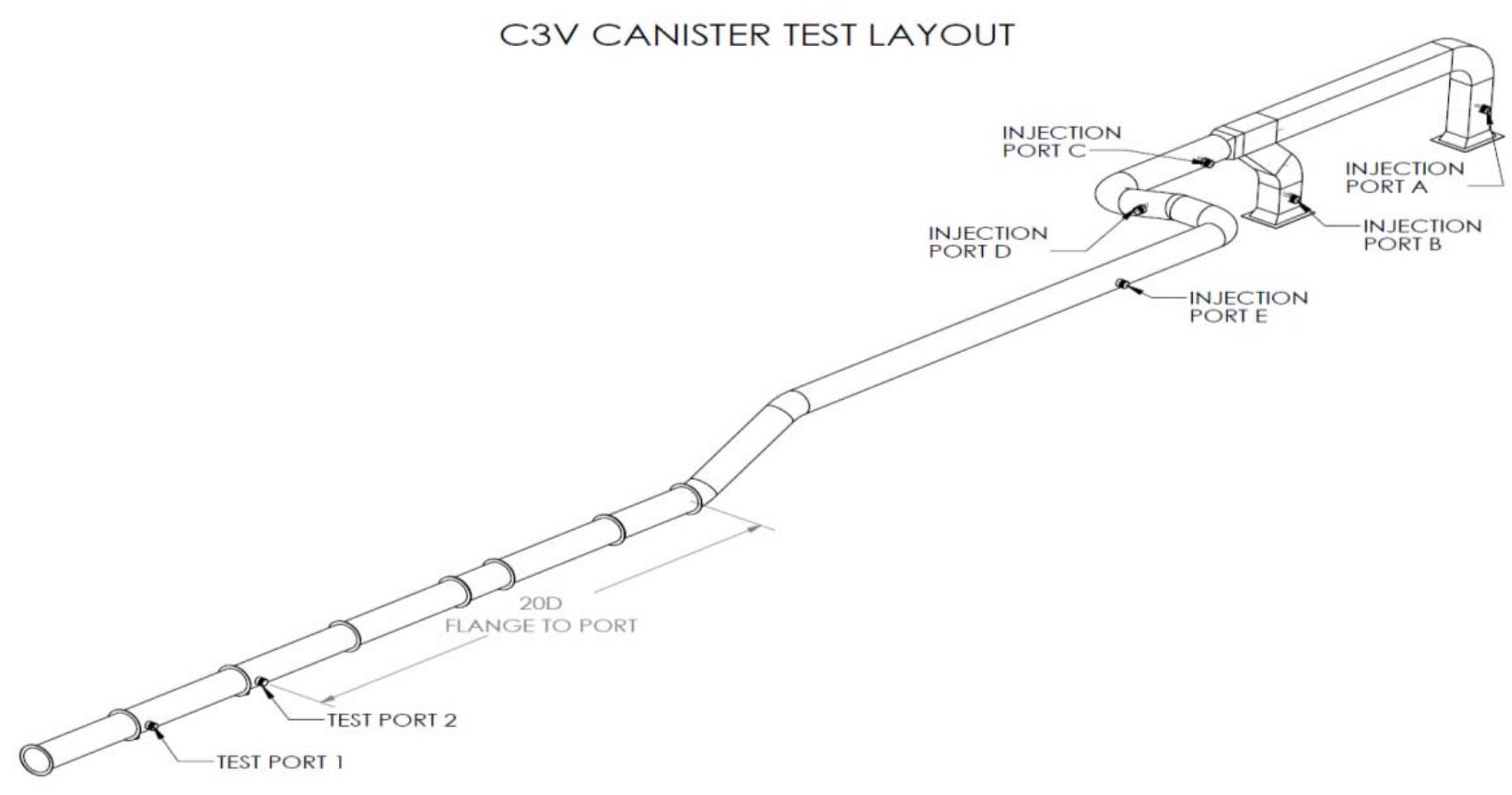

Figure 2.6. Scale Model Layout of the IHLW-S1 (IHLW-C3V-Canister) Model 


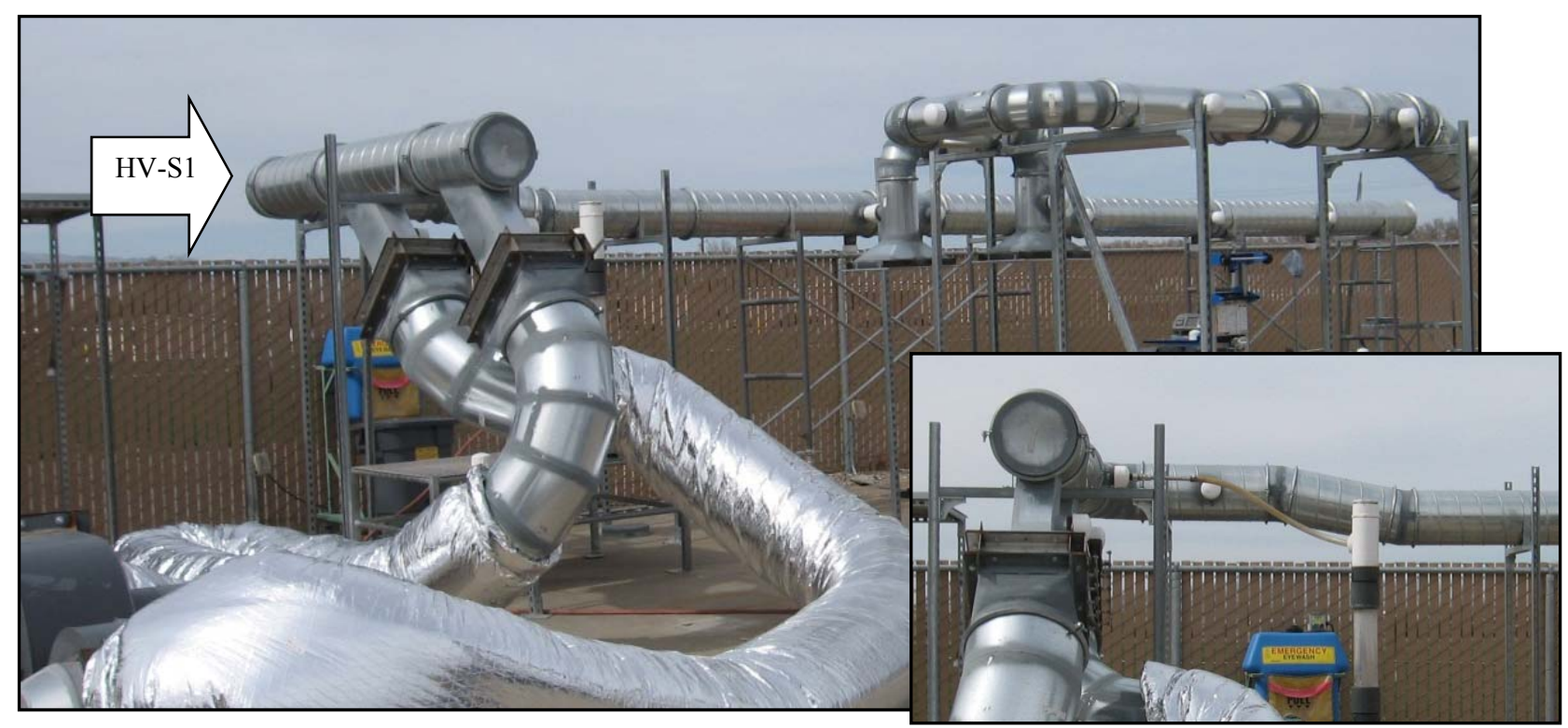

Figure 2.7. Photographs of the HV-S1 Test System

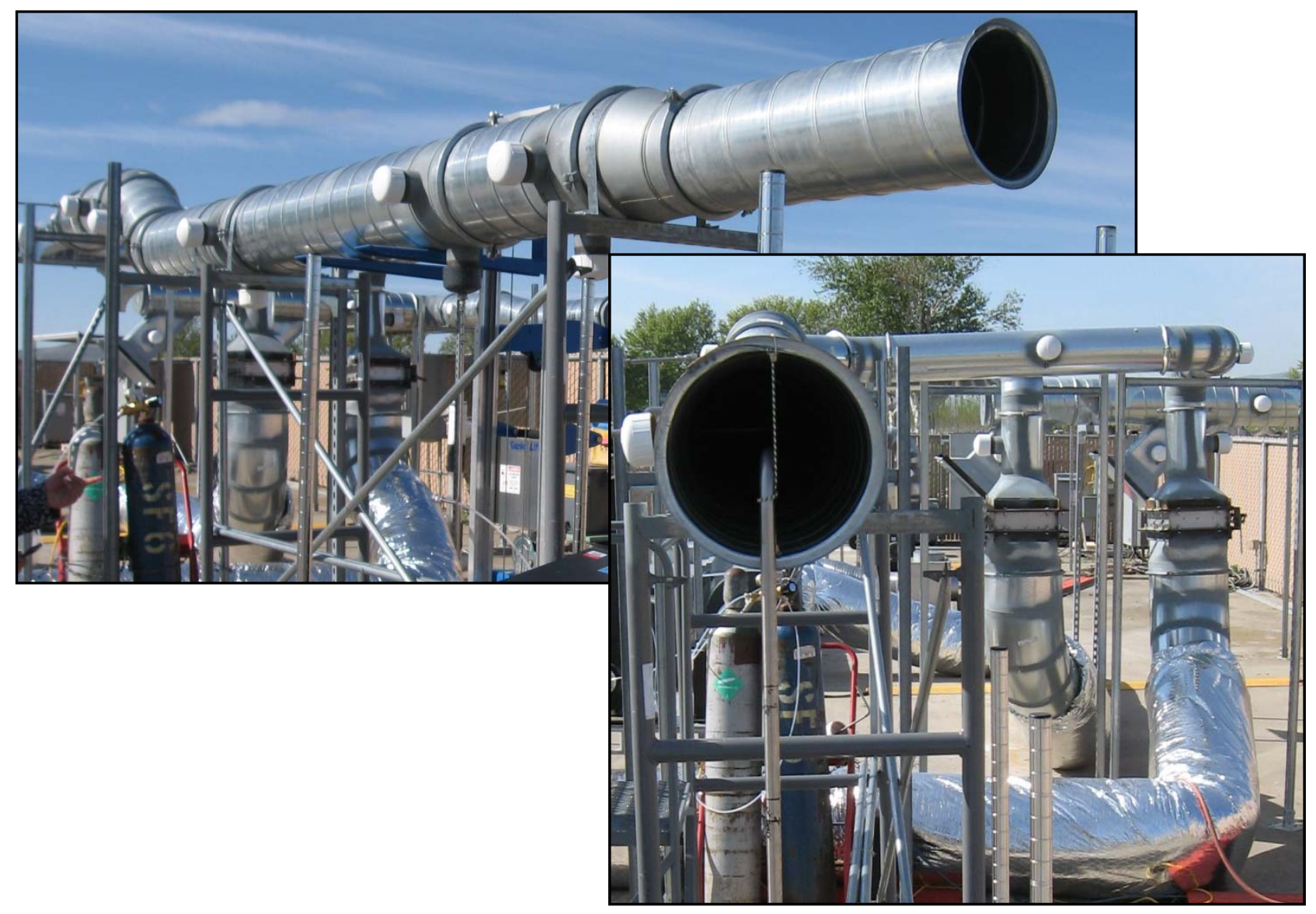

Figure 2.8. Photographs of the HV-S2 Test System 


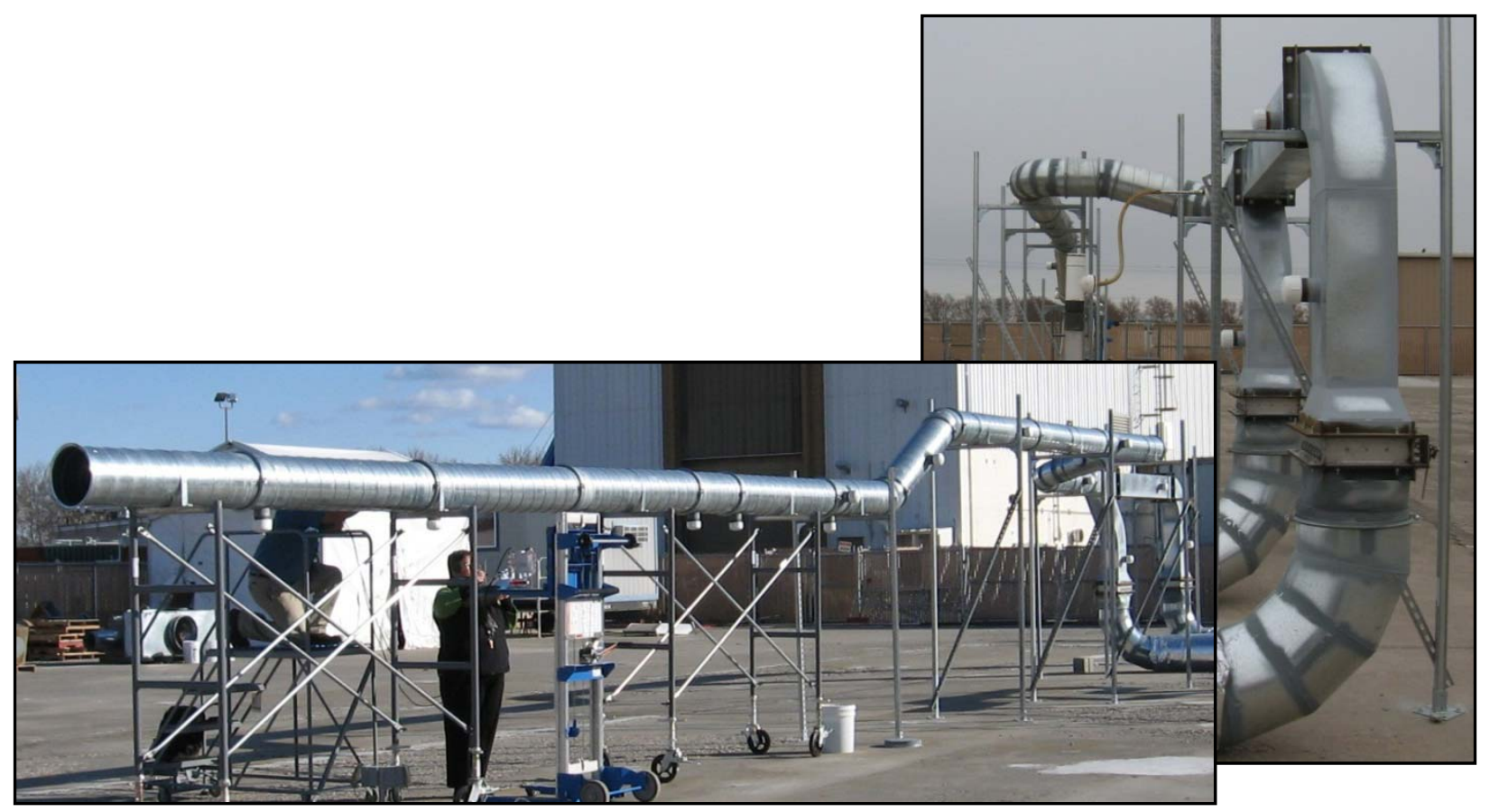

Figure 2.9. Photographs of the IHLW-S1 Test System

Table 2.1. Scaling Factor for 12-in.-Diameter Scale Model Stack

\begin{tabular}{lcc}
\hline & Actual Diameter & Scaling Factor \\
\hline HV-S1 & $60 \mathrm{in}$. & 5.00 \\
HV-S2 & $62 \mathrm{in.}$ & 5.17 \\
IHLW-S1 & $34 \mathrm{in.}$ & 2.83 \\
\hline
\end{tabular}

\subsection{Stack Flows}

Tests of scale model stacks were conducted at flow rates that bracket the range of expected normal and accident flow rates and operating configurations. Various combinations of flow rates and operating fans were tested. BNI provided normal, minimum, and maximum flow rates for each of the three systems tested in this group. Maximum flow rates are $125 \%$ of the normal flow rates, whereas minimum flow rates are $50 \%$ of normal.

Additional considerations come from the ANSI/HPS N13.1-1999 standard. The standard requires that the DV of the scale model be within a factor of six of the actual stack. For stacks with a circular cross section, this is equivalent to requiring that the ratio of flow rate to stack diameter be within a factor of six of the actual stack. The standard also requires that the Reynolds number for the prototype and model stacks must both exceed 10,000.

The WTP HV-S1 air exhaust system is equipped with two fans capable of 59,300 acfm (actual cubic feet per minute) flow each. The speed of both fans will be controlled with variable frequency drives to achieve the target flow rate. Only one fan will be operated at any given time, with the second fan in standby for use when maintenance needs arise on the primary fan. Each fan is equipped with an adjustable-speed drive to compensate for upstream filter loading and pressure variations. 
There are two fans available to power the WTP HV-S2 exhaust system, which exhausts air from the C5 ventilation system of the high-level waste facility. One fan will be operated at a time, and one will be on standby. Each fan is capable of providing the maximum flow rate of $63,750 \mathrm{acfm}$, and is equipped with an adjustable-speed drive to compensate for filter loading and pressure variations.

Two fans are available to power the IHLW-S1 exhaust system of the high-level waste facility, which exhausts air from the $\mathrm{C} 3$ ventilation system. Only one fan will be operated at a time, and one will be on standby. Each fan is capable of providing the maximum flow rate of 13,100 acfm. Each fan is equipped with an adjustable-speed drive to compensate for filter loading and pressure variations.

Table 2.2 lists the flow conditions for the actual stack as well as the scale model stack. The flow rates provided by BNI were in acfm and were converted to standard flow rates to account for temperature (see Appendix D). The minimum air flow (in standard feet per minute [scfm]) and air velocity (in standard feet per minute [sfpm]) to achieve the assumed minimum and maximum actual stack flow are listed. The tabulated values of flow and velocity in the "Scale Model Minimum" columns are the minimum scaled values that will meet the criterion listed in Section 1.1 that the DV product be within a factor of six of the prototype. The scale model Reynolds numbers are calculated for those minima. One of the qualification criteria listed in Section 1.1 was that the Reynolds number for both the actual and scale model stack must be greater than 10,000. Therefore, the Reynolds number for the actual and scale model stacks at the minimum and maximum flow rates are included in Table 2.2. The conditions prescribed for these scale model tests fulfill the criterion of a Reynolds number greater than 10,000.

Table 2.2. Summary of Flow Parameters for Scale Model Stacks

\begin{tabular}{|c|c|c|c|c|c|c|}
\hline \multirow[b]{2}{*}{ Fan(s)-Flow } & \multicolumn{2}{|c|}{ Air Flow (scfm) } & \multicolumn{2}{|c|}{ Air Velocity (sfpm) } & \multicolumn{2}{|c|}{ Reynolds Number } \\
\hline & $\begin{array}{l}\text { Actual } \\
\text { Stack }\end{array}$ & $\begin{array}{l}\text { Scale } \\
\text { Model } \\
\text { Minimum }\end{array}$ & $\begin{array}{l}\text { Actual } \\
\text { Stack }\end{array}$ & $\begin{array}{l}\text { Scale Model } \\
\text { Minimum }\end{array}$ & Actual Stack & $\begin{array}{l}\text { Scale Model } \\
\text { Minimum }\end{array}$ \\
\hline \multicolumn{7}{|l|}{$\mathrm{HV}-\mathrm{S} 1$} \\
\hline Single fan $-\max$ flow & 55627 & 1854 & 2833 & 2361 & $1.5 \mathrm{E}+06$ & $2.4 \mathrm{E}+05$ \\
\hline Single fan -norm flow & 45524 & 1517 & 2319 & 1932 & $1.2 \mathrm{E}+06$ & $2.0 \mathrm{E}+05$ \\
\hline Single fan $-\min$ flow & 24202 & 807 & 1233 & 1027 & $6.3 \mathrm{E}+05$ & $1.1 \mathrm{E}+05$ \\
\hline \multicolumn{7}{|l|}{ HV-S2 } \\
\hline Single fan $-\max$ flow & 53631 & 1730 & 2558 & 2203 & $1.4 \mathrm{E}+06$ & $2.6 \mathrm{E}+05$ \\
\hline Single fan -norm flow & 48096 & 1551 & 2294 & 1976 & $1.2 \mathrm{E}+06$ & $2.0 \mathrm{E}+05$ \\
\hline Single fan $-\min$ flow & 26040 & 840 & 1242 & 1070 & $6.6 \mathrm{E}+05$ & $1.1 \mathrm{E}+05$ \\
\hline \multicolumn{7}{|l|}{ IHLW-S1 } \\
\hline Single fan $-\max$ flow & 11198 & 659 & 1776 & 839 & $5.2 \mathrm{E}+05$ & $8.6 \mathrm{E}+04$ \\
\hline Single fan -norm flow & 9883 & 581 & 1567 & 740 & $4.6 \mathrm{E}+05$ & $7.6 \mathrm{E}+04$ \\
\hline Single fan $-\min$ flow & 5351 & 315 & 849 & 401 & $2.5 \mathrm{E}+05$ & $4.1 \mathrm{E}+04$ \\
\hline
\end{tabular}

Another qualification criterion listed in Section 1.1 pertains to the DV in the scale model relative to the stack. Table 2.3 lists the DV values for the stack as well as the DV values that are acceptable for the scale model. This minimum flow for the model is selected to be the lower boundary so the DV product is within a factor of six (i.e., one-sixth) of the DV product for the actual stack. Likewise, the maximum 
flow for the model is selected to be the upper boundary so the DV product is within a factor of six (i.e., six times) of the DV product for the actual stack.

Table 2.3. Summary of DV Values for Scale Model Stacks

\begin{tabular}{lccc}
\hline & \multicolumn{3}{c}{ DV } \\
\cline { 2 - 4 } \multicolumn{1}{c}{ System } & Predicted for Actual & \multicolumn{2}{c}{ Acceptable Range for Scale Model } \\
\cline { 2 - 4 } & Stack & Minimum & Maximum \\
\hline HV-S1 & $1.42 \mathrm{E}+04$ & $2.36 \mathrm{E}+03$ & $8.50 \mathrm{E}+04$ \\
HV-S2 & $1.32 \mathrm{E}+04$ & $2.20 \mathrm{E}+03$ & $7.93 \mathrm{E}+04$ \\
IHLW-S1 & $5.03 \mathrm{E}+03$ & $8.39 \mathrm{E}+02$ & $3.02 \mathrm{E}+04$ \\
\hline
\end{tabular}




\subsection{Testing Methods}

The testing methods were based on the requirements of ANSI/HPS N13.1-1999. A test plan, TP-WTPSP-032, Air Sampling Probe Location Tests for Waste Treatment Plant HV-S1, HV-S2, and IHLW-S-1 (Group 3-4) Air Exhaust Systems, was prepared by PNNL and approved by BNI. This plan referenced the use of PNNL procedures, which define how the test should be conducted in general. A test instruction (TI) was prepared for each test type and for each scale model stack. These TIs contain specific instructions pertaining to the tests that are not addressed in the general procedures. Such information includes the following:

- Layout of measurement points

- Location of tracer injection points

- List of equipment and instrumentation

- Safety requirements

- List of test runs

- Test description and measurement data sheets with hand entries

- Table of preliminary results.

Because the final data sheets and a description of the test methods are included in this report, the TIs are not included here. The QA program that is implemented for this project is described in Section 3.1 and a summary of the stack testing methods used for each of the four test types is presented in Section 3.2.

\subsection{Quality Assurance}

The PNNL QA program is based on the requirements defined in the U.S. Department of Energy Order 414.1D, Quality Assurance, and 10 CFR 830, Energy/Nuclear Safety Management, and Subpart A-Quality Assurance Requirements (a.k.a., the Quality Rule). PNNL has chosen to implement the following consensus standards in a graded approach:

- ASME NQA-1-2000, Quality Assurance Requirements for Nuclear Facility Applications, Part I, "Requirements for Quality Assurance Programs for Nuclear Facilities".

- ASME NQA-1-2000, Part II, Subpart 2.7, Quality Assurance Requirements for Computer Software for Nuclear Facility Applications.

- ASME NQA-1-2000, Part IV, Subpart 4.2, Graded Approach Application of Quality Assurance Requirements for Research and Development.

The procedures necessary to implement the requirements are documented through PNNL's "How Do I...?" (HDI), which is a system for managing the delivery of laboratory-level policies, requirements, and procedures.

The Waste Treatment Plant Support Program (WTPSP) implements an NQA-1-2000 QA program, using a graded approach presented in NQA-1-2000, Part IV, Subpart 4.2. The WTPSP Quality Assurance manual (QA-WTPSP-002) describes the technology life cycle stages under the WTPSP QA plan 
(QA-WTPSP-0001). The technology life cycle includes the progression of technology development, commercialization, and retirement in process phases of basic and applied research and development (R\&D), engineering and production, and operation until process completion. The life cycle is characterized by flexible and informal QA activities in basic research, which becomes more structured and formalized through the applied R\&D stages. The work described in this report has been completed under the QA Technology level of Developmental Work as the data will be used for applying air discharge permits.

- DEVELOPMENTAL WORK-Developmental work consists of research tasks moving toward technology commercialization. These tasks still require a degree of flexibility, and there is still a degree of uncertainty that exists in many cases. The role of quality on Developmental Work is to make sure that adequate controls exist to support movement into commercialization.

WTPSP addresses internal verification and validation activities by conducting an Independent Technical Review of the final data report in accordance with WTPSP's procedure QA-WTPSP-601, Document Preparation and Change. This review verifies that the reported results are traceable, that inferences and conclusions are soundly based, and the reported work satisfies the test plan objectives. Appendix E lists the reviewed test plan, test instructions, and calculation packages used for the tests documented in this report.

\subsection{Stack Tests}

The tests described in the following subsections were conducted under scale flow conditions between $50 \%$ and $125 \%$ of the design flow condition designed for each stack, which were listed in Table 2.2. The test matrix included with the test plan described the minimum number of tests that were planned for each stack. The actual number of tests typically differed from the test plan because tests were added to confirm results that had to be repeated.

Before conducting the tests to determine whether the four qualification criteria described in Section 1.1 were met for each stack, two other measurement sets were made. First, the major features of the stack were measured. The longitudinal distance from the fans to the bends, duct reducers, and ports were determined in addition to the duct diameter at each measurement port. The second set of measurements determined the fan frequency settings needed to achieve the desired flow rates. For these measurements, the location within the duct cross section that had velocity measurements closest to the mean velocity was determined for Port 2 . Then, velocity measurements were made at this single measurement point at 5-Hz increments in the fan frequency setting. By developing a frequency vs. velocity relationship for the scale model stack, the frequency setting needed to achieve the flow conditions could be pre-determined.

Measurements were made at specific locations within the duct for each of the four qualification criteria tests described in the following subsections. The number and distance between measurement points was based on the U.S. Environmental Protection Agency (EPA) procedure described in 40 CFR 60, Appendix A, Method 1, for circular stacks. For a 12- to 24-in. duct diameter, eight traverse points are required at the relative positions shown in Figure 3.1. Measurements also were made at the centerpoint. In lieu of making the two measurement points nearest to the walls at $3.2 \%$ of the duct diameter from the duct walls, the minimum distance from the wall was set to 0.5 in., as prescribed by EPA Method 1. The measurement point closest to the port was Point 1, while the point farthest from the port was Point 8. 


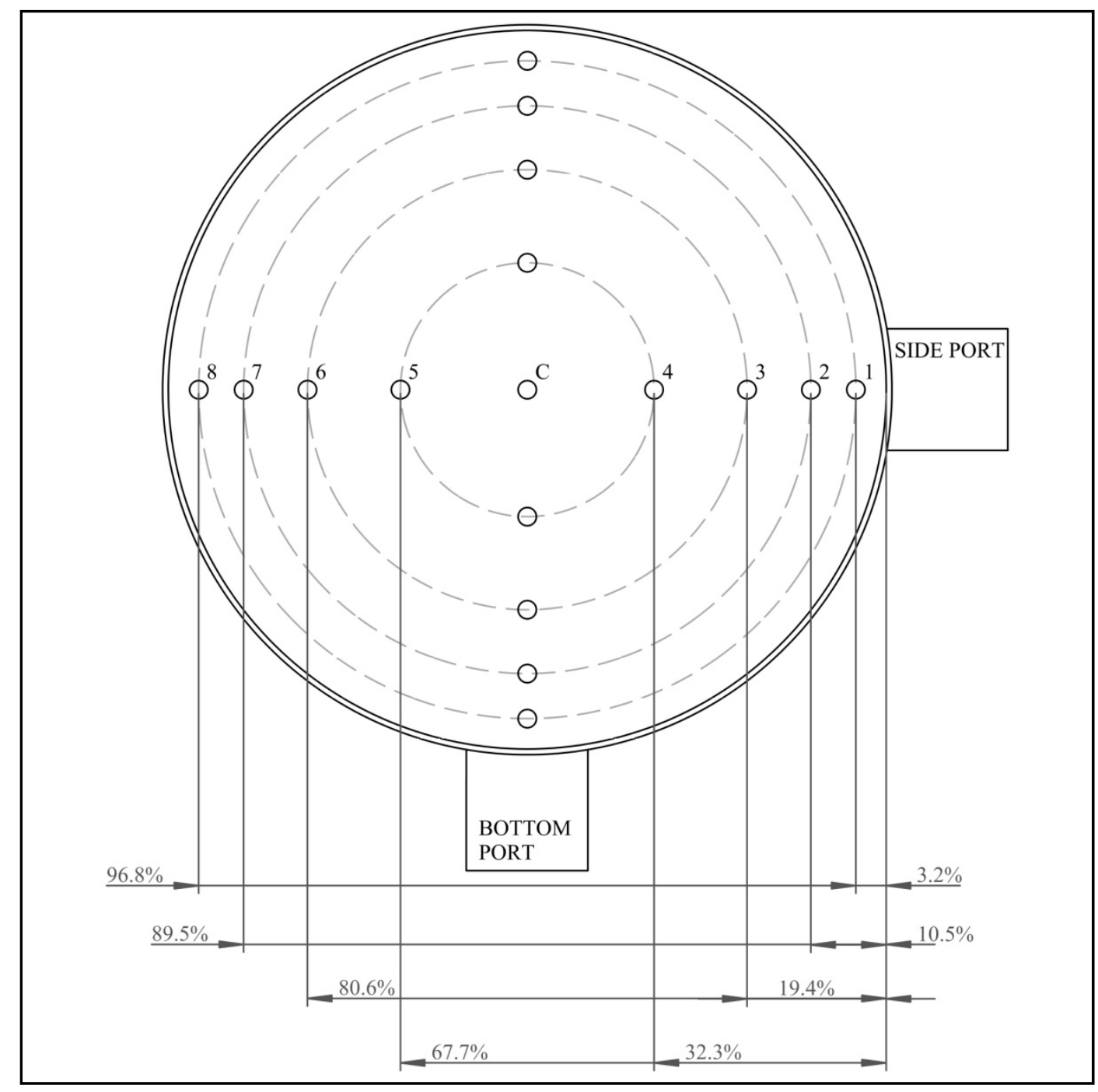

Figure 3.1. Cross Section of the Duct at the Testing Ports with Measurement Points

\subsubsection{Velocity Uniformity}

The uniformity of air velocity at the stack monitoring location indicates whether the momentum in the stack is well mixed. The method used to conduct the velocity uniformity tests was based on 40 CFR 60, Appendix A, Method 1. The velocity uniformity criterion is that the $\%$ COV should be less than $20 \%$ in the center two-thirds of the duct (measurement points 2 through 7 ).

For each run, three air velocity readings were obtained at each of the measurement points across the cross section of the duct. The measured velocity was the average of the three readings. The measured velocity for each point was used to determine the mean and standard deviation of the velocity across the cross-sectional plane. The \% COV (a.k.a., the percent relative standard deviation) was calculated as 100 times the standard deviation divided by the mean.

Air velocity measurements were made using a handheld thermal anemometer (TSI, Model 9545, Shoreview, Minnesota). Duct air temperature measurements also were made with the handheld thermal 
anemometer. The thermal anemometer reports velocity in standard feet per minute, with standard conditions defined as $1 \mathrm{~atm}$ and $70^{\circ} \mathrm{F}$. Figure 3.2 shows the thermal anemometer used for this test. The procedure EMS-JAG-04 and test instructions TI-RPP-WTP-676, TI-RPP-WTP-689, and TI-WTPSP-642 were followed to conduct this test for each of the three scale models.
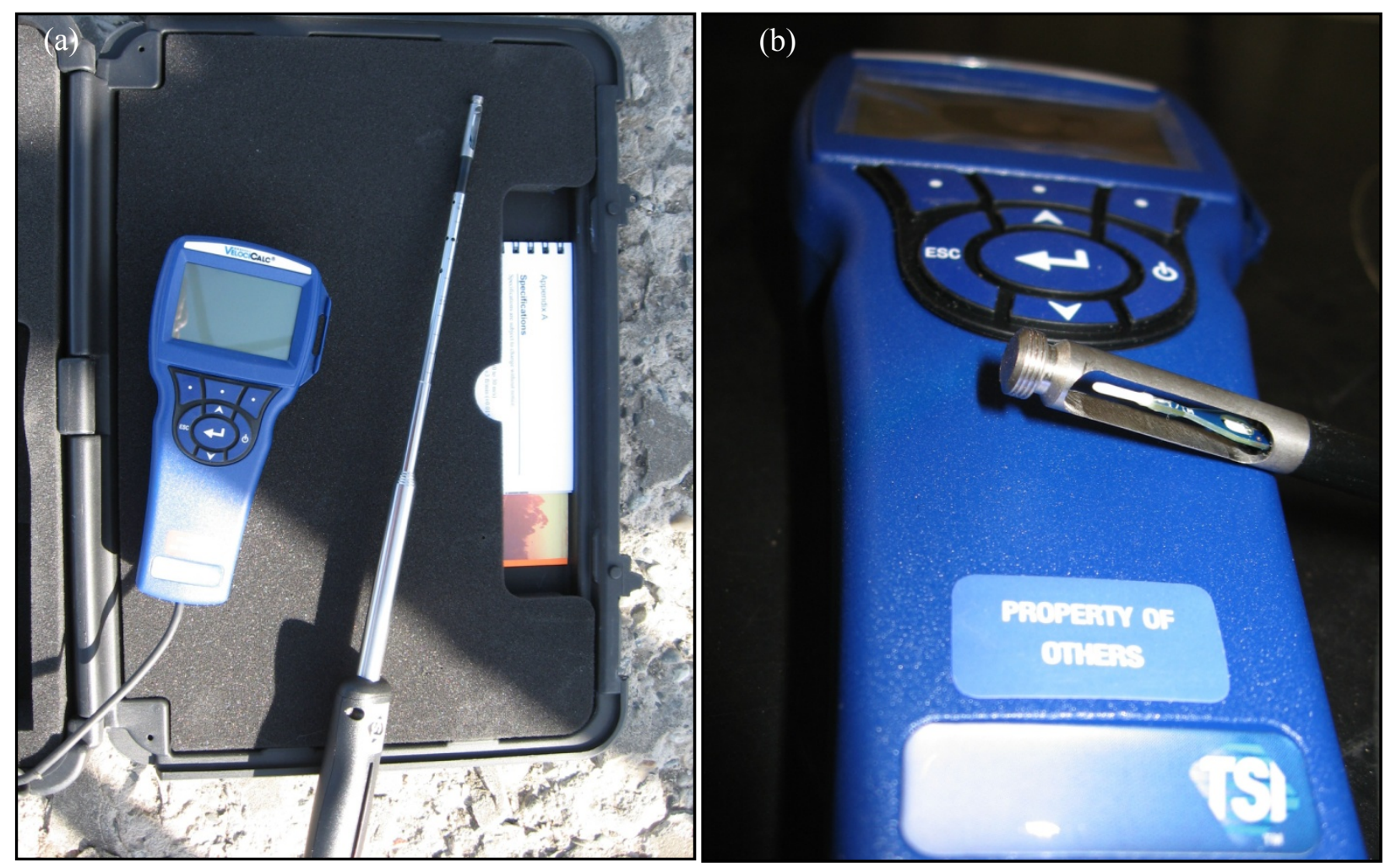

Figure 3.2. Equipment Used for the Velocity Uniformity Test: (a) Thermal Anemometer and (b) Close-Up View of Thermal Anemometer Probe Tip

\subsubsection{Flow Angle}

The air velocity vector approaching the sample nozzle should be aligned with the axis of the nozzle within an acceptable range so that the sample extraction performance is not degraded. The test method is based on 40 CFR 60, Appendix A, Method 1, Section 11.4, "Verification of the Absence of Cyclonic Flow." The term "flow angle" refers to the angle between the velocity vector of the flow in the duct and the axis of the sampling nozzle. For the stack testing activities, the flow angle was measured at a grid of nine points across two axes in a cross section of the duct (see Figure 3.1). The qualification criterion for the flow angle test is that the average angle should not exceed $20^{\circ}$.

The flow angle measurements were made using an S-type Pitot tube (Dwyer Instruments, 160S-36, Michigan City, Indiana) attached by flexible tubing to a slant-tube manometer (Dwyer Instruments, 400-5, Michigan City, Indiana) and an angle-indicating device attached to the sampling port as shown in Figure 3.3. For this test, the S-type pitot tube was rotated so that the planes of the two open ends of the two tubes are parallel to the long axis of the duct. The pitot tube is then rotated about its long axis until the differential pressure across the open ends of the tubes reads zero on the manometer. The rotation angle is read from the angle indicating device. The measured flow angle for each point is the average of 
the three readings. These measured values are used to calculate the mean absolute value of the flow angle across the duct. The procedure EMS-JAG-05 and test instructions TI-RPP-WTP-677, TI-RPP-WTP-689, and TI-WTPSP-018 were used to conduct this test for each of the three scale models.
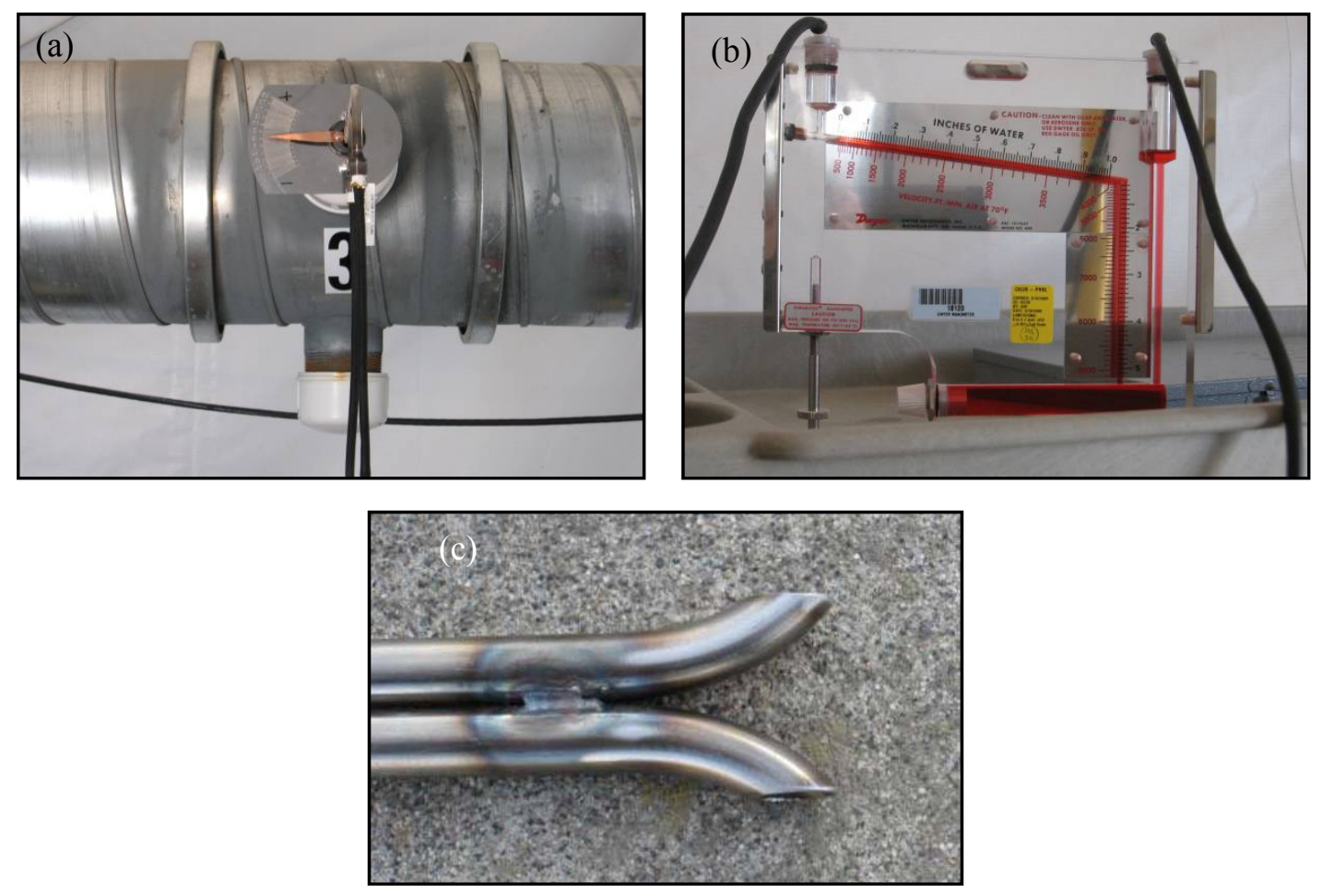

Figure 3.3. Equipment Used for the Flow Angle Test: (a) S-type Pitot Tube Inserted in a Measurement Port with the Protractor Plate, (b) Slant-Tube Manometer, and (c) Openings at Tip of S-Type Pitot Tube

\subsubsection{Gaseous Tracer Uniformity}

The gaseous contaminant concentration uniformity was demonstrated using the tracer gas sulfur hexafluoride $\left(\mathrm{SF}_{6}\right)$. A compressed gas cylinder and a flow controller were used to deliver a constant stream of $\mathrm{SF}_{6}$ into the duct. The gaseous tracer was typically injected into the duct at a point downstream of the fans. Figure 3.4 shows the injection locations with an injection probe positioned in the port. For separate test runs, the injection probe is positioned at one of five different locations in the duct cross section as illustrated in Figure 3.5. For some tests, just the centerline position is used. The remaining four injection locations are within a specified distance of the duct wall. For a nominally 12 -in.-diameter duct, the four "wall" injection locations were located within 2.4 in. of the wall.

For each test run, the tracer concentration was read three times at each of the measurement points across the duct. The measured concentration for each point is the average of the three readings. These measured concentrations are used to calculate the overall mean, standard deviation, and \%COV. These calculations also are performed just for the measurement points in the center two-thirds of the duct. The qualification criteria for the gaseous tracer test are that 1) the $\% \mathrm{COV}$ should be $\leq 20 \%$ within the center two-thirds of the duct and 2) the concentration at any measurement point should not deviate from the overall mean by more than $30 \%$. 

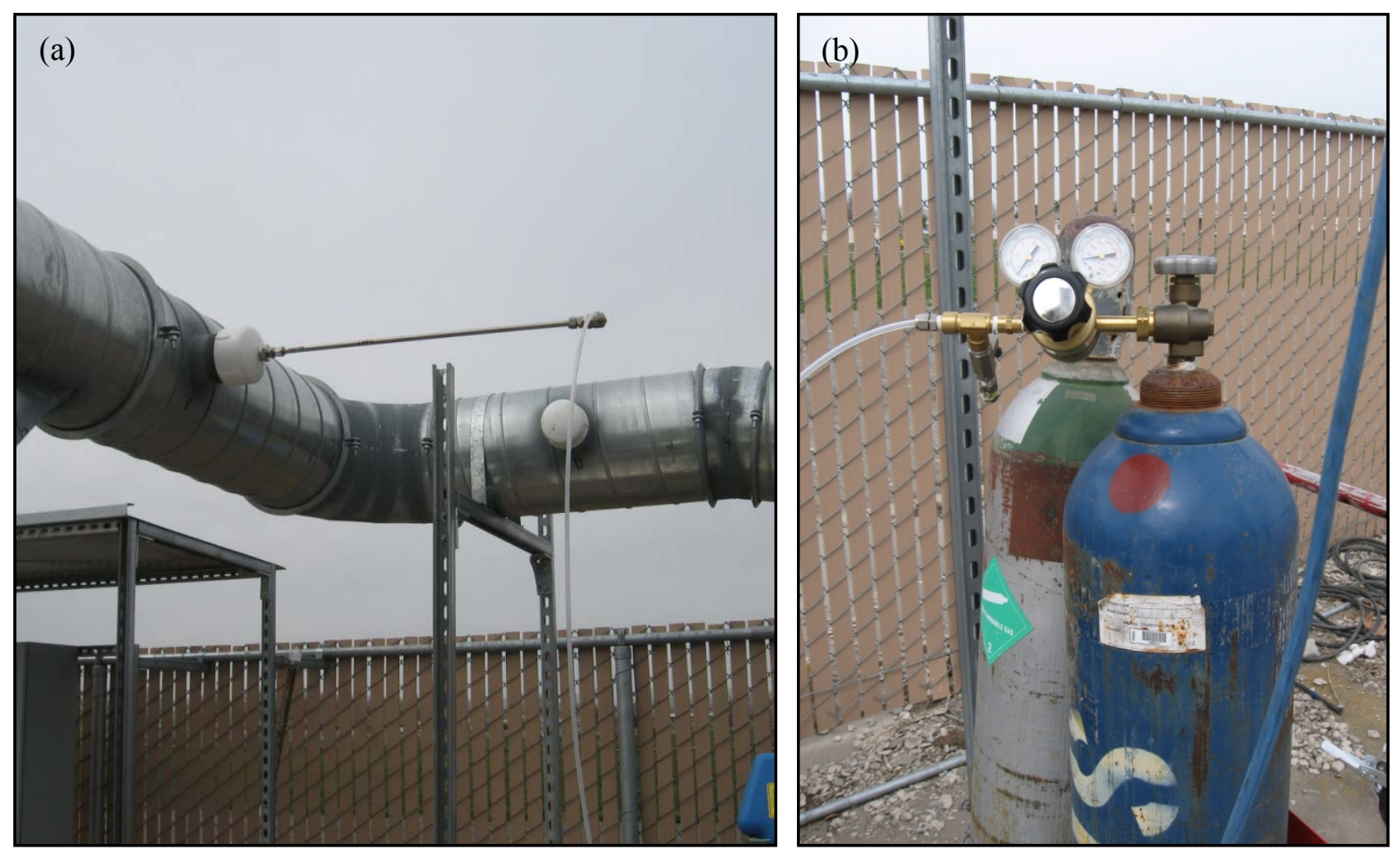

Figure 3.4. Equipment Used for the Gaseous Tracer Injection: (a) Injection Probe Installed in the HV-S1 Scale Model and (b) Cylinder of Pure $\mathrm{SF}_{6}$ with Regulator

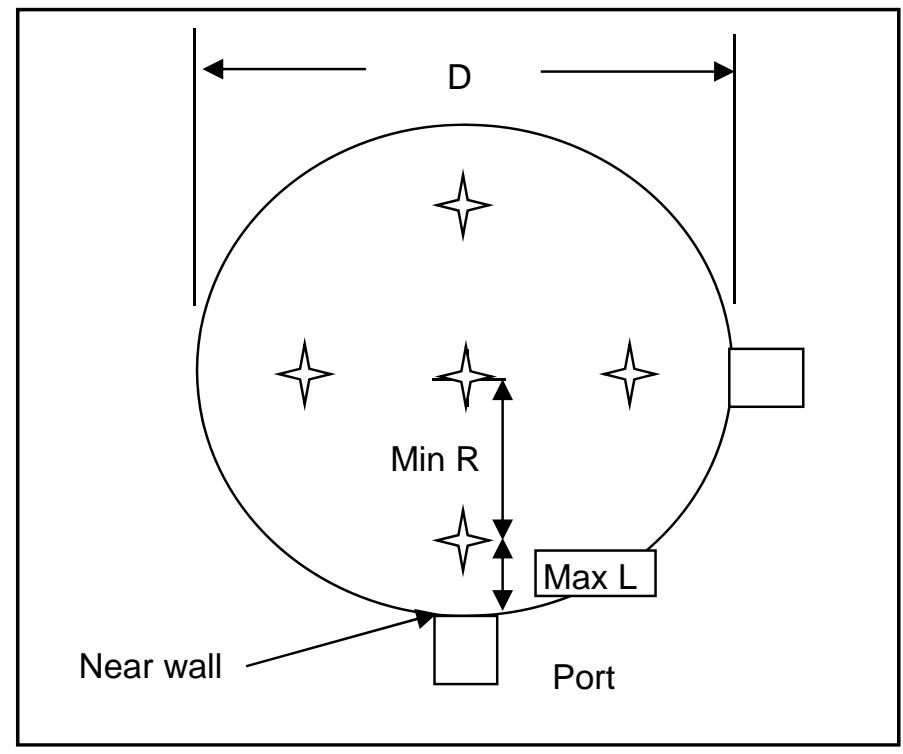

Figure 3.5. Illustration of Five Injection Points in a Circular Duct. Note: Max L is the maximum distance from the wall, which is $20 \%$ of the hydraulic diameter. Therefore, Min R, the minimum radius from the duct center, is $80 \%$ of the hydraulic diameter. In the case of a round duct, the hydraulic diameter is equal to the physical diameter (D). 
A photoacoustic gas analyzer (Brüel \& Kjær, Model 1302, Ballerup, Denmark) was used to measure tracer gas concentrations. The concentration variation is the important result for this test, so calibration bias is not important in the test results. However, the analyzer response was checked with calibration standards before and after conducting the test series (as well as weekly during the test series) to verify an adequate instrument response. The response was considered acceptable if the concentration from the instrument was within $10 \%$ of the calibration standard.

A simple probe was used to extract the sample and deliver it to the gas analyzer. A small pump drew air from within the stack through the probe. The gas analyzers then sampled the air from the sample line for analysis. Figure 3.6 shows the equipment setup for this test. The procedure EMS-JAG-01 and the test instructions TI-WTPSP-070, TI-WTPSP-078, and TI-WTPSP-084 were used to conduct this test for each of the three scale models.

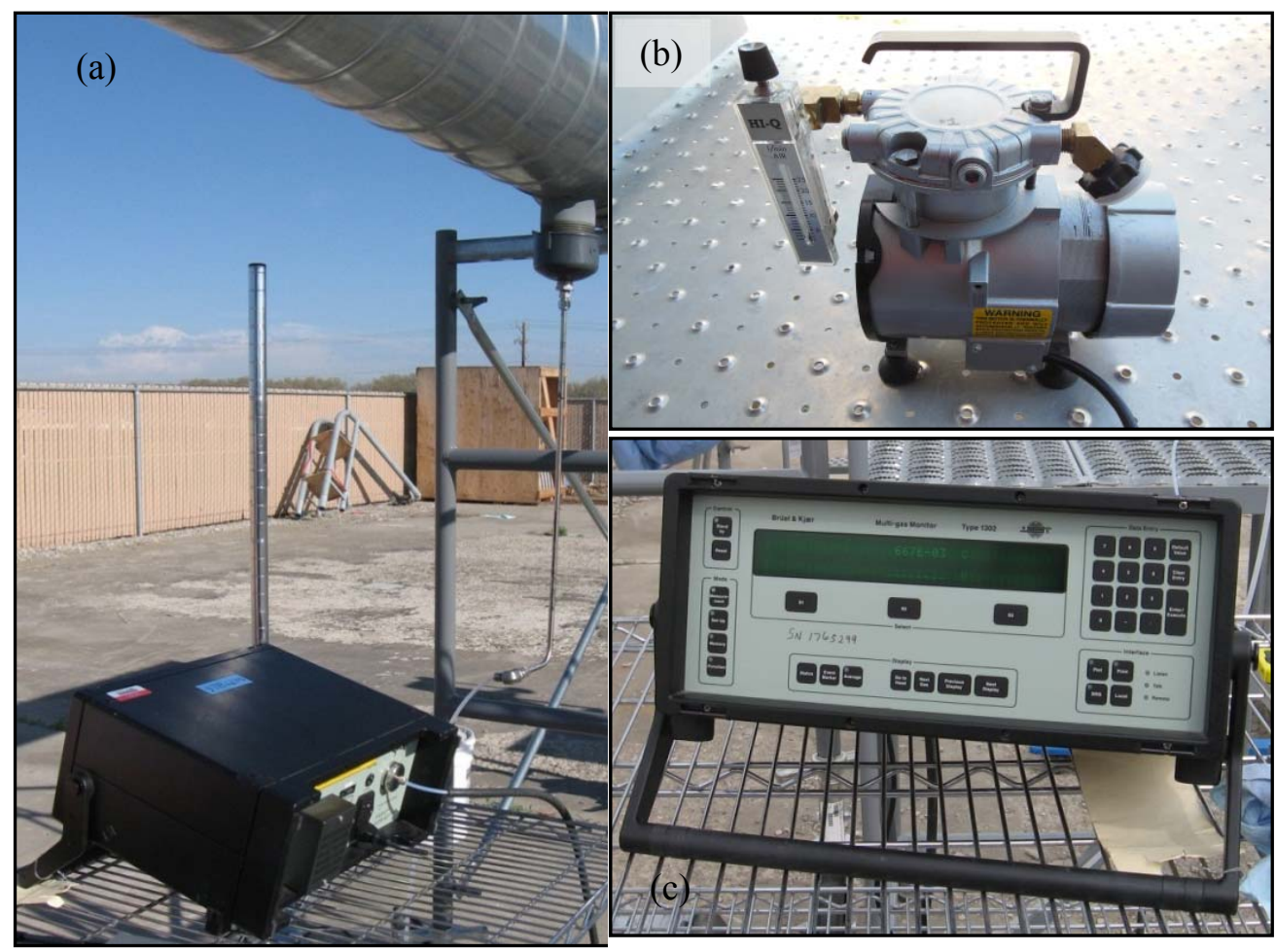

Figure 3.6. Equipment Used for the Gaseous Tracer Sampling: (a) Simple Sampling Probe Installed in a Port, (b) Sampling Pump, and (c) Gas Analyzer

\subsubsection{Particle Tracer Uniformity}

The uniformity of the particulate contaminant concentration was demonstrated using polydisperse pump oil particles as a particle tracer. Vacuum pump oil was drawn into a spray nozzle (driven by compressed air) housed in a plastic chamber. These aerosol particles were injected into the duct air at an injection point downstream of the fans as shown in Figure 3.7. Figure 3.7 shows the equipment setup for an aerosol injection in the IHLW-S1 scale model stack. The plastic chamber and spray nozzle assembly also is referred to as the aerosol generator. The aerosol was injected at the centerline of the duct, and this test was repeated to gain some sense of the variability of the results. 


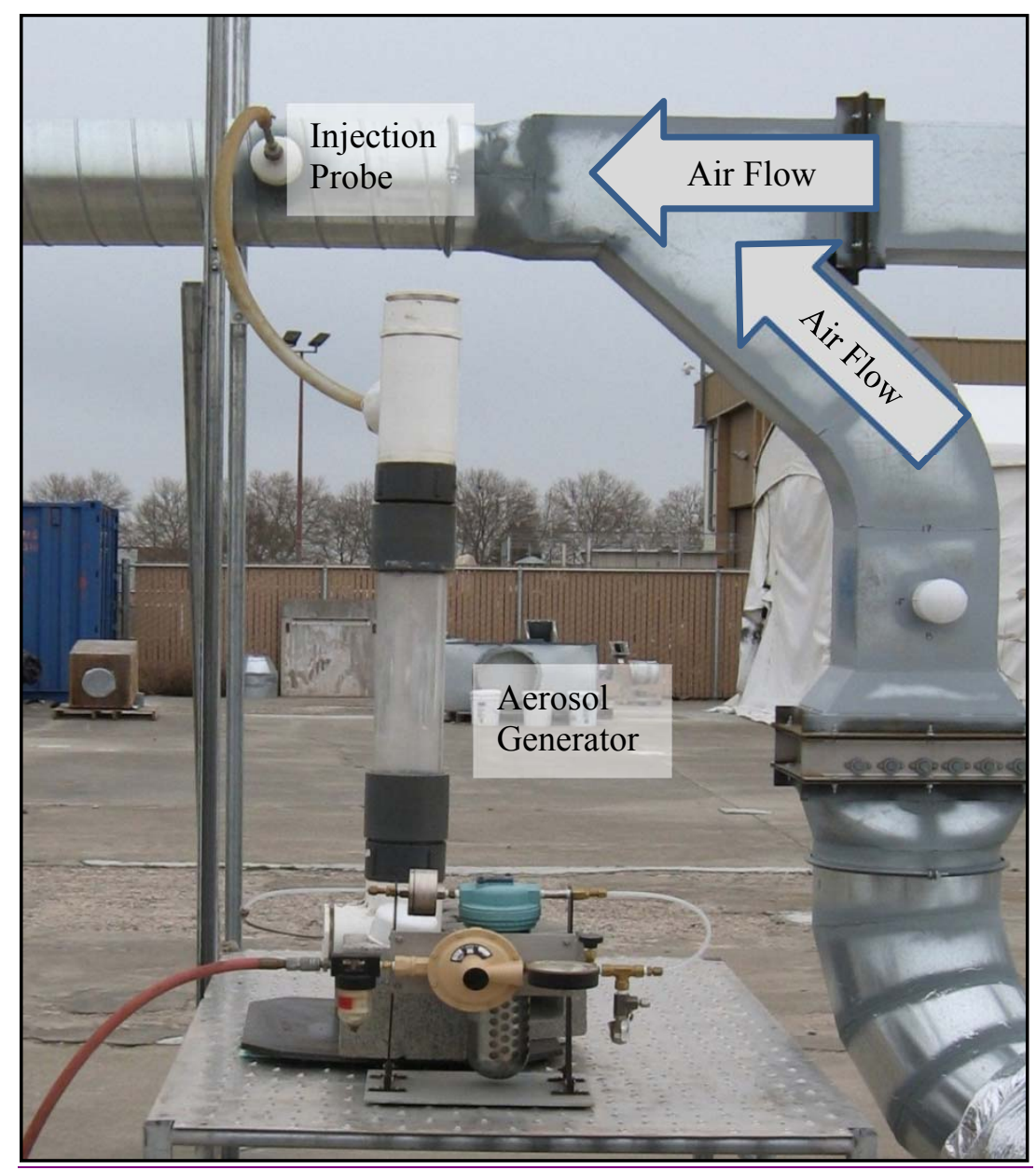

Figure 3.7. Equipment Used for Particle Injection (IHLW-S1)

The concentration of the particles is measured at the sampling grid points with a calibrated optical particle counter (OPC) (Hach, Met-One Model 3415, Loveland, Colorado). A simple probe was used to extract the sample and deliver it to the OPC. Figure 3.8 shows the sampling setup with the simple probe connected to the OPC. To identify potential inconsistencies in the aerosol output, tests were conducted with a reference instrument measuring the particle concentration at the centerpoint at a location downstream of the test port. During the first aerosol tests conducted in this group (PT-1 through PT-7 of IHLW-S1), the reference probe was slightly longer than the measurement probe, as shown in Figure 3.9. For consistency, a probe identical to the measurement probe was fabricated, and the majority of tests were conducted with probes of identical dimension for the measurement and reference locations. Figure 3.9 also shows the reference probe installed on the outlet of the HV-S2 stack, which does not have a port downstream of the measurement port. The OPC sorts the particles into six size channels. As mentioned in Section 1.1, the particles of interest have an $\mathrm{AD}$ of $10 \mu \mathrm{m}$. Therefore, only data in the 9- to 11- $\mu \mathrm{m}$ channel of the OPC were used.

The particle concentration was read three times at each of the measurement points across the cross section of the duct. The measured concentration for each point is the average of the three readings. From these measurements, the overall mean standard deviation, and \%COV were calculated for all of the points 
and also just for those within the center two-thirds of the duct. The qualification criterion for the particle tracer test is that the $\% \mathrm{COV}$ should be less than or equal to $20 \%$ within the center two-thirds of the duct. The procedure EMS-JAG-02 and test instructions TI-RPP-WTP-679, TI-RPP-WTP-691, and TI-WTPSP-021 were used to conduct this test for each of the three scale models.
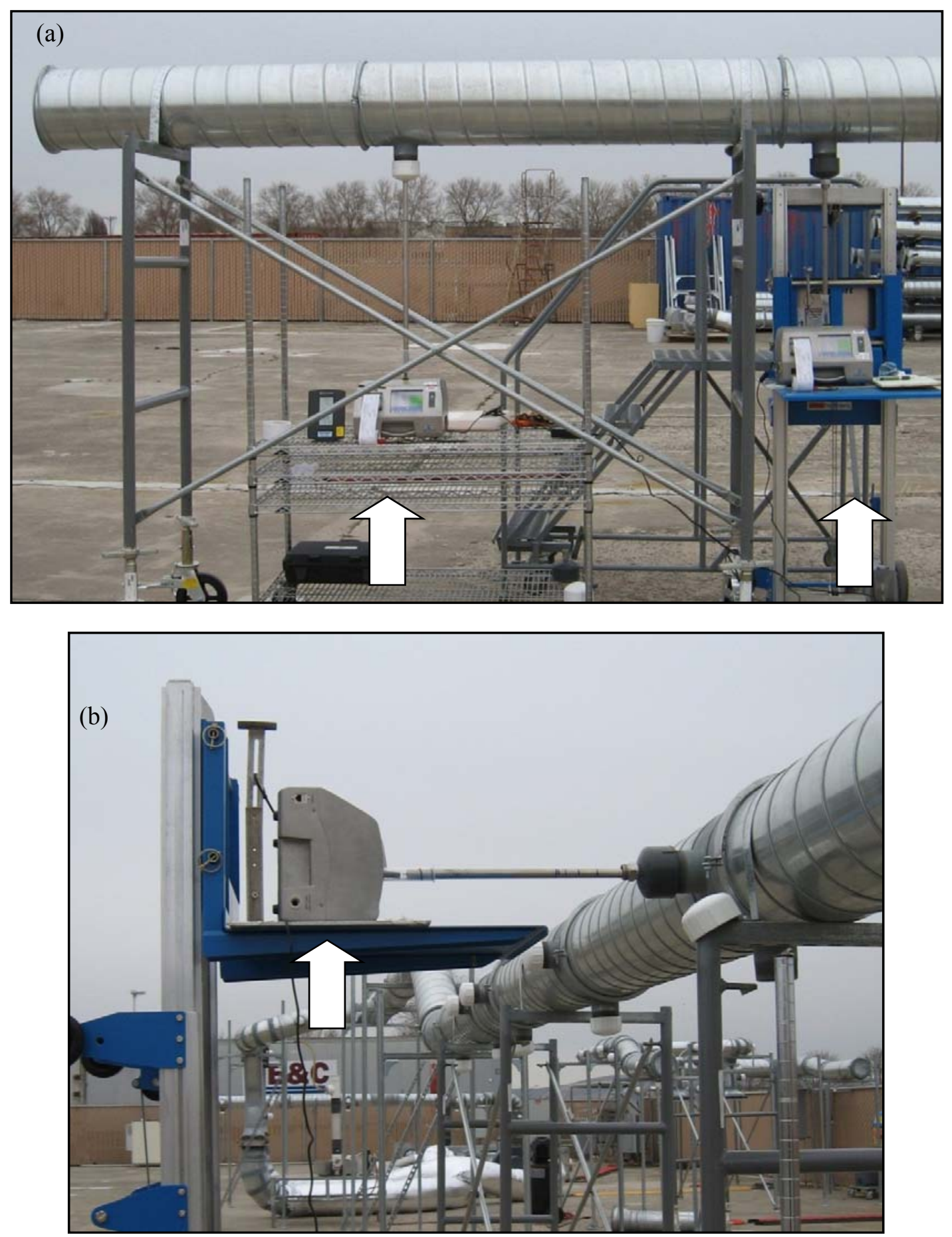

Figure 3.8. Particle Counters Used for the Particle Sampling: (a) Optical Particle Counters for Reference and Measurement Data in Bottom Ports and (b) Optical Particle Counter for Measurement Data in Side Port 

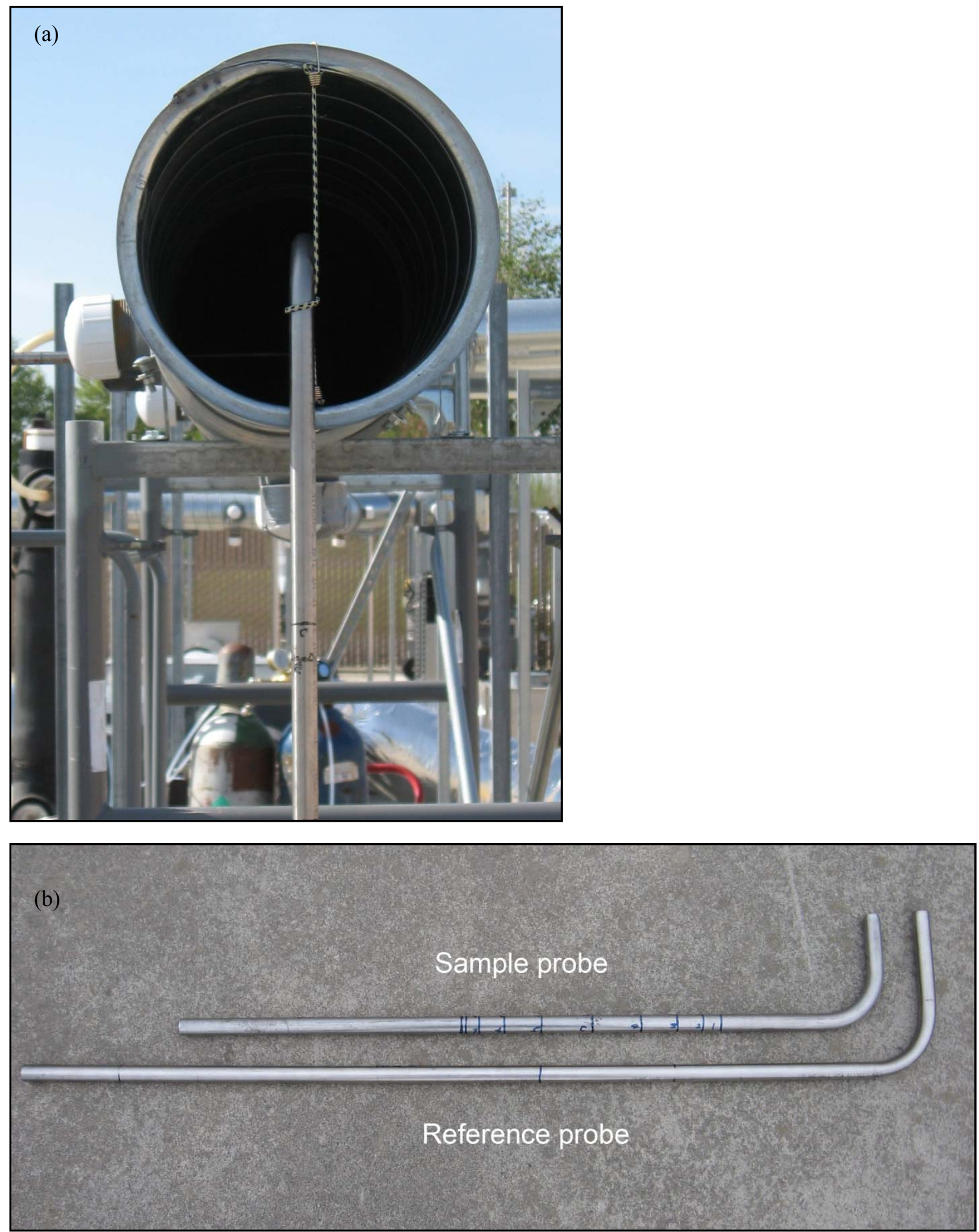

Figure 3.9. Probes Used for the Particle Sampling: (a) Reference Probe Installed in Outlet of HV-S2 Scale Model and (b) Sample and Reference Probe used in Initial Particle Tests. Subsequent tests used two probes identical to the sample probe shown here. 


\subsection{Stack Testing Results}

This section summarizes the results of the stack testing activities for the three scale model stacks in Group 3-4 (HV-S1, HV-S2, and IHLW-S1). The primary, reportable results are the data and data calculations to confirm that the requirements of the ANSI/HPS N13.1-1999 standard have been met. Independent reviews were performed to verify the data transcription and calculations. These calculations were performed using Microsoft Excel $(2007,2010)$ and documented in computer-assisted calculation packages (CCPs) in accordance with WTPSP procedures. The final data sheets are included in Appendices A through C. Appendix E contains a list of supporting documentation (such as the test plan and test instructions) used with this scale model test group. Each of the Group 3-4 scale model stacks underwent a series of velocity uniformity tests (designated VT), flow angle tests (designated FA), gas tracer tests (designated GT) and particle tracer tests (designated PT). Tables summarizing the results of tests for each scale model are presented in subsections of this chapter. During some tests the, scale model velocity values were higher than the actual stack flow for the conditions the test was meant to represent. This is acceptable because the DV value was still within a factor of six of the stack design values, and the facility flow conditions are estimates and may vary significantly from the design conditions at times for a variety of reasons.

\subsection{HV-S1 Stack Results}

Data tables, data plots, summary tables of the data for Test Ports 1 and 2 for HV-S1 flow angle, velocity, gas tracer, and particle tracer test results are presented in the following subsections. Some test combinations were repeated (i.e., performed more than once at different times) to quantify the testing and measurement uncertainty.

\subsubsection{HV-S1 Velocity Uniformity}

The initial test to determine the fan frequency setting for the HV-S1 model is included in Appendix A, Subsection A.1. Table 4.1 lists the results for the velocity uniformity tests performed on the scale model HV-S1 stack. In all cases, the results were well within the criterion of $\%$ COV values $\leq 20 \%$. $\mathrm{COV}(\% \mathrm{COV})$ values were typically less than $5 \%$, although they ranged from 2.4 to $6.6 \%$. The velocity through the stack ranged from 1136 to $2782 \mathrm{sfpm}$ (883 to $2162 \mathrm{scfm})$ with one fan operating.

Table 2.2 lists the desired range of minimum scale model flow rates as 1027 to $2361 \mathrm{sfpm}$ (807 to $1854 \mathrm{scfm})$. The desired testing conditions were between the minimum scale model flow rate and the actual stack velocity. The scale model test conditions meet both the Reynolds number and DV criteria required to represent the actual stack with one operating fan. The completed data sheets from these tests are available in Appendix A.2. 
Table 4.1. Summary of HV-S1 Velocity Uniformity Tests

\begin{tabular}{|c|c|c|c|c|c|c|}
\hline $\begin{array}{c}\text { Operating } \\
\text { Fan(s) }\end{array}$ & Test Port & $\begin{array}{c}\text { Flow } \\
\text { Condition }\end{array}$ & Run No. & $\begin{array}{l}\text { Flow } \\
\text { (scfm) }\end{array}$ & $\begin{array}{l}\text { Velocity } \\
\text { (sfpm) }\end{array}$ & $\% \mathrm{COV}$ \\
\hline \multirow{6}{*}{ A } & \multirow{6}{*}{2} & \multirow{3}{*}{ Max } & VT-1 & 2009 & 2585 & 3.6 \\
\hline & & & VT-13 & 1939 & 2494 & 2.8 \\
\hline & & & VT-14 & 1963 & 2526 & 3.4 \\
\hline & & \multirow{2}{*}{ Norm } & VT-15 & 1787 & 2299 & 3.9 \\
\hline & & & VT-16 & 1778 & 2288 & 3.5 \\
\hline & & Min & VT-2 & 883 & 1136 & 4.0 \\
\hline \multirow{11}{*}{ B } & 1 & Min & VT-12 & 911 & 1172 & 5.4 \\
\hline & \multirow{10}{*}{2} & \multirow{3}{*}{ Max } & VT-3 & 2105 & 2708 & 6.1 \\
\hline & & & VT-4 & 2128 & 2738 & 4.8 \\
\hline & & & VT-5 & 2162 & 2782 & 6.6 \\
\hline & & \multirow{4}{*}{ Norm } & VT-9 & 1900 & 2444 & 4.4 \\
\hline & & & VT-10 & 1952 & 2512 & 6.4 \\
\hline & & & VT-11 & 1881 & 2420 & 4.3 \\
\hline & & & VT-13 & 1939 & 2494 & 2.8 \\
\hline & & \multirow{3}{*}{ Min } & VT-6 & 894 & 1150 & 5.3 \\
\hline & & & VT-7 & 921 & 1184 & 5.1 \\
\hline & & & VT-8 & 940 & 1210 & 6.2 \\
\hline
\end{tabular}

Note: Individual or replicate sets of tests are alternately shaded and unshaded.

\subsubsection{HV-S1 Flow Angle}

Table 4.2 lists the results for the flow angle tests performed on the scale model HV-S1 stack. The results for all tests were well within the criterion of flow angle values $\leq 20^{\circ}$. Typical results were between $3^{\circ}$ and $5^{\circ}$. The completed data sheets from these two tests are available in Appendix A, Subsection A.3.

Table 4.2. Summary of HV-S1 Flow Angle Tests

\begin{tabular}{|c|c|c|c|c|c|}
\hline $\begin{array}{c}\text { Operating } \\
\text { Fan(s) } \\
\end{array}$ & $\begin{array}{l}\text { Test } \\
\text { Port } \\
\end{array}$ & Flow Condition & Run No. & $\begin{array}{c}\text { Approximate Air } \\
\text { Velocity (sfpm) }\end{array}$ & $\begin{array}{l}\text { Mean Absolute } \\
\text { Flow Angle }\left({ }^{\circ}\right) \\
\end{array}$ \\
\hline \multirow{2}{*}{ A } & \multirow{2}{*}{2} & Max & FA-1 & 2698 & 3.4 \\
\hline & & Min & FA-2 & 1141 & 3.1 \\
\hline \multirow{5}{*}{ B } & \multirow{4}{*}{2} & \multirow{2}{*}{ Max } & FA-3 & 2824 & 4.2 \\
\hline & & & FA-4 & 2881 & 3.4 \\
\hline & & Norm & FA-5 & 2427 & 4.7 \\
\hline & & Min & FA-6 & 1170 & 3.4 \\
\hline & 1 & Max & FA-7 & 2669 & 4.2 \\
\hline
\end{tabular}

Note: Individual or replicate sets of tests are alternately shaded and unshaded.

\subsubsection{HV-S1 Gaseous Tracer Uniformity}

During the gas tracer testing, the response of the gas analyzer was checked against calibration standards of appropriate concentrations, and the results met the requirements of the procedure. Table 4.3 lists the results for the gaseous tracer uniformity tests performed on the scale model HV-S1 stack for test Ports 1 and 2 and the tracer injection at Port C. In all cases, the tracer was well mixed, with results well 
within the criteria of $\% \mathrm{COV}$ values $\leq 20 \%$ and absolute values of maximum deviation $\leq 30 \%$. COV values were typically around $2 \%$, with maximum deviation values typically around $4 \%$. The "worst" mixing was observed for one of the bottom injection positions with the maximum velocity condition (GT-8). This test had nearly 5\% COV and 8\% maximum deviation from the mean concentration, which is still well within the bounds of the criteria. However, tests repeated at these conditions were nearly half of the GT-8 result, implying that GT-8 results may simply reveal the variability of the testing itself. Test results for GT-9 and GT-17 are not shown in Table 4.3 because the average velocity was well outside of the maximum flow conditions for the actual stack. The completed data sheets from these tests are available in Appendix A, Subsection A.4.

Table 4.3. Summary of HV-S1 Gas Tracer Uniformity Tests at Test Ports 1 and 2

\begin{tabular}{|c|c|c|c|c|c|c|c|}
\hline $\begin{array}{c}\text { Operating } \\
\text { Fan(s) }\end{array}$ & $\begin{array}{l}\text { Test } \\
\text { Port }\end{array}$ & $\begin{array}{c}\text { Flow } \\
\text { Condition }\end{array}$ & $\begin{array}{l}\text { Injection Port } \\
\& \text { Location }\end{array}$ & Run No. & $\begin{array}{l}\text { Avg Velocity } \\
\text { (sfpm) }\end{array}$ & $\% \mathrm{COV}$ & $\begin{array}{l}\text { Abs \% Max Dev } \\
\text { from Mean }\end{array}$ \\
\hline \multirow{3}{*}{ A } & \multirow{3}{*}{2} & Max & \multirow{3}{*}{ C Center } & GT-14 & 2543 & 1.3 & 3.4 \\
\hline & & Norm & & GT-1 & 2370 & 1.1 & 2.6 \\
\hline & & Min & & GT-2 & 1187 & 2.0 & 4.4 \\
\hline \multirow{12}{*}{ B } & \multirow{7}{*}{2} & \multirow{7}{*}{ Max } & C Center & GT-4 & 2965 & 1.7 & 3.5 \\
\hline & & & C Near & GT-5 & 2859 & 1.7 & 3.7 \\
\hline & & & C Far & GT-6 & 2900 & 2.3 & 4.6 \\
\hline & & & C Top & GT-7 & 3018 & 1.5 & 3.3 \\
\hline & & & \multirow{3}{*}{ C Bottom } & GT-8 & 2958 & 4.7 & 7.9 \\
\hline & & & & GT-15 & 2910 & 2.5 & 4.8 \\
\hline & & & & GT-16 & 3090 & 2.1 & 3.5 \\
\hline & \multirow{4}{*}{1} & \multirow{4}{*}{ Max } & C Center & GT-13 & 2863 & 3.2 & 5.7 \\
\hline & & & C Near & GT-11 & 3113 & 2.1 & 4.2 \\
\hline & & & C Far & GT-12 & 2811 & 1.3 & 5.5 \\
\hline & & & C Top & GT-10 & 3035 & 1.8 & 3.8 \\
\hline & 2 & Min & C Center & GT-3 & 1101 & 1.8 & 4.1 \\
\hline
\end{tabular}

Note: Individual or replicate sets of tests are alternately shaded and unshaded.

\subsubsection{HV-S1 Particle Tracer Uniformity}

Table 4.4 lists the results for the particle tracer uniformity tests performed on the scale model HV-S1 stack. Tests were conducted with the two fans running separately. The completed data sheets from these tests are available in Appendix A, Subsection A.5.

During some runs, the output of the aerosol generator varied with time. To observe the particle generator performance during a test run, a second OPC was set up to sample from the stack centerline, at an unused test port. Figure 4.1 shows the measurement data superimposed on the reference OPC data from run PT-3. In this case, the reference OPC shows a periodic variability in the aerosol generator output. Consequently, PT-3 results are not listed in Table 4.4. PT-3 was repeated as PT-5, and the normalized \%COV result for PT-5 was well within $20 \%$.

Previous testing has shown that the measured particle concentration was usually higher through the bottom port. A series of troubleshooting tests was unsuccessful in determining a consistent cause of this behavior. However, to mitigate errors, the concentration bias encountered between the two traverse 
directions at the measurement ports was removed by adjusting the data from the traverse with the lower concentration upward by a factor to match the concentrations at the center of the duct (the common point between the two traverses). These results were then termed "normalized."

The result of normalization is illustrated in Figure 4.2 for PT-6, the side traverse data were adjusted up by a factor of 1.28. For tests where there was a large discrepancy between the concentrations measured by the two traverses, the \%COV without normalization applied may exceed the qualification criterion. However, normalizing the data, helps meet the particle-tracer uniformity criterion of $\% \mathrm{COV} \leq 20 \%$. Table 4.4 shows the $\% \mathrm{COV}$ values both with and without normalization applied.

Table 4.4. Summary of HV-S1 Particle Tracer Uniformity Tests

\begin{tabular}{|c|c|c|c|c|c|c|c|}
\hline $\begin{array}{c}\text { Operating } \\
\text { Fan }\end{array}$ & $\begin{array}{c}\text { Injection Port \& } \\
\text { Location }\end{array}$ & $\begin{array}{l}\text { Test } \\
\text { Port }\end{array}$ & $\begin{array}{c}\text { Flow } \\
\text { Condition }\end{array}$ & $\begin{array}{l}\text { Run } \\
\text { No. }\end{array}$ & $\begin{array}{l}\text { Avg Velocity } \\
\text { (sfpm) }\end{array}$ & $\begin{array}{c}\text { Non- } \\
\text { Normalized } \\
\% \mathrm{COV}\end{array}$ & $\begin{array}{c}\text { Normalized } \\
\% \mathrm{COV}\end{array}$ \\
\hline \multirow{2}{*}{ A } & \multirow{2}{*}{ C Center } & \multirow{2}{*}{2} & Max & PT-5 & 2546 & 23.3 & 8.4 \\
\hline & & & Min & PT-4 & 1066 & 20.6 & 11.7 \\
\hline \multirow{5}{*}{ B } & \multirow{5}{*}{ C Center } & 1 & Max & PT-8 & 2933 & 25.1 & 14.8 \\
\hline & & \multirow{4}{*}{2} & Max & PT-1 & 2613 & 16.5 & 11.1 \\
\hline & & & Norm & PT-7 & 2396 & 17.0 & 9.5 \\
\hline & & & \multirow{2}{*}{ Min } & PT-2 & 931 & 12.7 & 4.4 \\
\hline & & & & PT-6 & 1298 & 13.5 & 4.6 \\
\hline
\end{tabular}

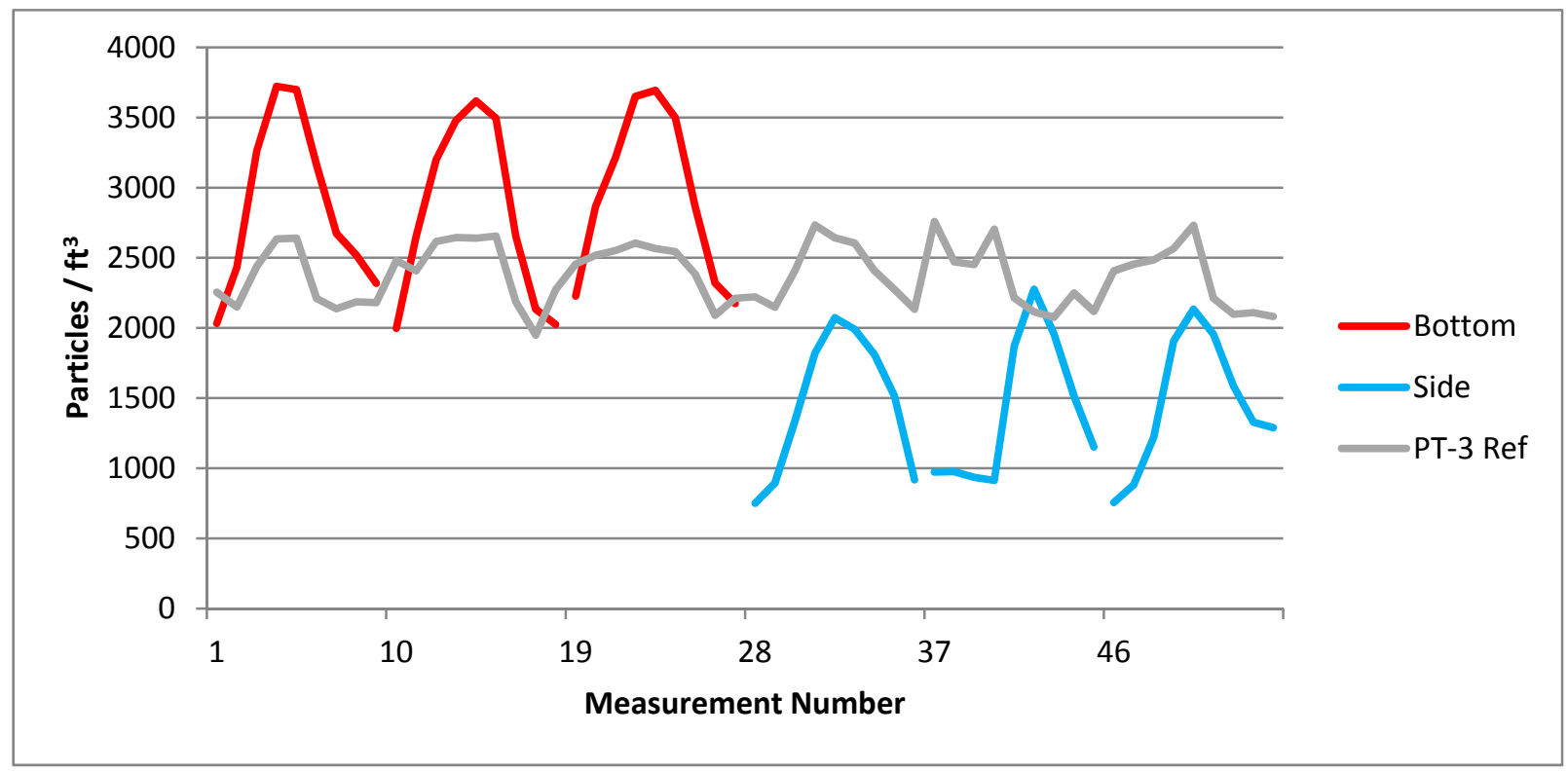

Figure 4.1. Measurement and Reference Particle Test Data from PT-3 on the HV-S1 Stack. Colored lines represent measurement traverses from the side and bottom ports, while the grey line represents the reference concentration at the centerpoint at a downstream location. This plot is an example of variability in the aerosol production and a systematic bias between the bottom and side port measurements. 


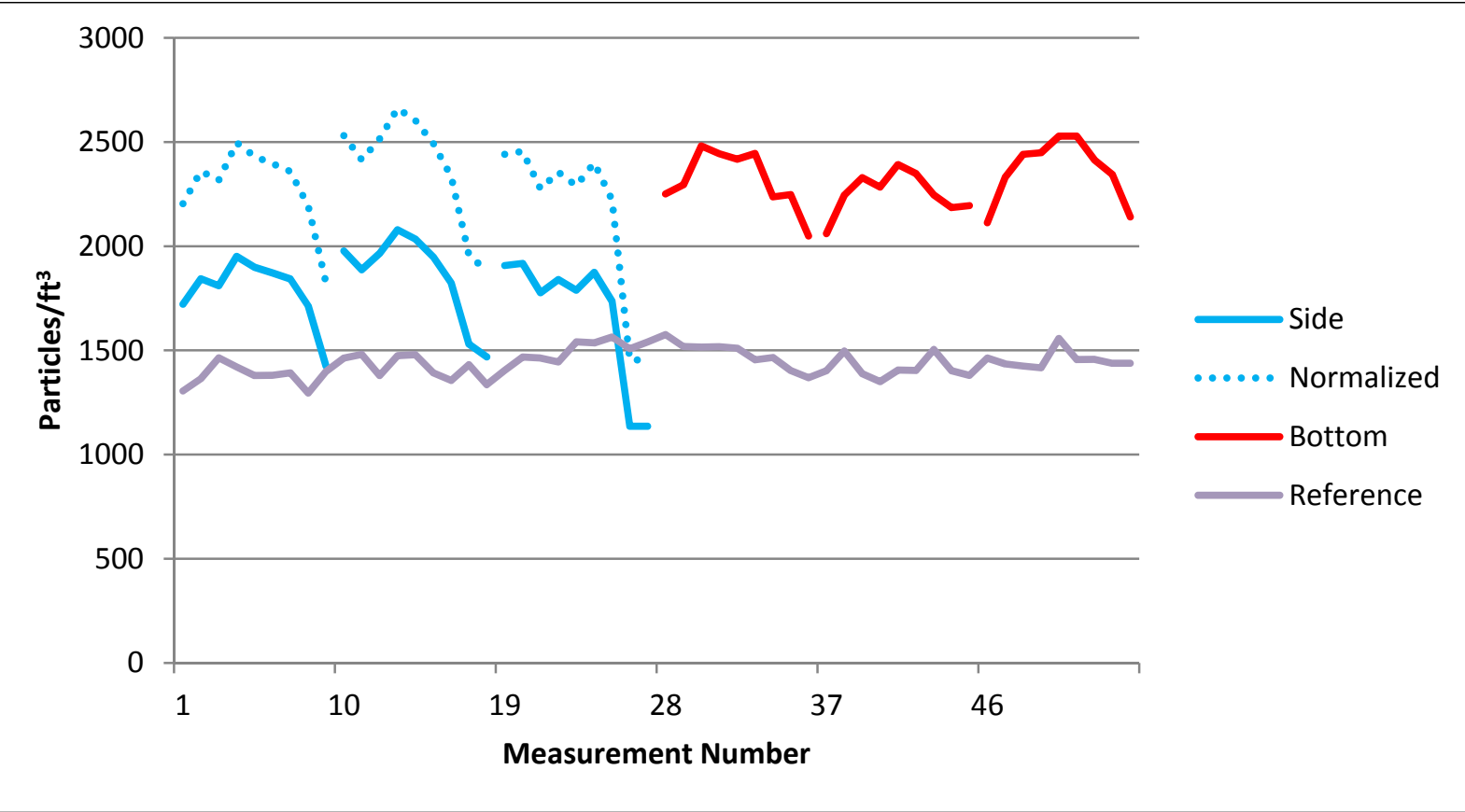

Figure 4.2. Measurement and Reference Particle Test Data from PT-6 on the HV-S1 Stack. Non-normalized data are shown with solid lines while normalized data are shown with dotted lines. Data collected from the side port have been adjusted up by a factor of 1.28.

\subsection{HV-S2 Stack Results}

Data tables, data plots, and summary tables of the data for Test Ports 1 and 2 for HV-S2 flow angle, velocity, gas tracer, and particle tracer test results are presented in the following subsections. Some test combinations were repeated (i.e., performed more than once at different times) to provide for quantifying the testing and response measurement uncertainty.

\subsubsection{HV-S2 Velocity Uniformity}

Table 4.5 lists the results for the velocity uniformity tests performed on the scale model HV-S2 stack. In all cases, the results were well within the criterion of $\% \mathrm{COV}$ values $>20 \%$. The velocity in the stack ranged from 1077 to $2877 \mathrm{sfpm}$ ( 837 to $2236 \mathrm{scfm}$ ). Table 2.2 lists the desired range of minimum scale model flow rates as 1047 to $2203 \mathrm{sfpm}$ ( 840 to $1730 \mathrm{scfm}$ ). The desired testing conditions were between the minimum scale model flow rate and the actual stack velocity. With these flow conditions, the scale model meets both the Reynolds number and DV criteria required to represent the actual stack. The completed data sheets from these tests are available in Appendix B, Subsection B.2. 
Table 4.5. Summary of HV-S2 Velocity Uniformity Tests

\begin{tabular}{|c|c|c|c|c|c|c|}
\hline $\begin{array}{c}\text { Operating } \\
\text { Fan }(s)\end{array}$ & $\begin{array}{l}\text { Test } \\
\text { Port }\end{array}$ & $\begin{array}{c}\text { Flow } \\
\text { Condition }\end{array}$ & Run No. & $\begin{array}{l}\text { Flow } \\
\text { (scfm) }\end{array}$ & $\begin{array}{l}\text { Velocity } \\
\text { (sfpm) }\end{array}$ & $\% \mathrm{COV}$ \\
\hline \multirow{4}{*}{ A } & \multirow{4}{*}{2} & Max & VT-10 & 2236 & 2877 & 2.5 \\
\hline & & Normal & VT-1 & 1810 & 2329 & 2.7 \\
\hline & & \multirow{2}{*}{ Min } & VT-3 & 838 & 1078 & 3.5 \\
\hline & & & VT-4 & 940 & 1210 & 3.5 \\
\hline \multirow{6}{*}{ B } & \multirow{6}{*}{2} & \multirow{2}{*}{ Max } & VT-2 & 2028 & 2609 & 4.7 \\
\hline & & & VT-9 & 2174 & 2796 & 4.5 \\
\hline & & \multirow{3}{*}{ Normal } & VT-6 & 1909 & 2456 & 7.8 \\
\hline & & & VT-7 & 1895 & 2437 & 6.2 \\
\hline & & & VT-8 & 1873 & 2409 & 4.7 \\
\hline & & Min & VT-5 & 837 & 1077 & 5.1 \\
\hline
\end{tabular}

Note: Individual and replicate sets of tests are alternately shaded and unshaded.

\subsubsection{HV-S2 Flow Angle}

Table 4.6 lists the results for the flow angle tests performed on the scale model HV-S2 stack. The results for all tests were well within the criterion of flow angle values $\leq 20^{\circ}$. Flow angles when Fan A was operating were markedly smaller than when Fan B was operating. Fan A flow angles ranged from $3.9^{\circ}$ to $7.1^{\circ}$, while Fan B flow angles range from $10.3^{\circ}$ to $12.5^{\circ}$. The geometry of the stack, in which the Fan B duct intersects the main stack ducting at a horizontal angle, followed immediately by a jog in the stack, is likely the source of the higher flow angles. The completed data sheets from these tests are available in Appendix B, Subsection B.3.

Table 4.6. Summary of HV-S2 Flow Angle Tests

\begin{tabular}{cccccc}
\hline $\begin{array}{c}\text { Test } \\
\text { Port }\end{array}$ & $\begin{array}{c}\text { Operating } \\
\text { Fan }\end{array}$ & $\begin{array}{c}\text { Flow } \\
\text { Condition }\end{array}$ & Run & $\begin{array}{c}\text { Approx. Air } \\
\text { Velocity (sfpm) }\end{array}$ & $\begin{array}{c}\text { Flow Angle } \\
\text { (Degrees) }\end{array}$ \\
\hline & Max & FA-5 & 3065 & 7.1 \\
\cline { 2 - 5 } & Norm & FA-2 & 2443 & 3.9 \\
\cline { 2 - 5 } & Min & FA-1 & 1063 & 6.1 \\
\cline { 3 - 5 } & \multirow{2}{*}{ Max } & FA-4 & 2627 & 11.0 \\
\cline { 3 - 5 } & & FA-8 & 3008 & 12.5 \\
\cline { 3 - 5 } & & Norm & FA-3 & 2471 & 10.3 \\
\cline { 3 - 5 } & \multirow{2}{*}{ Min } & FA-6 & 1084 & 11.5 \\
\cline { 3 - 5 } & & FA-7 & 1195 & 11.1 \\
\hline Note: Individual or replicate sets of tests are alternately shaded and unshaded.
\end{tabular}

Note: Individual or replicate sets of tests are alternately shaded and unshaded.

\subsubsection{HV-S2 Gaseous Tracer Uniformity}

During the gas tracer testing, the response of the gas analyzers was checked against calibration standards of appropriate concentrations, and the results met the requirements of the procedure. 
Table 4.7 lists the results for all of the gaseous tracer uniformity tests performed on the scale model HV-S2 stack. No contingency port was tested for this scale model; all tests were performed at Test Port 2.

In all cases, the tracer was well mixed, with results well within the qualification criteria of $\% \mathrm{COV}$ values less than $20 \%$ and absolute value of maximum deviation less than $30 \%$. COV values were typically less than $4 \%$, with maximum deviation values typically less than $10 \%$. The least amount of mixing was observed for the Fan A maximum flow conditions. For this condition, the COV ranged from $4.0 \%$ to $7.2 \%$, while the deviation from the mean ranged from $8.5 \%$ to $14.2 \%$. It is likely that Fan B conditions had increased mixing compared with similar Fan A conditions because of the geometry of the stack; the flow from Fan B enters the main stack from an angle, thus increasing the turbulence and mixing of the tracer near the injection point. The completed data sheets are available in Appendix B, Subsection B.4.

Table 4.7. Summary of HV-S2 Gas Tracer Uniformity Tests at Test Port 2

\begin{tabular}{|c|c|c|c|c|c|c|c|}
\hline Test Port & $\begin{array}{c}\text { Operating } \\
\text { Fan }\end{array}$ & $\begin{array}{c}\text { Flow } \\
\text { Condition } \\
(\%)\end{array}$ & $\begin{array}{l}\text { Injection } \\
\text { Port \& } \\
\text { Location }\end{array}$ & Run No. & $\begin{array}{c}\text { Avg } \\
\text { Velocity } \\
\text { (sfpm) }\end{array}$ & $\% \mathrm{COV}$ & $\begin{array}{l}\text { Absolute \% } \\
\text { Max. Dev. } \\
\text { from Mean }\end{array}$ \\
\hline \multirow{16}{*}{2} & \multirow{4}{*}{ A } & \multirow{4}{*}{ Max } & \multirow{4}{*}{ E Center } & GT-9 & 3103 & 7.2 & 14.2 \\
\hline & & & & GT-10 & 3052 & 5.0 & 11.9 \\
\hline & & & & GT-11 & 3060 & 4.2 & 10.5 \\
\hline & & & & GT-12 & 3094 & 4.0 & 8.5 \\
\hline & A & Min & E Center & GT-1 & 1084 & 4.0 & 12.5 \\
\hline & \multirow{9}{*}{ B } & \multirow{9}{*}{ Max } & E Center & GT-3 & 2651 & 2.2 & 4.6 \\
\hline & & & \multirow{2}{*}{ E Bottom } & GT-4 & 2573 & 3.5 & 5.9 \\
\hline & & & & GT-13 & 2934 & 4.5 & 7.2 \\
\hline & & & \multirow{2}{*}{ E Top } & GT-5 & 2602 & 3.4 & 6.7 \\
\hline & & & & GT-14 & 2999 & 3.1 & 6.0 \\
\hline & & & \multirow{2}{*}{ E Near } & GT-6 & 2660 & 2.8 & 6.1 \\
\hline & & & & GT-15 & 2973 & 2.7 & 5.2 \\
\hline & & & \multirow{2}{*}{ E Far } & GT-7 & 2661 & 3.0 & 7.2 \\
\hline & & & & GT-16 & 2943 & 1.7 & 5.4 \\
\hline & $\mathrm{B}$ & Norm & E Center & GT-8 & 2467 & 2.1 & 4.5 \\
\hline & $\mathrm{B}$ & Min & E Center & GT-2 & 1065 & 1.8 & 4.3 \\
\hline
\end{tabular}

Note: Individual or replicate sets of tests are alternately shaded and unshaded.

\subsubsection{HV-S2 Particle Tracer Uniformity}

Table 4.8 lists the normalized and non-normalized \%COV for each run at Test Port 2. In all cases, the uniformity criterion was met. As was the case for the gaseous tracer uniformity, the poorest mixing was observed under the Fan A Max condition. The \%COVs for this condition were $12.1 \%$ and $13.0 \%$. Other test conditions had COV values between 5.3 and 10.7\%. The completed data sheets from these tests are available in Appendix B, Subsection B.5. 
Table 4.8. Summary of HV-S2 Particle Tracer Uniformity Tests

\begin{tabular}{|c|c|c|c|c|c|c|c|}
\hline $\begin{array}{c}\text { Operating } \\
\text { Fan }\end{array}$ & $\begin{array}{c}\text { Injection } \\
\text { Port \& } \\
\text { Location } \\
\end{array}$ & $\begin{array}{l}\text { Test } \\
\text { Port }\end{array}$ & $\begin{array}{c}\text { Flow } \\
\text { Condition } \\
\end{array}$ & Run No. & $\begin{array}{c}\text { Avg } \\
\text { Velocity } \\
\text { (sfpm) }\end{array}$ & $\begin{array}{c}\text { Non- } \\
\text { Normalized } \\
\% \mathrm{COV} \\
\end{array}$ & $\begin{array}{c}\text { Normalized } \\
\% \mathrm{COV}\end{array}$ \\
\hline \multirow{3}{*}{ A } & \multirow{3}{*}{ E Center } & \multirow{3}{*}{2} & Max & PT-1 & 3069 & 15.5 & 13.0 \\
\hline & & & Norm & PT-8 & 2434 & 18.6 & 10.7 \\
\hline & & & Min & PT-4 & 1151 & 9.8 & 9.3 \\
\hline \multirow{4}{*}{ B } & \multirow{4}{*}{ E Center } & \multirow{4}{*}{2} & Max & PT-2 & 3073 & 18.0 & 8.7 \\
\hline & & & \multirow{2}{*}{ Norm } & PT-5 & 2413 & 25.3 & 10.0 \\
\hline & & & & PT-6 & 2403 & 34.9 & 6.1 \\
\hline & & & Min & PT-3 & 1034 & 18.4 & 5.3 \\
\hline
\end{tabular}

As was discussed for the HV-S1 scale model tests, the instrument response would change as it was moved between the bottom and side ports. Figure 4.3 shows the concentration profiles for the six traverses (trials) for PT-1. The concentration profile was clearly much lower for the side port as compared to the bottom port. The data were normalized as described in Section 4.1.4.

Although the aerosol generator was operated for 30 to $60 \mathrm{~min}$ prior to collecting measurements to allow aerosol output to stabilize, there were instances where the aerosol generator output changed during a run. For example, Figure 4.4 shows that the concentration declined by $50 \%$ during Run PT-7. The decline occurred during the side port measurements and then remained fairly constant for the bottom traverse. The PT-7 results have been omitted from Table 4.8 because of the decreasing aerosol output during the side traverses.

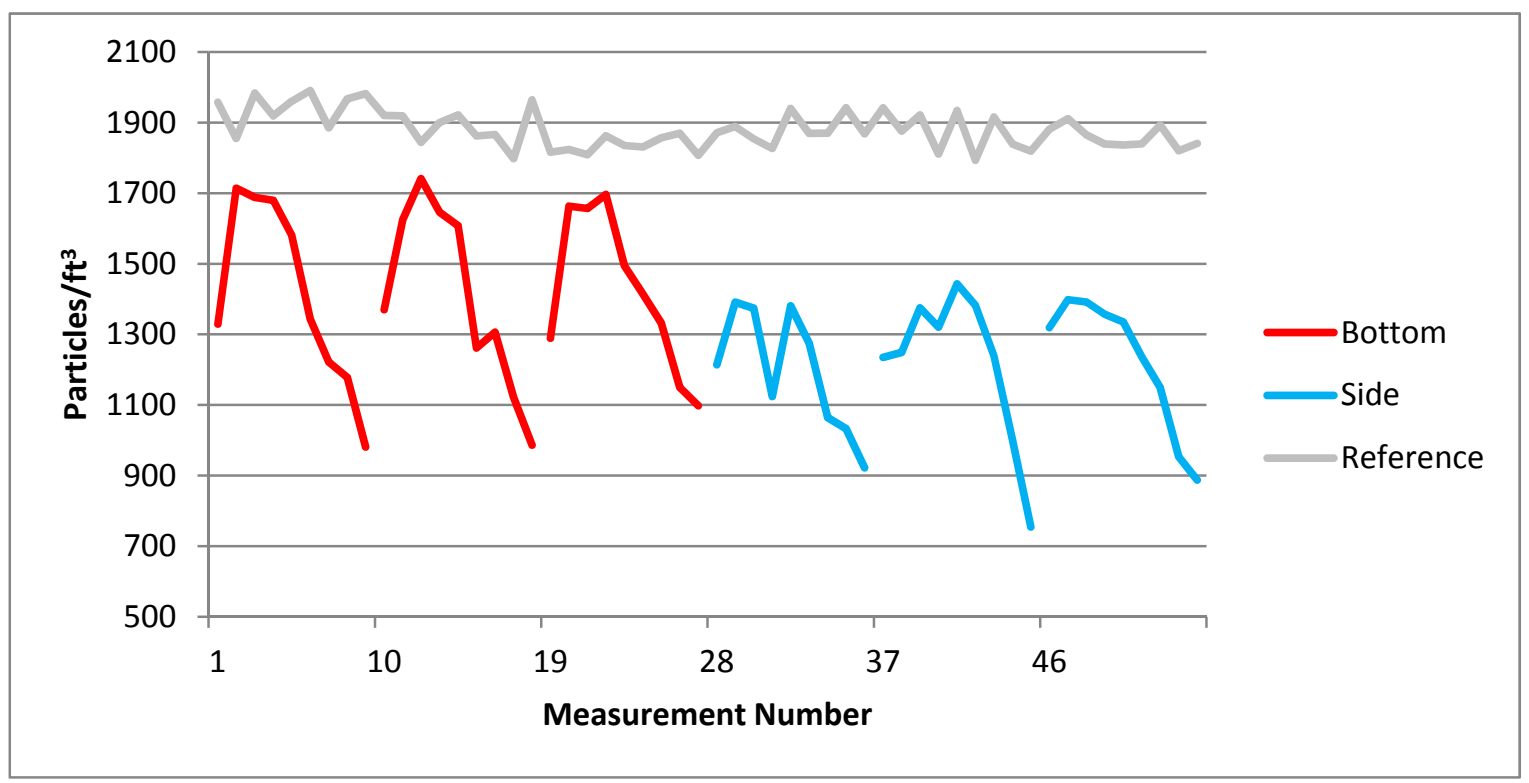

Figure 4.3. Measurement and Reference Particle Tracer Test Data from PT-1 of HV-S2. Colored lines represent measurement traverses from the side and bottom ports, while the grey line represents the reference concentration at the centerpoint at a downstream location. This plot is an example of very stable aerosol production and a small systematic bias between bottom and side port measurements. 


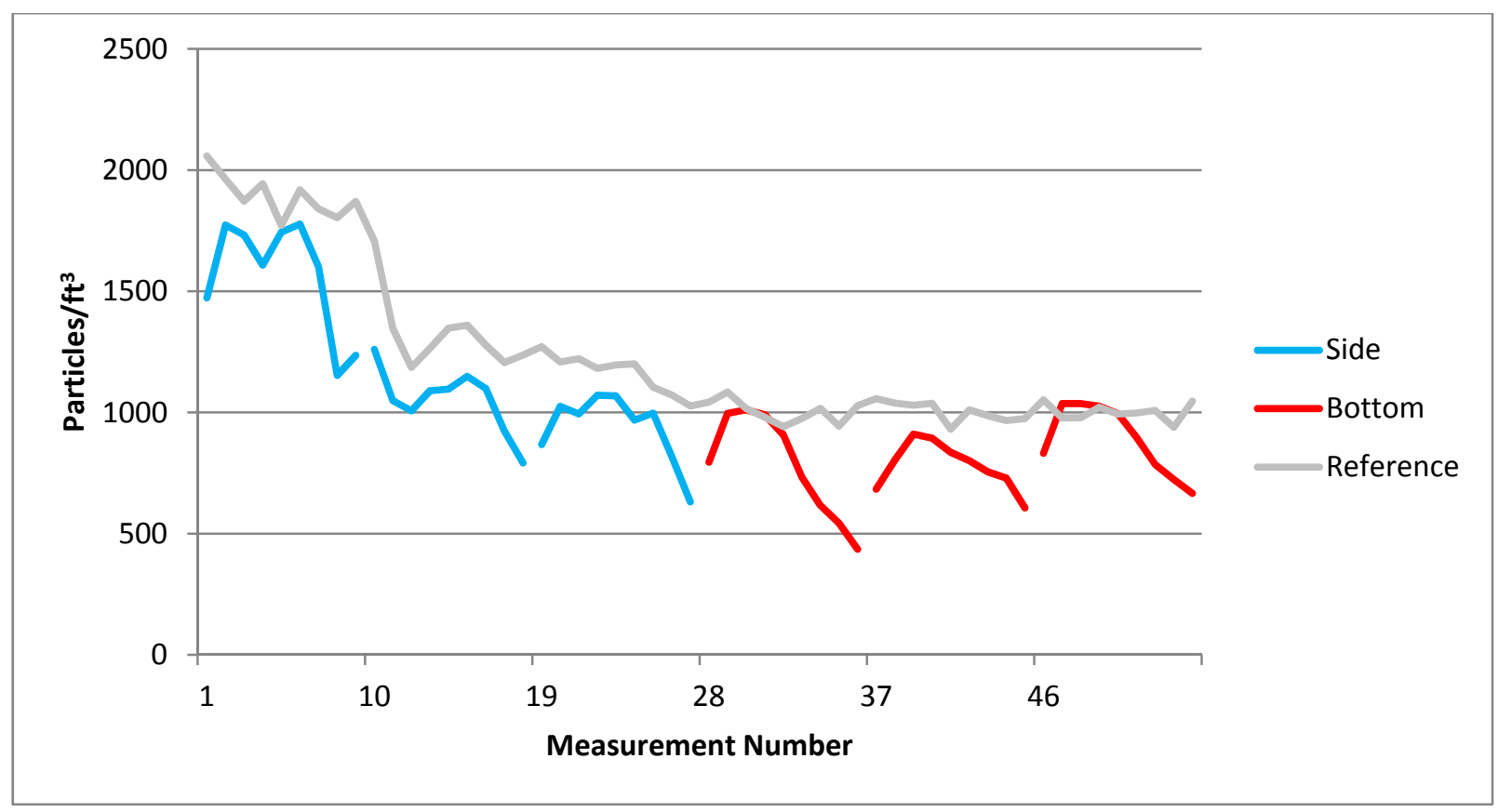

Figure 4.4. Measurement and Reference Particle Tracer Test Data from PT-7 of HV-S2. Colored lines represent measurement traverses from the side and bottom ports, while the grey line represents the reference concentration at the centerpoint at a downstream location. This plot is an example of a typical decline in aerosol production.

\subsection{IHLW-S1 Stack Results}

Data listings, data plots, and summary tables for IHLW-S1 flow angle, velocity, gas tracer, and particle tracer test results are presented in the following subsections. Some test combinations were repeated (performed more than once at different times) to provide for quantifying the testing and response measurement uncertainty.

\subsubsection{Velocity Uniformity}

The initial test to determine the fan frequency setting for the IHLW-S1 model to achieve the desired flow conditions is included in Appendix C, Subsection C.1. Fifteen velocity uniformity runs were performed for the IHLW-S1 model. Table 4.9 lists the results for all of the runs. Table 2.2 lists the range of minimum scale model flow rates as 401 to $839 \mathrm{sfpm}$ ( 315 to $659 \mathrm{scfm})$. The desired testing conditions were between the minimum scale model flow rate and the actual stack velocity. With these flow conditions, the scale model meets both the Reynolds number and DV criteria required to represent the actual stack with one operating fan.

All results were within the qualification criterion of $\% \mathrm{COV}$ values $\leq 20 \%$. COV values were typically less than $8 \%$, with values ranging from 4.2 to $10.0 \% \mathrm{COV}$. The largest $\mathrm{COV}$ value occurred for a minimum flow condition. The completed data sheets from these tests are available in Appendix C, Subsection C.2. 
Table 4.9. Summary of IHLW-S1 Velocity Uniformity Tests

\begin{tabular}{|c|c|c|c|c|c|c|}
\hline $\begin{array}{c}\text { Operating } \\
\text { Fan(s) }\end{array}$ & $\begin{array}{l}\text { Test } \\
\text { Port }\end{array}$ & $\begin{array}{c}\text { Flow } \\
\text { Condition }\end{array}$ & $\begin{array}{l}\text { Run } \\
\text { No. }\end{array}$ & $\begin{array}{l}\text { Flow } \\
(\mathrm{scfm})\end{array}$ & $\begin{array}{l}\text { Velocity } \\
\text { (sfpm) }\end{array}$ & $\% \mathrm{COV}$ \\
\hline \multirow{4}{*}{ A } & \multirow{4}{*}{2} & \multirow{3}{*}{ Max } & VT-1 & 1725 & 2219 & 4.6 \\
\hline & & & VT-11 & 1321 & 1700 & 4.7 \\
\hline & & & VT-12 & 1541 & 1983 & 4.2 \\
\hline & & Norm & VT-2 & 724 & 931 & 6.1 \\
\hline \multirow{11}{*}{ B } & \multirow{9}{*}{2} & \multirow{6}{*}{ Max } & VT-4 & 1853 & 2384 & 8.5 \\
\hline & & & VT-7 & 1605 & 2065 & 7.4 \\
\hline & & & VT-8 & 1605 & 2065 & 7.8 \\
\hline & & & VT-9 & 1606 & 2066 & 6.3 \\
\hline & & & VT-13 & 1390 & 1788 & 5.3 \\
\hline & & & VT-14 & 1410 & 1814 & 5.0 \\
\hline & & \multirow{2}{*}{ Norm } & VT-5 & 750 & 965 & 8.2 \\
\hline & & & VT-10 & 1294 & 1664 & 5.9 \\
\hline & & Min & VT-6 & 341 & 439 & 10.0 \\
\hline & \multirow{2}{*}{1} & \multirow{2}{*}{ Max } & VT-3 & 1881 & 2420 & 4.6 \\
\hline & & & VT-15 & 1557 & 2004 & 5.2 \\
\hline
\end{tabular}

Note: Individual and replicate sets of tests are alternately shaded and unshaded.

\subsubsection{IHLW-S1 Flow Angle}

Table 4.10 lists the results of the flow angle tests for the IHLW-S1 scale model. The qualification criterion of $\leq 20^{\circ}$ was met in all cases. The largest results of $9.3^{\circ}$ and $7.3^{\circ}$ occurred during tests of moderate flow rates representing maximum flow conditions for both Fan A and Fan B. The completed data sheets from these tests are available in Appendix C, Subsection C.3.

Table 4.10. Summary of IHLW-S1 Flow Angle Tests

\begin{tabular}{cccccc}
\hline $\begin{array}{c}\text { Operating } \\
\text { Fan }\end{array}$ & $\begin{array}{c}\text { Test } \\
\text { Port }\end{array}$ & $\begin{array}{c}\text { Flow } \\
\text { Condition }\end{array}$ & Run No. & $\begin{array}{c}\text { Approx. Air } \\
\text { Velocity (sfpm) }\end{array}$ & $\begin{array}{c}\text { Mean Absolute } \\
\text { Flow Angle }\left({ }^{\circ}\right)\end{array}$ \\
\hline \multirow{3}{*}{ A } & \multirow{2}{*}{2} & Max & FA-1 & 2500 & 4.0 \\
& & Min & FA-8 & 1797 & 9.3 \\
\cline { 3 - 6 } & & & FA-2 & 500 & 4.4 \\
\hline \multirow{3}{*}{ B } & \multirow{2}{*}{ Max } & FA-3 & 2570 & 3.3 \\
& & & FA-7 & 1800 & 1.9 \\
& & Min & FA-5 & 500 & 1.3 \\
\hline & & Max & FA-4 & 2590 & 1.9 \\
\hline
\end{tabular}

Note: Individual and replicate sets of tests are alternately shaded and unshaded.

\subsubsection{IHLW-S1 Gaseous Tracer Uniformity}

Twenty-seven gaseous tracer tests were conducted on the IHLW-S1 scale model. Table 4.11 lists the test results for the tests that are considered to have valid results. Test run results GT- 2 through GT- 8 were excluded from the table because the injection probe possibly was possibly positioned improperly. Many of the test results have very low \%COV values, which indicates a high level of tracer mixing at 
Test Port 2. This result was expected, given the number of bends and duct runs between the injection and sampling points. The completed data sheets from the IHLW-S1 gas tracer tests are available in Appendix C, Subsection C.4.

Table 4.11. Summary of IHLW-S1 Gas Tracer Uniformity at Test Ports 1 and 2

\begin{tabular}{|c|c|c|c|c|c|c|c|}
\hline $\begin{array}{c}\text { Operating } \\
\text { Fan }\end{array}$ & $\begin{array}{l}\text { Test } \\
\text { Port }\end{array}$ & $\begin{array}{c}\text { Flow } \\
\text { Condition } \\
\end{array}$ & Injection Point & Run No. & $\begin{array}{c}\text { Avg } \\
\text { Velocity } \\
\text { (sfpm) }\end{array}$ & $\% \mathrm{COV}$ & $\begin{array}{l}\text { Abs. \% Max. Dev. } \\
\text { from Mean }\end{array}$ \\
\hline \multirow{3}{*}{ A } & \multirow{3}{*}{2} & Max & C Center & GT-22 & 1997 & 0.9 & 2.5 \\
\hline & & Normal & C Center & GT-21 & 1630 & 0.9 & 1.9 \\
\hline & & Min & C Center & GT-23 & 406 & 4.0 & 7.5 \\
\hline \multirow{17}{*}{ B } & \multirow{8}{*}{2} & \multirow{8}{*}{$\operatorname{Max}$} & C Center & GT-1 & 2926 & 1.3 & 3.5 \\
\hline & & & C Far & GT-20 & 2137 & 0.9 & 1.8 \\
\hline & & & C Near & GT-19 & 2113 & 0.9 & 1.8 \\
\hline & & & C Top & GT-10 & 2215 & 3.4 & 6.2 \\
\hline & & & \multirow{4}{*}{ C Bottom } & GT-9 & 2200 & 3.1 & 8.2 \\
\hline & & & & GT-16 & 2058 & 5.8 & 11.2 \\
\hline & & & & GT-17 & 2192 & 3.4 & 8.1 \\
\hline & & & & GT-18 & 2179 & 3.4 & 7.5 \\
\hline & \multirow{5}{*}{1} & \multirow{5}{*}{ Max } & C Top & GT-11 & 2303 & 3.1 & 5.8 \\
\hline & & & C Bottom & GT-12 & 2228 & 3.3 & 6.1 \\
\hline & & & C Near & GT-13 & 2355 & 0.7 & 1.2 \\
\hline & & & C Far & GT-14 & 2454 & 0.6 & 1.3 \\
\hline & & & C Center & GT-15 & 2386 & 1.0 & 1.9 \\
\hline & \multirow{4}{*}{2} & Normal & C Center & GT-25 & 1617 & 1.2 & 2.6 \\
\hline & & \multirow{3}{*}{ Min } & C Center & GT-24 & 360 & 4.8 & 17.7 \\
\hline & & & C Center & GT-26 & 411 & 1.8 & 3.5 \\
\hline & & & C Center & GT-27 & 832 & 1.5 & 3.5 \\
\hline
\end{tabular}

Note: Individual or replicate sets of tests are alternately shaded and unshaded.

\subsubsection{IHLW-S1 Particle Tracer Uniformity}

Table 4.11 shows the particle tracer uniformity test results without and with normalization applied for the IHLW-S1 scale model. The normalized data show that the qualification criterion $(\leq 20 \% \mathrm{COV})$ is met for all of the runs. The completed data sheets from these tests are available in Appendix C, Subsection C.5.

As was observed in most particle tracer tests, the tracer aerosol output can vary with time during the run, and the OPC response can change when the instrument is reoriented. To track the output of the aerosol generator, a second OPC was used as the reference instrument sampling from the stack centerline at another test port throughout the run. PT-3 results were omitted from the table because of highly inconsistent aerosol production. Figure 4.5 shows the data collected from the measurement port during PT-3 along with the concurrent measurement at the center point at Port 1, downstream of the measurement port as a reference. The aerosol production was highly erratic, and the measurement data across the traverses track with the reference data, and does not provide useful information about the stack cross-sectional mixing of particulate tracer. PT- 8 has also been omitted from the summary of results 
tabulated in Table 4.12 because of a consistent decline in aerosol production throughout the test. PT- 8 results are driven primarily by the aerosol production rate, and is not a reliable measure of the stack mixing.

Table 4.12. Summary of IHLW-S1 Particle Tracer Uniformity Tests

\begin{tabular}{|c|c|c|c|c|c|c|c|}
\hline Test Port & $\begin{array}{c}\text { Operating } \\
\text { Fan }\end{array}$ & $\begin{array}{l}\text { Injection Port } \\
\& \text { Location }\end{array}$ & $\begin{array}{c}\text { Flow } \\
\text { Condition } \\
(\%)\end{array}$ & $\begin{array}{l}\text { Run } \\
\text { No. }\end{array}$ & $\begin{array}{c}\text { Avg } \\
\text { Velocity } \\
\text { (sfpm) }\end{array}$ & $\begin{array}{c}\text { Non-normalized } \\
\% \mathrm{COV}\end{array}$ & $\begin{array}{c}\text { Normalized } \\
\% \mathrm{COV}\end{array}$ \\
\hline \multirow{6}{*}{2} & \multirow{2}{*}{ A } & \multirow{2}{*}{ C Center } & Max & PT-1 & 2033 & 15.1 & 15.2 \\
\hline & & & Min & PT-7 & 398 & 16.7 & 5.3 \\
\hline & \multirow{4}{*}{ B } & \multirow{4}{*}{ C Center } & Max & PT-2 & 2085 & 8.3 & 8.5 \\
\hline & & & Norm & PT-9 & 849 & 5.5 & 3.3 \\
\hline & & & \multirow{2}{*}{ Min } & PT-4 & 378 & 9.0 & 7.9 \\
\hline & & & & PT-6 & 324 & 7.7 & 6.0 \\
\hline 1 & B & C Center & Max & PT-5 & 2104 & 16.6 & 9.2 \\
\hline
\end{tabular}

Note: Individual or replicate sets of tests are alternately shaded and unshaded.

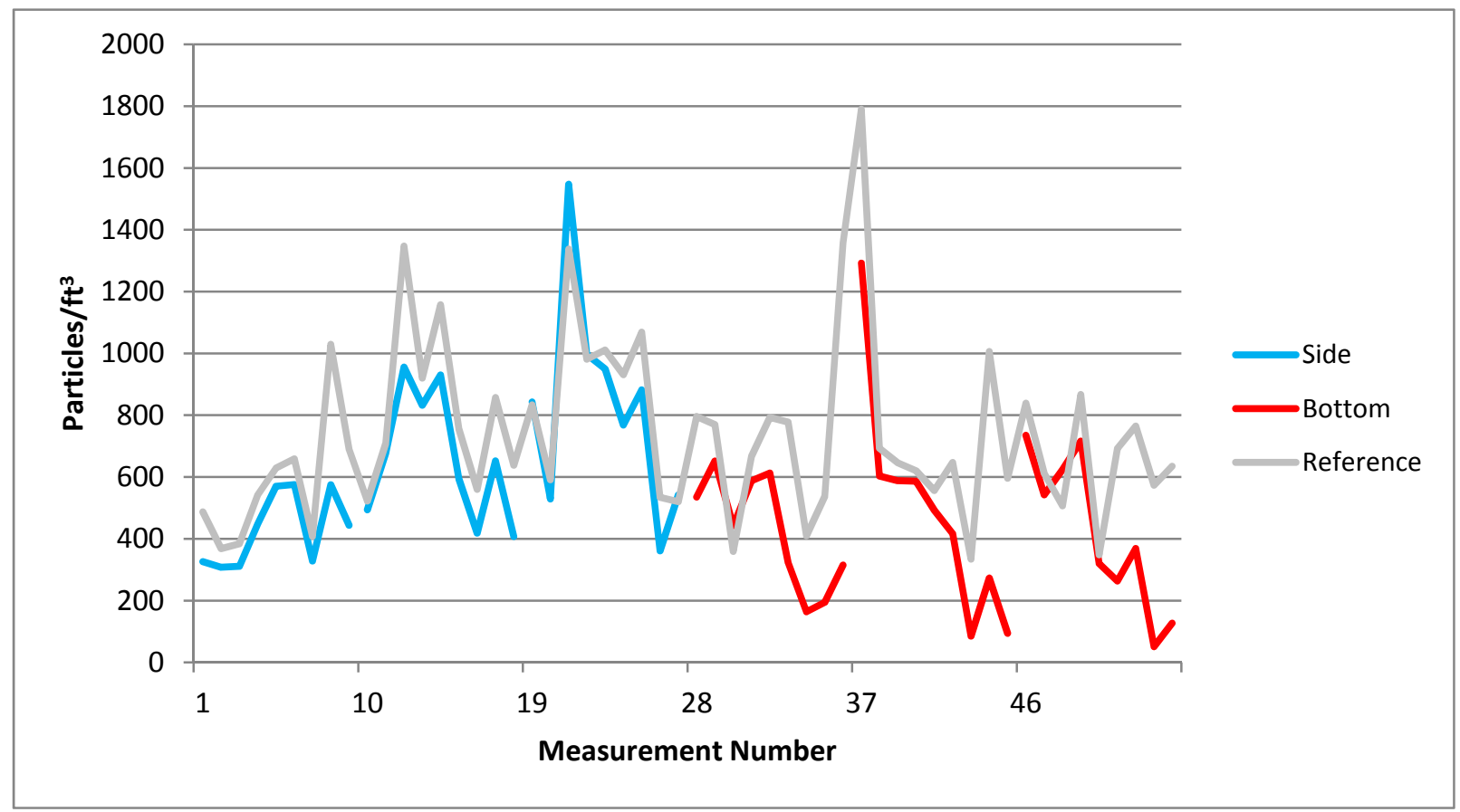

Figure 4.5. Measurement and Reference Particle Tracer Test Data from PT-3 of IHLW-S1. Colored lines represent measurement traverses from the side and bottom ports, while the grey line represents the reference concentration at the center point at a downstream location. This plot is an example of highly erratic aerosol production. 


\subsection{Conclusions}

The results of the tests for each scale model stack in Group 3-4 are summarized in Table 5.1 and Table 5.2. The criteria for sampling probe locations given in ANSI/HPS N13.1-1999, Sampling and Monitoring Releases of Airborne Radioactive Substances from the Stack and Ducts of Nuclear Facilities, were met in all cases. These criteria address the capability of the sampling probe to extract a sample that represents the effluent stream. The range of results presented in Table 5.1 for the Group 3-4 stacks covers the designed location for the air sampling probe, Test Port 2.

For the HV-S1 and IHLW-S1 stacks, a limited number of tests were also conducted at a location five duct diameters downstream of the primary location, Test Port 1. This allows for some variability that may occur because of design or construction changes. HV-S2 tests were conducted only at Test Port 2 because of client input that stated that no contingency ports are available for this stack. The results for Test Port 1 are included in Table 5.2. With the exception of the HV-S1 velocity uniformity test, the Test Port 1 conditions represent maximum stack flow conditions. The HV-S1 velocity uniformity test at Test Port 1 was conducted as a minimum flow case.

Table 5.1. Summary of Test Port 2 Results for the Group 3-4 Scale Model Stacks

\begin{tabular}{lclccc}
\hline & Acceptance Criteria & \multicolumn{1}{c}{ Units } & HV-S1 & HV-S2 & IHLW-S1 \\
\hline $\begin{array}{l}\text { Velocity } \\
\text { Uniformity }\end{array}$ & $\leq 20$ & \%COV & $2.8-6.6$ & $2.5-7.8$ & $4.2-10$ \\
\hline Flow Angle & $\leq 20$ & Degrees & $3.1-4.7$ & $3.9-12.5$ & $1.3-9.3$ \\
\hline $\begin{array}{l}\text { Gas Tracer } \\
\text { Uniformity }\end{array}$ & $\leq 20$ & \%COV & $1.1-4.7$ & $1.8-7.2$ & $0.9-5.8$ \\
\cline { 2 - 6 } & $\leq 30$ & $\begin{array}{l}\text { Maximum \% } \\
\text { Deviation from Mean }\end{array}$ & $2.6-7.9$ & $4.3-14.2$ & $1.8-11.2$ \\
\hline $\begin{array}{l}\text { Particle Tracer } \\
\text { Uniformity }\end{array}$ & $\leq 20$ & Normalized \%COV & $4.4-11.7$ & $5.3-13$ & $3.3-15.2$ \\
\hline
\end{tabular}

Table 5.2. Summary of Test Port 1 Results for the Group 3-4 Scale Model Stacks

\begin{tabular}{|c|c|c|c|c|}
\hline & Acceptance Criteria & Units & HV-S1 & IHLW-S1 \\
\hline $\begin{array}{l}\text { Velocity } \\
\text { Uniformity }\end{array}$ & $\leq 20$ & $\% \mathrm{COV}$ & $5.4^{\mathrm{a}}$ & $4.6-5.2$ \\
\hline Flow Angle & $\leq 20$ & Degrees & 4.2 & 1.9 \\
\hline \multirow{2}{*}{$\begin{array}{l}\text { Gas Tracer } \\
\text { Uniformity }\end{array}$} & $\leq 20$ & $\% \mathrm{COV}$ & $1.3-3.2$ & $0.6-3.3$ \\
\hline & $\leq 30$ & $\begin{array}{l}\text { Maximum \% Deviation from } \\
\text { Mean }\end{array}$ & $3.8-5.7$ & $1.2-6.1$ \\
\hline $\begin{array}{l}\text { Particle Tracer } \\
\text { Uniformity }\end{array}$ & $\leq 20$ & Normalized \%COV & 14.8 & 9.2 \\
\hline
\end{tabular}


The results at Test Port 1, which is 5 duct diameters downstream of the anticipated sampling location at Test Port 2 tend to be fairly similar to the Test Port 2 results. Without more extensive testing and statistical analysis to determine the effect of distance on the ANSI/HPS N13.1-1999 testing results, quantitative commentary cannot be made. However, qualitatively, the results indicate that there is minor difference between the two locations, and that either location (as well as locations in between) should be sufficient as a qualified sampling location.

Based on these scale model tests, the locations proposed for the air sampling probes in each of the three Group 3-4 stacks meet the requirements of the ANSI/HPS N13.1-1999 standard. Additional velocity uniformity and flow angle tests on the actual stacks will be necessary during cold startup to confirm the validity of the scale model results in representing the actual stacks. In particular, the velocity uniformity test results for the actual stacks must be within 5\%COV of the range of results listed above for the scale model so that scale model results can be said to be representative of the stack. For example, if the actual IHLW-S1 stack sampling probe is located in a position corresponding to Test Port 2, the measured velocity uniformity \% COV should be between 0.0 and $15 \% \mathrm{COV}$ (non-negative value for $4.3-5=0.0$, and $10+5=15$ ). The velocity uniformity test results summarized in Table 5.1 cover a range of flow conditions that are expected to bracket the conditions of the actual stack. For cold startup tests, the DV value and Reynolds number should meet the criteria listed in Section 1 (i.e., DV within a factor of six and Reynolds number $>10,000$ ). The velocity uniformity acceptance range would be constructed using the scale model results that correspond to the probe location and fan operating conditions present during the test on the actual stack. 


\subsection{References}

10 CFR 830, Subpart A. 2008. "Quality Assurance Requirements.” Code of Federal Regulations, U.S. Department of Energy.

40 CFR 60, Appendix A, Method 1. "Method 1-Sample and Velocity Traverses for Stationary

Sources.” Code of Federal Regulations, U.S. Environmental Protection Agency.

40 CFR 61, Subpart H. "National Emission Standard for Emissions of Radionuclides other than Radon from Department of Energy Facilities.” Code of Federal Regulations, U.S. Environmental Protection Agency.

ANSI/HPS N13.1-1999. Sampling and Monitoring Releases of Airborne Radioactive Substances from the Stacks and ducts of Nuclear Facilities. American National Standards Institute and the Health Physics Society, McLean, Virginia (reaffirmed in 2011 as ANSI/HPS N13.1-2011).

American Society of Mechanical Engineers (ASME). 2001. Quality Assurance Requirements for Nuclear Facility Applications. NQA-1-2000, New York, New York.

DOE Order 414.1D. April 25, 2011. "Quality Assurance.” U.S. Department of Energy, Washington, D.C. 

Appendix A

\section{HV-S1 Data Sheets}





\section{A.1 HV-S1 Calibration of Ventilation Flow Controller}

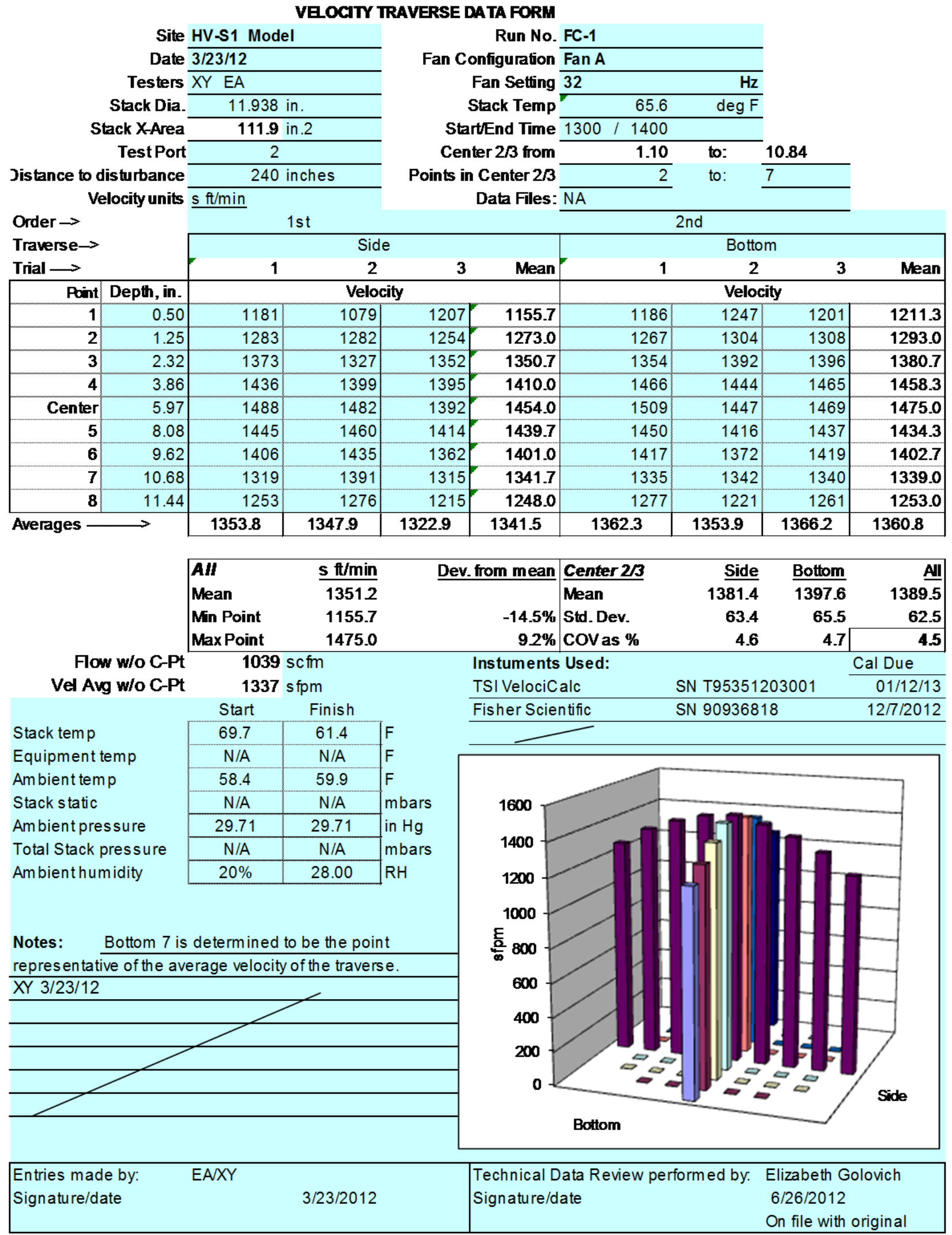




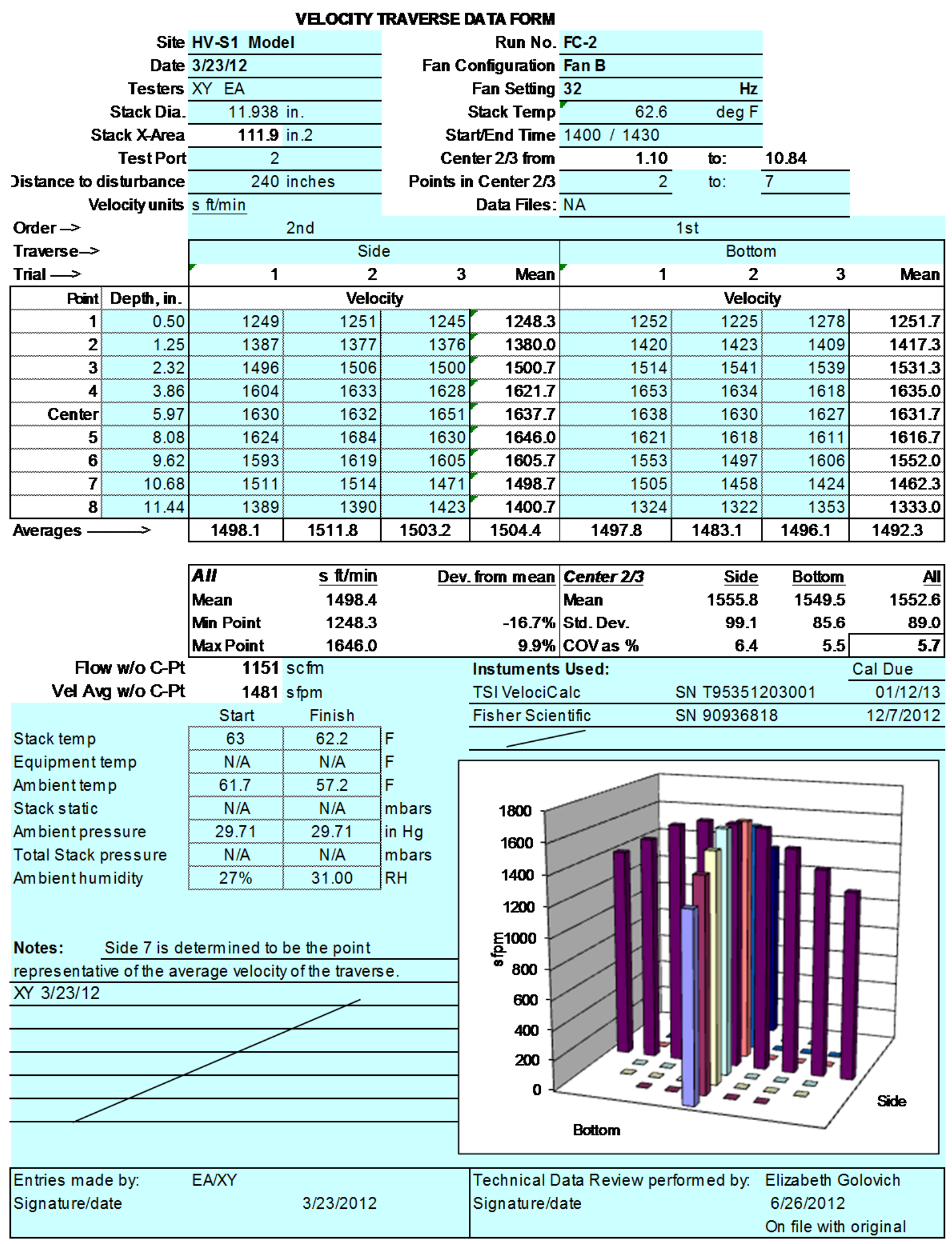




\section{VELOCITY vS. FREQUENCY DATA FORM}

\begin{tabular}{rll} 
Site & HV-S1 model \\
\cline { 2 - 2 } Date & \multicolumn{2}{c}{$3 / 23 / 2012$} \\
\cline { 2 - 2 } Tester & $\mathrm{EA}, \mathrm{XY}$ \\
\cline { 2 - 2 } Stack Dia. & 11.938 & in. \\
X-Area & 111.9 & in2 \\
\cline { 2 - 2 } Test Port & 2
\end{tabular}

\begin{tabular}{rc} 
Run No. VF-1 \\
Stack Temp & $63.0 \mathrm{~F} / 62.0 \mathrm{~F}$ \\
Stack RH\% & $30 \%$ \\
Baro Press & $29.71 \mathrm{in} \mathrm{Hg}$ \\
\cline { 2 - 2 } Fan Configuration & $\mathrm{A}$ only \\
Start/End Time & $15: 53 / 16: 10$
\end{tabular}

Dist. from disturbance $\overline{240}$ Reference point from velocity test VC : $\quad$ Bottom 7 Velocity Readings, units actual fpm

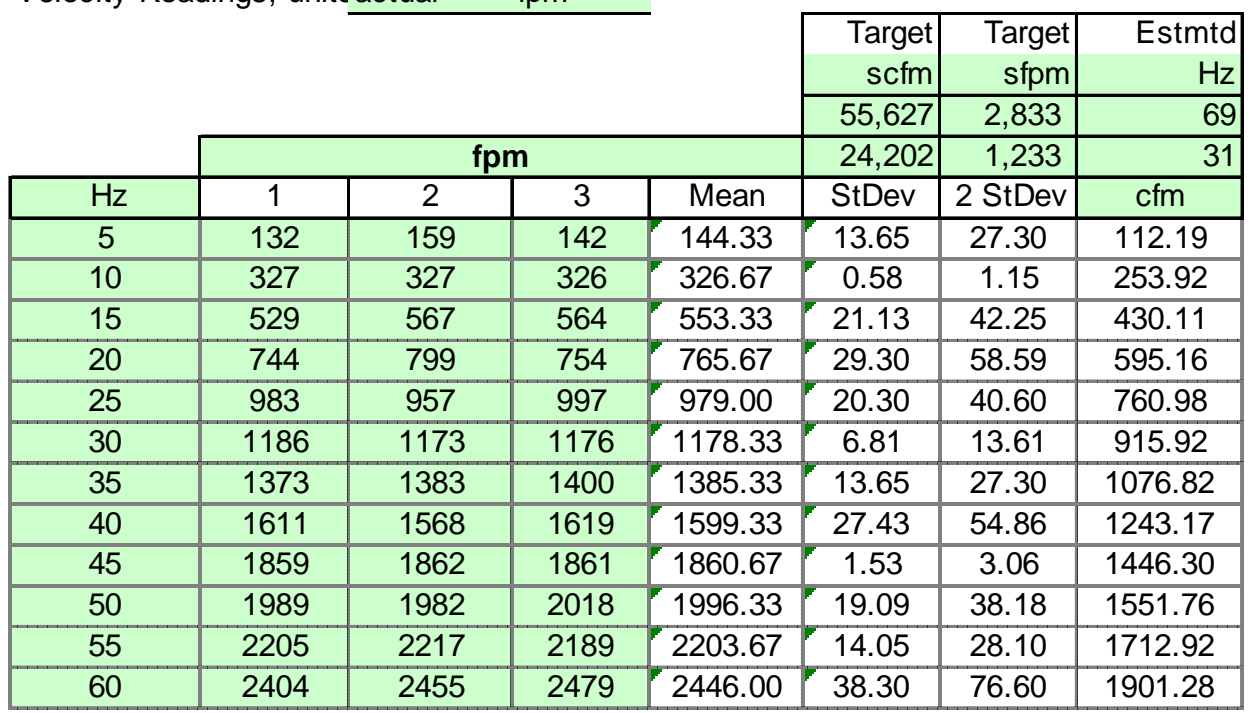

Instuments Used:

TSI VelociCalc Fisher Scientific
SN T95351203001 SN 90936818
Cal Exp. Date:

$01 / 12 / 13$

$12 / 7 / 2012$

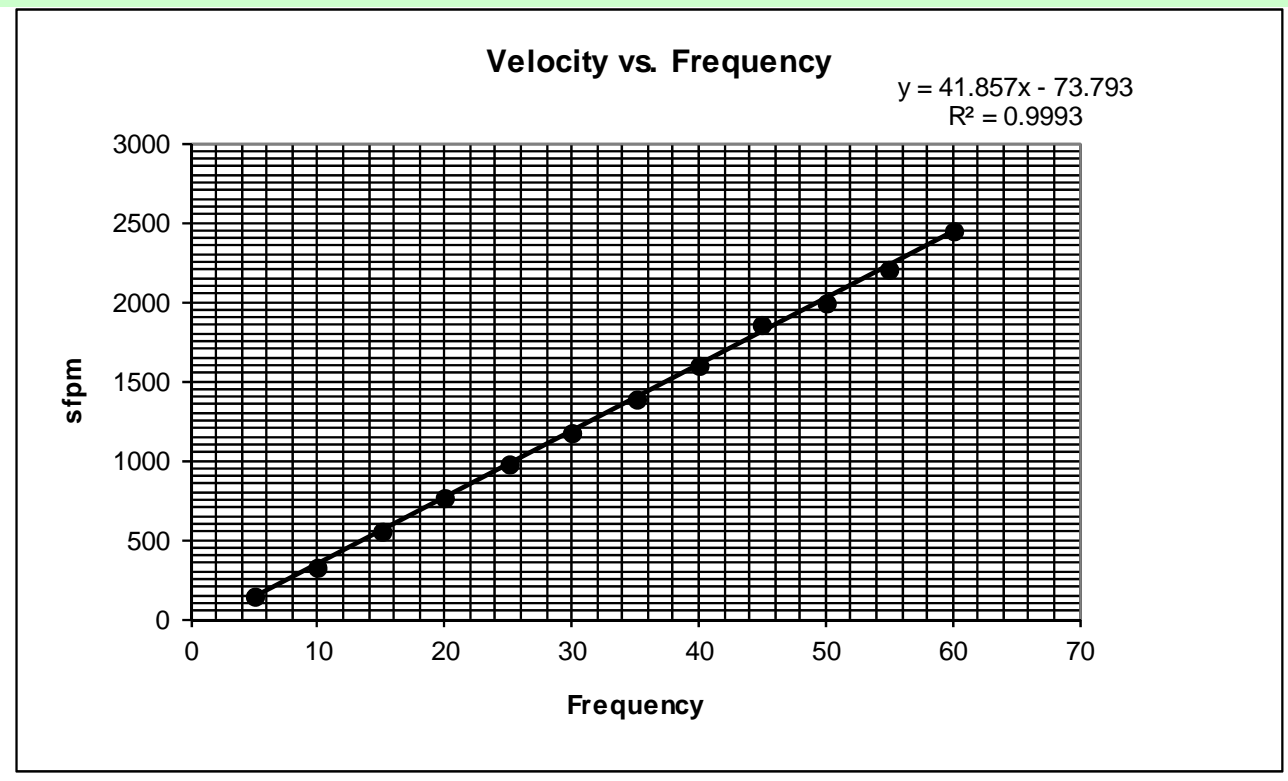

\begin{tabular}{|c|c|c|c|c|}
\hline \multirow{2}{*}{$\begin{array}{l}\text { Entries made by: } \\
\text { Signature/date }\end{array}$} & \multirow{3}{*}{$\begin{array}{l}\text { XY/EA } \\
3 / 23 / 2012\end{array}$} & \multicolumn{3}{|c|}{ Technical Data Review performed by: } \\
\hline & & Signature/date & Elizabeth Golovich & $6 / 26 / 2012$ \\
\hline & & \multicolumn{3}{|c|}{ Signaure on file with original } \\
\hline
\end{tabular}




\section{VELOCITY vS. FREQUENCY DATA FORM}

\begin{tabular}{|c|c|c|}
\hline Site & \multicolumn{2}{|c|}{ HV-S1 model } \\
\hline Date & \multicolumn{2}{|c|}{$3 / 23 / 2012$} \\
\hline Tester & \multicolumn{2}{|c|}{$E A, X Y$} \\
\hline Stack Dia. & 11.938 & in. \\
\hline Stack X-Area & 111.9 & in2 \\
\hline
\end{tabular}

\begin{tabular}{|c|c|}
\hline Run No. & \\
\hline Stack Temp & $64.8 \mathrm{~F} / 60.9 \mathrm{~F}$ \\
\hline Stack RH\% & $26 \%$ \\
\hline Baro Press & $29.71 \mathrm{in} \mathrm{Hg}$ \\
\hline an Configuration & B Only \\
\hline Start/End Time & $1525 / 1550$ \\
\hline
\end{tabular}

Dist. from disturbance $\begin{array}{llll}240 & \text { inches } & \text { Side } 7\end{array}$ Velocity Readings, units actual fpm

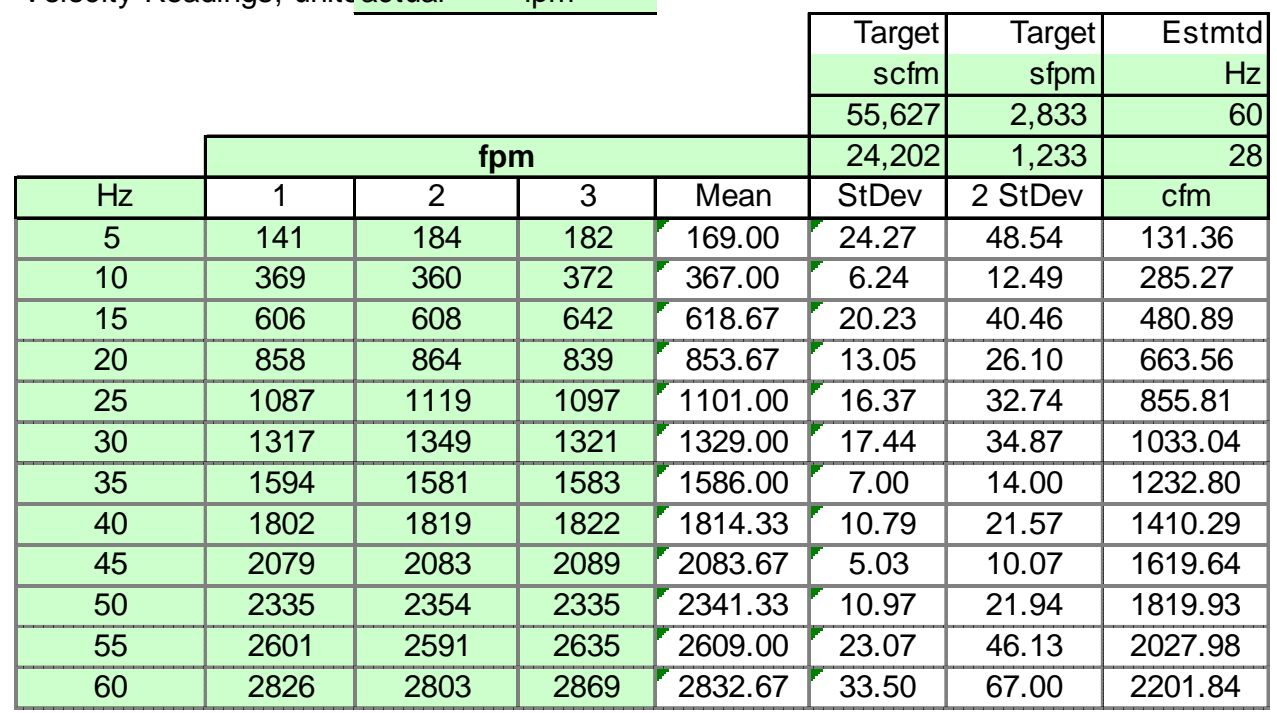

Instuments Used:

\begin{tabular}{ll} 
TSI VelociCalc & SN T95351203001 \\
\hline Fisher Scientific & SN 90936818 \\
\hline
\end{tabular}

Cal Exp. Date:

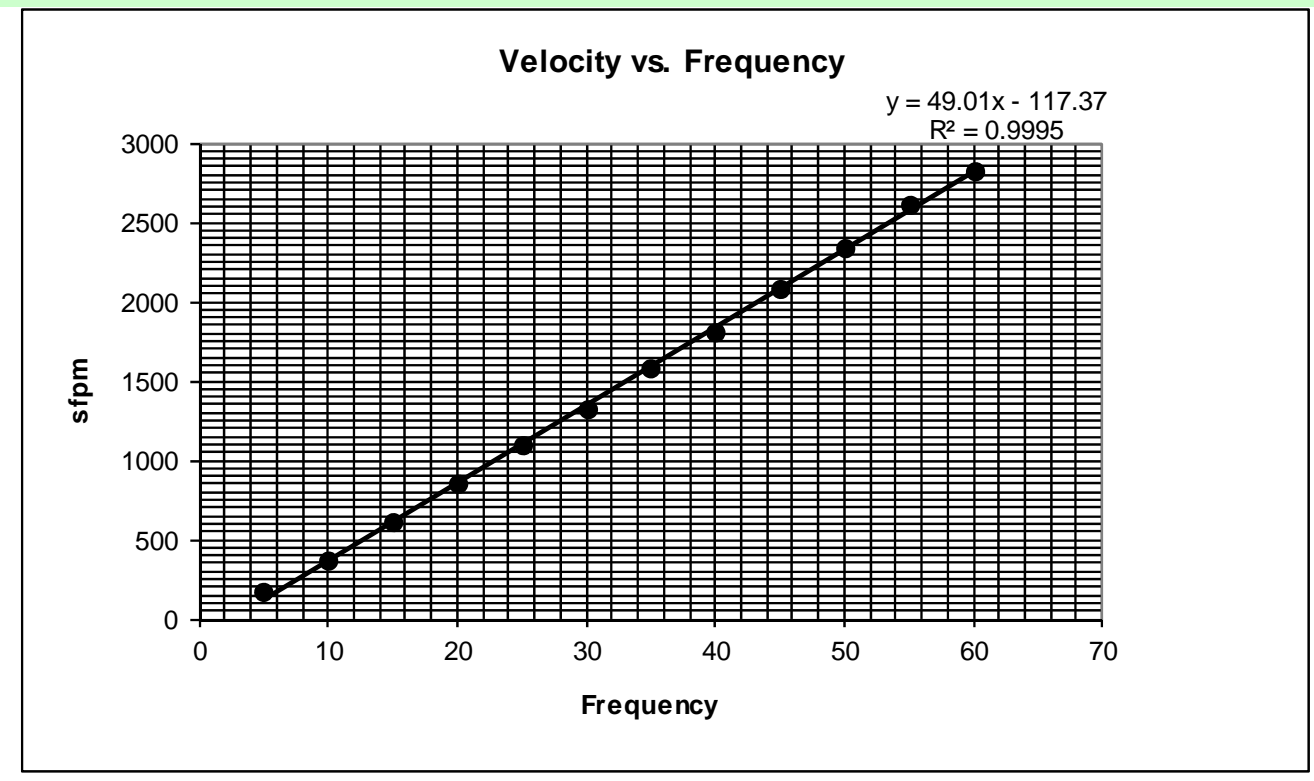

\section{Entries made by:}

Signature/date

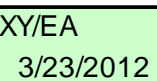

Technical Data Review performed by:

Signature/date Elizabeth Golovich

Signaure on file with original 


\section{A.2 HV-S1 Velocity Uniformity Data Sheets}

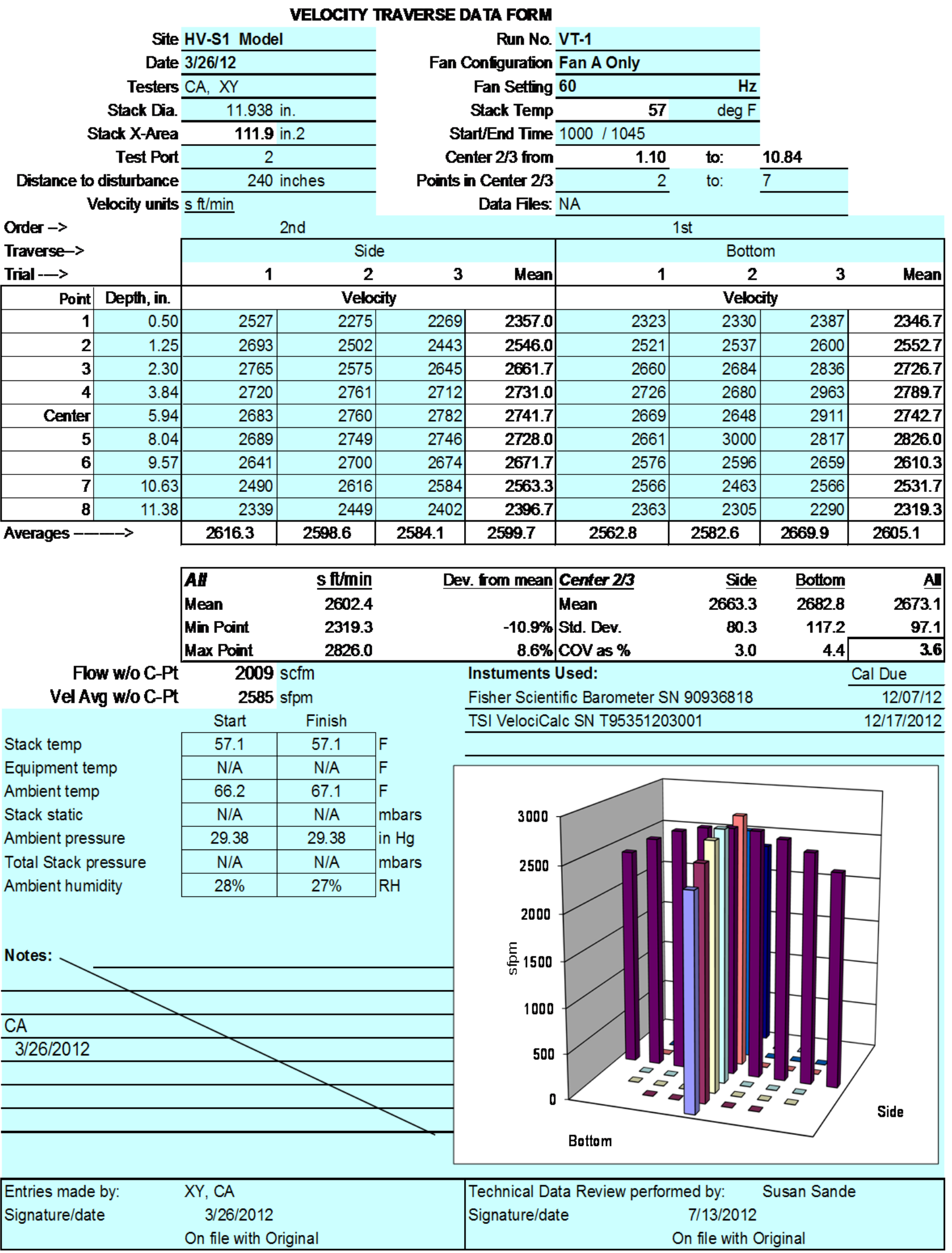




\section{VELOCITY TRAVERSE DATA FORM}

Site HV-S1 Model

Date 3/26/12

Testers CA XY

Stack Dia

Stack X-Area

Test Port

Distance to disturbance

Velocity units $\mathrm{s} \mathrm{ft} / \mathrm{min}$

Order $\rightarrow$

Traverse->

Trial $->$
11.938 in.

11.9 in. 2 240 inches

2nd
Run No. VT-2

Fan Confguration Fan A Only

Fan Setting 27

Stack Temp

Start/End Time 1050 / 1115

Center $2 / 3$ from $\quad 1.10$ to: 10.84

Points in Center $2 / 3$

Data Fles: NA

2 to: 7

1st

\begin{tabular}{|c|c|c|c|c|c|c|c|c|c|}
\hline \multicolumn{2}{|l|}{ Trial -—> } & 1 & 2 & 3 & Mean & 1 & 2 & 3 & Mean \\
\hline Point & Depth, in. & \multicolumn{4}{|c|}{ Velocity } & \multicolumn{4}{|c|}{ Velocity } \\
\hline 1 & 0.50 & 1027 & 987 & 1011 & 1008.3 & 1015 & 1046 & 1052 & 1037.7 \\
\hline 2 & 1.25 & 1111 & 1113 & 1102 & 1108.7 & 1136 & 1175 & 1095 & 1135.3 \\
\hline 3 & 2.30 & 1160 & 1213 & 1180 & 1184.3 & 1201 & 1193 & 1203 & 1199.0 \\
\hline 4 & 3.84 & 1181 & 1191 & 1222 & 1198.0 & 1234 & 1242 & 1234 & 1236.7 \\
\hline Center & 5.94 & 1217 & 1217 & 1245 & 1226.3 & 1211 & 1256 & 1229 & 12320 \\
\hline 5 & 8.04 & 1242 & 1197 & 1227 & 1222.0 & 1221 & 1187 & 1181 & 1196.3 \\
\hline 6 & 9.57 & 1171 & 1168 & 1231 & 1190.0 & 1205 & 1161 & 1189 & 1185.0 \\
\hline 7 & 10.63 & 1116 & 1125 & 1135 & 1125.3 & 1129 & 1029 & 1110 & 1089.3 \\
\hline 8 & 11.38 & 1007 & 988 & 1043 & 1012.7 & 1046 & 1040 & 1039 & 1041.7 \\
\hline Averages - & $\longrightarrow$ & 1136.9 & 1133.2 & 1155.1 & 1141.7 & 1155.3 & 1147.7 & 1148.0 & 1150.3 \\
\hline
\end{tabular}

\begin{tabular}{|lr}
\hline AH & s ft/min \\
Mean & 1146.0 \\
Min Point & 1008.3 \\
Max Point & 1236.7 \\
\hline
\end{tabular}

Flow w/o C-Pt Vel Avg w/o C-Pt

$883 \mathrm{scfm}$

1136 sfpm

\begin{tabular}{|c|c|l}
\multicolumn{1}{|c|}{ Start } & Finish & \\
\cline { 1 - 2 } 58.1 & 58.1 & $\mathrm{~F}$ \\
\cline { 1 - 2 } $\mathrm{N} / \mathrm{A}$ & $\mathrm{N} / \mathrm{A}$ & $\mathrm{F}$ \\
\cline { 1 - 2 } 68 & 65.3 & $\mathrm{~F}$ \\
\cline { 1 - 2 } $\mathrm{N} / \mathrm{A}$ & $\mathrm{N} / \mathrm{A}$ & $\mathrm{mbars}$ \\
\cline { 1 - 2 } 29.38 & 29.38 & in Hg \\
\cline { 1 - 2 } $\mathrm{N} / \mathrm{A}$ & $\mathrm{N} / \mathrm{A}$ & $\mathrm{mbars}$ \\
\cline { 1 - 2 } $27 \%$ & $29 \%$ & $\mathrm{RH}$ \\
\hline
\end{tabular}

Stack temp

Equipment temp

Ambient temp

Stack static

Ambient pressure

Total Stack pressure

Ambient humidity

\section{Dev. fom mean Center 2/3 \\ Mean \\ $-12.0 \%$ Std. Dev. \\ $7.9 \%$ COV as $\%$}

\begin{tabular}{lr} 
Instuments Used: & Cal Due \\
\cline { 2 - 2 } Fisher Scientific Barometer SN 90936818 & $12 / 07 / 12$ \\
\hline TSI VelociCalc SN T95351203001 & $12 / 17 / 2012$ \\
\hline
\end{tabular}

\begin{tabular}{rrr} 
Side & Bottom & Al \\
1179.2 & 1182.0 & 1180.6 \\
45.5 & 52.8 & 47.4 \\
3.9 & 4.5 & 4.0 \\
\hline
\end{tabular}

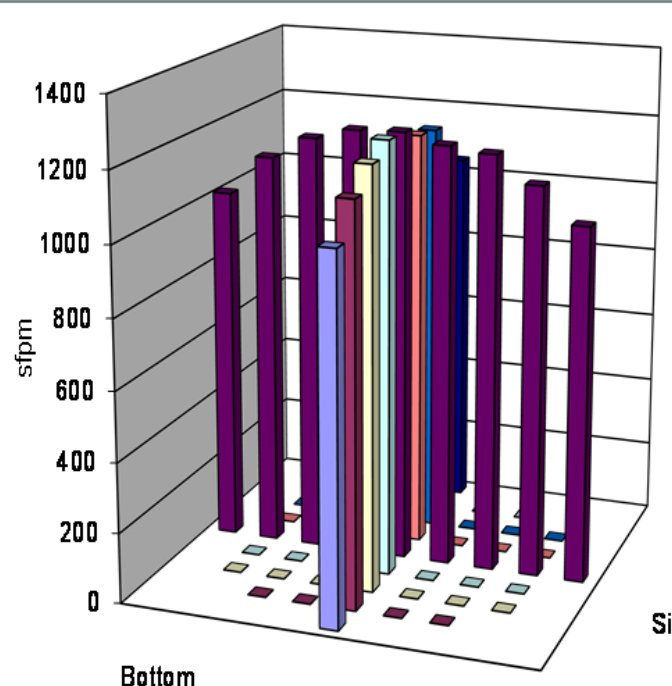

Side

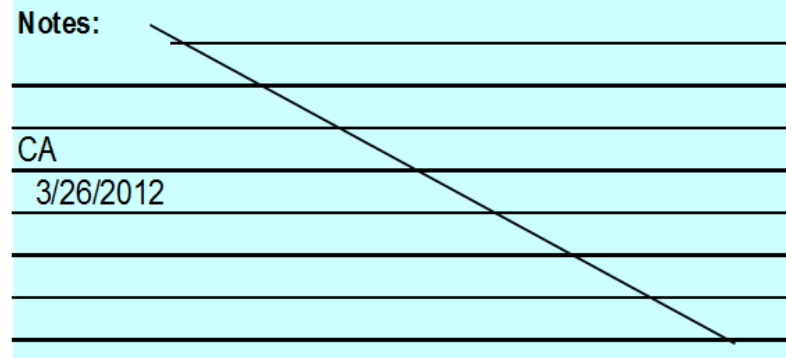

Entries made by:

Signature/date

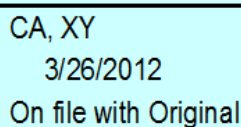

On file with Original \begin{tabular}{|l}
$\begin{array}{l}\text { Technical Data Review performed by: Susan Sande } \\
\text { Signature/date } \\
7 / 13 / 2012 \\
\text { On file with Original }\end{array}$ \\
\hline
\end{tabular} 


\section{VELOCITY TRAVERSE DATA FORM}

Site HV-S1 Model

Date 3/26/12

Testers CA, XY

Stack Dia.

Stack X-Area

Test Port

Distance to disturbance

Velocity units $\mathrm{s} \mathrm{ft} / \mathrm{min}$

Order $\rightarrow$

Traverse->

Trial $->$

\begin{tabular}{|r|r|}
\hline Point & Depth, in. \\
\hline $\mathbf{1}$ & 0.50 \\
\hline $\mathbf{2}$ & 1.25 \\
\hline $\mathbf{3}$ & 2.30 \\
\hline $\mathbf{4}$ & 3.84 \\
\hline Center & 5.94 \\
\hline $\mathbf{5}$ & 8.04 \\
\hline $\mathbf{6}$ & 9.57 \\
\hline $\mathbf{7}$ & 10.63 \\
\hline $\mathbf{8}$ & 11.38 \\
\hline
\end{tabular}

Averages -——>

11.38

$\frac{11.938 \text { in. }}{\frac{111.9 \text { in. } 2}{2}}$

$1 s t$

Run No. VT-3

Fan Configuration Fan B Only

Fan Setting 60

Stack Temp

$\mathrm{Hz}$

$62 \quad \operatorname{deg} \mathrm{F}$

Start/End Time $1120 / 1137$

Center $2 / 3$ from

Points in Center $2 / 3$

Data Fles: NA

\begin{tabular}{rll}
1.10 & to: & 10.84 \\
\hline & to: &
\end{tabular}

\section{Side}

2nd

\begin{tabular}{|r|r|r|r|r|r|}
\cline { 3 - 6 } & \multicolumn{5}{|c|}{ 2nd } \\
$\mathbf{3}$ & Mean & \multicolumn{5}{|c|}{$\begin{array}{c}\text { Bottom } \\
\mathbf{2}\end{array}$} \\
\hline 2447 & $\mathbf{2 4 3 1 . 0}$ & 2524 & 2470 & 2419 & $\mathbf{2 4 7 1 . 0}$ \\
\hline 2676 & $\mathbf{2 6 7 0 . 0}$ & 2730 & 2686 & 1678 & $\mathbf{2 3 6 4 . 7}$ \\
\hline 2839 & $\mathbf{2 8 3 5 . 0}$ & 2939 & 2812 & 2868 & $\mathbf{2 8 7 3 . 0}$ \\
\hline 2920 & $\mathbf{2 9 3 7 . 7}$ & 3101 & 2917 & 2917 & $\mathbf{2 9 7 8 . 3}$ \\
\hline 2935 & $\mathbf{2 9 4 0 . 3}$ & 3137 & 2921 & 2942 & $\mathbf{3 0 0 0 . 0}$ \\
\hline 2894 & $\mathbf{2 9 0 4 . 7}$ & 3040 & 2900 & 2888 & $\mathbf{2 9 4 2 7}$ \\
\hline 2824 & $\mathbf{2 8 2 3 . 7}$ & 2969 & 2746 & 2804 & $\mathbf{2 8 3 9 . 7}$ \\
\hline 2647 & $\mathbf{2 6 9 1 . 3}$ & 2694 & 2663 & 2647 & $\mathbf{2 6 6 8 . 0}$ \\
\hline 2412 & $\mathbf{2 4 5 3 . 0}$ & 2523 & 2386 & 2439 & $\mathbf{2 4 4 9 . 3}$ \\
\hline $\mathbf{2 7 3 2 . 7}$ & $\mathbf{2 7 4 3 . 0}$ & $\mathbf{2 8 5 0 . 8}$ & $\mathbf{2 7 2 2 . 3}$ & $\mathbf{2 6 2 2} \mathbf{4}$ & $\mathbf{2 7 3 1 . 9}$ \\
\hline
\end{tabular}

\begin{tabular}{|lrr|lrrr|}
\hline All & s ft/min & Dev. from mean & Center 2/3 & Side & Bottom & A \\
Mean & 2737.4 & & Mean & 2829.0 & 2809.5 & 2819.2 \\
Min Point & 2364.7 & $-13.6 \%$ & Std. Dev. & 111.2 & 225.6 & 171.2 \\
Max Point & 3000.0 & $9.6 \%$ & COV as $\%$ & 3.9 & 8.0 & $\mathbf{6 . 1}$ \\
\hline
\end{tabular}

Flow w/o C-Pt $\quad 2105 \mathrm{scfm}$

Vel Avg w/o C-Pt

$2708 \mathrm{sfpm}$

Instuments Used: Cal Due

\begin{tabular}{lr} 
Fisher Scientific Barometer SN 90936818 & $12 / 07 / 12$ \\
\hline TSI VelociCalc SN T95351203001 & $12 / 17 / 2012$ \\
\hline
\end{tabular}

Stack temp

Equipment temp

Ambient temp

Stack static

Ambient pressure

Total Stack pressure

Ambient humidity

\begin{tabular}{|c|c|}
\hline Start & Finish \\
\hline 60.5 & 63.3 \\
\hline$N / A$ & $N / A$ \\
\hline 68 & 68 \\
\hline$N / A$ & $N / A$ \\
\hline 29.38 & 29.38 \\
\hline$N / A$ & $N / A$ \\
\hline $27 \%$ & $24 \%$ \\
\hline
\end{tabular}

Notes:
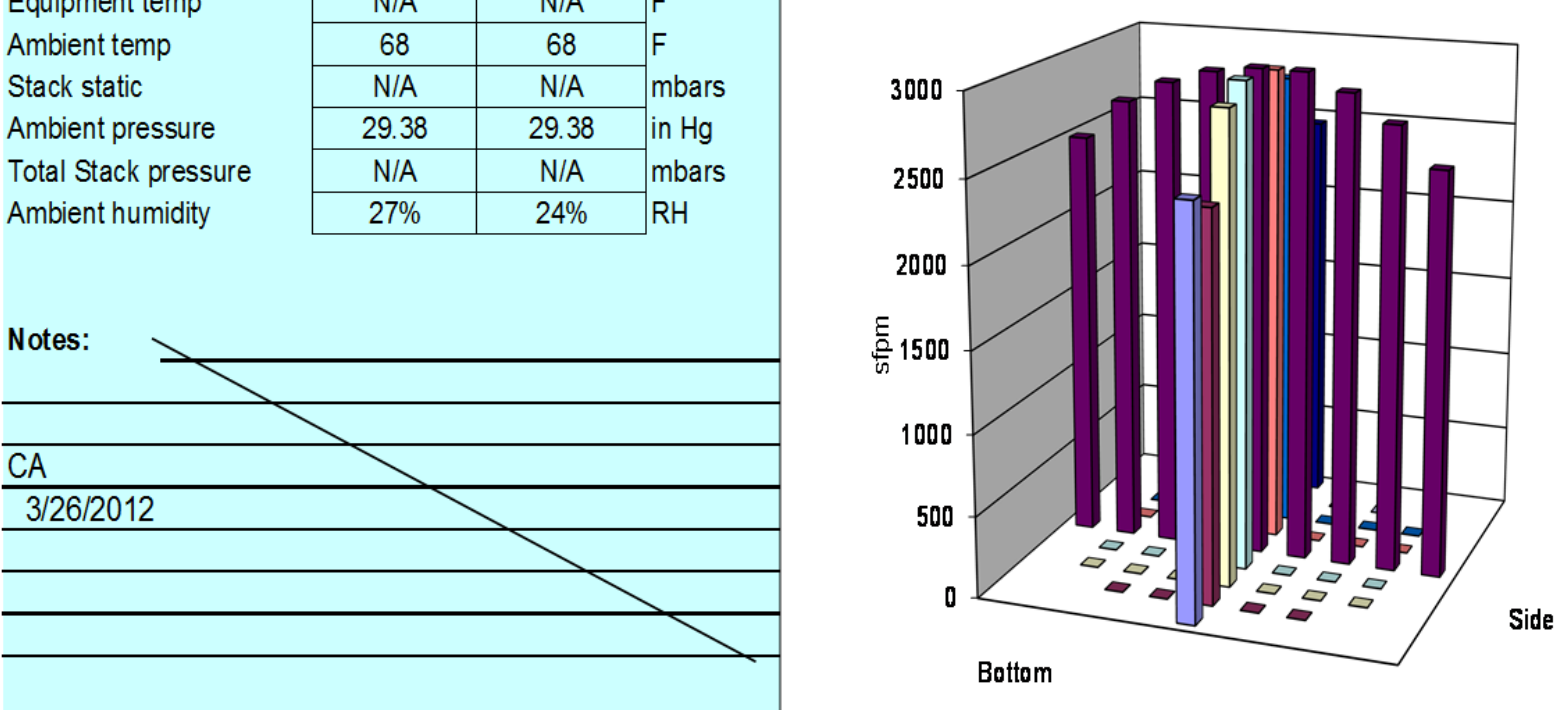

\begin{tabular}{|lc|}
\hline Entries made by: & CA, XY \\
Signature/date & $3 / 26 / 2012$ \\
& On file with Original
\end{tabular}

Technical Data Review performed by: Susan Sande

Signature/date

$7 / 13 / 2012$

On file with Original 


\section{VELOCITY TRAVERSE DATA FORM}

Site HV-S1 Model

Date 3/26/12

Testers $\mathrm{XY}, \mathrm{CA}$

Stack Dia.

Stack X-Area

Test Port

Distance to disturbance

\begin{tabular}{c}
\hline$X Y, C A$ \\
\hline 11.938 in. \\
\hline $\mathbf{1 1 1 . 9}$ in. 2 \\
\hline 2 \\
\hline 240 inches
\end{tabular}

Velocity units $\underline{\mathrm{sft} / \mathrm{min}}$

Order $\rightarrow$

Traverse->

Trial $-\longrightarrow$

\begin{tabular}{|r|r|}
\hline Point & Depth, in. \\
\hline $\mathbf{1}$ & 0.50 \\
\hline $\mathbf{2}$ & 1.25 \\
\hline $\mathbf{3}$ & 2.30 \\
\hline $\mathbf{4}$ & 3.84 \\
\hline Center & 5.94 \\
\hline $\mathbf{5}$ & 8.04 \\
\hline $\mathbf{6}$ & 9.57 \\
\hline $\mathbf{7}$ & 10.63 \\
\hline $\mathbf{8}$ & 11.38 \\
\hline
\end{tabular}

Averages -——

2nd

Side

Run No. VT-4

Fan Confguration Fan B Only

Fan Setting 60

Stack Temp

$\mathrm{Hz}$

$64 \operatorname{deg} \mathrm{F}$

Start/End Time 1140 / 1210

Center $2 / 3$ from 1.10 to: 10.84

Points in Center $2 / 3$

Data Files: NA

2

to: 7

$1 \mathrm{st}$

\begin{tabular}{|l|r|r|r|r|r|r|}
\hline \multicolumn{2}{|c|}{} & $\mathbf{3}$ & Mean & \multicolumn{5}{|c|}{$\mathbf{1}$} & \multicolumn{2}{c|}{$\mathbf{2}$} & Mean \\
\hline Velocity & & & \multicolumn{3}{|c|}{ Velocity } \\
\hline 2413 & 2364 & $\mathbf{2 3 9 0 . 0}$ & 2382 & 2382 & 2493 & $\mathbf{2 4 1 9 . 0}$ \\
\hline 2644 & 2702 & $\mathbf{2 6 6 9 . 0}$ & 2586 & 2636 & 2643 & $\mathbf{2 6 2 1 . 7}$ \\
\hline 2862 & 2883 & $\mathbf{2 8 7 2 . 3}$ & 2801 & 2856 & 2885 & $\mathbf{2 8 4 7 . 3}$ \\
\hline 2982 & 3031 & $\mathbf{3 0 0 1 . 3}$ & 2930 & 2965 & 3019 & $\mathbf{2 9 7 1 . 3}$ \\
\hline 3005 & 3075 & $\mathbf{3 0 3 3 . 3}$ & 2945 & 3008 & 2991 & $\mathbf{2 9 8 1 . 3}$ \\
\hline 2930 & 2996 & $\mathbf{2 9 6 0 . 3}$ & 2880 & 2985 & 2964 & $\mathbf{2 9 4 3 . 0}$ \\
\hline 2898 & 2870 & $\mathbf{2 8 8 0 . 0}$ & 2813 & 2886 & 2835 & $\mathbf{2 8 4 4 . 7}$ \\
\hline 2722 & 2743 & $\mathbf{2 7 4 3 . 0}$ & 2683 & 2669 & 2619 & $\mathbf{2 6 5 7 . 0}$ \\
\hline 2588 & 2516 & $\mathbf{2 5 7 1 . 0}$ & 2420 & 2434 & 2408 & $\mathbf{2 4 2 0 . 7}$ \\
\hline $\mathbf{7}$ & $\mathbf{2 7 9 7 . 8}$ & $\mathbf{2 7 9 1 . 1}$ & $\mathbf{2 7 1 5 . 6}$ & $\mathbf{2 7 5 7 . 9}$ & $\mathbf{2 7 6 1 . 9}$ & $\mathbf{2 7 4 5 . 1}$ \\
\hline
\end{tabular}

3

\begin{tabular}{c|cc} 
Mean & & Bottom \\
& 1 & 2
\end{tabular}

\begin{tabular}{|lr}
\hline AH & $\underline{\mathbf{s f t} / \mathrm{min}}$ \\
Mean & 2768.1 \\
Min Point & 2390.0 \\
Max Point & 3033.3 \\
\hline
\end{tabular}
Dev. fom mean Center 2/3
Mean
$-13.7 \%$ Std. Dev.
$9.6 \%$ COV as $\%$

\begin{tabular}{rrr} 
Side & Bottom & $\mathrm{Al}$ \\
2879.9 & 2838.0 & 2859.0 \\
134.2 & 146.7 & 136.8 \\
& 5.7 & $\mathbf{4 . 8}$ \\
\hline
\end{tabular}

Flow w/o C-Pt

$2128 \mathrm{scfm}$

Vel Avg w/o C-Pt

$2738 \mathrm{sfpm}$

\begin{tabular}{|c|c|}
\hline Start & Finish \\
\hline 65 & 63.8 \\
\hline $\mathrm{N} / \mathrm{A}$ & $\mathrm{N} / \mathrm{A}$ \\
\hline 63.2 & 62.6 \\
\hline $\mathrm{N} / \mathrm{A}$ & $\mathrm{N} / \mathrm{A}$ \\
\hline 29.38 & 29.38 \\
\hline $\mathrm{N} / \mathrm{A}$ & $\mathrm{N} / \mathrm{A}$ \\
\hline $24 \%$ & $29 \%$ \\
\hline
\end{tabular}

Stack temp

Equipment temp

Ambient temp

Stack static

Ambient pressure

Total Stack pressure

Ambient humidity

Instuments Used:

Fisher Scientific Barometer SN 90936818

Cal Due

TSI VelociCalc SN T95351203001

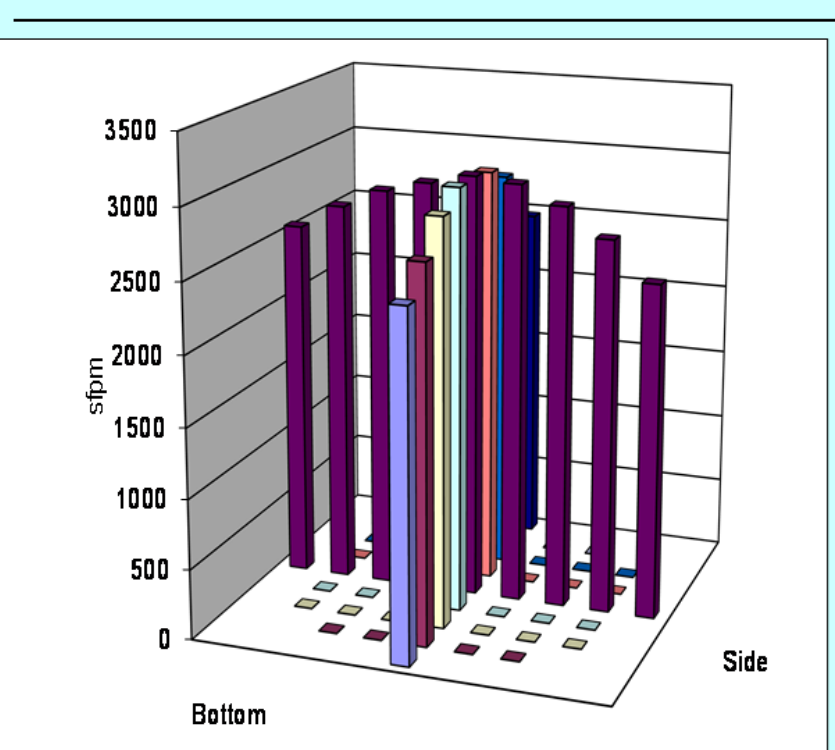

\begin{tabular}{|lc|}
\hline Entries made by: & XY, CA \\
Signature/date & $3 / 26 / 2012$ \\
& On file with Original
\end{tabular}

Technical Data Review performed by: Susan Sande

Signature/date

7/13/2012

On file with Original 


\section{VELOCITY TRAVERSE DATA FORM}

Site HV-S1 Model

Date $3 / 27 / 12$

Testers $\mathrm{CA}, \mathrm{XY}$

Stack Die

Stack X-Area

Test Port

Distance to disturbance

Velocity units $\underline{\mathrm{s} \mathrm{f} / \mathrm{min}}$

Order $\rightarrow$

Traverse->

Trial $->$

\begin{tabular}{|c|c|}
\hline Point & Depth, in. \\
\hline 1 & 0.50 \\
\hline 2 & 1.25 \\
\hline 3 & 2.30 \\
\hline 4 & 3.84 \\
\hline Center & 5.94 \\
\hline 5 & 8.04 \\
\hline 6 & 9.57 \\
\hline 7 & 10.63 \\
\hline 8 & 11.3 \\
\hline
\end{tabular}

Averages
11.38

\begin{tabular}{c}
\hline 11.938 in. \\
\hline $\mathbf{1 1 1 . 9}$ in. 2 \\
\hline 2 \\
\hline 240 inches
\end{tabular}

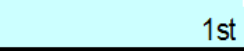

Side
$\mathbf{2}$
Velocily

Run No. VT-5

Fan Confguration FAN B ONLY

Fan Setting 60

Stack Temp

Start/End Time 947/ 1015

Center $2 / 3$ from

Points in Center $2 / 3$

Data Fles: NA

\begin{tabular}{|c|c|c|c|c|}
\hline & & \\
\hline In Setting & & $\mathrm{Hz}$ & & \\
\hline ack Temp & 49 & $\operatorname{deg} F$ & & \\
\hline End Time & $7 / 1015$ & & & \\
\hline 3 from & 1.10 & to: & 10.84 & \\
\hline $2 / 3$ & 2 & to: & 7 & \\
\hline Fles: & & & & \\
\hline & & 2nd & & \\
\hline & & Botto & & \\
\hline Mean & 1 & 2 & 3 & Mean \\
\hline & & Veloc & & \\
\hline 2239.7 & 2376 & 2251 & 2316 & 2314.3 \\
\hline 2537.7 & 2598 & 2574 & 2553 & 2575.0 \\
\hline 2808.0 & 2789 & 2769 & 2741 & 2766.3 \\
\hline 2975.0 & 2978 & 3093 & 3004 & 3025.0 \\
\hline 3101.0 & 3093 & 3087 & 3065 & 3081.7 \\
\hline 3124.3 & 3031 & 3067 & 3018 & 3038.7 \\
\hline 3066.7 & 2971 & 3027 & 2956 & 2984.7 \\
\hline 2938.7 & 2739 & 2865 & 2741 & 2781.7 \\
\hline 2737.0 & 2604 & 2661 & 2528 & 2597.7 \\
\hline 2836.4 & 2797.7 & 2821.6 & 2769.1 & 2796.1 \\
\hline
\end{tabular}

\begin{tabular}{|lr}
\hline All & s ft/min \\
Mean & 2816.3 \\
Min Point & 2239.7 \\
Max Point & 3124.3 \\
\hline
\end{tabular}

Flow w/o C-Pt $2162 \mathrm{scfm}$ Vel Avg w/o C-Pt

$2782 \mathrm{sfpm}$

\begin{tabular}{|c|c|}
\hline Start & Finish \\
\hline 50.3 & 48.6 \\
\hline $\mathrm{N} / \mathrm{A}$ & $\mathrm{N} / \mathrm{A}$ \\
\hline 58.9 & 47.3 \\
\hline $\mathrm{N} / \mathrm{A}$ & $\mathrm{N} / \mathrm{A}$ \\
\hline 29.58 & 29.53 \\
\hline $\mathrm{N} / \mathrm{A}$ & $\mathrm{N} / \mathrm{A}$ \\
\hline $62 \%$ & $84 \%$ \\
\hline
\end{tabular}

Stack temp

Equipment temp Ambient temp

Stack static

Ambient pressure

Total Stack pressure

Ambient humidity

$62 \%$

\begin{tabular}{|r|r|}
\hline 2253 \\
\hline 2497 \\
\hline 2773 \\
\hline 2938 \\
\hline 3084 \\
\hline 3120 \\
\hline 3085 \\
\hline 2929 \\
\hline 2677 \\
\hline $\mathbf{1 7 . 3}$ \\
\hline $\mathbf{s f t / m i n}$ \\
\hline $\mathbf{2 8 1 6 . 3}$ \\
$\mathbf{2 2 3 9 . 7}$ \\
$\mathbf{3 1 2 4 . 3}$ \\
\hline
\end{tabular}

3

\begin{tabular}{|l|r|}
\hline 2264 & $\mathbf{2 2 3 9 . 7}$ \\
\hline 2609 & $\mathbf{2 5 3 7 . 7}$ \\
\hline 2785 & $\mathbf{2 8 0 8 . 0}$ \\
\hline 3003 & $\mathbf{2 9 7 5 . 0}$ \\
\hline 3103 & $\mathbf{3 1 0 1 . 0}$ \\
\hline 3133 & $\mathbf{3 1 2 4 . 3}$ \\
\hline 3062 & $\mathbf{3 0 6 6 . 7}$ \\
\hline 2984 & $\mathbf{2 9 3 8 . 7}$ \\
\hline 2730 & $\mathbf{2 7 3 7 . 0}$ \\
\hline $\mathbf{2 . 6}$ & $\mathbf{2 8 3 6 . 4}$ \\
\hline
\end{tabular}

\begin{tabular}{|c|c|}
\hline Dev. from mean & Center $2 / 3$ \\
\hline$m 50$ & Mean \\
\hline $10.9 \%$ & cov as $\%$ \\
\hline
\end{tabular}

\begin{tabular}{|c|c|c|}
\hline Side & Bottom & A \\
\hline 2935.9 & 2893.3 & 2914.6 \\
\hline 206.7 & 188.1 & 191.1 \\
\hline 7.0 & 6.5 & 6.6 \\
\hline
\end{tabular}

\begin{tabular}{lr} 
Instuments Used: & Cal Due \\
\cline { 2 - 2 } Fisher Scientific Barometer SN 90936818 & $12 / 07 / 12$ \\
\hline TSI VelociCalc SN T95351203001 & $12 / 17 / 2012$ \\
\hline
\end{tabular}
TSI VelociCalc SN T95351203001
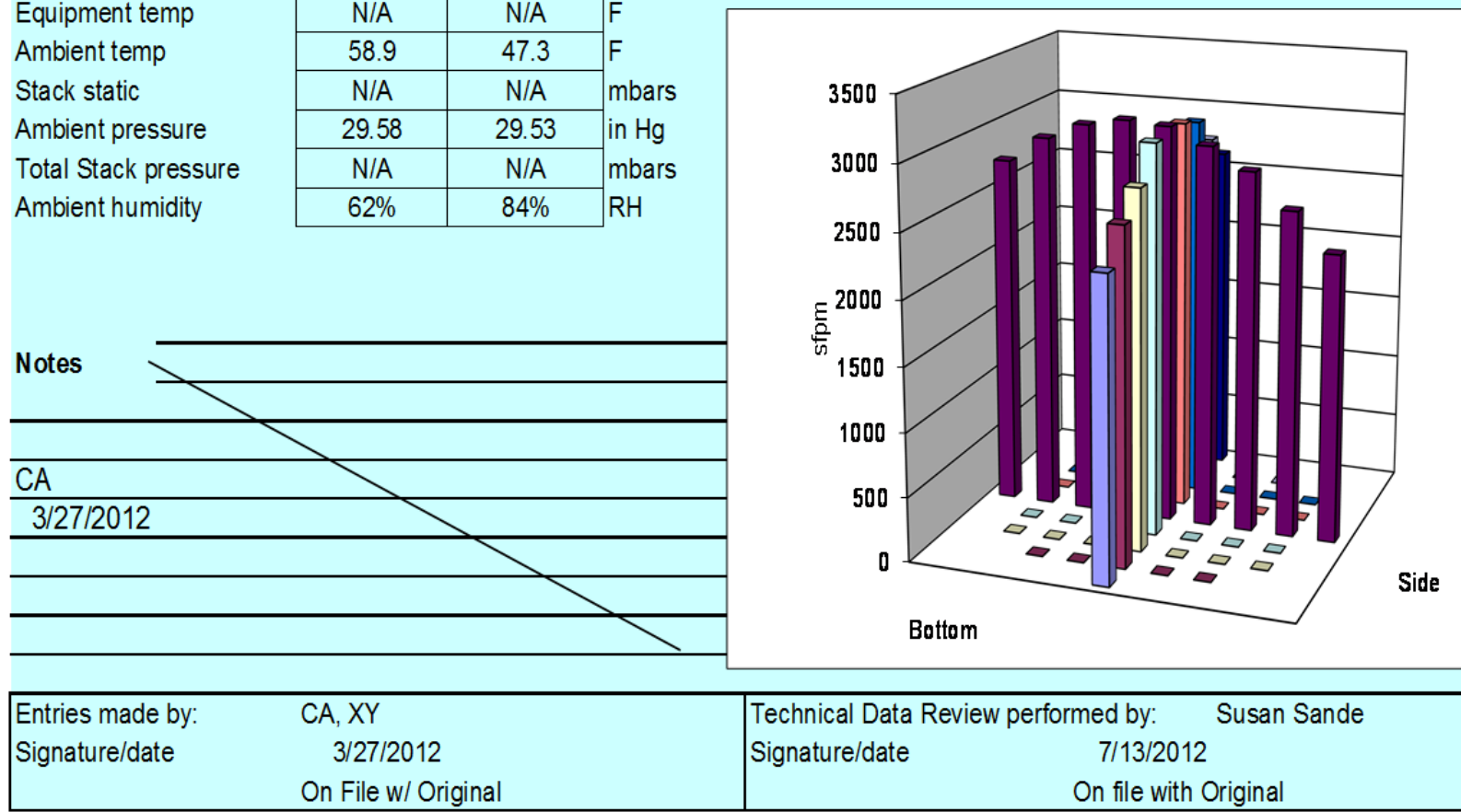
Technical Data
Signature/date
ed by:
7/13/2012
On file with Original 


\section{VELOCITY TRAVERSE DATA FORM}

Site HV-S1 Model

Date 2/27/12

Testers XY, CA

Stack Dia

Stack X-Area

Test Port

Distance to disturbance

Velocity units $\underline{\mathrm{s} f t / m i n}$

Order $\rightarrow$

Traverse->

Trial $->$

\begin{tabular}{|r|r|}
\hline Point & Depth, in. \\
\hline $\mathbf{1}$ & 0.50 \\
\hline $\mathbf{2}$ & 1.25 \\
\hline $\mathbf{3}$ & 2.30 \\
\hline $\mathbf{4}$ & 3.84 \\
\hline Center & 5.94 \\
\hline $\mathbf{5}$ & 8.04 \\
\hline $\mathbf{6}$ & 9.57 \\
\hline $\mathbf{7}$ & 10.63 \\
\hline $\mathbf{8}$ & 11.38 \\
\hline
\end{tabular}

Averages -- -

11.38

\begin{tabular}{|c|}
\hline 11.938 in. \\
\hline 111.9 in. 2 \\
\hline 2 \\
\hline 240 inches \\
\hline
\end{tabular}

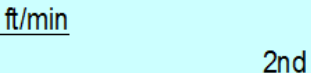

2nd

2nd

1

Velocity

981

1067

109

1168

1217

1243

1200

112

1076

1146.7

\begin{tabular}{|lr}
\hline AH & s ft/min \\
Mean & 1161.8 \\
Min Point & 1003.7 \\
Max Point & 1288.0 \\
\hline
\end{tabular}

Flow w/o C-Pt

$894 \mathrm{scfm}$

Vel Avg w/o C-Pt

1150 sfpm

\begin{tabular}{|c|c|}
\hline Start & Finish \\
\hline 47.8 & 47.6 \\
\hline $\mathrm{N} / \mathrm{A}$ & N/A \\
\hline 47.3 & 48.2 \\
\hline $\mathrm{N} / \mathrm{A}$ & $\mathrm{N} / \mathrm{A}$ \\
\hline 29.53 & 29.53 \\
\hline $\mathrm{N} / \mathrm{A}$ & $\mathrm{N} / \mathrm{A}$ \\
\hline $85 \%$ & $83 \%$ \\
\hline
\end{tabular}

Stack temp

Equipment temp

Ambient temp

Stack static

Ambient pressure

Total Stack pressure

Ambient humidity

\begin{tabular}{|r|r|}
\hline 1135 & 11 \\
\hline 1215 & 1214 \\
\hline 1216 & 121 \\
\hline 1201 & 1204 \\
1198 & 11 \\
\hline 1108 & 11 \\
\hline 1079 & 1081 \\
\hline 133.3 & $\mathbf{1 1 3 5 . 8}$ \\
\hline
\end{tabular}

1288.0

Run No. VT-6

Fan Confguration FAN B ONLY

Fan Setting 28

Stack Temp

$\mathrm{Hz}$

Start/End Time 1019/1030

Center $2 / 3$ from

Points in Center $2 / 3$

Data Fles: NA

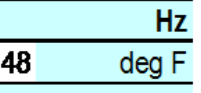

1st

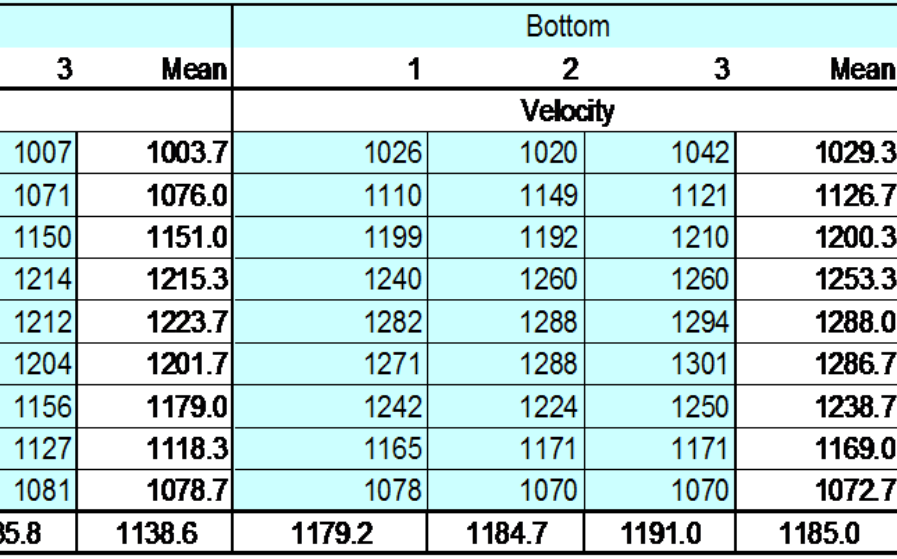

\begin{tabular}{r|lrrrr|} 
Dev. from mean & Center 2/3 & Side & Bottom & A] \\
$-13.6 \%$ & Mean & 1166.4 & 1223.2 & 1194.8 \\
$10.9 \%$ & Std. Dev. & 54.5 & 60.8 & 628 \\
\hline
\end{tabular}

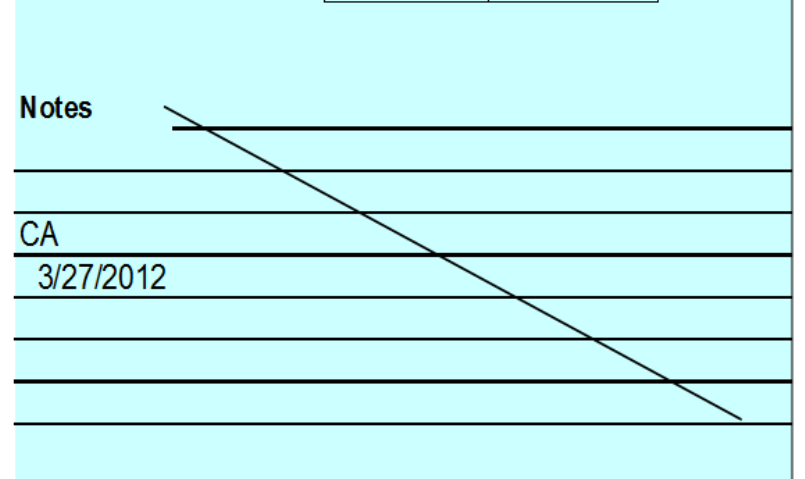

Fisher Scientific Barometer SN 90936818

TSI VelociCalc SN T95351203001

to: 10.84

2 to: 7

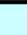

TSIVelociCalc SN T95351203001 12/17/2012

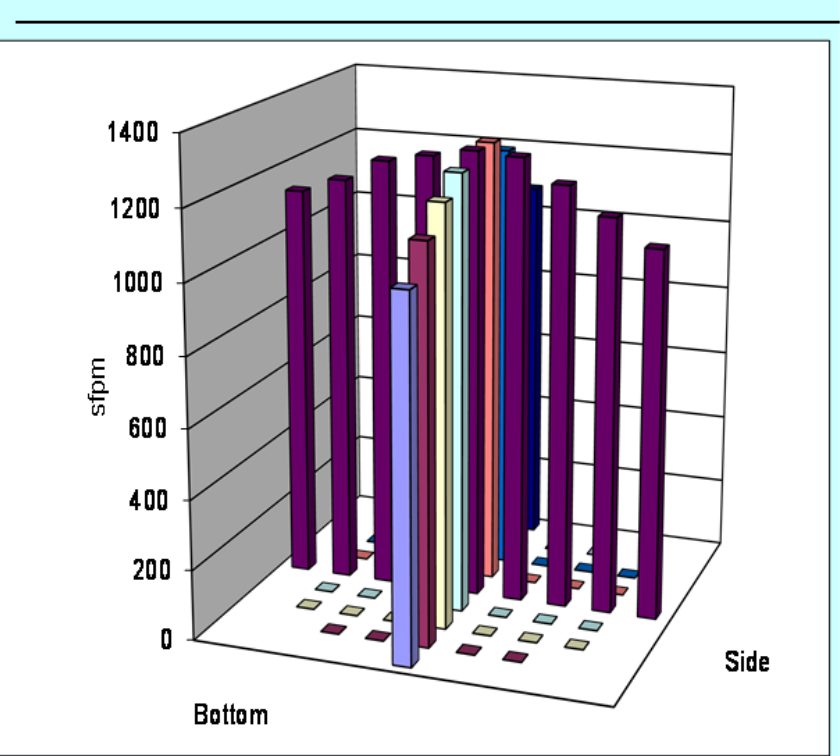

\begin{tabular}{|lc|}
\hline Entries made by: & CA, XY \\
Signature/date & $3 / 27 / 2012$ \\
& On File w/ Original \\
\hline
\end{tabular}

Technical Data Review performed by: Susan Sande

Signature/date

7/13/2012

On file with Original 


\section{VELOCITY TRAVERSE DATA FORM}

Site HV-S1 Model

Date $2 / 27 / 12$

Testers $X Y, C A$

Stack Dia

Stack X-Area

Test Port

Distance to disturbance

Velocity units $\mathrm{s} \mathrm{ft} / \mathrm{min}$

Run No. VT-7

Fan Confguration FAN B ONLY

Fan Setting 28

Stack Temp

Start/End Time 1040/ 1115

Center $2 / 3$ from

Points in Center $2 / 3$

Data Fles: NA

Order $\rightarrow$

Traverse->

Trial $\rightarrow$

\begin{tabular}{|r|r|}
\hline Point & Depth, in. \\
\hline $\mathbf{1}$ & 0.50 \\
\hline $\mathbf{2}$ & 1.2 \\
\hline $\mathbf{3}$ & 2.30 \\
\hline $\mathbf{4}$ & 3.8 \\
\hline Center & 5.94 \\
\hline $\mathbf{5}$ & 8.04 \\
\hline $\mathbf{6}$ & 9.5 \\
\hline $\mathbf{7}$ & 10.63 \\
\hline $\mathbf{8}$ & 11.38 \\
\hline Averages - - & \\
\hline
\end{tabular}

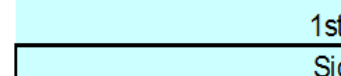

Side

12

3

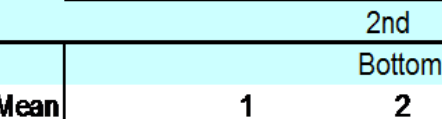

\begin{tabular}{|r|r|r|r|r|r|r|}
\hline \multicolumn{2}{|c|}{ Velocity } & \multicolumn{4}{|c|}{ Velocity } \\
\hline 1007 & 995 & $\mathbf{1 0 1 1 . 3}$ & 1074 & 1116 & 1047 & $\mathbf{1 0 7 9 . 0}$ \\
\hline 1112 & 1115 & $\mathbf{1 1 1 4 . 3}$ & 1136 & 1214 & 1182 & $\mathbf{1 1 7 7 . 3}$ \\
\hline 1164 & 1191 & $\mathbf{1 1 8 8 . 3}$ & 1247 & 1298 & 1242 & $\mathbf{1 2 6 2 3}$ \\
\hline 1328 & 1257 & $\mathbf{1 2 8 6 . 3}$ & 1314 & 1317 & 1293 & $\mathbf{1 3 0 8 . 0}$ \\
\hline 1285 & 1293 & $\mathbf{1 2 9 1 . 7}$ & 1319 & 1318 & 1313 & $\mathbf{1 3 1 6 . 7}$ \\
\hline 1283 & 1280 & $\mathbf{1 2 8 4 . 3}$ & 1276 & 1294 & 1293 & $\mathbf{1 2 8 7 . 7}$ \\
\hline 1261 & 1239 & $\mathbf{1 2 5 2 . 7}$ & 1264 & 1235 & 1261 & $\mathbf{1 2 5 3 . 3}$ \\
\hline 1175 & 1191 & $\mathbf{1 1 7 9 . 7}$ & 1171 & 1149 & 1187 & $\mathbf{1 1 6 9 . 0}$ \\
\hline 1068 & 1079 & $\mathbf{1 0 7 5 . 3}$ & 1049 & 1008 & 1002 & $\mathbf{1 0 1 9 . 7}$ \\
\hline $\mathbf{7 . 0}$ & $\mathbf{1 1 8 2 . 2}$ & $\mathbf{1 1 8 7 . 1}$ & $\mathbf{1 2 0 5 . 6}$ & $\mathbf{1 2 1 6 . 6}$ & $\mathbf{1 2 0 2 2}$ & $\mathbf{1 2 0 8 . 1}$ \\
\hline
\end{tabular}

\begin{tabular}{|lr}
\hline All & s ft/min \\
Mean & 1197.6 \\
Min Point & 1011.3 \\
Max Point & 1316.7 \\
\hline
\end{tabular}

Flow w/o C-Pt $921 \mathrm{scfm}$ Vel Avg w/o C-Pt

$1184 \mathrm{sfpm}$

\begin{tabular}{|c|c|}
\hline Start & Finish \\
\hline 48.3 & 49.9 \\
\hline $\mathrm{N} / \mathrm{A}$ & $N / A$ \\
\hline 49 & 50.9 \\
\hline $\mathrm{N} / \mathrm{A}$ & $\mathrm{N} / \mathrm{A}$ \\
\hline 29.53 & 29.50 \\
\hline $\mathrm{N} / \mathrm{A}$ & $\mathrm{N} / \mathrm{A}$ \\
\hline $80 \%$ & $75 \%$ \\
\hline
\end{tabular}

Stack temp

Equipment temp

Ambient temp

Stack static

Ambient pressure

Total Stack pressure

Ambient humidity

1316.7

\begin{tabular}{r|l} 
Dev. fom mean & $\frac{\text { Center } 23}{\text { Mean }}$ \\
$-15.6 \%$ & Std. Dev. \\
$9.9 \%$ & COV as \%
\end{tabular}

\begin{tabular}{rrr}
$\underline{\text { Side }}$ & Bottom & $\underline{\text { Al }}$ \\
1228.2 & 1253.5 & 1240.8 \\
68.4 & 59.4 & 629 \\
5.6 & 4.7 & 51 \\
\hline
\end{tabular}

\begin{tabular}{lr} 
Instuments Used: & Cal Due \\
\cline { 2 - 2 } Fisher Scientific Barometer SN 90936818 & $12 / 07 / 12$ \\
\hline TSI VelociCalc SN T95351203001 & $12 / 17 / 2012$ \\
\hline
\end{tabular}

TSI VelociCalc SN T95351203001

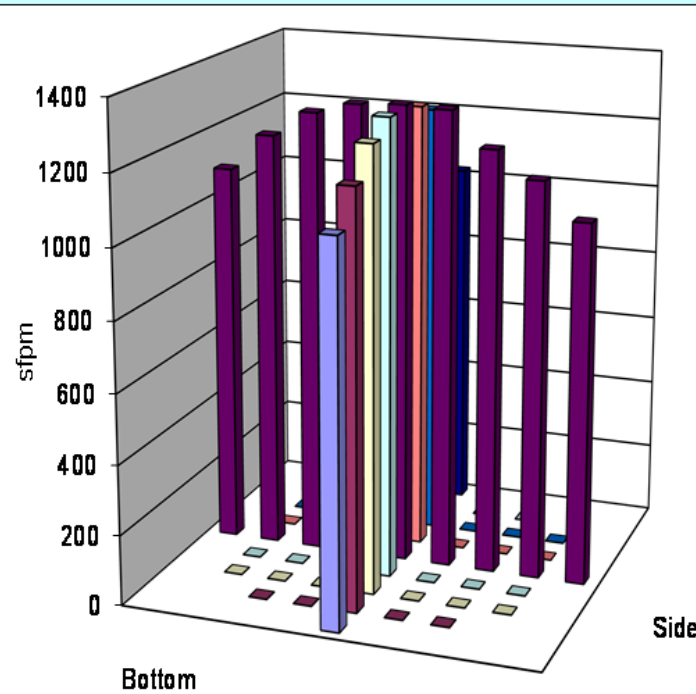

Notes

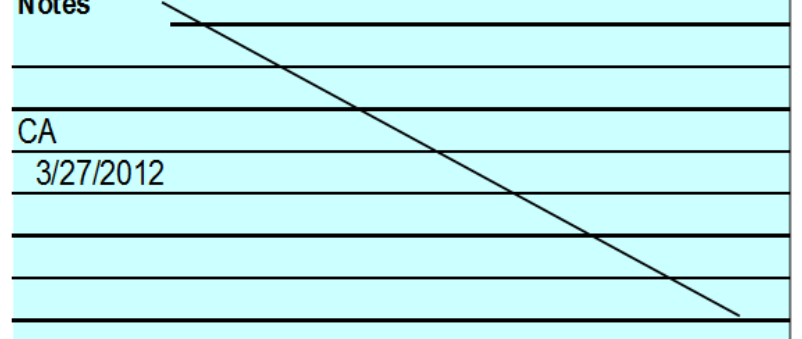
Technical Data
Signature/date
Signature/date

\section{7/13/2012}
On file with Original 


\section{VELOCITY TRAVERSE DATA FORM}

Site HV-S1 Model

Date 3/27/12

Testers $\mathrm{CA}, \mathrm{XY}$

Stack Die

Stack X-Area

Test Port

\begin{tabular}{c}
\hline 11.938 in. \\
\hline 111.9 in. 2 \\
\hline 2 \\
\hline 240 inches
\end{tabular}

Distance to disturbance

Velocity units $\underline{\mathrm{s} f t / m i n}$

Order $\rightarrow>$

Traverse->

Trial $-\rightarrow$

\begin{tabular}{|c|c|}
\hline Point & Depth, in. \\
\hline 1 & 0.50 \\
\hline 2 & 1.25 \\
\hline 3 & 2.30 \\
\hline 4 & 3.84 \\
\hline Center & 5.94 \\
\hline 5 & 8.04 \\
\hline 6 & 9.57 \\
\hline 7 & 10.63 \\
\hline 8 & 11.38 \\
\hline
\end{tabular}

Averages
Run No. VT-8

Fan Confguration FAN B ONLY

Fan Setting 28

Stack Temp

Start/End Time 1120/1135

Center $2 / 3$ from

Points in Center $2 / 3$

Data Fles: NA

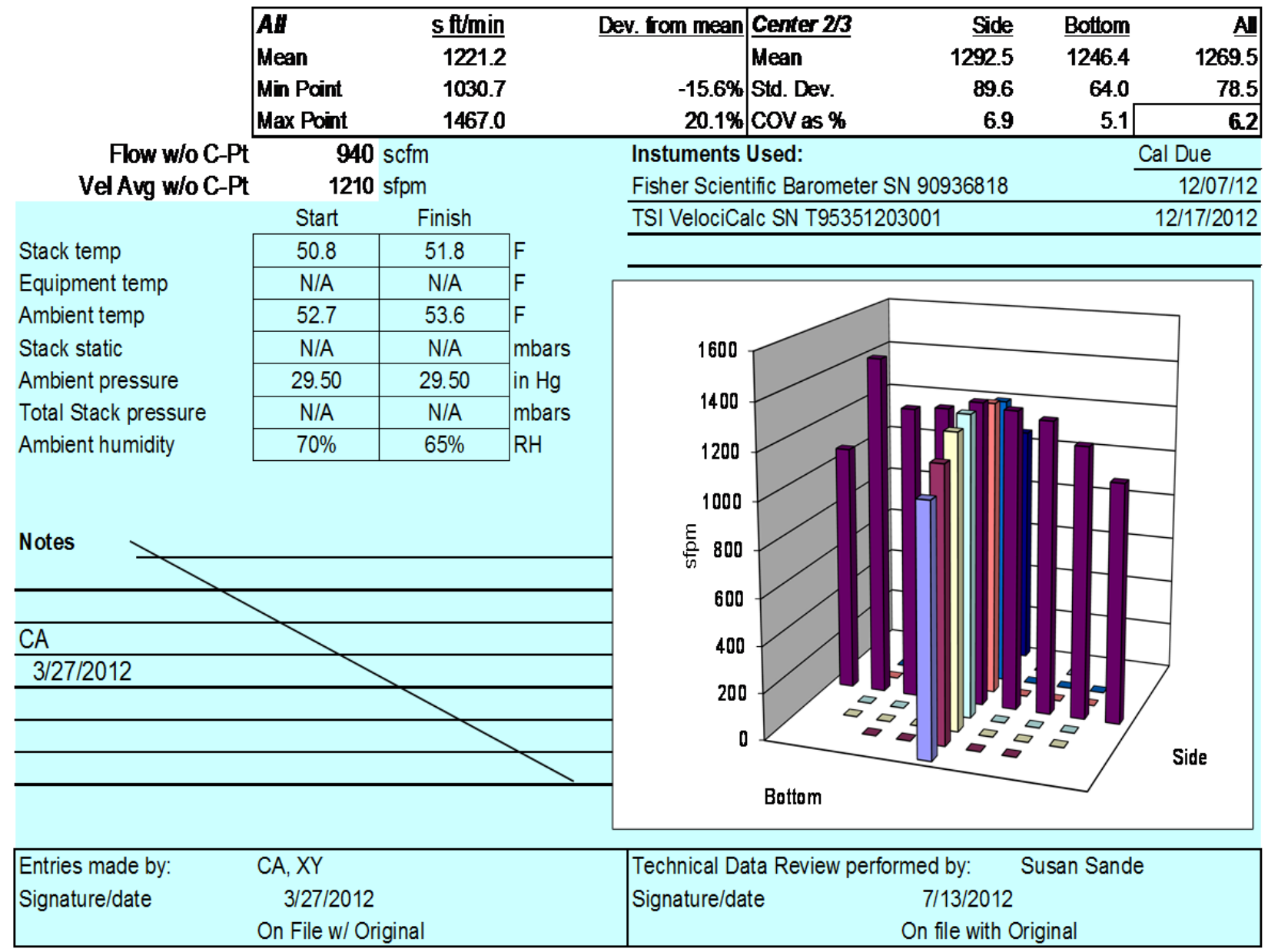




\section{VELOCITY TRAVERSE DATA FORM}

Site HV-S1 Model

Date $3 / 27 / 12$

Testers $\mathrm{XY}, \mathrm{CA}$

Stack Dia.

Stack X-Area

Test Port

Distance to disturbance

Velocity units $\mathrm{s} \mathrm{ft} / \mathrm{min}$

Order $\rightarrow$

Traverse->

Trial $->$
11.938 in.

11.9 in 2

240 inches

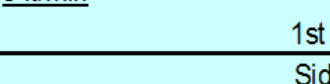

12

Run No. VT-9

Fan Confguration FAN B ONLY

Fan Setting 55

Stack Temp

$\frac{\mathrm{Hz}}{\operatorname{deg} \mathrm{F}}$

Start/End Time 1140/ 1200

Center $2 / 3$ from

Points in Center $2 / 3$

Data Fles: NA

\begin{tabular}{|c|c|c|c|c|c|c|c|c|c|}
\hline Trial -—> & & 1 & 2 & 3 & Mean & 1 & 2 & 3 & Mean \\
\hline Point & Depth, in. & \multicolumn{4}{|c|}{ Velocity } & \multicolumn{4}{|c|}{ Velocity } \\
\hline 1 & 0.50 & 2092 & 2103 & 2143 & 2112.7 & 2182 & 2148 & 2159 & 2163.0 \\
\hline 2 & 1.25 & 2302 & 2405 & 2323 & 2343.3 & 2349 & 2369 & 2406 & 2374.7 \\
\hline 3 & 2.30 & 2496 & 2513 & 2511 & 2506.7 & 2549 & 2604 & 2629 & 2594.0 \\
\hline 4 & 3.84 & 2590 & 2613 & 2605 & 2602.7 & 2677 & 2652 & 2681 & 2670.0 \\
\hline Center & 5.94 & 2641 & 2629 & 2638 & 2636.0 & 2720 & 2647 & 2714 & 2693.7 \\
\hline 5 & 8.04 & 2603 & 2609 & 2619 & 2610.3 & 2619 & 2651 & 2681 & 2650.3 \\
\hline 6 & 9.57 & 2541 & 2539 & 2538 & 2539.3 & 2551 & 2566 & 2599 & 25720 \\
\hline 7 & 10.63 & 2433 & 2456 & 2417 & 2435.3 & 2396 & 2421 & 2493 & 2436.7 \\
\hline 8 & 11.38 & 2181 & 2270 & 2280 & 2243.7 & 2258 & 2250 & 2228 & 2245.3 \\
\hline Averages - & $\rightarrow$ & 2431.0 & 2459.7 & 2452.7 & 2447.8 & 2477.9 & 2478.7 & 2510.0 & 2488.9 \\
\hline
\end{tabular}

\begin{tabular}{|lr}
\hline Af & s ft/min \\
Mean & 2468.3 \\
Min Point & 2112.7 \\
Max Point & 2693.7 \\
\hline
\end{tabular}

Flow w/o C-Pt Vel Avg w/o C-Pt

Stack temp

Equipment temp Ambient temp

Stack static

Ambient pressure

Total Stack pressure

Ambient humidity

$1900 \mathrm{scfm}$

2444 sfpm

\begin{tabular}{|c|c|}
\hline Start & Finish \\
\hline 53.1 & 55 \\
\hline$N / A$ & $\mathrm{~N} / \mathrm{A}$ \\
\hline 55.4 & 56.3 \\
\hline$N / A$ & N/A \\
\hline 29.53 & 29.50 \\
\hline$N / A$ & N/A \\
\hline $63 \%$ & $56 \%$ \\
\hline
\end{tabular}

Notes

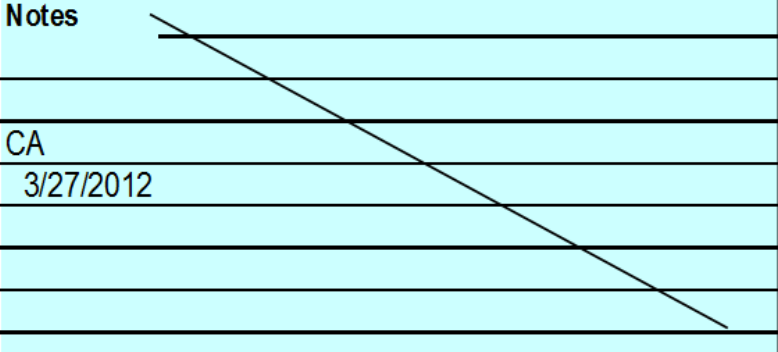
Dev. from mean Center 2/3
Mean
$-14.4 \%$ Std. Dev.
$9.1 \%$ COV as $\%$

$\frac{1.10}{2}$ to: $\quad$ to: $\underline{10.84}$

2nd

Bottom

\begin{tabular}{lr} 
Instuments Used: & Cal Due \\
\cline { 2 - 2 } Fisher Scientific Barometer SN 90936818 & $12 / 07 / 12$ \\
\hline TSI VelociCalc SN T95351203001 & $12 / 17 / 2012$
\end{tabular}

TSI VelociCalc SN T95351203001

\begin{tabular}{|c|c|c|}
\hline Side & Bottom & $\mathrm{A}$ \\
\hline 2524.8 & 2570.2 & 2547.5 \\
\hline 105.8 & 121.3 & 11. \\
\hline 4.2 & 4.7 & \\
\hline
\end{tabular}

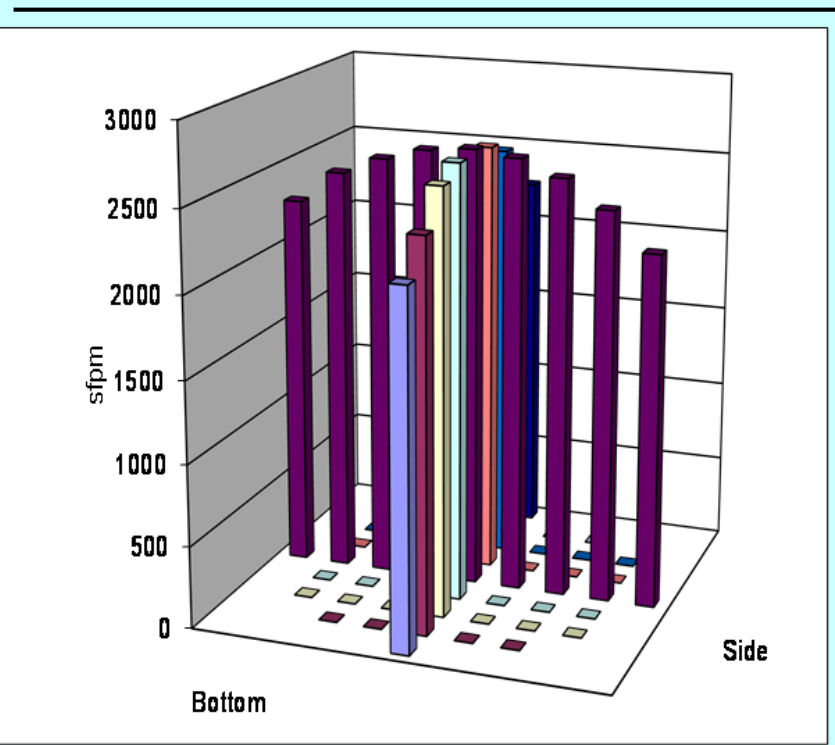

\begin{tabular}{|lc|}
\hline $\begin{array}{l}\text { Entries made by: } \\
\text { Signature/date }\end{array}$ & $3 / 27 / 2012$ \\
& On File w/ Original \\
\hline
\end{tabular}

\begin{tabular}{|l} 
Technical Data Review performed by: Susan Sande \\
$\begin{array}{l}\text { Signature/date } \\
7 / 13 / 2012 \\
\text { On file with Original }\end{array}$
\end{tabular}




\section{VELOCITY TRAVERSE DATA FORM}

Site HV-S1 Model

Date 3/27/12

Testers $\mathrm{CA}, \mathrm{XY}$

Stack Dia

Stack X-Area

Test Port

Distance to disturbance

Velocity units $\mathrm{s} \mathrm{ft} / \mathrm{min}$

Order $\rightarrow$

Traverse->

Trial $->$

\begin{tabular}{|r|r|}
\hline Point & Depth, in. \\
\hline $\mathbf{1}$ & 0.50 \\
\hline $\mathbf{2}$ & 1.25 \\
\hline $\mathbf{3}$ & 2.30 \\
\hline $\mathbf{4}$ & 3.84 \\
\hline Center & 5.94 \\
\hline $\mathbf{5}$ & 8.04 \\
\hline $\mathbf{6}$ & 9.57 \\
\hline $\mathbf{7}$ & 10.63 \\
\hline $\mathbf{8}$ & 11.38 \\
\hline
\end{tabular}

Averages -——>

11.38

\begin{tabular}{c}
\hline 11.938 in. \\
\hline $\mathbf{1 1 1 . 9}$ in. 2 \\
\hline 2 \\
\hline 240 inches
\end{tabular}

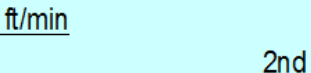

2nd

\begin{tabular}{rrr}
\cline { 2 - 3 } & & Sid \\
& $\mathbf{1}$ & $\mathbf{2}$ \\
\hline
\end{tabular}

Side
$\mathbf{2}$
Velocity

\begin{tabular}{|l|l|l|}
\hline 50 & 2187 & 2129 \\
\hline 25 & 2390 & 2367 \\
\hline 30 & 2620 & 2635 \\
\hline
\end{tabular}

84

\begin{tabular}{l|l}
\hline .94 & 2938 \\
\hline
\end{tabular}

\begin{tabular}{r|r}
\hline .04 & 293 \\
\hline .63 & 283 \\
\hline 38 & 252 \\
\hline
\end{tabular}

\begin{tabular}{|l|l|}
\hline 826 & 2800 \\
\hline 938 & 2886 \\
\hline
\end{tabular}

\begin{tabular}{l|l}
2938 & 2886 \\
2934 & 2874 \\
\hline
\end{tabular}

\begin{tabular}{l|l}
2832 & 2779 \\
\hline 2667 & 2594 \\
\hline
\end{tabular}

$\mathbf{2 6 5 7 . 7}$

$2525 \quad 2486$

\begin{tabular}{|lr}
\hline All & s ft/min \\
Mean & 2541.7 \\
Min Point & 2120.0 \\
Max Point & 2895.7 \\
\hline
\end{tabular}

Flow w/o C-Pt

$1952 \mathrm{scfm}$ Vel Avg w/o C-Pt

2512 sfpm

\begin{tabular}{|c|c|}
\hline Start & Finish \\
\hline 54.8 & 57.3 \\
\hline$N / A$ & $\mathrm{~N} / \mathrm{A}$ \\
\hline 57.2 & 60.8 \\
\hline $\mathrm{N} / \mathrm{A}$ & $\mathrm{N} / \mathrm{A}$ \\
\hline 29.50 & 29.47 \\
\hline$N / A$ & N/A \\
\hline $53 \%$ & $48 \%$ \\
\hline
\end{tabular}

Stack temp

Equipment temp

Ambient temp

Stack static

Ambient pressure

Total Stack pressure

Ambient humidity

$53 \%$

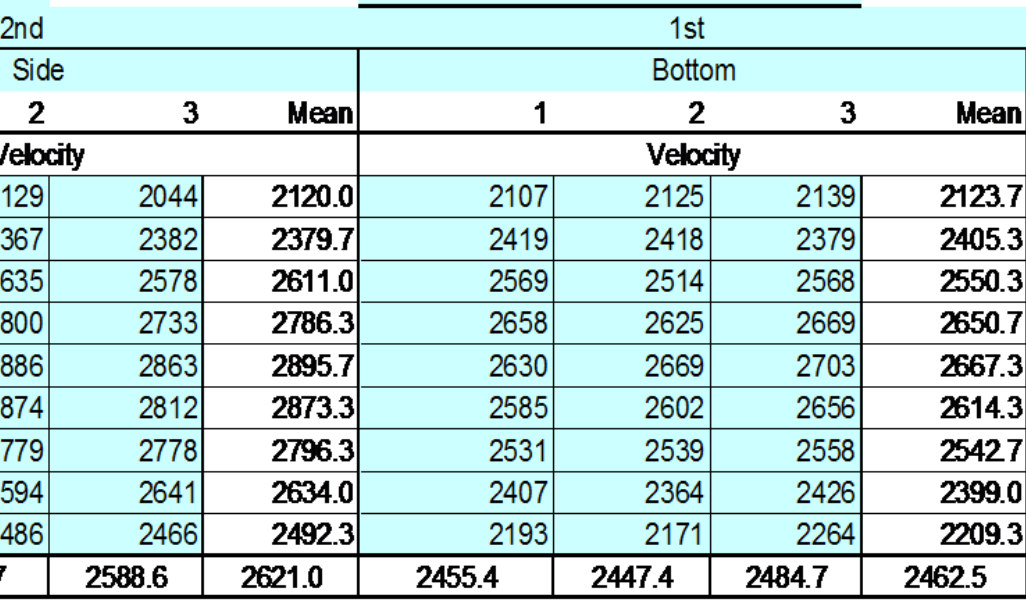

Run No. VT-10

Fan Confguration FAN B ONLY

Fan Setting 55

Stack Temp

$1205 / 1230$

Center $2 / 3$ from

Points in Center $2 / 3$

Data Files: $\frac{\overline{N A}}{1 \mathrm{st}}$ 


\section{VELOCITY TRAVERSE DATA FORM}

Site HV-S1 Model

Date 3/27/12

Testers CA, EA

Stack Dia

Stack X-Area

Test Port

Distance to disturbance

Velocity units $\underline{\mathrm{s} f t / m i n}$

Order $\rightarrow$

Traverse->

Trial $->$

\begin{tabular}{|r|r|}
\hline Point & Depth, in. \\
\hline $\mathbf{1}$ & 0.50 \\
$\mathbf{2}$ & 1.25 \\
$\mathbf{3}$ & 2.30 \\
\hline $\mathbf{4}$ & 3.84 \\
\hline Center & 5.94 \\
\hline $\mathbf{5}$ & 8.04 \\
\hline $\mathbf{6}$ & 9.57 \\
\hline $\mathbf{7}$ & 10.63 \\
\hline $\mathbf{8}$ & 11.38 \\
\hline
\end{tabular}

Averages -- -
Run No. VT-11

Fan Confguration FAN B ONLY

Fan Setting 55

Stack Temp

$\mathrm{Hz}$

$59 \quad \operatorname{deg} \mathrm{F}$

Start/End Time 1354/ 1415

Center $2 / 3$ from

Points in Center $2 / 3$

Data Fles: NA

$\begin{array}{rll}1.10 & \text { to: } & 10.84 \\ 2 & \text { to: } & \end{array}$
1st Bottom

$\begin{array}{lllllll}2 & 3 & \text { Mean } & 1 & 2 & 3 & \text { Mean }\end{array}$

$1 \quad 2$

\begin{tabular}{c|c}
1 & 2 \\
\hline & Velocity
\end{tabular}

\begin{tabular}{|l|r|r|r|r|r|r|}
\hline 2082 & 2117 & $\mathbf{2 0 8 8 . 7}$ & 2026 & 2140 & 2111 & $\mathbf{2 0 9 2 3}$ \\
\hline 2338 & 2270 & $\mathbf{2 3 1 9 . 0}$ & 2325 & 2338 & 2341 & $\mathbf{2 3 3 4 . 7}$ \\
\hline 2512 & 2500 & $\mathbf{2 4 9 3 . 7}$ & 2521 & 2505 & 2493 & $\mathbf{2 5 0 6 . 3}$ \\
\hline 2612 & 2619 & $\mathbf{2 5 9 8 . 3}$ & 2645 & 2627 & 2592 & $\mathbf{2 6 2 1 . 3}$ \\
\hline 2619 & 2658 & $\mathbf{2 6 3 2 . 7}$ & 2637 & 2610 & 2614 & $\mathbf{2 6 2 0 . 3}$ \\
\hline 2631 & 2632 & $\mathbf{2 6 2 8 . 3}$ & 2608 & 2610 & 2578 & $\mathbf{2 5 9 8 . 7}$ \\
\hline 2548 & 2567 & $\mathbf{2 5 4 4 . 3}$ & 2542 & 2502 & 2522 & $\mathbf{2 5 2 2 0}$ \\
\hline 2452 & 2470 & $\mathbf{2 4 5 9 . 7}$ & 2390 & 2402 & 2422 & $\mathbf{2 4 0 4 . 7}$ \\
\hline 2290 & 2320 & $\mathbf{2 2 8 8 . 3}$ & 2255 & 2212 & 2213 & $\mathbf{2 2 6 . 7}$ \\
\hline $\mathbf{3 . 8}$ & $\mathbf{2 4 6 1 . 4}$ & $\mathbf{2 4 5 0 . 3}$ & $\mathbf{2 4 3 8 . 8}$ & $\mathbf{2 4 3 8 . 4}$ & $\mathbf{2 4 3 1 . 8}$ & $\mathbf{2 4 3 6 . 3}$ \\
\hline
\end{tabular}

\begin{tabular}{|lrr|lrrr|}
\hline All & s ft/min & Dev. from mean & Center 2/3 & Side & Bottom & Al \\
Mean & 2443.3 & Mean & 2525.1 & 2515.4 & 2520.3 \\
Min Point & 2088.7 & $-14.5 \%$ & Std. Dev. & 112.3 & 111.2 & 107.5 \\
Max Point & 2632.7 & $7.7 \%$ & COV as $\%$ & 4.4 & 4.4 & 4.3 \\
\hline
\end{tabular}

Flow w/o C-Pt $\quad 1881 \mathrm{scfm}$ Vel Avg w/o C-Pt $2420 \mathrm{sfpm}$

\begin{tabular}{|c|c|}
\hline Start & Finish \\
\hline 59 & 58 \\
\hline $\mathrm{N} / \mathrm{A}$ & $N / A$ \\
\hline 59.9 & 59 \\
\hline $\mathrm{N} / \mathrm{A}$ & $\mathrm{N} / \mathrm{A}$ \\
\hline 29.44 & 29.44 \\
\hline $\mathrm{N} / \mathrm{A}$ & $\mathrm{N} / \mathrm{A}$ \\
\hline $46 \%$ & $50 \%$ \\
\hline
\end{tabular}

Stack temp

Equipment temp Ambient temp Stack static Ambient pressure Total Stack pressure Ambient humidity

$$
\text { Instuments Used: }
$$
Fisher Scientific Barometer SN 90936818 Cal Due TSI VelociCalc SN T95351203001

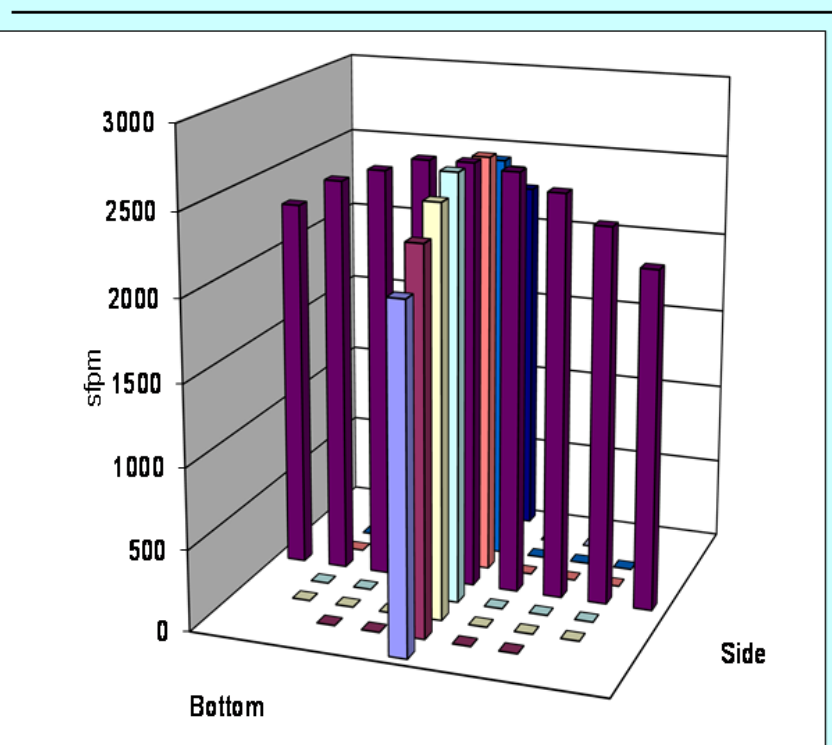

\begin{tabular}{|lc|}
\hline Entries made by: & CA, EA \\
Signature/date & $3 / 27 / 2012$ \\
& On File w/ Original
\end{tabular}

Technical Data Review performed by: Susan Sande Signature/date 7/13/2012 On file with Original 


\section{VELOCITY TRAVERSE DATA FORM}

Site HV-S1 Model

Date 3/27/12

Testers CA, EA

Stack Dia

Stack X-Area

Test Port

Distance to disturbance

Velocity units $\underline{\mathrm{s} \mathrm{f} / \mathrm{min}}$

Order $\rightarrow$

Traverse->

Trial $-\rightarrow$

\begin{tabular}{|r|r|}
\hline Point & Depth, in. \\
\hline $\mathbf{1}$ & 0.50 \\
\hline $\mathbf{2}$ & 1.25 \\
\hline $\mathbf{3}$ & 2.30 \\
\hline $\mathbf{4}$ & 3.84 \\
\hline Center & 5.94 \\
\hline $\mathbf{5}$ & 8.04 \\
\hline $\mathbf{6}$ & 9.57 \\
\hline $\mathbf{7}$ & 10.63 \\
\hline $\mathbf{8}$ & 11.38 \\
\hline
\end{tabular}

\begin{tabular}{c}
\hline 11.938 in. \\
\hline $\mathbf{1 1 1 . 9}$ in. 2 \\
\hline 1 \\
\hline 300 inches
\end{tabular}

Averages 11.38

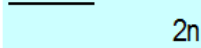

2nd

Run No. VT-12

Fan Confguration FAN B ONLY

Fan Setting 28

Stack Temp $57 \quad \mathrm{~Hz}$

Start/End Time 1418/1440

Center 2/3 from

Points in Center $2 / 3$

Data Fles: NA

\begin{tabular}{|c|c|c|}
\hline 1.10 & to: & 10.84 \\
\hline 2 & to: & 7 \\
\hline
\end{tabular}

$1 \mathrm{st}$

Side

\begin{tabular}{c|cc} 
1st \\
\hline
\end{tabular}

$1 \quad$ Side

2

3

\begin{tabular}{c|cc} 
Mean & 1 & 2 \\
& & Velocity
\end{tabular}

\begin{tabular}{|l|l|}
\hline 50 & 1024 \\
\hline 35 & 1121 \\
\hline 30 & 1183 \\
\hline 94 & 1269 \\
\hline 9.04 & 13 \\
\hline 57 & 1289 \\
\hline 63 & 1242 \\
\hline 38 & 1154 \\
\hline
\end{tabular}

\begin{tabular}{|r|r|r|r|}
\hline 1024 & 973 & 1027 & $\mathbf{1 0 0 8 . 0}$ \\
\hline 121 & 1142 & 1141 & $\mathbf{1 1 3 4 . 7}$ \\
\hline 183 & 1185 & 1202 & $\mathbf{1 1 9 0 . 0}$ \\
\hline 1269 & 1252 & 1271 & $\mathbf{1 2 6 4 . 0}$ \\
\hline 1289 & 1291 & 1312 & $\mathbf{1 3 0 4 . 7}$ \\
\hline 1242 & 1289 & 1281 & $\mathbf{1 2 8 6 . 3}$ \\
\hline 154 & 1211 & 1223 & $\mathbf{1 2 2 5 . 3}$ \\
\hline $\mathbf{3}$ & 1145 & 1163 & $\mathbf{1 1 5 4 . 0}$ \\
\hline $\mathbf{1 1 7 2 7}$ & 1066 & 1066 & $\mathbf{1 0 7 2 . 0}$ \\
\hline
\end{tabular}

\begin{tabular}{|c|}
\hline 104 \\
\hline 1142 \\
\hline 118 \\
\hline 1294 \\
\hline 129 \\
\hline 1288 \\
\hline 1247 \\
\hline 1139 \\
\hline 1030 \\
\hline $\mathbf{1 1 8 3 . 8}$ \\
\hline
\end{tabular}

\begin{tabular}{|l|l|l|l|}
\hline \multicolumn{4}{|c|}{ Velocity } \\
\hline 1041 & 1082 & 1077 & $\mathbf{1 0 6 6 . 7}$ \\
\hline 142 & 1155 & 1148 & $\mathbf{1 1 4 8 . 3}$ \\
\hline 182 & 1252 & 1230 & $\mathbf{1 2 2 1 . 3}$ \\
\hline 294 & 1318 & 1313 & $\mathbf{1 3 0 8 . 3}$ \\
\hline 291 & 1331 & 1306 & $\mathbf{1 3 0 9 . 3}$ \\
\hline 288 & 1283 & 1282 & $\mathbf{1 2 8 4 . 3}$ \\
\hline 247 & 1208 & 1205 & $\mathbf{1 2 2 0 . 0}$ \\
\hline 139 & 1153 & 1129 & $\mathbf{1 1 4 0 . 3}$ \\
\hline 030 & 1040 & 1028 & $\mathbf{1 0 3 2 7}$ \\
\hline & $\mathbf{1 2 0 2 . 4}$ & $\mathbf{1 1 9 0 . 9}$ & $\mathbf{1 1 9 2 . 4}$ \\
\hline
\end{tabular}

\begin{tabular}{|lr}
\hline All & s ft/min \\
Mean & 1187.2 \\
Min Point & 1008.0 \\
Max Point & 1309.3 \\
\hline
\end{tabular}

Flow w/o C-Pt $\quad 911 \mathrm{scfm}$

Vel Avg w/o C-Pt

1172 sfpm

\begin{tabular}{|c|c|l}
\multicolumn{1}{c|}{ Start } & \multicolumn{1}{c}{ Finish } & \\
\cline { 1 - 2 } 57.2 & 57 & $F$ \\
\cline { 1 - 2 } N/A & N/A & F \\
\cline { 1 - 2 } 58.1 & 58.1 & F \\
\cline { 1 - 2 } N/A & N/A & mbars \\
\cline { 1 - 2 } 29.44 & 29.44 & in Hg \\
\cline { 1 - 2 } N/A & N/A & mbars \\
\cline { 1 - 2 } $50 \%$ & $52 \%$ & RH \\
\hline
\end{tabular}

Stack temp

Equipment temp

Ambient temp

Stack static

Ambient pressure

Total Stack pressure

Ambient humidity

$50 \%$

(2)

\begin{tabular}{r|l} 
Dev. from mean & $\frac{\text { Center 2/3 }}{\text { Mean }}$ \\
$-15.1 \%$ & Std. Dev.
\end{tabular}

\begin{tabular}{rrr|} 
Side & Bottom & A \\
1222.7 & 1233.1 & 1227.9 \\
65.9 & 70.9 & 66.0 \\
& 5.4 & 5.4
\end{tabular}

\begin{tabular}{lr} 
Instuments Used: & Cal Due \\
\cline { 2 - 2 } Fisher Scientific Barometer SN 90936818 & $12 / 07 / 12$ \\
\hline TSI VelociCalc SN T95351203001 & $12 / 17 / 2012$ \\
\hline
\end{tabular}

TSI VelociCalc SN T95351203001

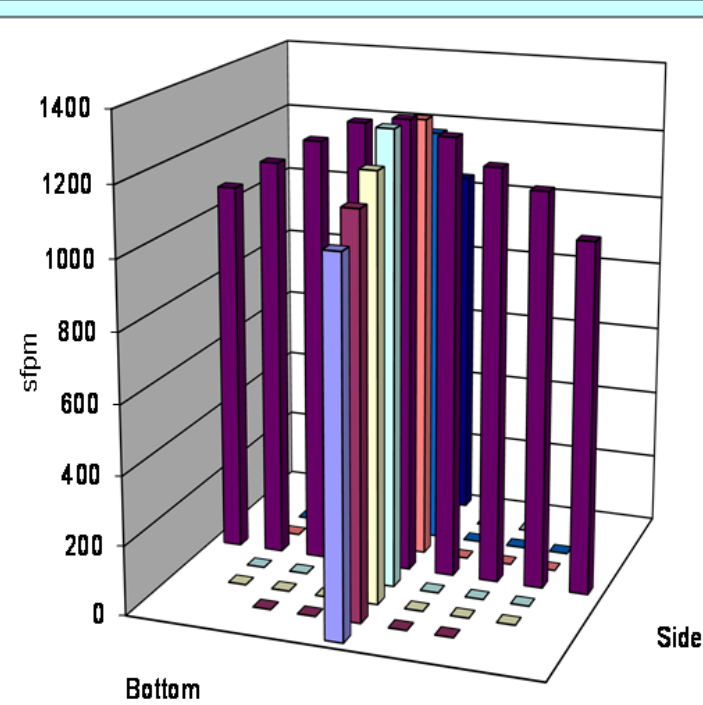

Notes

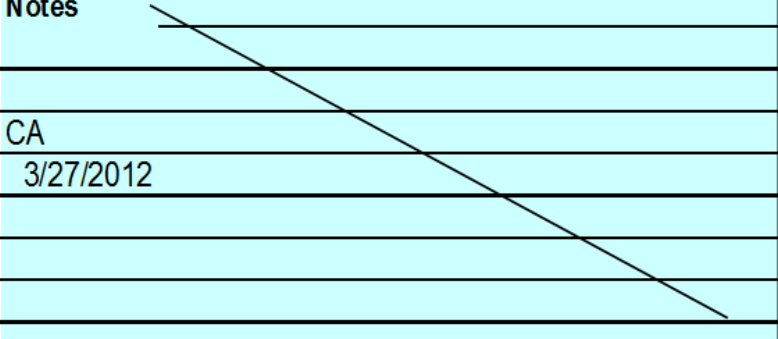

Bottom

\begin{tabular}{|lc|}
\hline Entries made by: & EA, CA \\
Signature/date & $3 / 27 / 2012$ \\
& On File w/ Original \\
\hline
\end{tabular}

Technical Data Review performed by: Susan Sande

Signature/date

$7 / 13 / 2012$

On file with Original 


\section{VELOCITY TRAVERSE DATA FORM}

Site HV-S1 Model

Date $3 / 28 / 12$

Testers YFS, EA

Stack Dia

11.938 in

Stack X-Area

Test Port 111.9 in. 2

Distance to disturbance

Velocity units $\mathrm{s} \mathrm{f} / \mathrm{min}$

Order $\rightarrow$

Traverse->

Trial $\rightarrow$ 240 inches

2nd
Run No. VT-13

Fan Confguration Fan A Only

Fan Setting 60

Stack Temp

Start/End Time $1330 / 1413$

Center $2 / 3$ from

Points in Center $2 / 3$

Data Fles: NA

\begin{tabular}{|c|c|c|c|c|c|c|c|c|c|}
\hline \multicolumn{2}{|l|}{ Trial -—> } & 1 & 2 & 3 & Mean & 1 & 2 & 3 & Mean \\
\hline Point & Depth, in. & \multicolumn{4}{|c|}{ Velocity } & \multicolumn{4}{|c|}{ Velocity } \\
\hline 1 & 0.50 & 2234 & 2201 & 2262 & 2232.3 & 2236 & 2231 & 2223 & 2230.0 \\
\hline 2 & 1.25 & 2459 & 2412 & 2455 & 2442.0 & 2500 & 2475 & 2461 & 2478.7 \\
\hline 3 & 2.30 & 2568 & 2632 & 2637 & 2612.3 & 2542 & 2590 & 2602 & 2578.0 \\
\hline 4 & 3.84 & 2617 & 2656 & 2662 & 2645.0 & 2440 & 2658 & 2613 & 2570.3 \\
\hline Center & 5.94 & 2679 & 2646 & 2668 & 2664.3 & 2611 & 2606 & 2613 & 2610.0 \\
\hline 5 & 8.04 & 2697 & 2660 & 2681 & 2679.3 & 2564 & 2570 & 2574 & 2569.3 \\
\hline 6 & 9.57 & 2600 & 2641 & 2654 & 2631.7 & 2516 & 2546 & 2563 & 2541.7 \\
\hline 7 & 10.63 & 2544 & 2570 & 2555 & 2556.3 & 2464 & 2469 & 2501 & 2478.0 \\
\hline 8 & 11.38 & 2365 & 2328 & 2415 & 2369.3 & 2330 & 2249 & 2303 & 2294.0 \\
\hline Averages - & $\longrightarrow$ & 2529.2 & 2527.3 & 2554.3 & 2537.0 & 2467.0 & 2488.2 & 2494.8 & 2483.3 \\
\hline
\end{tabular}

\begin{tabular}{|lr}
\hline All & s ft/min \\
Mean & 2510.1 \\
Min Point & 2230.0 \\
Max Point & 2679.3
\end{tabular}

Flow w/o C-Pt Vel Avg w/o C-Pt

$1939 \mathrm{scfm}$

2494 sfpm

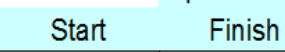

Stack temp

Equipment temp Ambient temp

Stack static

Ambient pressure

Total Stack pressure

Ambient humidity

\begin{tabular}{|c|c|}
\hline 66.7 & 67.6 \\
\hline$N / A$ & $N / A$ \\
\hline 70 & 77 \\
\hline $\mathrm{N} / \mathrm{A}$ & $\mathrm{N} / \mathrm{A}$ \\
\hline 29.47 & 29.47 \\
\hline $\mathrm{N} / \mathrm{A}$ & $\mathrm{N} / \mathrm{A}$ \\
\hline $26 \%$ & $24 \%$ \\
\hline
\end{tabular}

Notes: Barometer in direct sun. Overcast skies.

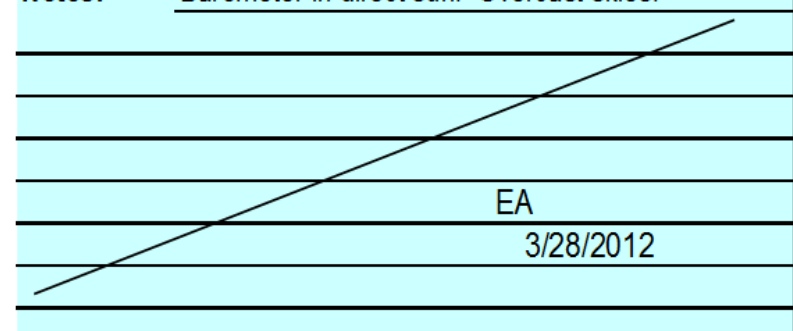

Entries made by:

Signature/date

\begin{tabular}{r|lrrr|} 
Dev. fom mean & Center 2/3 & Side & Bottom & Al \\
$-11.2 \%$ & Mean & 2604.4 & 2546.6 & 2575.5 \\
$6.7 \%$ & Std. Dev. & 82.0 & 50.7 & 721 \\
Cov as \% & 3.1 & 2.0 & 28 \\
\hline
\end{tabular}

Instuments Used:

Fisher Scientific Barometer SN 90936818 $12 / 07 / 12$ TSI VelociCalc SN T95351203001 $12 / 17 / 2012$

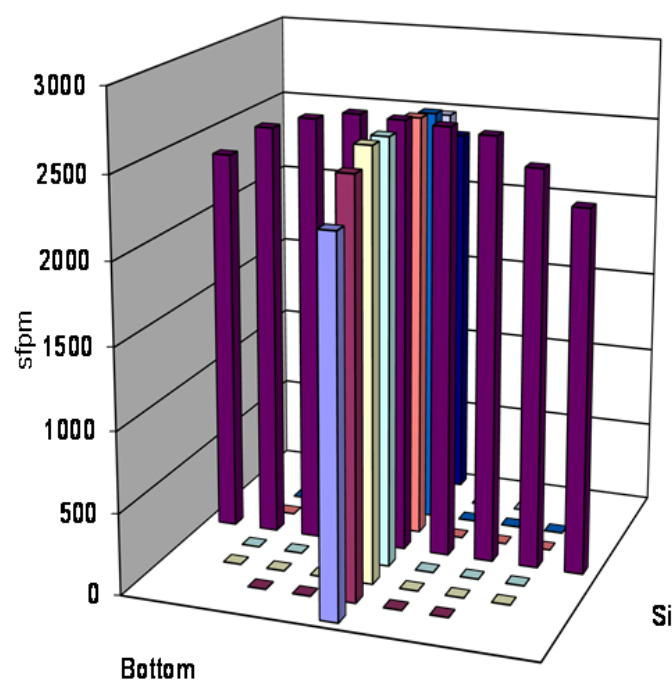

Side

\begin{tabular}{|l|l|}
\hline Technical Data Review performed by: $\quad$ Susan Sande \\
Signature/date & $7 / 13 / 2012$ \\
& On file with Original
\end{tabular}




\section{VELOCITY TRAVERSE DATA FORM}

Site HV-S1 Model

Date 3/28/12

Testers YFS EA

Stack Dia

Stack X-Area

Test Port

Distance to disturbance

Velocity units $\mathrm{s} \mathrm{ft} / \mathrm{min}$

Order $\rightarrow$

Traverse->

Trial $->$
11.938 in 111.9 in. 2 240 inches

$1 \mathrm{st}$
Run No. VT-14

Fan Confguration Fan A Only

Fan Setting 60

Stack Temp

Start/End Time 1415 / 1441

Center $2 / 3$ from

Points in Center $2 / 3$

Data Fles: NA

\begin{tabular}{|c|c|c|c|c|c|c|c|c|c|}
\hline Trial $-\longrightarrow$ & & 1 & 2 & 3 & Mean & 1 & 2 & 3 & Mean \\
\hline Point & Depth, in. & \multicolumn{4}{|c|}{ Velocity } & \multicolumn{4}{|c|}{ Velocity } \\
\hline 1 & 0.50 & 2293 & 2260 & 2226 & 2259.7 & 2231 & 2270 & 2291 & 2264.0 \\
\hline 2 & 1.25 & 2463 & 2393 & 2391 & 2415.7 & 2479 & 2507 & 2477 & 2487.7 \\
\hline 3 & 2.30 & 2585 & 2608 & 2563 & 2585.3 & 2662 & 2675 & 2646 & 2661.0 \\
\hline 4 & 3.84 & 2689 & 2686 & 2606 & 2660.3 & 2695 & 2711 & 2685 & 2697.0 \\
\hline Center & 5.94 & 2661 & 2682 & 2637 & 2660.0 & 2678 & 2668 & 2680 & $\mathbf{2 6 7 5 . 3}$ \\
\hline 5 & 8.04 & 2689 & 2716 & 2667 & 2690.7 & 2655 & 2692 & 2674 & 2673.7 \\
\hline 6 & 9.57 & 2643 & 2682 & 2610 & 2645.0 & 2565 & 2594 & 2608 & 2589.0 \\
\hline 7 & 10.63 & 2526 & 2532 & 2557 & 2538.3 & 2524 & 2466 & 2495 & 2495.0 \\
\hline 8 & 11.38 & 2456 & 2346 & 2401 & 2401.0 & 2330 & 2364 & 2354 & 2349.3 \\
\hline \multicolumn{2}{|c|}{ Averages $-\longrightarrow \longrightarrow$} & 2556.1 & 2545.0 & 2517.6 & 2539.6 & 2535.4 & 2549.7 & 2545.6 & 2543.6 \\
\hline
\end{tabular}

Flow w/o C-Pt Vel Avg w/o C-Pt

Stack temp
Equipment temp
Ambient temp
Stack static
Ambient pressure
Total Stack pressure
Ambient humidity

Notes: Overcast skies. Getting windy.

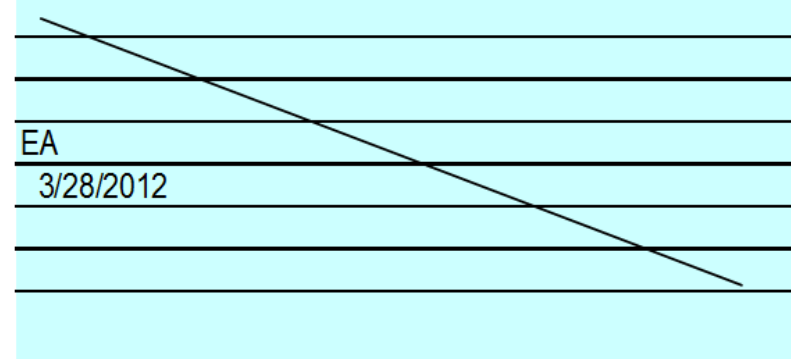

\section{$2526 \mathrm{sfpm}$}

\begin{tabular}{|c|c|l}
\multicolumn{1}{c|}{ Start } & \multicolumn{1}{c}{ Finish } & \\
\cline { 1 - 2 } 66.9 & 66.6 & $F$ \\
\cline { 1 - 2 } N/A & N/A & F \\
\cline { 1 - 2 } 77.9 & 76.1 & F \\
\cline { 1 - 2 } N/A & N/A & mbars \\
\cline { 1 - 2 } 29.47 & 29.47 & in Hg \\
\cline { 1 - 2 } N/A & N/A & mbars \\
\cline { 1 - 2 } $23 \%$ & $23 \%$ & RH \\
\hline
\end{tabular}

\begin{tabular}{|lr}
\hline All & s ft/min \\
Mean & 2541.6 \\
Min Point & 2259.7 \\
Max Point & 2697.0 \\
\hline
\end{tabular}

\begin{tabular}{r|l} 
Dev. fom mean & Center $2 / 3$ \\
$-11.1 \%$ & Mean \\
$6.1 \%$ & Cov. Dev. \\
Cov $\%$
\end{tabular}
Instuments Used: Fisher Scientific Barometer SN 90936818 TSI VelociCalc SN T95351203001

\begin{tabular}{rrrr}
$\underline{\text { Side }}$ & & Bottom & $\underline{\text { A }}$ \\
2599.3 & & 2611.2 & 2605.3 \\
$\mathbf{9 6 . 2}$ & 88.6 & 89.0 \\
3.7 & 3.4 & $\mathbf{3 . 4}$ \\
\hline
\end{tabular}

to: 10.84

2

to:

7

2nd

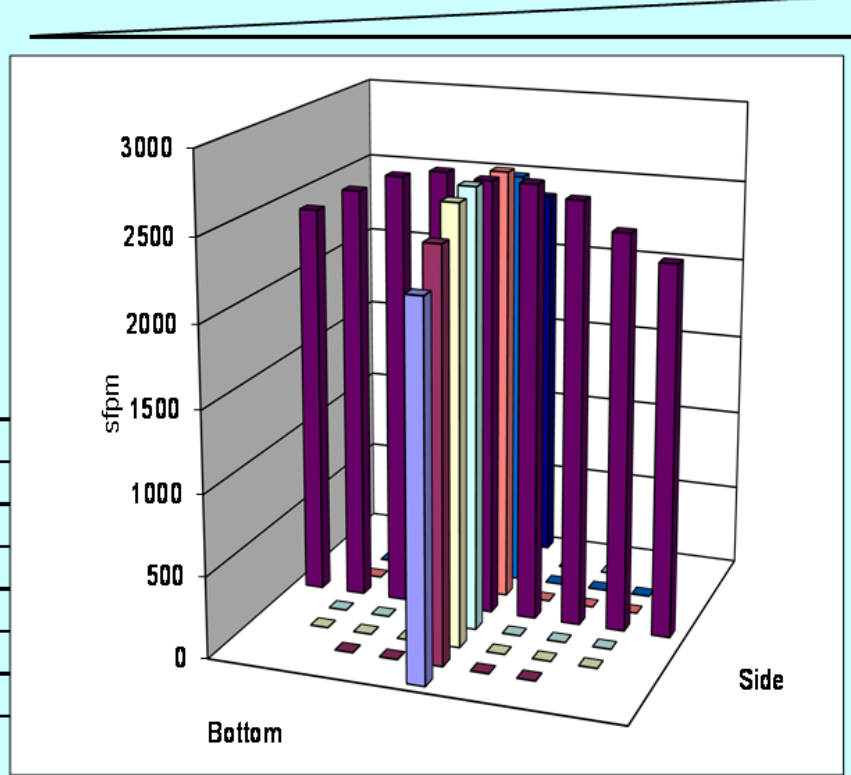

Entries made by:

Signature/date
EA
$3 / 28 / 2012$
On File w/ Origina

Technical Data Review performed by: Susan Sande Signature/date $7 / 13 / 2012$ On file with Original 


\section{VELOCITY TRAVERSE DATA FORM}

Site HV-S1 Model

Date 4/5/12

Testers JEF/XY

Stack Die

Stack X-Area

Test Port

Distance to disturbance

Velocity units $\mathrm{s} \mathrm{ft} / \mathrm{min}$

Order $\rightarrow$

Traverse->

Trial $\longrightarrow$
Run No. VT-15

Fan Confguration Fan A Only

Fan Setting 55

Stack Temp

Start/End Time 1119/1139

Center $2 / 3$ from

Points in Center $2 / 3$

Data Fles: NA

\begin{tabular}{|c|c|c|c|c|c|c|c|c|c|}
\hline \multicolumn{2}{|l|}{ Trial $\longrightarrow$} & 1 & \multirow{2}{*}{\multicolumn{2}{|c|}{$\frac{2}{\text { Velocity }}$}} & \multirow[t]{2}{*}{ Mean } & \multirow[t]{2}{*}{1} & 2 & 3 & \multirow[t]{2}{*}{ Mean } \\
\hline Point & Depth, in. & & & & & & Veloc & & \\
\hline 1 & 0.50 & 1921 & 1998 & 2005 & 1974.7 & 2127 & 2120 & 2094 & 2113.7 \\
\hline 2 & 1.25 & 2261 & 2247 & 2177 & 2228.3 & 2308 & 2299 & 2299 & 23020 \\
\hline 3 & 2.30 & 2424 & 2394 & 2441 & 2419.7 & 2489 & 2471 & 2495 & 2485.0 \\
\hline 4 & 3.84 & 2524 & 2538 & 2469 & 2510.3 & 2474 & 2445 & 2509 & 2476.0 \\
\hline Center & 5.94 & 2435 & 2471 & 2445 & 2450.3 & 2428 & 2455 & 2461 & 2448.0 \\
\hline 5 & 8.04 & 2395 & 2450 & 2420 & 2421.7 & 2405 & 2441 & 2406 & 2417.3 \\
\hline 6 & 9.57 & 2354 & 2386 & 2359 & 2366.3 & 2359 & 2346 & 2337 & 2347.3 \\
\hline 7 & 10.63 & 2312 & 2246 & 2269 & 2275.7 & 2249 & 2234 & 2220 & 2234.3 \\
\hline 8 & 11.38 & 2134 & 2167 & 2143 & 2148.0 & 2044 & 2090 & 2066 & 2066.7 \\
\hline \multicolumn{2}{|c|}{ Averages $-\longrightarrow \longrightarrow$} & 2306.7 & 2321.9 & 2303.1 & 2310.6 & 2320.3 & 2322.3 & 2320.8 & 2321.1 \\
\hline
\end{tabular}

\begin{tabular}{|lr}
\hline AH & s ft/min \\
Mean & 2315.9 \\
Min Point & 1974.7 \\
Max Point & 2510.3 \\
\hline
\end{tabular}

Flow w/o C-Pt Vel Avg w/o C-Pt

$1787 \mathrm{scfm}$

2299 sfpm

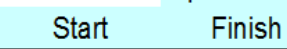

Stack temp

Equipment temp

Ambient temp

Stack static

Ambient pressure

Total Stack pressure

Ambient humidity

\begin{tabular}{|c|c|l|}
\hline 61.7 & 62 & $F$ \\
\cline { 1 - 2 } N/A & N/A & F \\
\cline { 1 - 2 } 71 & 67.1 & F \\
\cline { 1 - 2 } N/A & N/A & mbars \\
\cline { 1 - 2 } 29.74 & 29.74 & in Hg \\
\cline { 1 - 2 } N/A & N/A & mbars \\
\cline { 1 - 2 } $24 \%$ & $25 \%$ & RH \\
\hline
\end{tabular}

Notes:

Light winds

Warming up aerosol generator during test. Shut off after complete.

\section{Dev. from mean Center 2/3 Mean \\ $-14.7 \%$ Std. Dev. \\ $8.4 \%$ COV as $\%$}

Instuments Used:

Fisher Scientific Barometer SN 90936818

TSI VelociCalc SN T95351203001

\begin{tabular}{|c|c|c|}
\hline Side & Bottom & Al \\
\hline 2381.8 & 2387.1 & 2384.5 \\
\hline 99.4 & 95.1 & 93.5 \\
\hline 4.2 & 4.0 & 3.9 \\
\hline
\end{tabular}

to: $\quad 10.84$

2 to:

$1 \mathrm{st}$

o: 7

Bottom

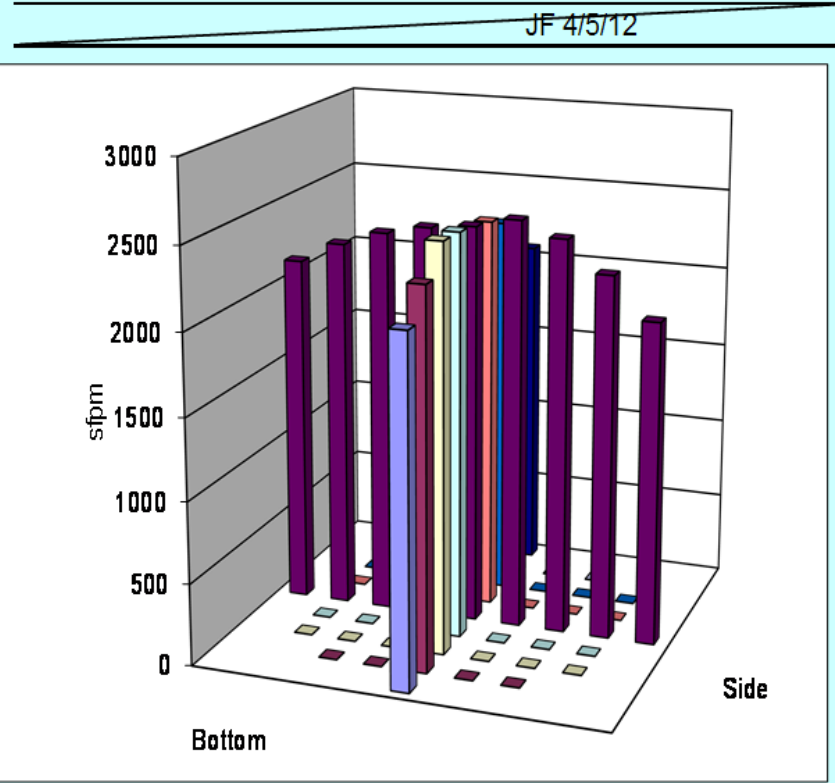

Entries made by:

JEF, XY

Signature/date

$4 / 5 / 2012$ 


\section{VELOCITY TRAVERSE DATA FORM}

Site HV-S1 Model

Date 4/5/12

Testers JEF, XY

Stack Dia.

Stack X-Area

Test Port

$\frac{11.938 \text { in. }}{\frac{111.9 \text { in. } 2}{2}}$

Distance to disturbance

Velocity units $\underline{\mathrm{s} f t / m i n}$

Order $\rightarrow$

Traverse->

Trial $->$

\begin{tabular}{|r|r|}
\hline Point & Depth, in. \\
\hline $\mathbf{1}$ & 0.50 \\
\hline $\mathbf{2}$ & 1.25 \\
\hline $\mathbf{3}$ & 2.30 \\
\hline $\mathbf{4}$ & 3.84 \\
\hline Center & 5.94 \\
\hline $\mathbf{5}$ & 8.04 \\
\hline $\mathbf{6}$ & 9.57 \\
\hline $\mathbf{7}$ & 10.63 \\
\hline $\mathbf{8}$ & 11.38 \\
\hline
\end{tabular}

Averages -—— $>$

$>$

$1 s t$

290.9

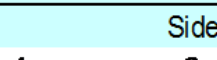

2

\begin{tabular}{l|l}
1938 & 2101 \\
\hline 2194 & 2261
\end{tabular}

234

$2552 \quad 2475$

2261

2381

2475

244

2421

\begin{tabular}{l|l}
2421 & 2402 \\
\hline 2333 & 2383 \\
\hline
\end{tabular}

$2288 \quad 2316$

\begin{tabular}{|r|r|}
\hline 21 & 21 \\
\hline & 2321.6 \\
\hline
\end{tabular}

Run No. VT-16

Fan Configuration Fan A Only

Fan Setting 55

Stack Temp

$\mathrm{Hz}$

$62 \quad \operatorname{deg} \mathrm{F}$

Start/End Time $1140 / 1155$

Center $2 / 3$ from

\begin{tabular}{rll}
1.10 & to: & 10.84 \\
\hline & to: &
\end{tabular}

Points in Center $2 / 3$

Data Files: NA

\begin{tabular}{|c|c|c|c|c|c|}
\hline & \multicolumn{5}{|c|}{ 2nd } \\
\hline \multirow[b]{2}{*}{3} & \multirow[b]{2}{*}{ Mean } & \multicolumn{3}{|c|}{ Bottom } & \multirow[b]{2}{*}{ Mean } \\
\hline & & \multirow[t]{2}{*}{1} & 2 & 3 & \\
\hline & & & \multicolumn{2}{|c|}{ Velocity } & \\
\hline 2057 & 2032.0 & 2015 & 2118 & 2082 & 2071.7 \\
\hline 2265 & 2240.0 & 2278 & 2287 & 2234 & 2266.3 \\
\hline 2348 & 2357.7 & 2486 & 2434 & 2409 & 2443.0 \\
\hline 2446 & 2491.0 & 2469 & 2436 & 2459 & 2454.7 \\
\hline 2455 & 2452.7 & 2426 & 2461 & 2423 & 2436.7 \\
\hline 2417 & 2413.3 & 2408 & 2405 & 2368 & 2393.7 \\
\hline 2356 & 2357.3 & 2349 & 2377 & 2307 & 2344.3 \\
\hline 2330 & 2311.3 & 2248 & 2235 & 2259 & 2247.3 \\
\hline 2197 & 2139.0 & 2017 & 2059 & 2058 & 2044.7 \\
\hline 9.0 & 2310.5 & 2299.6 & 2312.4 & 2288.8 & 2300.3 \\
\hline
\end{tabular}

$2101 \quad 205$

\begin{tabular}{|lr}
\hline All & s tt/min \\
Mean & 2305.4 \\
Min Point & 2032.0 \\
Max Point & 2491.0 \\
\hline
\end{tabular}

Flow w/o C-Pt

$1778 \mathrm{scfm}$

Vel Avg w/o C-Pt

2288 sfpm

\begin{tabular}{|c|c|}
\hline Start & Finish \\
\hline 62 & 61.1 \\
\hline$N / A$ & $\mathrm{~N} / \mathrm{A}$ \\
\hline 65.3 & 65.3 \\
\hline $\mathrm{N} / \mathrm{A}$ & $\mathrm{N} / \mathrm{A}$ \\
\hline 29.74 & 29.74 \\
\hline$N / A$ & N/A \\
\hline $24 \%$ & $25 \%$ \\
\hline
\end{tabular}

Stack temp

Equipment temp

Ambient temp

Stack static

Ambient pressure

Total Stack pressure

Ambient humidity

2491.0

\begin{tabular}{r|lrrrr|} 
Dev. from mean & Center 2/3 & $\underline{\text { Side }}$ & & Bottom & $\underline{\text { A }}$ \\
$-11.9 \%$ & Mean & 2374.8 & 2369.4 & 23721 \\
$8.1 \%$ & Std. Dev. & 85.5 & 85.6 & 822 \\
COV as \% & 3.6 & 3.6 & 3.5 \\
\hline
\end{tabular}

\begin{tabular}{lc} 
Instuments Used: & Cal Due \\
Fisher Scientific Barometer SN 90936818 & $12 / 07 / 12$ \\
\hline TSI VelociCalc SN T95351203001 & $12 / 17 / 2012$ \\
\hline
\end{tabular}

Notes: Light winds.
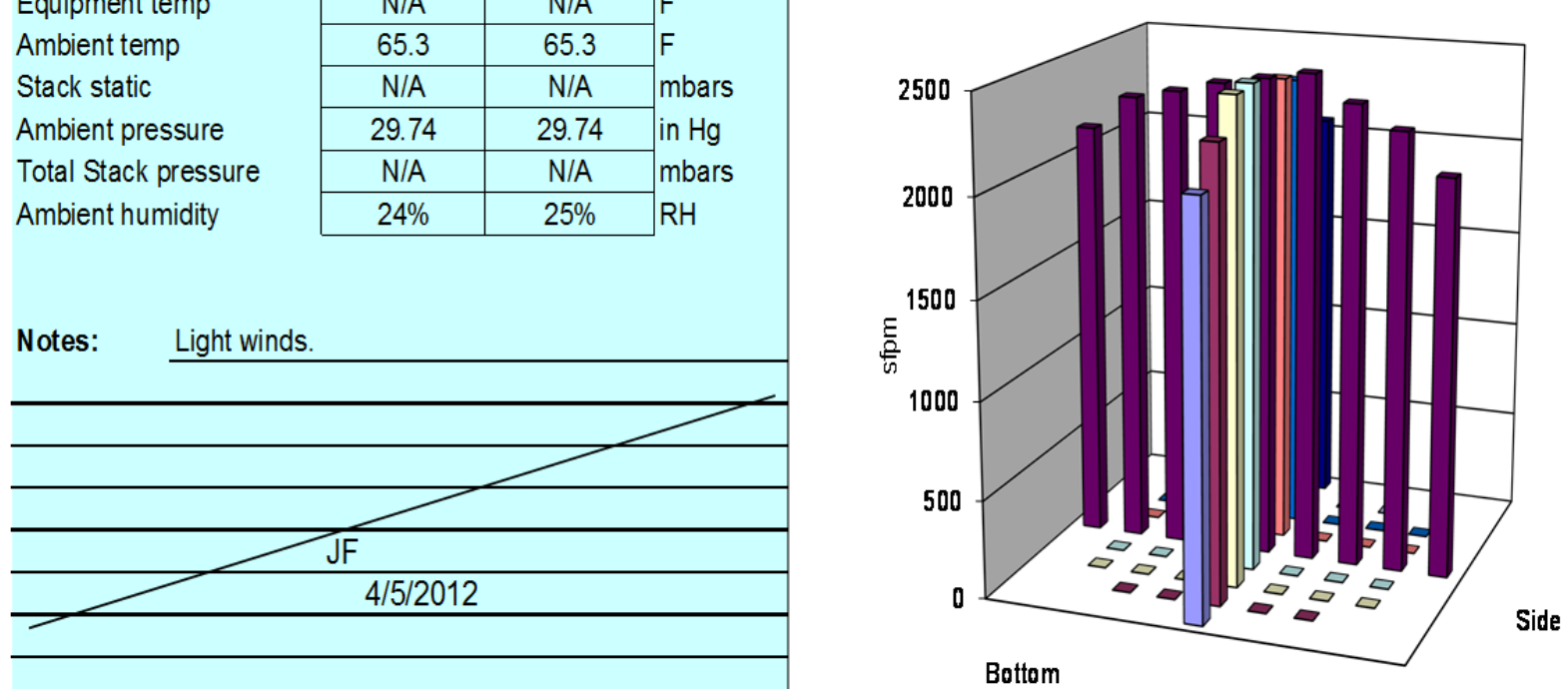

\begin{tabular}{lr}
\hline Entries made by: & $\mathrm{JF}, \mathrm{XY}$ \\
Signature/date & $4 / 5 / 2012$
\end{tabular}

Technical Data Review performed by: Susan Sande Signature/date

7/13/2012

On file with Original 


\section{A.3 HV-S1 Flow Angle Data Sheets}

FLOW ANGLE DATA FORM

\begin{tabular}{|c|c|c|}
\hline $\begin{array}{r}\text { Site } \\
\text { Date } \\
\text { Tester }\end{array}$ & $\frac{\text { HV-S1 scale }}{\frac{3 / 28 / 2012}{C A, X Y}}$ & model \\
\hline Stack Dia. & 11.938 & in \\
\hline Stack XArea & 111.9 & in2 \\
\hline Eevation & N.A. & ft \\
\hline istance to disturbance & 240 & in \\
\hline Start/End Time & 10:15/11:00 & \\
\hline
\end{tabular}

HV-S1_FlowAngle.xds

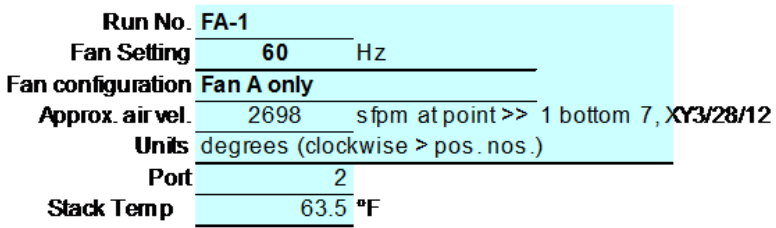

Run No. FA-1

$\begin{aligned} & \text { Fan Setting } \mathbf{6 0} \mathrm{Hz} \\ & \text { Fan configuration } \text { Fan A only } \\ & \text { Approx.air vel. } \frac{2698 \mathrm{sfpm} \text { at point }>>}{\text { Units }} \text { 1 bottom 7, XY3/28/12 } \\ & \text { Port } \\ & \text { Stack Temp (clockwise > pos. nos.) }\end{aligned}$

1 st

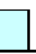

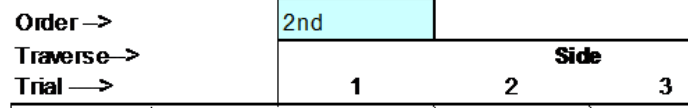

\begin{tabular}{|c|r|c|c|}
\hline Point & Depth, in. & deg. w & deg. \\
\hline $\mathbf{1}$ & 0.50 & -3 \\
\hline $\mathbf{2}$ & 1.25 & 7 \\
\hline $\mathbf{3}$ & 2.30 & 8 \\
\hline $\mathbf{4}$ & 3.84 & 5 \\
\hline Center & 5.94 & 2 \\
\hline $\mathbf{5}$ & 8.04 & 0 \\
\hline $\mathbf{6}$ & 9.57 & 1 \\
\hline $\mathbf{7}$ & 10.63 & 2 \\
\hline $\mathbf{8}$ & 11.38 & 4 & \\
\hline
\end{tabular}

Mean of absolute velues:

- $\quad$ w/o points bywall

Instuments Used:

S-type pitot

Velocitysens or

Angle indicator

Manom eter

Fisher Scientific

Note:

To assure similarhose connections

between the manom eter and pitot tube, rotating

the pitot tube assemblyclockwise drives the

meniscus to the right (to higherpos. numbers)

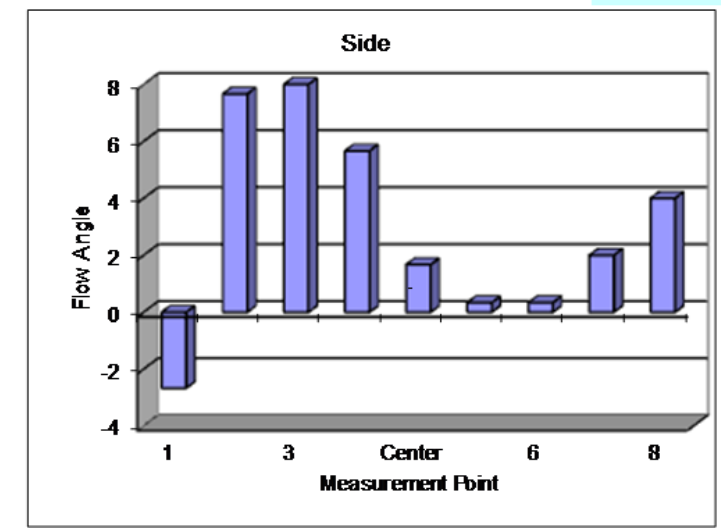

Entries made by.

Signature/date

\begin{tabular}{ll} 
Dwyer 24-inch S-type Pitot\#10 & Cal.Due \\
\cline { 2 - 2 } TSI Velocicalc SN\#T95351203001 & Cert. of conform ance \\
\hline Shop built & 17-Dec-12 \\
\hline Dwyer 400-5, S36N & Cat. 3 \\
\hline S/N 90936818 -5 & Cat. 3 \\
\hline
\end{tabular}

\section{Bottom}

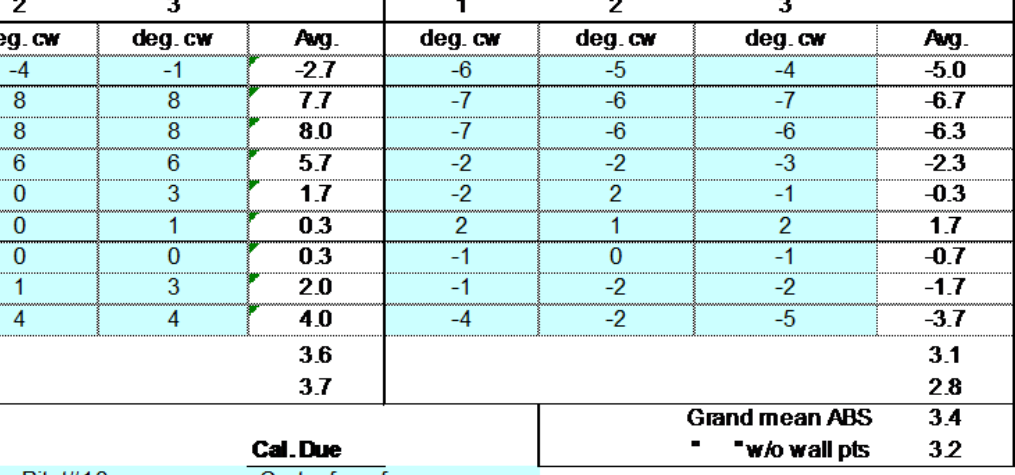

Notes: $2586 \mathrm{fpm}, 66.3 \mathrm{~F}$. This were the ending conditions
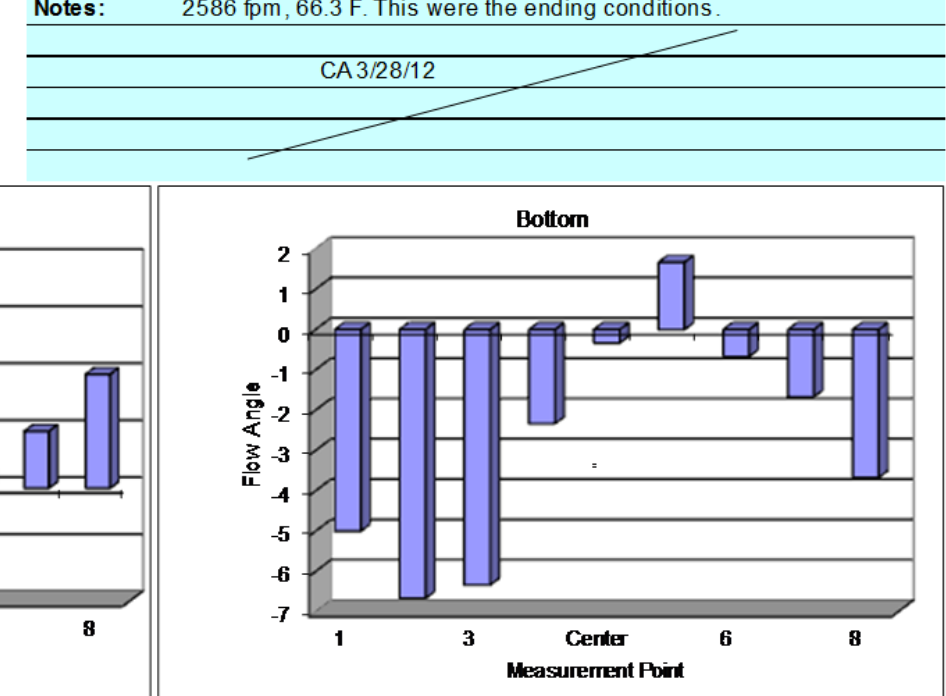

on file w/original

Technical Data Review performed by. Elizabeth Golovich Signature/date

On file with original 
FLOW ANGLE DATA FORM

Site HV-S1 scale model

Date 3/28/2012

Tester $\mathrm{XY}, \mathrm{CA}$

Stack Dia. 11.938 in

Stack X-Area 111.9 in2

Elevation N.A. $\mathrm{ft}$

Distance b disturbance 240 in

Start/End Tme 11:02/11:50
HV-S1_FlowAngle.xls

Run No. FA-2

Fan Setting $28 \mathrm{~Hz}$

Fan configuration Fan A only

Approx air vel. $1141 \quad \mathrm{sfpm}$ at point $>1$ bottom 7 Units degrees (clockwise > pos. nos. XY 3/28/12 Port

Stack Temp $\frac{2.7}{62}{ }^{\circ} \mathrm{F}$

Order $\rightarrow$

Traverse $\rightarrow$

Trial $\longrightarrow$

$$
1 \text { st }
$$

2nd

\begin{tabular}{|c|r|c|c|}
\hline Point & Depth, in. & deg.cw & deg. \\
\hline $\mathbf{1}$ & 0.50 & 2 & \\
\hline $\mathbf{2}$ & 1.25 & 8 & \\
\hline $\mathbf{3}$ & 2.30 & 8 & \\
\hline $\mathbf{4}$ & 3.84 & -3 & \\
\hline Center & 5.94 & 0 & \\
\hline $\mathbf{5}$ & 8.04 & 2 & \\
\hline $\mathbf{6}$ & 9.57 & 1 & \\
\hline $\mathbf{7}$ & 10.63 & 1 & \\
\hline $\mathbf{8}$ & 11.38 & 3 & \\
\hline
\end{tabular}

Mean of absclute velues:

- w/o points bywall:

Instuments Used

S-type pitot

Velocity sensor

Angle indicator

Manometer

Fisher Scientific

Note:

To assure similar hose connections

between the manometer and pitot tube, rotating

the pitot tube assembly clockwise drives the

meniscus to the right (to higher pos. numbers).

\section{side}

$2 n$

\begin{tabular}{ll|lll}
2 & 3 & 1 & $2^{\text {Bottom }}$
\end{tabular}

3

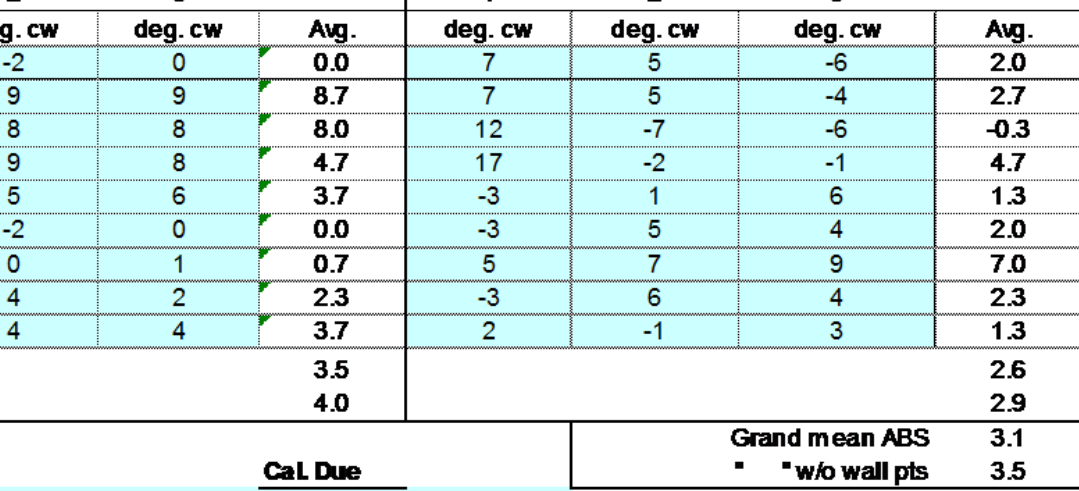

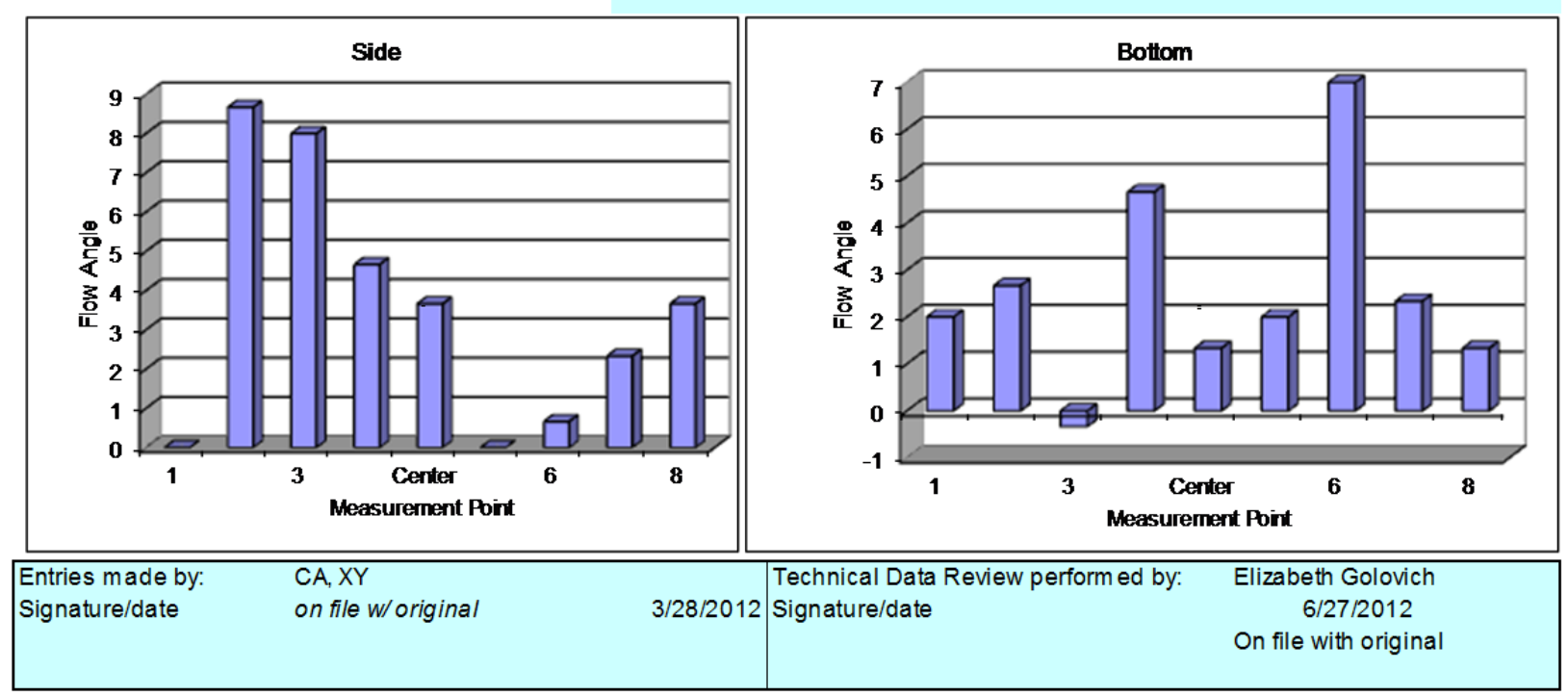


FLOW ANGLE DATA FORM

Site HV-S1 scale model

Date 3/28/2012

Tester CA, XY

Stack Dia. 11.938 in

Stack X-Area 111.9 in2

Elevation N.A. $f$ t

Distance to disturbance 240 in

StartEnd Trme 12:00/12:45
HV-S1_HowAnglexts

Run No. FA-3

Fan Seting $\quad 60 \mathrm{~Hz}$

Fan configuration B only

Approx.air vel. $2824 \quad \mathrm{sfpm}$ at point $\gg 1$ side 7

Units degrees (clockwise > pos. nos.)

Port

Stack Temp $\frac{2}{66.1}{ }^{\circ} \mathrm{F}$

Order $\rightarrow$

Traverse $\rightarrow$

Trial $\longrightarrow$

$$
\text { 2nd }
$$

\begin{tabular}{|c|c|c|c|c|}
\hline Point & Depth, in. & deg. cw & deg. cw & deg. cw \\
\hline $\mathbf{1}$ & 0.50 & 6 & -1 & 1 \\
\hline $\mathbf{2}$ & 1.25 & 1 & 2 & 2 \\
\hline $\mathbf{3}$ & 2.30 & 1 & 2 & 1 \\
\hline $\mathbf{4}$ & 3.84 & 1 & 3 & 3 \\
\hline Center & 5.94 & 0 & 3 & 3 \\
\hline $\mathbf{5}$ & 8.04 & 4 & 3 & 3 \\
\hline $\mathbf{6}$ & 9.57 & 5 & 5 & 4 \\
\hline $\mathbf{7}$ & 10.63 & 8 & 6 & 6 \\
\hline $\mathbf{8}$ & 11.38 & 6 & 6 & 7 \\
\hline
\end{tabular}

Mean of absclute velues:

- " wo points by wall:

Instuments Used

S-type pitot

Velocity sensor

Angle indicator

Manometer

Fisher Scientific

Note:

To assure similar hose connections

between the manometer and pitot tube, rotating

the pitot tube assembly clockwise drives the

meniscus to the right (to higherpos. numbers). 1st

\begin{tabular}{|c|c|c|}
\hline & \\
\hline & 1st & \\
\hline Side & & Bottom \\
\hline 3 & 1 & 2 \\
\hline
\end{tabular}

3

\begin{tabular}{|c|c|c|c|c|}
\hline Avg. & deg. cw & deg. cw & deg. cw & Avg. \\
\hline 2.0 & 9 & 3 & 6 & 6.0 \\
\hline 1.7 & 1 & 1 & -11 & -3.0 \\
\hline 1.3 & 2 & 3 & 4 & 3.0 \\
\hline 2.3 & -3 & 7 & 7 & 3.7 \\
\hline 2.0 & 5 & 1 & 4 & 3.3 \\
\hline 3.3 & 7 & 4 & 6 & 5.7 \\
\hline 4.7 & 7 & 6 & 7 & 6.7 \\
6.7 & 8 & 5 & 7 & 6.7 \\
\hline 6.3 & 9 & 9 & 9 & 9.0 \\
\hline 3.4 & & & & 5.2 \\
3.1 & & & & 4.6 \\
\hline
\end{tabular}

Cert. of conform ance

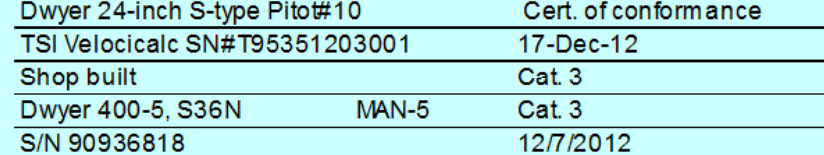
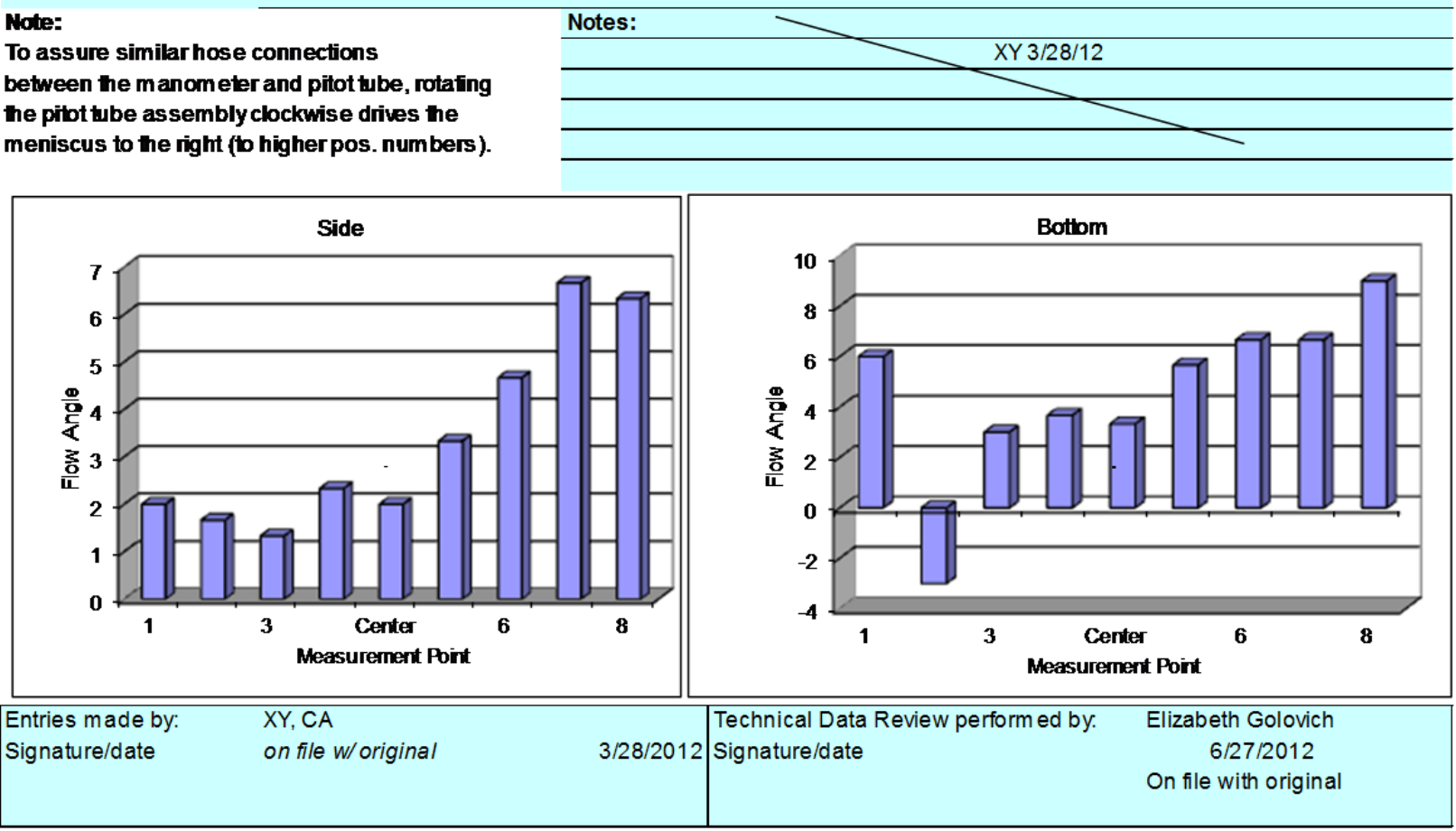
FLOW ANGLE DATA FORM

Site HV-S1 scale model

Date 3/3/0/2012

Tester XY, JEF

Stack Dia. 11.938 in

Stack X-Area 111.9 in2

Elevation N.A. $f$ t

Distance to disturbance 240 in

Start/End Tme 10:50/11:30
HV-S1_RowAnglexts

Run No. FA-4

Fan Seting $\quad 60 \mathrm{~Hz}$

Fan configuration Fan B only

Approx. air vel. $\frac{2881 \quad \mathrm{sfpm} \text { at point } \gg>}{1 \text { side } 7}$

Units degrees (clockwise > pos. nos.)

Port

Stack Temp $\frac{2}{5.5^{\circ} \mathrm{F}}$

Onder $\rightarrow$

Traverse $\rightarrow$

Trial $\longrightarrow$

$$
\text { 2nd }
$$

1 st

\begin{tabular}{|c|c|c|c|c|}
\hline Point & Depth, in. & deg. cw & deg. cw & deg. cw \\
\hline $\mathbf{1}$ & 0.50 & 6 & 2 & 4 \\
\hline $\mathbf{2}$ & 1.25 & 1 & -1 & 0 \\
\hline $\mathbf{3}$ & 2.30 & 1 & 0 & 1 \\
\hline $\mathbf{4}$ & 3.84 & 2 & 0 & 1 \\
\hline Center & 5.94 & 3 & 3 & 0 \\
\hline $\mathbf{5}$ & 8.04 & 5 & 5 & 4 \\
\hline $\mathbf{6}$ & 9.57 & 6 & 6 & 7 \\
\hline $\mathbf{7}$ & 10.63 & 7 & 7 & 8 \\
\hline $\mathbf{8}$ & 11.38 & 7 & 8 & 7 \\
\hline
\end{tabular}

Mean of absclute velues:

- " wo points by wall:

Instuments Used

S-type pitot

Velocity sensor

Angle indicator

Manometer

Fisher Scientific

Note:

To assure similar hose connections

between the manometer and pitot tube, rotaing

the pitot tube assembly clockwise drives the

meniscus to the right (to higher pos. numbers).

\begin{tabular}{l} 
Dwyer 24-inch S-type P \\
\hline TSI Velocicalc SN\#T953 \\
\hline Shop built \\
\hline Dwyer 400-5, S36N \\
\hline S/N 90936818 \\
connections \\
r and pitot tube, rotating \\
clockwise drives the \\
o higher pos. numbers).
\end{tabular}

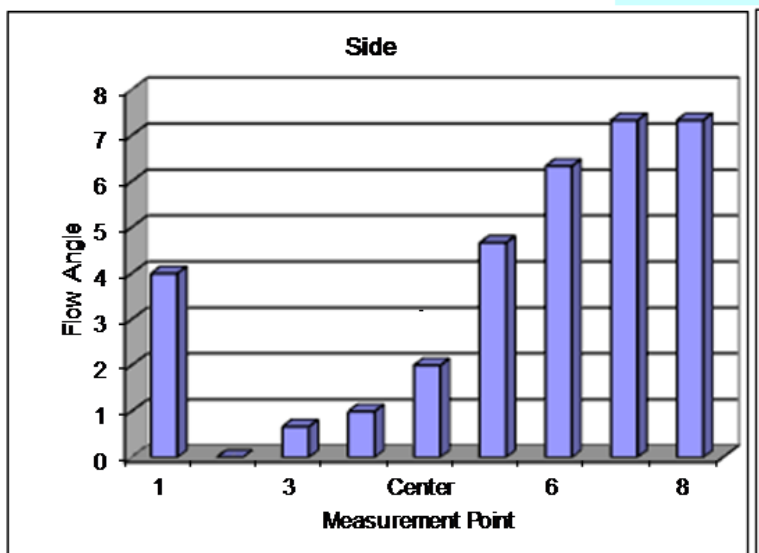

Entries made by:

Signature/date

JF

on file $w$ /original

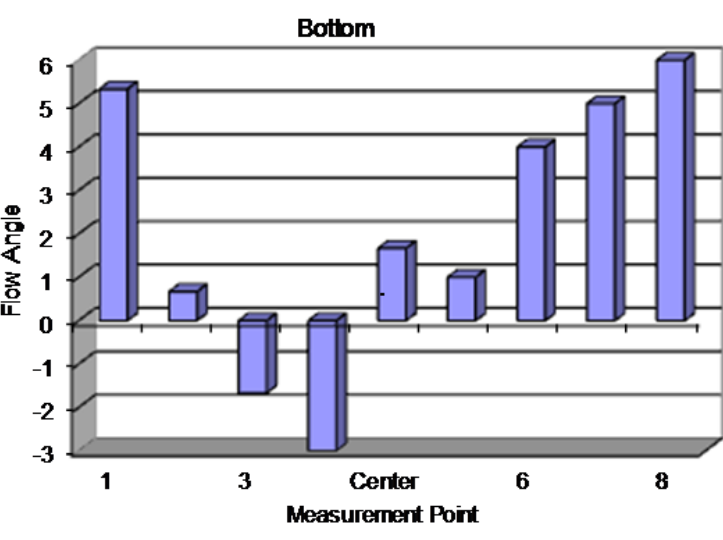

Technical Data Review perform ed by.

Elizabeth Golovich

$6 / 27 / 2012$

On file with original 
FLOW ANGLE DATA FORM

Site HV-S1 scale model

Date 3/30/2012

Tester JEF, XY

Stack Dia. 11.938 in

Stack X-Area 111.9 in2

Elevation N.A. $\mathrm{ft}$

Distance bo disturbance 240 in

Start/End Tme 11:30/12:00
HV-S1_FlowAngle.xls

Run No. FA-5

Fan Setting $\quad 55 \mathrm{~Hz}$

Fan configuration Fan B only

Approx airvel. 2427 sfpm at point $>>$

.

Port $\frac{2}{553}$

Stack Temp

$55.3^{\circ} \mathrm{F}$

2nd

Order $\rightarrow$

Traverse->

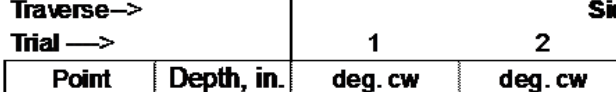

Side $\quad$ Bottom

3

\begin{tabular}{|c|r|c|c|c|}
\hline Point & Depth, in. & deg. $\mathbf{c w}$ & deg. cw & deg. $\mathbf{c w}$ \\
\hline $\mathbf{1}$ & 0.50 & 8 & 7 & 8 \\
\hline $\mathbf{2}$ & 1.25 & 1 & 0 & -1 \\
\hline $\mathbf{3}$ & 2.30 & 1 & -1 & 1 \\
\hline $\mathbf{4}$ & 3.84 & -1 & -3 & 2 \\
\hline Center & 5.94 & 10 & 11 & 11 \\
\hline $\mathbf{5}$ & 8.04 & 8 & 7 & 9 \\
\hline $\mathbf{6}$ & 9.57 & 10 & 10 & 9 \\
\hline $\mathbf{7}$ & 10.63 & 8 & 9 & 10 \\
\hline $\mathbf{8}$ & 11.38 & 10 & 10 & 8 \\
\hline
\end{tabular}

Mean of absclute velues:

- w/o points bywal:
Instuments Used

S-type pitot

Velocity sensor

Angle indicator

Manometer

Fisher Scientific

Note:

To assure similar hose connections

between the manometer and pitot tube, rotating

the pitot tube assembly clockwise drives the

meniscus to the right (to higher pos. numbers).

Shop built

Dwyer 400-5, S36N

S/N 90936818
Dwyer 24-inch S-type Pito\#10 TSI Velocicalc SN\#T95351203001

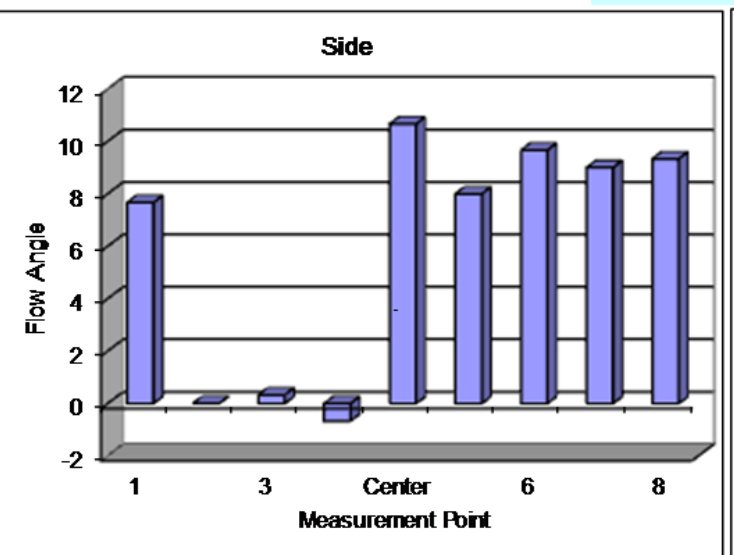

Entries made by:

Signature/date

on file $w /$ original

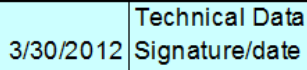

CaL Due

Cert. of conform ance

17-Dec-12

Cat. 3

Cat. 3

$12 / 7 / 2012$

Notes: Side center takes a lot to get to zero, even though the oil level is pretty close.

$\mathrm{XY} 3 / 30 / 12$

\begin{tabular}{l|l|l|l} 
deg. cw & deg. cw & deg. cw & Avg.
\end{tabular}

\begin{tabular}{c|c|c|c}
\hline-2 & 9 & 0 & 2.3 \\
\hline 1 & 2 & 2 & 17
\end{tabular}

\begin{tabular}{|l|l|l|l|}
\hline 1 & 2 & 2 & 1.7 \\
1 & 2 & 1 & 13
\end{tabular}

$2 \quad 2$

5

$\frac{5}{4}$

$4 \quad \frac{4}{4}$

\begin{tabular}{c|c|c}
4 & 5 \\
5 & 6 & 6 \\
5 & 6
\end{tabular}

5

\begin{tabular}{|c|c}
6 & 5.7 \\
\hline 7 & 5.3 \\
& 32
\end{tabular}


FLOW ANGLE DATA FORM

Site HV-S1 scale model

Date 4/2/2012

Tester XY, CA

Stack Dia. 11.938

Stack X-Area 1119

Elevation $\mathrm{N}$.A. $\mathrm{ft}$

Distance to distumance 240 in

Start/End Time 940/1020
HV-S1 RowAngle xts

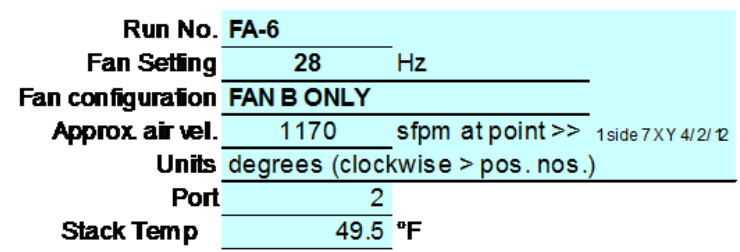

Run No. FA-6

Fan Seting 28

Approx air vel. $1170 \quad$ sfpm at point $>>1$ side 7 XY 4/2/ R Units degrees (clockwise > pos. nos.)

Stack Temp $49.5{ }^{\circ} \mathrm{F}$

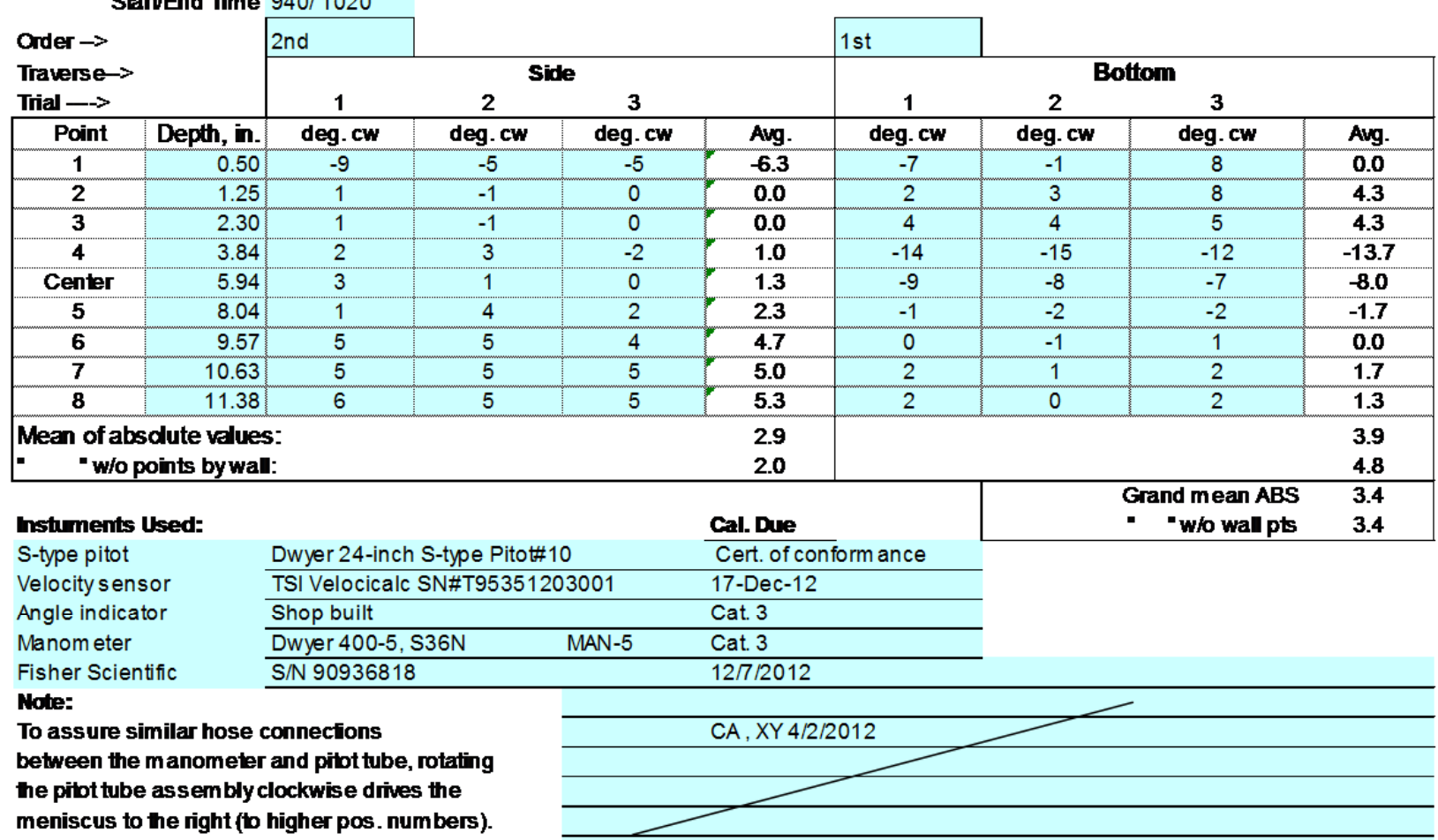

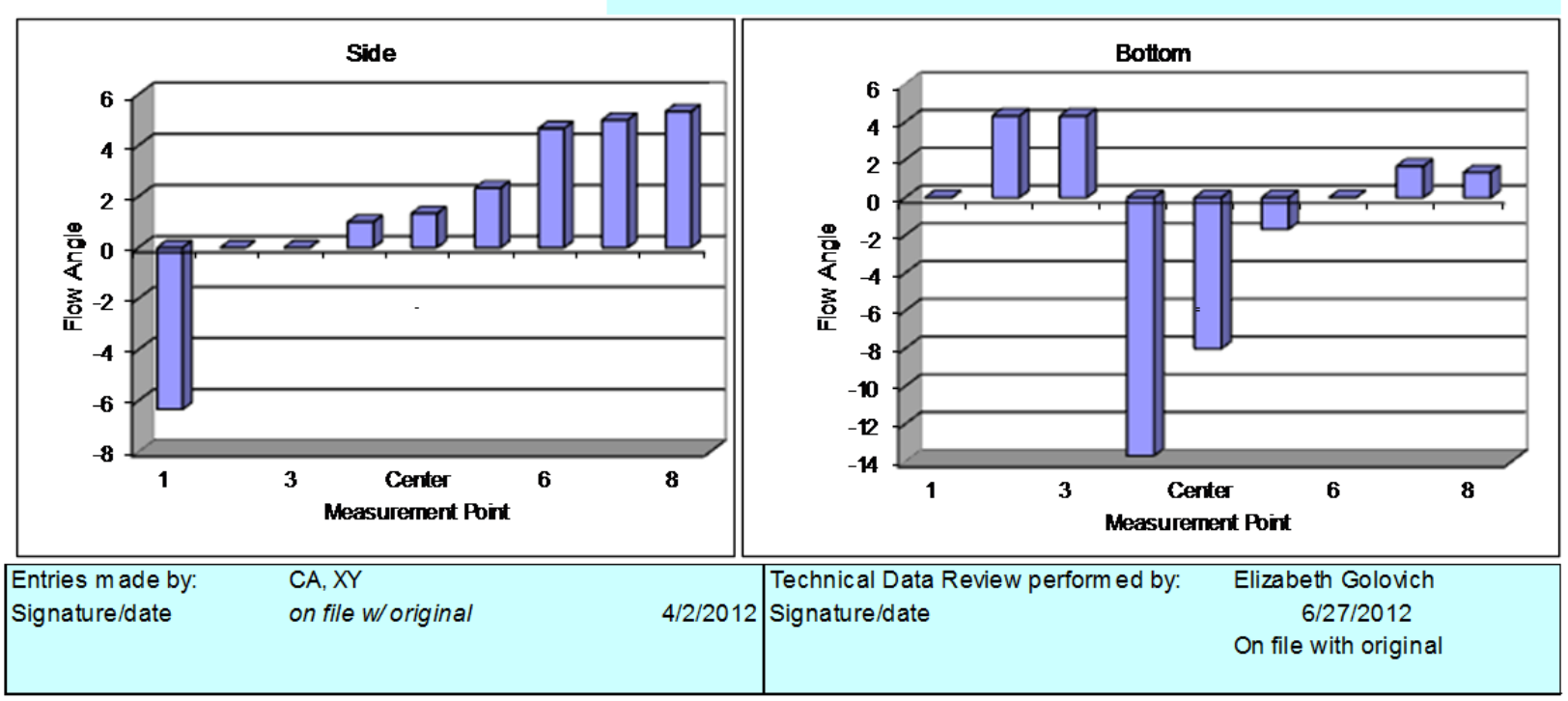


FLOW ANGLE DATA FORM

Site HV-S1 scale mode

Date $4 / 2 / 2012$

Tester $\mathrm{CA}, \mathrm{XY}$

Stack Dia. 11.938 in

Stack X-Area 111.9 in2

Elevation N.A. $\mathrm{ft}$

Distance to disturbance 300 in

Start/End Tme $1030 / 1130$
HV-S1_FowAngle.xls

Run No. FA-7

Fan Seting $\quad 60 \mathrm{~Hz}$

Fan configuration FAN B ONLY

Approx air vel. 2669 sfpm at point $\gg$ 1 1 side $7 \times$ XY 4/212 Units degrees (clockwise $>$ pos. nos.)

Port

Stack Temp ${ }^{51.7}{ }^{\circ} \mathrm{F}$

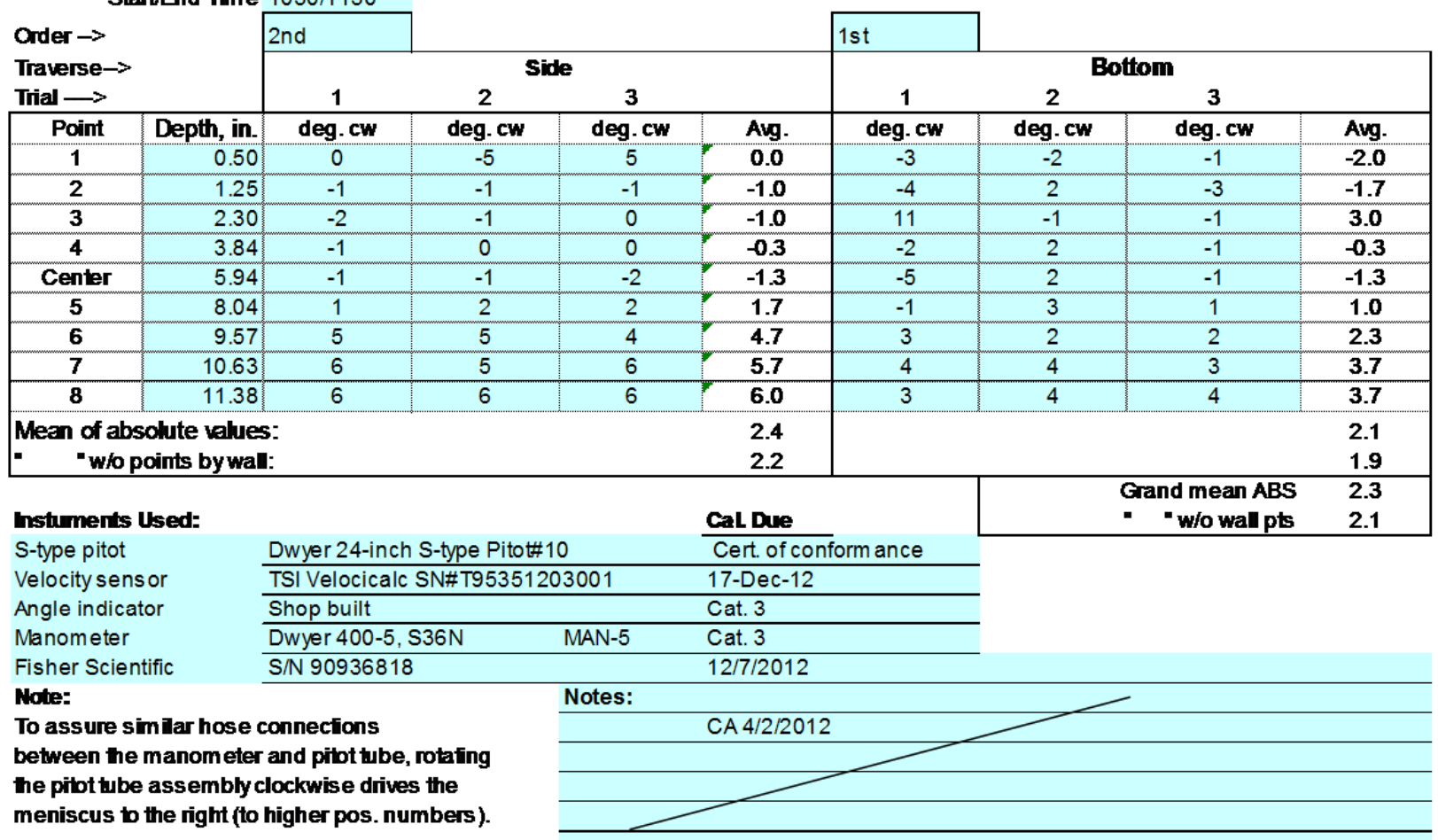

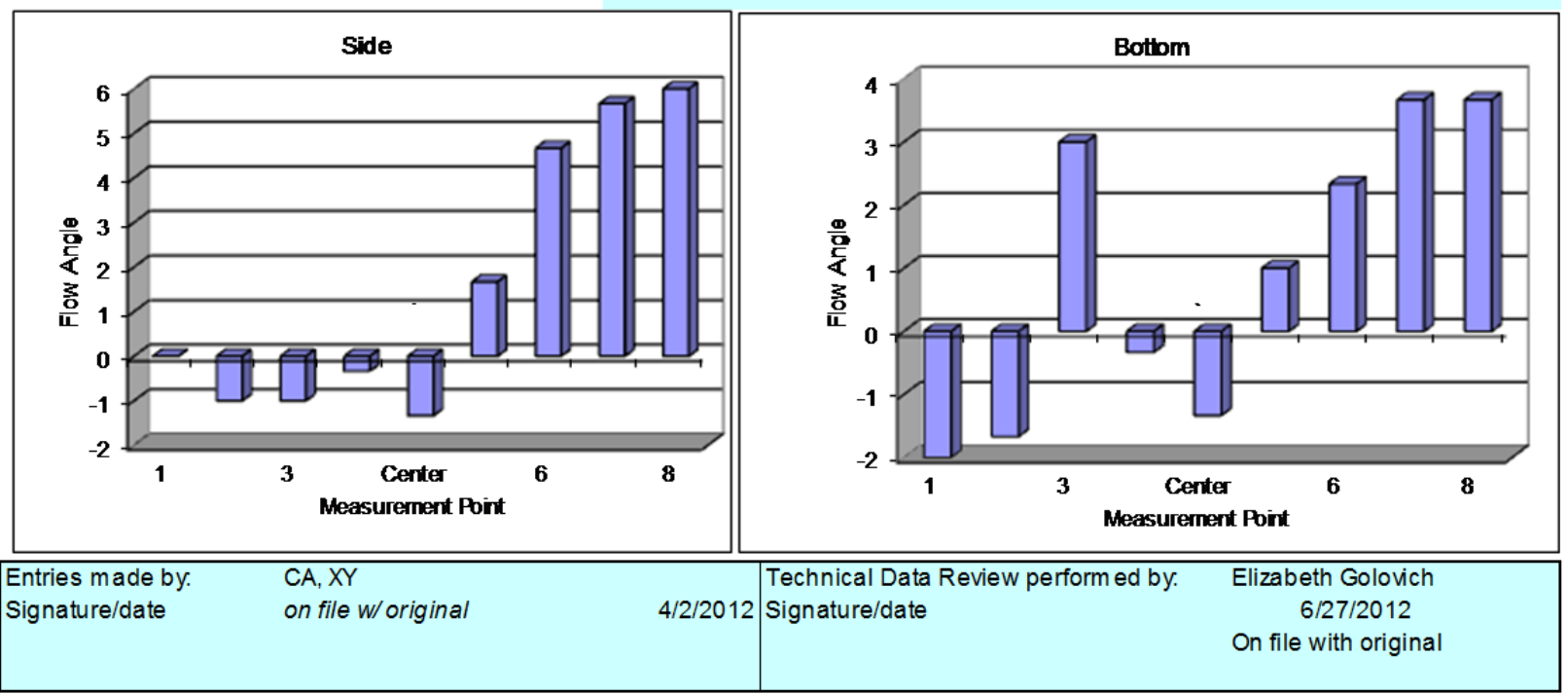




\section{A.4 HV-S1 Gas Tracer Calibration and Uniformity Data Sheets}

\section{SULFUR HEXAFLUORIDE GAS INSTRUMENT CALIBRATION}

\begin{tabular}{|c|c|c|}
\hline \multicolumn{2}{|c|}{ Site HV-S1 Model } & Instrument B\&K Model 1302 \\
\hline Date/Time & 4/9/2012 14:35 & Serial No. 1765299 \\
\hline Testers & CA, JEF, YS, XY & Property No. WD17210 \\
\hline Setup: & $7.7 \mathrm{ft}$ & B\&K sample inlet tube length \\
\hline \multicolumn{3}{|c|}{1008 mbar station pressure } \\
\hline \multicolumn{3}{|c|}{77.00 deg F ambient temp analyzer corrects to 20 deg $C$} \\
\hline \multicolumn{3}{|c|}{$22 \%$ percent $\mathrm{RH}$ ambient humidity } \\
\hline \multicolumn{3}{|c|}{$\begin{array}{l}\text { Pre-Test background, ppb } \\
\text { Not compensating for water vapor, monitoring task } 2\end{array}$} \\
\hline \multicolumn{3}{|c|}{$\begin{array}{r}17.9,17.4,20.7,20.1,15.1 \\
\text { Compensating for water vapor, monitoring task } 1\end{array}$} \\
\hline & $8.25,6.09,5.39,6.8 \varepsilon$ & $3,1.15$ \\
\hline
\end{tabular}

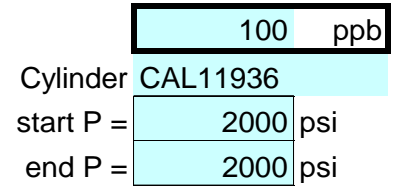

$B \& K$

Calibration

readings: (ppb)

Compensating for water vapor

\begin{tabular}{|r|}
\hline 105 \\
\hline 105 \\
\hline 102 \\
\hline 104 \\
\hline 102 \\
\hline
\end{tabular}

Not compensating for water vapor

\begin{tabular}{|r|}
\hline 103 \\
\hline 99.8 \\
\hline 99.0 \\
\hline 100 \\
\hline 100 \\
\hline $102=a v g$ \\
$1.02=$ avg/standard
\end{tabular}

Standards Used:

Air Liquide 0.1 ppm SF6 in air, CAL11936

Air Liquide 4.97 ppm SF6 in air, FF34346

Weather Station Used:

Fisher Scientific S/N 90936818

\begin{tabular}{|c|c|c|}
\hline Entries made by: & Carmen Arimescu & Technical Data Review performed by: \\
\hline Signature/date & Signature on file with original & $\begin{array}{ll}\text { Signature/date } & 7 / 16 / 2012 \\
& \text { Signature on file with Original } \\
\text { TI-WTPSP-070 }\end{array}$ \\
\hline
\end{tabular}

\begin{tabular}{|c|c|c|}
\hline & \multicolumn{2}{|c|}{ ppm } \\
\hline Cylinder & FF34346 & \\
\hline start $\mathrm{P}=$ & 1750 & ps \\
\hline end $P=$ & 1650 & ps \\
\hline
\end{tabular}

B\&K

Calibration

readings: (ppm)

Compensating for water vapor

\begin{tabular}{|r|}
\hline 5.17 \\
\hline 5.16 \\
\hline 5.15 \\
\hline 5.14 \\
\hline 5.13 \\
\hline
\end{tabular}

Not compensating for water vapor

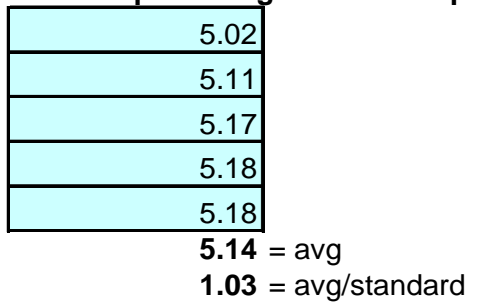

Expiration date:

$3 / 19 / 2013$

$3 / 19 / 2014$

$12 / 7 / 2012$ 


\section{SULFUR HEXAFLUORIDE GAS INSTRUMENT CALIBRATION}

$\begin{aligned} \text { Site } & \frac{\text { HV-S1 Model }}{\text { Date/Time }} \underline{\text { 4/16/2012 9:00am }} \\ \text { Testers } \underline{\text { CA, XY }} & \end{aligned}$

Instrument B\&K Model 1302

Serial No. 1765299

Property No. WD17210

Setup:

$7.7 \mathrm{ft} \quad$ B\&K sample inlet tube length

1004 mbar station pressure

59.9 deg F ambient temp analyzer corrects to 20 deg C

$43 \%$ percent $\mathrm{RH}$ ambient humidity

Pre-Test background, ppb
Not compensating for water vapor, monitoring task
$29.8,31.8,36.6,31.3,32.5$
Compensating for water vapor, monitoring task 1
$5.41,5.27,1.94,4.71,4.28$

$\begin{aligned} & 100 \mathrm{ppb} \\ \text { Cylinder CAL11936 } & \\ \text { start } P= & 1710 \text { psi } \\ \text { end } P= & 1710 \text { psi }\end{aligned}$

B\&K

Calibration

readings: (ppb)

Compensating for water vapor

\begin{tabular}{|r|}
\hline 104 \\
\hline 103 \\
\hline 101 \\
\hline 101 \\
\hline 102 \\
\hline
\end{tabular}

Not compensating for water vapor

\begin{tabular}{|r|}
\hline 103 \\
\hline 101 \\
\hline 101 \\
\hline 101 \\
\hline 101 \\
\hline $102=a v g$ \\
$1.02=$ avg/standard
\end{tabular}

Standards Used: \begin{tabular}{ll}
\hline Air Liquide & 0.1 ppm SF6 in air, CAL11936 \\
\hline Air Liquide & 4.97 ppm SF6 in air, FF34346
\end{tabular} Weather Station Used:

Fisher Scientific S/N 90936818

\begin{tabular}{|c|c|c|}
\hline & 4.97 & ppm \\
\hline \multicolumn{3}{|c|}{ Cylinder FF34346 } \\
\hline st & 1600 & psi \\
\hline end $P=$ & 1600 & psi \\
\hline
\end{tabular}

B\&K

Calibration

readings: (ppm)

Compensating for water vapor

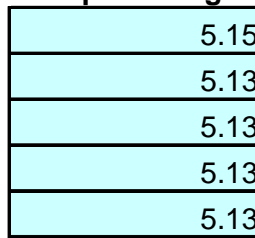

Not compensating for water vapor

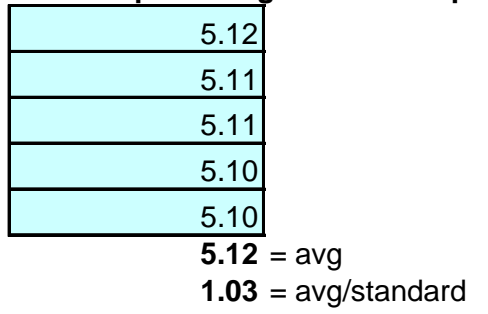

Expiration date:

$3 / 19 / 2013$

$3 / 19 / 2014$

$12 / 7 / 2012$

\begin{tabular}{|ll|lc|}
\hline $\begin{array}{l}\text { Entries made by: } \\
\text { Signature/date }\end{array}$ & Carmen Arimescu & \multicolumn{1}{|c|}{$\begin{array}{l}\text { Technical Data Review performed by: } \\
\text { Signature/date }\end{array}$} & $\begin{array}{c}\text { Susan Sande } \\
\text { Signature on file with Original } \\
\text { TI-WTPSP-070 }\end{array}$ \\
\hline
\end{tabular}




\section{SULFUR HEXAFLUORIDE GAS INSTRUMENT CALIBRATION}

$\begin{array}{rll}\text { Site } & \text { HV-S1 Model } \\ \text { Date/Time } & \text { 4/16/2012 } & 12: 35 \text { PM } \\ \text { Testers } \mathrm{CA}, \mathrm{XY}, \mathrm{EA} & \end{array}$

$\begin{aligned} & \text { Instrument } \text { B\&K Model } 1302 \\ & \text { Serial No. } 1765299 \\ & \text { Property No. WD17210 }\end{aligned}$

Setup: $7.7 \mathrm{ft}$

B\&K sample inlet tube length 1004 mbar station pressure 59.9 deg F ambient temp analyzer corrects to 20 deg C $65 \%$ percent $\mathrm{RH}$ ambient humidity

\begin{tabular}{|}
$\begin{array}{c}\text { Pre-Test background, ppb } \\
\text { Not compensating for water vapor, monitoring task } 2 \\
34,33,32,29,34 \\
\quad \text { Compensating for water vapor, monitoring task } 1 \\
-3,6,-5,0,0\end{array}$ \\
\hline
\end{tabular}

\begin{tabular}{rrr|}
\cline { 2 - 2 } & 100 & $\mathrm{ppb}$ \\
Cylinder CAL11936 & \\
start $\mathrm{P}=$ & $1600 \mathrm{psi}$ \\
end $\mathrm{P}=$ & $1600 \mathrm{psi}$
\end{tabular}

B\&K

Calibration readings: (ppb)

Compensating for water vapor

\begin{tabular}{|r|}
\hline 108 \\
\hline 110 \\
\hline 106 \\
\hline 105 \\
\hline 104 \\
\hline
\end{tabular}

\begin{tabular}{|r|}
\hline Not compensating for water vapor \\
\hline 108 \\
\hline 108 \\
\hline 110 \\
\hline 107 \\
\hline 107 \\
\hline $107=$ avg \\
$1.07=$ avg/standard
\end{tabular}

Standards Used:

Air Liquide 0.1 ppm SF6 in air, CAL11936

Air Liquide 4.97 ppm SF6 in air, FF34346

Weather Station Used:

Fisher Scientific S/N 90936818

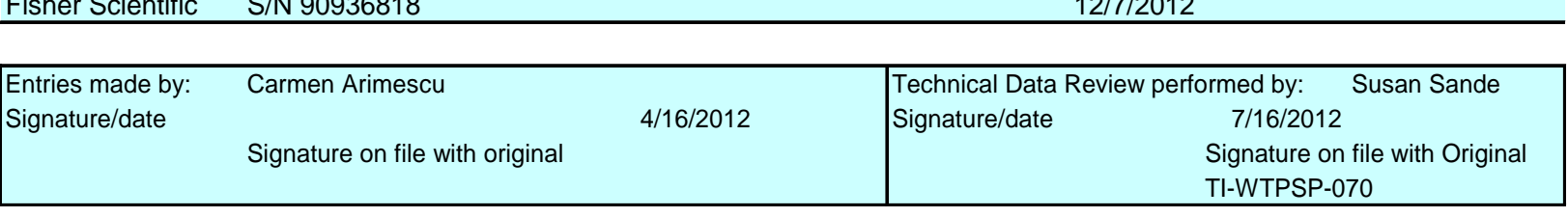

$4.97 \mathrm{ppm}$

Cylinder FF34346

\begin{tabular}{rl|r|} 
start $P=$ & 1500 psi \\
end $P=$ & 1500 psi
\end{tabular}

$B \& K$

readings: (ppm)

Compensating for water vapor

\begin{tabular}{|r|}
\hline 5.04 \\
\hline 5.03 \\
\hline 5.02 \\
\hline 5.01 \\
\hline 5.04 \\
\hline
\end{tabular}

Not compensating for water vapor

\begin{tabular}{|l|}
\hline 5.01 \\
\hline 5.01 \\
\hline 5.00 \\
\hline 5.00 \\
\hline 5.00 \\
\hline $\mathbf{5 . 0 2}$ \\
\hline $\mathbf{1 . 0 1}=$ avg $\mathrm{avg} /$ standard
\end{tabular}

Expiration date:

$3 / 19 / 2013$

$3 / 19 / 2014$

12/7/2012
Calibration 
SULFUR HEXAFLUORIDE GAS INSTRUMENT CALIBRATION

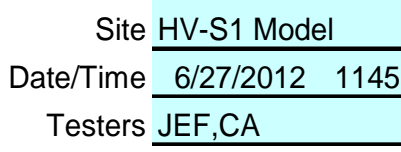

\author{
Instrument B\&K Model 1302 \\ Serial No. \\ 1765299 \\ Property No. WO17210
}

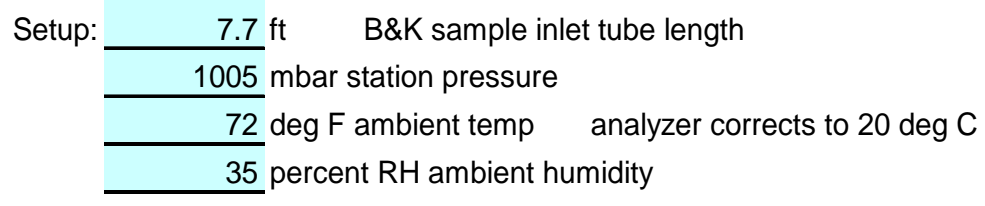

Setup: $7.7 \mathrm{ft} \quad$ B\&K sample inlet tube length 1005 mbar station pressure

72 deg $\mathrm{F}$ ambient temp analyzer corrects to $20 \mathrm{deg} \mathrm{C}$ 35 percent $\mathrm{RH}$ ambient humidity

Pre-Test background, ppb

Not compensating for water vapor, monitoring task 2

38.4, 36.1, 34.3, 35.5, 33.8

Compensating for water vapor, monitoring task 1

$2.6,1.6,2.80,1.83,4.50$

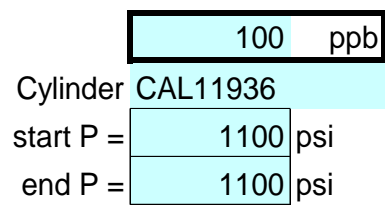

B\&K

Calibration

readings: (ppb)

Compensating for water vapor

\begin{tabular}{|r|}
\hline 100 \\
\hline 95 \\
\hline 99 \\
\hline 99 \\
\hline 101 \\
\hline
\end{tabular}

Not compensating for water vapor

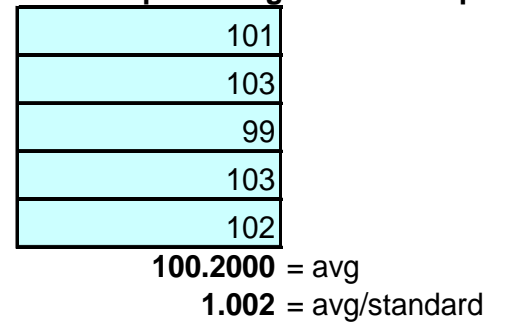

Standards Used:

Air Liquide 0.1 ppm SF6 in air, CAL11936

Air Liquide 4.97 ppm SF6 in air, FF34346

Weather Station Used:

Fisher Scientific S/N 90936818

$12 / 7 / 2012$

\section{B\&K}

Calibration

readings: (ppm)

Compensating for water vapor

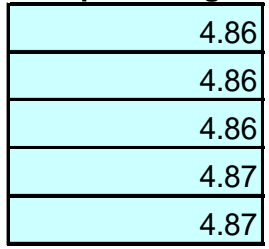

Not compensating for water vapor

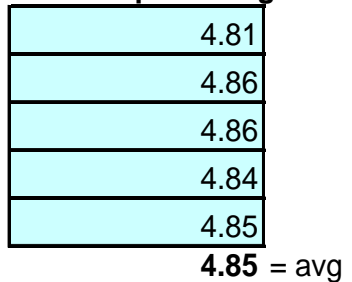

$0.97665996=\mathrm{avg} / \mathrm{standard}$

Expiration date:

$3 / 19 / 2013$

$3 / 19 / 2014$

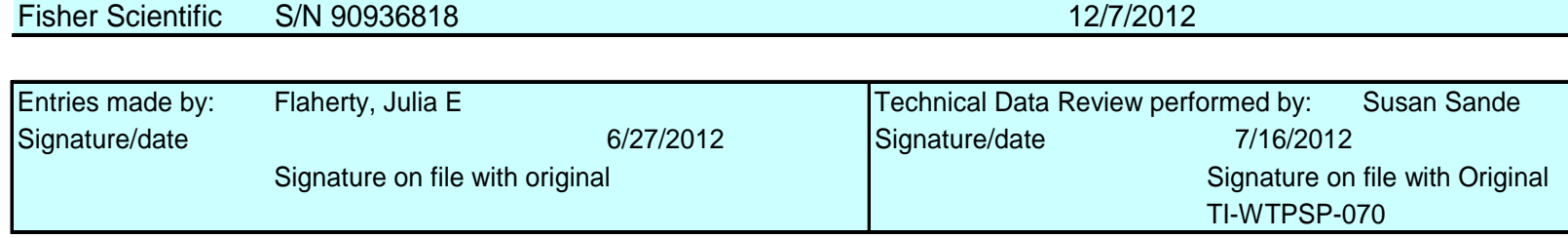




\section{SULFUR HEXAFLUORIDE GAS INSTRUMENT CALIBRATION}
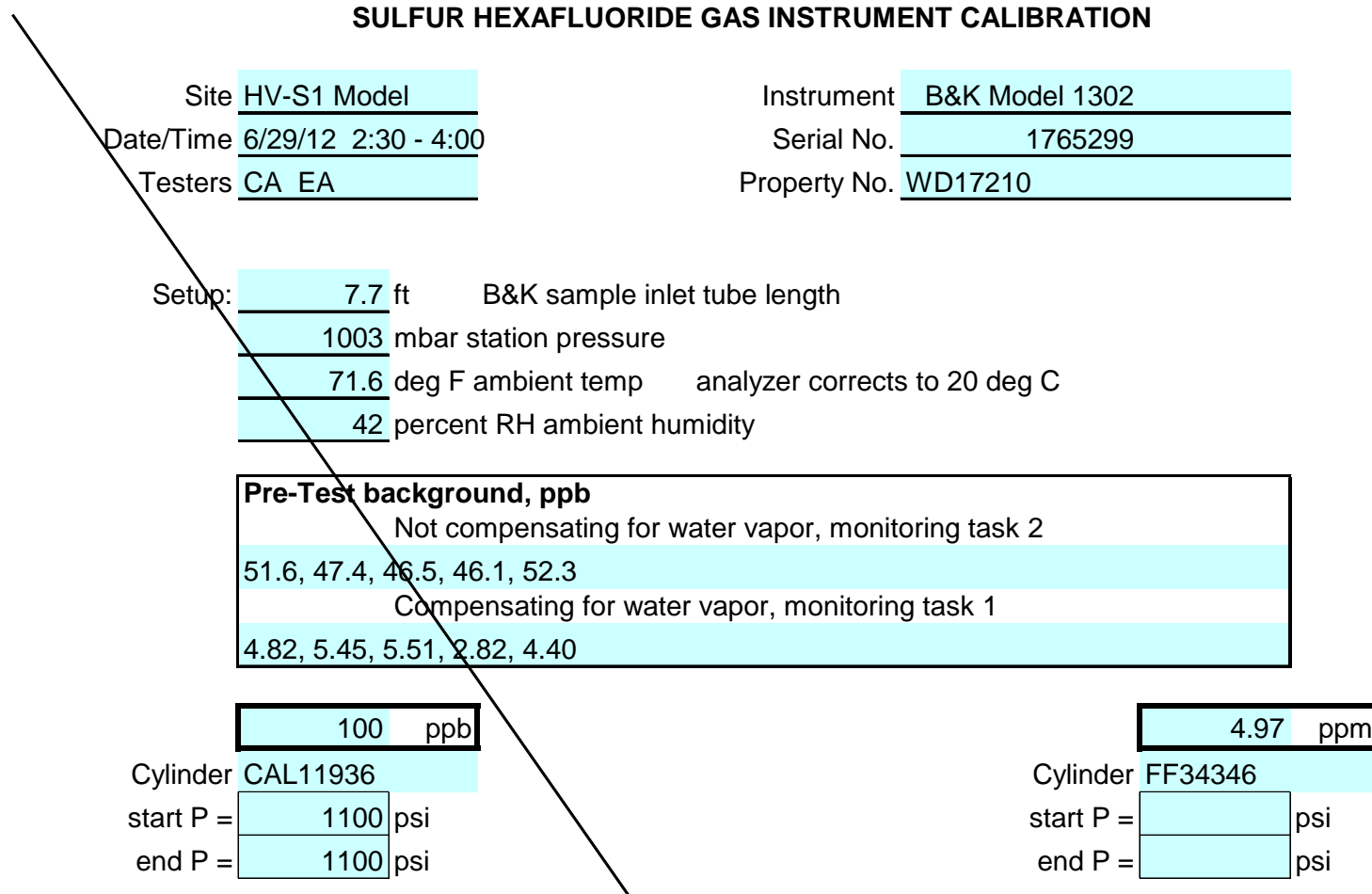

B\&K

Calibration

readings: (ppb)

Compensating for water vapor

\begin{tabular}{|r|}
\hline 91.4 \\
\hline 90.5 \\
\hline 88.0 \\
\hline 83.6 \\
\hline 83.3 \\
\hline
\end{tabular}

Not compensating for water vapor

\begin{tabular}{|r|}
\hline 87.4 \\
\hline 84.2 \\
\hline 80.8 \\
\hline 79.0 \\
\hline
\end{tabular}

Data invalid due to improper set up on B\&K Gas Analyzer.

Do not use. EA 7/3/12

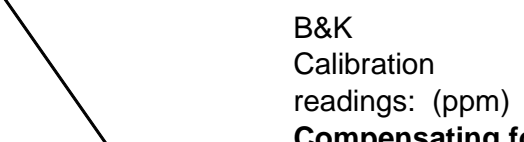

Compensating for water vapor

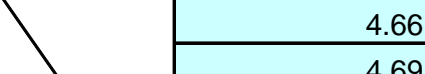

4.69

4.70

$4 \begin{array}{r}4.68 \\ \hline 4.69\end{array}$

Not compensating for water vapor

4.24

$\begin{array}{r}4.46 \\ \hline 4.56 \\ \hline\end{array}$

\begin{tabular}{|r|}
\hline \\
\hline \\
\hline \\
4.58 \\
\hline
\end{tabular}

Standards Used: Expiration date:

\begin{tabular}{l} 
Air Liquide 0.1 ppm SF6 in air, CAL11936 \\
\hline Air Liquide 4.97 ppm SF6 in air, FF34346 \\
\hline Weather Station Used: \\
\hline Fisher Scientific $\quad$ S/N 90936818 \\
\hline $\begin{array}{ll}\text { Entries made by: } & \text { CA } \\
\text { Signature/date } & \text { Signature on file with original } \\
& 6 / 26 / 2012\end{array}$
\end{tabular}

' = avg/standard $3 / 19 / 2013$

Air Liquide 4.97 ppm SF6 in air, FF34346 $3 / 19 / 2014$

Weather Station Used:

\begin{tabular}{|l} 
Technical Data Review performed by: Susan Sande \\
Signature/date \\
S/16/2012 \\
Signature on file with Original \\
TI-WTPSP-070
\end{tabular}




\section{SULFUR HEXAFLUORIDE GAS INSTRUMENT CALIBRATION}

$\begin{aligned} \text { Site } & \text { HV-S1 Model } \\ \text { Date/Time } & \frac{7 / 2 / 121050}{\text { Testers }}\end{aligned}$

$\begin{aligned} & \text { Instrument } \text { B\&K Model } 1302 \\ & \text { Serial No. } 1765299 \\ & \text { Property No. WD17210 }\end{aligned}$

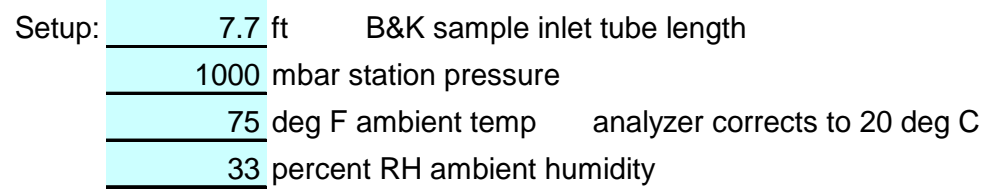

\begin{tabular}{rr|} 
& $100 \quad \mathrm{ppb}$ \\
Cylinder CAL11936 & \\
start $\mathrm{P}=$ & $1000 \mathrm{psi}$ \\
end $\mathrm{P}=$ & $990 \mathrm{psi}$
\end{tabular}

B\&K

Calibration

readings: (ppb)

Compensating for water vapor

\begin{tabular}{|r|}
\hline 99.6 \\
\hline 98.2 \\
\hline 97.8 \\
\hline 99.3 \\
\hline 95.7 \\
\hline
\end{tabular}

Not compensating for water vapor

\begin{tabular}{|r|}
\hline 99.7 \\
\hline 101.0 \\
\hline 105.0 \\
\hline 103.0 \\
\hline 102.0 \\
\hline $100.1300=$ avg \\
$1.0013=$ avg/standard
\end{tabular}

Standards Used:

Air Liquide 0.1 ppm SF6 in air, CAL11936

Air Liquide 4.97 ppm SF6 in air, FF34346

Weather Station Used:

Fisher Scientific S/N 90936818
$12 / 7 / 2012$

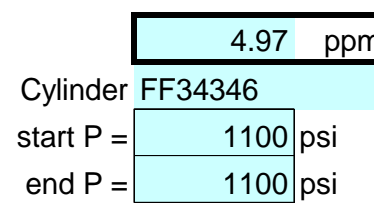

B\&K

Calibration

readings: (ppm)

Compensating for water vapor

\begin{tabular}{|r|}
\hline 4.86 \\
\hline 4.86 \\
\hline 4.87 \\
\hline 4.86 \\
\hline 4.85 \\
\hline
\end{tabular}

Not compensating for water vapor

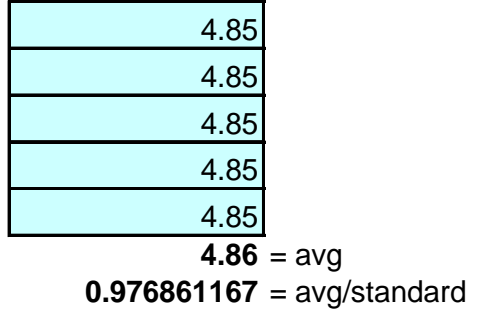

Expiration date:

$3 / 19 / 2013$

$3 / 19 / 2014$

$\begin{aligned} & \text { Technical Data Review performed by: Susan Sande } \\ & \text { Signature/date } \\ & 7 / 16 / 2012 \\ & \\ & \text { Signature on file with Original } \\ & \text { TI-WTPSP-070 }\end{aligned}$


Rev. 0

31-JuH06

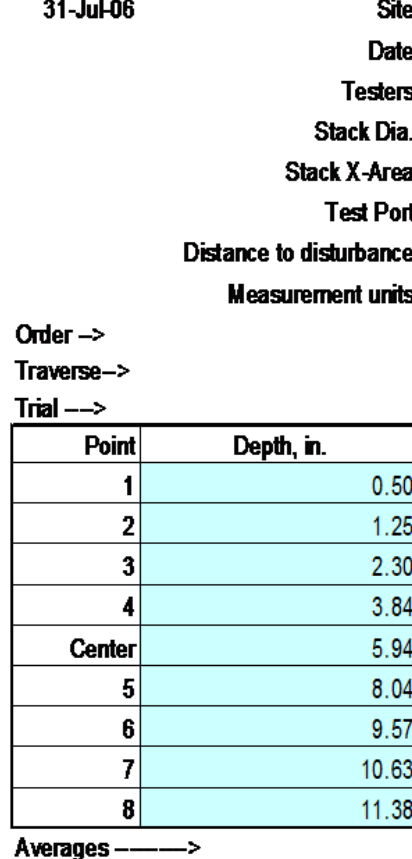

Site HV-S1 Model

Date 4/10/2012

Testers $\mathrm{CA}, \mathrm{XY}$

Stack Dia. 11.938 in.

X-Area 111.9 in

Port

2

240 inches

ppb SF6

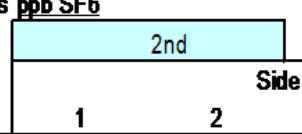

RACER GAS TRAVERSE DATA FORU

Run No. GT-1

Fan Configuration Fan A only

Fan Setting 55

Stack Temp

StartEnd Time 930/ 1120

Center $2 / 3$ from

Points in Center 2/3

Injection Point $\mathrm{C}$ center

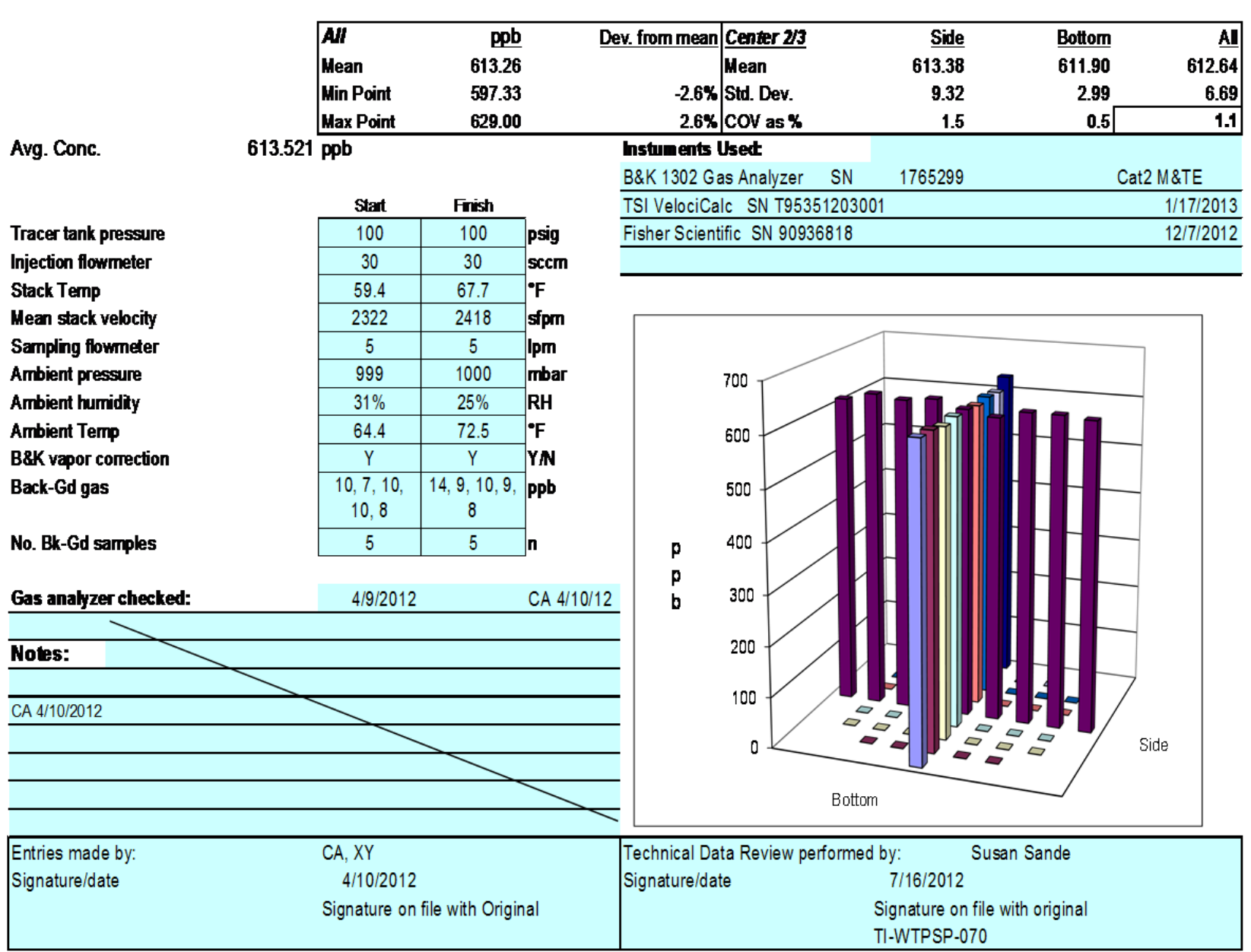


Rev. 0

31-JuL06

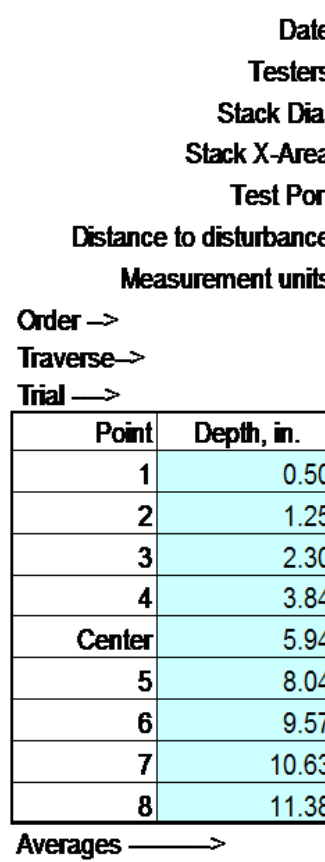

TRACER GAS TRAVERSE DATA FORU

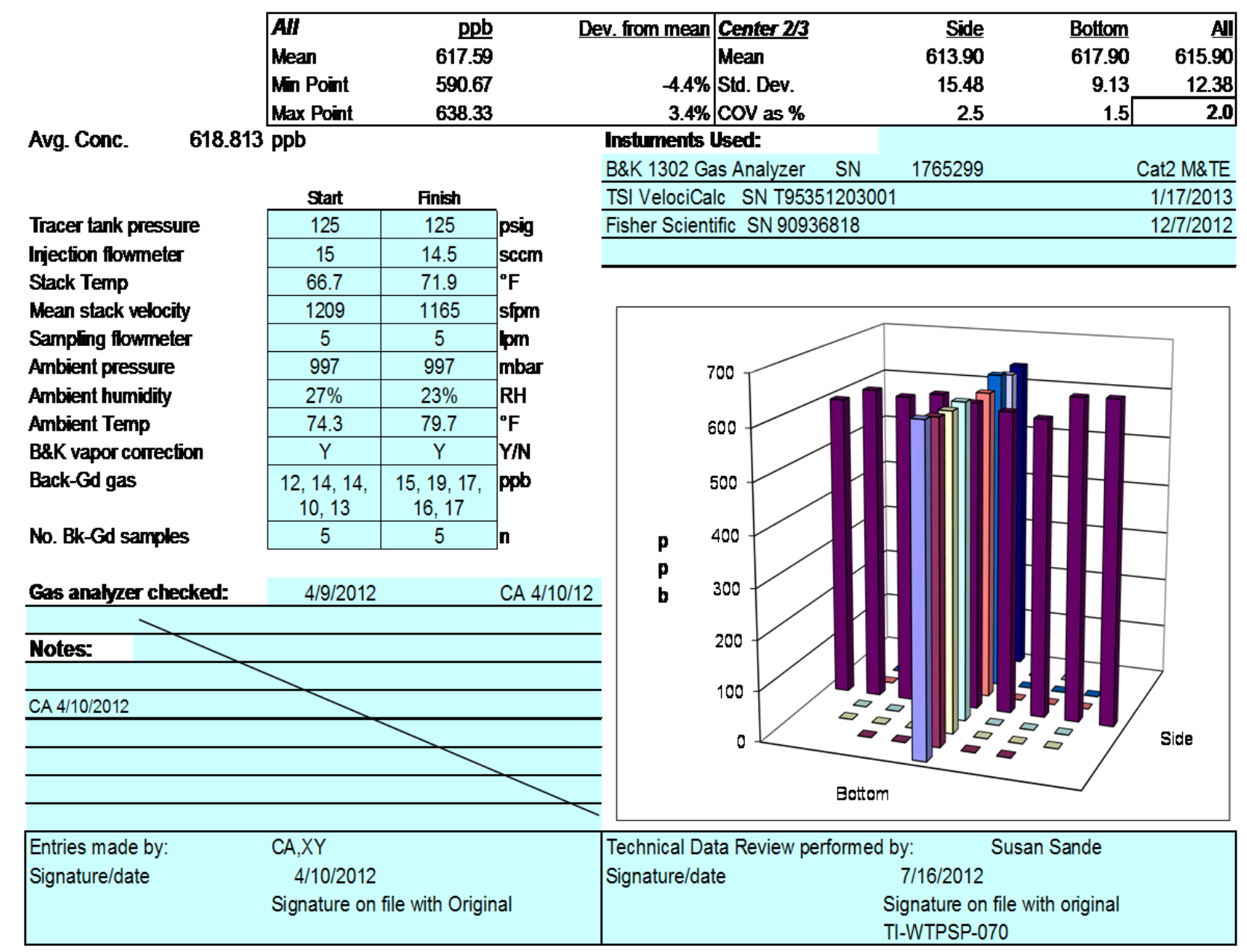


Rev. 0

31-JuL06

$\begin{aligned} & \text { Site HV -S1 Model } \\ & \text { Date } \frac{4 / 11 / 2012}{\mathrm{C}, \mathrm{XY}} \\ & \text { Testers } \frac{11.938 \text { in. }}{\mathrm{CA}} \\ & \text { Stack Dia. } \frac{11.93 \text { in. }^{2}}{111.9} \\ & \text { Stack X-Area } 2 \\ & \text { Test Port } 2\end{aligned}$

Distance to disturbance 240 inches

Measurement units pob SF6

TRACER GAS TRAVERSE DATA FORU

\begin{tabular}{|c|c|c|c|c|c|c|c|c|c|}
\hline \multicolumn{4}{|c|}{ Measurement units ppb SF6 } & \multicolumn{5}{|c|}{ Injection Point C center } & \\
\hline \multirow{3}{*}{$\begin{array}{l}\text { Order } \rightarrow \\
\text { Traverse } \rightarrow \\
\text { Trial } \longrightarrow\end{array}$} & & \multicolumn{2}{|c|}{ 2nd } & & & \multicolumn{2}{|c|}{ 1st } & & \\
\hline & & \multicolumn{4}{|c|}{ Side } & \multicolumn{4}{|c|}{ Bottom } \\
\hline & & 1 & 2 & 3 & Mean & 1 & 2 & 3 & Mean \\
\hline Point & Depth, in. & \multicolumn{4}{|c|}{ ppb } & \multicolumn{4}{|c|}{ ppb } \\
\hline 1 & 0.50 & 609 & 597 & 621 & 609.0 & 620 & 626 & 623 & 623.0 \\
\hline 2 & 1.25 & 629 & 627 & 613 & 623.0 & 602 & 628 & 628 & 619.3 \\
\hline 3 & 2.30 & 632 & 605 & 608 & 615.0 & 613 & 623 & 640 & 625.3 \\
\hline 4 & 3.84 & 635 & 647 & 605 & 629.0 & 616 & 635 & 608 & 619.7 \\
\hline Center & 5.94 & 629 & 605 & 597 & 610.3 & 619 & 608 & 615 & 614.0 \\
\hline 5 & 8.04 & 596 & 598 & 631 & 608.3 & 646 & 627 & 621 & 631.3 \\
\hline 6 & 9.57 & 591 & 596 & 587 & 591.3 & 639 & 646 & 584 & 623.0 \\
\hline 7 & 10.63 & 611 & 590 & 599 & 600.0 & 625 & 639 & 606 & 623.3 \\
\hline 8 & 11.38 & 649 & 616 & 588 & 617.7 & 623 & 626 & 608 & 619.0 \\
\hline Averages & $\longrightarrow$ & 620.1 & 609.0 & 605.4 & 611.5 & 622.6 & 628.7 & 614.8 & 622.0 \\
\hline
\end{tabular}

\begin{tabular}{|c|c|c|c|c|c|c|c|c|c|}
\hline \multirow{4}{*}{$\begin{array}{l}\text { Order } \rightarrow \\
\text { Traverse } \rightarrow \\
\text { Trial } \longrightarrow\end{array}$} & & \multirow{2}{*}{\multicolumn{2}{|c|}{ 2nd }} & & \\
\hline & & & & & & & st & & \\
\hline & & \multicolumn{4}{|c|}{ Side } & \multicolumn{3}{|c|}{ Bottom } & \\
\hline & & 1 & 2 & 3 & Mean & 1 & 2 & 3 & \\
\hline Point & Depth, in. & \multicolumn{4}{|c|}{ ppb } & \multicolumn{4}{|c|}{ ppb } \\
\hline 1 & 0.50 & 609 & 597 & 621 & 609.0 & 620 & 626 & 623 & 623.0 \\
\hline 2 & 1.25 & 629 & 627 & 613 & 623.0 & 602 & 628 & 628 & 619.3 \\
\hline 3 & 2.30 & 632 & 605 & 608 & 615.0 & 613 & 623 & 640 & 625.3 \\
\hline 4 & 3.84 & 635 & 647 & 605 & 629.0 & 616 & 635 & 608 & 619.7 \\
\hline Center & 5.94 & 629 & 605 & 597 & 610.3 & 619 & 608 & 615 & 614.0 \\
\hline 5 & 8.04 & 596 & 598 & 631 & 608.3 & 646 & 627 & 621 & 631.3 \\
\hline 6 & 9.57 & 591 & 596 & 587 & 591.3 & 639 & 646 & 584 & 623.0 \\
\hline 7 & 10.63 & 611 & 590 & 599 & 600.0 & 625 & 639 & 606 & 623.3 \\
\hline 8 & 11.38 & 649 & 616 & 588 & 617.7 & 623 & 626 & 608 & 619.0 \\
\hline Averages & $\rightarrow$ & 620.1 & 609.0 & 605.4 & 611.5 & 622.6 & 628.7 & 614.8 & 622.0 \\
\hline
\end{tabular}

\section{- Fan}

Run No. GT-3

Fan Configuration Fan B only

Fan Setting 26

Stack Temp

Start/End Tme 936/1110

Center 2/3 from

Points in Center $2 / 3$

Injection Point $\mathrm{C}$ center

\begin{tabular}{r|rl|}
\cline { 2 - 4 } & \multicolumn{3}{|c|}{ 1st Bottom }
\end{tabular}

\begin{tabular}{|c|c|c|}
\hline \multicolumn{2}{|c|}{ Hz } & \\
\hline & & \\
\hline 10 & to: & 10.84 \\
\hline 2 & to: & 7 \\
\hline
\end{tabular}

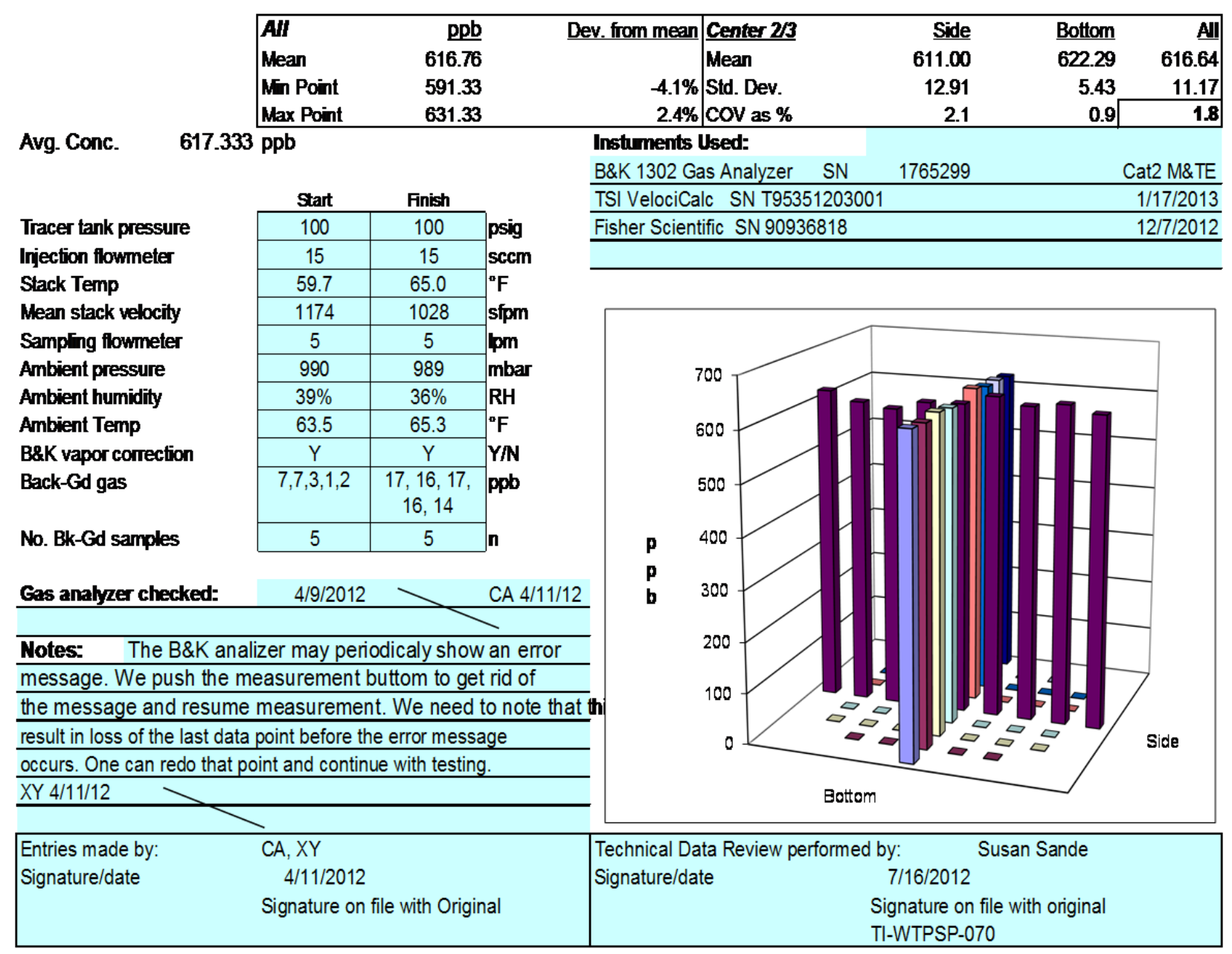


Rev. 0

31-JuL06

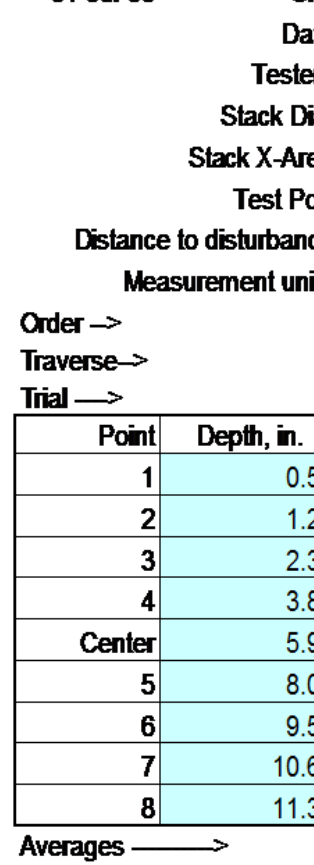

TRACER GAS TRAVERSE DATA FORU

Site HV -S1 Model

Date $4 / 11 / 2012$

Testers $X Y, C A$

11.938 in.

240 inches

Run No. GT-4

Fan Configuration Fan B only

Fan Setting 60

Stack Temp

Start/End Tme $1110 / 1210$

Center $2 / 3$ from

Points in Center $2 / 3$

Injection Point $\mathbf{C}$ center

$\mathrm{Hz}$

$65.2 \operatorname{deg} F$

1.10 to: 10.84

2 to: 7

2nd 1

Bottom

\begin{tabular}{|c|c|c|c|c|c|c|c|c|}
\hline & \multicolumn{4}{|c|}{ ) } & \multicolumn{4}{|c|}{. } \\
\hline & 1 & 2 & 3 & Mean & 1 & 2 & 3 & Mean \\
\hline & \multicolumn{4}{|c|}{ ppb } & \multicolumn{4}{|c|}{ ppb } \\
\hline 50 & 481 & 505 & 512 & 499.3 & 500 & 507 & 513 & 506.7 \\
\hline 25 & 465 & 528 & 515 & 502.7 & 535 & 503 & 501 & 513.0 \\
\hline 30 & 521 & 498 & 514 & 511.0 & 523 & 515 & 523 & 520.3 \\
\hline 84 & 498 & 520 & 522 & 513.3 & 515 & 547 & 535 & 532.3 \\
\hline 94 & 504 & 510 & 525 & 513.0 & 518 & 540 & 520 & 526.0 \\
\hline .04 & 516 & 502 & 502 & 506.7 & 520 & 506 & 518 & 514.7 \\
\hline 57 & 516 & 508 & 535 & 519.7 & 533 & 506 & 500 & 513.0 \\
\hline 63 & 494 & 499 & 530 & 507.7 & 523 & 528 & 539 & 530.0 \\
\hline 8 & 507 & 526 & 522 & 518.3 & 502 & 536 & 496 & 511.3 \\
\hline & 500.2 & 510.7 & 519.7 & 510.2 & 518.8 & 520.9 & 516.1 & 518.6 \\
\hline
\end{tabular}

\begin{tabular}{|lr}
\hline $\boldsymbol{A} I I$ & $\mathrm{ppb}$ \\
Mean & 514.39 \\
Min Point & 499.33 \\
Max Point & 532.33 \\
\hline
\end{tabular}

Avg. Conc. $\quad 513.750 \mathrm{ppb}$

\begin{tabular}{r|l} 
Dev. from mean & Center 2/3 \\
$-2.9 \%$ & Mean \\
$3.5 \%$ & Std. Dev. \\
Cov as \%
\end{tabular}

\begin{tabular}{|rrr|}
\hline Side & Bottom & All \\
510.57 & 521.33 & 515.95 \\
5.52 & 8.19 & 8.73 \\
1.1 & 1.6 & 1.7 \\
\hline
\end{tabular}

Instuments Used:

$\begin{array}{lll}\text { B\&K } 1302 \text { Gas Analyzer SN } 1765299 & \text { Cat2 M\&TE }\end{array}$

TSI VelociCalc SN T95351203001 $1 / 17 / 2013$

Fisher Scientific SN 90936818

$12 / 7 / 2012$

Tracer tank pressure Injection flowmeter

Stack Temp

Mean stack velocity

Sampling flowmeter

Ambient pressure

Ambient humidity

Ambient Temp

B\&K vapor correction

Back-Gd gas

No. Bk-Gd samples

\begin{tabular}{|c|c|}
\hline Start & Finish \\
\hline 100 & 125 \\
\hline 30 & 30 \\
\hline 65.6 & 64.8 \\
\hline 3121 & 2808 \\
\hline 5 & 5 \\
\hline 989 & 989 \\
\hline $34 \%$ & $36 \%$ \\
\hline 68.9 & 65.3 \\
\hline$Y$ & $Y$ \\
\hline $4,7,6,6,7$ & $9,12,10,6,9$ \\
\hline 5 & 5 \\
\hline
\end{tabular}

Gas anabzer checked:

$4 / 9 / 2012$

CA $4 / 11 / 12$

Notes: The injection flow was increased to $30 \mathrm{sccm}$ to reach about $500 \mathrm{ppb}$ at center of the stack. $X Y 4 / 11 / 12$

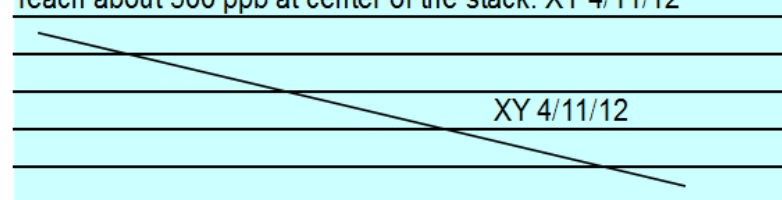

CA, XY

4/11/2012

Signature on file with Original

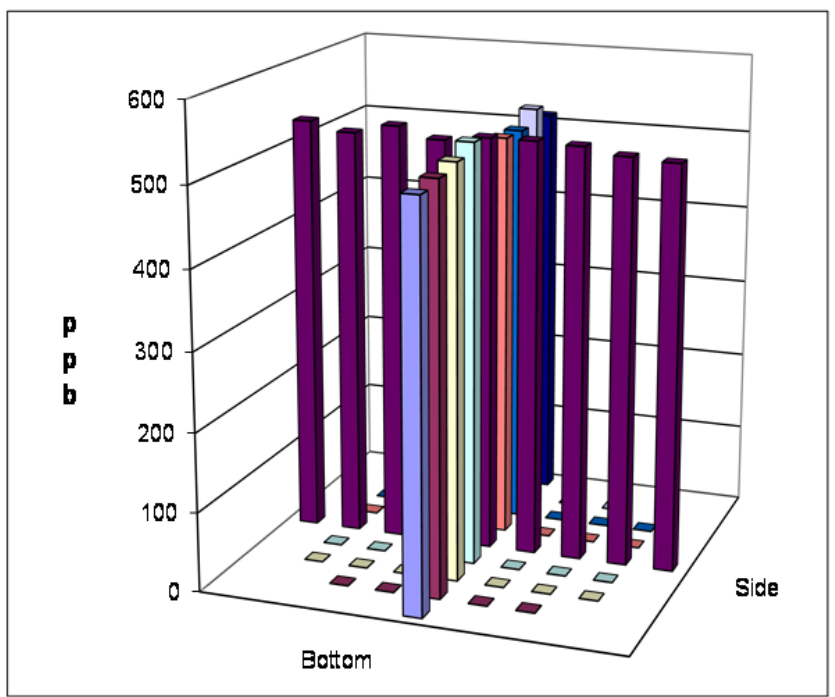

Technical Data Review performed by: Signature/date 7/16/2012

Signature on file with original TI-WTPSP-070 
Rev. 0

31-JuL06

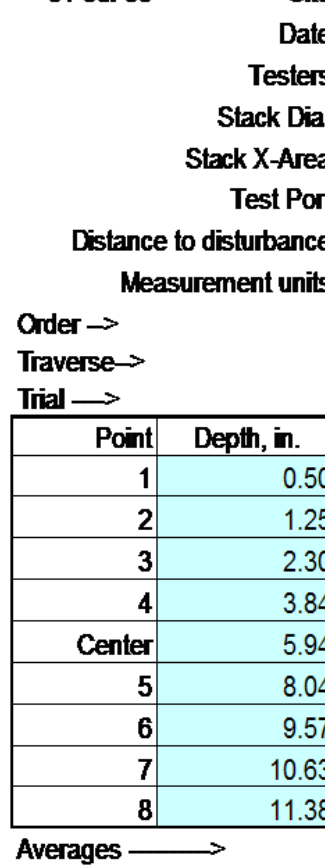

TRACER GAS TRAVERSE DATA FORU

\begin{tabular}{|c|c|c|c|c|c|}
\hline \multicolumn{5}{|c|}{ TRACER GAS TRAVERSE DATA FORU } & \\
\hline \multicolumn{2}{|c|}{ Site HV -S1 Model } & \multirow{2}{*}{\multicolumn{3}{|c|}{$\begin{array}{c}\text { Run No. GT-5 } \\
\text { Fan Configuration Fan B only }\end{array}$}} & \\
\hline Date & $4 / 11 / 2012$ & & & & \\
\hline \multicolumn{2}{|c|}{ Testers CA, JEF } & Fan Setfing 60 & \multicolumn{2}{|c|}{ Hz } & \\
\hline Stack Dia. & 11.938 in. & Stack Temp. & \multicolumn{2}{|c|}{$65.25 \operatorname{deg} F$} & \\
\hline Stack X-Area & $111.9 \mathrm{in}^{2}$ & \multicolumn{3}{|c|}{ Start/End Tme $1220 / 1335$} & \\
\hline \multirow{2}{*}{ Test Port } & 2 & Center $2 / 3$ from & 1.10 & to: & 10.84 \\
\hline & 240 inches & Points in Center $2 / 3$ & 2 & to: & 7 \\
\hline \multicolumn{2}{|c|}{ surement units pob SF6 } & Injection Point $\overline{C N}$ & ALL & & \\
\hline
\end{tabular}

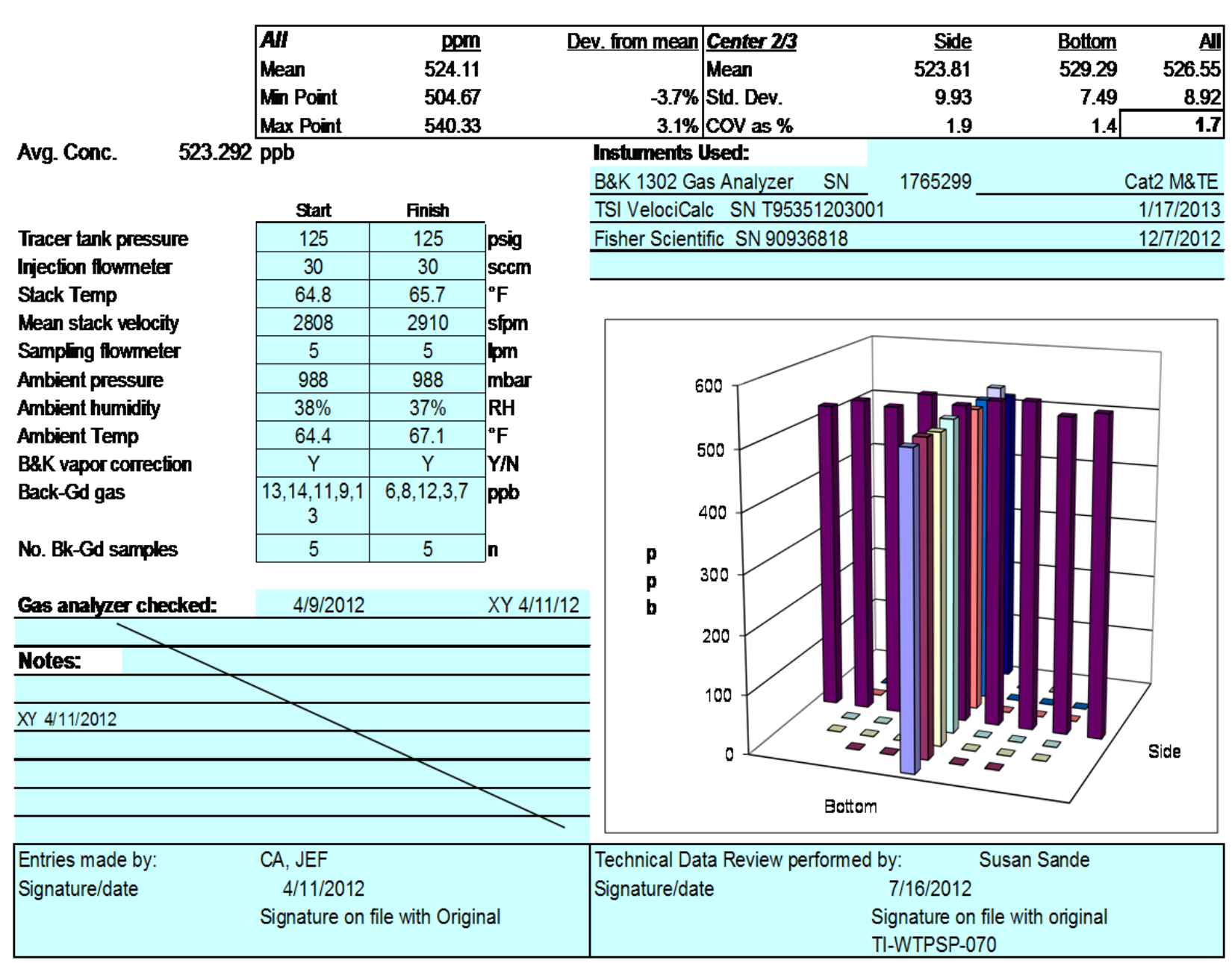


Rev. 0

31-JuL06

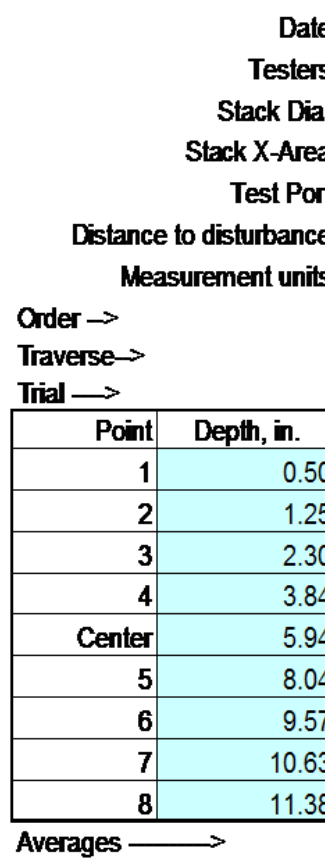

\section{TRACER GAS TRAVERSE DATA FORU}

Site HV -S1 Model

Date $4 / 11 / 2012$

Testers CA. JAG

tack Dia. 11.938 in.

X-Area 111.9 in. $^{2}$

240 inches

its pob SF6
Run No. GT-6

Fan Configuration Fan B only

Fan Seting 60

Stack Temp

Start/End Tme 148/315

Center $2 / 3$ from

Points in Center $2 / 3$

Injection Point C Far Wall
$\mathrm{Hz}$

1.10 to: 10.84

2 to: 7

2nd

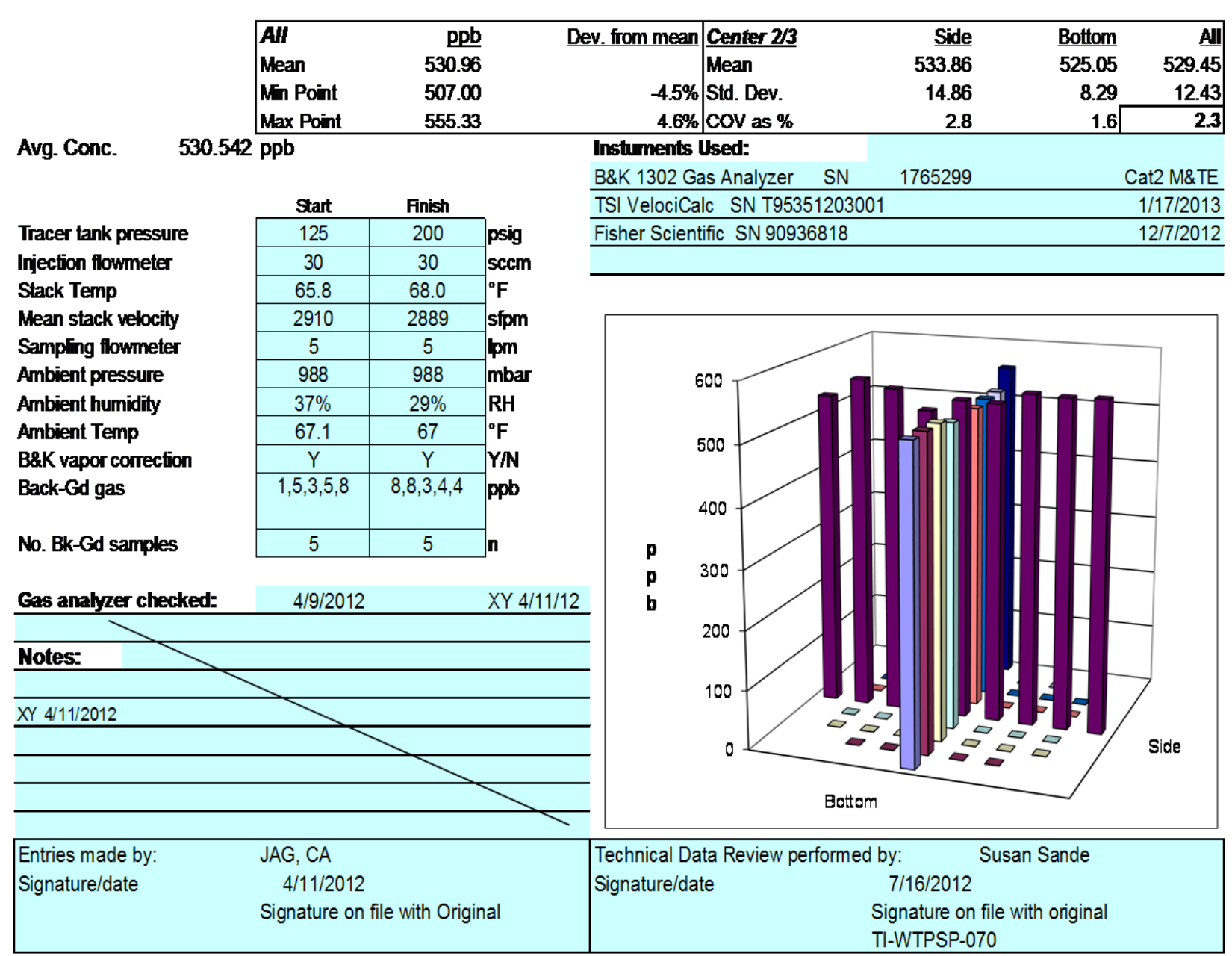


Rev. 0

31-JuL06

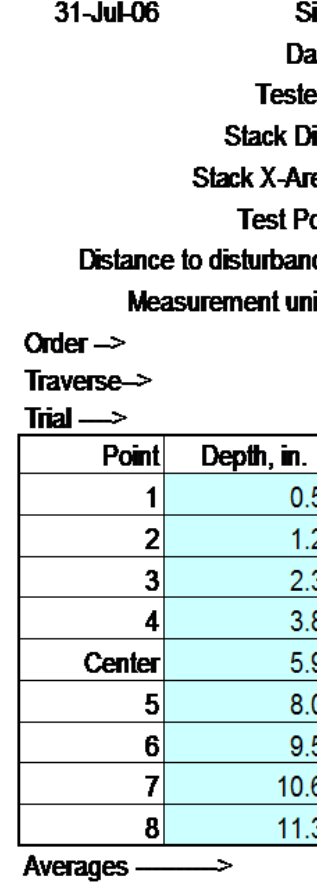

TRACER GAS TRAVERSE DATA FORU

Sïte HV -S1 Model

Date 4/12/2012

Testers EA, YFS, JEF

Stack Dia. 11.938 in

ck X-Area 111.9 in.

240 inches

Run No. GT-7

Fan Configuration Fan B only

Fan Setting 60

Stack Temp

Start/End Tme 0925 / 1055

Center $2 / 3$ from

Points in Center $2 / 3$

Injection Point C TOP
$\mathrm{Hz}$

$66.35 \operatorname{deg} F$

\begin{tabular}{|r|}
\hline 1 st \\
\hline
\end{tabular}

\begin{tabular}{c}
$n$ \\
\cline { 2 - 2 }
\end{tabular}

1.10 to: 10.84

2 to: 7

2nd $\quad$ Bottom

\begin{tabular}{|c|c|c|c|c|c|c|c|c|}
\hline & \multicolumn{4}{|r|}{ Mean } & \multicolumn{4}{|r|}{ Mean } \\
\hline & \multicolumn{4}{|c|}{ ppb } & \multicolumn{4}{|c|}{ ppb } \\
\hline .50 & 503 & 495 & 497 & 498.3 & 502 & 511 & 527 & 513.3 \\
\hline .25 & 520 & 487 & 518 & 508.3 & 510 & 506 & 519 & 511.7 \\
\hline 30 & 510 & 527 & 484 & 507.0 & 521 & 532 & 527 & 526.7 \\
\hline 84 & 527 & 493 & 504 & 508.0 & 491 & 487 & 539 & 505.7 \\
\hline .94 & 503 & 508 & 499 & 503.3 & 516 & 518 & 538 & 524.0 \\
\hline .04 & 513 & 510 & 512 & 511.7 & 532 & 525 & 515 & 524.0 \\
\hline .57 & 513 & 507 & 527 & 515.7 & 494 & 512 & 506 & 504.0 \\
\hline 63 & 514 & 518 & 504 & 512.0 & 495 & 521 & 496 & 504.0 \\
\hline .38 & 501 & 482 & 498 & 493.7 & 501 & 521 & 531 & 517.7 \\
\hline & 511.6 & 503.0 & 504.8 & 506.4 & 506.9 & 514.8 & 522.0 & 514.6 \\
\hline
\end{tabular}

\begin{tabular}{|lr}
\hline $\boldsymbol{A l l}$ & $\mathrm{ppb}$ \\
Mean & 510.50 \\
Mn Point & 493.67 \\
Max Point & 526.67 \\
\hline
\end{tabular}

Avg. Conc. $\quad 510.104 \mathrm{ppb}$

\begin{tabular}{r|l} 
Dev. from mean & Center 2/3 \\
$-3.3 \%$ & Mean \\
$3.2 \%$ & Std. Dev. \\
Cov as \%
\end{tabular}

\begin{tabular}{rrr|} 
Side & Bottom & All \\
509.43 & 514.29 & 511.86 \\
4.02 & 10.29 & 7.91 \\
0.8 & 2.0 & 1.5 \\
\hline
\end{tabular}

Instuments Used:

$\begin{array}{lll}\text { B\&K } 1302 \text { Gas Analyzer SN } 1765299 & \text { Cat2 M\&TE }\end{array}$

TSI VelociCalc SN T95351203001 $1 / 17 / 2013$

Fisher Scientific SN 90936818

$12 / 7 / 2012$

Tracer tank pressure Injection flowmeter

Stack Temp

Mean stack velocity

Sampling flowmeter

Ambient pressure

Ambient humidity

Ambient Temp

B\&K vapor correction

Back-Gd gas

No. Bk-Gd samples

\begin{tabular}{|c|c|}
\hline Start & Finish \\
\hline 125 & 125 \\
\hline 30 & 30 \\
\hline 63.6 & 69.1 \\
\hline 2930 & 3106 \\
\hline 5 & 5 \\
\hline 996 & 996 \\
\hline $28 \%$ & $31 \%$ \\
\hline 72 & 69 \\
\hline$Y$ & $Y$ \\
\hline $7,4,6,4,3$ & $\begin{array}{c}13,16,13,9, \\
20\end{array}$ \\
\hline 5 & 5 \\
\hline
\end{tabular}

Gas anahzer checked:

$4 / 9 / 2012$

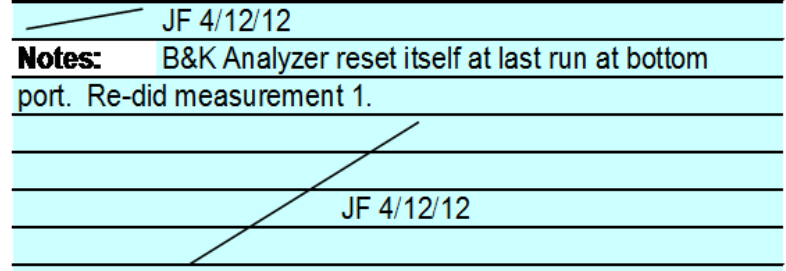

Entries made by:

Signature/date

EA

4/12/2012

Signature on file with Original

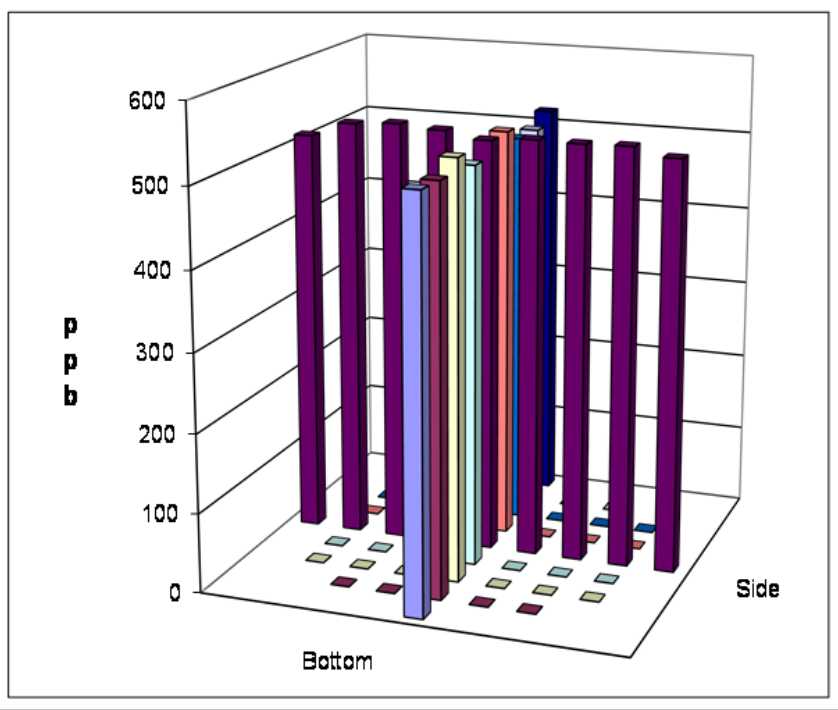

Technical Data Review performed by:

Signature/date $7 / 16 / 2012$

Signature on file with original TI-WTPSP-070 
Rev. 0

31-JuL06

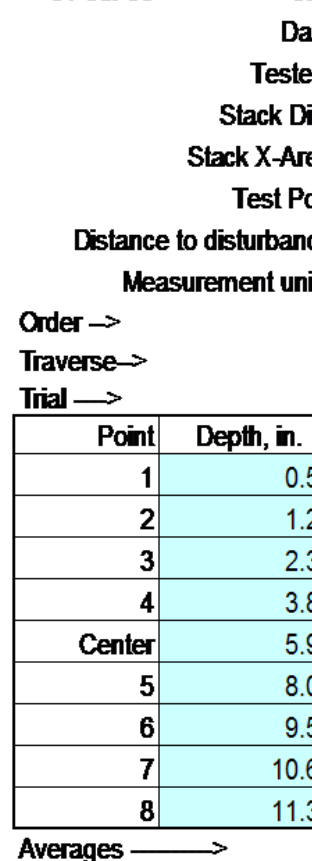

\section{TRACER GAS TRAVERSE DATA FORU}

Site HV -S1 Model

Date 4/12/2012

Testers EA, YFS

tack Dia. 11.938 in.

$\begin{array}{ll}\text { Test Port } & 111.9 \text { in. } \\ & \end{array}$

bance 240 inches

units pobsF6
Run No. GT-8

Fan Configuration Fan B only

Fan Setiing 60

Stack Temp

Start/End Tme $1100 / 1225$

Center $2 / 3$ from

Points in Center $2 / 3$

Injection Point $\mathrm{C}$ bottom

\begin{tabular}{|l|l|}
\hline 527.6 & 492.7 \\
\hline
\end{tabular}

Side

\begin{tabular}{|c|c|}
\hline \multicolumn{2}{|c|}{ 2nd } \\
\hline 1 & 2 \\
\hline
\end{tabular}

Side 3

1st

\begin{tabular}{r|r|r|r|}
\hline & \multicolumn{3}{|c|}{ ppb } \\
\hline .50 & 542 & 457 & 52 \\
.25 & 509 & 453 & 605 \\
\hline .30 & 595 & 482 & 508 \\
\hline .84 & 546 & 568 & 512 \\
5.94 & 470 & 487 & 523 \\
\hline .04 & 452 & 523 & 444 \\
\hline .57 & 579 & 476 & 51 \\
\hline .63 & 567 & 487 & 542 \\
\hline .38 & 488 & 501 & 530 \\
\hline & $\mathbf{5 2 7 . 6}$ & $\mathbf{4 9 2 . 7}$ & $\mathbf{5 2 2 . 2}$ \\
\hline
\end{tabular}

Mean $\quad 1 \quad 2^{\text {Bottom }}$

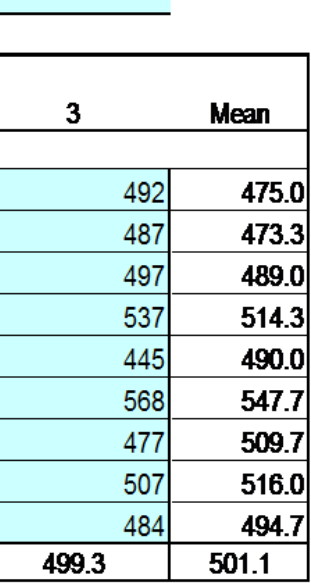

\begin{tabular}{|lr}
\hline $\boldsymbol{A l l}$ & $\mathrm{ppb}$ \\
Mean & 507.61 \\
Min Point & 473.00 \\
Max Point & 547.67 \\
\hline
\end{tabular}

Avg. Conc. $509.604 \mathrm{ppb}$

\begin{tabular}{r|l} 
Dev. from mean & Center 2/3 \\
$-6.8 \%$ & Mean \\
$7.9 \%$ & Std. Dev. \\
CoV as \% \\
\hline
\end{tabular}

Side
516.33
24.29
4.7

\begin{tabular}{rr|}
\hline Bottom & All \\
\hline 505.71 & 511.02 \\
24.26 & 23.96 \\
& 4.7 \\
\hline
\end{tabular}

\section{Instuments Used:}

B\&K 1302 Gas Analyzer $\quad$ SN $1765299 \quad$ Cat2 M\&TE TSI VelociCalc SN T95351203001 $1 / 17 / 2013$ Fisher Scientific SN 90936818

Tracer tank pressure Injection flowmeter Stack Temp

Mean stack velocity Sampling flowmeter Ambient pressure Ambient humidity Ambient Temp B\&K vapor correction Back-Gd gas

No. Bk-Gd samples

\begin{tabular}{|c|c|}
\hline Start & Finish \\
\hline 125 & 125 \\
\hline 30 & 30 \\
\hline 68.4 & 73.2 \\
\hline 3006 & 2910 \\
\hline 5 & 5 \\
\hline 996 & 996 \\
\hline $31 \%$ & $26 \%$ \\
\hline 68 & 71 \\
\hline$Y$ & $Y$ \\
\hline $\begin{array}{c}13,12,11 \\
9,11\end{array}$ & $\begin{array}{c}13,12,16 \\
10,8\end{array}$ \\
\hline 5 & 5 \\
\hline
\end{tabular}

Gas analyzer checked:

\section{$4 / 9 / 2012$}

Notes: At beginning of first side traverse, B\&K Analyzer reset itself. Redid side 1 measurement.

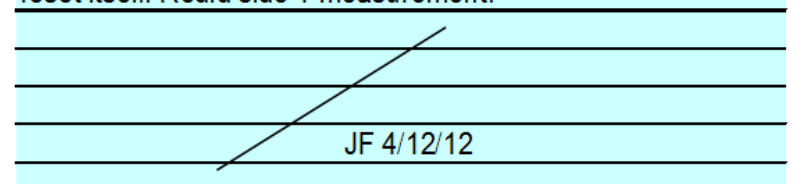

\begin{tabular}{|lc}
\hline $\begin{array}{l}\text { Entries made by: } \\
\text { Signature/date }\end{array}$ & EA \\
& Signature on file with Original
\end{tabular}

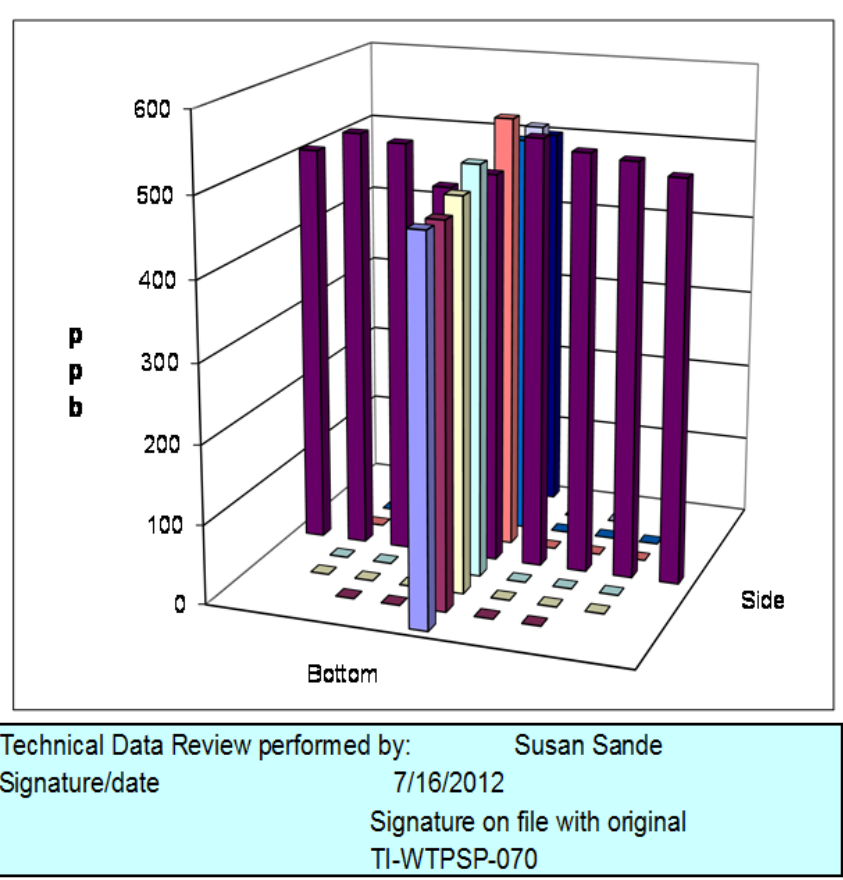


Rev. 0

31-JuL06

Date
Testers $\frac{4 / 12 / 2012}{\mathrm{CA}, \mathrm{XY}}$
Stack Dia.
Stack X-Area
Test Port $\frac{11.938 \mathrm{in} .}{111.9 \text { in. }^{2}}$
Distance to disturbance
Measurement units pob SF6

\section{TRACER GAS TRAVERSE DATA FORU}

Run No. GT-9

Fan Configuration Fan B only

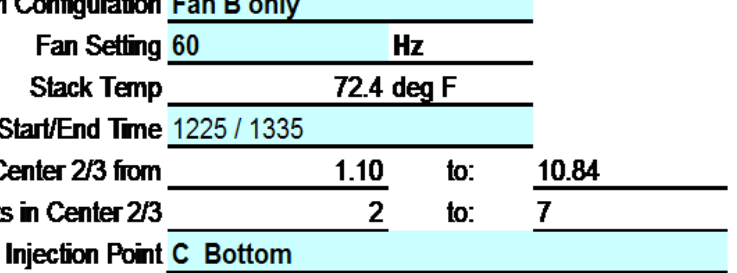

\begin{tabular}{|c|c|c|c|c|c|c|c|c|c|}
\hline \multirow{4}{*}{\multicolumn{2}{|c|}{$\begin{array}{l}\text { Order } \rightarrow \\
\text { Traverse } \rightarrow \\
\text { Trial } \rightarrow\end{array}$}} & & & & \multirow{4}{*}{ Mean } \\
\hline & & \multicolumn{2}{|c|}{ 1st } & & & \multicolumn{2}{|c|}{ 2nd } & & \\
\hline & & \multicolumn{4}{|c|}{ Side } & \multicolumn{3}{|c|}{ Bottom } & \\
\hline & & 1 & 2 & 3 & Mean & 1 & 2 & 3 & \\
\hline Point & Depth, in. & \multicolumn{4}{|c|}{ ppb } & \multicolumn{4}{|c|}{ ppb } \\
\hline 1 & 0.50 & 503 & 536 & 466 & 501.7 & 510 & 489 & 497 & 498.7 \\
\hline 2 & 1.25 & 480 & 491 & 474 & 481.7 & 602 & 606 & 555 & 587.7 \\
\hline 3 & 2.30 & 551 & 522 & 539 & 537.3 & 589 & 477 & 492 & 519.3 \\
\hline 4 & 3.84 & 547 & 443 & 484 & 491.3 & 530 & 484 & 556 & 523.3 \\
\hline Center & 5.94 & 555 & 521 & 525 & 533.7 & 530 & 460 & 539 & 509.7 \\
\hline 5 & 8.04 & 566 & 496 & 451 & 504.3 & 481 & 575 & 428 & 494.7 \\
\hline 6 & 9.57 & 572 & 533 & 557 & 554.0 & 566 & 506 & 474 & 515.3 \\
\hline 7 & 10.63 & 490 & 535 & 515 & 513.3 & 610 & 512 & 634 & 585.3 \\
\hline 8 & 11.38 & 549 & 509 & 496 & 518.0 & 502 & 521 & 498 & 507.0 \\
\hline Averages - & $\longrightarrow$ & 534.8 & 509.6 & 500.8 & 515.0 & 546.7 & 514.4 & 519.2 & 526.8 \\
\hline
\end{tabular}

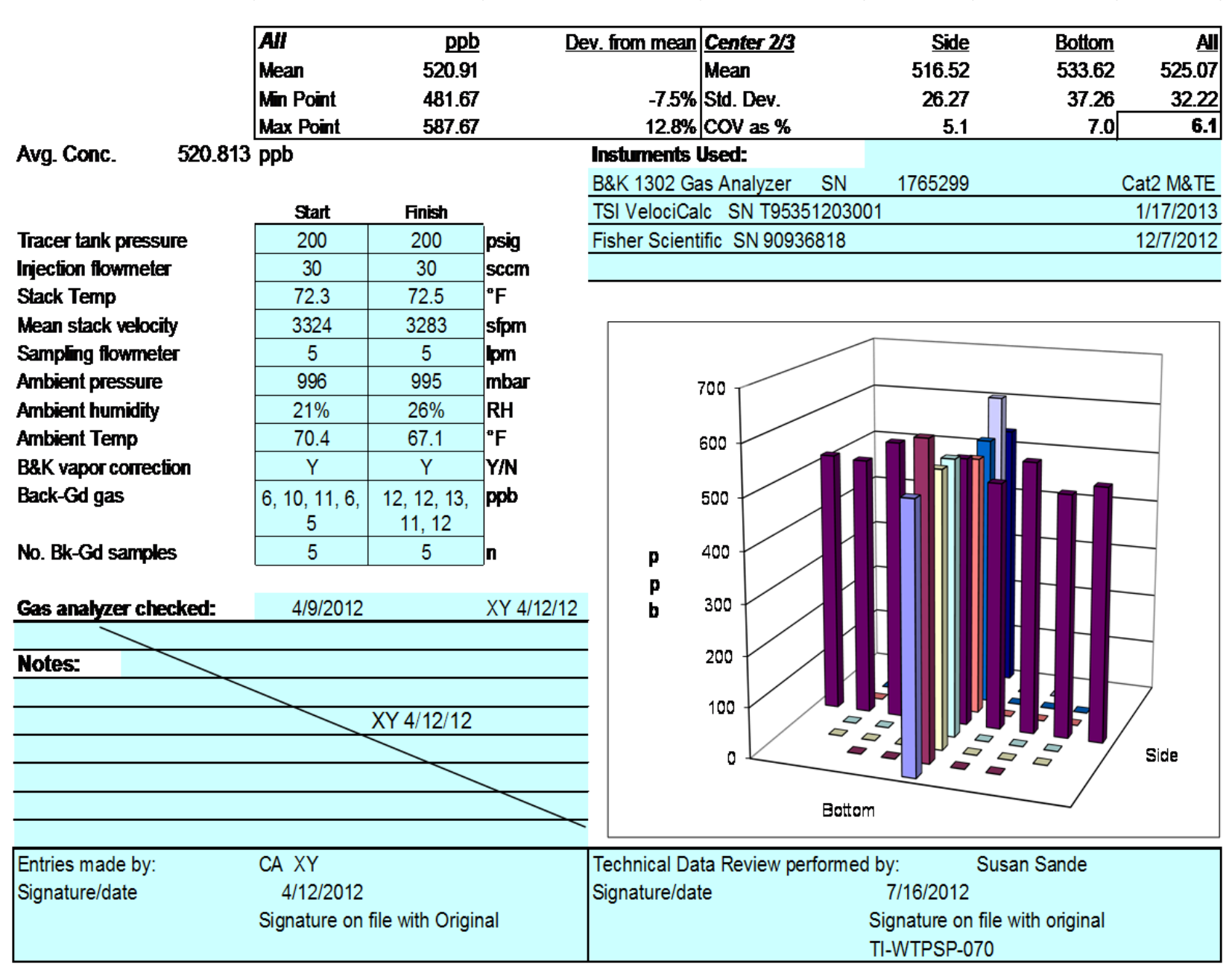


Rev. 0
31-JuL06

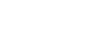

Site HV-S1 Model

Date 4/12/2012

Testers CA, XY

Stack Dia. Stack X-Area

Test Port

$\frac{11.938 \text { in. }}{111.9 \text { in. }^{2}}$

Order $\rightarrow$

Traverse $\rightarrow$

Trial $\longrightarrow$ 300 inches
TRACER GAS TRAVERSE DATA FORU

Run No. GT-10

Fan Configuration Fan B only

Fan Seting 60

Stack Temp

Start/End Tme $1345 / 1457$

Center $2 / 3$ from

Points in Center 2/3

Injection Point C Top

\begin{tabular}{|c|c|c|}
\hline \multicolumn{2}{|c|}{ Hz } & \\
\hline \multicolumn{2}{|c|}{$73.4 \operatorname{deg} F$} & \\
\hline & & \\
\hline 1.10 & to: & 10.84 \\
\hline$\overline{2}$ & to: & 7 \\
\hline
\end{tabular}

\begin{tabular}{|c|c|c|c|c|c|c|c|c|c|}
\hline Trial $\longrightarrow$ & & 1 & 2 & 3 & Mean & 1 & 2 & 3 & Mean \\
\hline Point & Depth, in. & \multicolumn{4}{|c|}{ ppb } & \multicolumn{4}{|c|}{ ppb } \\
\hline 1 & 0.50 & 489 & 496 & 522 & 502.3 & 504 & 517 & 509 & 510.0 \\
\hline 2 & 1.25 & 513 & 492 & 512 & 505.7 & 524 & 514 & 492 & 510.0 \\
\hline 3 & 2.30 & 483 & 509 & 473 & 488.3 & 514 & 523 & 497 & 511.3 \\
\hline 4 & 3.84 & 504 & 509 & 491 & 501.3 & 517 & 511 & 513 & 513.7 \\
\hline Center & 5.94 & 485 & 492 & 535 & 504.0 & 503 & 516 & 493 & 504.0 \\
\hline 5 & 8.04 & 501 & 498 & 530 & 509.7 & 486 & 509 & 499 & 498.0 \\
\hline 6 & 9.57 & 496 & 521 & 561 & 526.0 & 505 & 518 & 490 & 504.3 \\
\hline 7 & 10.63 & 504 & 494 & 535 & 511.0 & 507 & 540 & 514 & 520.3 \\
\hline 8 & 11.38 & 496 & 511 & 517 & 508.0 & 509 & 493 & 485 & 495.7 \\
\hline Averages - & $\rightarrow$ & 496.8 & 502.4 & 519.6 & 506.3 & 507.7 & 515.7 & 499.1 & 507.5 \\
\hline
\end{tabular}

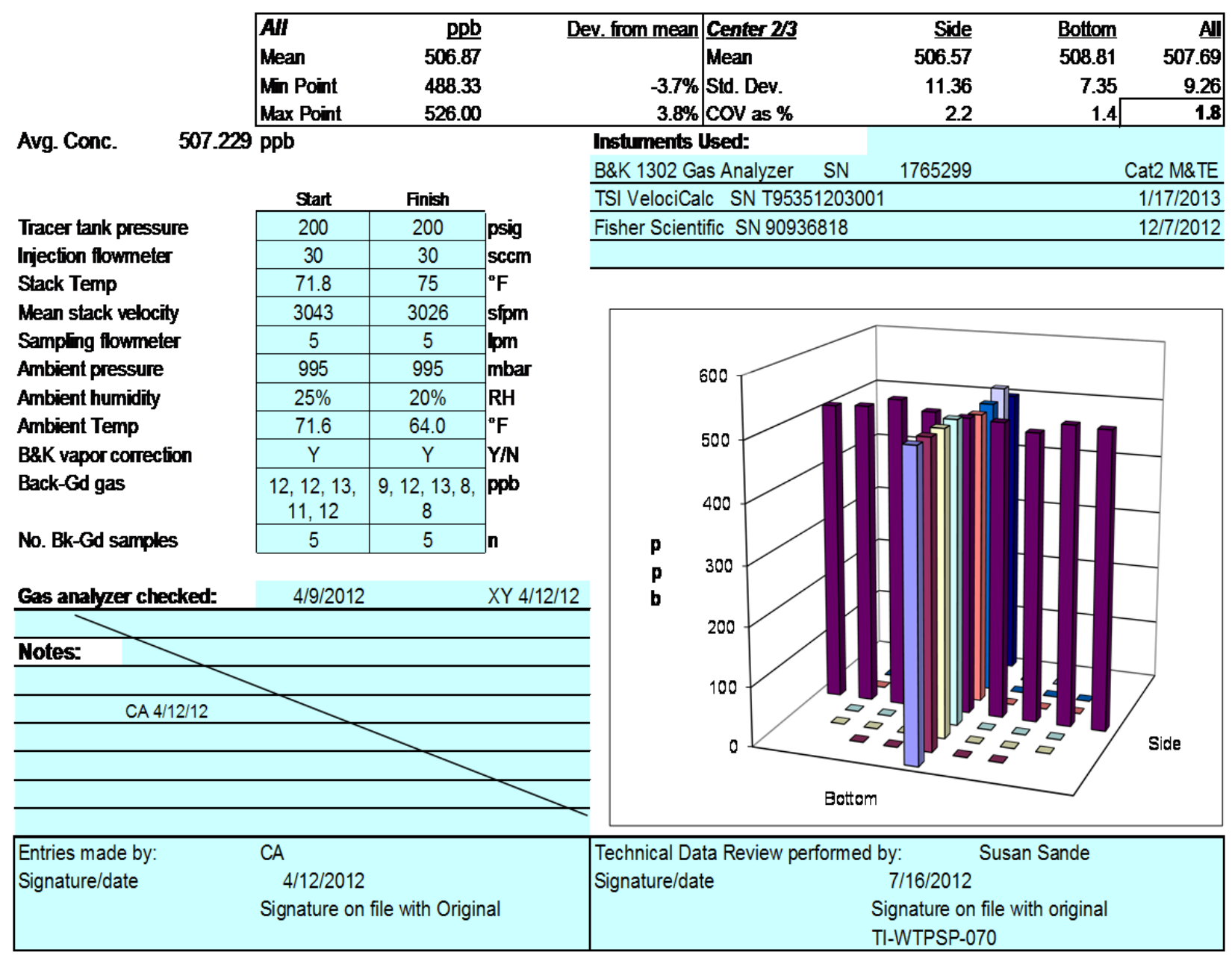


Rev. 0

31-JuL06

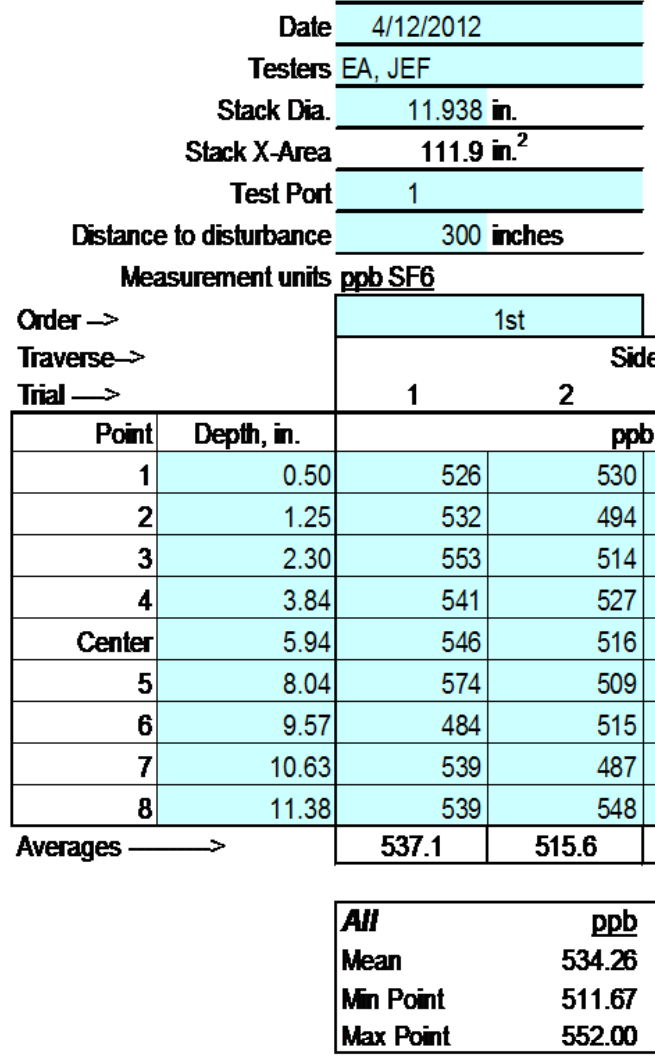

Avg. Conc.

$534.125 \mathrm{ppb}$

Tracer tank pressure Injection flowmeter Stack Temp Mean stack velocity Sampling flowmeter Ambient pressure Ambient humidity Ambient Temp B\&K vapor correction Back-Gd gas

No. Bk-Gd samples

\begin{tabular}{|c|c|c|}
\hline Start & Finish & \\
\hline 240 & 125 & \\
\hline 30 & 30 & \\
\hline 69.7 & 64.6 & \\
\hline 3117 & 3108 & \\
\hline 5 & 5 & \\
\hline 995 & 995 & \\
\hline $23 \%$ & $27 \%$ & \\
\hline 75 & 64 & \\
\hline$Y$ & $Y$ & \\
\hline $\begin{array}{c}9,12,5,10 \\
11\end{array}$ & $\begin{array}{c}20,19,12,6 \\
12 \\
\end{array}$ & \\
\hline 5 & 5 & \\
\hline
\end{tabular}

Gas anahzer checked:

\section{$4 / 9 / 2012$}

JEF 4/12/12

Notes: Wind getting pretty gusty during second traverse.

Side, Trial 3, Point 4 discovered the SF6 cylinder was closed.

Had been running on back pressure. Appears that delivery of

SF6 dropped in one sample cycle. Run trial 4 just in case.

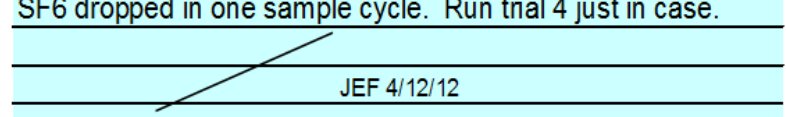

Entries made by:

Signature/date

Julia Flaherty

Signature on file with original

$4 / 12 / 2012$
Run No. GT-11

Fan Configuration Fan B only

Fan Setting 60

Stack Temp

Start/End Tme 1500 / 1625

Center $2 / 3$ from

Points in Center 2/3

Injection Point C NEAR WALL
$\mathrm{Hz}$

$67.15 \mathrm{deg} F$

1.10 to: 10.84

2 to: 7

2nd


Rev. 0

31-JuL06

\begin{tabular}{|c|c|c|c|}
\hline & Date & $4 / 13 / 2012$ & \\
\hline & Testers & $E A, X Y$ & \\
\hline & Stack Dia. & 11.938 & \\
\hline & Stack X-Area & 111.9 & \\
\hline & Test Port & 1 & \\
\hline Distance & to disturbance & 300 & ches \\
\hline Mea & urement units & pobSF6 & \\
\hline Order $\rightarrow$ & & & $\mathrm{nd}$ \\
\hline Traverse $\rightarrow$ & & & Side \\
\hline Trial $\longrightarrow$ & & 1 & 2 \\
\hline Point & Depth, in. & & ppb \\
\hline 1 & 0.50 & 554 & 511 \\
\hline 2 & 1.25 & 524 & 539 \\
\hline 3 & 2.30 & 550 & 544 \\
\hline 4 & 3.84 & 517 & 553 \\
\hline Center & 5.94 & 529 & 488 \\
\hline 5 & 8.04 & 536 & 534 \\
\hline 6 & 9.57 & 509 & 511 \\
\hline 7 & 10.63 & 545 & 501 \\
\hline 8 & 11.38 & 513 & 527 \\
\hline Averages - & $\longrightarrow$ & 530.8 & 523.1 \\
\hline & & $\boldsymbol{A I I}$ & $\mathrm{ppb}$ \\
\hline & & Mean & 527.50 \\
\hline & & Mn Point & 512.33 \\
\hline & & Max Point & 556.33 \\
\hline
\end{tabular}

Avg. Conc. $\quad 528.208 \mathrm{ppb}$

\begin{tabular}{|c|c|c|c|}
\hline \multirow{3}{*}{$\begin{array}{l}\text { Tracer tank pressure } \\
\text { Injection flowmeter }\end{array}$} & Start & Finish & \\
\hline & 100 & 100 & psig \\
\hline & 30 & 30 & scem \\
\hline Stack Temp & 61.0 & 63.2 & ${ }^{\circ} \mathrm{F}$ \\
\hline Mean stack velocity & 2799 & 2822 & sfpm \\
\hline Sampling flowmeter & 5 & 5 & lpm \\
\hline Ambient pressure & 998 & 998 & mbal \\
\hline Ambient humidity & $44 \%$ & $34 \%$ & RH \\
\hline Ambient Temp & 64.4 & 63.5 & ${ }^{\circ} \mathrm{F}$ \\
\hline B\&K vapor correction & $Y$ & $\mathrm{Y}$ & $Y / N$ \\
\hline Back-Gd gas & $10,10,13,9,7$ & $7,9,5,5,10$ & ppb \\
\hline o. Bk-Gd samples & 5 & 5 & n \\
\hline
\end{tabular}

Gas anahzer checked:

$4 / 9 / 2012$

$X Y 4 / 13 / 12$

Notes: $\quad$ Reduce injection flow meter from 30 to $15 \mathrm{accm}$ to reach $\sim 600-700$ ooob at center. We foiund this was caused by not completely opening the cover of the fan. Corrected. Reset the injection flow meter back to $30 \mathrm{sccm}$, center concentration is about $500 \mathrm{ppb}$. XY $4 / 13 / 12$

\begin{tabular}{|c|c|}
\hline 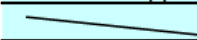 & $X Y 4 / 13 / 12$ \\
\hline Entries made by: & Xiao-Ying Yu \\
\hline Signature/date & $\begin{array}{l}\text { Signature on file with original } \\
4 / 13 / 2012\end{array}$ \\
\hline
\end{tabular}

20.1
Run No. GT-12

Fan Configuration Fan B only

Fan Seting 60

Stack Temp

Hz

Start/End Tme 9:40/10:46

Center 2/3 from

Points in Center $2 / 3$

Injection Point C FAR WALL

$62.1 \mathrm{deg} F$

1.10 to: 10.84

2

to: 7

1 st

\begin{tabular}{|c|c|c|c|c|}
\hline \multirow[b]{3}{*}{ Mean } & \multicolumn{2}{|c|}{$1 \mathrm{st}$} & & \multirow[b]{3}{*}{ Mean } \\
\hline & \multicolumn{3}{|c|}{ Bottom } & \\
\hline & 1 & 2 & 3 & \\
\hline & \multicolumn{3}{|c|}{ ppb } & \\
\hline 538.0 & 543 & 565 & 561 & 556.3 \\
\hline 527.3 & 522 & 523 & 525 & 523.3 \\
\hline 528.7 & 534 & 534 & 529 & 532.3 \\
\hline 525.0 & 519 & 542 & 545 & 535.3 \\
\hline 512.3 & 513 & 527 & 554 & 531.3 \\
\hline 530.3 & 531 & 504 & 512 & 515.7 \\
\hline 519.0 & 513 & 526 & 508 & 515.7 \\
\hline 522.0 & 526 & 539 & 517 & 527.3 \\
\hline 519.3 & 524 & 537 & 546 & 535.7 \\
\hline 524.7 & 525.0 & 533.0 & 533.0 & 530.3 \\
\hline
\end{tabular}

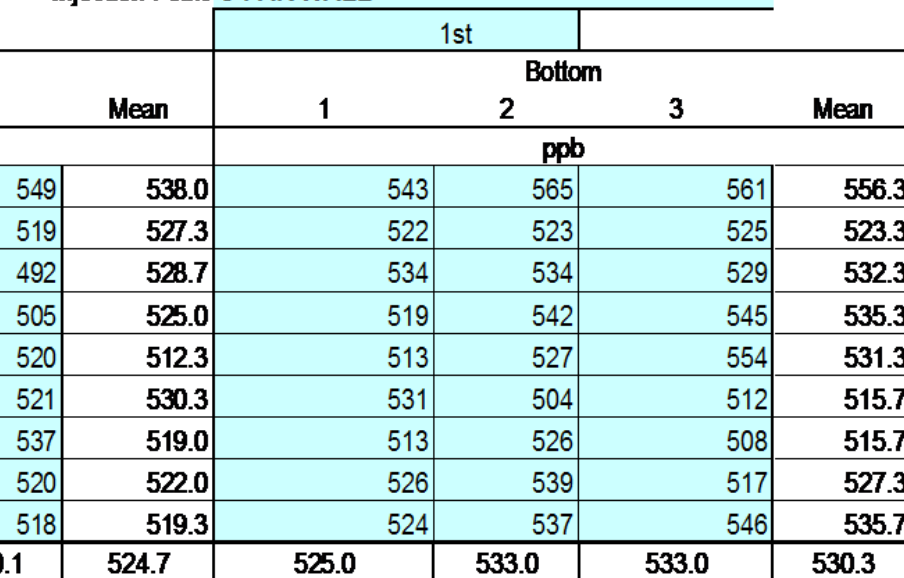

\begin{tabular}{r|lrrr|} 
Dev. from mean & Center 2/3 & Side & Bottom & All \\
$-2.9 \%$ & Mean & 523.52 & 525.86 & 524.69 \\
$5.5 \%$ & Std. Dev. & 6.29 & 7.94 & 6.99 \\
\hline Cov as \% & 1.2 & 1.5 & 1.3 \\
\hline
\end{tabular}

\begin{tabular}{lr} 
Instuments Used: & \\
B\&K 1302 Gas Analyzer SN $\quad 1765299$ & Cat2 M\&TE \\
\hline TSI VelociCalc SN T95351203001 & $1 / 17 / 2013$ \\
\hline Fisher Scientific SN 90936818 & $12 / 7 / 2012$ \\
\hline
\end{tabular}

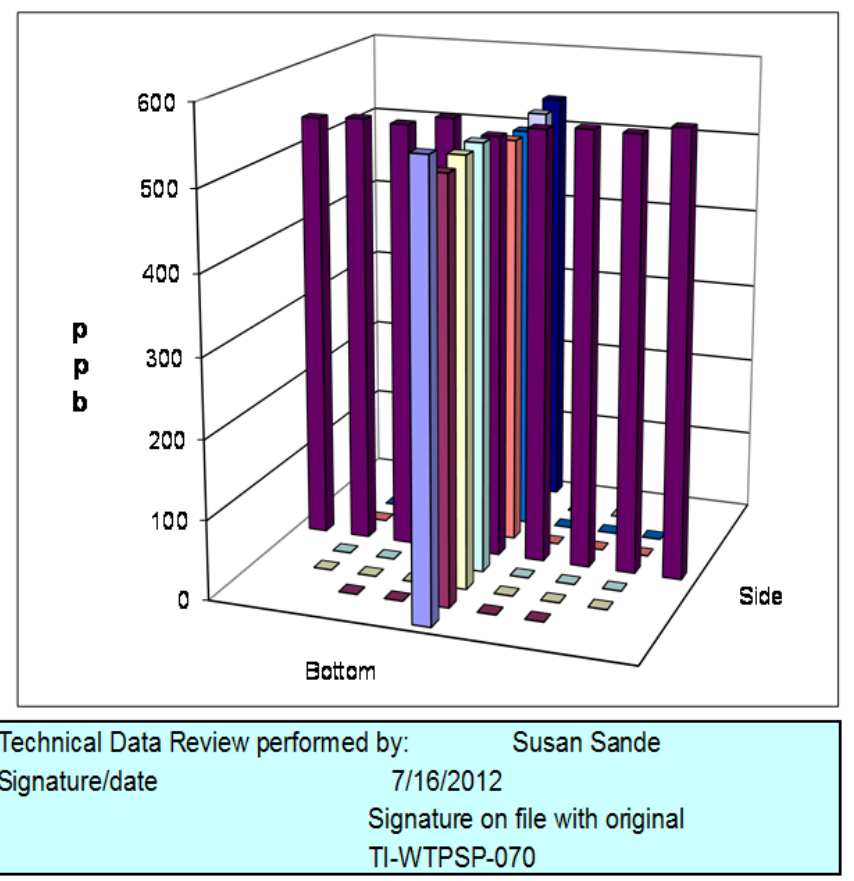


Rev. 0

31-JuL06

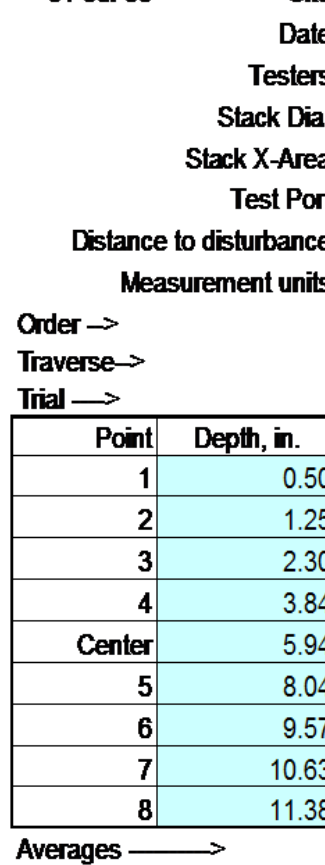

TRACER GAS TRAVERSE DATA FORU

Site HV -S1 Model

Date $4 / 13 / 2012$

Testers EA, XY

11.938 in.

X-Area 111.9 in. $^{2}$

1 300 inches

ppb SF6
Run No. GT-13

Fan Configuration Fan B only

Fan Setting 60

Stack Temp

Start/End Tme 10:46/12:20

Center $2 / 3$ from

Points in Center $2 / 3$

Injection Point $\mathbf{C}$ center

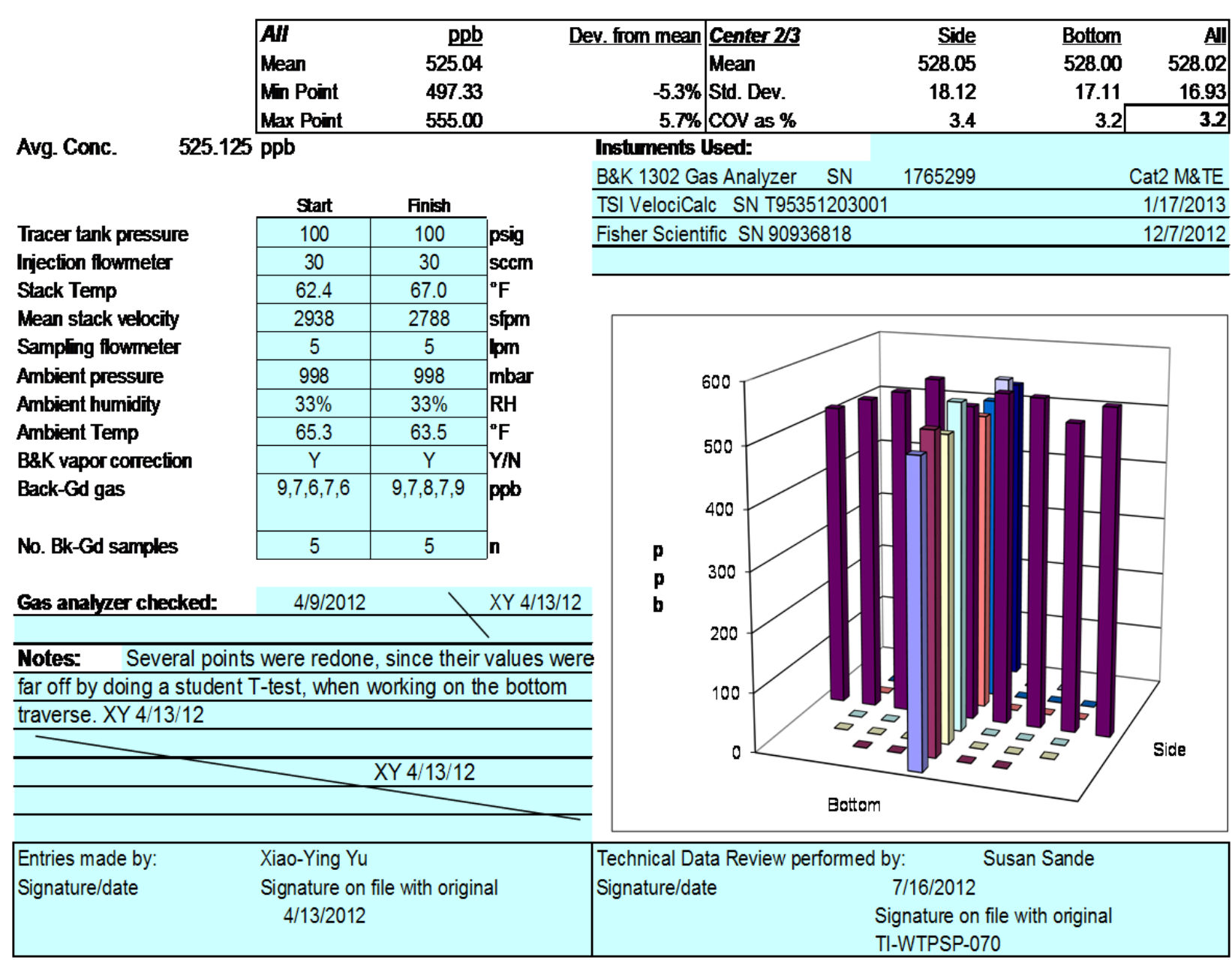


Rev. 0

31-JuL06

Run No. GT-14

Fan Configuration Fan A only

\begin{tabular}{|c|c|c|c|}
\hline Fan Setting 60 & \multicolumn{2}{|c|}{$\mathrm{Hz}$} & \\
\hline Stack Temp & \multicolumn{2}{|c|}{$73.6 \operatorname{deg} F$} & \\
\hline \multicolumn{4}{|c|}{ Start/End Tme $1300 / 1415$} \\
\hline Senter $2 / 3$ from & 1.10 & to: & 10.84 \\
\hline s in Center $2 / 3$ & 2 & to: & 7 \\
\hline
\end{tabular}

Injection Point $\mathrm{C}$ center

\begin{tabular}{|c|c|c|c|c|c|c|}
\hline $\boldsymbol{A l l}$ & ppb & Dev. from mean & Center 2/3 & Side & Bottom & All \\
\hline Mean & 559.56 & & Mean & $55 \overline{9.43}$ & 557.67 & $558 . \overline{55}$ \\
\hline Mn Point & 552.00 & $-1.4 \%$ & Std. Dev. & 8.81 & 5.21 & 7.02 \\
\hline Max Point & 578.33 & $3.4 \%$ & cov as $\%$ & 1.6 & 0.9 & 1.3 \\
\hline
\end{tabular}

Avg. Conc. $\quad 559.375$ ppb

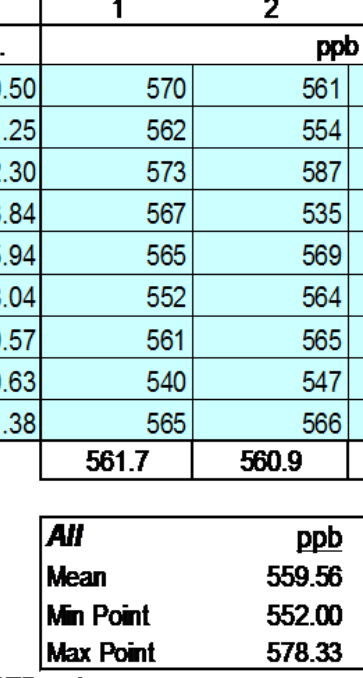

Tracer tank pressure Injection flowmeter Stack Temp

Mean stack velocity Sampling flowmeter Ambient pressure Ambient humidity Ambient Temp B\&K vapor correction Back-Gd gas

No. Bk-Gd samples

\begin{tabular}{|c|c|}
\hline Start & Finish \\
\hline 125 & 200 \\
\hline 30 & 30 \\
\hline 71.8 & 75.4 \\
\hline 2525 & 2560 \\
\hline 5 & 5 \\
\hline 996 & 996 \\
\hline $23 \%$ & $24 \%$ \\
\hline 75 & 71 \\
\hline $\mathrm{Y}$ & $Y$ \\
\hline $4,8,6,4,3$ & $\begin{array}{c}12,14,13 \\
11,10\end{array}$ \\
\hline 5 & 5 \\
\hline
\end{tabular}

Gas anahzer checked:

EA 4/13/12

Notes: $\quad$ B\&K Analyzer reset on final traverse side traverse center. Re-did reading 4.

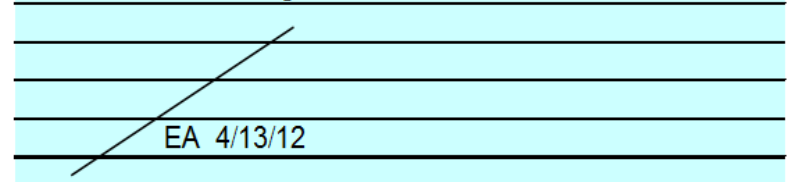

\section{Instuments Used:}

\begin{tabular}{llll} 
B\&K 1302 Gas Analyzer & SN $\quad 1765299$ & Cat2 M\&TE \\
\hline
\end{tabular} TSI VelociCalc SN T95351203001 $1 / 17 / 2013$ Fisher Scientific SN 90936818 $12 / 7 / 2012$

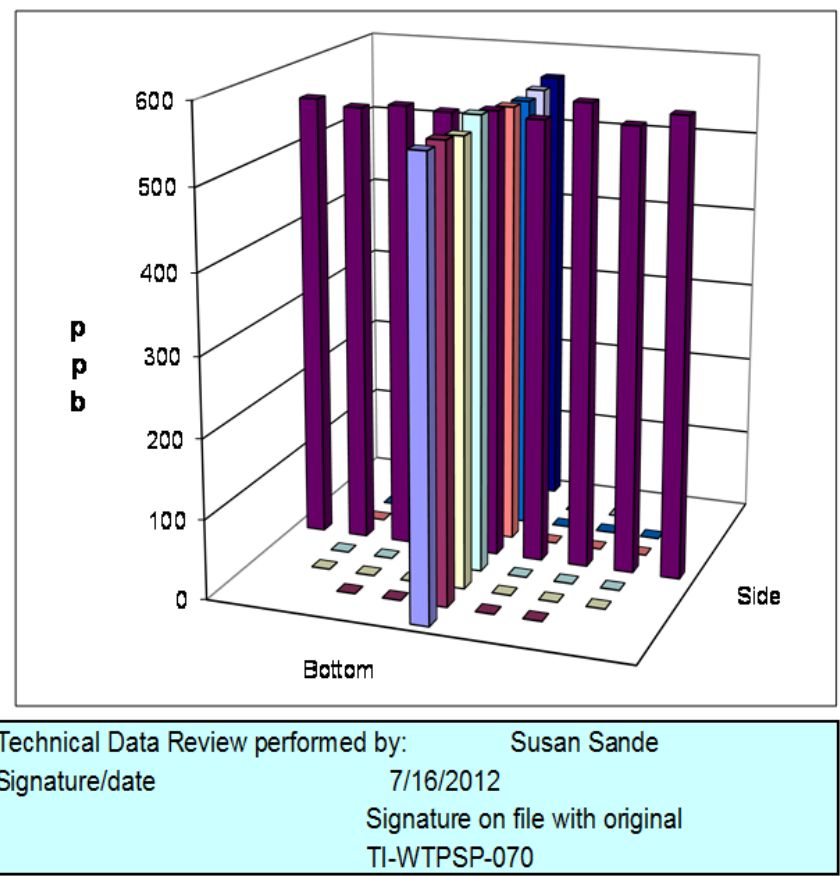


Rev. 0

31-JuL06

\begin{tabular}{|c|c|}
\hline \multicolumn{2}{|c|}{ Site HV-S1 Model } \\
\hline Date & $4 / 13 / 2012$ \\
\hline \multicolumn{2}{|c|}{ Testers EA, YFS } \\
\hline Stack Dia. & 11.938 in. \\
\hline Stack X-Area & 111.9 in. $^{2}$ \\
\hline Test Port & 2 \\
\hline
\end{tabular}

Distance to disturbance 240 inches Measurement units pob SF6

TRACER GAS TRAVERSE DATA FORU

Order $\rightarrow$

Traverse $\rightarrow$

Tíal $\longrightarrow$

\begin{tabular}{|c|c|}
\hline Point & Depth, in. \\
\hline 1 & 0.5 \\
\hline 2 & 1.2 \\
\hline 3 & 2.3 \\
\hline 4 & 3.8 \\
\hline Center & 5.8 \\
\hline 5 & 8.0 \\
\hline 6 & 9.5 \\
\hline 7 & 10.6 \\
\hline 8 & 11.3 \\
\hline
\end{tabular}

Averages

Run No. GT-15

Fan Configuration Fan B only

Fan Setting 60

Stack Temp $\mathrm{Hz}$

Start/End Tme $1510 / 1630$

Center $2 / 3$ from

Points in Center 2/3

$73.55 \operatorname{deg} F$

Injection Point

$\begin{array}{llll} & 1.10 & \text { to: } & 10.84 \\ & 2 & \text { to: } & \end{array}$

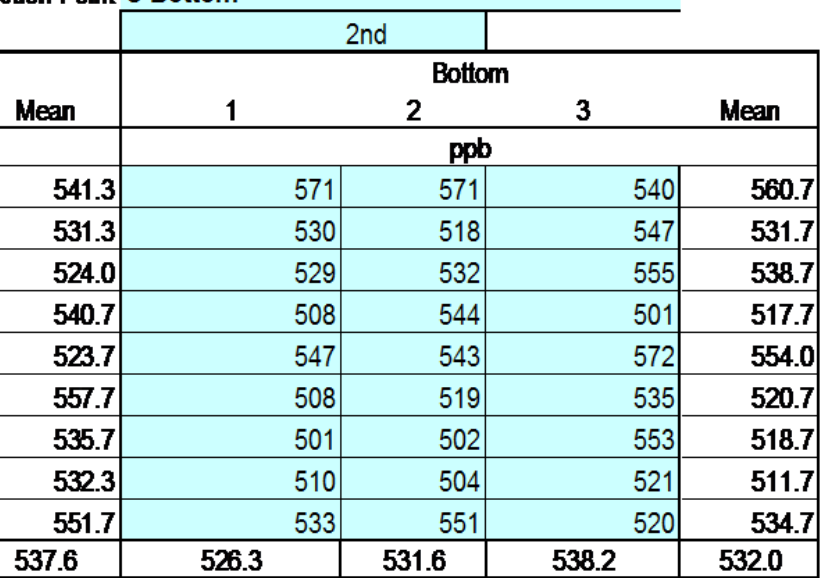

Avg. Conc. $\quad 534.313 \mathrm{ppb}$

\begin{tabular}{|rr}
\hline $\boldsymbol{A l l}$ & $\mathrm{ppb}$ \\
Mean & 534.81 \\
Mn Point & 511.67 \\
Max Point & 560.67 \\
\hline
\end{tabular}

\begin{tabular}{r|l} 
Dev. from mean & Center $2 / 3$ \\
$-4.3 \%$ & Mean \\
$4.8 \%$ & Std. Dev. \\
Cov as \% \\
\hline
\end{tabular}

\begin{tabular}{rrr|} 
Side & Bottom & All \\
535.05 & 527.57 & 531.31 \\
11.66 & 14.81 & 13.38 \\
2.2 & 2.8 & 2.5 \\
\hline
\end{tabular}

\begin{tabular}{|c|c|c|}
\hline \multirow{3}{*}{$\begin{array}{l}\text { Tracer tank pressure } \\
\text { Injection flowmeter }\end{array}$} & Start & Finish \\
\hline & 200 & 125 \\
\hline & 30 & 30 \\
\hline Stack Temp & 76 & 71.1 \\
\hline Mean stack velocity & 3021 & 2798 \\
\hline Sampling flowmeter & 5 & 5 \\
\hline Ambient pressure & 995 & 995 \\
\hline Ambient humidity & $25 \%$ & $24 \%$ \\
\hline Ambient Temp & 69.8 & 70.7 \\
\hline B\&K vapor conrection & $Y$ & $\mathrm{Y}$ \\
\hline Back-Gd gas & $\begin{array}{c}10,6,6,10 \\
6\end{array}$ & $9,3,6,5,3$ \\
\hline No. Bk-Gd samples & 5 & 5 \\
\hline
\end{tabular}

Gas analyzer checked: $\quad$ 4/9/2012

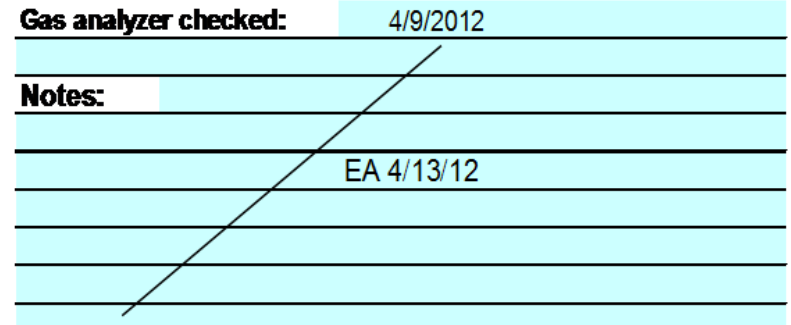

\begin{tabular}{lr} 
Instuments Used: & \\
B\&K 1302 Gas Analyzer SN 1765299 & Cat2 M\&TE \\
\hline TSI VelociCalc SN T95351203001 & $1 / 17 / 2013$ \\
\hline Fisher Scientific SN 90936818 & $12 / 7 / 2012$ \\
\hline
\end{tabular}

Entries made by:

Signature/date
EA

Signature on file with original $4 / 13 / 2012$

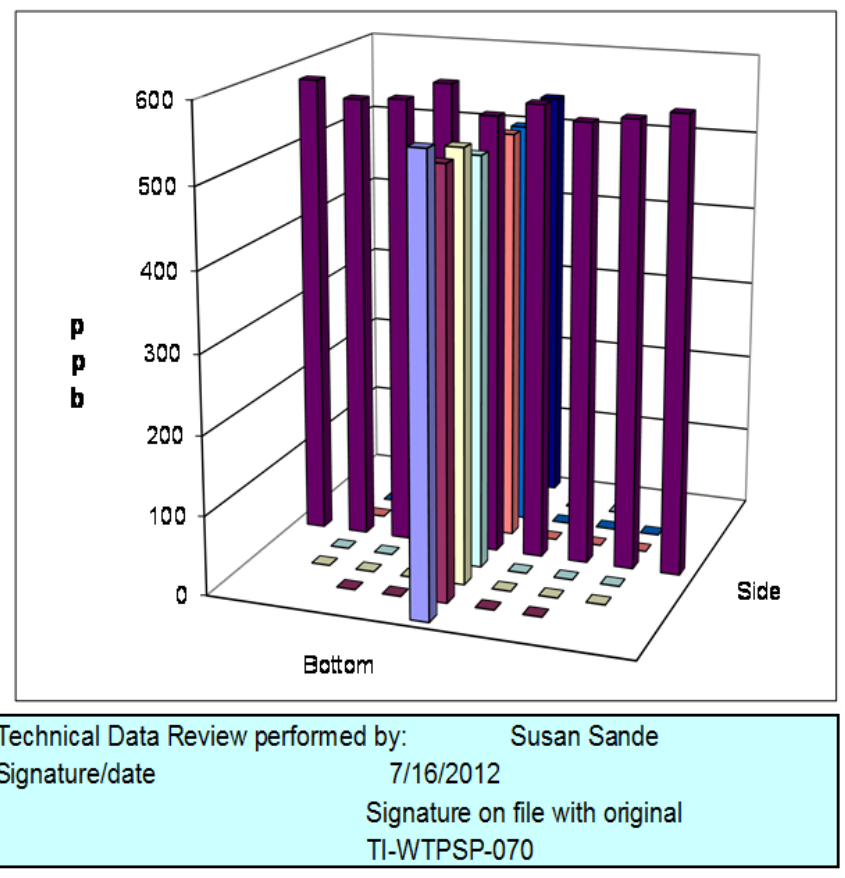


Rev. 0

31-JuL06

$\begin{aligned} & \text { Site HV -S1 Model } \\ & \text { Date } \frac{4 / 16 / 2012}{\mathrm{C}, \mathrm{XY}} \\ & \text { Testers } \frac{11.938 \text { in. }}{\mathrm{CA}} \\ & \text { Stack Dia. } \frac{11.93 \text { in. }^{2}}{111.9} \\ & \text { Stack X-Area } 2 \\ & \text { Test Port } 2\end{aligned}$

Distance to disturbance 240 inches Measurement units pob SF6

TRACER GAS TRAVERSE DATA FORU

Order $\rightarrow$

Traverse $\rightarrow$

Tíal $\longrightarrow$

\begin{tabular}{|c|c|}
\hline Point & Depth, in. \\
\hline 1 & 0. \\
\hline 2 & 1. \\
\hline 3 & 2. \\
\hline 4 & 3. \\
\hline Center & 5. \\
\hline 5 & 8. \\
\hline 6 & 9. \\
\hline 7 & 10. \\
\hline 8 & 11. \\
\hline
\end{tabular}

Averages

Run No. GT-16

Fan Configuration Fan B only

Fan Setting 60 $\mathrm{Hz}$

Points in Center $2 / 3$

Stack Temp

Start/End Tme 11:00/12:30

Center $2 / 3$ from

Injection Point C Bottom

$57.8 \operatorname{deg} F$

1.10 to: 10.84

2 to: 7

$2 \mathrm{nd}$

\begin{tabular}{|c|c|c|c|}
\hline \multirow[b]{2}{*}{ Avg. Conc. } & \begin{tabular}{|l|}
$A l l$ \\
Mean \\
Min Point \\
Max Point \\
\end{tabular} & \multicolumn{2}{|l|}{$\begin{array}{l}537.00 \\
518.67 \\
555.67\end{array}$} \\
\hline & \multicolumn{3}{|c|}{$536.000 \mathrm{ppb}$} \\
\hline & Start & Finish & \\
\hline \multirow{5}{*}{$\begin{array}{l}\text { Tracer tank pressure } \\
\text { Injection flowmeter } \\
\text { Stack Temp } \\
\text { Mean stack velocity } \\
\text { Sampha flowmeter }\end{array}$} & 100 & 100 & psig \\
\hline & 30 & 30 & scem \\
\hline & 57.1 & 58.5 & ${ }^{\circ} \mathrm{F}$ \\
\hline & 3175 & 3004 & sfpm \\
\hline & 5 & 5 & lom \\
\hline \multirow{2}{*}{$\begin{array}{l}\text { Ambient pressure } \\
\text { Ambient humidity }\end{array}$} & 1004 & 1004 & mbar \\
\hline & $79 \%$ & $68 \%$ & $\mathbf{R H}$ \\
\hline \multirow{2}{*}{$\begin{array}{l}\text { Ambient Temp } \\
\text { B\&K vapor correction }\end{array}$} & 59 & 57.2 & ${ }^{\circ} \mathrm{F}$ \\
\hline & $Y$ & $Y$ & Y/N \\
\hline Back-Gd gas & $3,4,5,2,4$ & $10,11,9,5,7$ & ppb \\
\hline No. Bk-Gd samples & 5 & 5 & \\
\hline
\end{tabular}

Gas analyzer checked:

$4 / 16 / 2012$

CA XY $4 / 16 / 12$

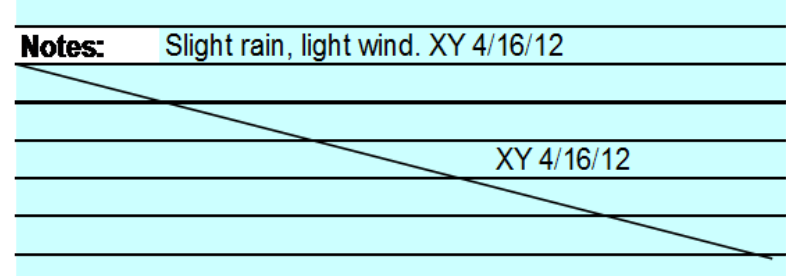

Entries made by:

Signature/date

\begin{tabular}{|c|c|}
\hline Dev. from mean & Center 2/3 \\
\hline & Mean \\
\hline $\begin{array}{r}-3.4 \% \\
3.5 \%\end{array}$ & $\begin{array}{l}\text { Std. Dev. } \\
\text { COV as \% }\end{array}$ \\
\hline
\end{tabular}

Instuments Used:

TSI VelociCalc SN T95351203001

Fisher Scientific SN 90936818

\begin{tabular}{rrr|}
\hline Side & Bottom & All \\
534.10 & 541.62 & 537.86 \\
11.32 & 10.72 & 11.29 \\
2.1 & 2.0 & 2.1 \\
\hline
\end{tabular}

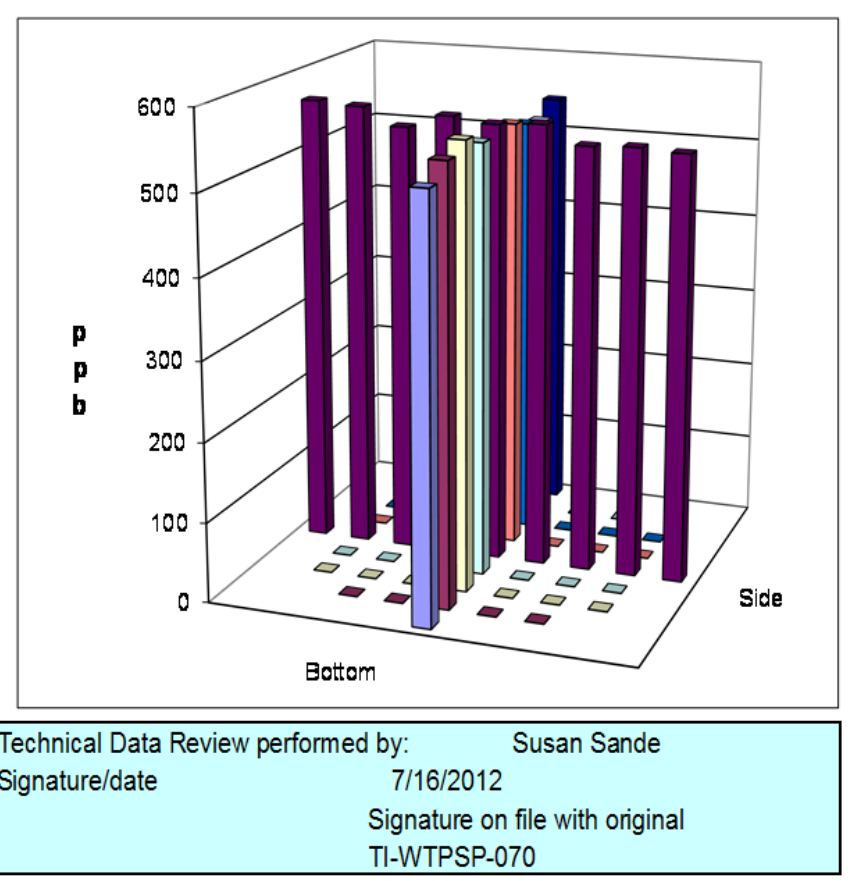


Rev. 0

31-JuL06

\begin{tabular}{|c|c|}
\hline \multicolumn{2}{|c|}{ Site HV-S1 Model } \\
\hline Date & $6 / 28 / 2012$ \\
\hline \multicolumn{2}{|c|}{ Testers $\mathrm{Ca}, \mathrm{EA}$} \\
\hline Stack Dia. & 11.938 in. \\
\hline Stack X-Area & 111.9 in. $^{2}$ \\
\hline Test Port & 2 \\
\hline
\end{tabular}

Distance to disturbance 240 inches

Measurement units pob SF6

TRACER GAS TRAVERSE DATA FORU

Order $\rightarrow$

Traverse $\rightarrow$

Tinal $\longrightarrow$

\begin{tabular}{|c|c|}
\hline Point & Depth, in. \\
\hline 1 & 0. \\
\hline 2 & 1. \\
\hline 3 & 2. \\
\hline 4 & 3. \\
\hline Center & 5. \\
\hline 5 & 8. \\
\hline 6 & 9. \\
\hline 7 & 10. \\
\hline 8 & 11. \\
\hline
\end{tabular}

Averages

\begin{tabular}{|ccc|}
\hline & & \\
\hline & & \\
1 & & 2
\end{tabular}

\author{
Fan
}

Run No. GT-17

Fan Configuration Fan A only

Fan Seting 60

Stack Temp

Start/End Tme 1000/1130

Center $2 / 3$ from

Points in Center $2 / 3$

\begin{tabular}{cc}
\cline { 2 - 2 } Injection Point & C-C \\
\cline { 2 - 3 } & 2nd
\end{tabular}

3 \begin{tabular}{|lr}
\hline$A I I$ & ppb \\
Mean & 467.06 \\
Mn Point & 455.00 \\
Max Point & 473.00 \\
\hline
\end{tabular}

Avg. Conc.

466.917 ppb

Tracer tank pressure Injection flowmeter Stack Temp

Mean stack velocity Samping flowmeter Ambient pressure Ambient humidity Ambient Temp B\&K vapor correction Back-Gd gas

No. Bk-Gd samples

\begin{tabular}{|c|c|}
\hline Start & Finish \\
\hline 125 & 125 \\
\hline 30 & 30 \\
\hline 80 & 86 \\
\hline 3317 & 3307 \\
\hline 5 & 5 \\
\hline 1002 & 1001 \\
\hline $32 \%$ & $32 \%$ \\
\hline 75.2 & 77.0 \\
\hline$Y$ & $Y$ \\
\hline $\begin{array}{c}1,1.27, .3 \\
4.0,4.1 \\
\end{array}$ & $\begin{array}{c}6.8,8.3,8.3, \\
2.08,4.28 \\
\end{array}$ \\
\hline 5 & 5 \\
\hline
\end{tabular}

Gas anabyer checked:

$6 / 27 / 2012$

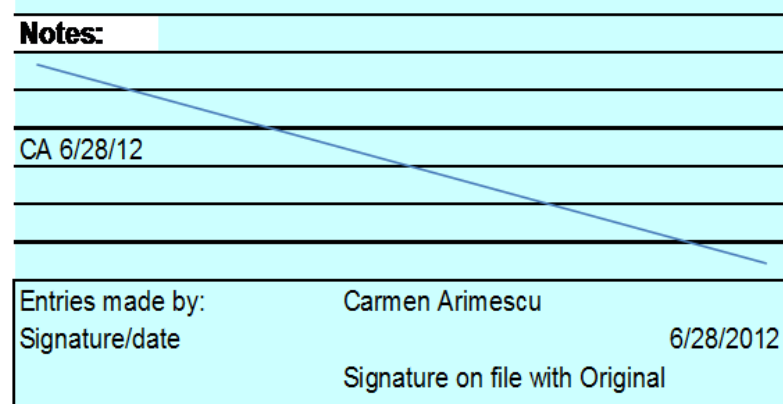

\begin{tabular}{r|l} 
Dev. from mean & Center 2/3 \\
$-2.6 \%$ & Mean \\
$1.3 \%$ & Std. Dev. \\
Cov as \%
\end{tabular}
Instuments Used:

B\&K 1302 Gas Analyzer SN TSI VelociCalc SN T95351203001 Fisher Scientific SN 90936818

Side
466.95
5.96
1.3

\begin{tabular}{rr|}
\hline Bottom & All \\
\hline 468.10 & 467.52 \\
4.08 & 4.94 \\
0.9 & 1.1 \\
\hline
\end{tabular}

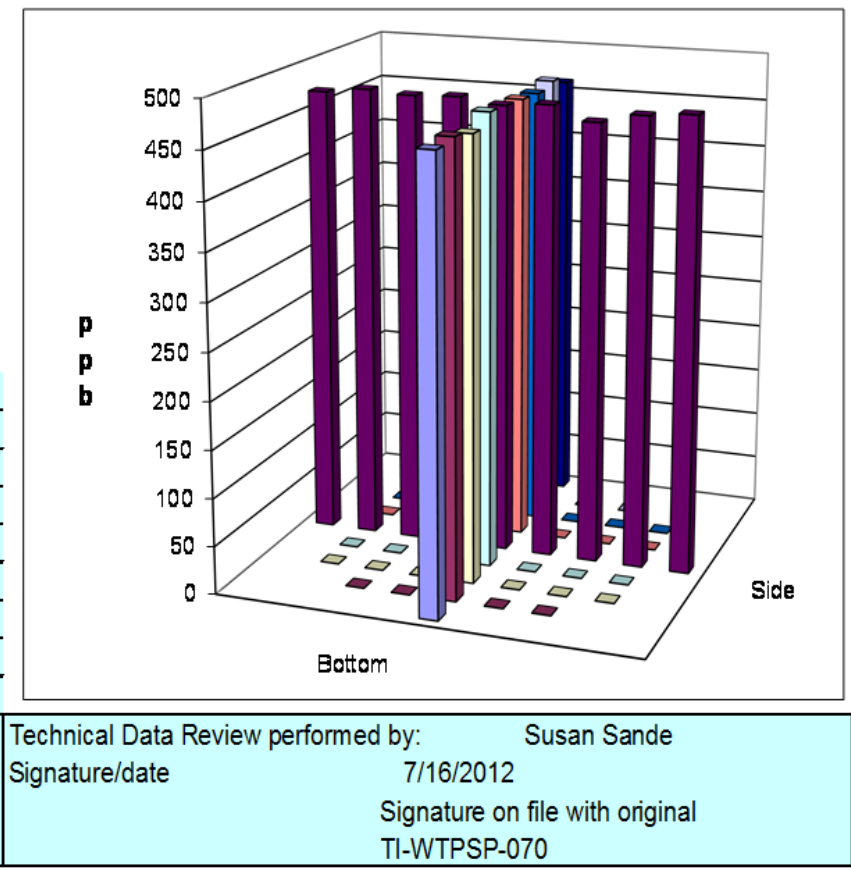




\section{A.5 HV-S1 Particle Tracer Uniformity Data Sheets}

Rev. 0

3 Aug. 2006

\begin{tabular}{|c|c|}
\hline & \\
\hline Date & $4 / 3 / 2012$ \\
\hline Tester & JAG,CA \\
\hline Stack Dia. & 11.938 in. \\
\hline Stack X-Area & 111.9 in. 2 \\
\hline Test Port & 2 \\
\hline Distance to disturbance & 240 inches \\
\hline Measurement units & particles $\mathrm{fl3}$ \\
\hline
\end{tabular}

\section{PARTICLE TRACER TRAVERE DATA FORM}

Fan configuration FAN B ONLY

Fan Setting 60

Stack Temp $\mathbf{7 2 . 4 5 ~ d e g ~ F ~}$

Start/End Time 1:48/3:48

Center $2 / 3$ from

Points in Center $2 / 3$ Injection Point C Center

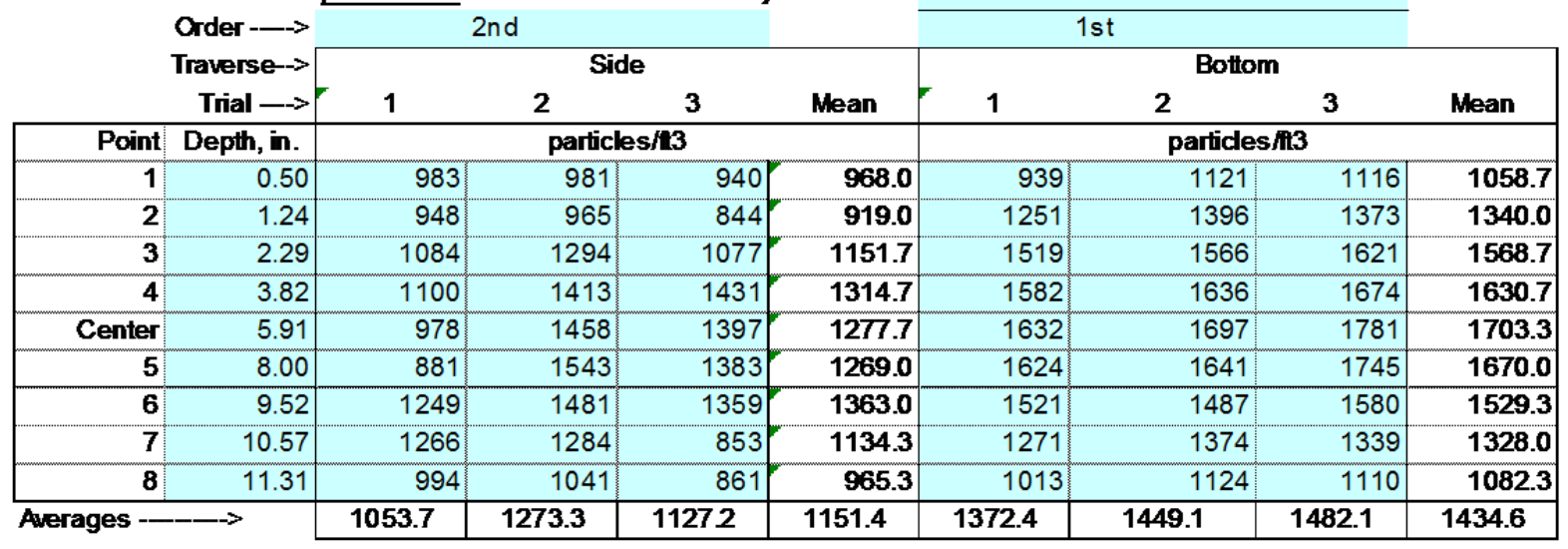

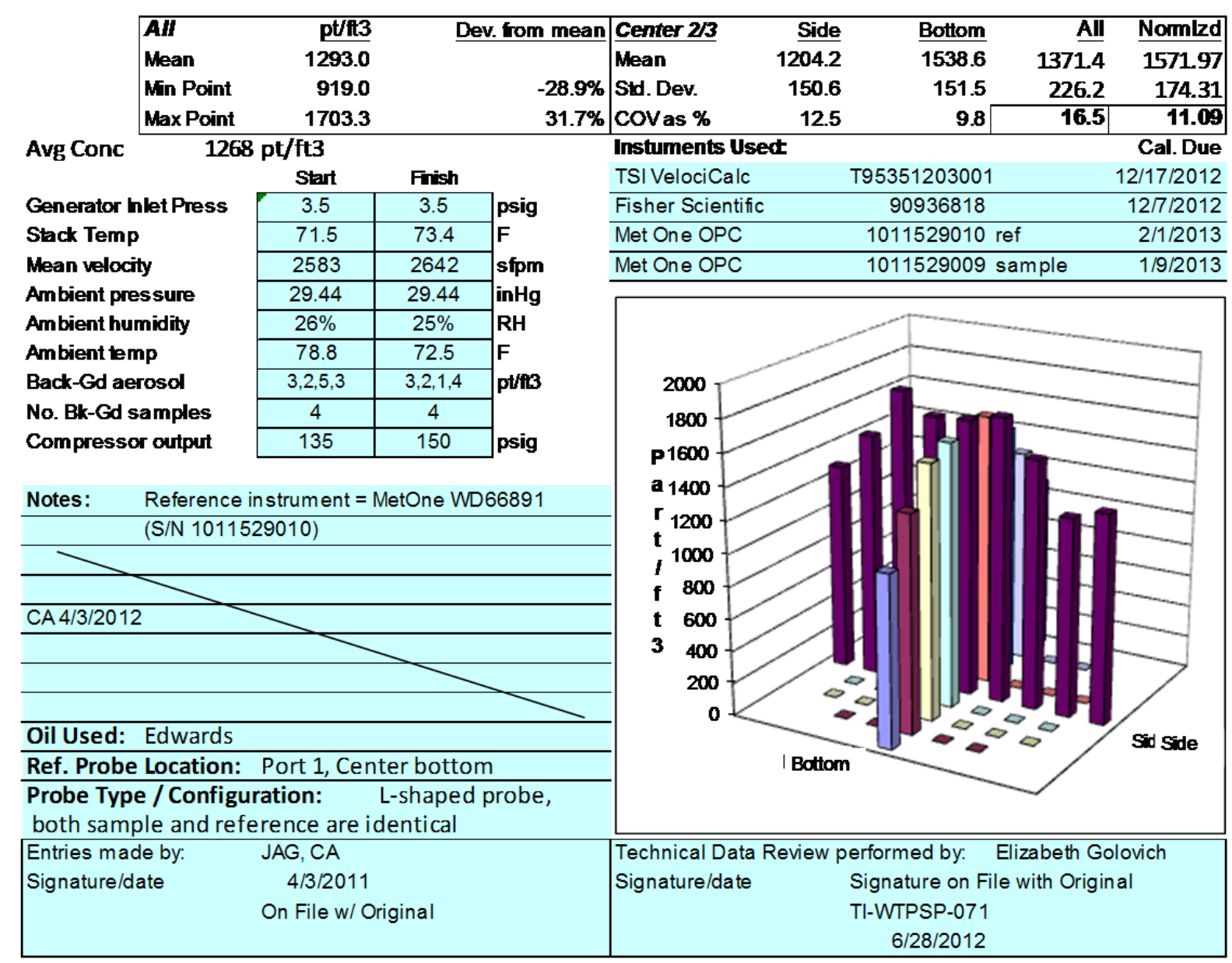


Rev. 0

3 Aug. 2006
PARTICLE TRACER TRAVERE DATA FORM

Site HV-S1 Model Date 4/3/2012

Tester JAG ,CA

Stack Dia. 11.938 in.

Stack X-Area 111.9 in 2

TestPort

2

Distance to disturbance 240 inches

Measurement units particles $/$ th 3

Order $\longrightarrow$

Traverse-

Trial $\longrightarrow$

\begin{tabular}{|ll}
\multicolumn{3}{|c|}{1 st } & \\
1 & $2^{\text {Side }}$
\end{tabular}
Run No. PT-2

Fan configuration FAN B ONLY

Fan Setting 23

Stack Temp

$67.85 \operatorname{deg} \mathrm{F}$

StartEnd Time 3:40/ 5:45

Center 23 from

Points in Center 23 mjection Point Center

\begin{tabular}{|c|c|c|c|c|c|c|c|c|c|}
\hline \multirow{3}{*}{\multicolumn{2}{|c|}{ Trave }} & \multirow{2}{*}{\multicolumn{4}{|c|}{ Side }} & \multirow{2}{*}{\multicolumn{4}{|c|}{ Bottom }} \\
\hline & & & & & & & & & \\
\hline & & 1 & 2 & 3 & Mean & 1 & 2 & 3 & Mean \\
\hline Point & Depth, in. & \multicolumn{4}{|c|}{ particles $/ 1$ 3 } & \multicolumn{4}{|c|}{ particles/ft3 } \\
\hline 1 & 0.50 & 1377 & 1525 & 1515 & 1472.3 & 1095 & 1570 & 1321 & 1328.7 \\
\hline 2 & 1.24 & 1509 & 1515 & 1534 & 1519.3 & 1166 & 1564 & 1299 & 13430 \\
\hline 3 & 2.29 & 1538 & 1585 & 1613 & 1578.7 & 1141 & 1574 & 1301 & 1338.7 \\
\hline 4 & 3.82 & 1517 & 1670 & 1723 & 1636.7 & 1223 & 1414 & 1356 & 13310 \\
\hline Center & 5.91 & 1612 & 1691 & 1748 & 1683.7 & 1193 & 1558 & 1259 & 1336.7 \\
\hline 5 & 8.00 & 1683 & 1734 & 1776 & 17310 & 1203 & 1530 & 1285 & 1339.3 \\
\hline 6 & 9.52 & 1666 & 1718 & 1701 & 16950 & 1126 & 1339 & 1219 & 1228.0 \\
\hline 7 & 10.57 & 1593 & 1701 & 1660 & 1651.3 & 1126 & 1368 & 1107 & 1200.3 \\
\hline 8 & 11.31 & 1490 & 1575 & 1438 & 15010 & 1069 & 1231 & 1064 & 11213 \\
\hline Averages & $\longrightarrow$ & 1553.9 & 1634.9 & 16342 & 1607.7 & 1149.1 & 1460.9 & 1245.7 & 12852 \\
\hline
\end{tabular}

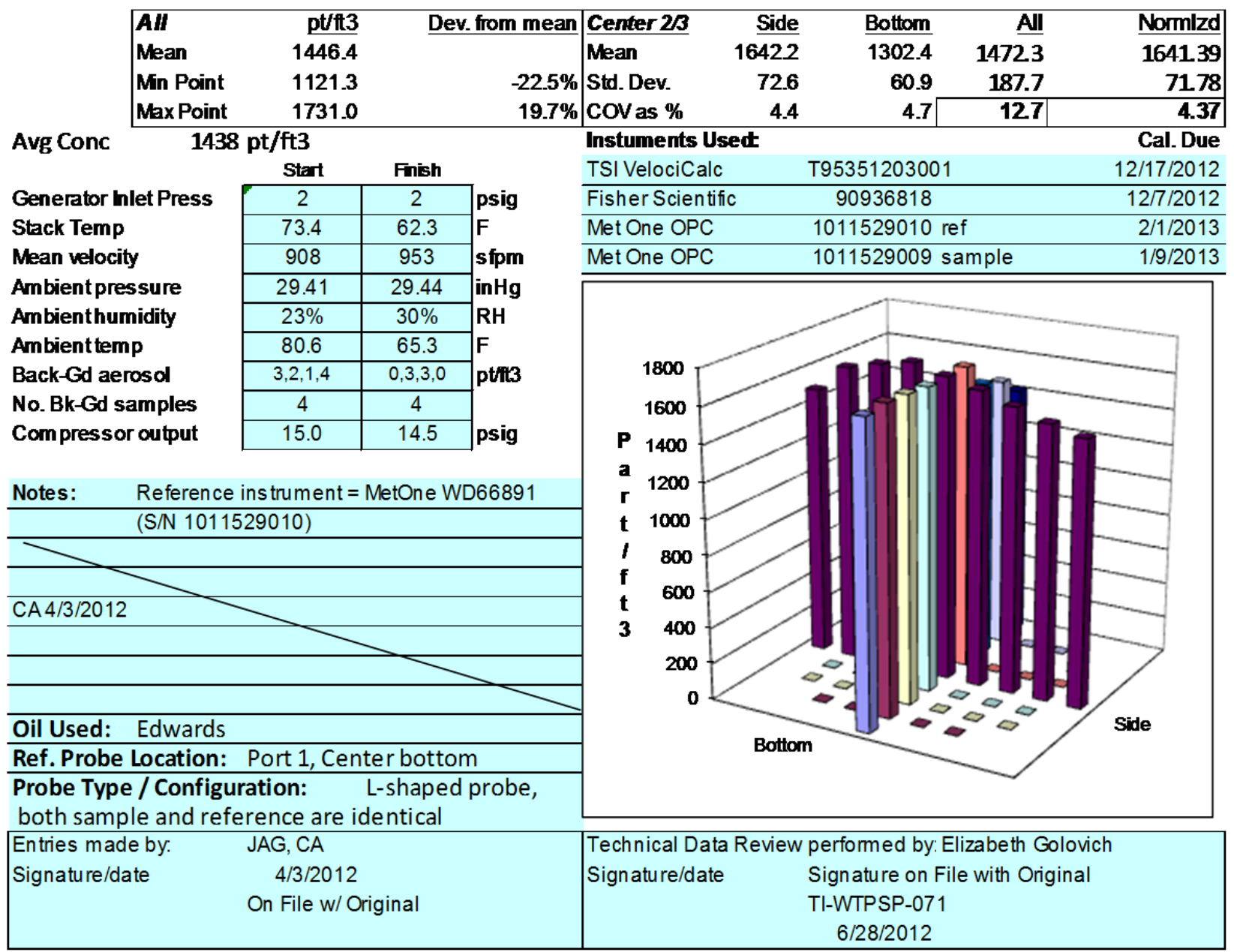


Rev. 0

3 Aug. 2006

Site HV-S1 Model

Date 4/4/2012

Tester CA, XY, JAG

Stack Dia. 11.938 in.

Stack X-Area 111.9 in 2

TestPort

Distance to disturbance

Measurement units particlesfit?
ARTICLE TRACER TRAVERE DATA FORM

Run No. PT-3

Fan configuration FAN A ONLY

Fan Setting 60

Stack Temp

StartEnd Time 9:45/12:45

Center 23 from

Points in Center 23 njection Point Center

\begin{tabular}{|c|c|c|c|c|c|c|c|c|c|}
\hline \multirow{3}{*}{\multicolumn{2}{|c|}{$\begin{array}{c}\text { Order } \longrightarrow \\
\text { Traverse } \rightarrow \\
\text { Trial } \longrightarrow\end{array}$}} & \multicolumn{3}{|c|}{ 2nd } & \multicolumn{4}{|c|}{$1 \mathrm{st}$} & \multirow[b]{3}{*}{ Mean } \\
\hline & & \multicolumn{3}{|c|}{ Side } & \multirow{3}{*}{ Mean } & \multicolumn{3}{|c|}{ Bottom } & \\
\hline & & 1 & 2 & 3 & & 1 & 2 & 3 & \\
\hline Point & Depth, in. & \multicolumn{3}{|c|}{ particles/fi3 } & & \multicolumn{4}{|c|}{ particles fit3 } \\
\hline 1 & 0.50 & 750 & 973 & 756 & 826.3 & 2032 & 1998 & 2227 & 2085.7 \\
\hline 2 & 1.24 & 895 & 976 & 882 & 917.7 & 2433 & 2655 & 2867 & 2651.7 \\
\hline 3 & 2.29 & 1335 & 935 & 1221 & 1163.7 & 3261 & 3197 & 3214 & 3224.0 \\
\hline 4 & 3.82 & 1819 & 913 & 1906 & 1546.0 & 3723 & 3478 & 3650 & 36170 \\
\hline Center & 5.91 & 2073 & 1867 & 2134 & 2024.7 & 3700 & 3619 & 3694 & 3671.0 \\
\hline 5 & 8.00 & 1991 & 2275 & 1955 & 2073.7 & 3162 & 3495 & 3498 & 33850 \\
\hline 6 & 9.52 & 1809 & 1957 & 1585 & 1783.7 & 2673 & 2652 & 2863 & 2729.3 \\
\hline 7 & 10.57 & 1514 & 1515 & 1328 & 1452.3 & 2518 & 2134 & 2320 & 23240 \\
\hline 8 & 11.31 & 917 & 1152 & 1289 & 1119.3 & 2318 & 2025 & 2174 & 2172.3 \\
\hline Averages - & > & 1455.9 & 1395.9 & 1450.7 & 1434.1 & 2868.9 & 2805.9 & 29452 & 2873.3 \\
\hline
\end{tabular}

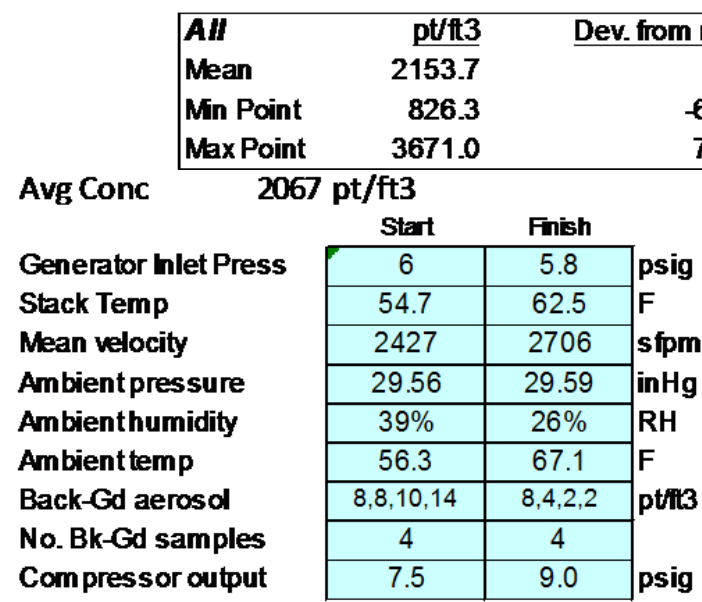

Notes: Compressor pressure $150 \mathrm{psi}$

The particle generator seems to be very sensitive to ambient temperaturechange. For example, outdoor temperature changes from $56 \mathrm{~F}$ to $72 \mathrm{~F}$, the particle number concentration at center raised from $\sim 2700$ to 3700 . Thus, we redid traverse 1 st and 2 nd for bottom.

\begin{tabular}{l}
\hline \multicolumn{1}{|c|}{$\mathrm{XY} 4 / 4 / 12$} \\
\hline Oil Used: Edwards \\
\hline Ref. Probe Location: Port 1, Center bottom \\
\hline Probe Type / Configuration: L-shaped probe, \\
both sample and reference are identical \\
$\begin{array}{ll}\text { Entries made by: } \quad \mathrm{CA}, \mathrm{XY} \\
\text { Signature/date } \quad 4 / 4 / 2012 \\
\end{array} \quad$ On File w/ Original
\end{tabular}

\begin{tabular}{|c|c|c|c|c|c|}
\hline Iean & Center 23 & Side & Bottom & All & Normlzd \\
\hline & Mean & 1566.0 & 3086.0 & 2326.0 & 296264 \\
\hline 1.6 & Std. Dev. & 430.3 & 521.1 & 9126 & 650.12 \\
\hline 70.4 & COV as \% & 27.5 & 16.9 & 39.2 & 21.94 \\
\hline
\end{tabular}

\begin{tabular}{lr} 
Instuments Usedt & Cal. Due \\
\hline
\end{tabular}

\begin{tabular}{lcr} 
TSI VelociCalc & T95351203001 & $12 / 17 / 2012$ \\
\hline Fisher Scientific & 90936818 & $12 / 7 / 2012$ \\
\hline Met One OPC & 1011529010 sample & $2 / 1 / 2013$ \\
\hline Met One OPC & 1011529009 ref & $1 / 9 / 2013$ \\
\hline
\end{tabular}

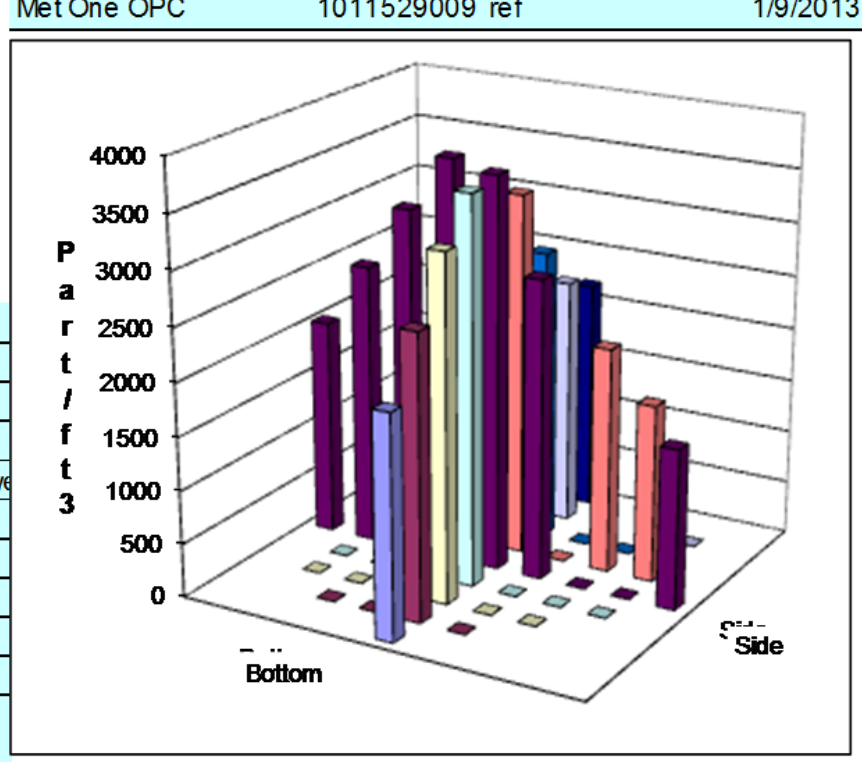

Technical Data Review performed by: Elizabeth Golovich Signatu re/date

Signatu re on File with Original

TI-WTPSP-071

$6 / 28 / 2012$ 
Rev. 0

3 Aug. 2006
PARTICLE TRACER TRAVERE DATA FORM

Site HV-S1 Model

Date 4/4/2012

Tester JAG, CA

Stack Dia. 11.938 in.

Stack X-Area 111.9 in 2

TestPort

2

Distance to disturbance 240 inches

Measurement units particles $/$ th 3

Order $\longrightarrow$

Traverse-

Trial $\longrightarrow$

\begin{tabular}{|r|r|}
\hline Point & Depth, in \\
\hline $\mathbf{1}$ & 0.50 \\
\hline $\mathbf{2}$ & 1.2 \\
\hline $\mathbf{3}$ & 2.29 \\
\hline $\mathbf{4}$ & 3.82 \\
\hline Center & 5.9 \\
\hline $\mathbf{5}$ & 8.00 \\
\hline $\mathbf{6}$ & 9.52 \\
\hline $\mathbf{7}$ & 10.5 \\
\hline $\mathbf{8}$ & 11.3 \\
\hline
\end{tabular}

Averages

$1 \mathrm{st}$
Run No. PT-4

Fan configuration FAN A ONLY

Fan Setting 27.1

Stack Temp $68.95 \mathrm{deg} F$

Start/End Time 1:40/4:00

Center 23 from 1.10

Points in Center $2 3 \longdiv { 2 }$

mjection Point Center

\begin{tabular}{|c|c|c|c|c|c|c|c|}
\hline \multicolumn{4}{|c|}{$1 \mathrm{st}$} & \multicolumn{3}{|c|}{ 2nd } & \\
\hline \multicolumn{3}{|c|}{ Side } & \multirow[b]{2}{*}{ Mean } & \multicolumn{4}{|c|}{ Bottom } \\
\hline 1 & 2 & 3 & & 1 & 2 & 3 & Mean \\
\hline \multicolumn{4}{|c|}{ particles $/$ t3 } & \multicolumn{4}{|c|}{ particles $/ \mathrm{t} 3$} \\
\hline 864 & 853 & 848 & 855.0 & 1497 & 1399 & 1459 & 1451.7 \\
\hline 991 & 973 & 826 & 930.0 & 1530 & 1550 & 1467 & 1515.7 \\
\hline 982 & 976 & 930 & 9627 & 1515 & 1549 & 1467 & 1510.3 \\
\hline 1012 & 1016 & 935 & 987.7 & 1491 & 1596 & 1476 & 15210 \\
\hline 1120 & 1159 & 1174 & 1151.0 & 1422 & 1587 & 1469 & 14927 \\
\hline 1297 & 1304 & 1283 & 1294.7 & 1514 & 1552 & 1418 & 1494.7 \\
\hline 1273 & 1008 & 1205 & 11620 & 1479 & 1521 & 1496 & 1498.7 \\
\hline 929 & 693 & 794 & 805.3 & 1390 & 1436 & 1417 & 14143 \\
\hline 799 & 833 & 731 & 787.7 & 1357 & 1340 & 1331 & 13427 \\
\hline 1029.7 & 979.4 & 969.6 & 992.9 & 1466.1 & 1503.3 & 1444.4 & 1471.3 \\
\hline
\end{tabular}

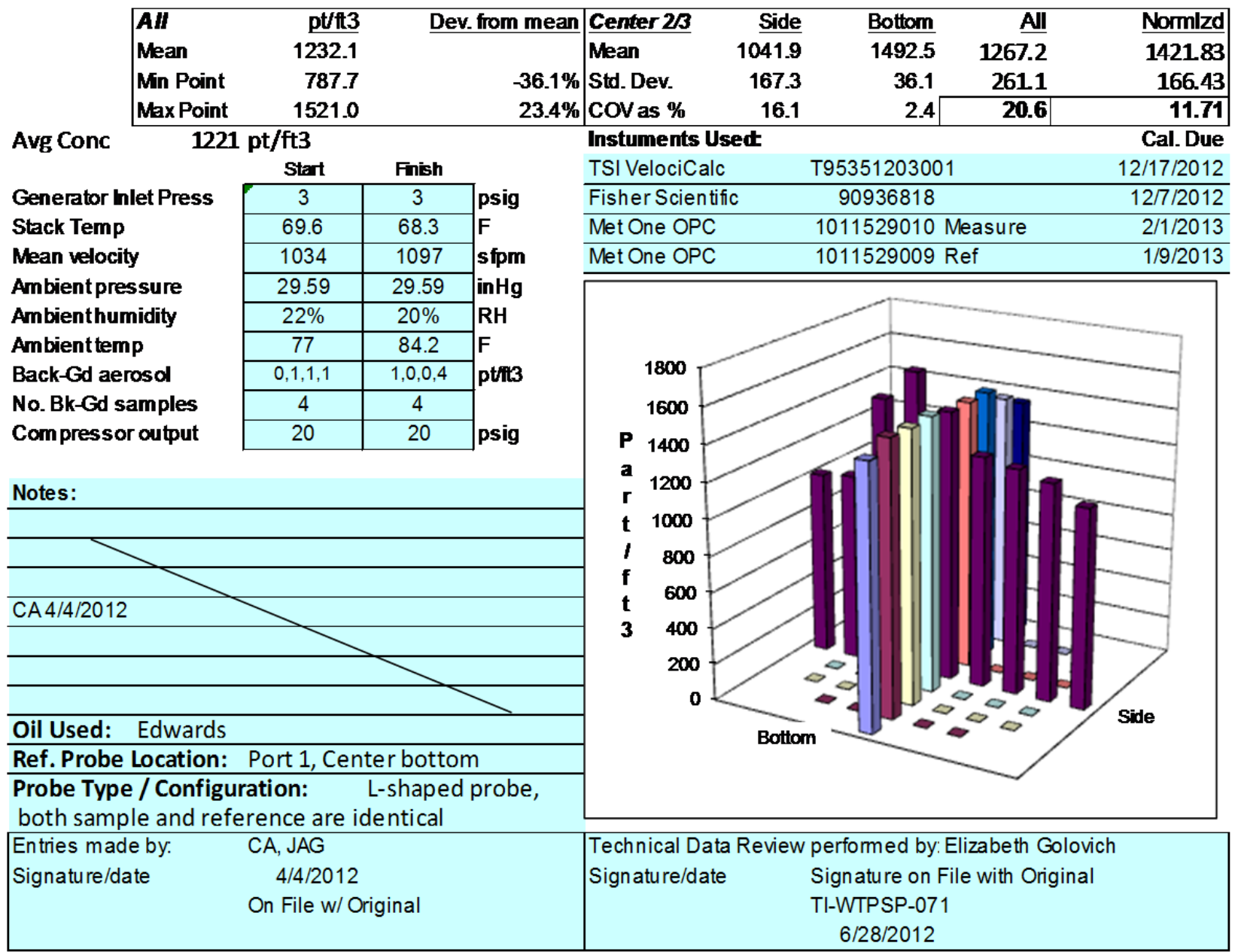


Rev. 0

3 Aug. 2006

PARTICLE TRACER TRAVERE DATA FORM

6 Site HV-S1 Model

Date 4/5/2012

Tester JEF, XY, CA

Stack Dia. 11.938 in.

Stack X-Area 111.9 in 2

TestPort

Distance to disturbance

Measurement units particles/t3

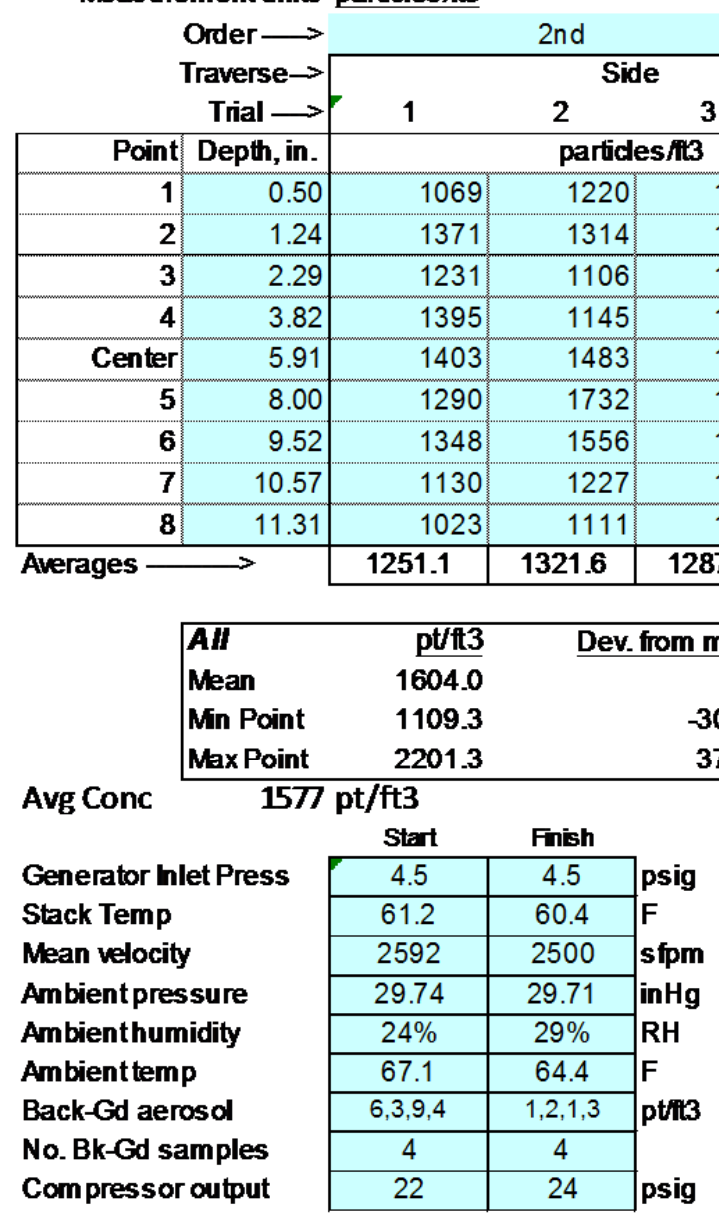

Notes: $\quad$ WD66891 (S/N 1011529010) used as Reference instrument

\section{RE-do PT-3}

\begin{tabular}{l} 
Oil Used: Edwards \\
Ref. Probe Location: Port1, Bottom C \\
\hline Probe Type / Configuration: L-shaped probe
\end{tabular}

\begin{tabular}{|lc}
\hline Entries made by: & $\mathrm{CA}, \mathrm{XY}$ \\
Signatu re/date & $4 / 5 / 2012$ \\
& On File w/ Original
\end{tabular}

Run No. PT-5

Fan configuration FAN A ONLY

Fan Setting 60

Stack Temp Hz

Start/End Time 1218/230

Center 23 from

Points in Center 23

njection Point Center

1st

\begin{tabular}{|c|c|c|c|c|}
\hline \multirow[b]{3}{*}{ Mean } & \multicolumn{3}{|c|}{ 1st } & \multirow[b]{3}{*}{ Mean } \\
\hline & \multicolumn{3}{|c|}{ Bottom } & \\
\hline & 1 & 2 & 3 & \\
\hline & \multicolumn{4}{|c|}{ particles fit3 } \\
\hline 1143.0 & 1320 & 1564 & 1516 & 1466.7 \\
\hline 1314.7 & 1843 & 1804 & 1832 & 1826.3 \\
\hline 1229.3 & 2082 & 2095 & 2179 & 2118.7 \\
\hline 1288.3 & 2164 & 2206 & 2196 & 2188.7 \\
\hline 1437.0 & 2169 & 2159 & 2276 & 22013 \\
\hline 1502.3 & 2073 & 2193 & 2133 & 2133.0 \\
\hline 1405.3 & 1861 & 2091 & 2025 & 1992.3 \\
\hline 1150.3 & 1807 & 1732 & 1890 & 1809.7 \\
\hline 1109.3 & 1403 & 1624 & 1639 & 1555.3 \\
\hline 1286.6 & 1858.0 & 1940.9 & 1965.1 & 1921.3 \\
\hline
\end{tabular}

\begin{tabular}{|c|c|c|c|c|c|}
\hline iean & Center 23 & Side & Bottom & All & Normlzd \\
\hline & Mean & 1332.5 & 2038.6 & 1685.5 & 2039.89 \\
\hline 8 & Std. Dev. & 123.3 & 165.3 & 3922 & 170.50 \\
\hline $372 \%$ & COV as \% & 9.3 & 8.1 & 23.3 & 8.36 \\
\hline
\end{tabular}

\begin{tabular}{lcr} 
Instuments Used & & Cal. Due \\
TSI VelociCalc & T95351203001 & $12 / 17 / 2012$ \\
\hline Fisher Scientific & 90936818 & $12 / 7 / 2012$ \\
\hline Met One OPC & 1011529010 & $2 / 1 / 2013$ \\
\hline Met One OPC & 1011529009 & $1 / 9 / 2013$
\end{tabular}

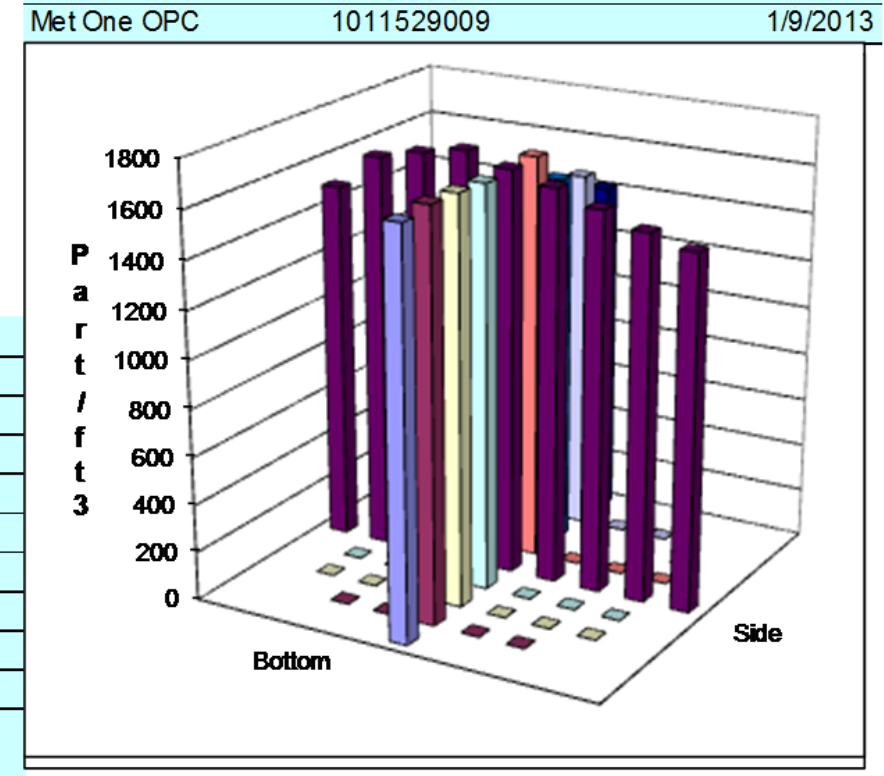

Technical Data Review performed by: Elizabeth Golovich Signatu re/date

Signatu re on File with Original

TI-WTPSP-071

$6 / 28 / 2012$ 
Rev. 0

3 Aug. 2006

\begin{tabular}{|r|r|}
\hline Date & $4 / 6 / 2012$ \\
\hline Tester JEF, $X Y$ & JY \\
\hline Stack Dia. & 11.938 in. \\
\hline Stack X-Area & $\mathbf{1 1 1 . 9}$ in 2 \\
\hline TestPort & 2 \\
\hline Distance to disturbance & 240 inches \\
\hline Measurement units particles $\mathrm{ft3}$ \\
\hline
\end{tabular}

PARTICLE TRACER TRAVESE DATA FORM

Run No. PT-6

Fan configuration FAN B ONLY

Fan Setting 28 Hz

Stack Temp $59.35 \mathrm{deg} F$

Start/End Time 1055/1225

Center 2/3 from 1.10 to: 10.84

Points in Center $2 3 \longdiv { 3 }$ to: $\overline{7}$

Injection Point C, CENTER

\begin{tabular}{|c|c|c|c|c|c|c|c|c|c|}
\hline & & \multirow{2}{*}{\multicolumn{3}{|c|}{$1 \mathrm{st}$}} & \multirow{2}{*}{\multicolumn{4}{|c|}{ 2nd }} & \\
\hline \multirow{2}{*}{\multicolumn{2}{|c|}{$\begin{array}{l}\text { Order } \longrightarrow \\
\text { Traverse } \rightarrow\end{array}$}} & & & & & & & & \\
\hline & & \multicolumn{4}{|c|}{ Side } & \multicolumn{4}{|c|}{ Bottom } \\
\hline & Trial $\longrightarrow$ & 1 & 2 & 3 & Mean & 1 & 2 & 3 & Mean \\
\hline Point & Depth, in. & \multicolumn{4}{|c|}{ particles $/ \mathrm{ft} 3$} & \multicolumn{4}{|c|}{ particles fit3 } \\
\hline 1 & 0.50 & 1722 & 1977 & 1907 & 1868.7 & 2251 & 2060 & 2113 & 2141.3 \\
\hline 2 & 1.24 & 1844 & 1887 & 1918 & 1883.0 & 2295 & 2245 & 2331 & 22903 \\
\hline 3 & 2.29 & 1810 & 1965 & 1777 & 1850.7 & 2481 & 2329 & 2441 & 24170 \\
\hline 4 & 3.82 & 1951 & 2079 & 1840 & 1956.7 & 2444 & 2283 & 2449 & 23920 \\
\hline Center & 5.91 & 1899 & 2034 & 1789 & 1907.3 & 2418 & 2392 & 2528 & 24460 \\
\hline 5 & 8.00 & 1872 & 1948 & 1875 & 1898.3 & 2445 & 2349 & 2528 & 2440.7 \\
\hline 6 & 9.52 & 1843 & 1823 & 1736 & 1800.7 & 2237 & 2246 & 2413 & 2298.7 \\
\hline 7 & 10.57 & 1713 & 1530 & 1636 & 1626.3 & 2248 & 2185 & 2344 & 22590 \\
\hline 8 & 11.31 & 1426 & 1469 & 1136 & 1343.7 & 2049 & 2195 & 2141 & 2128.3 \\
\hline \multicolumn{2}{|c|}{ Averages $\longrightarrow$} & 1786.7 & 1856.9 & 1734.9 & 1792.8 & 2318.7 & 2253.8 & 2365.3 & 2312.6 \\
\hline
\end{tabular}

\begin{tabular}{|c|c|c|c|c|c|c|c|c|c|}
\hline & $\mathbf{A H}$ & pt/f3 & \multicolumn{2}{|c|}{ Dev. from mean } & \multirow{2}{*}{$\begin{array}{l}\text { Center 23 } \\
\text { Mean }\end{array}$} & \multirow{2}{*}{$\begin{array}{r}\text { Side } \\
1846.1\end{array}$} & \multirow{2}{*}{$\frac{\text { Bottom }}{2363.4}$} & \multirow{2}{*}{2104.8} & \multirow{2}{*}{$\frac{\text { Nomlzd }}{2365.45}$} \\
\hline & Mean & 2052.7 & & & & & & & \\
\hline & Min Point & 1343.7 & & $-34.5 \%$ & Std. Dev. & 108.4 & 78.4 & 283.3 & 108.43 \\
\hline & Mex Point & 2446.0 & & $192 \%$ & COV as \% & 5.9 & 3.3 & 13.5 & 4.58 \\
\hline \multirow[t]{2}{*}{ Avg Conc } & \multicolumn{2}{|c|}{2037 pt/ft3 } & & & \multicolumn{2}{|c|}{ Instuments Used } & \multirow{2}{*}{\multicolumn{2}{|c|}{ T95351203001 }} & Cal. Due \\
\hline & & Start & Finish & & \multicolumn{2}{|c|}{ TSI VelociCalc } & & & $12 / 17 / 2012$ \\
\hline \multicolumn{2}{|c|}{ Generator Inlet Press } & 3.8 & 3.8 & psig & \multicolumn{2}{|c|}{ Fisher Scientific } & \multicolumn{2}{|l|}{90936818} & $12 / 7 / 2012$ \\
\hline \multicolumn{2}{|c|}{ Stack Temp } & 61.1 & 57.6 & $F$ & Met One OI & & \multicolumn{2}{|c|}{1011529010 Ref. } & $2 / 1 / 2013$ \\
\hline \multicolumn{2}{|c|}{ Mean velocity } & 1281 & 1314 & sfpm & \multicolumn{2}{|c|}{ Met One OPC } & \multicolumn{2}{|c|}{1011529009 Sample } & $1 / 9 / 2013$ \\
\hline
\end{tabular}

Ambient pressure

Ambienthumidity

Ambient temp

Back-Gd aerosol

No. Bk-Gd samples

Compressor output

\begin{tabular}{|c|c|l|}
\hline 29.88 & 29.88 & inHg \\
\hline $28 \%$ & $26 \%$ & RH \\
\hline 59 & 68.9 & F \\
\hline $1,0,2,0$ & $1,1,0,1$ & ptft3 \\
\hline 4 & 4 & \\
\cline { 1 - 2 } 23 & 23 & psig \\
\hline
\end{tabular}

Notes: Cloudyday

Rain drops during the last measurements points.
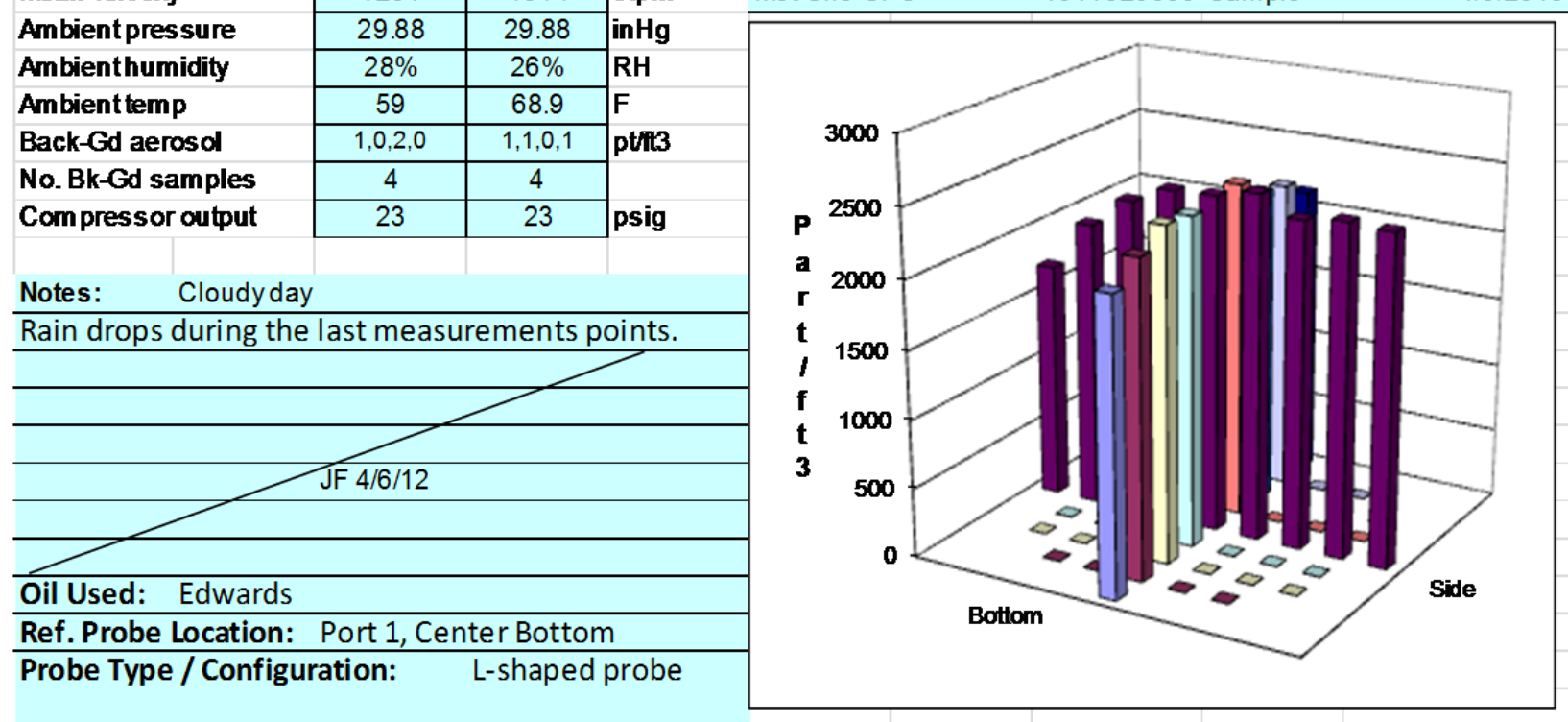

\begin{tabular}{|lc|}
\hline Entries made by: & JEF \\
Signature/date & $4 / 6 / 2012$ \\
& On File w/ Original
\end{tabular}

Technical Data Review performed by: Elizabeth Golovich

Signature/date Signature on File with Original

TI-WTPSP-071

$6 / 28 / 2012$ 
Rev. 0

3 Aug. 2006

PARTICLE TRACER TRAVERE DATA FORM

6 Site HV-S1 Model

Date 4/6/2012

Tester JEF, XY, CA

Stack Dia. 11.938 in.

Stack X-Area 111.9 in 2

TestPort

Distance to disturbance

Measurement units particles fit3

240 inches

\begin{tabular}{|r|r|}
\multicolumn{2}{c|}{$\begin{array}{c}\text { Order } \\
\text { Traverse- } \\
\text { Trial } \longrightarrow\end{array}$} \\
\hline Point & Depth, in \\
\hline $\mathbf{1}$ & 0.5 \\
\hline $\mathbf{2}$ & 1.2 \\
\hline $\mathbf{3}$ & 2.2 \\
\hline $\mathbf{4}$ & 3.8 \\
\hline Center & 5.9 \\
\hline $\mathbf{5}$ & 8.00 \\
\hline $\mathbf{6}$ & 9.5 \\
\hline $\mathbf{7}$ & 10.5 \\
\hline $\mathbf{8}$ & 11.3 \\
\hline &
\end{tabular}

Run No. PT-7

Fan configuration Fan B only

Fan Setting 55

Stack Temp $62.75 \mathrm{deg} F$

Start/End Time 1230/217

Center $\mathbf{2} 3$ from

Points in Center 23

Ijection Point CENTER PORT C

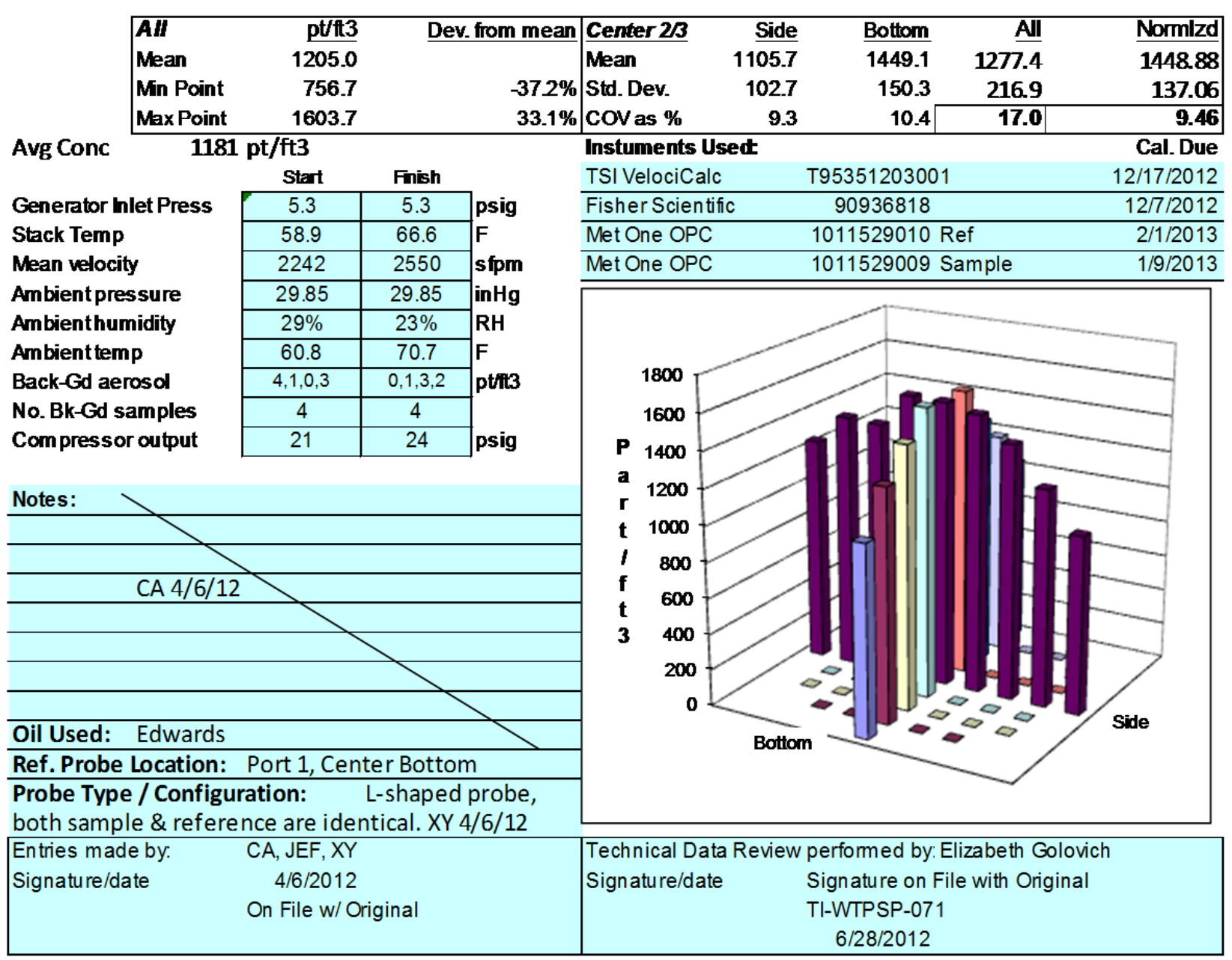


Rev. 0

3 Aug. 2006

PARTICLE TRACER TRAVERSDATA FORM

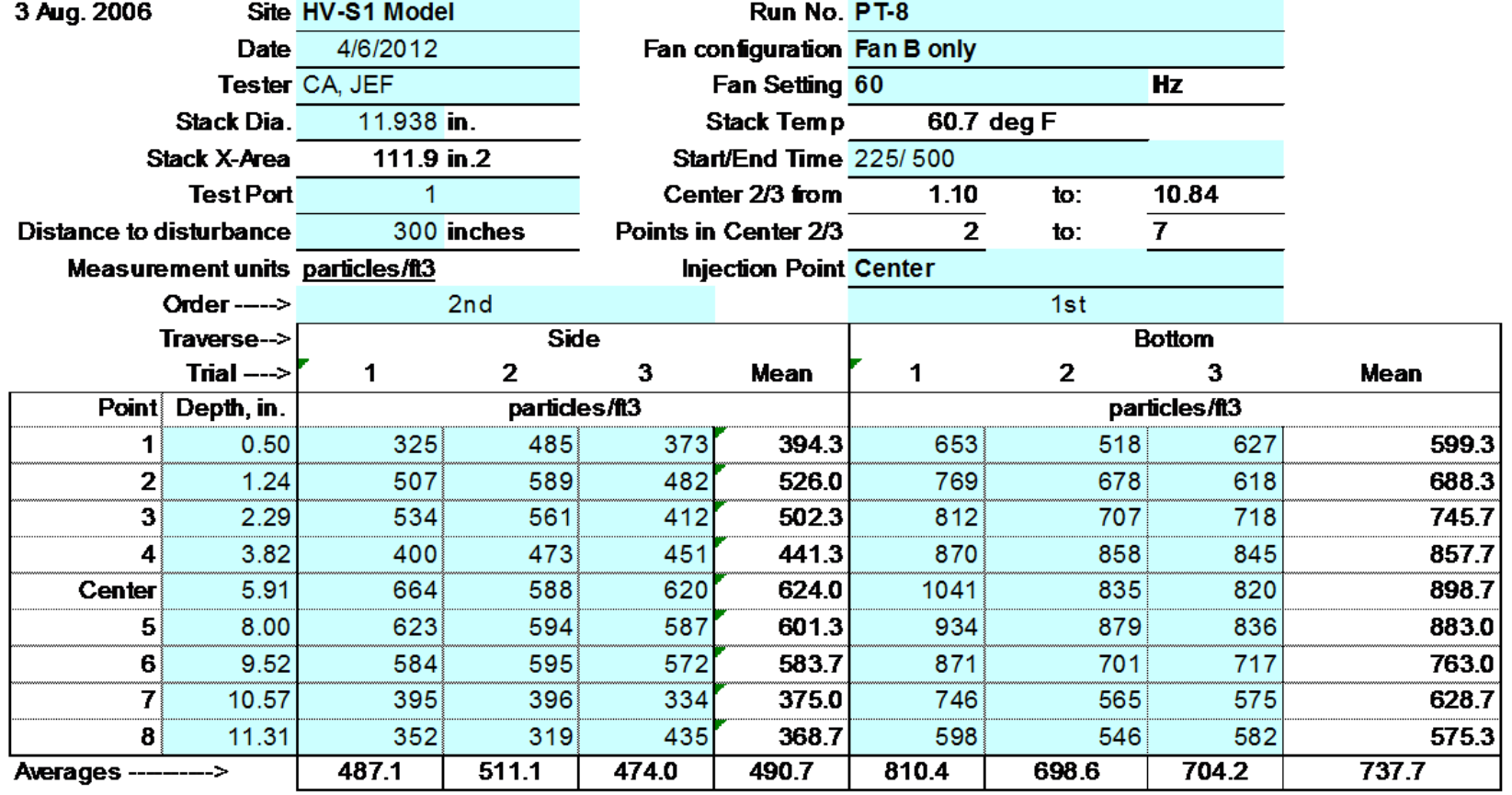

\begin{tabular}{|c|c|c|c|}
\hline$\overline{A H}$ & pt/ft: & & from $\mathrm{m}$ \\
\hline Mean & 614.2 & & \\
\hline Min Point & 368.7 & & -40 \\
\hline Max Poin & 898.7 & & 4 \\
\hline & $\mathrm{t} / \mathrm{ft3}$ & & \\
\hline & Start & Finish & \\
\hline Press & 6.9 & 6.9 & Tpsig \\
\hline & 61.1 & 60.3 & $F$ \\
\hline & 3055 & 2810 & sfpm \\
\hline iure & 29.85 & 29.85 & in $\mathrm{Hg}$ \\
\hline dity & $22 \%$ & $26 \%$ & RH \\
\hline & 75.2 & 63.5 & $F$ \\
\hline & $2,8,4,14$ & $4,0,0,0$ & $\mathrm{pt} / \mathrm{ta}$ \\
\hline les & 4 & 4 & \\
\hline itput & 23 & 24 & psig \\
\hline
\end{tabular}

Avg Conc

Generator Inlet Press

Stack Temp

Mean velocity

Ambient pressure

Ambient humidity

Ambient temp

Back-Gd aerosol

No. Bk-Gd samples

Compressor output

Notes: Cloudy, varied temperatures som etimes light gusts...

Aerosol counts appear to have temperature

dependence.

Run 4 trials on bottom.

\begin{tabular}{l}
\hline JF 4/6/12 \\
\hline Oil Used: Edwards \\
\hline Ref. Probe Location: Port 2, Bottom Center \\
\hline Probe Type / Configuration: L-shaped probe
\end{tabular}

Entries made by:

Signature/date

CA, JEF
$4 / 6 / 2012$
On File w/ Original

Center 2/3

Mean

Std. Dev.

$46.3 \%$ COV as $\%$

Instuments Used

\begin{tabular}{lcr} 
TSI VelociCalc & T95351203001 & $12 / 17 / 2012$ \\
\hline Fisher Scientific & 90936818 & $12 / 7 / 2012$ \\
\hline Met One OPC & 1011529010 Ref & $2 / 1 / 2013$ \\
\hline Met One OPC & 1011529009 Sample & $1 / 9 / 2013$ \\
\hline
\end{tabular}

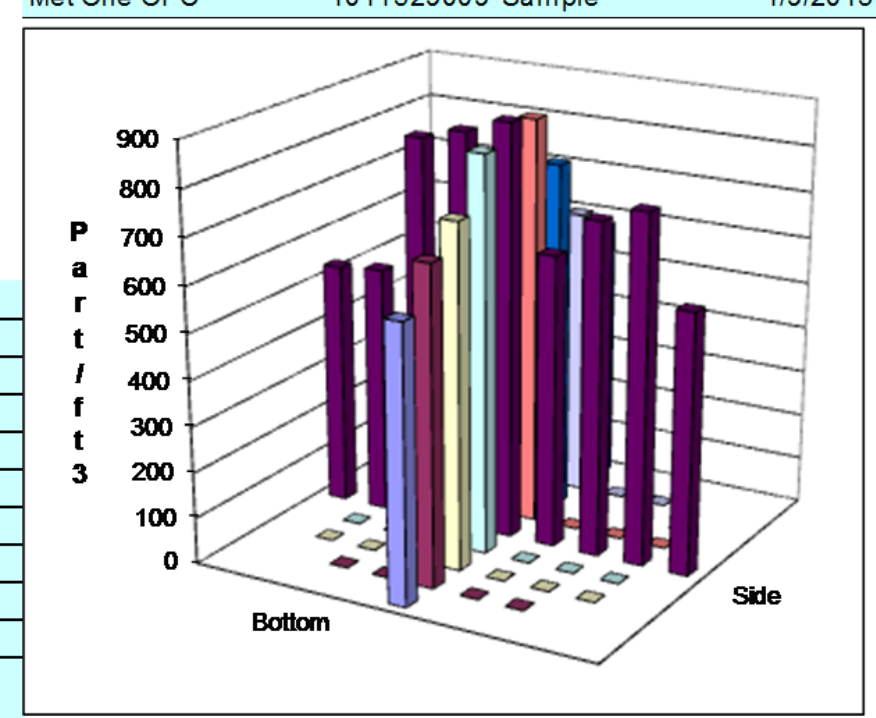

Technical Data Review performed by: Elizabeth Golovich Signature/date

TI-WTPSP-071

$6 / 28 / 2012$ 
Appendix B

HV-S2 Data Sheets 



\section{B.1 HV-S2 Calibration of Ventilation Flow Controller}

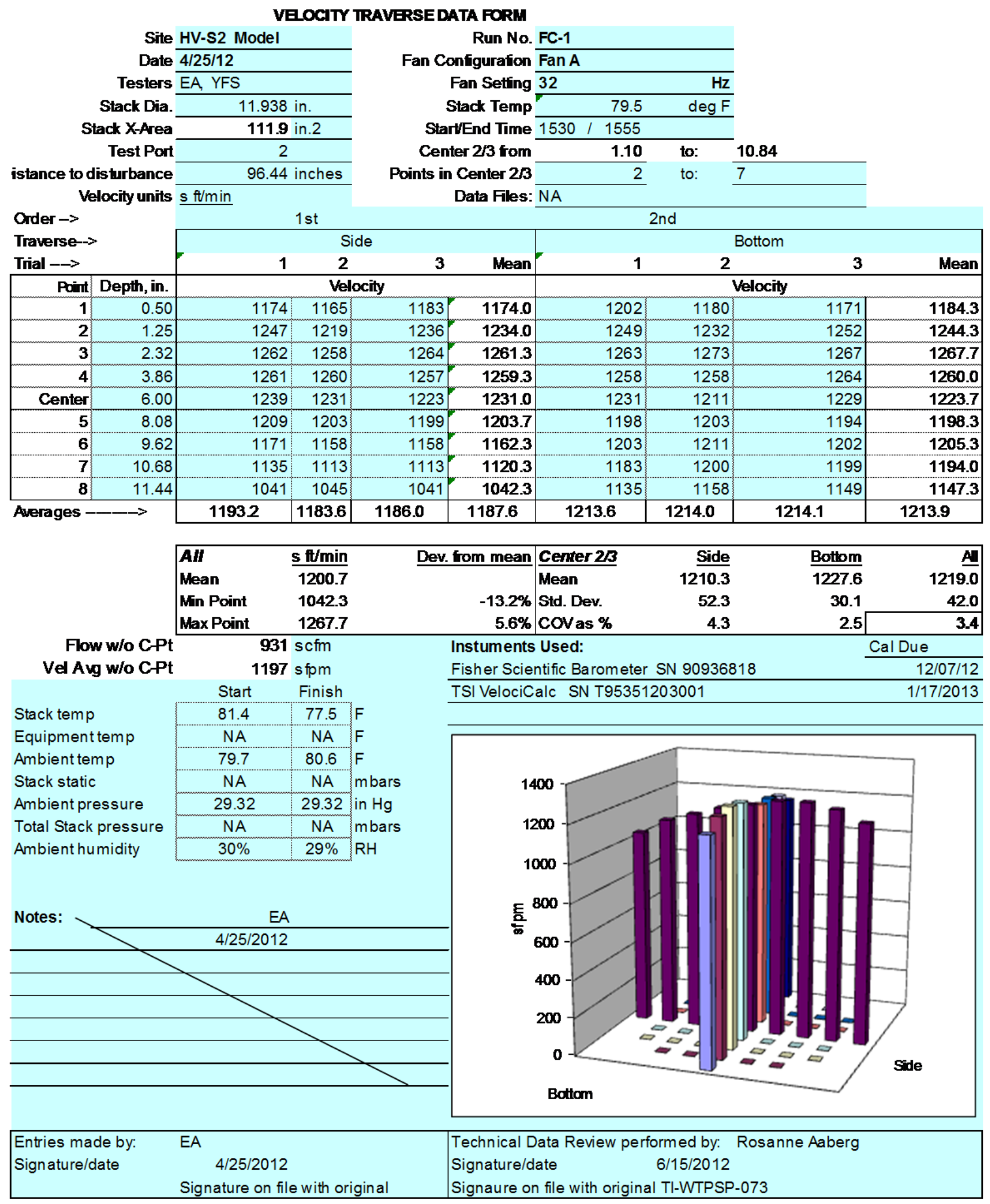




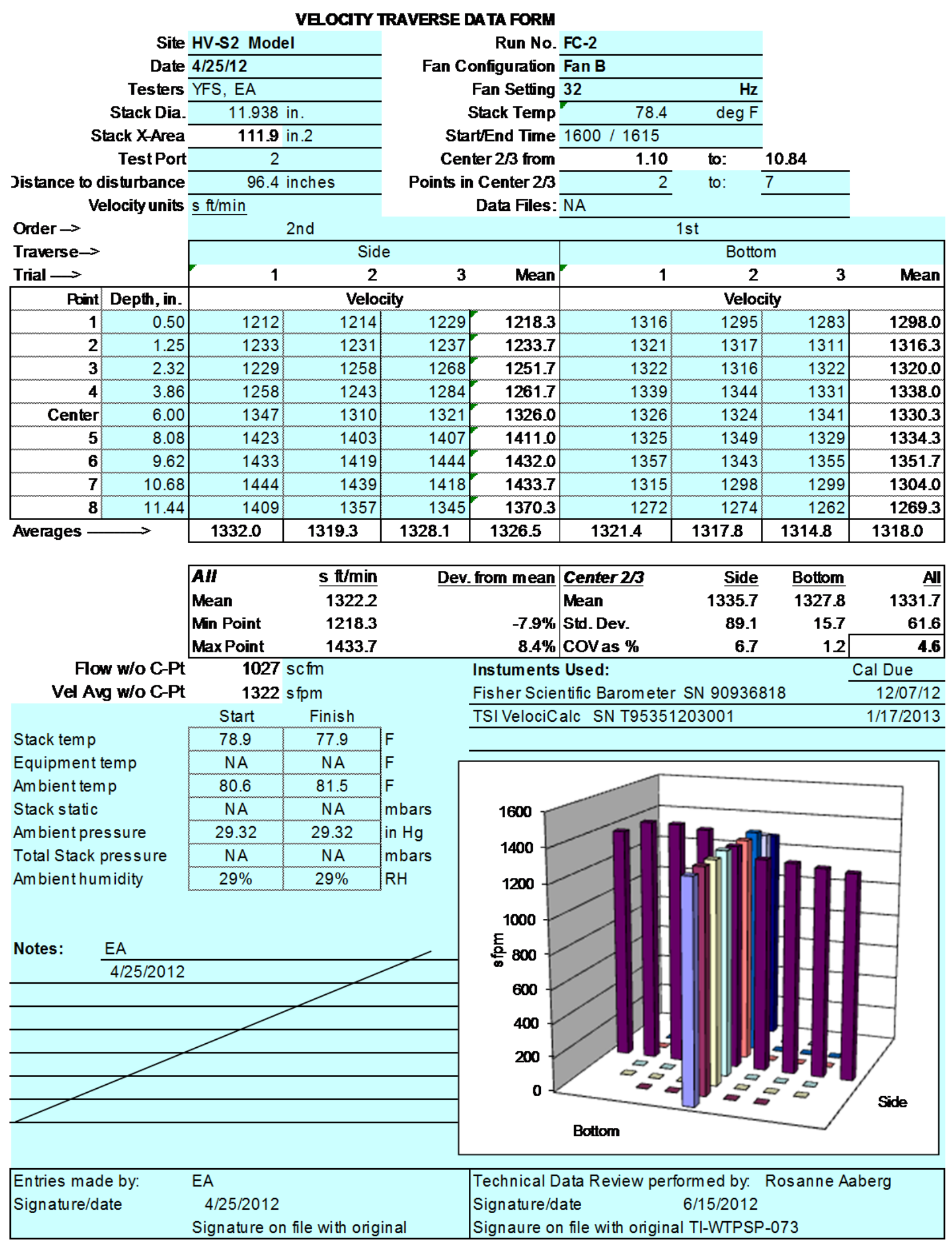




\section{VELOCITY VS. FREQUENCY DATA FORM}

\begin{tabular}{|c|c|c|}
\hline Site & \multicolumn{2}{|c|}{ HV-S2 model } \\
\hline Date & \multicolumn{2}{|c|}{$4 / 27 / 2012$} \\
\hline Tester & \multicolumn{2}{|c|}{ YFS, EA } \\
\hline Stack Dia. & 11.938 & in. \\
\hline Stack X-Area & 111.9 & in2 \\
\hline
\end{tabular}

\begin{aligned} & Run No. VF-1 \\ & \cline { 2 - 2 } Stack Temp $61.6 \quad$ deg F \\ & Stack RH\% $37 \% \\ &$ Baro Press 29.85 in Hg \\ & \cline { 2 - 2 } Fan Configuration Fan A Only \\ & \cline { 2 - 2 } Start/End Time $0855 / 0930\end{aligned}$

Dist. from disturbance 96.4 Velocity Readings, units $=\quad$ sfpm

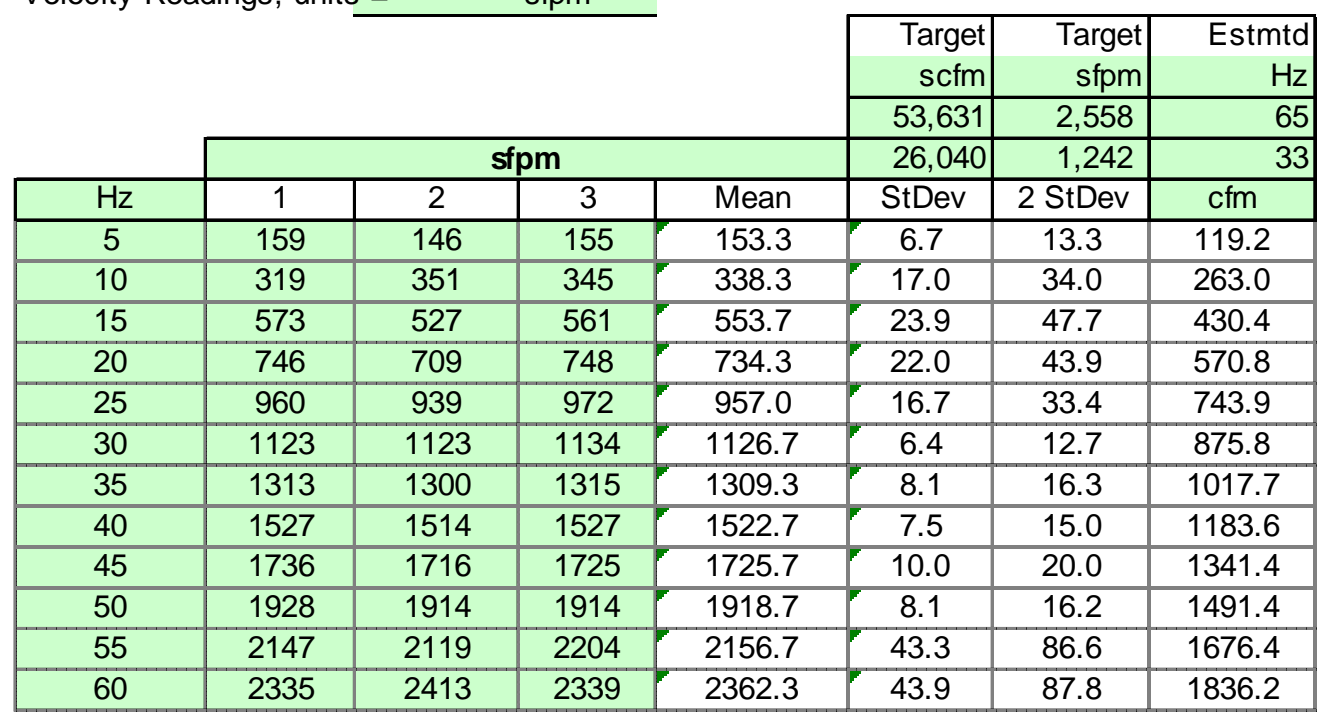

Instuments Used:

Cal Exp. Date:

Fisher Scientific Barometer SN 90936818

TSI VelociCalc SN T95351203001

$12 / 07 / 12$

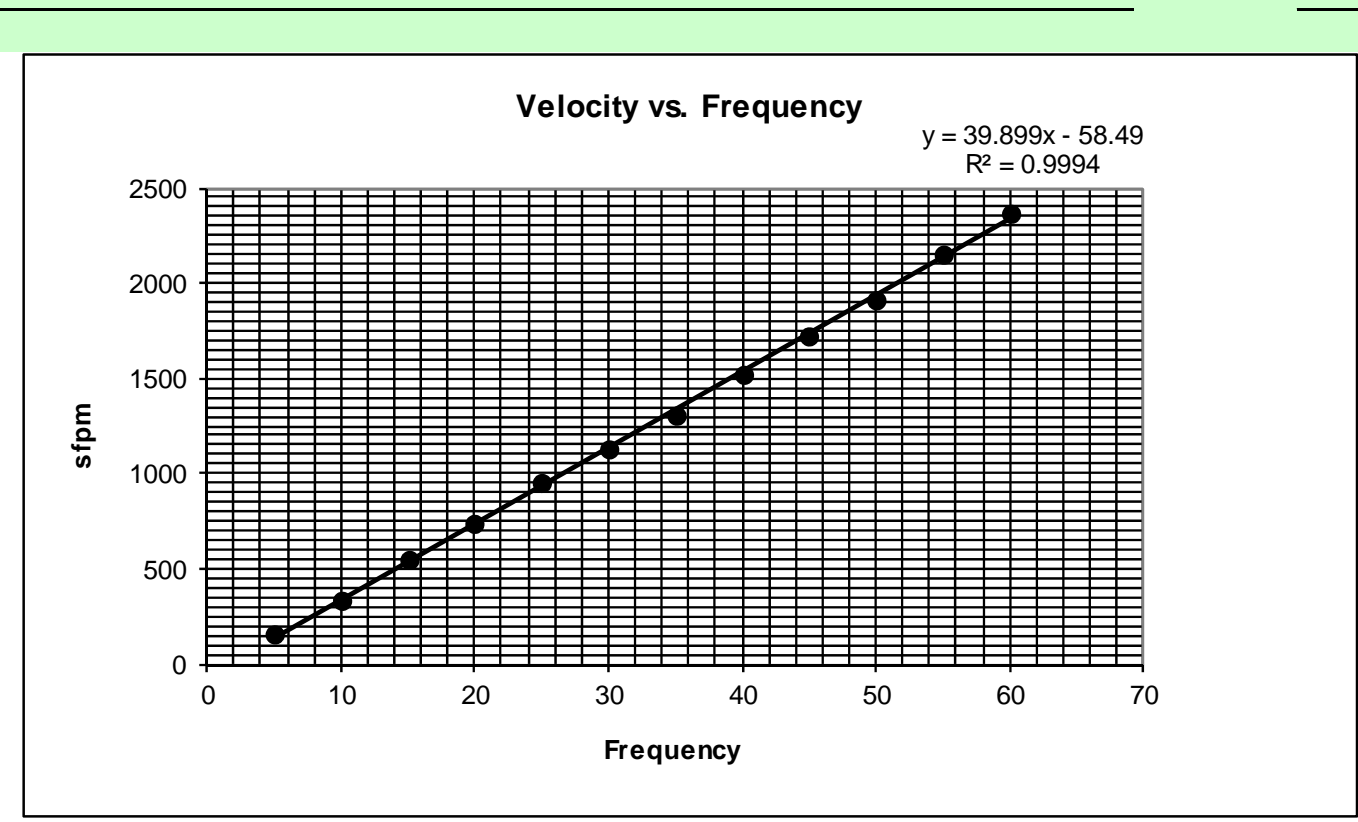

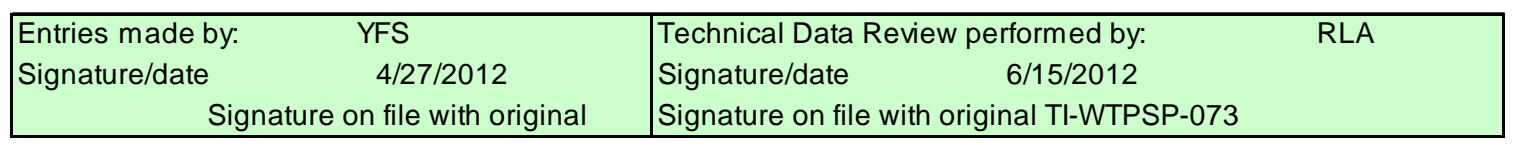




\section{VELOCITY vS. FREQUENCY DATA FORM}

\begin{tabular}{|c|c|c|}
\hline Site & \multicolumn{2}{|c|}{ HV-S2 model } \\
\hline Date & \multicolumn{2}{|c|}{$4 / 27 / 2012$} \\
\hline Tester & \multicolumn{2}{|c|}{ YFS, EA } \\
\hline Stack Dia. & 11.938 & in. \\
\hline Stack X-Area & 111.9 & in2 \\
\hline
\end{tabular}

\begin{aligned} & Run No. VF-2 \\ & Stack Temp $61 \quad$ deg F \\ & Stack RH\% $31 \% \\ &$\cline { 2 - 2 } Baro Press $29.85 \mathrm{inHg} \\ &$\cline { 2 - 2 } Fan Configuration Fan B only \\ &${ } } 0935 / 1000\end{aligned}$

Dist. from disturbance $\overline{96.4 \quad \text { inches }}$ Reference point from velocity test VC : $\quad$ Bottom 3 Velocity Readings, units $=\quad \mathrm{sfpm}$

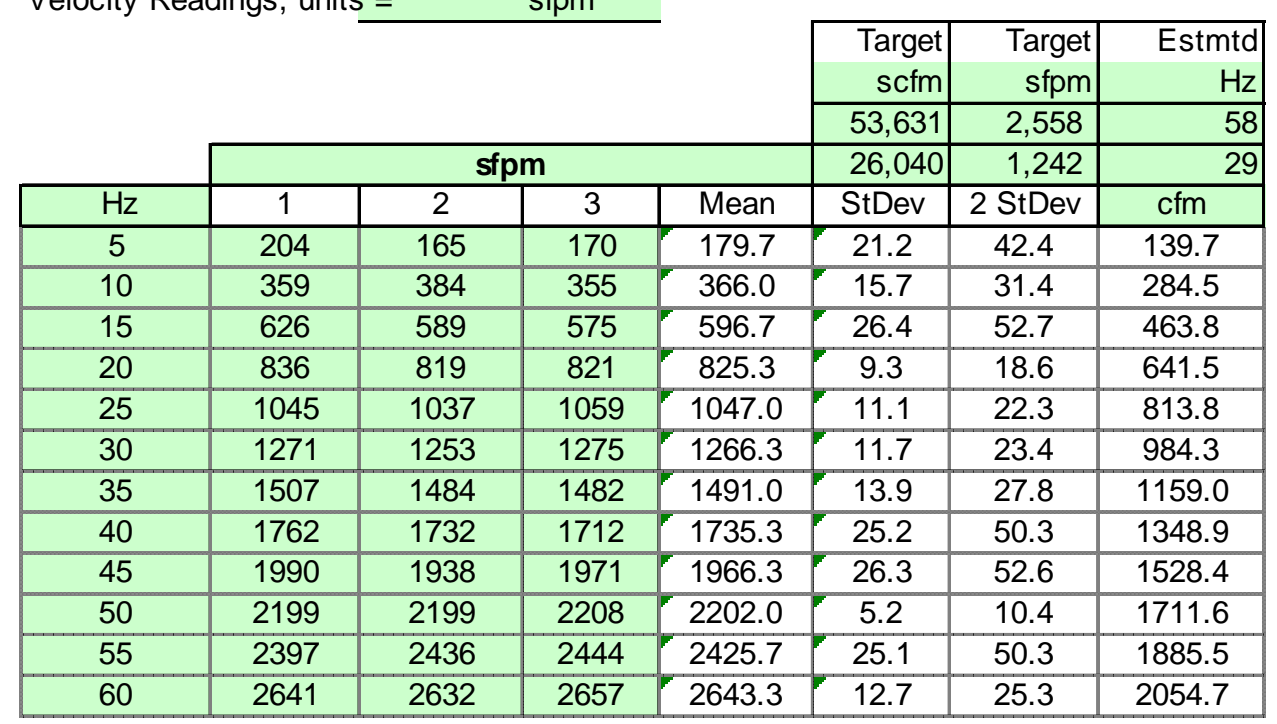

Instuments Used:

Fisher Scientific Barometer SN 90936818

TSI VelociCalc SN T95351203001
Cal Exp. Date:

$\frac{12 / 07 / 12}{1 / 17 / 2013}$

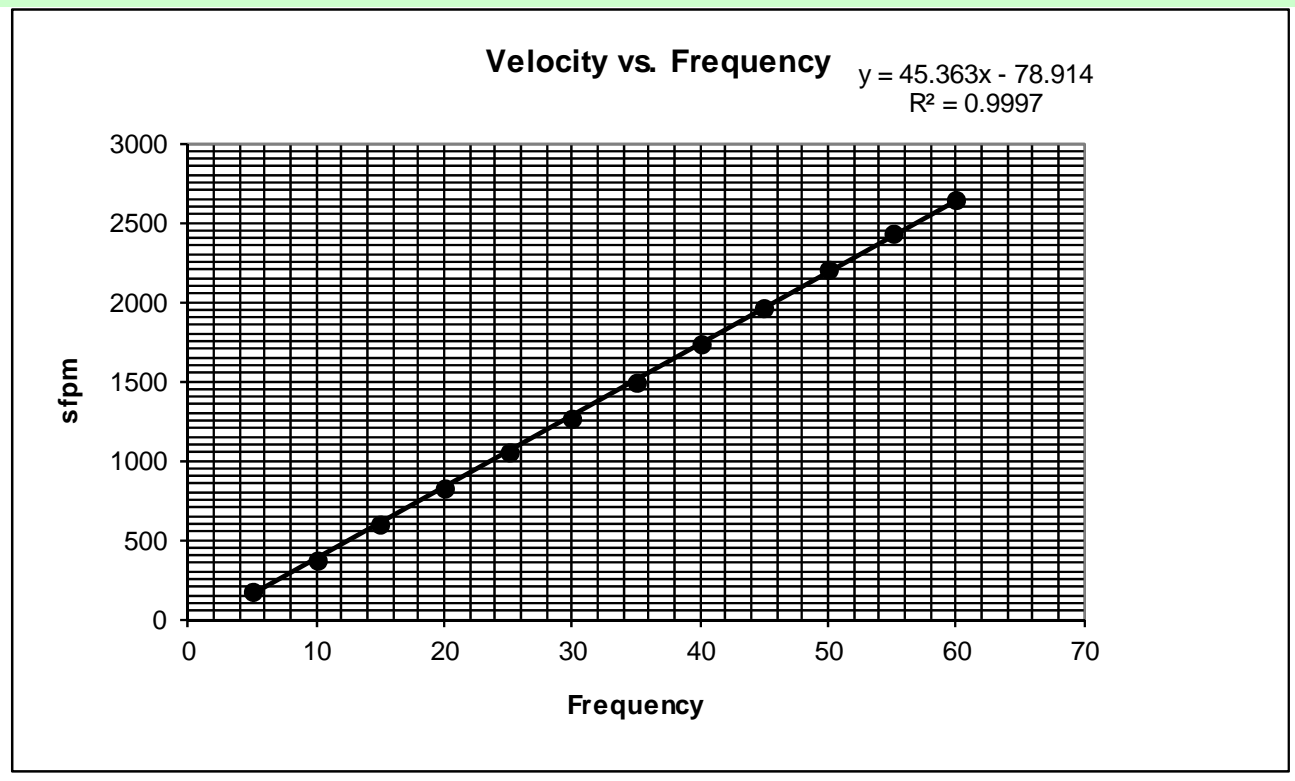

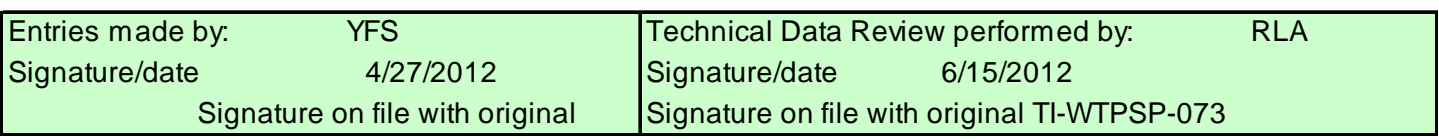




\section{B.2 HV-S2 Velocity Uniformity Data Sheets}

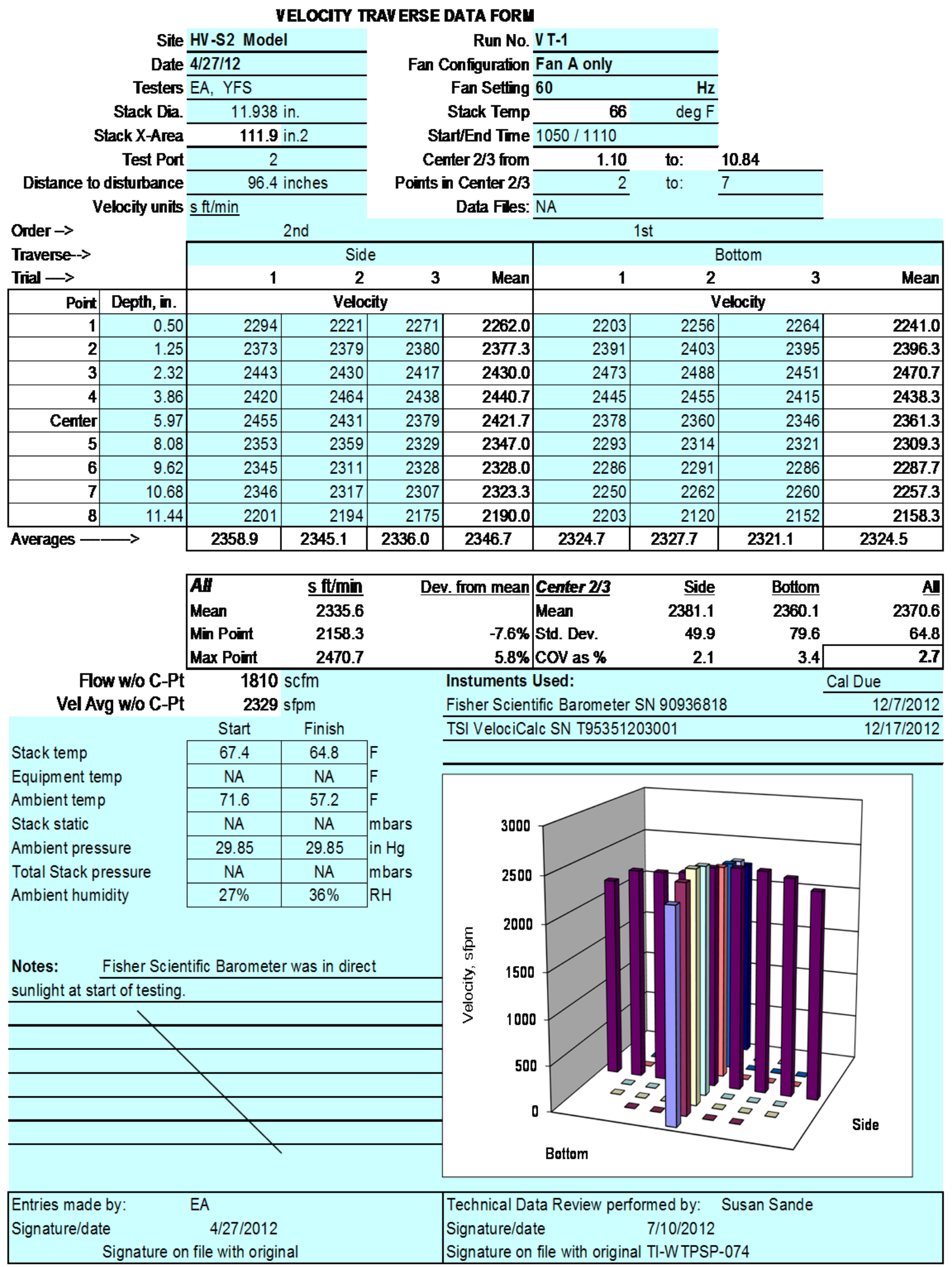




\section{VELOCITY TRAVERSE DATA FORM}

Site HV-S2 Model

Date 4/27/12

Testers YFS, EA

Stack Dia.

Stack X-Area

Test Port

\begin{tabular}{c}
\hline 11.938 in. \\
\hline $\mathbf{1 1 1 . 9}$ in 2 \\
\hline 2 \\
\hline 96.4 inches \\
\hline
\end{tabular}

Distance to disturbance

Velocity units $\underline{\mathrm{s} \mathrm{f} / \mathrm{min}}$

Run No. VT-2

Fan Confguration Fan B only

Fan Setting 60

Stack Temp

Center $2 / 3$ from

Points in Center $2 / 3$

Data Fles: NA

Order $\rightarrow$

$1 \mathrm{st}$

Traverse->

Trial $-\longrightarrow$

\begin{tabular}{|c|c|}
\hline Point & Depth, in. \\
\hline 1 & 0.5 \\
\hline 2 & 1.2 \\
\hline 3 & 2.3 \\
\hline 4 & 3.8 \\
\hline Center & 5.9 \\
\hline 5 & 8.0 \\
\hline 6 & 9.6 \\
\hline 7 & 10.6 \\
\hline 8 & 11.4 \\
\hline
\end{tabular}

Averages

11.44

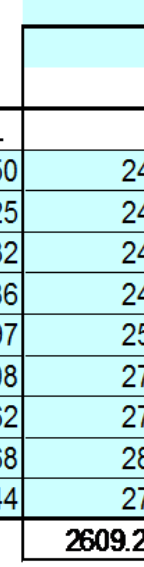
1 Side

\section{Side}

2

3

2nd

\begin{tabular}{|r|r|r|r|r|}
\hline & \multicolumn{5}{|c|}{$\begin{array}{c}\text { Bottom } \\
\text { Mean }\end{array}$} & $\mathbf{2}$ & $\mathbf{3}$ & Mean \\
\hline & 2502 & 2498 & 2464 & $\mathbf{2 4 8 8 . 0}$ \\
\hline $\mathbf{2 4 5 5 . 3}$ & 2566 & 2601 & 2560 & $\mathbf{2 5 7 5 . 7}$ \\
\hline $\mathbf{2 4 5 9 . 0}$ & 2567 & 2620 & 2589 & $\mathbf{2 5 9 2 0}$ \\
\hline $\mathbf{2 4 6 1 . 0}$ & 2608 & 2598 & 2600 & $\mathbf{2 6 0 2 0}$ \\
\hline $\mathbf{2 4 3 6 . 0}$ & 2565 & 2608 & 2599 & $\mathbf{2 5 9 0 . 7}$ \\
\hline $\mathbf{2 5 6 0 . 7}$ & 2585 & 2601 & 2629 & $\mathbf{2 6 0 5 . 0}$ \\
\hline $\mathbf{2 7 5 3 . 0}$ & 2707 & 2669 & 2662 & $\mathbf{2 6 7 9 . 3}$ \\
\hline $\mathbf{2 8 1 1 . 3}$ & 2666 & 2624 & 2680 & $\mathbf{2 6 5 6 . 7}$ \\
\hline $\mathbf{2 8 2 9 . 0}$ & 2533 & 2553 & 2585 & $\mathbf{2 5 5 7 . 0}$ \\
\hline $\mathbf{2 7 8 9 . 7}$ & $\mathbf{2 5 8 8 . 8}$ & $\mathbf{2 5 9 6 . 9}$ & $\mathbf{2 5 9 6 . 4}$ & $\mathbf{2 5 9 4 . 0}$ \\
\hline $\mathbf{2 6 1 7 . 2}$ & & &
\end{tabular}

\begin{tabular}{|lr}
\hline AH & s ft/min \\
Mean & $\mathbf{2 6 0 5 . 6}$ \\
Min Point & 2436.0 \\
Max Point & 2829.0 \\
\hline
\end{tabular}

Flow w/o C-Pt $2028 \mathrm{scfm}$ $\mathbf{2 6 0 9} \mathrm{sfpm}$ Vel Avg w/o C-Pt

2609 sfpm

\begin{tabular}{|c|c|c|}
\hline \multicolumn{1}{c|}{ Start } & \multicolumn{1}{c}{ Finish } & \\
\hline 67.7 & 66 & $F$ \\
\hline NA & NA & F \\
\hline 58.1 & 72.5 & F \\
\hline NA & NA & mbars \\
\hline 29.85 & 29.88 & in Hg \\
\cline { 1 - 2 } NA & NA & mbars \\
\hline $34 \%$ & $30 \%$ & RH \\
\hline
\end{tabular}

Stack temp

Equipment temp

Ambient temp

Stack static

Ambient pressure

Total Stack pressure

Ambient humidity

Notes: $\quad$ Fisher Scientific Barometer was in direct sunlight at end of the testing.

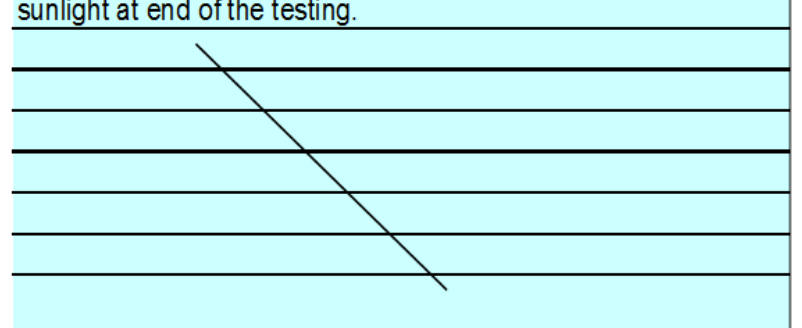

\begin{tabular}{r|l} 
Dev. from mean & Center 2/3 \\
$-6.5 \%$ & Mean \\
$8.6 \%$ & Std. Dev. \\
Cov as \% \\
\hline
\end{tabular}

\begin{tabular}{rrr|} 
Side & Bottom & Al \\
2615.7 & 2614.5 & 2615.1 \\
176.3 & 38.3 & 1226 \\
6.7 & 1.5 & 4.7 \\
\hline
\end{tabular}

\begin{tabular}{lc} 
Instuments Used: & Cal Due \\
\cline { 2 - 2 } Fisher Scientific Barometer SN 90936818 & $12 / 7 / 2012$ \\
\hline TSI VelociCalc SN T95351203001 & $12 / 17 / 2012$ \\
\hline
\end{tabular}

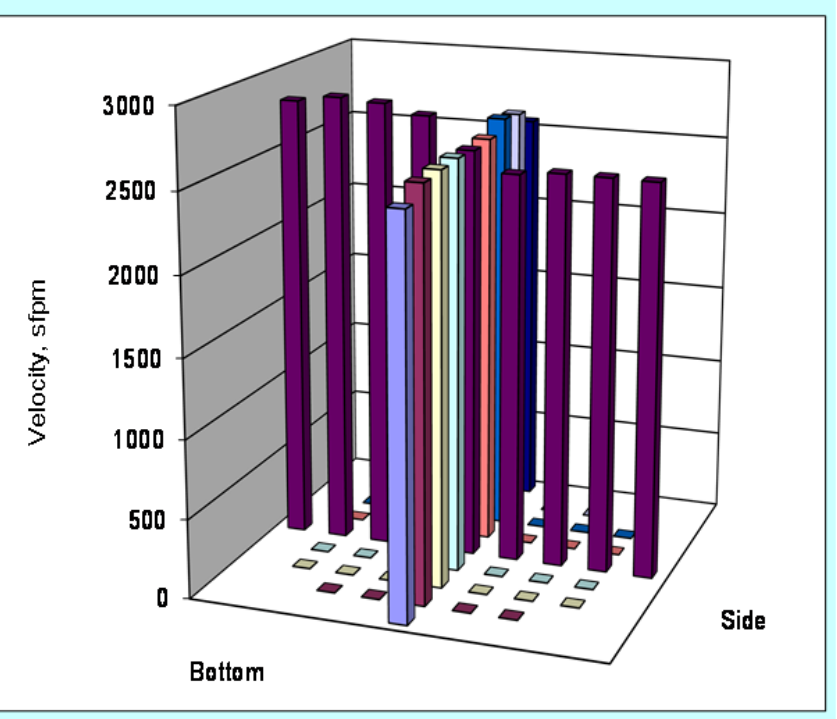

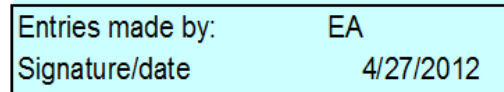

Signature on file with original

\begin{tabular}{|l|l|}
\hline Technical Data Review performed by: Susan Sande \\
Signature/date $\quad 7 / 10 / 2012$ \\
Signature on file with original TI-WTPSP-074
\end{tabular}




\section{VELOCITY TRAVERSE DATA FORM}

Site HV-S2 Model

Date 4/27/12

Testers JAG, XYY

Stack Dia. 11.938 in

Stack X-Area $\quad 111.9$ in. 2

Test Port

Distance to disturbance

Velocity units $\mathrm{s} \mathrm{ft} / \mathrm{min}$

Order $\rightarrow$

Traverse->

Trial $-\longrightarrow$

\begin{tabular}{|r|r|}
\hline Point & Depth, in. \\
\hline $\mathbf{1}$ & 0.50 \\
\hline $\mathbf{2}$ & 1.25 \\
\hline $\mathbf{3}$ & 2.32 \\
\hline $\mathbf{4}$ & 3.86 \\
\hline Center & 5.97 \\
\hline $\mathbf{5}$ & 8.08 \\
\hline $\mathbf{6}$ & 9.62 \\
\hline $\mathbf{7}$ & \multicolumn{2}{|c|}{10.68} \\
\hline $\mathbf{8}$ & \multicolumn{2}{|c}{11.44} \\
\hline Averages - - -
\end{tabular}

Averages 2nd
Run No. VT-3

Fan Configuration Fan A

Fan Setting 28.9

Stack Temp

Start/End Time 1315/ 1348

Center $2 / 3$ from

Points in Center $2 / 3$

Data Fles: NA

Side

1st

st

\begin{tabular}{|c|c|c|c|c|c|c|c|}
\hline 1 & 2 & 3 & Mean & 1 & 2 & 3 & Mean \\
\hline \multicolumn{4}{|c|}{ Velocity } & \multicolumn{4}{|c|}{ Velocity } \\
\hline 1057 & 1070 & 1081 & \begin{tabular}{|l|}
1069.3 \\
\end{tabular} & 1038 & 1045 & 1025 & 1036.0 \\
\hline 1118 & 1135 & 1121 & 1124.7 & 1098 & 1140 & 1115 & 1117.7 \\
\hline 1126 & 1151 & 1124 & 1133.7 & 1144 & 1149 & 1133 & 11420 \\
\hline 1142 & 1124 & 1143 & 1136.3 & 1134 & 1167 & 1147 & 1149.3 \\
\hline 1129 & 1124 & 11117 & 1123.3 & 1117 & 1129 & 1097 & 1114.3 \\
\hline 1101 & 1092 & 1098 & 1097.0 & 1096 & 1073 & 1067 & 1078.7 \\
\hline 1088 & 1069 & 1084 & 1080.3 & 1072 & 1086 & 1088 & 10820 \\
\hline 1022 & 997 & 1016 & 1011.7 & 1060 & 1062 & 1028 & 1050.0 \\
\hline 936 & 958 & 939 & 944.3 & 979 & 1020 & 967 & 988.7 \\
\hline 1079.9 & 1080.0 & 1080.3 & 1080.1 & 1082.0 & 1096.8 & 1074.1 & 1084.3 \\
\hline
\end{tabular}

\begin{tabular}{|lr}
\hline All & $\mathrm{s} \mathrm{tt/min}$ \\
Mean & 1082.2 \\
Min Point & 944.3 \\
Max Point & 1149.3 \\
\hline
\end{tabular}

Flow w/o C-Pt Vel Avg w/o C-Pt

$838 \mathrm{scfm}$

$1078 \mathrm{spm}$

\begin{tabular}{|c|c|l}
\hline \multicolumn{1}{|c|}{ Start } & Finish & \\
\hline 67.9 & 67.9 & $\mathrm{~F}$ \\
\cline { 1 - 2 } NA & NA & $\mathrm{F}$ \\
\cline { 1 - 2 } 84.2 & 72.5 & $\mathrm{~F}$ \\
\cline { 1 - 2 } NA & NA & mbars \\
\cline { 1 - 2 } 1011 & 1011 & in Hg \\
\cline { 1 - 2 } NA & NA & mbars \\
\cline { 1 - 2 } $20 \%$ & $23 \%$ & RH \\
\hline
\end{tabular}

Stack temp

Equipment temp Ambient temp

Stack static

Ambient pressure

Total Stack pressure

Ambient humidity

Notes: $\quad$ Bottom 5 for Fan A; Bottom 3 for Fan B Target flow is $1047-1216 \mathrm{fpm}$.

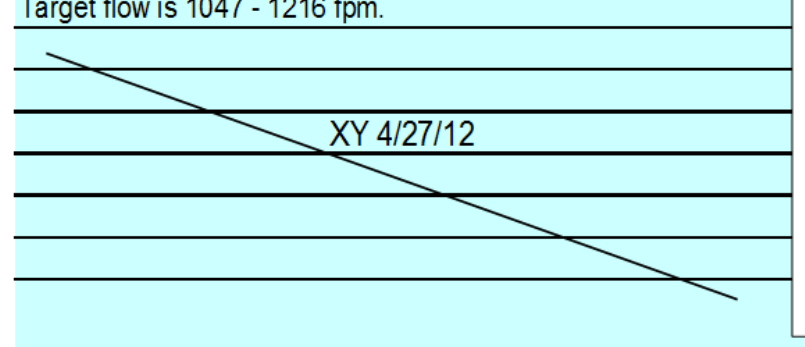

\begin{tabular}{|lc|}
\hline $\begin{array}{l}\text { Entries made by: } \\
\text { Signature/date }\end{array}$ & John Glyssmeyer \\
& $4 / 27 / 2012$ \\
& signature on file with original
\end{tabular}

\begin{tabular}{|c|c|c|c|c|}
\hline Dev. from mean & Center 2/3 & Side & Bottom & A) \\
\hline & Mean & 1101.0 & 1104.9 & 1102 \\
\hline$-12.7 \%$ & Std. Dev. & 44.3 & 36.1 & 38 \\
\hline $6.2 \%$ & $\operatorname{cov}$ as $\%$ & 4.0 & 3.3 & 3.5 \\
\hline
\end{tabular}

Instuments Used: $\quad$ Cal Due

Fisher Scientific Barometer SN $90936818 \quad \frac{12 / 7 / 2012}{$\cline { 2 - 2 }}

TSI VelociCalc SN T95351203001

$12 / 17 / 2012$

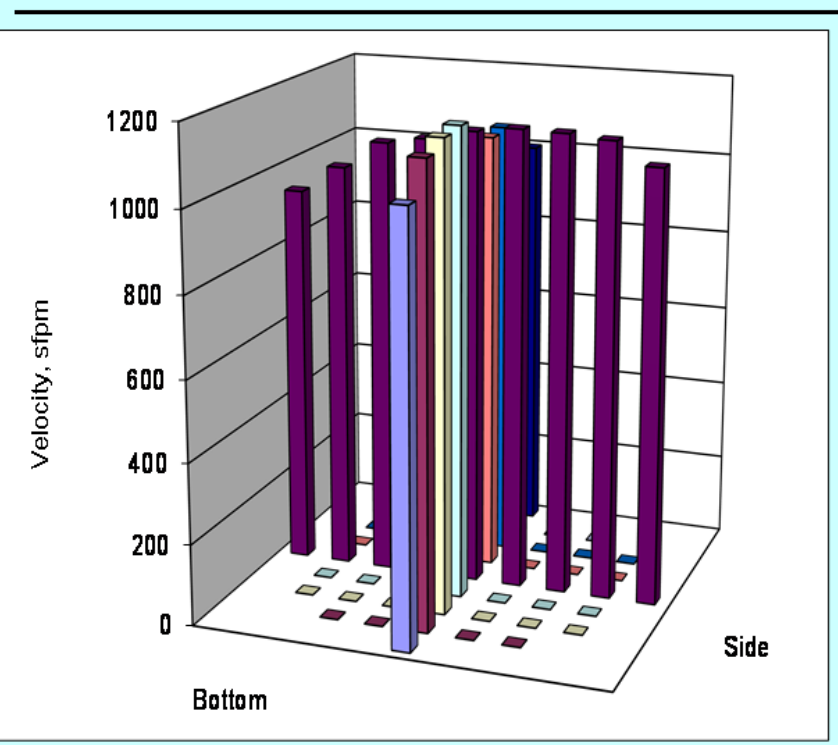

\begin{tabular}{|l} 
Technical Data Review performed by: Susan Sande \\
Signature/date $\quad 7 / 10 / 2012$ \\
Signature on file with original TI-WTPSP-074
\end{tabular} 


\section{VELOCITY TRAVERSE DATA FORM}

Site HV-S2 Model

Date 4/27/12

Testers JAG / XYY

Stack Dia.

Stack X-Area

Test Port

\begin{tabular}{|c|}
\hline 11.938 in. \\
\hline 111.9 in. 2 \\
\hline 2 \\
\hline
\end{tabular}

Distance to disturbance

Velocity units $\underline{\mathrm{s} \mathrm{f} / \mathrm{min}}$

Order $\rightarrow$

Traverse->

Trial $-\rightarrow$

\begin{tabular}{|c|c|}
\hline Point & Depth, in. \\
\hline 1 & 0.5 \\
\hline 2 & 1.2 \\
\hline 3 & 2.3 \\
\hline 4 & 3.8 \\
\hline Center & 5.9 \\
\hline 5 & 8.0 \\
\hline 6 & 9.6 \\
\hline 7 & 10.6 \\
\hline 8 & 11.4 \\
\hline
\end{tabular}
2nd

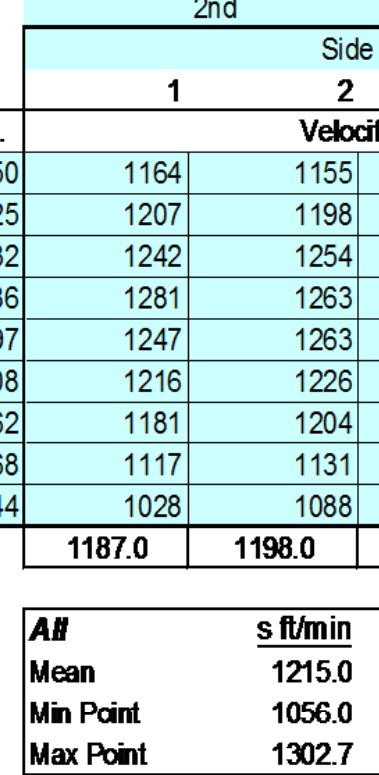

\section{Flow w/o C-Pt Vel Avg w/o C-Pt}

$940 \mathrm{scfm}$

$1210 \mathrm{sfpm}$

\begin{tabular}{|c|c|c|}
\hline \multicolumn{1}{l|}{ Start } & \multicolumn{1}{c}{ Finish } & \\
\hline 72.0 & 72.8 & F \\
\cline { 1 - 2 } N.A. & N.A. & F \\
\hline 75.2 & 70.7 & F \\
\hline N.A. & N.A. & mbars \\
\cline { 1 - 2 } 1011 & 1011 & in Hg \\
\cline { 1 - 2 } N.A. & N.A. & mbars \\
\cline { 1 - 2 } $22 \%$ & $24 \%$ & RH \\
\hline
\end{tabular}

Stack temp

Equipment temp

Ambient temp

Stack static

Ambient pressure

Total Stack pressure

Ambient humidity

Notes: Light wind.

Target fkiw us 1216 fpm. XY 4/27/12

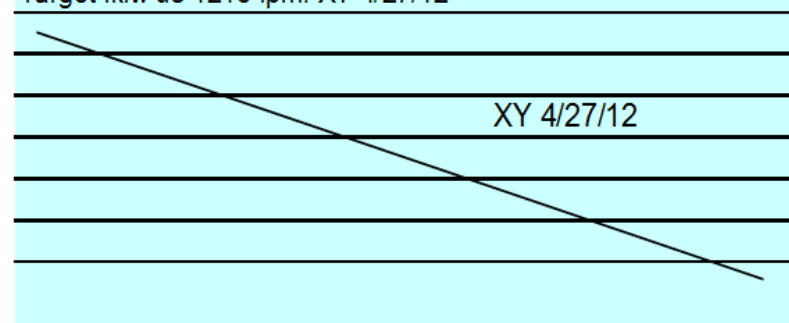

Entries made by:

Signature/date

4/27/2012

signature on file with original
Run No. VT-4

Fan Configuration Fan A Only

Fan Setting 32.5

Stack Temp

Start/End Time 1350 / 1407

Center $2 / 3$ from

Points in Center $2 / 3$

Data Fles: NA

$1 \mathrm{st}$

\begin{tabular}{|r|r|r|r|r|r|}
\multicolumn{5}{|c|}{ 1st } \\
$\mathbf{3}$ & Mean & \multicolumn{5}{|c|}{$\begin{array}{c}\text { Bottom } \\
\mathbf{2}\end{array}$} \\
\hline & & \multicolumn{5}{|c|}{ Velocity } & Mean \\
\hline 1153 & $\mathbf{1 1 5 7 . 3}$ & 1152 & 1149 & 1165 & $\mathbf{1 1 5 5 . 3}$ \\
\hline 1213 & $\mathbf{1 2 0 6 . 0}$ & 1237 & 1264 & 1271 & $\mathbf{1 2 5 7 . 3}$ \\
\hline 1250 & $\mathbf{1 2 4 8 . 7}$ & 1307 & 1302 & 1299 & $\mathbf{1 3 0 2 7}$ \\
\hline 1246 & $\mathbf{1 2 6 3 . 3}$ & 1283 & 1283 & 1303 & $\mathbf{1 2 8 9 . 7}$ \\
\hline 1260 & $\mathbf{1 2 5 6 . 7}$ & 1246 & 1243 & 1282 & $\mathbf{1 2 5 7 . 0}$ \\
\hline 1224 & $\mathbf{1 2 2 2 . 0}$ & 1233 & 1238 & 1243 & $\mathbf{1 2 3 8 . 0}$ \\
\hline 1184 & $\mathbf{1 1 8 9 . 7}$ & 1237 & 1242 & 1240 & $\mathbf{1 2 3 9 . 7}$ \\
\hline 1146 & $\mathbf{1 1 3 1 . 3}$ & 1213 & 1229 & 1243 & $\mathbf{1 2 2 8 . 3}$ \\
\hline 1052 & $\mathbf{1 0 5 6 . 0}$ & 1172 & 1171 & 1172 & $\mathbf{1 1 7 1 . 7}$ \\
\hline $\mathbf{1 1 9 2 . 0}$ & $\mathbf{1 1 9 2 . 3}$ & $\mathbf{1 2 3 1 . 1}$ & $\mathbf{1 2 3 5 . 7}$ & $\mathbf{1 2 4 6 . 4}$ & $\mathbf{1 2 3 7 . 7}$ \\
\hline
\end{tabular}

\begin{tabular}{r|lrrr|} 
Dev. from mean & Center 2/3 & Side & Bottom & Al \\
\cline { 2 - 3 } & Mean & 1216.8 & 1259.0 & 1237.9 \\
$7.2 \%$ & Std. Dev. & 46.5 & 27.7 & 428 \\
\hline
\end{tabular}

\begin{tabular}{lc} 
Instuments Used: & Cal Due \\
\cline { 2 - 2 } Fisher Scientific Barometer SN 90936818 & $12 / 7 / 2012$ \\
\hline TSI VelociCalc SN T95351203001 & $12 / 17 / 2012$ \\
\hline
\end{tabular}

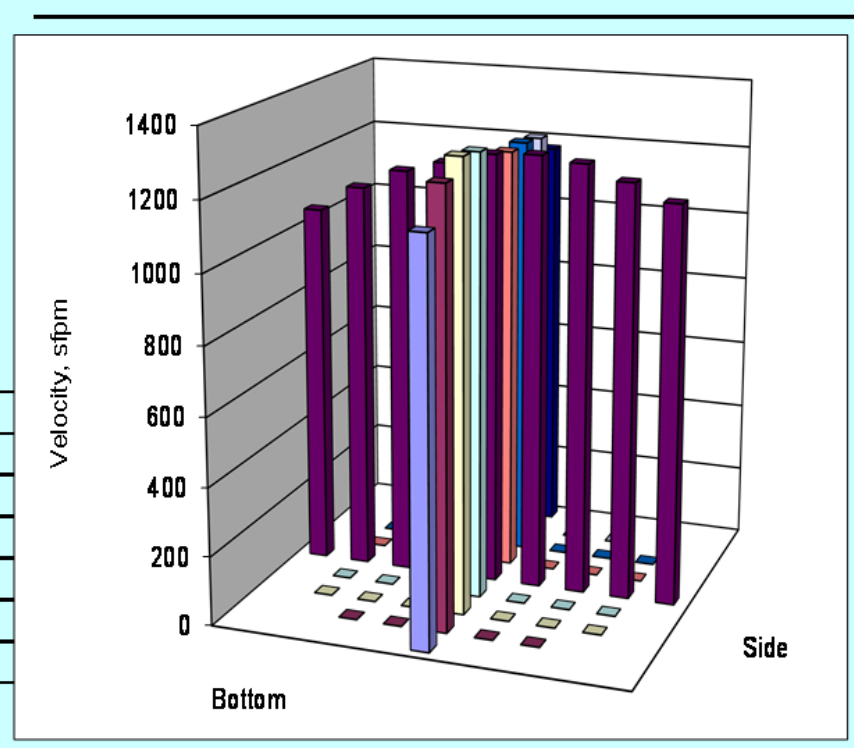

Technical Data Review performed by: Susan Sande Signature/date 7/10/2012 Signature on file with original TI-WTPSP-074 


\section{VELOCITY TRAVERSE DATA FORM}

Site HV-S2 Model

Date 4/27/12

Testers JAG/XYY

Stack Dia.

Stack X-Area

Test Port

\begin{tabular}{c}
\hline 11.938 in. \\
\hline $\mathbf{1 1 1 . 9}$ in. 2 \\
\hline 2 \\
\hline 96.4 inhes
\end{tabular}

Distance to disturbance 96.4 inches

Velocity units $\underline{\mathrm{s} \mathrm{ft} / \mathrm{min}}$

Order $\rightarrow$

Traverse->

Trial $-\longrightarrow$

\begin{tabular}{|r|r|}
\hline Point & Depth, in. \\
\hline $\mathbf{1}$ & 0.50 \\
\hline $\mathbf{2}$ & 1.25 \\
\hline $\mathbf{3}$ & 2.32 \\
\hline $\mathbf{4}$ & 3.86 \\
\hline Center & 5.97 \\
\hline $\mathbf{5}$ & 8.08 \\
\hline $\mathbf{6}$ & 9.62 \\
\hline $\mathbf{7}$ & 10.68 \\
\hline $\mathbf{8}$ & \multicolumn{2}{|c|}{11.44} \\
\hline Averages -- -
\end{tabular}

2nd
Run No. VT-5

Fan Configuration $\mathrm{B}$

Fan Setting 26.0

Stack Temp

Start/End Time $1410 / 1434$

Center $2 / 3$ from

Points in Center $2 / 3$

Data Fles: NA

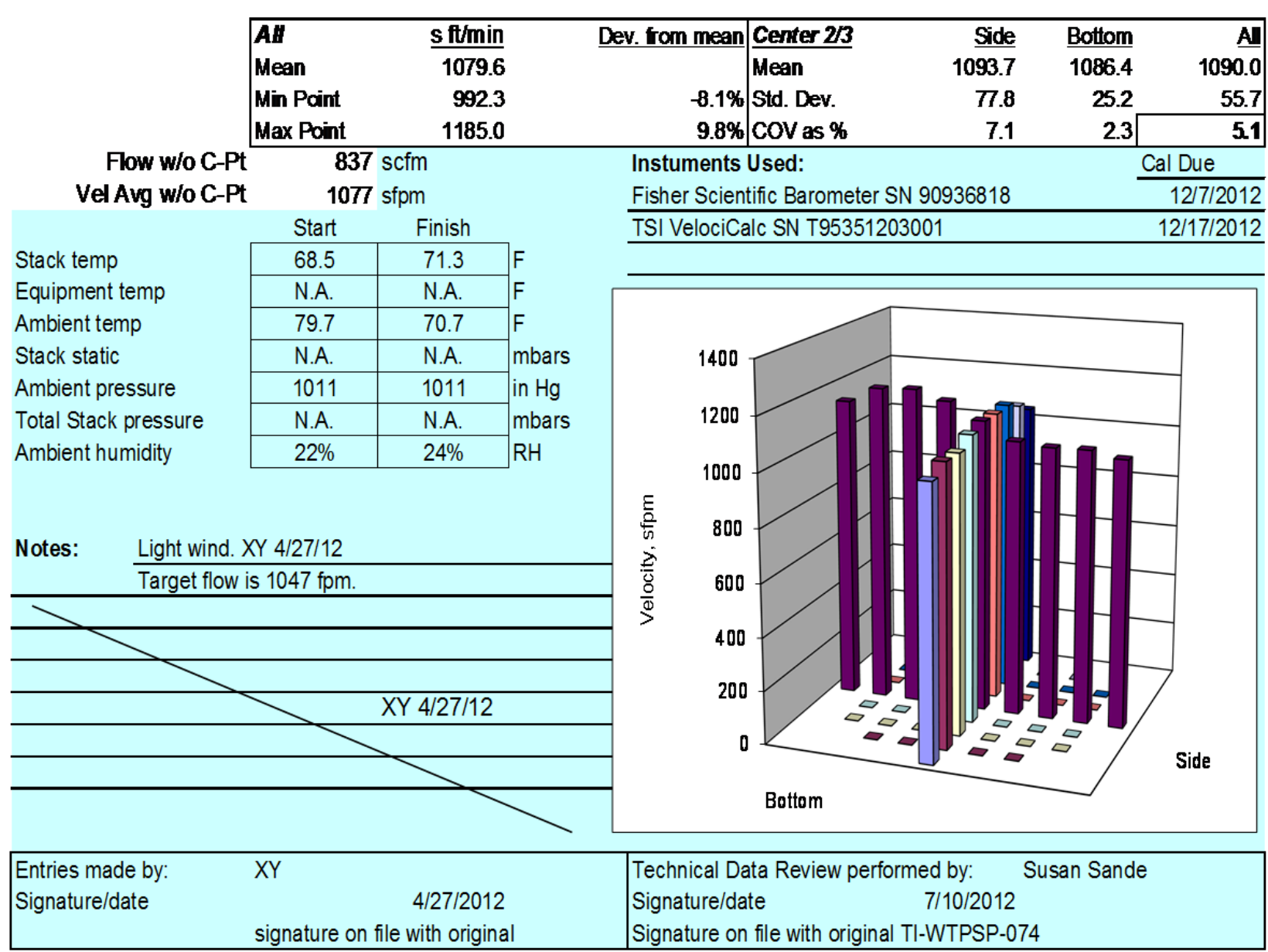




\section{VELOCITY TRAVERSE DATA FORM}

Site HV-S2 Model

Date 4/27/12

Testers JAG, XY

Stack Dia

Stack X-Area

Test Port

Distance to disturbance

Velocity units $\mathrm{s} \mathrm{ft} / \mathrm{min}$

Order $\rightarrow$

Traverse->

Trial $->$
11.938 in. 111.9 in. 2

2
96.4 inches

2nd
Run No. VT-6

Fan Confguration Fan B Only

Fan Setting 56.0

Stack Temp

Start/End Time $1437 / 1500$

Center $2 / 3$ from

Points in Center $2 / 3$

Data Fles: NA

\begin{tabular}{|c|c|c|c|}
\hline & $\mathrm{Hz}$ & & \\
\hline 70 & $\operatorname{deg} F$ & & \\
\hline 1500 & & & \\
\hline 1.10 & to: & 10.84 & \\
\hline 2 & to: & 7 & \\
\hline & & & \\
\hline & Bottc & & \\
\hline 1 & 2 & 3 & Mean \\
\hline & Veloc & & \\
\hline 2305 & 2356 & 2280 & 2313.7 \\
\hline 2381 & 2374 & 2362 & 23723 \\
\hline 2435 & 2427 & 2415 & 2425.7 \\
\hline 2430 & 2422 & 2382 & 2411.3 \\
\hline 2405 & 2387 & 2425 & 2405.7 \\
\hline 2436 & 2451 & 2415 & 2434.0 \\
\hline 2475 & 2430 & 2531 & 2478.7 \\
\hline 2507 & 2420 & 2467 & 2464.7 \\
\hline 2354 & 2370 & 2356 & 2360.0 \\
\hline 414.2 & 2404.1 & 2403.7 & 2407.3 \\
\hline
\end{tabular}

\begin{tabular}{|lr}
\hline All & s ft/min \\
Mean & 2457.9 \\
Min Point & 2174.0 \\
Max Point & 2832.3 \\
\hline
\end{tabular}

Flow w/o C-Pt Vel Avg w/o C-Pt

$1909 \mathrm{scfm}$
$2456 \mathrm{sfpm}$

\begin{tabular}{|c|c|l}
\multicolumn{1}{|c|}{ Start } & Finish & \\
\cline { 1 - 2 } 68.6 & 71.3 & F \\
\cline { 1 - 2 } N.A. & N.A. & F \\
\cline { 1 - 2 } 69.8 & 72.5 & F \\
\cline { 1 - 2 } N.A. & N.A. & mbars \\
\cline { 1 - 2 } 1011 & 1011 & in Hg \\
\cline { 1 - 2 } N.A. & N.A. & mbars \\
\cline { 1 - 2 } $24 \%$ & $24 \%$ & RH \\
\hline
\end{tabular}

Stack temp

Equipment temp Ambient temp

Stack static

Ambient pressure

Total Stack pressure

Ambient humidity

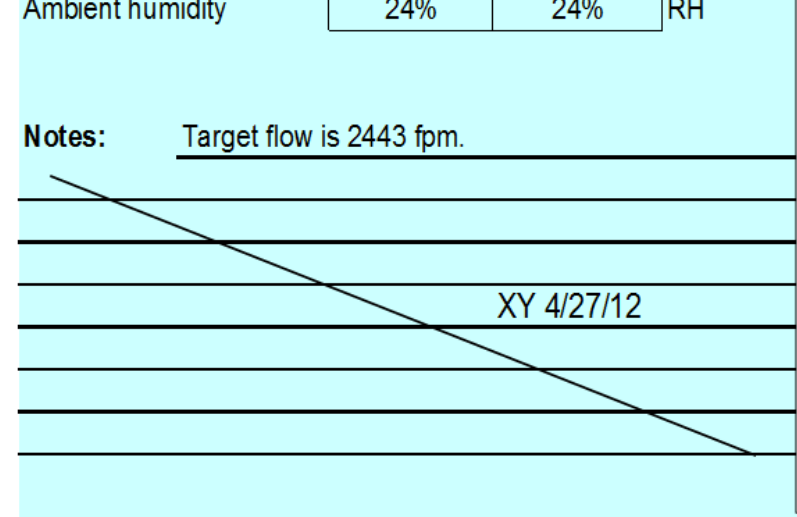

Entries made by:

Signature/date

\begin{abstract}
$X Y$
\end{abstract}
4/27/2012

signature on file with original

\begin{tabular}{r|lrrrr|} 
Dev. from mean & Center 2/3 & $\underline{\text { Side }}$ & Bottom & $\underline{\text { A }}$ \\
\cline { 2 - 3 } & Mean & 2528.4 & & 2427.5 & 2478.0 \\
$11.6 \%$ & Std. Dev. & 270.9 & 36.1 & 1929 \\
\hline Cov as $\%$ & 10.7 & 1.5 & 7.8 \\
\hline
\end{tabular}

Instuments Used:

Fisher Scientific Barometer SN 90936818

TSI VelociCalc SN T95351203001

$12 / 7 / 2012$

$12 / 17 / 2012$

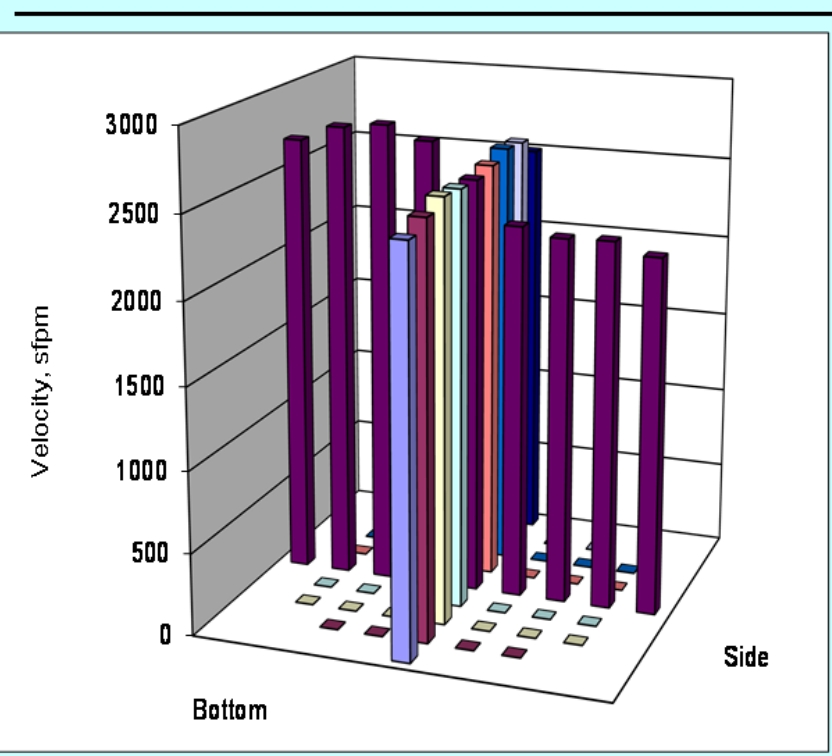

Technical Data Review performed by: Susan Sande Signature/date 7/10/2012 Signature on file with original TI-WTPSP-074 


\section{VELOCITY TRAVERSE DATA FORM}

Site HV-S2 Model

Date 4/27/12

Testers JAG/XYY

Stack Dia

Stack X-Area

Test Port

\begin{tabular}{c}
\hline 11.938 in. \\
\hline $\mathbf{1 1 1 . 9}$ in. 2 \\
\hline 2 \\
\hline 96.4 inches
\end{tabular}

Distance to disturbance

Velocity units $\underline{\mathrm{s} f t / m i n}$

Order $\rightarrow$

Traverse->

Trial $->$

\begin{tabular}{|r|r|}
\hline Point & Depth, in. \\
\hline $\mathbf{1}$ & 0.50 \\
\hline $\mathbf{2}$ & 1.2 \\
$\mathbf{3}$ & 2.3 \\
\hline $\mathbf{4}$ & 3.8 \\
\hline Center & 5.9 \\
\hline $\mathbf{5}$ & 8.08 \\
\hline $\mathbf{6}$ & 9.62 \\
\hline $\mathbf{7}$ & 10.68 \\
\hline $\mathbf{8}$ & 11.4 \\
\hline
\end{tabular}

Averages -- -
$1 \mathrm{st}$
1.44

\begin{tabular}{|l|r|}
\cline { 2 - 2 } & \\
\hline 50 & 220 \\
\hline 32 & 22 \\
\hline 86 & 219 \\
\hline 97 & 247 \\
\hline 62 & 268 \\
\hline & 27 \\
\hline
\end{tabular}

24522

1

Side
2

Run No. VT-7

Fan Configuration $B$

Fan Setting 56.0

Stack Temp

$\mathrm{Hz}$

$70 \quad \operatorname{deg} \mathrm{F}$

Start/End Time 1504 / 1522

Center $2 / 3$ from

Points in Center $2 / 3$

Data Files: NA

\begin{tabular}{rll}
1.10 & to: & 10.84 \\
\hline & to: &
\end{tabular}

2nd

\begin{tabular}{|c|c|c|c|c|}
\hline \multicolumn{5}{|c|}{ 2nd } \\
\hline \multirow{3}{*}{ Mean } & \multicolumn{4}{|c|}{ Bottom } \\
\hline & \multicolumn{2}{|c|}{1} & 2 & Mean \\
\hline & \multicolumn{4}{|c|}{ Velocity } \\
\hline 2206.3 & 2275 & 2332 & 2271 & 22927 \\
\hline 2263.7 & 2407 & 2360 & 2389 & 2385.3 \\
\hline 2237.0 & 2451 & 2420 & 2467 & 2446.0 \\
\hline 2243.0 & 2425 & 2478 & 2466 & 2456.3 \\
\hline 2437.3 & 2437 & 2380 & 2395 & 2404.0 \\
\hline 2652.7 & 2458 & 2465 & 2384 & 2435.7 \\
\hline 2719.7 & 2538 & 2507 & 2451 & 2498.7 \\
\hline 2686.3 & 2522 & 2489 & 2422 & 2477.7 \\
\hline 2612.3 & 2418 & 2402 & 2336 & 2385.3 \\
\hline 2450.9 & 2436.8 & 2425.9 & 2397.9 & 2420.2 \\
\hline
\end{tabular}

\begin{tabular}{|lr}
\hline AH & s ft/min \\
Mean & 2435.6 \\
Min Point & 2206.3 \\
Max Point & 2719.7 \\
\hline
\end{tabular}

Flow w/o C-Pt $1895 \mathrm{scfm}$ Vel Avg w/o C-Pt

$2437 \mathrm{sfpm}$

\begin{tabular}{|c|c|}
\hline Start & Finish \\
\hline 68.5 & 72.3 \\
\hline N.A. & N.A. \\
\hline 73.4 & 71.6 \\
\hline N.A. & N.A. \\
\hline 1011 & 1011 \\
\hline N.A. & N.A. \\
\hline $24 \%$ & $24 \%$ \\
\hline
\end{tabular}

Stack temp

Equipment temp

Ambient temp

Stack static

Ambient pressure

Total Stack pressure

Ambient humidity

2719.7

2451.2

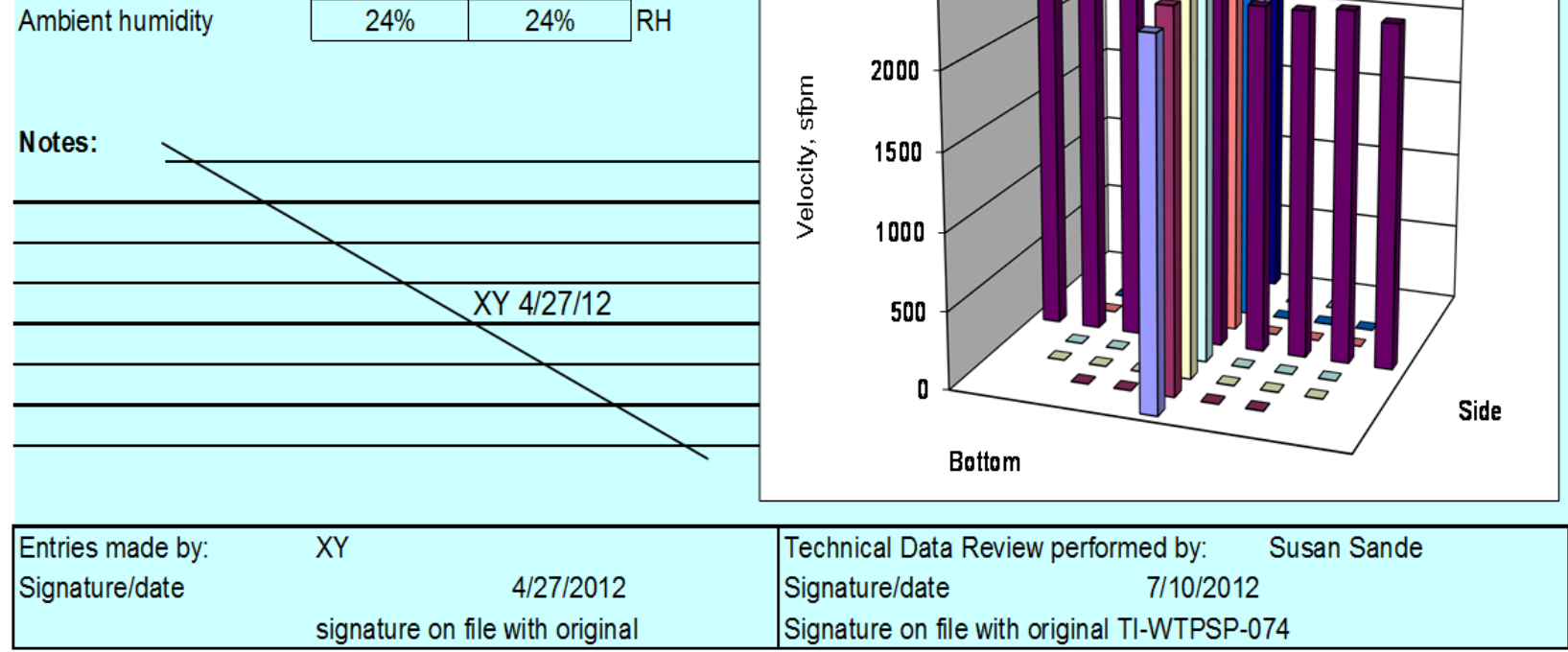




\section{VELOCITY TRAVERSE DATA FORM}

Site HV-S2 Model

Date 4/27/12

Testers JAG, XYY

Stack Dia

Stack X-Area

Test Port

Distance to disturbance

Velocity units $\mathrm{s} \mathrm{ft} / \mathrm{min}$

Order $\rightarrow$

Traverse->

Trial $->$
11.938 in 111.9 in. 2 96.4 inches

2nd
Run No. VT-8

Fan Confguration Fan B only

Fan Setting 56.0

Stack Temp

Start/End Time $1523 / 1547$

Center $2 / 3$ from $\quad 1.10$ to: 10.84

Points in Center $2 / 3$

Data Fles: NA

\begin{tabular}{|c|c|c|c|c|c|c|c|c|c|}
\hline \multicolumn{2}{|l|}{ Trial -—> } & 1 & 2 & \multirow{2}{*}{\multicolumn{2}{|c|}{3 Mean }} & \multirow[t]{2}{*}{1} & 2 & 3 & \multirow[t]{2}{*}{ Mean } \\
\hline Point & Depth, in. & \multicolumn{2}{|c|}{ Velocity } & & & & \multicolumn{2}{|c|}{ Velocity } & \\
\hline 1 & 0.50 & 2189 & 2202 & 2220 & 2203.7 & 2333 & 2356 & 2332 & 2340.3 \\
\hline 2 & 1.25 & 2261 & 2251 & 2273 & 2261.7 & 2355 & 2386 & 2416 & 2385.7 \\
\hline 3 & 2.32 & 2311 & 2299 & 2282 & 2297.3 & 2386 & 2413 & 2396 & 2398.3 \\
\hline 4 & 3.86 & 2262 & 2240 & 2242 & 2248.0 & 2445 & 2409 & 2385 & 2413.0 \\
\hline Center & 5.97 & 2416 & 2346 & 2381 & 2381.0 & 2399 & 2396 & 2444 & 2413.0 \\
\hline 5 & 8.08 & 2525 & 2597 & 2548 & 2556.7 & 2426 & 2440 & 2449 & 2438.3 \\
\hline 6 & 9.62 & 2651 & 2625 & 2602 & 2626.0 & 2434 & 2411 & 2458 & 2434.3 \\
\hline 7 & 10.68 & 2613 & 2612 & 2569 & 2598.0 & 2460 & 2422 & 2377 & 2419.7 \\
\hline 8 & 11.44 & 2532 & 2586 & 2517 & 2545.0 & 2382 & 2368 & 2389 & 2379.7 \\
\hline Averages - & $\longrightarrow$ & 2417.8 & 2417.6 & 2403.8 & 2413.0 & 2402.2 & 2400.1 & 2405.1 & 2402.5 \\
\hline
\end{tabular}

\begin{tabular}{|lr}
\hline AH & s ft/min \\
Mean & 2407.8 \\
Min Point & 2203.7 \\
Max Point & 2626.0 \\
\hline
\end{tabular}

Flow w/o C-Pt Vel Avg w/o C-Pt

$1873 \mathrm{scfm}$

2409 sfpm

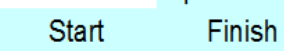

Stack temp

Equipment temp

Ambient temp

Stack static

Ambient pressure

Total Stack pressure

Ambient humidity

\begin{tabular}{|l|l|l|}
\hline 68.2 & 71 & $F$ \\
\cline { 1 - 2 } N.A. & N.A. & F \\
\cline { 1 - 2 } 71.6 & 76.1 & F \\
\cline { 1 - 2 } N.A. & N.A. & mbars \\
\cline { 1 - 2 } 1011 & 1011 & in Hg \\
\cline { 1 - 2 } N.A. & N.A. & mbars \\
\cline { 1 - 2 } $24 \%$ & $22 \%$ & RH \\
\hline
\end{tabular}

Notes:

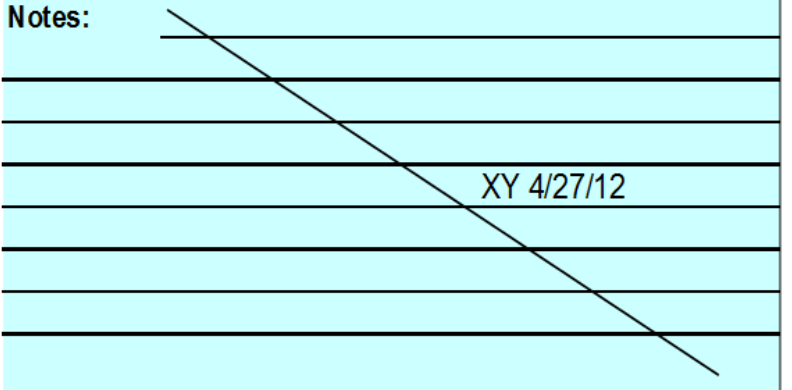

\begin{tabular}{r|l} 
Dev. fom mean & Center 2/3 \\
$-8.5 \%$ & Mean \\
$9.1 \%$ & Cov. Dev. \\
\hline
\end{tabular}

Side
2424.1
165.3
6.8

Instuments Used:

Fisher Scientific Barometer SN 90936818

TSI VelociCalc SN T95351203001

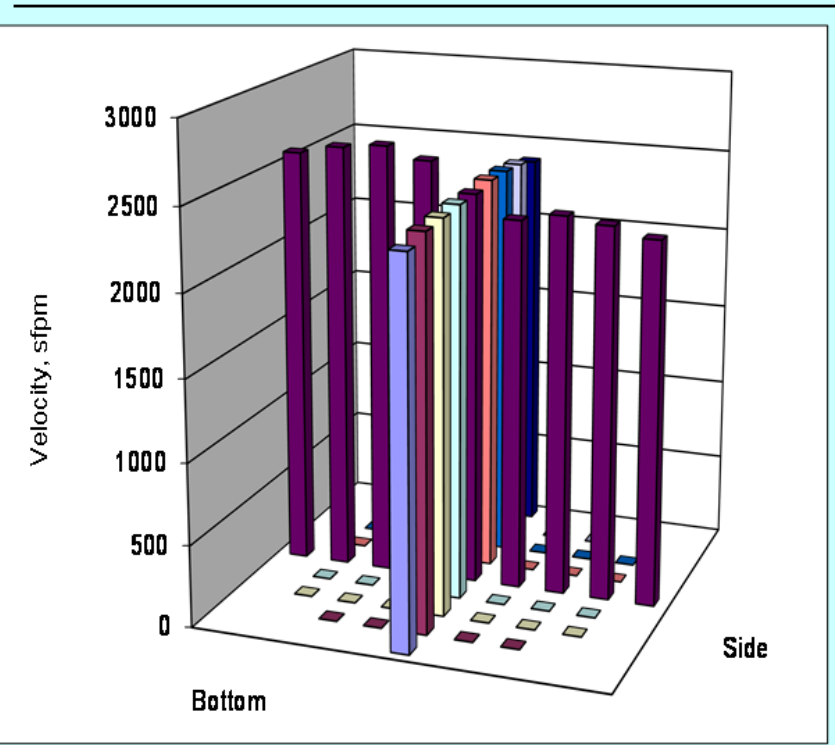

Entries made by:

Signature/date
$X Y Y$

4/27/2012
Technical Data Review performed by: Susan Sande Signature/date

7/10/2012

Signature on file with original TI-WTPSP-074 


\section{VELOCITY TRAVERSE DATA FORM}

\begin{tabular}{|c|c|}
\hline \multicolumn{2}{|c|}{ Site HV-S2 Model } \\
\hline \multicolumn{2}{|c|}{ Date $6 / 19 / 12$} \\
\hline Testers & $\mathrm{CA}$ \\
\hline Stack Dia. & 11.938 in. \\
\hline Stack X-Area & 111.9 in. 2 \\
\hline Test Port & 2 \\
\hline Distance to disturbance & 96.4 inches \\
\hline
\end{tabular}

Order $\rightarrow$

Traverse->

Trial $\rightarrow$

\begin{tabular}{|c|c|}
\hline Point & Depth, in. \\
\hline 1 & 0.5 \\
\hline 2 & 1.2 \\
\hline 3 & 2.3 \\
\hline 4 & 3.8 \\
\hline Center & 5.9 \\
\hline 5 & 8.0 \\
\hline 6 & 9.6 \\
\hline 7 & 10.6 \\
\hline 8 & 11.4 \\
\hline
\end{tabular}

2nd

Run No. VT-9

Fan Configuration Fan B only

Fan Setting 59.1

Stack Temp

\begin{tabular}{lr} 
& $\mathrm{Hz}$ \\
\hline & $\operatorname{deg} \mathrm{F}$ \\
\hline
\end{tabular}

Start/End Time 925/948

Center $2 / 3$ from $\quad 1.10 \quad$ to: 10.84

Points in Center $2 / 3 \overline{2}$ to: $\overline{7}$

Data Files: NA

$1 \mathrm{st}$

\begin{tabular}{|c|c|c|c|c|c|c|}
\hline \multirow{2}{*}{\multicolumn{3}{|c|}{ Side }} & \multicolumn{3}{|c|}{$1 \mathrm{st}$} & \multirow[b]{3}{*}{ Mean } \\
\hline & & & \multicolumn{3}{|c|}{ Bottom } & \\
\hline 2 & 3 & Mean & 1 & 2 & 3 & \\
\hline \multicolumn{3}{|c|}{ Velocity } & \multicolumn{4}{|c|}{ Velocity } \\
\hline 598 & 2616 & 2606.7 & 2717 & 2726 & 2739 & 2727.3 \\
\hline 39 & 2625 & 2619.0 & 2786 & 2797 & 2821 & 2801.3 \\
\hline 585 & 2647 & 2612.3 & 2834 & 2853 & 2836 & 2841.0 \\
\hline 2553 & 2633 & 2593.7 & 2826 & 2860 & 2863 & 2849.7 \\
\hline 2721 & 2686 & 2717.3 & 2757 & 2774 & 2752 & 2761.0 \\
\hline 2902 & 2934 & 2939.3 & 2813 & 2725 & 2815 & 2784.3 \\
\hline 3015 & 2937 & 2975.3 & 2928 & 2807 & 2916 & 2883.7 \\
\hline 2922 & 2864 & 2943.3 & 2924 & 2871 & 2897 & 2897.3 \\
\hline 2810 & 2875 & 2871.3 & 2860 & 2768 & 2763 & 2797.0 \\
\hline & 2757.4 & 2764.3 & 2827.2 & 2797.9 & 28224 & 2815.9 \\
\hline
\end{tabular}

\begin{tabular}{|lrr|lrrr|}
\hline All & s ft/min & Dev. from mean & Center 2/3 & $\underline{\text { Side }}$ & $\underline{\text { Bottom }}$ & A \\
Mean & 2790.1 & Mean & 2771.5 & 2831.2 & 2801.3 \\
Min Point & 2593.7 & $-7.0 \%$ & Std. Dev. & 174.4 & 51.0 & 127.2 \\
Max Point & 2975.3 & $6.6 \%$ & CoV as $\%$ & 6.3 & 1.8 & 4.5 \\
\hline
\end{tabular}

Flow w/o C-Pt $2174 \mathrm{scfm}$

Vel Avg w/o C-Pt 2796 sfpm

\begin{tabular}{ll} 
Instuments Used: & Cal Due \\
Fisher Scientific Barometer SN 90936818 & $12 / 7 / 2012$ \\
\hline
\end{tabular}

Stack temp

Equipment temp

Ambient temp

Stack static

Ambient pressure

Total Stack pressure

Ambient humidity

\begin{tabular}{|c|c|l}
\multicolumn{1}{c|}{ Start } & \multicolumn{1}{c|}{ Finish } & \\
\hline 65.0 & 68 & $\mathrm{~F}$ \\
\cline { 1 - 2 } N.A. & N.A. & $\mathrm{F}$ \\
\hline 66 & 65 & $\mathrm{~F}$ \\
\cline { 1 - 2 } N.A. & N.A. & mbars \\
\hline 29.68 & 29.68 & in Hg \\
\cline { 1 - 2 } N.A. & N.A. & mbars \\
\cline { 1 - 2 } $29 \%$ & $23 \%$ & RH \\
\hline
\end{tabular}

Notes: Fan B max flow target is 2619 to $3041 \mathrm{fpm}$ at

Bottom 3

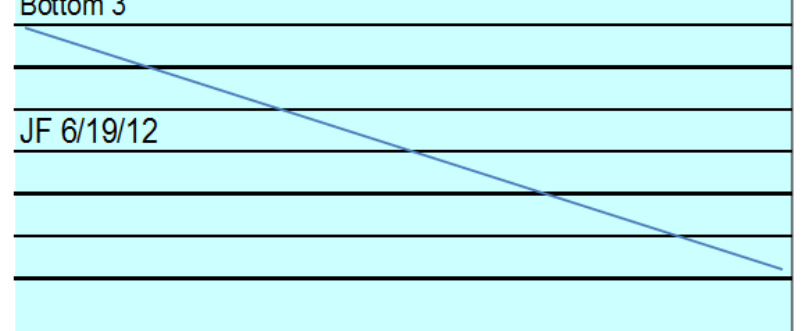

TSI VelociCalc SN T95351203001

$12 / 17 / 2012$

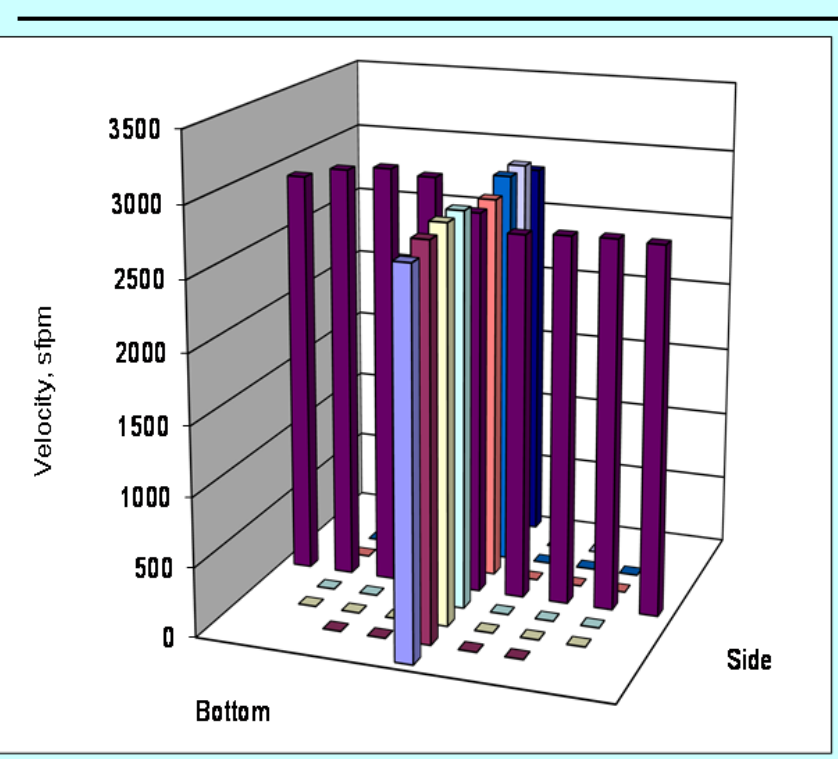

Entries made by:

Carmen Arimescu 6/19/2012

Signature/date

signature on file with original

Technical Data Review performed by: Susan Sande

Signature/date

7/10/2012

Signature on file with original TI-WTPSP-074 


\section{VELOCITY TRAVERSE DATA FORM}

Site HV-S2 Model

Date 6/19/12

Testers JEF, CA

Stack Die

Stack X-Area

Test Port

Distance to disturbance

\begin{tabular}{|c|}
\hline 11.938 in. \\
\hline 111.9 in. 2 \\
\hline 2 \\
\hline
\end{tabular}

Velocity units $\mathrm{s} \mathrm{ft} / \mathrm{min}$

Order $\rightarrow$

Traverse->

Trial $->$

\begin{tabular}{|c|c|}
\hline Point & Depth, in. \\
\hline 1 & 0.50 \\
\hline 2 & 1.25 \\
\hline 3 & 2.3 \\
\hline 4 & 3.8 \\
\hline Center & 5.97 \\
\hline 5 & 8.08 \\
\hline 6 & 9.62 \\
\hline 7 & 10.68 \\
\hline 8 & 11.4 \\
\hline
\end{tabular}

Averages 1st
Run No. VT-10

Fan Confguration Fan A only

Fan Setting 60.0

Stack Temp

Start/End Time 950/1018

Center $2 / 3$ from

Points in Center $2 / 3$

Data Fles: NA

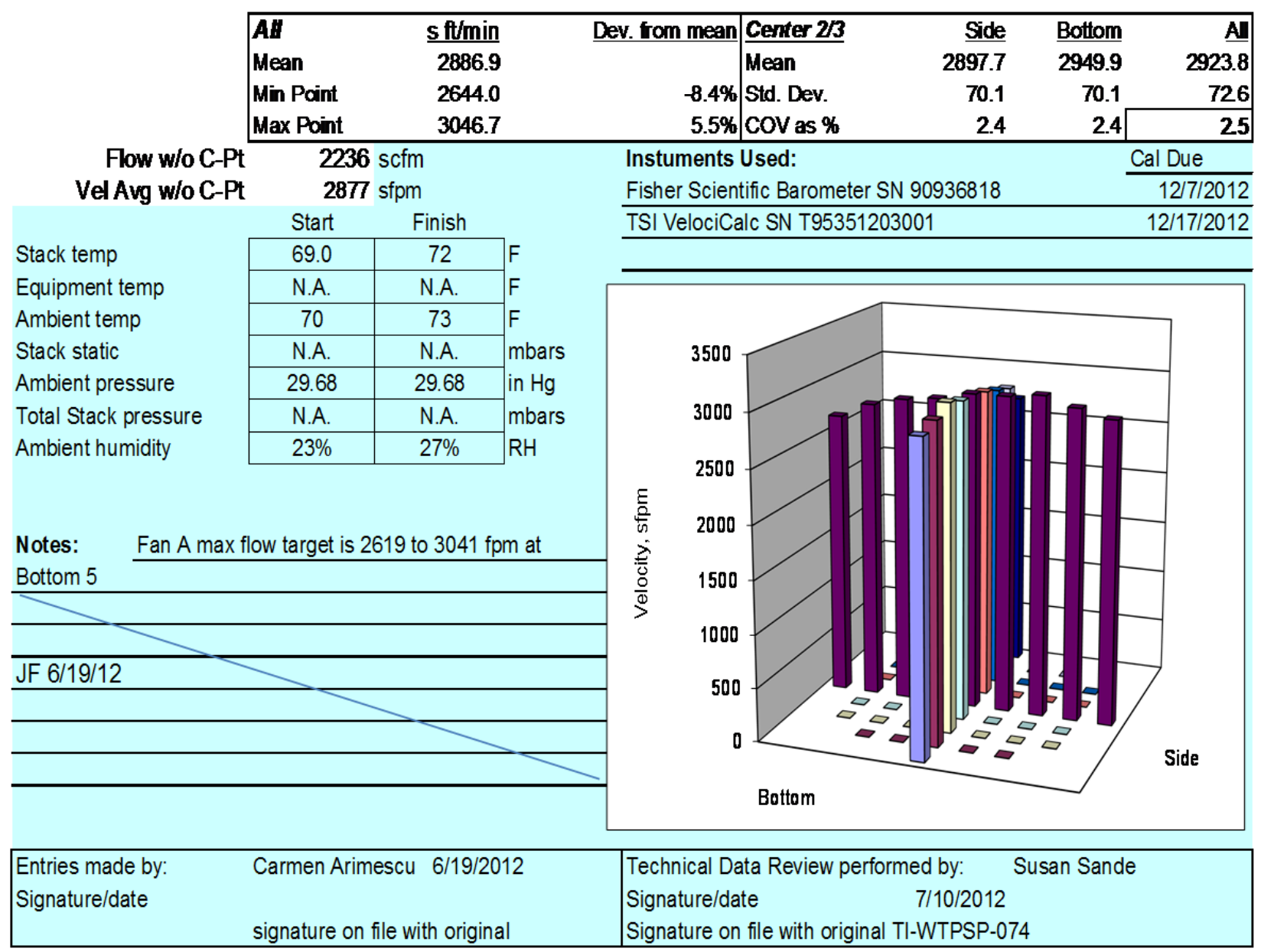




\section{B.3 HV-S2 Flow Angle Data Sheets}

\section{FLOW ANGLE DATA FORM}

Site HV-S2 scale model

Date 5/2/2012

Tester JAG EA

Stack Dia. 11.938 in

Stack X-Area 111.9 in2

Elevation N.A ft

Distance to disturbance $\frac{96.4}{10}$ in

Start/End Time $1340 / 1455$
HV-S2_RowAngle.xds

\section{Fan}

Fan

Fan confguration Fan A Only

Approx air vel. $1063 \mathrm{sfpm}$ at point $\gg>$ Bottom 5

Units degrees (clockwise > pos. nos.)

Port

Stack Temp $\quad 70.9^{\circ} \mathrm{F}$

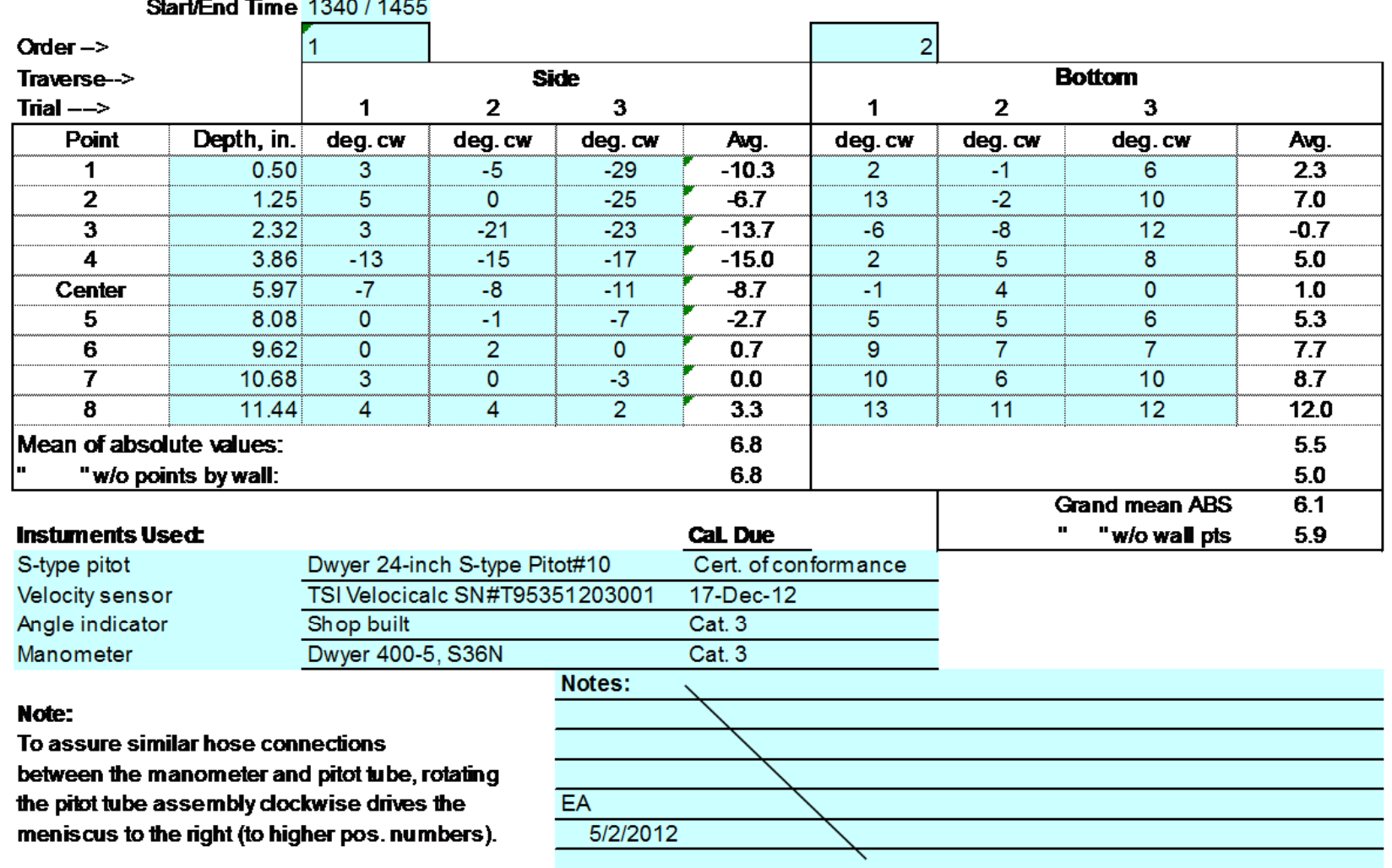

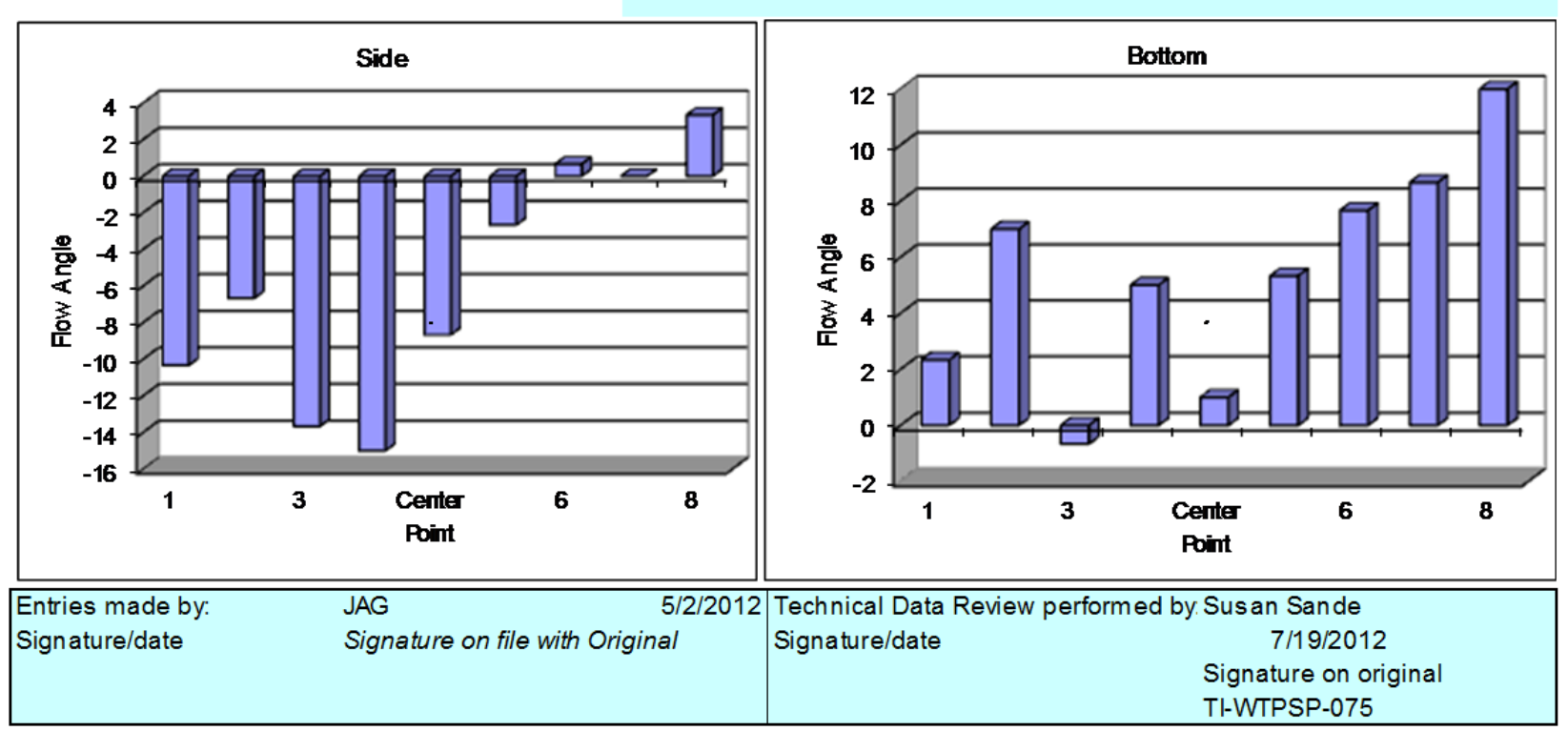


FLOW ANGLE DATA FORM

Site HV-S2 scale model

Date 5/2/2012

Tester JAG EA

Stack Dia. 11.938 in

Stack X-Area 111.9

Elevafion N.A. ft

Distance to distubance 96.4 in

Start/End Time $1500 / 1521$
HV-S2_FlowAngle.xds

Run No. FA-2

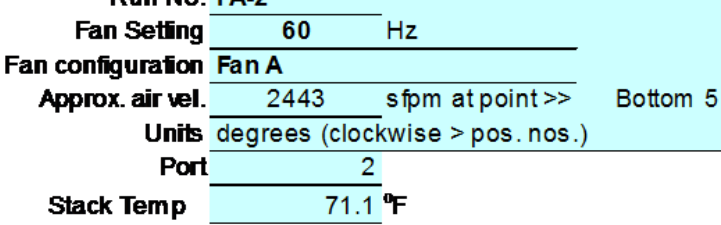

Order $\rightarrow$

Traverse- $\rightarrow$

Trial $\longrightarrow$

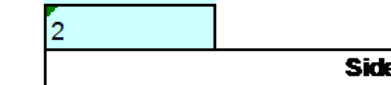

Side

1

$\begin{array}{llll}1 & 2 & 3\end{array}$

3

\begin{tabular}{|c|}
\hline 3 \\
\hline deg. $\mathbf{c w}$ \\
\hline 0 \\
\hline 8 \\
\hline 8 \\
\hline 0 \\
\hline 1 \\
\hline 4 \\
\hline 5 \\
\hline 8 \\
\hline 9 \\
\hline
\end{tabular}

\begin{tabular}{|c|r|c|c|}
\hline Point & Depth, in. & deg. cw & de \\
\hline $\mathbf{1}$ & 0.50 & 1 & \\
\hline $\mathbf{2}$ & 1.25 & -6 & \\
\hline $\mathbf{3}$ & 2.32 & -6 & \\
\hline $\mathbf{4}$ & 3.86 & -1 & \\
\hline Center & 5.97 & 0 & \\
\hline $\mathbf{5}$ & 8.08 & 2 & \\
\hline $\mathbf{6}$ & 9.62 & 5 & \\
\hline $\mathbf{7}$ & 10.68 & 6 & \\
\hline $\mathbf{8}$ & 11.44 & 9 & \\
\hline
\end{tabular}

Mean of absolute velues:

- w/o points bywal:

\begin{tabular}{l} 
g. cw \\
\hline 3 \\
-5 \\
-5 \\
0 \\
0 \\
\hline 4 \\
\hline 6 \\
\hline 7 \\
9 \\
\hline
\end{tabular}

\begin{tabular}{|c|c|}
\hline Avg. & deg. cw \\
\hline 1.3 & 0 \\
\hline-1.0 & -2 \\
\hline-1.0 & -2 \\
\hline-0.3 & 5 \\
\hline $\mathbf{0 . 3}$ & 4 \\
\hline $\mathbf{3 . 3}$ & 5 \\
\hline $\mathbf{5 . 3}$ & 8 \\
\hline $\mathbf{7 . 0}$ & 11 \\
\hline $\mathbf{9 . 0}$ & 13 \\
\hline 3.2 & \\
\hline
\end{tabular}

\begin{tabular}{c|c}
\hline deg. cw & deg. \\
-1 \\
-3 \\
-2 \\
2 \\
0 \\
4 \\
\hline 7 \\
11 \\
11
\end{tabular}

Instuments Used: S-type pitot Velocity sensor Angle indicator Manometer

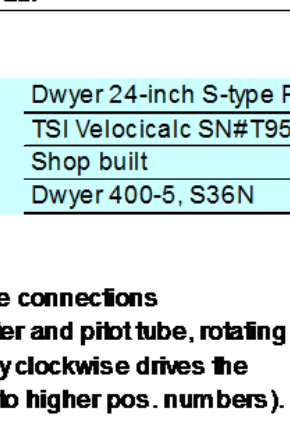

To assure similar hose connections between the manometer and pibt tube, rotaing the pitot tube assem bly clockwise drives the meniscus to the right (to higher pos. numbers).

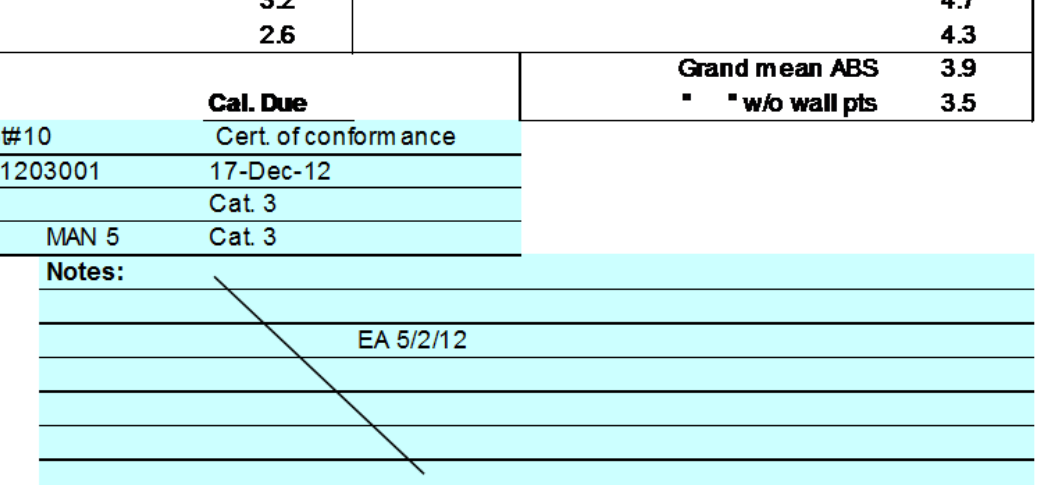

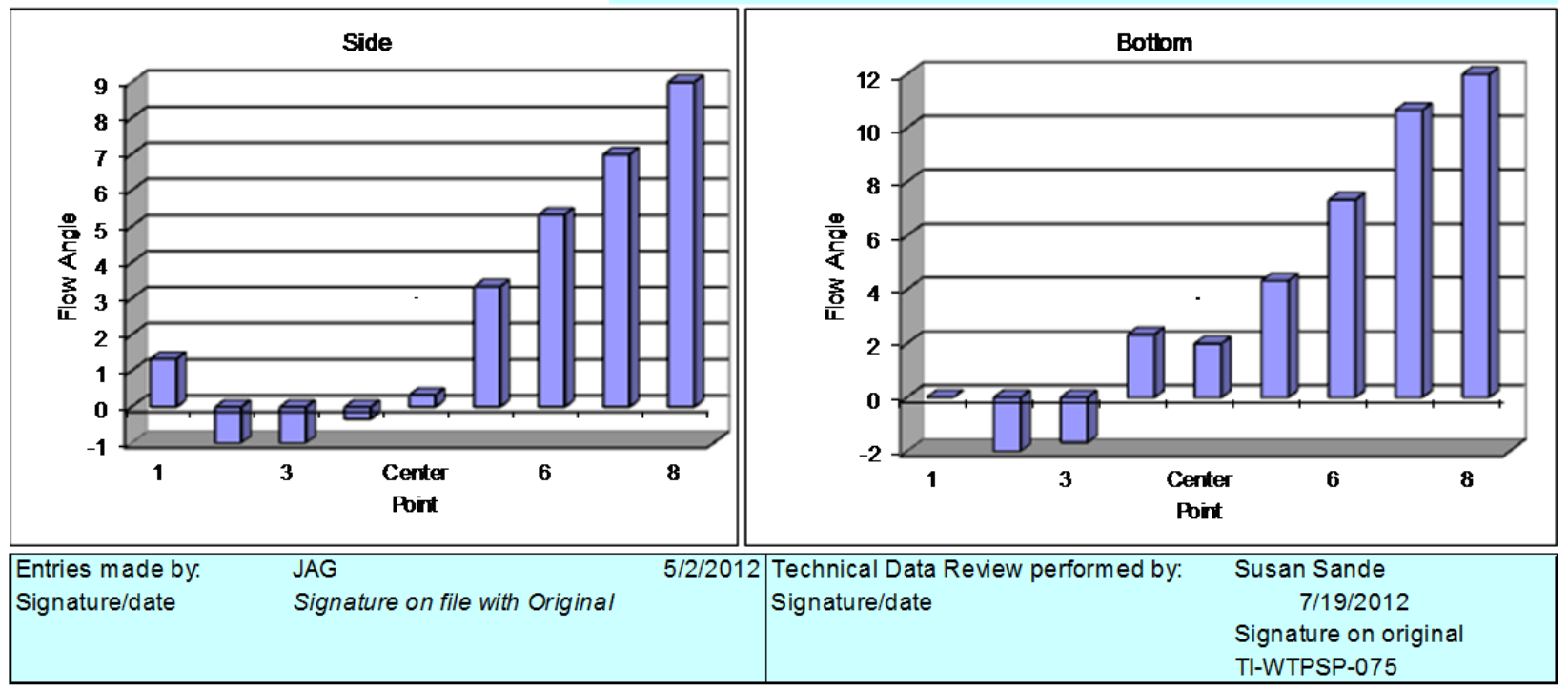


FLOW ANGLE DATA FORM

Site HV-S2 scale model

Date 5/2/2012

Tester JAG, EA

Stack Dia. 11.938 in

Stack X-Area 111.9 in2

Elevation N.A. ft

Distance to distubance 96.4 in

Start/End Time $1530 / 1555$

HV-S2_FlowAngle.xds

Fan Setting $55.9 \mathrm{~Hz}$

Fan configuration Fan B

Approx.air vel. $2471 \mathrm{sfpm}$ at point $\gg$ Bottom 3 Units degrees (clockwise > pos. nos.)

Port $\frac{2}{74^{\circ}}$

Stack Temp

2

Order $\rightarrow$

Traverse $\rightarrow$

Trial $\longrightarrow$

\begin{tabular}{|l|c|c|} 
& & \multicolumn{1}{c}{2} \\
\hline Point & Depth, in. & 1 \\
\hline
\end{tabular}

Side

\begin{tabular}{l|lr}
\cline { 2 - 3 } & \multicolumn{2}{r}{2} \\
\hline 3 & 1 & $2^{\text {Bottom }}$ \\
\hline
\end{tabular}

\begin{tabular}{|c|r|c|c|c|}
\hline Point & Depth, $\mathbf{i}$. & deg. cw & deg. cw & deg. cw \\
\hline $\mathbf{1}$ & 0.50 & -17 & -18 & -17 \\
\hline $\mathbf{2}$ & 1.25 & -10 & -10 & -10 \\
\hline $\mathbf{3}$ & 2.32 & -8 & -7 & -7 \\
\hline $\mathbf{4}$ & 3.86 & -2 & -3 & -4 \\
\hline Center & 5.97 & 4 & 4 & 4 \\
\hline $\mathbf{5}$ & 8.08 & 10 & 11 & 8 \\
\hline $\mathbf{6}$ & 9.62 & 13 & 13 & 12 \\
\hline $\mathbf{7}$ & 10.68 & 15 & 14 & 15 \\
\hline $\mathbf{8}$ & 11.44 & 16 & 16 & 16 \\
\hline
\end{tabular}

Mean of absolute velues:

- w/o points bywall:

16

16

\begin{tabular}{|c|c|c|}
\hline Avg. & deg. cw & deg \\
\hline-17.3 & -12 & -1 \\
\hline-10.0 & 0 & -3 \\
\hline-7.3 & -15 & 2 \\
\hline-3.0 & -5 & -3 \\
\hline 4.0 & 2 & 3 \\
\hline 9.7 & 10 & 1 \\
\hline 12.7 & 17 & 1 \\
\hline 14.7 & 20 & 2 \\
\hline 16.0 & 21 & 22 \\
\hline
\end{tabular}

10.5

8.8

Instuments Used: S-type pitot

Velocity sensor

Angle indicator

Manom eter

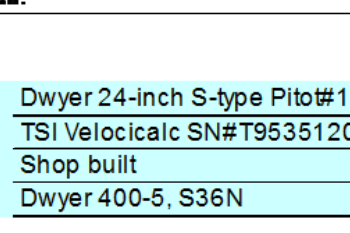

Note:

To assure similarhose connections between the manometer and pibt tube, rotaing the pitot tube assembly clockwise drives the meniscus to the right (to higher pos. numbers).

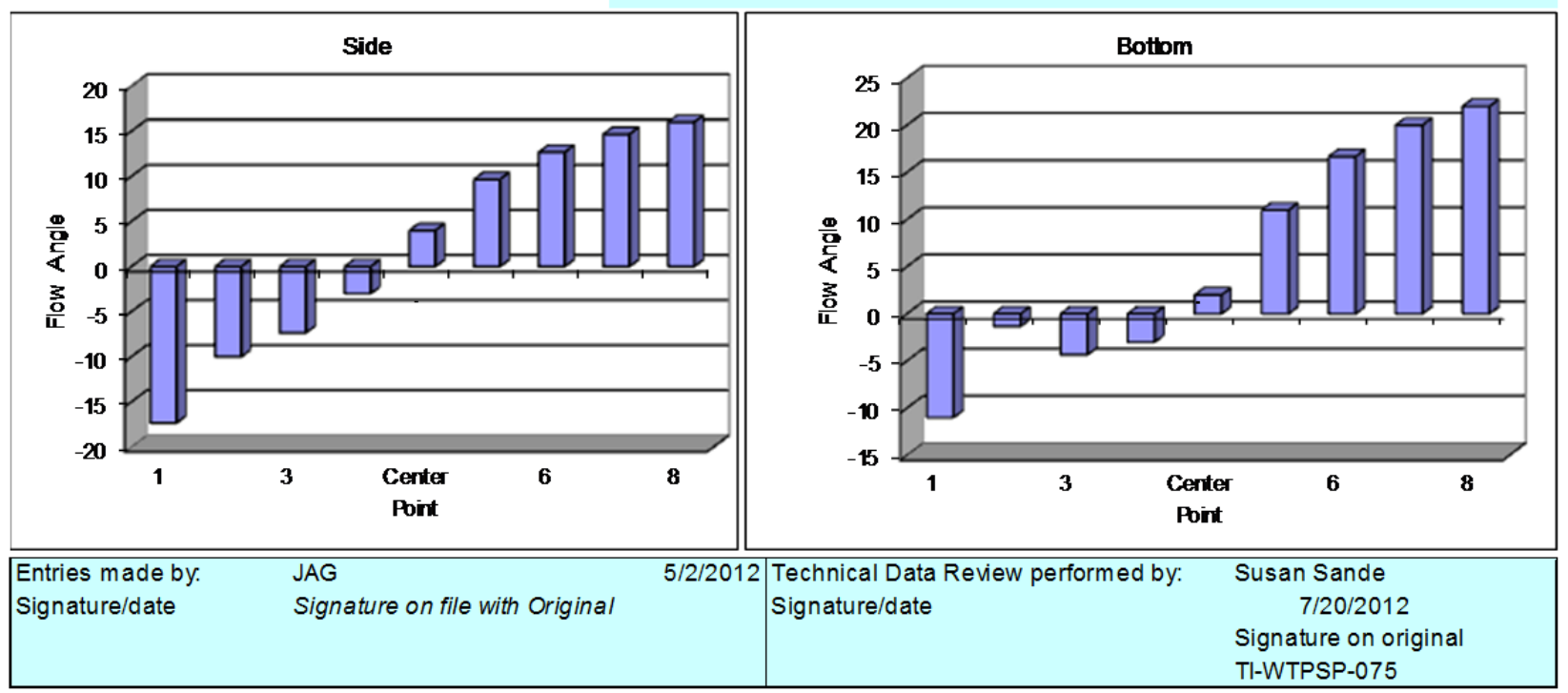


FLOW ANGLE DATA FORM

Site HV-S2 scale model

Date 5/4/2012

Tester EA, CA

Stack Dia. 11.938 in

Stack X-Area 111.9 in2

Elevation N.A. ft

Distance to distubance 96.4 in

Start/End Time $1135 / 1155$
HV-S2_FlowAngle.xds

Run No. FA-4

Fan Setting $53 \quad \mathrm{~Hz}$

Fan configuration Fan B only

Approx.air vel. $2627 \mathrm{sfpm}$ at point $>$ Bottom 3 Units degrees (clockwise > pos. nos.)
Por 2

Stack Temp

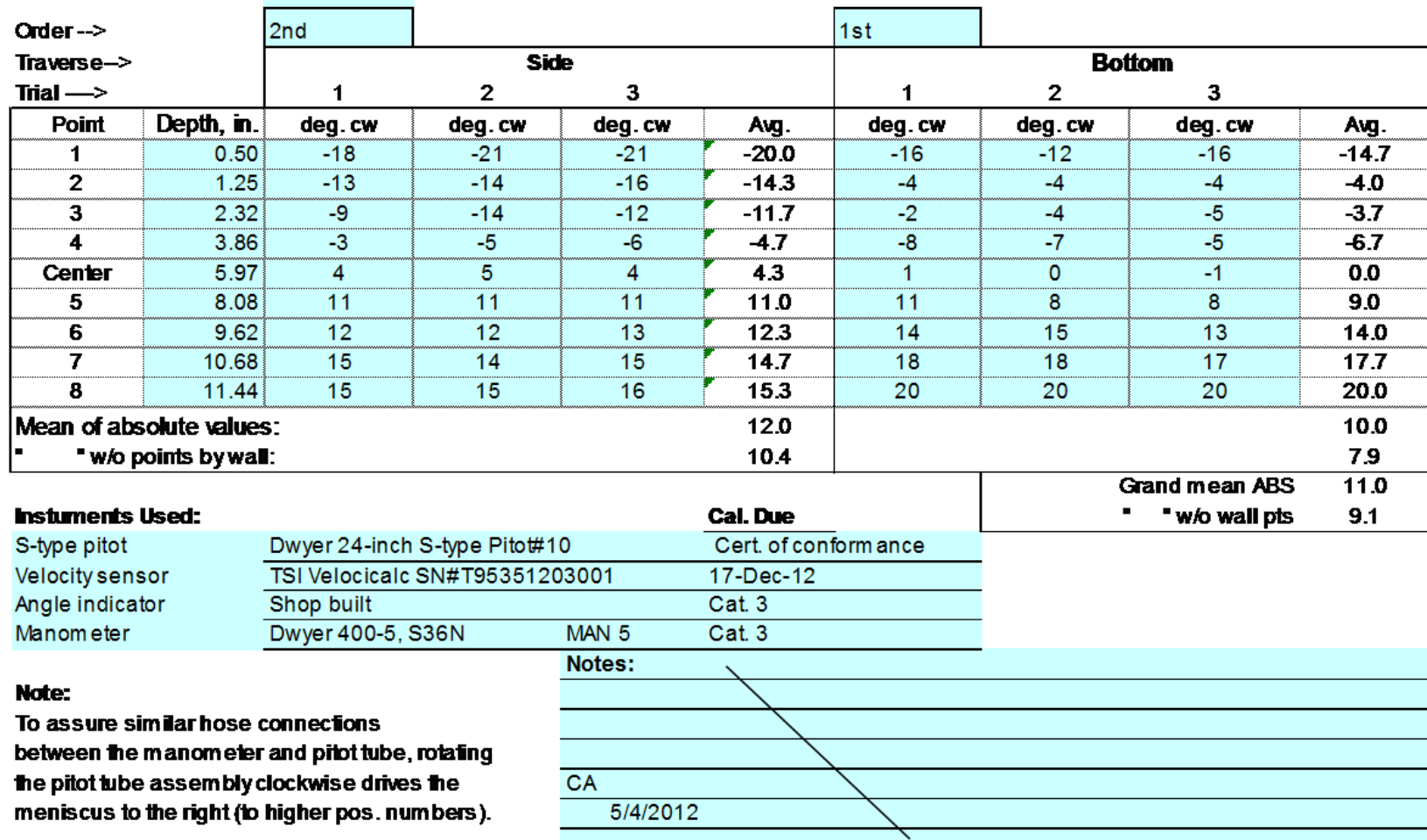

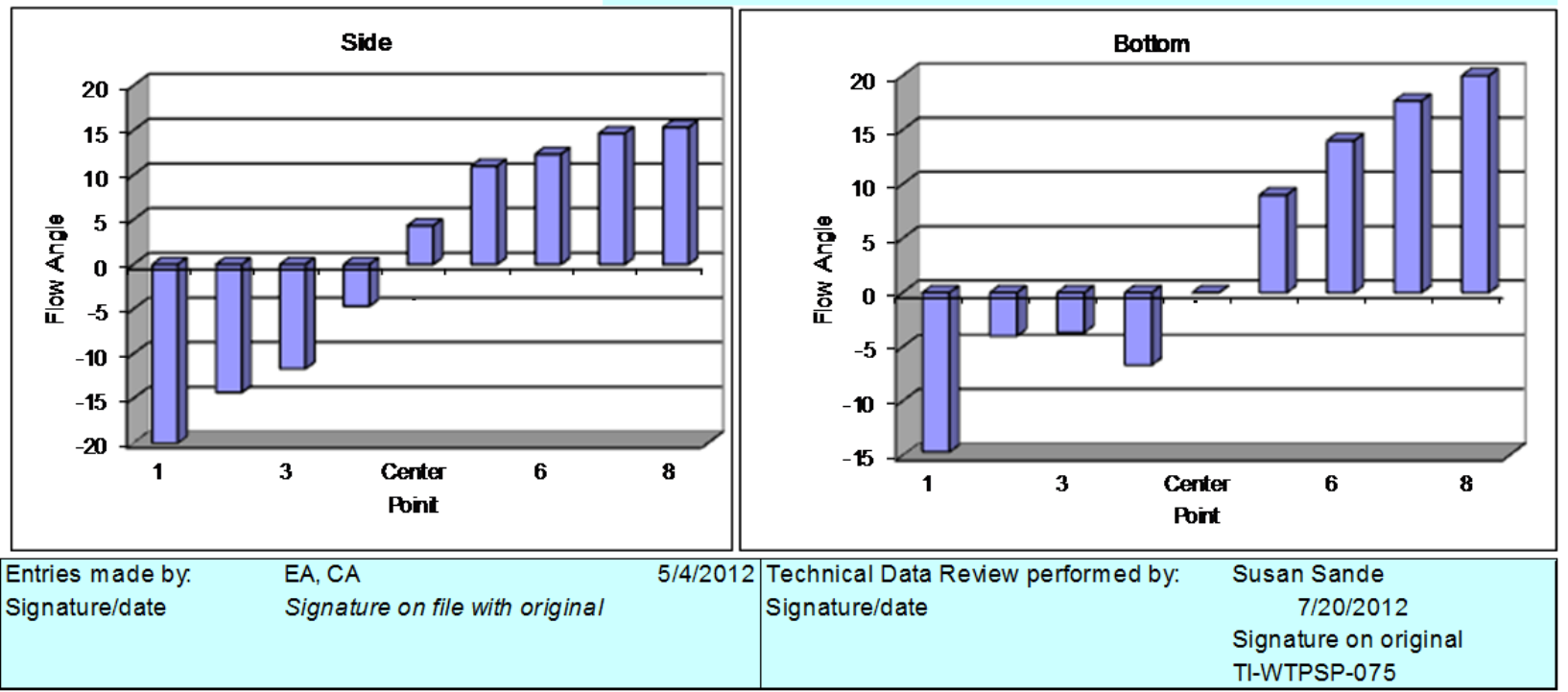


FLOW ANGLE DATA FORM

Site HV-S2 scale model

Date 5/4/2012

Tester EA, CA

Stack Dia. 11.938 in

Stack X-Area 111.9 in2

Elevation N.A. ft

Distance to distubance 96.4 in

Start/End Time 10301130
HV-S2_FlowAngle.xls

Run No. FA-5

Fan Seting $\quad 60 \mathrm{~Hz}$

Fan configuration Fan A Only

Approx.air vel. $3065 \mathrm{sfpm}$ at point $>$ Bottom 5 Units degrees (clockwise > pos. nos.)

Port 2

Stack Temp $63.5^{\circ} \mathrm{F}$ 2nd

Order $-\rightarrow$

Traverse $\rightarrow$

Trial $\longrightarrow$

$1 \mathrm{st}$

\begin{tabular}{|c|r|c|c|c|}
\hline Point & Depth, in. & deg. cw & deg. cw & deg. cw \\
\hline $\mathbf{1}$ & 0.50 & -4 & 8 & 7 \\
\hline $\mathbf{2}$ & 1.25 & 0 & 13 & 10 \\
\hline $\mathbf{3}$ & 2.32 & 8 & 13 & 11 \\
\hline $\mathbf{4}$ & 3.86 & 9 & 10 & 7 \\
\hline Center & 5.97 & 9 & 10 & 5 \\
\hline $\mathbf{5}$ & 8.08 & 8 & 5 & 5 \\
\hline $\mathbf{6}$ & 9.62 & 8 & 9 & 9 \\
\hline $\mathbf{7}$ & 10.68 & 11 & 11 & 9 \\
\hline $\mathbf{8}$ & 11.44 & 13 & 13 & 12 \\
\hline
\end{tabular}

Mean of absolute velues:

- "w/o points bywal:

Instuments Used: S-type pitot

Velocity sensor Angle indicator

Manom eter

side

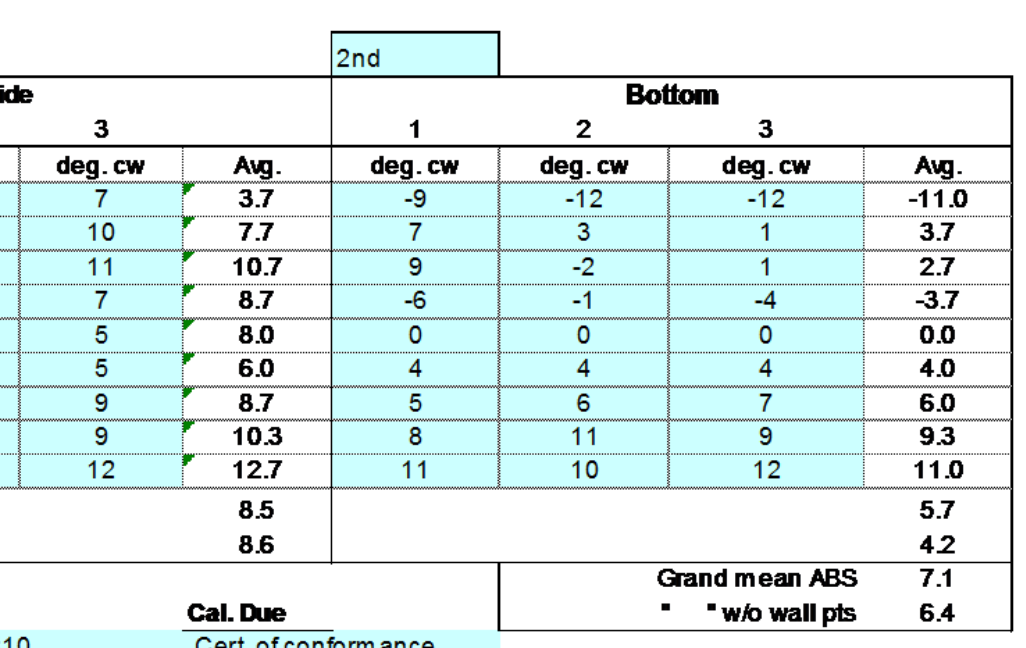

Note:

To assure similarhose connections between the manometer and pibt tube, rotaing the pitot tube assembly clockwise drives the meniscus to the right (to higher pos. numbers).
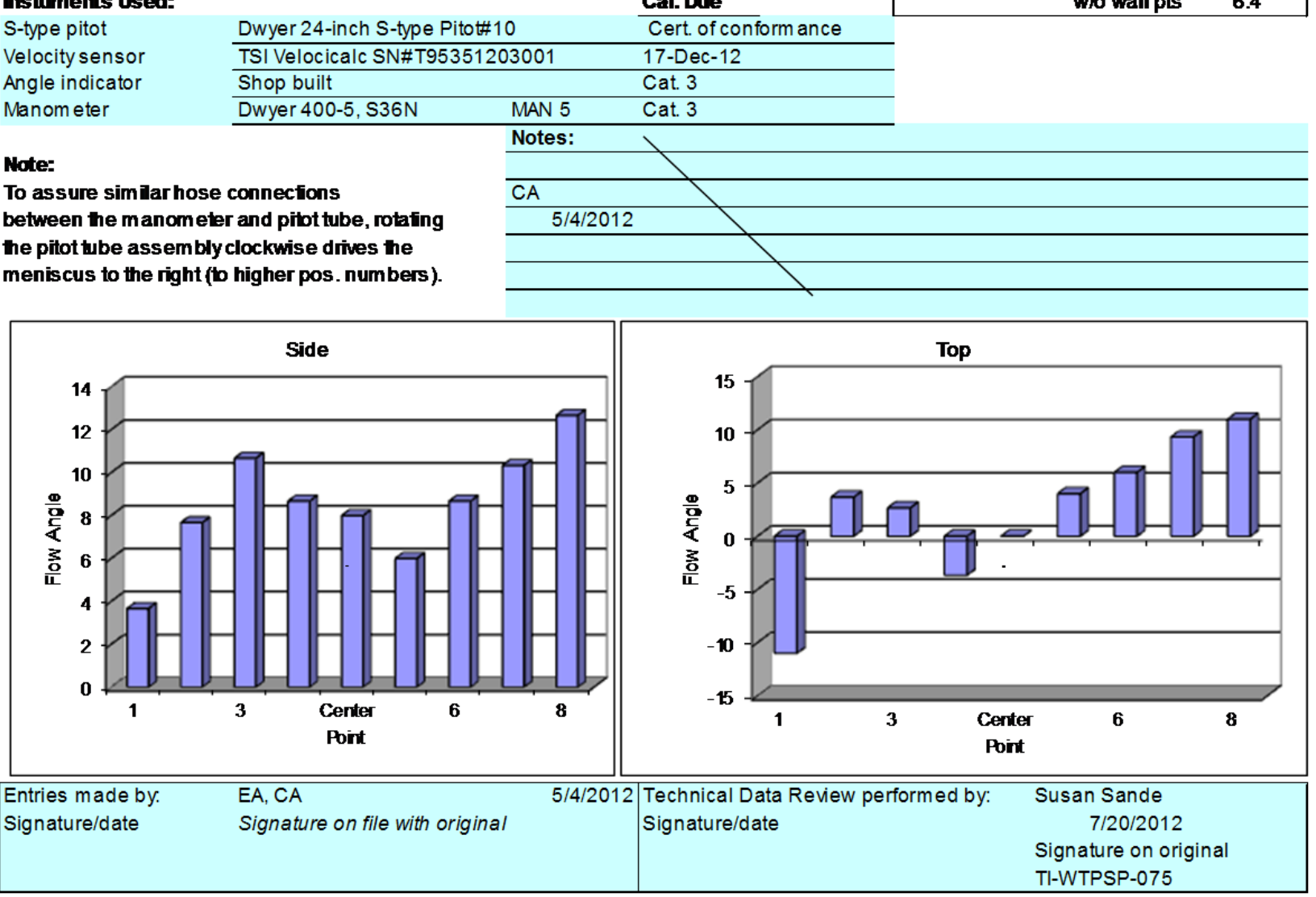
FLOW ANGLE DATA FORM

Site HV-S2 scale model

Date 5/4/2012

Tester YFS, XYY

Stack Dia. 11.938 in

Stack X-Area 111.9 in2

Elevation N.A. fi

Distance to disturbance 96.4 in

Start/End Time 13:15/14:33
HV-S2_FlowAngle.xls

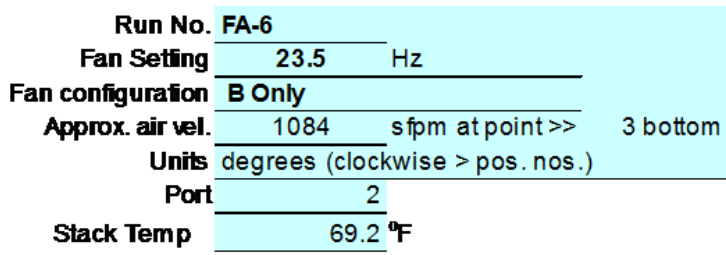

Run No. FA-6 2nd

Order $\rightarrow$

Traverse $\rightarrow$

Trial $\longrightarrow$

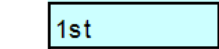

\begin{tabular}{|l|l|l|l}
\hline st & \\
\hline
\end{tabular}

3

nd

\begin{tabular}{|c|r|c|c|c|}
\hline Point & Depth, in. & deg. cw & deg. cw & deg. cw \\
\hline $\mathbf{1}$ & 0.50 & 3 & 10 & 4 \\
\hline $\mathbf{2}$ & 1.25 & -4 & 1 & 8 \\
\hline $\mathbf{3}$ & 2.32 & -1 & 0 & 3 \\
\hline $\mathbf{4}$ & 3.86 & -5 & 8 & 2 \\
\hline Center & 5.97 & 7 & 6 & 8 \\
\hline $\mathbf{5}$ & 8.08 & 16 & 14 & 17 \\
\hline $\mathbf{6}$ & 9.62 & 19 & 23 & 23 \\
\hline $\mathbf{7}$ & 10.68 & 22 & 20 & 29 \\
\hline $\mathbf{8}$ & 11.44 & 24 & 22 & 29 \\
\hline
\end{tabular}

Mean of absolute velues:

- w/o points bywall:

\begin{tabular}{|l|c|c|c|c|}
\hline Avg. & deg. cw & deg. cw & deg. cw & Avg. \\
\hline 5.7 & -20 & -14 & -22 & -18.7 \\
\hline 1.7 & -15 & -11 & -16 & -14.0 \\
\hline 0.7 & -8 & -5 & -16 & -9.7 \\
\hline 1.7 & -5 & -7 & -11 & -7.7 \\
\hline 7.0 & -15 & 6 & 8 & -0.3 \\
\hline 15.7 & 10 & 12 & 11 & 11.0 \\
\hline 21.7 & 15 & 12 & 12 & 13.0 \\
\hline 23.7 & 17 & 15 & 13 & 15.0 \\
\hline 25.0 & 15 & 15 & 15 & 15.0 \\
\hline 11.4 & & & & 11.6 \\
10.3 & & & & 10.1 \\
\hline
\end{tabular}

Instuments Used: S-type pitot Velocity sensor Angle indicator Manom eter

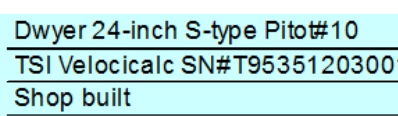

Shop built Dwyer 400-5, S36N

Note:

To assure similarhose connections between the manometer and pibt tube, rotaing the pitot tube assem bly clockwise drives the meniscus to the right (to higher pos. numbers).

\section{Cert. of conform ance}

\section{7-Dec-12}

Cat. 3

Cat. 3

MAN 5 Cat. 3

Notes: Mild wind, overcast

Ambient pressure $1006 \mathrm{mb}, \mathrm{RH} 29 \%$, am bient tem perature: $67 \mathrm{~F}$

Vel: initial $1088 \mathrm{fpm}$; ini. stack tem p: 67.4F.

Vel: end $1079 \mathrm{fpm}$; end stack temp: $71.0 \mathrm{~F}$.

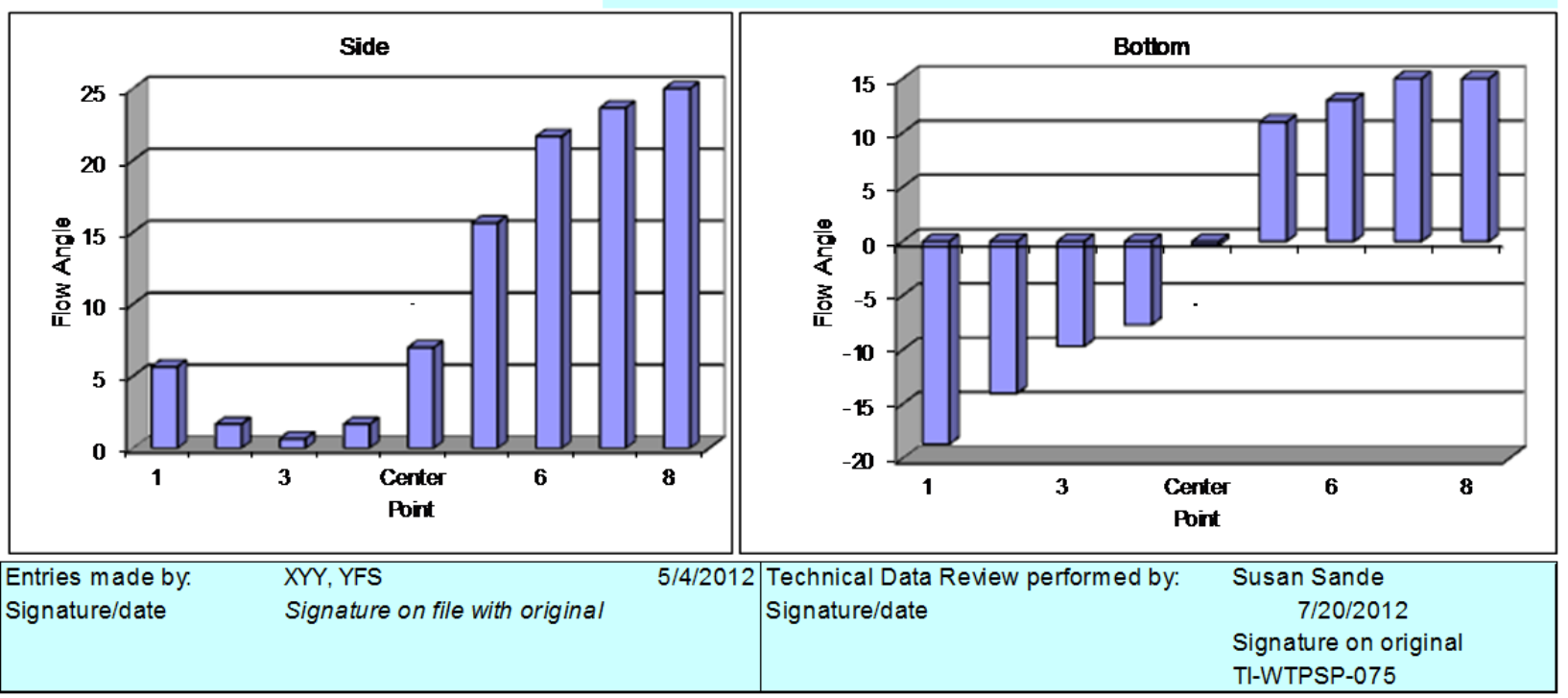


FLOW ANGLE DATA FORM

\begin{tabular}{|c|c|c|}
\hline $\begin{array}{l}\text { Site } \\
\text { Date }\end{array}$ & $\frac{\text { IV-S2 sca }}{/ 19 / 2012}$ & model \\
\hline Tester & $\overline{E F, C A}$ & \\
\hline Stack Dia. & 11.938 & in \\
\hline Stack X-Area " & 111.9 & in2 \\
\hline Elevation & N.A. & ft \\
\hline $\begin{array}{r}\text { Distance to disturbance } \\
\text { Start/End Time }\end{array}$ & $\frac{96.4}{: 30 / 8: 57}$ & in \\
\hline
\end{tabular}

Run No. FA-7

Fan Seting $24.4 \mathrm{~Hz}$

Fan configuration B Only

Approx. air vel. $1195 \mathrm{sfpm}$ at point $\gg \quad 3$ bottom Units degrees (clockwise > pos. nos.) Port 2

Stack Temp $66^{\circ} \mathrm{F}$

\begin{tabular}{|c|c|c|c|c|}
\hline \multirow{3}{*}{$\begin{array}{l}\text { Order }-> \\
\text { Traverse-> } \\
\text { Trial } \longrightarrow\end{array}$} & & 2nd & & \\
\hline & & \multirow[b]{2}{*}{1} & \multicolumn{2}{|c|}{ Side } \\
\hline & & & 2 & 3 \\
\hline Point & Depth, in. & deg. cw & deg. cw & deg. cw \\
\hline 1 & 0.50 & -14 & -15 & -16 \\
\hline 2 & 1.25 & -5 & -14 & -15 \\
\hline 3 & 2.32 & -2 & -5 & -6 \\
\hline 4 & 3.86 & 4 & -4 & -6 \\
\hline Center & 5.97 & 12 & 8 & 9 \\
\hline 5 & 8.08 & 20 & 15 & 12 \\
\hline 6 & 9.62 & 16 & 15 & 15 \\
\hline 7 & 10.68 & 16 & 15 & 16 \\
\hline 8 & 11.44 & 18 & 16 & 18 \\
\hline
\end{tabular}

Mean of absolute velues:

- "w/o points bywal:

Instuments Used: S-type pitot Velocity sensor Angle indicator Manom eter

\section{Note:}

To assure similar hose connections between the manometer and pibt tube, rotaing the pitot tube assem bly clockwise drives the meniscus to the right (to higher pos. numbers).

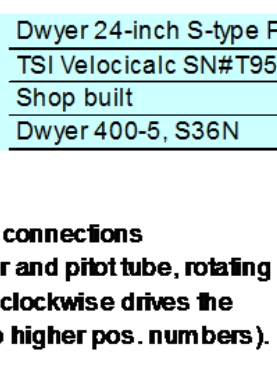

Dwyer 24-inch S-type Pito\#10

203001

MAN 5

Notes:

\section{Repeat of FA-6.}

Cal. Due

Cert. of conform ance

17-Dec-12

Cat. 3 1 st

$\begin{array}{lll} & 2^{\text {Bottom }} & \\ & & \end{array}$

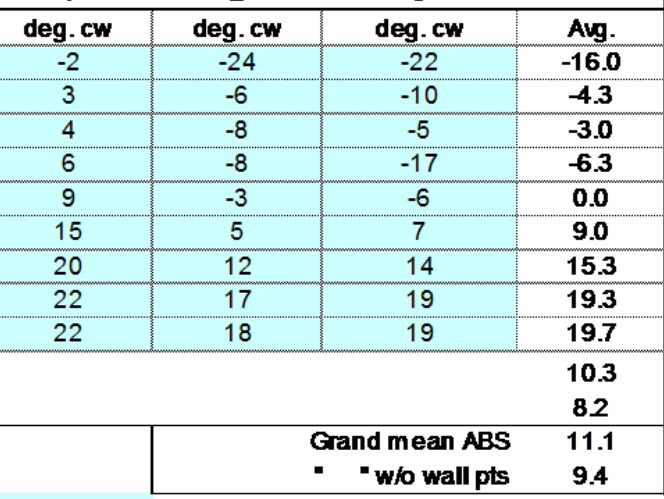

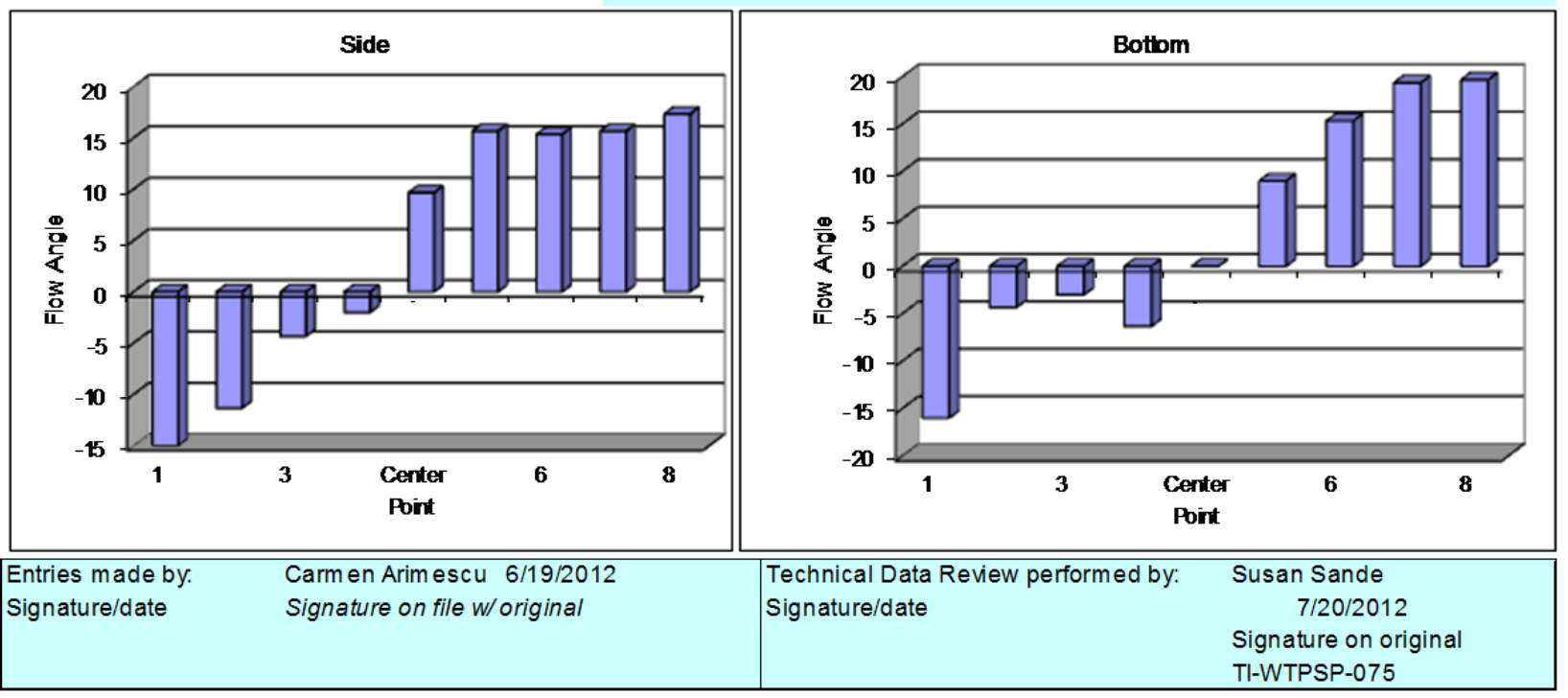


FLOW ANGLE DATA FORM

\begin{tabular}{|c|c|c|}
\hline $\begin{array}{l}\text { Site } \\
\text { Date }\end{array}$ & $\frac{\text { IV-S2 sca }}{3 / 19 / 2012}$ & model \\
\hline Tester & $\mathrm{EF}, \mathrm{CA}$ & \\
\hline Stack Dia. & 11.938 & in \\
\hline Stack X-Area " & 111.9 & in2 \\
\hline Elevation & N.A. & ft \\
\hline $\begin{array}{l}\text { Distance to disturbance } \\
\text { Start/End Time }\end{array}$ & $\begin{array}{c}96.4 \\
00 / 9: 21\end{array}$ & in \\
\hline
\end{tabular}

Run No. FA-8

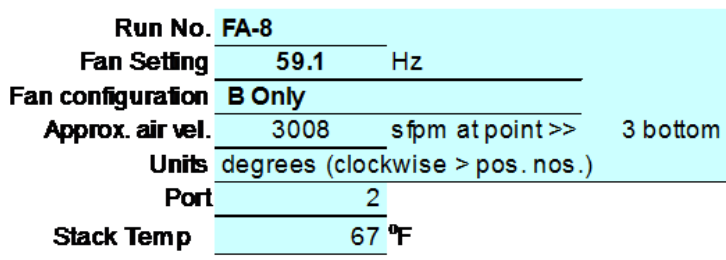

Order $\rightarrow$

Traverse $\rightarrow$

Trial $\longrightarrow$

Stack Temp 2nd

\begin{tabular}{|c|r|c|c|c|}
\hline Point & Depth, in. & deg. cw & deg. cw & deg. cw \\
\hline $\mathbf{1}$ & 0.50 & -20 & -21 & -19 \\
\hline $\mathbf{2}$ & 1.25 & -11 & -14 & -13 \\
\hline $\mathbf{3}$ & 2.32 & -9 & -10 & -9 \\
\hline $\mathbf{4}$ & 3.86 & -3 & -4 & -4 \\
\hline Center & 5.97 & 6 & 7 & 6 \\
\hline $\mathbf{5}$ & 8.08 & 12 & 12 & 12 \\
\hline $\mathbf{6}$ & 9.62 & 15 & 14 & 14 \\
\hline $\mathbf{7}$ & 10.68 & 16 & 17 & 16 \\
\hline $\mathbf{8}$ & 11.44 & 16 & 17 & 18 \\
\hline
\end{tabular}

Mean of absolute velues:

- "w/o points bywall:

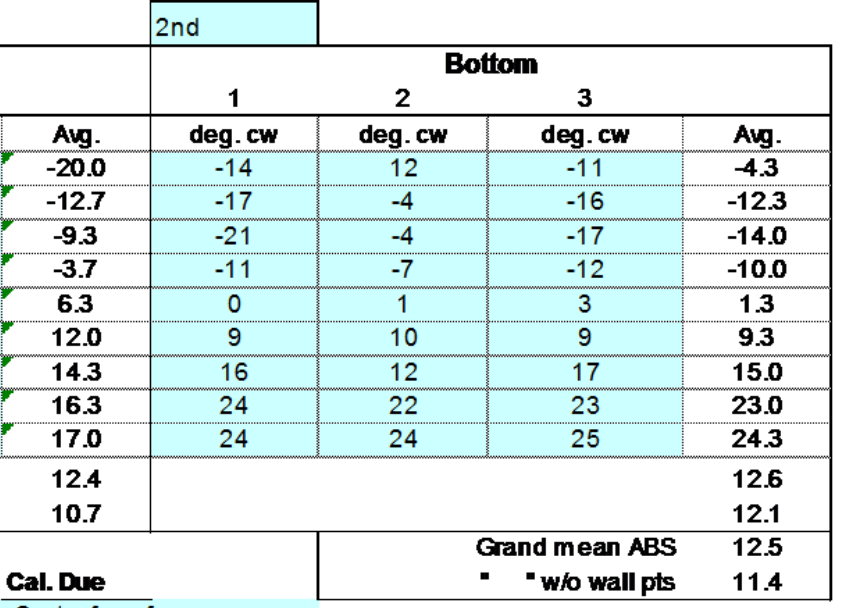

Instuments Used: S-type pitot Velocity sensor Angle indicator Manometer

\begin{tabular}{|c|c|}
\hline & \\
\hline Dwyer 24-inch S-type Pito \#10 & Cert. of conform ance \\
\hline TSI Velocicalc SN\#T95351203001 & 17-Dec-12 \\
\hline Shop built & Cat. 3 \\
\hline Dwyer 400-5, S36N & Cat. 3 \\
\hline
\end{tabular}

Notes: $\quad$ Fan B m ax flow target is 2619 to $3041 \mathrm{fpm}$ at Bottom 3.

Note:

To assure similarhose connections between the manometer and pibt tube, rotaing the pitot tube assem bly clockwise drives the meniscus to the right (to higher pos. numbers). Mostly light winds, occassional gusts, overcast skies.

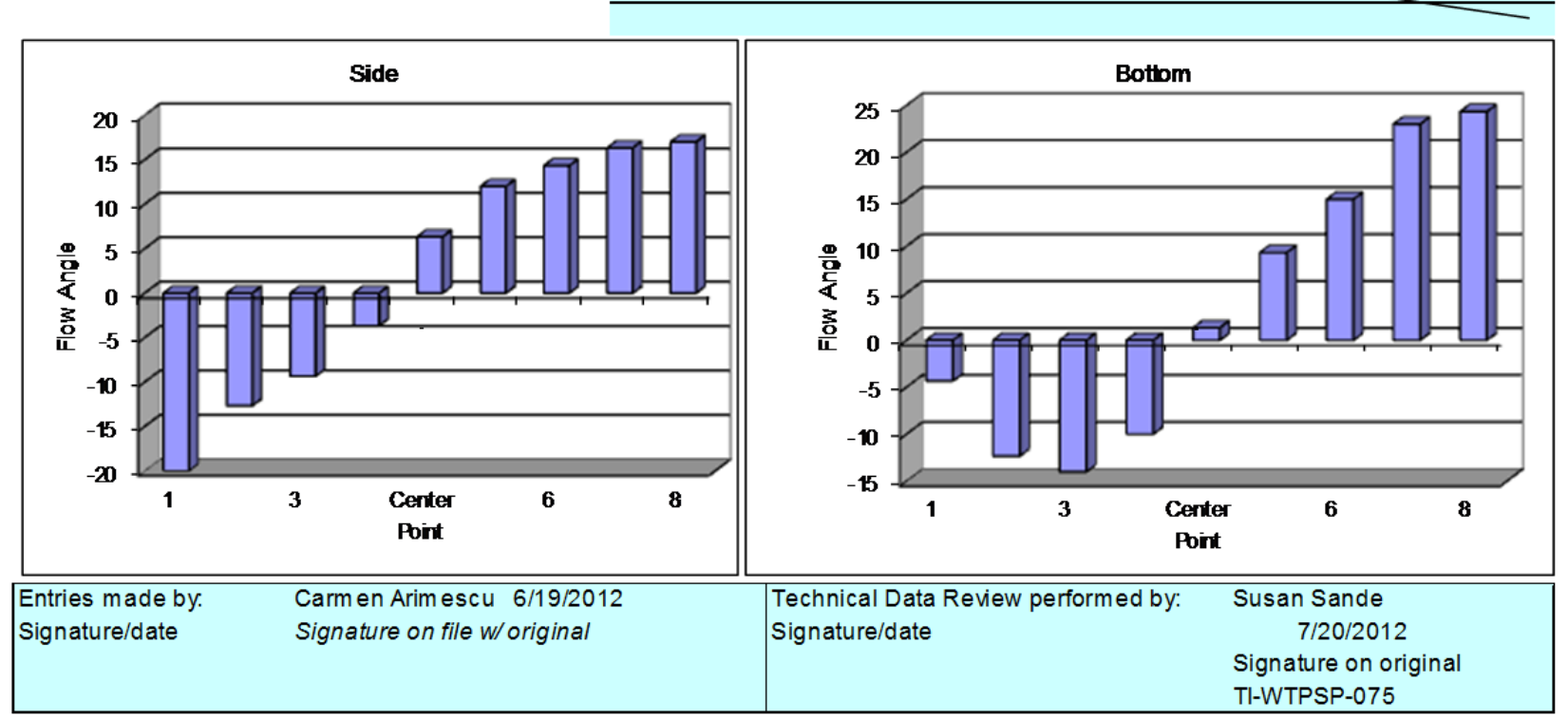




\section{B.4 HV-S2 Gas Tracer Calibration and Uniformity Data Sheets}

\section{SULFUR HEXAFLUORIDE GAS INSTRUMENT CALIBRATION}

$\begin{aligned} & \text { Site } \text { HV-S2 Model } \\ & \text { Date/Time } \underline{4 / 30 / 128: 15} \\ & \text { Testers } \underline{\text { CA, JEF }}\end{aligned}$

Setup:

$7.7 \mathrm{ft} \quad$ B\&K sample inlet tube length

992 mbar station pressure

65 deg F ambient temp analyzer corrects to $20 \operatorname{deg} \mathrm{C}$

45 percent $\mathrm{RH}$ ambient humidity
Instrument < Model 1302

Serial No. 1765299

Property No. WD17210

Pre-Test background, ppb

Not compensating for water vapor, monitoring task 2

$37.0,35.3,32.0,34.2,35.4$

Compensating for water vapor, monitoring task 1

$7.4,4.2,3.2,7.8,5.4$

\section{$100 \mathrm{ppb}$}

Cylinder CAL11936

start $P=$

end $P=$ 1600 psi 1500 psi

$B \& K$

Calibration

readings: (ppb)

Compensating for water vapor

\begin{tabular}{|c|}
\hline 107 \\
\hline 106 \\
\hline 107 \\
\hline 104 \\
\hline 107 \\
\hline
\end{tabular}

Not compensating for water vapor

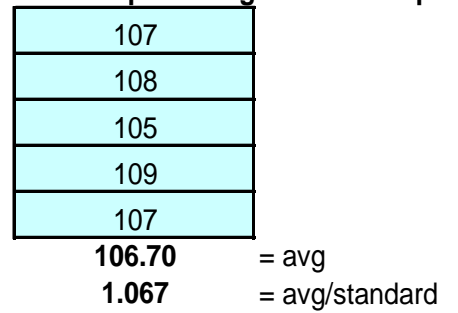

Standards Used:

Air Liquide 0.1 ppm SF6 in air, CAL11936

Air Liquide 4.97 ppm SF6 in air, FF34346

Weather Station Used:

Fisher Scientific S/N 90936818

\begin{tabular}{|c|c|}
\hline & 4.97 \\
\hline \multicolumn{2}{|c|}{ Cylinder FF34346 } \\
\hline start $P=$ & 1490 psi \\
\hline end $P=$ & $1450 \mathrm{psi}$ \\
\hline
\end{tabular}

B\&K

Calibration

readings:

Compensating for water vapor

\begin{tabular}{|l|}
\hline 5.10 \\
\hline 5.09 \\
\hline 5.08 \\
\hline 5.09 \\
\hline 5.08 \\
\hline
\end{tabular}

Not compensating for water vapor

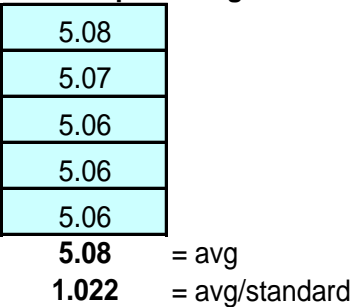

Expiration date:

$3 / 19 / 2013$

$3 / 19 / 2014$

\section{$12 / 7 / 2012$}

Technical Data Review performed by:

5/10/2012 Signature/date
Susan Sande

$7 / 10 / 2012$

Signature on file with Original TI-WTPSP-078 


\section{SULFUR HEXAFLUORIDE GAS INSTRUMENT CALIBRATION}

$\begin{aligned} & \text { Site } \frac{H V-S 2}{} \text { Model } \\ & \text { Date/Time } \text { 5/7/12 8:00 } \\ & \text { Testers } \mathrm{CA}, \mathrm{JEF}\end{aligned}$

Instrument B\&K Model 1302

Serial No. 1765299

Property No. WD17210

Setup: $\quad 7.7 \mathrm{ft} \quad$ B\&K sample inlet tube length

1016 mbar station pressure

58 deg F ambient temp analyzer corrects to 20 deg C

34 percent $\mathrm{RH}$ ambient humidity

Pre-Test background, ppb
Not compensating for water vapor, monitoring task 2

Not compensating for water

Compensating for water vapor, monitoring task 1

$5.23,3.36,5.69,6.09,9.12$

\section{$100 \mathrm{ppb}$}

Cylinder CAL11936

\begin{tabular}{r|r} 
start $P=$ & 1400 psi \\
end $P=$ & 1400 psi
\end{tabular}

B\&K

Calibration

readings: (ppb)

Compensating for water vapor

\begin{tabular}{|c|}
\hline 108 \\
\hline 109 \\
\hline 107 \\
\hline 107 \\
\hline 111 \\
\hline
\end{tabular}

Not compensating for water vapor

\begin{tabular}{|c|}
\hline 107 \\
\hline 105 \\
\hline 108 \\
\hline 105 \\
\hline 107 \\
\hline 107.40 \\
\hline 1.074 \\
\hline
\end{tabular}

Standards Used:

Air Liquide 0.1 ppm SF6 in air, CAL11936

Air Liquide 4.97 ppm SF6 in air, FF34346

Weather Station Used:

Fisher Scientific S/N 90936818

\begin{tabular}{ll}
\hline Entries made by: & Julia Flaherty \\
Signature/date & On File w/ Original
\end{tabular}

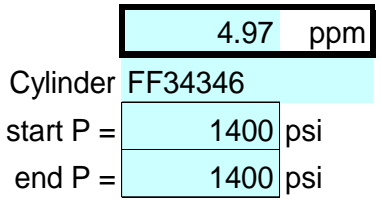

B\&K

Calibration readings: (ppm)

Compensating for water vapor

\begin{tabular}{|c|}
\hline 5.12 \\
\hline 5.13 \\
\hline 5.20 \\
\hline 5.21 \\
\hline 5.21 \\
\hline
\end{tabular}

Not compensating for water vapor

\begin{tabular}{|c|c|}
\hline 5.19 & \\
\hline 5.18 & \\
\hline 5.19 & \\
\hline 5.18 & \\
\hline 5.15 & \\
\hline 5.18 & \\
\hline 1.041 & \\
\hline
\end{tabular}

Expiration date: $3 / 19 / 2013$ $3 / 19 / 2014$ $12 / 7 / 2012$ 


\section{SULFUR HEXAFLUORIDE GAS INSTRUMENT CALIBRATION}

$\begin{aligned} \text { Site } & \frac{H V-S 2 ~ M o d e l}{5 / 10 / 12 ~ 13: 45} \\ \text { Date/Time } & \text { Testers }\end{aligned}$

Instrument B\&K Model 1302

Serial No. 1765299

Property No. WD17210

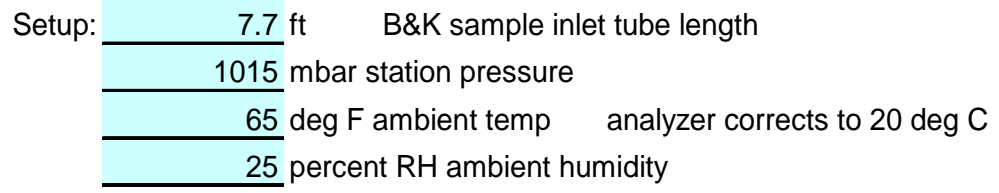

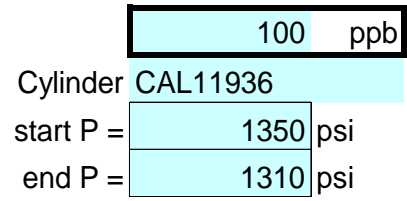

B\&K

Calibration

readings: (ppm)

Compensating for water vapor

\begin{tabular}{|c|}
\hline 103 \\
\hline 103 \\
\hline 105 \\
\hline 105 \\
\hline 104 \\
\hline
\end{tabular}

Not compensating for water vapor

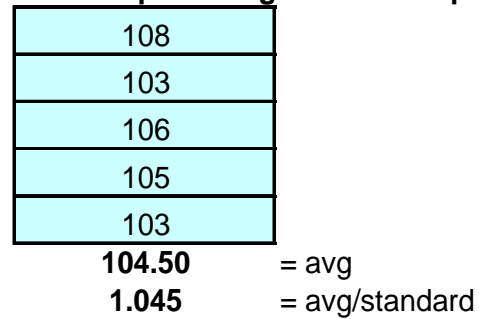

Standards Used:

Air Liquide 0.1 ppm SF6 in air, CAL11936

Air Liquide 4.97 ppm SF6 in air, FF34346

Weather Station Used:

Fisher Scientific S/N 90936818

\begin{tabular}{|ll}
\hline Entries made by: & Julia Flaherty \\
Signature/date & On File w/ Original
\end{tabular}

\begin{tabular}{|c|c|}
\hline & 4.97 \\
\hline linder & FF34346 \\
\hline start $P=$ & 1400 \\
\hline end $P=$ & 1390 \\
\hline
\end{tabular}

B\&K

Calibration

readings: (ppm)

Compensating for water vapor

\begin{tabular}{|c|}
\hline 4.98 \\
\hline 4.90 \\
\hline 5.03 \\
\hline 5.03 \\
\hline 5.02 \\
\hline
\end{tabular}

Not compensating for water vapor

\begin{tabular}{|c|}
\hline 5.02 \\
\hline 4.95 \\
\hline 5.03 \\
\hline 4.96 \\
\hline 4.96 \\
\hline 4.99 \\
\hline 1.004 \\
\hline
\end{tabular}

Expiration date: $3 / 19 / 2013$ $3 / 19 / 2014$ 


\section{SULFUR HEXAFLUORIDE GAS INSTRUMENT CALIBRATION}

\begin{aligned} & Site $\frac{H V-S 2 \text { Model }}{\text { Date/Time }} \\ &$ Testers 6/19/12 1025 \\ & \hline CAEF \end{aligned}

$\begin{aligned} & \text { Instrument } \text { B\&K Model } 1302 \\ & \text { Serial No. } 1765299 \\ & \text { Property No. WD17210 }\end{aligned}$
Setup: $\quad 7.7 \mathrm{ft} \quad$ B\&K sample inlet tube length 996 mbar station pressure
65 deg F ambient temp analyzer corrects to 20 deg C
28 percent $\mathrm{RH}$ ambient humidity

Pre-Test background, ppb
Not compensating for water vapor, monitoring task 2
$30,27,28,29,29$
Compensating for water vapor, monitoring task 1
$8.3,6.6,4.3,5.3,6.1$

\section{$100 \mathrm{ppb}$}

Cylinder CAL11936

$\begin{aligned} \text { start } P & =1300 \text { psi } \\ \text { end } P & =1250 \text { psi }\end{aligned}$

B\&K

Calibration

readings: (ppm)

Compensating for water vapor

\begin{tabular}{|c|}
\hline 102 \\
\hline 103 \\
\hline 104 \\
\hline 102 \\
\hline 102 \\
\hline
\end{tabular}

Not compensating for water vapor

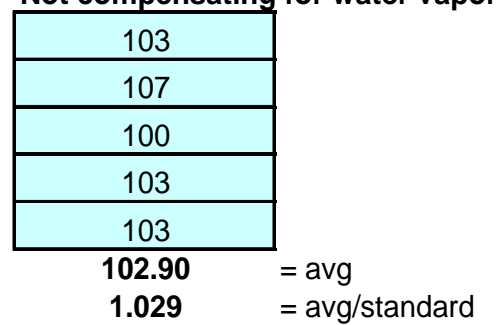

Standards Used:

Air Liquide 0.1 ppm SF6 in air, CAL11936

Air Liquide 4.97 ppm SF6 in air, FF34346

Weather Station Used:

Fisher Scientific S/N 90936818

$12 / 7 / 2012$
B\&K

Calibration

readings: (ppm)

Compensating for water vapor

\begin{tabular}{|l|}
\hline 4.90 \\
\hline 4.89 \\
\hline 4.89 \\
\hline 4.89 \\
\hline 4.89 \\
\hline
\end{tabular}

Not compensating for water vapor

\begin{tabular}{|c|c|}
\hline 4.90 & \\
\hline 4.90 & \\
\hline 4.90 & \\
\hline 4.89 & \\
\hline 4.90 & \\
\hline 4.90 & \\
\hline 0.985 & \\
\hline
\end{tabular}

Expiration date:

$3 / 19 / 2013$

$3 / 19 / 2014$

Technical Data Review performed by: Susan Sande
Signature/date $\quad 7 / 10 / 2012$

Signature on file with Original

Signature on file with Original TI-WTPSP-078 


\section{SULFUR HEXAFLUORIDE GAS INSTRUMENT CALIBRATION}

$\begin{aligned} \text { Site } & \frac{\mathrm{HV} \text {-S2 Model }}{\text { Date/Time }} \\ \text { Testers } & \underline{\mathrm{CA}, \mathrm{JEF}}\end{aligned}$

Instrument B\&K Model 1302

Serial No. 1765299

Property No. WD17210
Setup: $\quad 7.7 \mathrm{ft} \quad$ B\&K sample inlet tube length 1005 mbar station pressure
72 deg F ambient temp analyzer corrects to 20 deg C
35 percent $\mathrm{RH}$ ambient humidity

Pre-Test background, ppb
Not compensating for water vapor, monitoring task 2

$38.4,36.1,34.3,35.5,33.8$

Compensating for water vapor, monitoring task 1

2.6, 1.6, 2.8, 1.83, 4.05

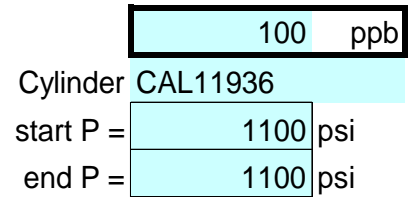

B\&K

Calibration

readings: (ppm)

Compensating for water vapor

\begin{tabular}{|c|}
\hline 100 \\
\hline 95 \\
\hline 99 \\
\hline 99 \\
\hline 101 \\
\hline
\end{tabular}

Not compensating for water vapor

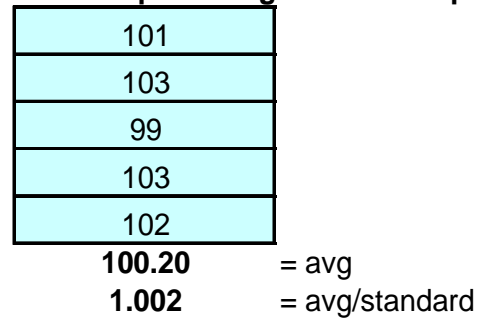

Standards Used:

Air Liquide 0.1 ppm SF6 in air, CAL11936

Air Liquide 4.97 ppm SF6 in air, FF34346

Weather Station Used:

Fisher Scientific S/N 90936818

\begin{tabular}{|ll}
\hline $\begin{array}{l}\text { Entries made by: } \\
\text { Signature/date }\end{array}$ & Julia Flaherty \\
& Signature on file with Original
\end{tabular}

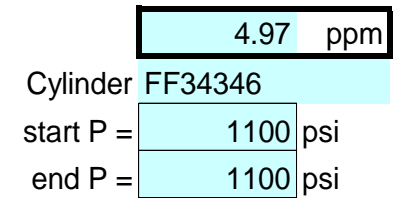

B\&K

Calibration

readings: (ppm)

Compensating for water vapor

\begin{tabular}{|l|}
\hline 4.86 \\
\hline 4.86 \\
\hline 4.86 \\
\hline 4.87 \\
\hline 4.87 \\
\hline
\end{tabular}

Not compensating for water vapor

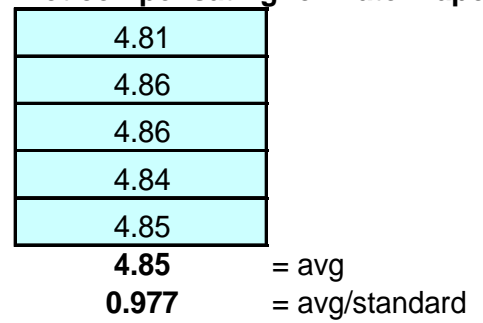

Expiration date:

$3 / 19 / 2013$

$3 / 19 / 2014$ 


\section{SULFUR HEXAFLUORIDE GAS INSTRUMENT CALIBRATION}

$\begin{aligned} \text { Site } & \frac{\mathrm{HV} \text {-S2 Model }}{\text { Date/Time }} \\ \text { Testers } & \underline{\mathrm{CA}, \mathrm{JEF}}\end{aligned}$

Instrument B\&K Model 1302

Serial No. 1765299

Property No. WD17210

Setup:

$7.7 \mathrm{ft} \quad$ B\&K sample inlet tube length

1005 mbar station pressure

72 deg F ambient temp analyzer corrects to 20 deg C

35 percent $\mathrm{RH}$ ambient humidity

Pre-Test background, ppb
Not compensating for water vapor, monitoring task 2

$38.4,36.1,34.3,35.5,33.8$

Compensating for water vapor, monitoring task 1

$2.6,1.6,2.8,1.83,4.05$

$100 \mathrm{ppb}$

Cylinder CAL11936

$\begin{array}{rr}\text { start } P= & 1100 \text { psi } \\ \text { end } P= & 1100 \text { psi }\end{array}$

B\&K

Calibration

readings: (ppm)

Compensating for water vapor

\begin{tabular}{|c|}
\hline 100 \\
\hline 95 \\
\hline 99 \\
\hline 99 \\
\hline 101 \\
\hline
\end{tabular}

Not compensating for water vapor

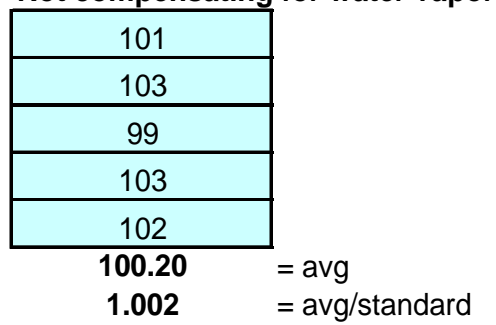

Standards Used:

Air Liquide 0.1 ppm SF6 in air, CAL11936

Air Liquide 4.97 ppm SF6 in air, FF34346

Weather Station Used:

Fisher Scientific S/N 90936818

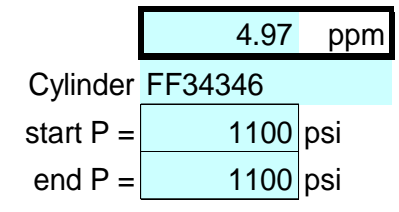

B\&K

Calibration

readings: (ppm)

Compensating for water vapor

\begin{tabular}{|l|}
\hline 4.86 \\
\hline 4.86 \\
\hline 4.86 \\
\hline 4.87 \\
\hline 4.87 \\
\hline
\end{tabular}

Not compensating for water vapor

\begin{tabular}{|c|}
\hline 4.81 \\
\hline 4.86 \\
\hline 4.86 \\
\hline 4.84 \\
\hline 4.85 \\
\hline 4.85 \\
\hline 0.977 \\
\hline
\end{tabular}

Expiration date:

$3 / 19 / 2013$

$3 / 19 / 2014$

\section{$12 / 7 / 2012$}

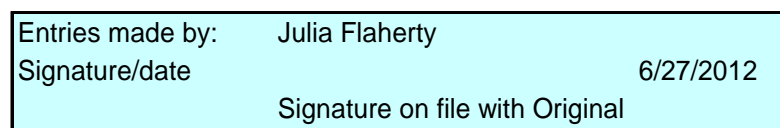

Technical Data Review performed by: Susan Sande

Signature/date 7/10/2012

Signature on file with Original TI-WTPSP-078 
Rev. 0

31-Jul06

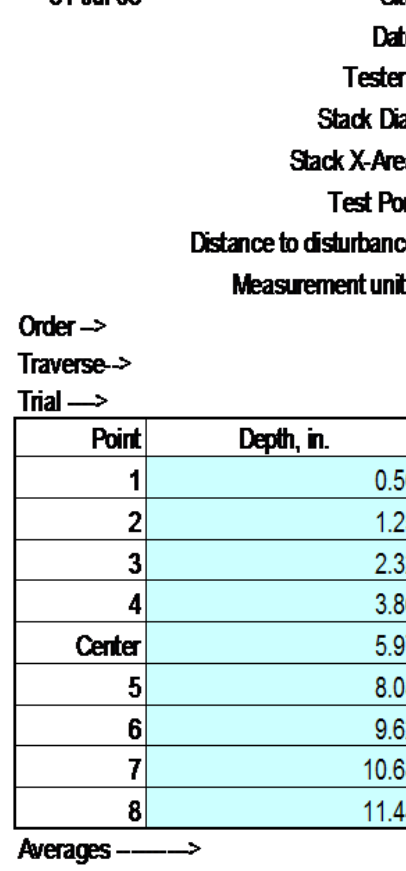

Avg. Conc.

$657.08 \mathrm{ppb}$

Tracer tank pressure Injection flometer

Stack Temp

Mean stack velocity

Samping flometer

Ambient pressure

Ambient humidty

Ambient Temp

B\&Kvapor correction

Back-Gd gas

No. Bk-Gd samples

\begin{tabular}{|c|c|c|}
\hline Stat & Frish & \\
\hline 10 & 10 & psig \\
\hline 15 & 15 & $\mathrm{sccm}$ \\
\hline 58.8 & 62.8 & ${ }^{\circ} \mathrm{F}$ \\
\hline 1072 & 1095 & sfpm \\
\hline 5 & 5 & pm \\
\hline 1002 & 1002 & mbar \\
\hline $35 \%$ & $28 \%$ & RH \\
\hline 57.2 & 72.5 & ${ }^{\circ} \mathrm{F}$ \\
\hline$Y$ & $Y$ & YN \\
\hline $6,5,7,6,13$ & $\begin{array}{c}11,6,13,10,1 \\
1\end{array}$ & ppb \\
\hline 5 & 5 & n \\
\hline
\end{tabular}

Gas anabzer checked

4/30/2012

CA 5/1/12

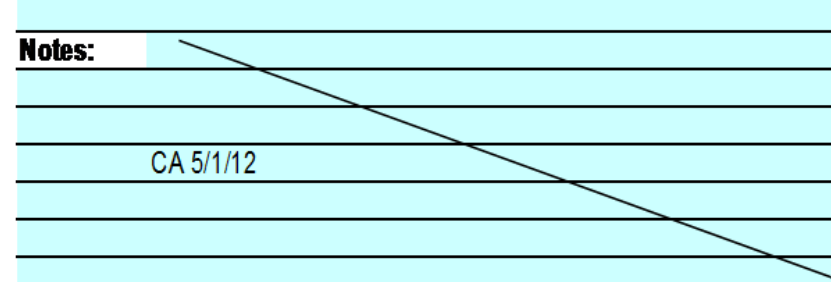

Entries made by:

Signature/date

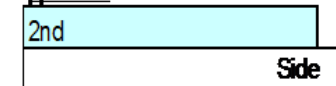

TRACER GAS TRAVERSE DATA FORU

Run No. GT-1

Fan Configuration Fan A

Fan Selting 29

Stack Temp

Hz

StartiEnd Time $827 / 1000$

Center 2/3 from

Points in Center 23

Injection Point E -Center

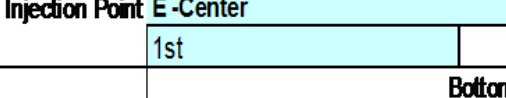

\begin{tabular}{|c|c|c|c|c|}
\hline Mean & 1 & 2 & 3 & Mean \\
\hline & \multicolumn{4}{|c|}{ ppb } \\
\hline 7367 & 628 & 710 & 711 & 683.0 \\
\hline 690.3 & 666 & 709 & 691 & 688.7 \\
\hline 685.0 & 665 & 669 & 676 & 670.0 \\
\hline 640.0 & 661 & 644 & 599 & 634.7 \\
\hline 6487 & 650 & 629 & 591 & 623.3 \\
\hline 621.3 & 655 & 584 & 605 & 614.7 \\
\hline 6340 & 631 & 624 & 672 & 6423 \\
\hline 6267 & 675 & 631 & 651 & 6523 \\
\hline 610.7 & 727 & 618 & 704 & 683.0 \\
\hline 654.8 & 6620 & 6464 & 655.6 & 654.7 \\
\hline
\end{tabular}

\begin{tabular}{|lrr|lrrr|}
\hline All & ppb & Dev. from mean & Certer 2/3 & Side & Bottom & All \\
Mean & 654.7 & & Mean & 649.4 & 646.6 & 648.0 \\
Min Point & 610.7 & $-67 \%$ & Std Dev. & 27.6 & 26.1 & 25.8 \\
Max Point & 736.7 & $125 \%$ & $\operatorname{Cov~as~\% ~}$ & 4.3 & 4.0 & 4.0 \\
\hline
\end{tabular}

Instuments Usect

$\begin{array}{lll}\text { B\&K } 1302 \text { Gas Analyzer } & \text { SN } 1765299 & \text { Cat2 M\&TE }\end{array}$

\begin{tabular}{ll}
\hline TSI VelociCalc SN T95351203001 & $1 / 17 / 2013$ \\
\hline Fisher Scientific SN 90936818 & $127 / 2012$
\end{tabular}

Fisher Scientific SN 90936818

$12 / 7 / 2012$

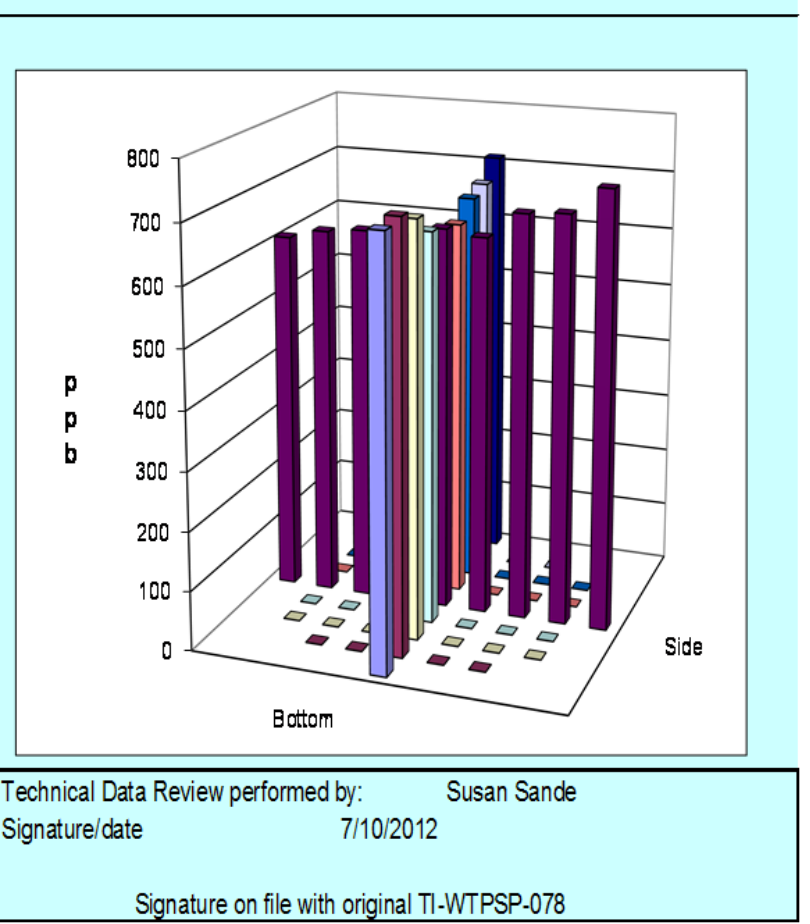


Rev. 0

31-JuL06

\begin{aligned} Site HV-S2 Model & \multicolumn{1}{c}{$\frac{\text { H/1/2012 }}{\text { Date }}$} \\ Testers & XY, CA \\ Stack Dia. & 11.938 in. \\ Stack X-Area & 111.9 in. $^{2} \\$ Test Port & 2\end{aligned}

Distance to disturbance 96.4 inches

Measurement units pob SF6

TRACER GAS TRAVERSE DATA FORI

Order $\rightarrow$

Traverse $\rightarrow$

Trial $\longrightarrow$

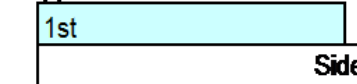

\begin{tabular}{|r|r|}
\hline Point & Depth, in. \\
\hline $\mathbf{1}$ & 0.50 \\
$\mathbf{2}$ & 1.2 \\
\hline $\mathbf{3}$ & 2.32 \\
\hline $\mathbf{4}$ & 3.8 \\
\hline Center & 5.97 \\
\hline $\mathbf{5}$ & 8.08 \\
\hline $\mathbf{6}$ & 9.62 \\
\hline $\mathbf{7}$ & 10.68 \\
\hline $\mathbf{8}$ & 11.44 \\
\hline
\end{tabular}

Averages

11.44

$\begin{array}{lll}1 & 2 & 3\end{array}$

Run No. GT-2

Fan Configuration Fan B only

Fan Setting 26

Stack Temp

Start/End Tme $1013 / 1130$

Center 2/3 from

Points in Center $2 / 3$

Injection Point E -Center

\begin{tabular}{|lr}
\hline$A l l$ & ppb \\
Mean & 728.5 \\
Mn Point & 710.3 \\
Max Point & 759.7 \\
\hline
\end{tabular}

Avg. Conc.

728.38 ppb

Tracer tank pressure Injection flowmeter Stack Temp

Mean stack velocity Sampling flowmeter Ambient pressure Ambient humidity Ambient Temp B\&K vapor correction Back-Gd gas

No. Bk-Gd samples

\begin{tabular}{|c|c|}
\hline Start & Fnish \\
\hline 125 & 125 \\
\hline 15 & 15 \\
\hline 65.8 & 68.6 \\
\hline 1042 & 1087 \\
\hline 5 & 5 \\
\hline 1002 & 1002 \\
\hline $28 \%$ & $24 \%$ \\
\hline 72.5 & 76.1 \\
\hline $\mathrm{Y}$ & $Y$ \\
\hline $\begin{array}{c}9,11,11,10,1 \\
0 \\
\end{array}$ & $3,7,8,10,8$ \\
\hline 5 & 5 \\
\hline
\end{tabular}

Gas anahzer checked:

$4 / 30 / 2012$

CA $5 / 1 / 12$

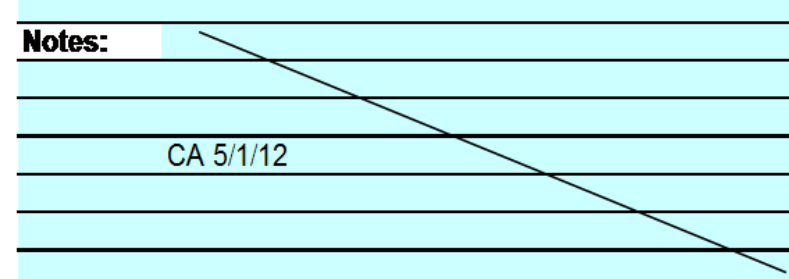

Entries made by: Signature/date

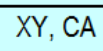

$5 / 1 / 2012$

Signature on file with original

\begin{tabular}{r|l} 
Dev. from mean & Center 2/3 \\
$-2.5 \%$ & Mean \\
$4.3 \%$ & Std. Dev. \\
COV as \%
\end{tabular}

Instuments Used:

B\&K 1302 Gas Analyzer SN 1765299 TSI VelociCalc SN T95351203001

Fisher Scientific SN 90936818

\begin{tabular}{rrr|} 
Side & Bottom & Al \\
728.9 & 730.3 & 729.6 \\
15.4 & 11.8 & 13.2 \\
2.1 & 1.6 & 1.8 \\
\hline
\end{tabular}

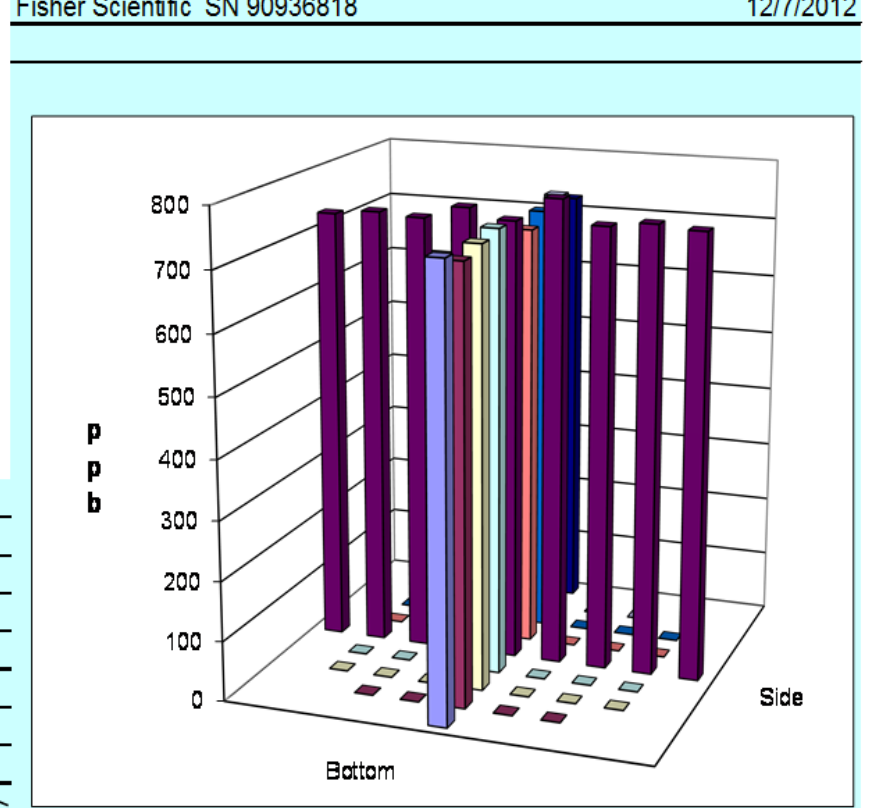

Technical Data Review performed by: Susan Sande Signature/date

7/10/2012 
Rev. 0

31-JuL06

\begin{aligned} Site HV-S2 Model & \multicolumn{1}{c}{$\frac{\text { H/1/2012 }}{\text { Date }}$} \\ Testers & XY, CA \\ Stack Dia. & 11.938 in. \\ Stack X-Area & 111.9 in. $^{2} \\$ Test Port & 2\end{aligned}

Distance to disturbance 96.4 inches

Measurement units pob SF6

TRACER GAS TRAVERSE DATA FORI

Order $\rightarrow$

Traverse $\rightarrow$

Trial $\longrightarrow$

\begin{tabular}{|c|c|}
\hline Point & Depth, in. \\
\hline 1 & 0 \\
\hline 2 & 1 \\
\hline 3 & 2 \\
\hline 4 & 3 \\
\hline Center & 5 \\
\hline 5 & 8 \\
\hline 6 & $\theta$ \\
\hline 7 & 10 \\
\hline 8 & 11 \\
\hline
\end{tabular}

1 st

1st

Run No. GT-3

Fan Configuration Fan B only

Fan Setting 60 Hz

Stack Temp

$65.5 \operatorname{deg} F$

Start/End Tme 11:43/1300

Center $2 / 3$ from

Points in Center 2/3

1.10 to: 10.84

Injection Point E -Center

Averages

11.44

\begin{tabular}{l|l}
\hline 50 & 634 \\
\hline 25 & 6 \\
\hline 32 & 6 \\
\hline 86 & 635 \\
\hline 97 & 6 \\
\hline 08 & 6 \\
\hline 62 & 6 \\
\hline 68 & 6 \\
\hline 44 & 6 \\
\hline
\end{tabular}

638.1

$2 \quad 3$

\begin{tabular}{|c|c|c|c|c|}
\hline \multirow[b]{3}{*}{ Mean } & 2nd & & & \\
\hline & \multicolumn{4}{|c|}{ Bottom } \\
\hline & 1 & 2 & 3 & Mean \\
\hline & \multicolumn{4}{|c|}{ ppb } \\
\hline 647.7 & 633 & 647 & 641 & 640.3 \\
\hline 650.0 & 629 & 615 & 624 & 622.7 \\
\hline 650.3 & 632 & 644 & 642 & 639.3 \\
\hline 659.7 & 646 & 624 & 650 & 640.0 \\
\hline 664.3 & 666 & 631 & 630 & 642.3 \\
\hline 643.0 & 633 & 620 & 632 & 628.3 \\
\hline 632.3 & 633 & 632 & 654 & 639.7 \\
\hline 610.3 & 622 & 643 & 637 & 634.0 \\
\hline 648.0 & 623 & 638 & 616 & 625.7 \\
\hline 645.1 & 635.2 & 632.7 & 636.2 & 634.7 \\
\hline
\end{tabular}

\begin{tabular}{|lr}
\hline $\boldsymbol{A l l}$ & $\mathrm{ppb}$ \\
Mean & 639.9 \\
Min Point & 610.3 \\
Max Point & 664.3 \\
\hline
\end{tabular}

Avg. Conc

$638.21 \mathrm{ppb}$

Tracer tank pressure Injection flowmeter Stack Temp

Mean stack velocity Sampling flowmeter Ambient pressure Ambient humidity Ambient Temp B\&K vapor correction Back-Gd gas

No. Bk-Gd samples

\begin{tabular}{|c|c|}
\hline Start & Finish \\
\hline 125 & 125 \\
\hline 30 & 30 \\
\hline 66.5 & 64.5 \\
\hline 2615 & 2686 \\
\hline 5 & 5 \\
\hline 1002 & 1001 \\
\hline $26 \%$ & $27 \%$ \\
\hline 69.8 & 67.1 \\
\hline$Y$ & $Y$ \\
\hline $9,6,6,7,10$ & $10,10,6,9,7$ \\
\hline 5 & 5 \\
\hline
\end{tabular}

Gas analyzer checked:

$4 / 30 / 2012$

CA $5 / 1 / 12$

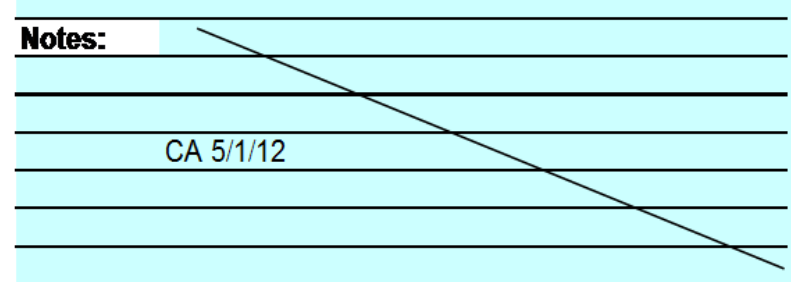

Entries made by:

Signature/date

\section{Dev. from mean Center 2/3 \\ Mean \\ $-4.6 \%$ Std. Dev. \\ $3.8 \%$ COV as \%}

Instuments Used:

B\&K 1302 Gas Analyzer S

TSI VelociCalc SN T95351203001

Fisher Scientific SN 90936818

\begin{tabular}{|c|c|c|}
\hline Side & Bottom & A \\
\hline 644.3 & 635.2 & 639.7 \\
\hline 18.3 & 7.3 & 14.2 \\
\hline 2.8 & 1.1 & 2.2 \\
\hline
\end{tabular}

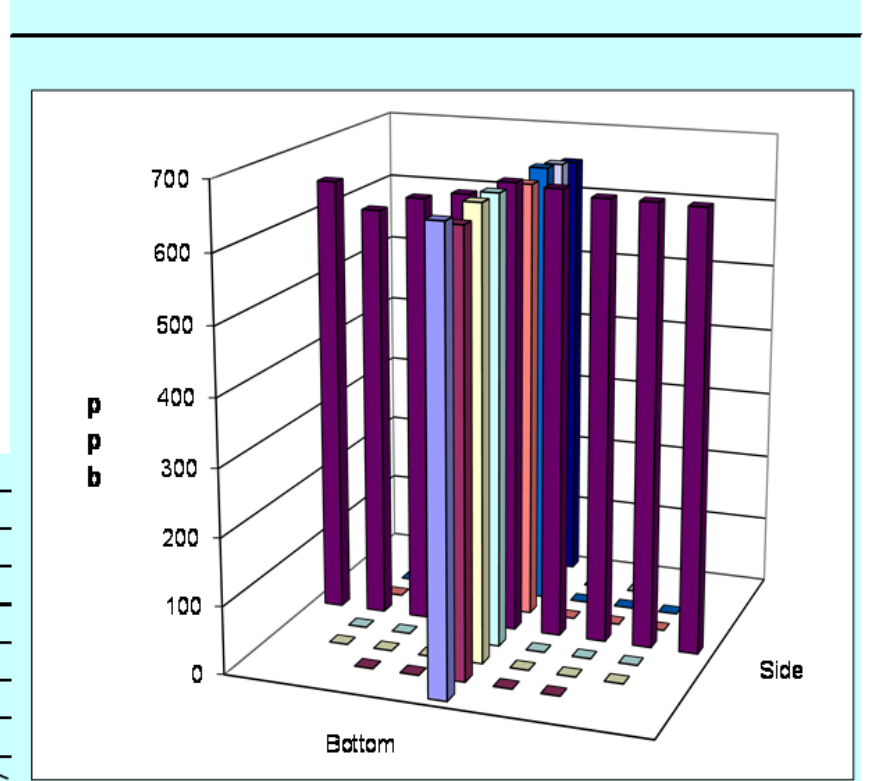

Technical Data Review performed by: Susan Sande Signature/date $7 / 10 / 2012$

Signature on file with original TI-WTPSP-078 
Rev. 0

31-JuL06

$\begin{aligned} & \text { Site HV-S2 Model } \\ & \text { Date } 5 / 1 / 2012 \\ & \text { Testers } \text { EA, JAG } \\ & \text { Stack Dia. } 11.938 \text { in. } \\ & \text { Stack X-Area } 111.9 \text { in. }^{2} \\ & \text { Test Port } 2\end{aligned}$

Distance to disturbance 96.4 inches

Measurement units pob SF6

TRACER GAS TRAVERSE DATA FORI

Order $\rightarrow$

Traverse $\rightarrow$

Trial $\longrightarrow$

\begin{tabular}{|c|c|}
\hline Point & Depth, in. \\
\hline 1 & 0 \\
\hline 2 & 1 \\
\hline 3 & 2 \\
\hline 4 & 3 \\
\hline Center & 5 \\
\hline 5 & 8 \\
\hline 6 & 9 \\
\hline 7 & 10 \\
\hline 8 & 11 \\
\hline
\end{tabular}

2nd

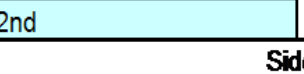

Run No. GT-4

Fan Configuration Fan B only

Fan Seting 60

Stack Temp

Hz

Start/End Tme 1300 / 1418

Center $2 / 3$ from

$66.95 \operatorname{deg} F$

Points in Center 2/3

1.10 to: 10.84

Injection Point Port E, Bottom

2

to: 7

\begin{tabular}{|c|c|c|c|c|}
\hline & $1 \mathrm{st}$ & & & \\
\hline \multirow{3}{*}{ Mean } & \multicolumn{4}{|c|}{ Bottom } \\
\hline & 1 & 2 & 3 & Mean \\
\hline & \multicolumn{4}{|c|}{ ppb } \\
\hline 627.0 & 651 & 640 & 606 & 632.3 \\
\hline 620.3 & 602 & 563 & 603 & 589.3 \\
\hline 592.7 & 619 & 659 & 609 & 629.0 \\
\hline 636.0 & 604 & 659 & 664 & 642.3 \\
\hline 620.7 & 662 & 677 & 598 & 645.7 \\
\hline 584.7 & 653 & 581 & 624 & 619.3 \\
\hline 653.7 & 615 & 627 & 608 & 616.7 \\
\hline 610.3 & 634 & 598 & 577 & 603.0 \\
\hline 601.7 & 634 & 629 & 711 & 658.0 \\
\hline 316.3 & 630.4 & 62 & 622.2 & 6262 \\
\hline
\end{tabular}

\begin{tabular}{|c|c|c|c|}
\hline \multirow{3}{*}{ Avg. Conc. } & \begin{tabular}{|l}
$A I I$ \\
Mean \\
Min Point \\
Max Point \\
\end{tabular} & $\begin{array}{r}\mathrm{ppb} \\
621.3 \\
584.7 \\
658.0\end{array}$ & \\
\hline & \multicolumn{3}{|c|}{$619.77 \mathrm{ppb}$} \\
\hline & Start & Fnish & \\
\hline \multirow{5}{*}{$\begin{array}{l}\text { Tracer tank pressure } \\
\text { Injection flowmeter } \\
\text { Stack Temp } \\
\text { Mean stack velocity } \\
\text { Sampling flowmeter }\end{array}$} & 125 & 125 & psig \\
\hline & 30 & 30 & scem \\
\hline & 64.9 & 69 & ${ }^{\circ} \mathrm{F}$ \\
\hline & 2546 & 2599 & sfpm \\
\hline & 5 & 4 & $1 \mathrm{~mm}$ \\
\hline Ambient pressure & 1001 & 1000 & mbar \\
\hline Ambient humidity & $27 \%$ & $26 \%$ & RH \\
\hline Ambient Temp & 65.3 & 67.1 & ${ }^{\circ} \mathrm{F}$ \\
\hline B\&K vapor correction & $Y$ & $Y$ & YIN \\
\hline Back-Gd gas & $9,10,5,8,7$ & $8,7,1,6,2$ & ppb \\
\hline No. Bk-Gd samples & 5 & 5 & \\
\hline
\end{tabular}

Gas analyzer checked:

$4 / 30 / 2012$

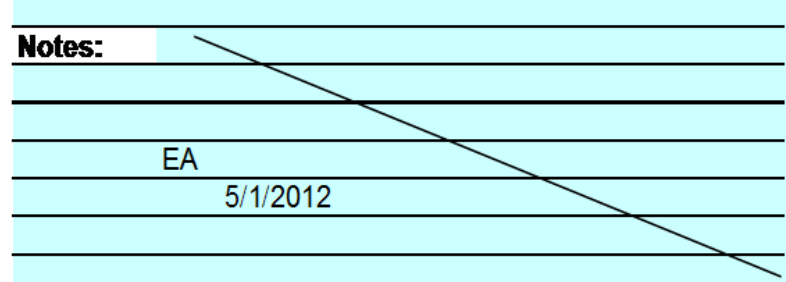

Entries made by:

Signature/date

$$
\text { John Glissmeyer } 5 / 1 / 2012
$$

\begin{tabular}{|c|c|c|c|c|}
\hline Dev. from mean & Center 2/3 & Side & Bottom & Al \\
\hline & Mean & 616.9 & 620.8 & 618.8 \\
\hline$-5.9 \%$ & Std. Dev. & 23.8 & 20.3 & 21.4 \\
\hline $5.9 \%$ & CON as $\%$ & 3.9 & 3.3 & 3.5 \\
\hline
\end{tabular}

Signature on file with original
Instuments Used:

B\&K 1302 Gas Analyzer SN $1765299 \quad$ Cat2 M\&TE TSI VelociCalc SN T95351203001 $1 / 17 / 2013$

Fisher Scientific SN $90936818 \quad 12 / 7 / 2012$

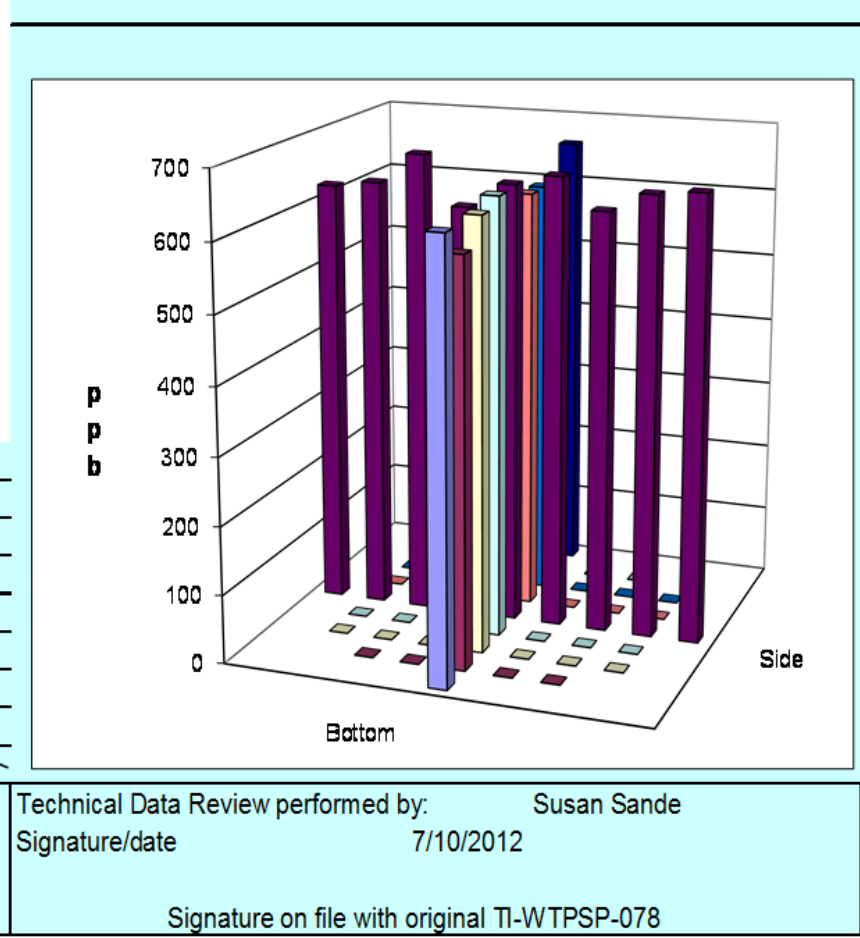


Rev. 0

31-JuL06

\begin{tabular}{r|r} 
& $\begin{array}{r}\text { Date } \\
\text { Testers } \\
\text { Stack Dia } \\
\text { Stack X-Area } \\
\text { Test Por }\end{array}$ \\
Distance to disturbance \\
Measurement units
\end{tabular}

\section{TRACER GAS TRAVERSE DATA FORU}

Site HV-S2 Model

Date $5 / 1 / 2012$

esters EA, JAG

tack Dia. 11.938 in.

$\begin{array}{ll}\text { Test Port } & 111.9 \text { in. }^{2} \\ \end{array}$

pgob SF
Run No. GT-5

Fan Configuration Fan B only

Fan Setting 60

Stack Temp

Hz

Start/End Tme 1419 / 1526

Center $2 / 3$ from

Points in Center $2 / 3$

Injection Point Port E, Top

$70.05 \operatorname{deg} F$

1.10 to: 10.84

2 to: 7

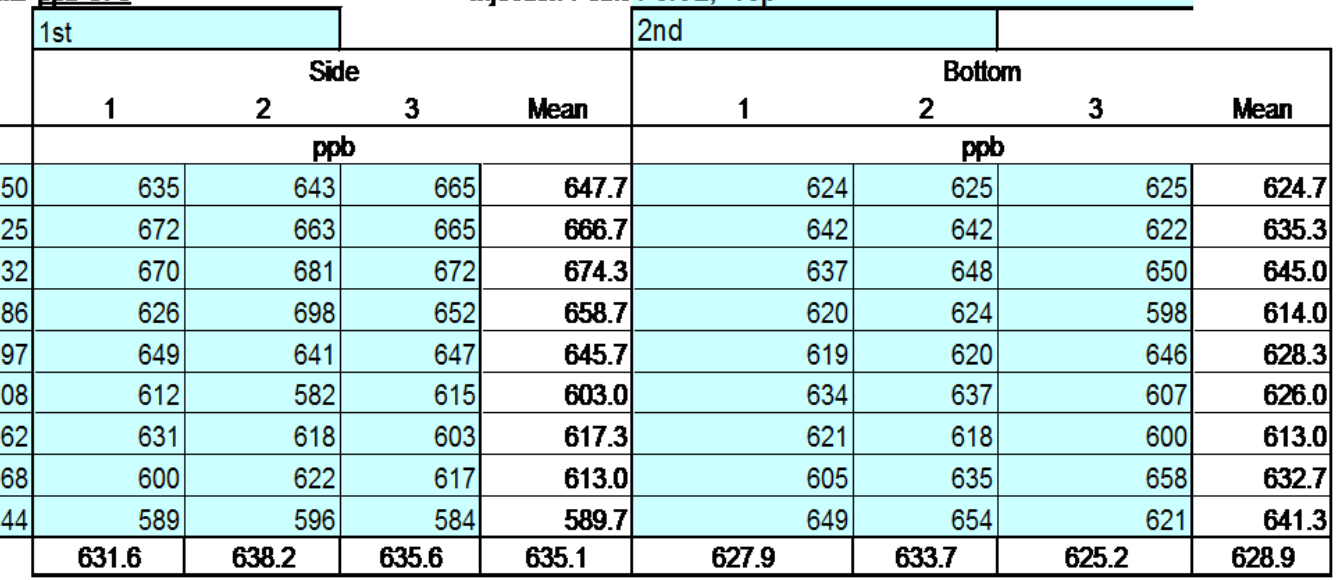

Avg. Conc.

\begin{tabular}{|lr}
\hline$A I I$ & $\mathrm{ppb}$ \\
Mean & 632.0 \\
Min Point & 589.7 \\
Max Point & 674.3 \\
\hline
\end{tabular}

$631.40 \mathrm{ppb}$

Tracer tank pressure Injection flowmeter Stack Temp

Mean stack velocity Sampling flowmeter Ambient pressure Ambient humidity Ambient Temp B\&K vapor correction Back-Gd gas

No. Bk-Gd samples

\begin{tabular}{|c|c|}
\hline Start & Finish \\
\hline 125 & 125 \\
\hline 30 & 30 \\
\hline 69 & 71.1 \\
\hline 2594 & 2609 \\
\hline 5 & 5 \\
\hline 1000 & 999 \\
\hline $26 \%$ & $26 \%$ \\
\hline 67.1 & 67 \\
\hline$Y$ & $Y$ \\
\hline $8,7,1,6,2$ & $2,5,3,8,3$ \\
\hline 5 & 5 \\
\hline
\end{tabular}

Gas analyzer checked: $4 / 30 / 2012$

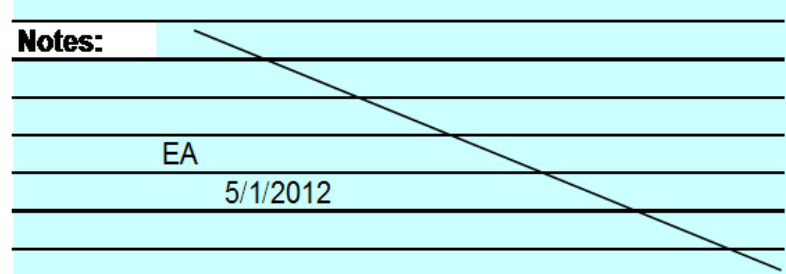
Entries made by: Signature/date

$$
\text { John Glissmeyer }
$$

Signature on file with original

\section{Dev. from mean Center 2/3 \\ Mean \\ $-6.7 \%$ Std. Dev. \\ $6.7 \%$ COV as \%}

\begin{abstract}
Instuments Used:
\end{abstract}
\begin{tabular}{llr} 
B\&K 1302 Gas Analyzer & SN 1765299 & Cat2 M\&TE \\
\hline TSIV &
\end{tabular} TSI VelociCalc SN T95351203001 $1 / 17 / 2013$

Fisher Scientific SN 90936818

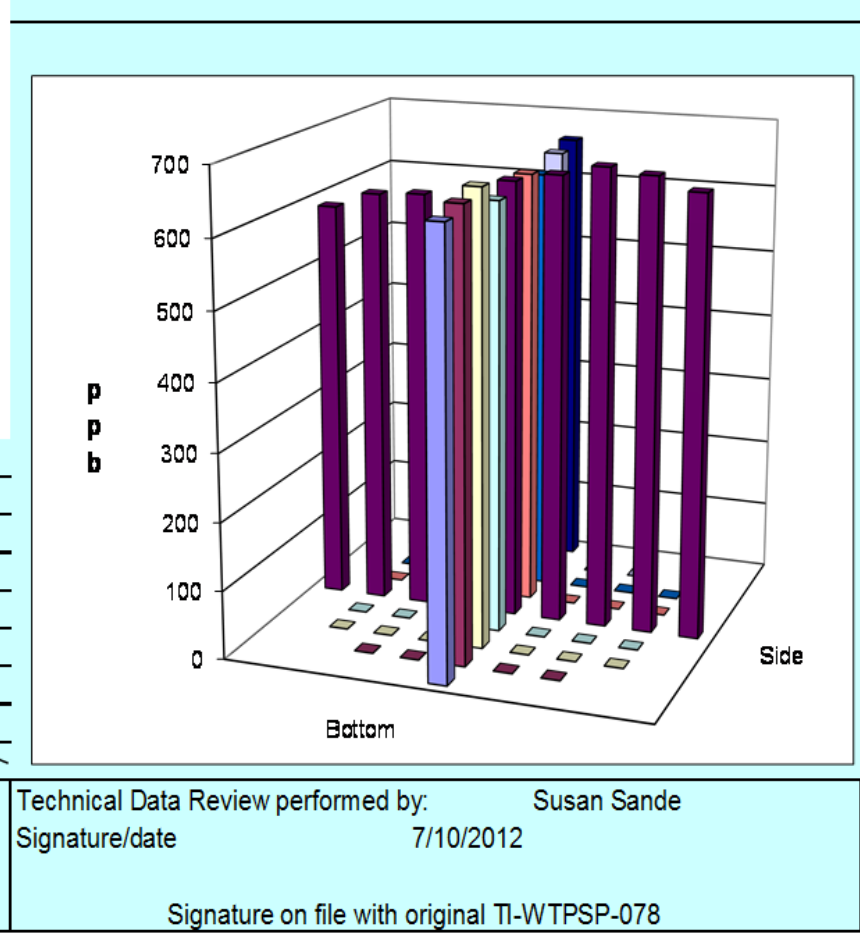


Rev. 0

31-JuL06

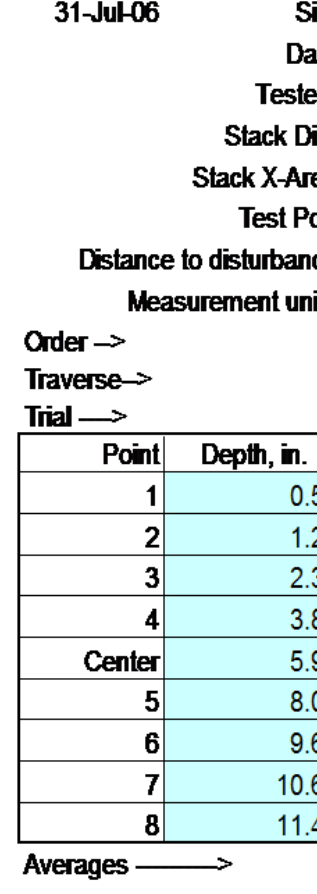

TRACER GAS TRAVERSE DATA FORI

Site HV-S2 Model

Date $5 / 2 / 2012$

Testers CA, JEF, XY

Stack Dia. 11.938 in.

\begin{tabular}{l} 
Test Port 111.9 in. $^{2}$ \\
\hline
\end{tabular}

pob SF6

Run No. GT-6

Fan Configuration Fan B only

Fan Setting 60

Stack Temp Hz

Start/End Tme $830 / 955$

Center $2 / 3$ from

Points in Center $2 / 3$

Injection Point Port E, Near

\begin{tabular}{|c|c|c|c|c|c|c|c|c|}
\hline \multicolumn{5}{|c|}{ 2nd } & \multicolumn{4}{|l|}{$1 \mathrm{st}$} \\
\hline & \multicolumn{4}{|c|}{ Side } & \multicolumn{4}{|c|}{ Bottom } \\
\hline & 1 & 2 & 3 & Mean & 1 & 2 & 3 & Mean \\
\hline & \multicolumn{4}{|c|}{ ppb } & \multicolumn{4}{|c|}{ ppb } \\
\hline & 593 & 604 & 590 & 595.7 & 637 & 617 & 615 & 623.0 \\
\hline & 596 & 609 & 572 & 592.3 & 603 & 606 & 631 & 613.3 \\
\hline & 574 & 597 & 590 & 587.0 & 614 & 610 & 612 & 612.0 \\
\hline & 575 & 590 & 598 & 587.7 & 616 & 603 & 634 & 617.7 \\
\hline & 568 & 586 & 594 & 582.7 & 611 & 608 & 588 & 602.3 \\
\hline & 616 & 635 & 623 & 624.7 & 599 & 593 & 606 & 599.3 \\
\hline & 645 & 615 & 623 & 627.7 & 609 & 618 & 609 & 612.0 \\
\hline 8 & 654 & 646 & 627 & 642.3 & 617 & 603 & 610 & 610.0 \\
\hline & 641 & 648 & 653 & 647.3 & 617 & 589 & 608 & 604.7 \\
\hline & 06.9 & 614.4 & 607.8 & 609.7 & 613.7 & 6052 & 612.6 & 610.5 \\
\hline
\end{tabular}

\begin{tabular}{|lrr|lrrr|}
\hline All & $\mathrm{ppb}$ & Dev. from mean & Center 2/3 & Side & Bottom & Al \\
Mean & 610.1 & & Mean & 606.3 & 609.5 & 607.9 \\
Mn Point & 582.7 & $-4.5 \%$ & Std. Dev. & 24.4 & 6.4 & 17.2 \\
Max Point & 647.3 & $6.1 \%$ & COV as $\%$ & 4.0 & 1.1 & 2.8 \\
\hline
\end{tabular}

Avg. Conc.

Instuments Used:

\begin{tabular}{llr} 
B\&K 1302 Gas Analyzer SN 1765299 & Cat2 M\&TE \\
\hline
\end{tabular}

Tracer tank pressure Injection flowmeter Stack Temp

Mean stack velocity Sampling flowmeter Ambient pressure Ambient humidity Ambient Temp B\&K vapor correction Back-Gd gas

No. Bk-Gd samples

\begin{tabular}{|c|c|}
\hline Start & Finish \\
\hline 50 & 50 \\
\hline 30 & 30 \\
\hline 55.5 & 60.5 \\
\hline 2641 & 2679 \\
\hline 5 & 5 \\
\hline 1003 & 1003 \\
\hline $37 \%$ & $37 \%$ \\
\hline 55.4 & 55.4 \\
\hline$Y$ & $Y$ \\
\hline $3,1,3,3,2$ & $9,9,12,9,11$ \\
\hline 5 & 5 \\
\hline
\end{tabular}

Gas anahzer checked: $4 / 30 / 2012$ CA $5 / 2 / 12$

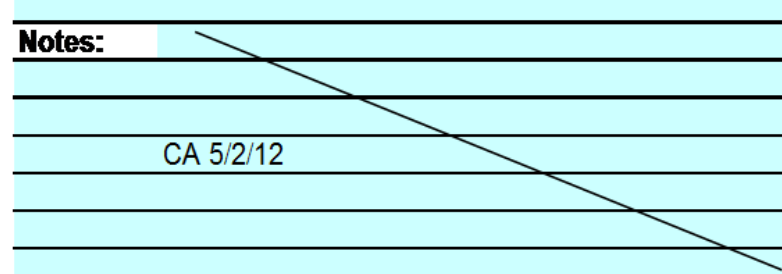
TSI VelociCalc SN T95351203001 Fisher Scientific SN 90936818 $1 / 17 / 2013$

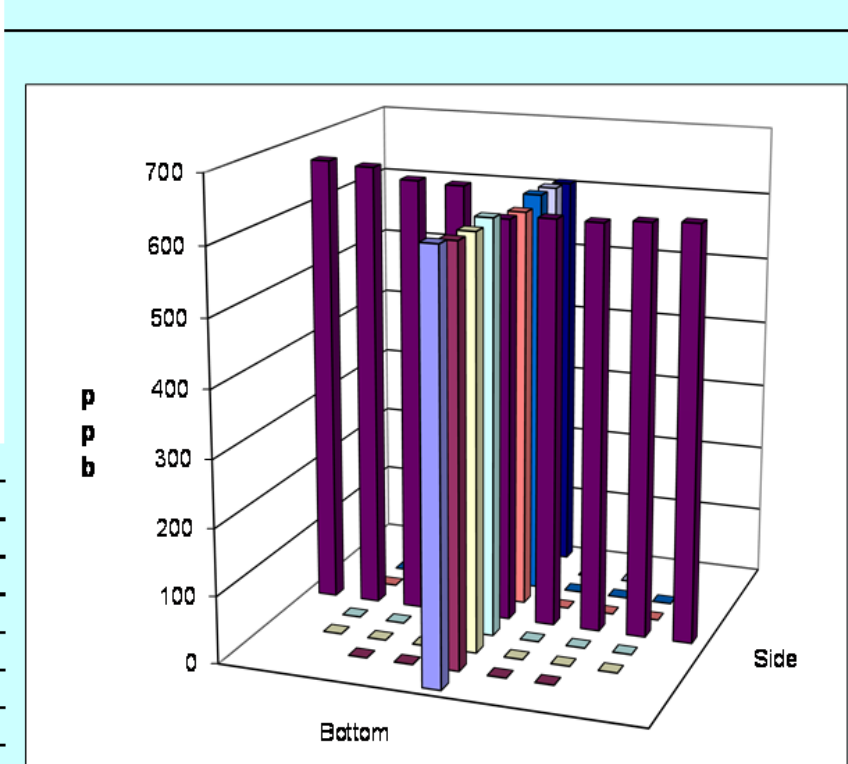

Entries made by: $5 / 2 / 2012$

Technical Data Review performed by: Signature/date 7/10/2012

Signature on file with original 
Rev. 0

31-JuL06

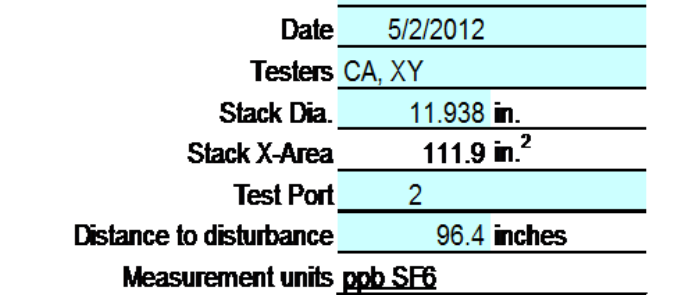

\section{TRACER GAS TRAVERSE DATA FORU}

\begin{tabular}{|c|c|c|c|c|c|c|c|c|c|}
\hline \multirow{4}{*}{$\begin{array}{l}\text { Mea } \\
\text { Order } \rightarrow \\
\text { Traverse } \rightarrow \\
\text { Trial } \longrightarrow\end{array}$} & Urement unis & 00050 & & & \multirow{4}{*}{ Mean } \\
\hline & & 1st & & & & 2nd & & & \\
\hline & & \multicolumn{4}{|c|}{ Side } & \multicolumn{3}{|c|}{ 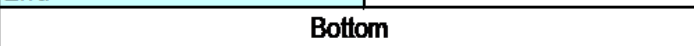 } & \\
\hline & & 1 & 2 & 3 & Mean & 1 & 2 & 3 & \\
\hline Point & Depth, in. & \multicolumn{4}{|c|}{ ppb } & \multicolumn{4}{|c|}{ ppb } \\
\hline 1 & 0.50 & 564 & 693 & 625 & 627.3 & 644 & 619 & 639 & 634.0 \\
\hline 2 & 1.25 & 604 & 608 & 605 & 605.7 & 603 & 616 & 606 & 608.3 \\
\hline 3 & 2.32 & 591 & 620 & 636 & 615.7 & 658 & 658 & 669 & 661.7 \\
\hline 4 & 3.86 & 617 & 651 & 636 & 634.7 & 639 & 592 & 623 & 618.0 \\
\hline Center & 5.97 & 596 & 653 & 604 & 617.7 & 651 & 623 & 659 & 644.3 \\
\hline 5 & 8.08 & 595 & 587 & 578 & 586.7 & 645 & 602 & 633 & 626.7 \\
\hline 6 & 9.62 & 607 & 613 & 619 & 613.0 & 602 & 633 & 599 & 611.3 \\
\hline 7 & 10.68 & 574 & 616 & 620 & 603.3 & 599 & 647 & 583 & 609.7 \\
\hline 8 & 11.44 & 581 & 605 & 591 & 592.3 & 617 & 611 & 584 & 604.0 \\
\hline \multicolumn{2}{|c|}{ Averages $\longrightarrow$} & 592.1 & 627.3 & 612.7 & 610.7 & 628.7 & 622.3 & 621.7 & 624.2 \\
\hline
\end{tabular}

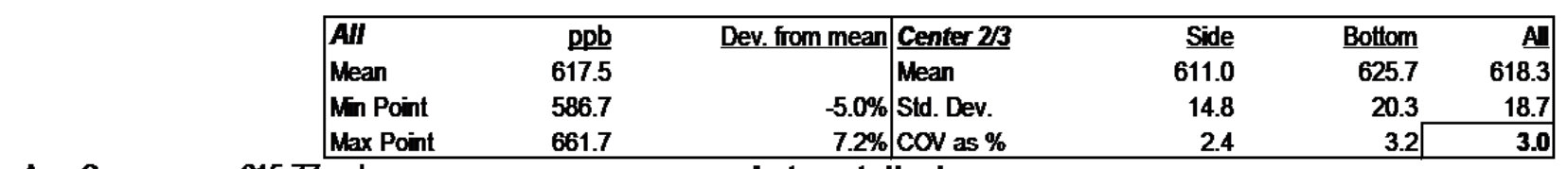

Avg. Conc. $\quad 615.77 \mathrm{ppb}$

\begin{tabular}{|c|c|c|c|}
\hline \multirow{4}{*}{$\begin{array}{l}\text { Tracer tank pressure } \\
\text { Injection flowmeter } \\
\text { Stack Temp }\end{array}$} & Start & Finish & \multirow{4}{*}{$\begin{array}{l}\text { psig } \\
\text { sccm } \\
\text { "F }\end{array}$} \\
\hline & 100 & 100 & \\
\hline & 30 & 30 & \\
\hline & 60.7 & 63.8 & \\
\hline Mean stack velocity & 2654 & 2668 & sfpm \\
\hline Sampling flowmeter & 5 & 5 & $\mathrm{lpm}$ \\
\hline Ambient pressure & 1003 & 1003 & mbar \\
\hline Ambient humidity & $35 \%$ & $34 \%$ & RH \\
\hline Ambient Temp & 58.1 & 61.7 & ${ }^{\circ} \mathrm{F}$ \\
\hline B\&K vapor correction & $\mathrm{Y}$ & $\mathrm{Y}$ & YIN \\
\hline Back-Gd gas & $9,9,12,9,11$ & $8,8,10,10,9$ & ppb \\
\hline No. Bk-Gd samples & 5 & 5 & n \\
\hline
\end{tabular}

Gas anahzer checked: 4/30/2012

CA $5 / 2 / 12$

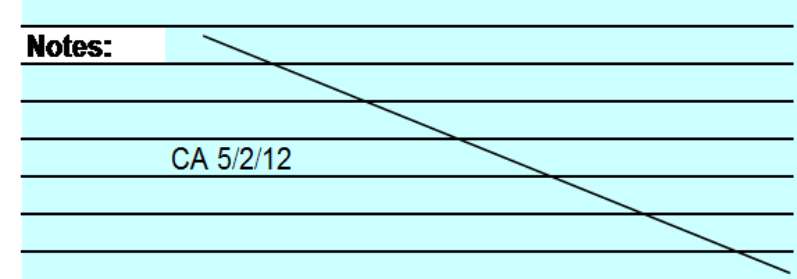

\begin{tabular}{|lc|}
\hline $\begin{array}{l}\text { Entries made by: } \\
\text { Signature/date }\end{array}$ & $\mathrm{XY}, \mathrm{CA}$ \\
& Signature on file with original \\
\hline
\end{tabular}

Run No. GT-7

Fan Configuration Fan B only

Fan Setting 60 Hz

Stack Temp $62.25 \operatorname{deg} F$

Start/End Tme 10:00/10:55

Center $2 / 3$ from

Points in Center $2 / 3$

Injection Point Port E, Fa
1.10 to: 10.84 2 to: $\underline{7}$

\section{Instuments Used:}

\begin{tabular}{llll} 
B\&K 1302 Gas Analyzer SN $\quad 1765299$ & Cat2 M\&TE \\
\hline
\end{tabular}

\begin{tabular}{ll}
\hline TSI VelociCalc SN T95351203001 & $1 / 17 / 2013$ \\
\hline Fisher Scientific SN 90936818 & $12 / 7 / 2012$ \\
\hline
\end{tabular}

Fisher Scientific SN 90936818

$12 / 7 / 2012$

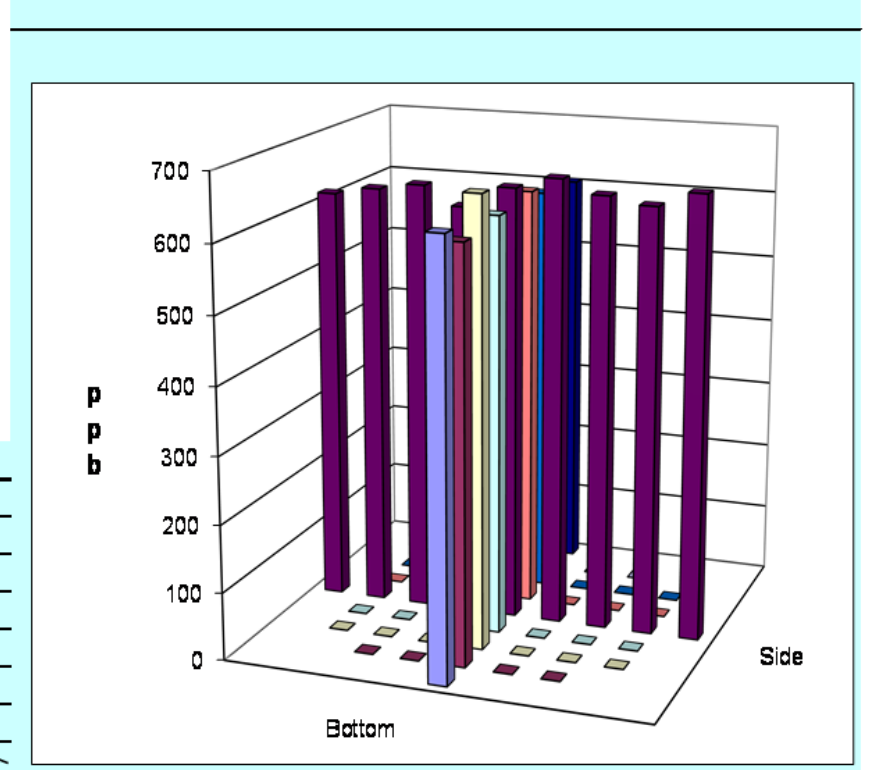

Technical Data Review performed by: Susan Sande

Signature/date 7/10/2012

Signature on file with original TI-WTPSP-078 
Rev. 0

31-JuL06

\begin{tabular}{|c|c|}
\hline \multicolumn{2}{|c|}{ Site HV-S2 Model } \\
\hline Date & $5 / 2 / 2012$ \\
\hline \multicolumn{2}{|c|}{ Testers $X Y, C A$} \\
\hline Stack Dia. & 11.938 in. \\
\hline Stack X-Area & 111.9 in. $^{2}$ \\
\hline Test Port & 2 \\
\hline
\end{tabular}

Distance to disturbance 96.4 inches

Measurement units pob SF6

TRACER GAS TRAYERSE DATA FORI

\begin{tabular}{|c|c|c|c|}
\hline \multirow{4}{*}{$\begin{array}{l}\text { Mea } \\
\text { Order } \rightarrow \\
\text { Traverse- } \\
\text { Trial } \longrightarrow \\
\end{array}$} & & 然 & \\
\hline & & \multicolumn{2}{|l|}{1 st } \\
\hline & & \multirow[b]{2}{*}{1} & \multirow[t]{2}{*}{ Sid } \\
\hline & & & \\
\hline Point & Depth, in. & & ppl \\
\hline 1 & 0.50 & 637 & 698 \\
\hline 2 & 1.25 & 669 & 681 \\
\hline 3 & 2.32 & 660 & 664 \\
\hline 4 & 3.86 & 705 & 670 \\
\hline Center & 5.97 & 652 & 685 \\
\hline 5 & 8.08 & 636 & 666 \\
\hline 6 & 9.62 & 619 & 655 \\
\hline 7 & 10.68 & 652 & 687 \\
\hline 8 & 11.44 & 729 & 655 \\
\hline \multirow{5}{*}{\multicolumn{2}{|c|}{ Averages $\longrightarrow$}} & 662.1 & 673.4 \\
\hline & & $\boldsymbol{A l l}$ & ppb \\
\hline & & Mean & 669.9 \\
\hline & & Mn Point & 639.7 \\
\hline & & Max Point & 685.3 \\
\hline
\end{tabular}

Avg. Conc.

$669.35 \mathrm{ppb}$

\begin{tabular}{|c|c|c|c|}
\hline \multirow{4}{*}{$\begin{array}{l}\text { Tracer tank pressure } \\
\text { Injection flowmeter } \\
\text { Stack Temp }\end{array}$} & Start & Finish & \multirow{4}{*}{$\begin{array}{l}\text { psig } \\
\text { sccm } \\
{ }^{\circ} \mathrm{F}\end{array}$} \\
\hline & 125 & 125 & \\
\hline & 30 & 30 & \\
\hline & 64.0 & 66.3 & \\
\hline \multirow{2}{*}{$\begin{array}{l}\text { Mean stack velocity } \\
\text { Sampling flowmeter }\end{array}$} & 2434 & 2499 & sfpm \\
\hline & 5 & 5 & lpm \\
\hline \multirow{2}{*}{$\begin{array}{l}\text { Ambient pressure } \\
\text { Ambient humidity }\end{array}$} & 1003 & 1003 & mbar \\
\hline & $32 \%$ & $29 \%$ & RH \\
\hline \multirow{3}{*}{$\begin{array}{l}\text { Ambient Temp } \\
\text { B\&K vapor correction } \\
\text { Back-Gd gas }\end{array}$} & 60.7 & 63.5 & ${ }^{\circ} \mathrm{F}$ \\
\hline & $\mathrm{Y}$ & $Y$ & Y/N \\
\hline & $8,6,8,8,10$ & $5,7,10,8,9$ & ppb \\
\hline No. Bk-Gd samples & 5 & 5 & n \\
\hline
\end{tabular}

Gas anahzer checked: $4 / 30 / 2012$

CA $5 / 2 / 12$

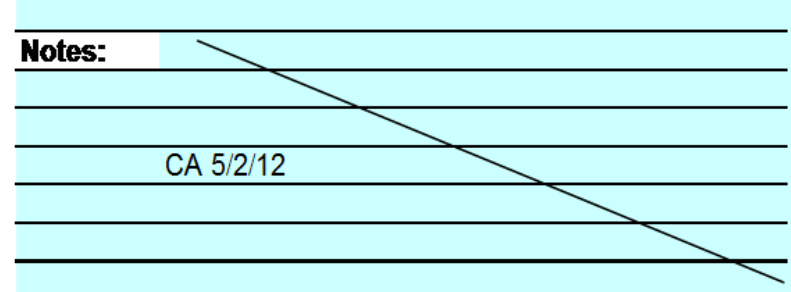

\begin{tabular}{|lc}
\hline $\begin{array}{l}\text { Entries made by: } \\
\text { Signature/date }\end{array}$ & $\mathrm{XY}, \mathrm{CA}$ \\
& Signature on file with original
\end{tabular}

Run No. GT-8

Fan Configuration Fan B only

Fan Setting 56

Stack Temp

Start/End Tme 11:11/12:45

Center 2/3 from

Points in Center $2 / 3$

Injection Point Port E, Center

Hz

$65.15 \operatorname{deg} F$

$\begin{array}{rl}1.10 & \text { to: } \\ 2 & 10.84\end{array}$

Porte, Center \begin{tabular}{ll|}
\hline 2nd & \\
Bottom
\end{tabular}

\begin{tabular}{|c|c|c|c|c|c|}
\hline & & \multicolumn{4}{|c|}{ Bottom } \\
\hline 3 & Mean & 1 & 2 & 3 & Mean \\
\hline
\end{tabular}

\begin{tabular}{r|r|r|r|}
648 & \multicolumn{2}{|c}{ pp } \\
\hline 661.0 & 681 & 649
\end{tabular}

\begin{tabular}{|c|c|c|c|c|c|}
\hline 648 & 661.0 & 681 & 649 & 679 & 669.7 \\
\hline 699 & 683.0 & 669 & 689 & 653 & 670.3 \\
\hline 690 & 671.3 & 705 & 645 & 692 & 680.7 \\
\hline 670 & 681.7 & 689 & 653 & 686 & 676.0 \\
\hline 717 & 684.7 & 656 & 662 & 673 & 663.7 \\
\hline 662 & 654.7 & 667 & 670 & 719 & 685.3 \\
\hline 645 & 639.7 & 645 & 664 & 677 & 662.0 \\
\hline 630 & 656.3 & 699 & 674 & 669 & 680.7 \\
\hline 640 & 674.667 & 673 & 654 & 661 & 662.7 \\
\hline 6.8 & 667.4 & 676.0 & 6622 & 678.8 & 672.3 \\
\hline
\end{tabular}

Dev. from mean $\mid \frac{\text { Center 2/3 }}{\text { Mean }}$ $-4.5 \%$ Std. Dev. $2.3 \%$ COV as \%

Instuments Used:

\begin{tabular}{llll} 
B\&K 1302 Gas Analyzer & SN 1765299 & Cat2 M\&TE \\
\hline
\end{tabular}

\begin{tabular}{ll}
\hline TSI VelociCalc SN T95351203001 & $1 / 17 / 2013$ \\
\hline Fisher Scientific SN 90936818 & $12 / 7 / 2012$ \\
\hline
\end{tabular}

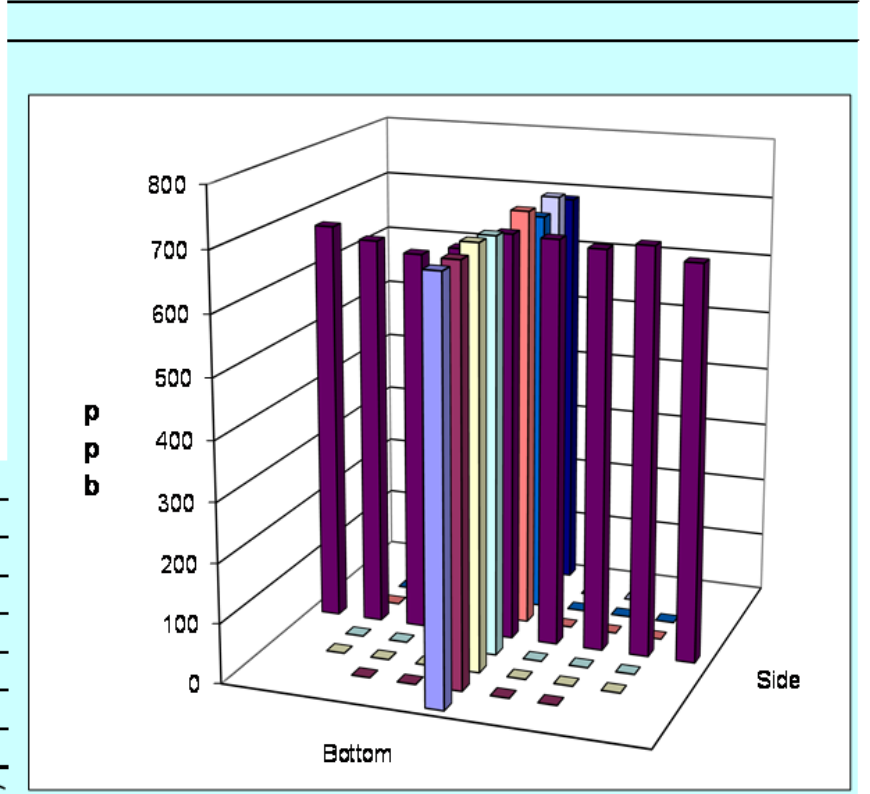

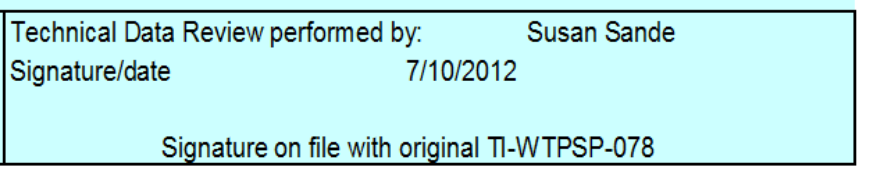


Rev. 0

31-JuL06

Date
Testers $\frac{5 / 4 / 2012}{\mathrm{CA}, \mathrm{EA}}$
Stack Dia.
Stack X-Area
Test Port $\frac{11.938 \text { in. }}{111.9 \text { in. }^{2}}$
Distance to disturbance
Measurement units pob SF 6

TRACER GAS TRAVERSE DATA FORI

\begin{tabular}{|c|c|c|c|c|c|c|c|c|c|}
\hline \multirow{4}{*}{$\begin{array}{l}\quad \text { Me } \\
\text { Order } \rightarrow \\
\text { Traverse } \rightarrow \\
\text { Trial } \longrightarrow\end{array}$} & \multicolumn{3}{|c|}{ easurement units pob SF6 } & \multicolumn{5}{|c|}{ Injection Point Port E Center } & \\
\hline & & 2nd & & & & \multicolumn{2}{|c|}{ 1st } & & \\
\hline & & \multicolumn{4}{|c|}{ Side } & \multicolumn{4}{|c|}{ Bottom } \\
\hline & & 1 & 2 & 3 & Mean & 1 & 2 & 3 & Mean \\
\hline Point & Depth, in. & \multicolumn{4}{|c|}{ ppb } & \multicolumn{4}{|c|}{$\mathbf{p p b}$} \\
\hline 1 & 0.50 & 582 & 558 & 599 & 579.7 & 530 & 511 & 462 & 501.0 \\
\hline 2 & 1.25 & 564 & 583 & 549 & 565.3 & 491 & 501 & 532 & 508.0 \\
\hline 3 & 2.32 & 557 & 551 & 562 & 556.7 & 469 & 476 & 492 & 479.0 \\
\hline 4 & 3.86 & 567 & 539 & 511 & 539.0 & 483 & 473 & 469 & 475.0 \\
\hline Center & 5.97 & 485 & 505 & 506 & 498.7 & 492 & 481 & 509 & 494.0 \\
\hline 5 & 8.08 & 465 & 460 & 478 & 467.7 & 494 & 512 & 513 & 506.3 \\
\hline 6 & 9.62 & 482 & 466 & 450 & 466.0 & 564 & 541 & 566 & 557.0 \\
\hline 7 & 10.68 & 475 & 490 & 511 & 492.0 & 560 & 571 & 563 & 564.7 \\
\hline 8 & 11.44 & 475 & 473 & 432 & 460.0 & 580 & 587 & 603 & 590.0 \\
\hline \multicolumn{2}{|c|}{ Averages $\longrightarrow$} & 516.9 & 513.9 & 510.9 & 513.9 & 518.1 & 517.0 & 523.2 & 519.4 \\
\hline
\end{tabular}

Fan Configuration Fan A Only

Fan Setting $\frac{\text { Hz }}{60}$
Stack Temp

Center $2 / 3$ from $\quad 1.10$ to: 10.84

Points in Center 2/3 2 to: $\overline{7}$

Injection Point Port E Center

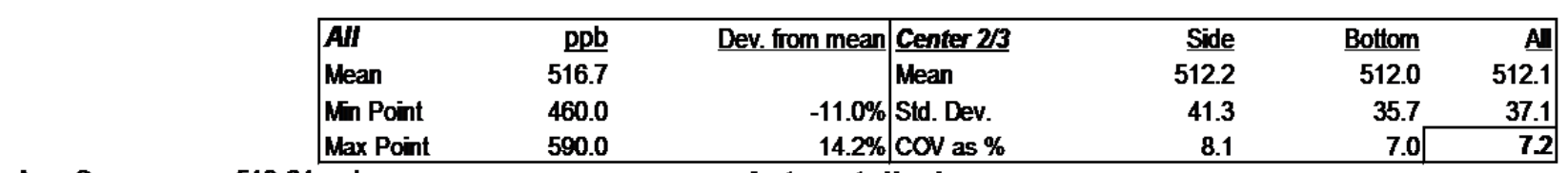

Avg. Conc. $\quad 519.21 \mathrm{ppb}$

\begin{tabular}{|c|c|c|c|}
\hline \multirow{4}{*}{$\begin{array}{l}\text { Tracer tank pressure } \\
\text { Injection flowmeter } \\
\text { Stack Temp }\end{array}$} & Start & Finish & \\
\hline & 100 & 75 & psig \\
\hline & 30 & 30 & sccm \\
\hline & 57.9 & 63.2 & ${ }^{\circ} \mathrm{F}$ \\
\hline Mean stack velocity & 3076 & 3130 & sfpm \\
\hline Sampling flowmeter & 5 & 5 & Ipm \\
\hline Ambient pressure & 1005.00 & 1006.00 & mbar \\
\hline Ambient humidity & $44 \%$ & $30 \%$ & $\mathbf{R H}$ \\
\hline Ambient Temp & 59.0 & 59 & " $\mathrm{F}$ \\
\hline B\&K vapor correction & $Y$ & $\mathrm{Y}$ & YIN \\
\hline Back-Gd gas & $6,4,4,5,8$ & $6,3,2,4,0.3$ & 3 ppb \\
\hline No. Bk-Gd samples & 5 & 5 & n \\
\hline
\end{tabular}

Gas analyzer checked: $\quad 4 / 30 / 152 \quad$ CA 5/4/12

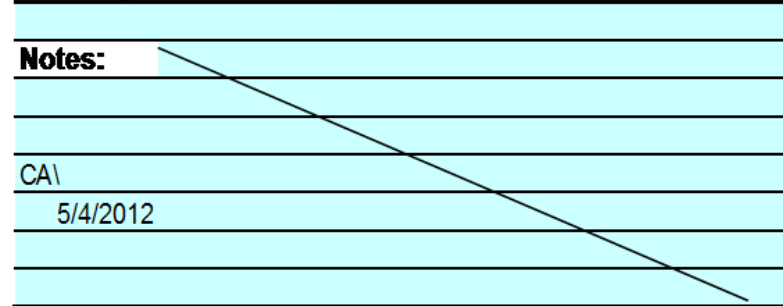

\begin{tabular}{|lc|}
\hline Entries made by: & EA, CA \\
Signature/date & $5 / 4 / 2012$ \\
& Signature on fiel with original
\end{tabular}

Instuments Used:

B\&K 1302 Gas Analyzer SN $1765299 \quad$ Cat2 M\&TE TSI VelociCalc SN T95351203001 $1 / 17 / 2013$ Fisher Scientific SN $90936818 \quad 12 / 7 / 2012$
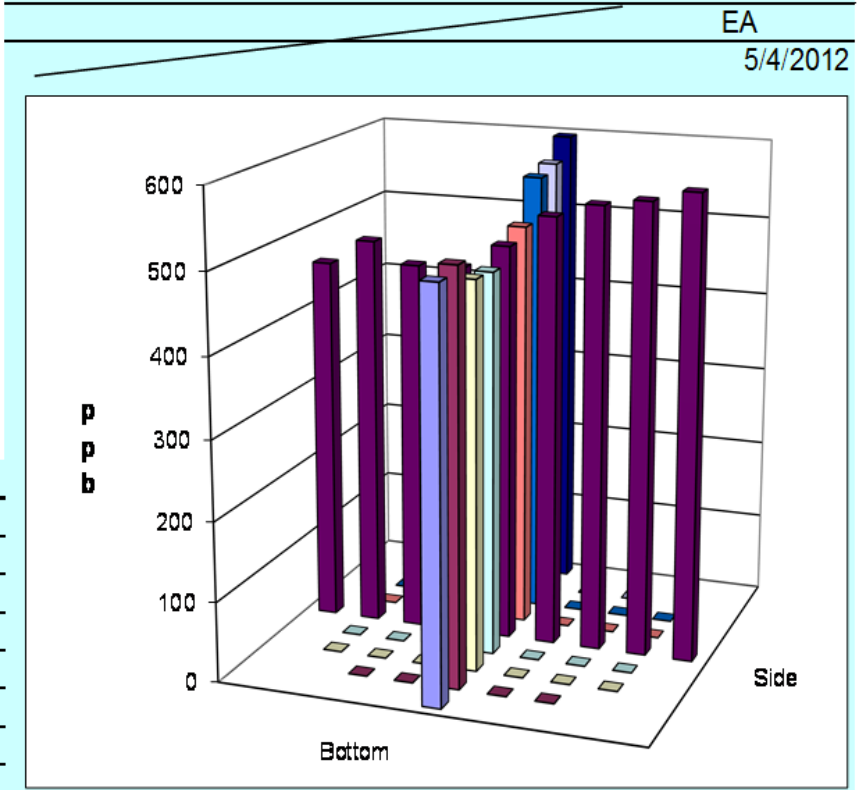

Technical Data Review performed by: Susan Sande Signature/date 7/10/2012

Signature on file with original TT-WTPSP-078 
Rev. 0

31-JuL06

$\begin{aligned} & \text { Site HV-S2 Model } \\ & \text { Date } 5 / 4 / 2012 \\ & \text { Testers } \text { YFS, XYY } \\ & \text { Stack Dia. } 11.938 \text { in. } \\ & \text { Stack X-Area } 111.9 \text { in. }^{2} \\ & \text { Test Port } 2\end{aligned}$

Distance to disturbance 96.4 inches

Measurement units pob SF6

TRACER GAS TRAVERSE DATA FORI

Order $\rightarrow$

Traverse $\rightarrow$

Tíal $\longrightarrow$

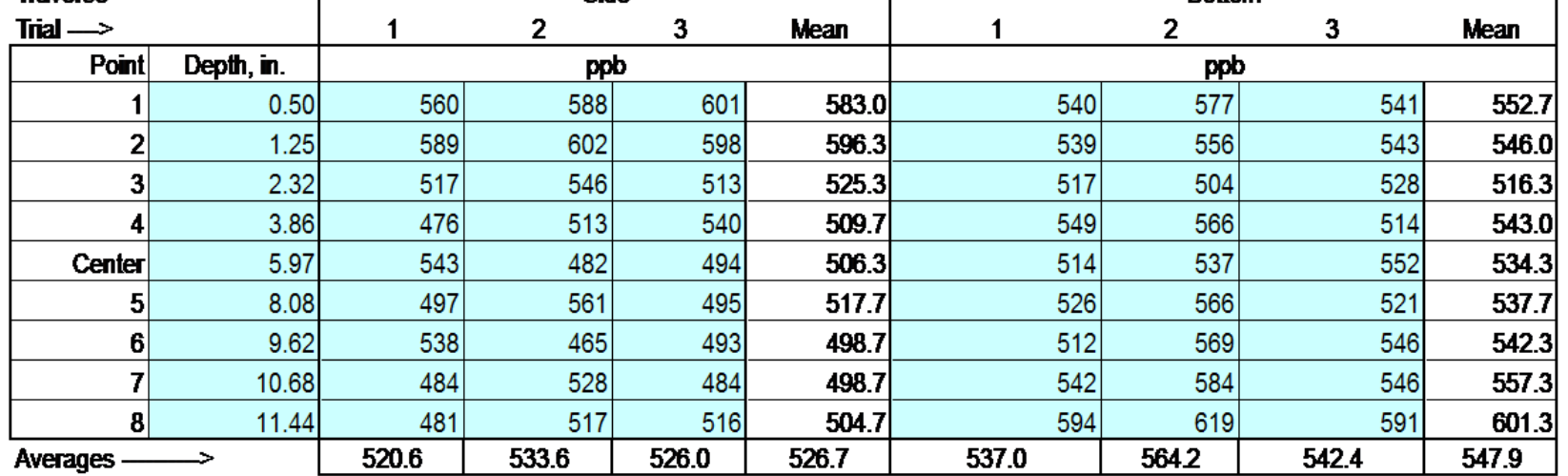

Avg. Conc.

\begin{tabular}{|lr}
\hline All & ppb \\
Mean & 537.3 \\
Min Point & 498.7 \\
Max Point & 601.3 \\
\hline
\end{tabular}

$539.42 \mathrm{ppb}$

Tracer tank pressure Injection flowmeter

Stack Temp

Mean stack velocity

Sampling flowmeter

Ambient pressure

Ambient humidity

Ambient Temp

B\&K vapor correction

Back-Gd gas

No. Bk-Gd samples

\begin{tabular}{|c|c|}
\hline Start & Finish \\
\hline 150 & 150 \\
\hline 30 & 30 \\
\hline 67.1 & 68.7 \\
\hline 3073 & 3030 \\
\hline 5 & 5 \\
\hline 1008 & 1007 \\
\hline $25 \%$ & $33 \%$ \\
\hline 71.6 & 62.6 \\
\hline Y & $Y$ \\
\hline $5,7,5,7,5$ & $6,10,6,4,4$ \\
\hline 5 & 5 \\
\hline
\end{tabular}

Gas analyzer checked: $\quad 4 / 30 / 152 \quad X Y$ 5/4/12

Notes: Light rain and wind.

Repeat of GT-9, A max flow. Reduce frequency to be close to rtarget velocity of $3040 \mathrm{fpm}$

\begin{tabular}{l}
$\frac{X Y Y 5 / 4 / 12}{}$ \\
\hline $5 / 7 / 12$ JEF found injection probe pointed upsteam, suspect \\
\hline injection probe oriented incorrectly during this test. \\
$\begin{array}{ll}\text { Entries made by: } & X Y Y \\
\text { Signature/date } & 5 / 4 / 2012 \\
& \text { Signature on file with original }\end{array}$
\end{tabular}

\begin{tabular}{|c|c|c|c|c|}
\hline Dev. from mear & Center 2/3 & Side & Bottom & A] \\
\hline & Mean & 521.8 & 539.6 & 530.7 \\
\hline$-7.2 \%$ & Std. Dev. & 34.3 & 12.6 & 26.4 \\
\hline $11.9 \%$ & COV as \% & 6.6 & 2.3 & 5.0 \\
\hline
\end{tabular}

\section{Instuments Used:}

$\begin{array}{lll}\text { B\&K } 1302 \text { Gas Analyzer SN } 1765299 & \text { Cat2 M\&TE }\end{array}$ TSI VelociCalc SN T95351203001 $1 / 17 / 2013$

Fisher Scientific SN $90936818 \quad 12 / 7 / 2012$

\begin{tabular}{cc}
\hline EA \\
\hline $5 / 4 / 2012$
\end{tabular}

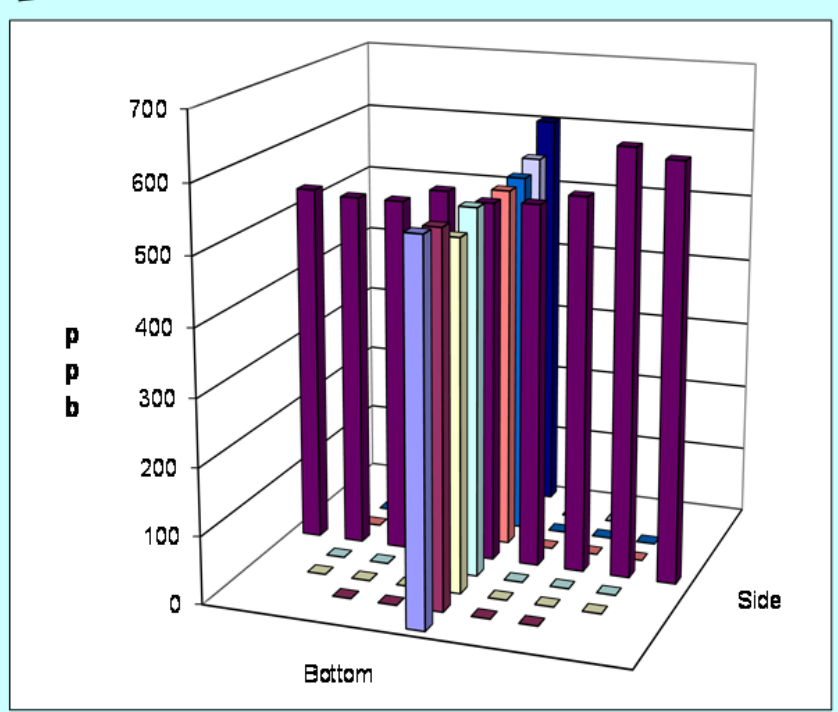

Technical Data Review performed by: Susan Sande

Signature/date 7/10/2012

Signature on file with original TI-WTPSP-078 
Rev. 0

31-JuL06

$$
\begin{aligned}
& \text { Date } \multicolumn{1}{c}{5 / 7 / 2012} \\
& \text { Testers } \text { CA, EA } \\
& \text { Stack Dia. } 11.938 \text { in. } \\
& \text { Stack X-Area } \mathbf{1 1 1 . 9} \text { in. }^{2} \\
& \text { Test Port } 2 \\
& \text { Distance to disturbance } 96.4 \text { inches } \\
& \text { Measurement units pob SF } 6
\end{aligned}
$$$$
\text { Site HV-S2 Mode }
$$

Order $\rightarrow$

Traverse $\rightarrow$

Tỉal $\rightarrow$

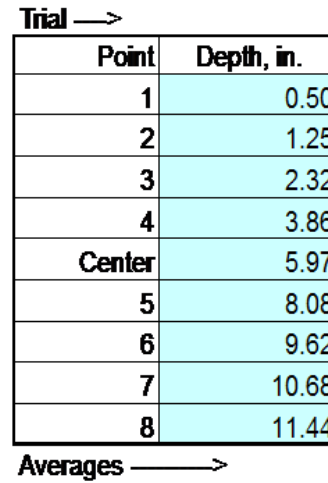

Avg. Conc.

$$
512.50 \mathrm{ppb}
$$

\begin{tabular}{|lr}
\hline All & $\mathrm{ppb}$ \\
Mean & 509.9 \\
Min Point & 468.7 \\
Max Point & 563.3 \\
\hline
\end{tabular}

Tracer tank pressure Injection flowmeter Stack Temp Mean stack velocity Sampling flowmeter

\begin{tabular}{|c|c|c|}
\hline Start & Finish & \\
\hline 10 & 10 & \\
\hline 30 & 30 & \\
\hline 63 & 68.3 & \\
\hline 3067 & 3053 & \\
\hline 5 & 5 & \\
\hline 1016 & 1016 & \\
\hline $32 \%$ & $20 \%$ & \\
\hline 66.2 & 76.1 & \\
\hline Y & Y & \\
\hline $4, .6,5,2,4$ & $4,3,6,5, .6$ & \\
\hline 5 & 5 & \\
\hline
\end{tabular}
Ambient pressure Ambient humidity Ambient Temp B\&K vapor correction Back-Gd gas

No. Bk-Gd samples

Gas analyzer checked: $\quad$ 5/7/2012 CA 5/7/12

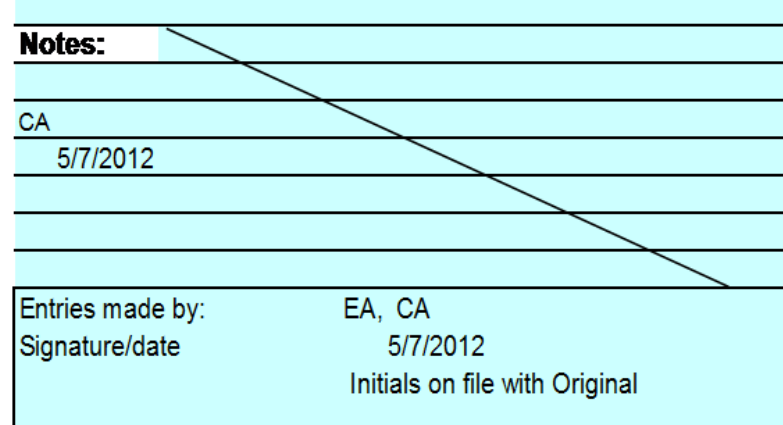

Run No. GT-11

Fan Configuration Fan A

Fan Setting 60

Stack Temp

Start/End Tme 0846 / 1015

Center 2/3 from

Points in Center $2 / 3$

Injection Point Center, Port E
66 deg $F$

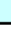

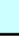

F

1.10 to: 10.84

2 to: 7

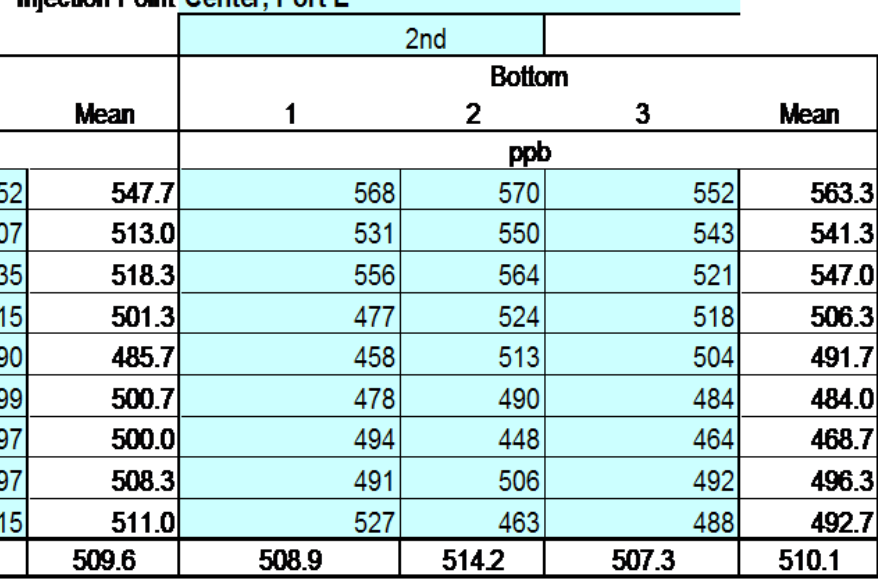

\begin{tabular}{r|lrrr|} 
Dev. from mean & Center 2/3 & Side & Bottom & Al \\
\cline { 2 - 5 } & Mean & 503.9 & 505.0 & 504.5 \\
$10.1 \%$ & Std. Dev. & 10.6 & 29.2 & 21.1 \\
$10.5 \%$ & COV as \% & 2.1 & 5.8 & 4.2 \\
\hline
\end{tabular}

\section{Instuments Used:}

\begin{tabular}{llll} 
B\&K 1302 Gas Analyzer & SN & 1765299 & Cat2 M\&TE \\
\hline TSIV
\end{tabular}

\begin{tabular}{lc}
\hline TSI VelociCalc SN T95351203001 & $1 / 17 / 2013$ \\
\hline Fisher Scientific SN 90936818 & $12 / 7 / 2012$ \\
\hline & EA \\
\hline
\end{tabular}

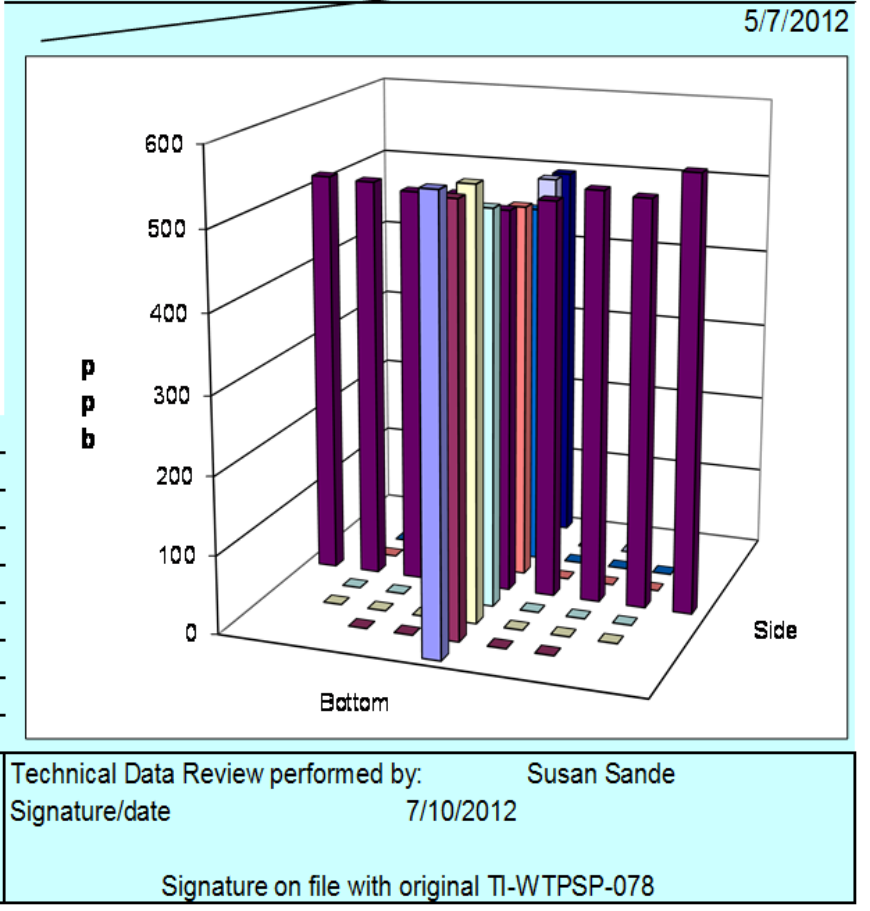


Rev. 0

31-JuL06

\begin{tabular}{|c|c|}
\hline \multicolumn{2}{|c|}{ Site HV-S2 Model } \\
\hline Date & $5 / 7 / 2012$ \\
\hline \multicolumn{2}{|c|}{ Testers CA, EA } \\
\hline Stack Dia. & 11.938 in. \\
\hline Stack X-Area & 111.9 in. $^{2}$ \\
\hline Test Port & 2 \\
\hline
\end{tabular}

Distance to disturbance 96.4 inches

Measurement units pob SF6

$$
\text { ppb SF6 }
$$

TRACER GAS TRAVERSE DATA FORI

Oder $\rightarrow$

Traverse $\rightarrow$

Tíal $\rightarrow$

\begin{tabular}{|c|c|}
\hline Point & Depth, in. \\
\hline 1 & 0. \\
\hline 2 & 1. \\
\hline 3 & 2. \\
\hline 4 & 3. \\
\hline Center & 5. \\
\hline 5 & 8. \\
\hline 6 & 9. \\
\hline 7 & 10. \\
\hline 8 & 11. \\
\hline
\end{tabular}

\begin{tabular}{|c|c|}
\hline \multicolumn{2}{|c|}{ 2nd } \\
\hline 1 & 2 \\
\hline
\end{tabular}

\section{S TRAVERS}

Run No. GT-12

Fan Configuration Fan A

\begin{aligned} Fan Setting & Hz \\ Stack Temp & H2 deg F \\ Start/End Tme & \\ \hline $1018 / 1125 & \end{aligned}$

Center 2/3 from

Points in Center $2 / \overline{2}$ to: $\overline{7}$

Injection Point Center, Port E

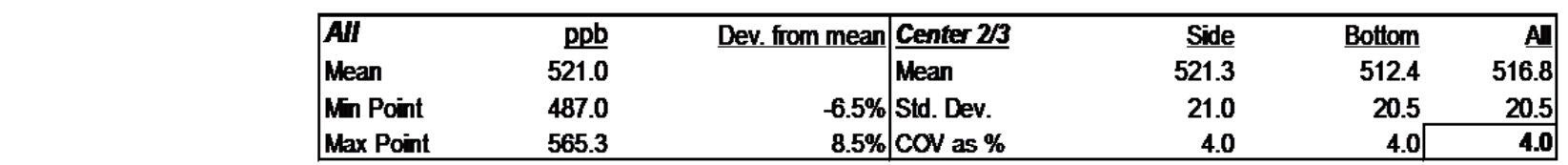

Avg. Conc. $\quad 523.17 \mathrm{ppb}$

Tracer tank pressure Injection flowmeter Stack Temp Mean stack velocity Sampling flowmeter Ambient pressure Ambient humidity Ambient Temp B\&K vapor correction Back-Gd gas

No. Bk-Gd samples

\begin{tabular}{|c|c|}
\hline Start & Finish \\
\hline 10 & 10 \\
\hline 30 & 30 \\
\hline 68.3 & 75.6 \\
\hline 3053 & 3134 \\
\hline 5 & 5 \\
\hline 1016 & 1015 \\
\hline $20 \%$ & $29 \%$ \\
\hline 76.1 & 70.7 \\
\hline$Y$ & $Y$ \\
\hline $\begin{array}{c}4,3,6,5 \\
0.6\end{array}$ & $2,3,6,8,2$ \\
\hline 5 & 5 \\
\hline
\end{tabular}

Gas anahzer checked: $5 / 7 / 2012$ CA $5 / 7 / 12$

Notes: Re-do of GT-10 due to finding injection probe in Port pointing upstream into the flow.

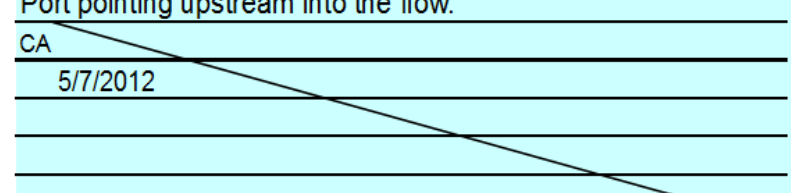

\begin{tabular}{|lc}
\hline Entries made by: & EA, CA \\
Signature/date & $5 / 7 / 2012$ \\
& Initials on file with Original \\
\hline
\end{tabular}

\section{Instuments Used:}

\begin{tabular}{llll} 
B\&K 1302 Gas Analyzer & SN & 1765299 & Cat2 M\&TE \\
\hline
\end{tabular}

\begin{tabular}{lc}
\hline TSI VelociCalc SN T95351203001 & $1 / 17 / 2013$ \\
\hline Fisher Scientific SN 90936818 & $12 / 7 / 2012$ \\
\hline & EA \\
\hline
\end{tabular}

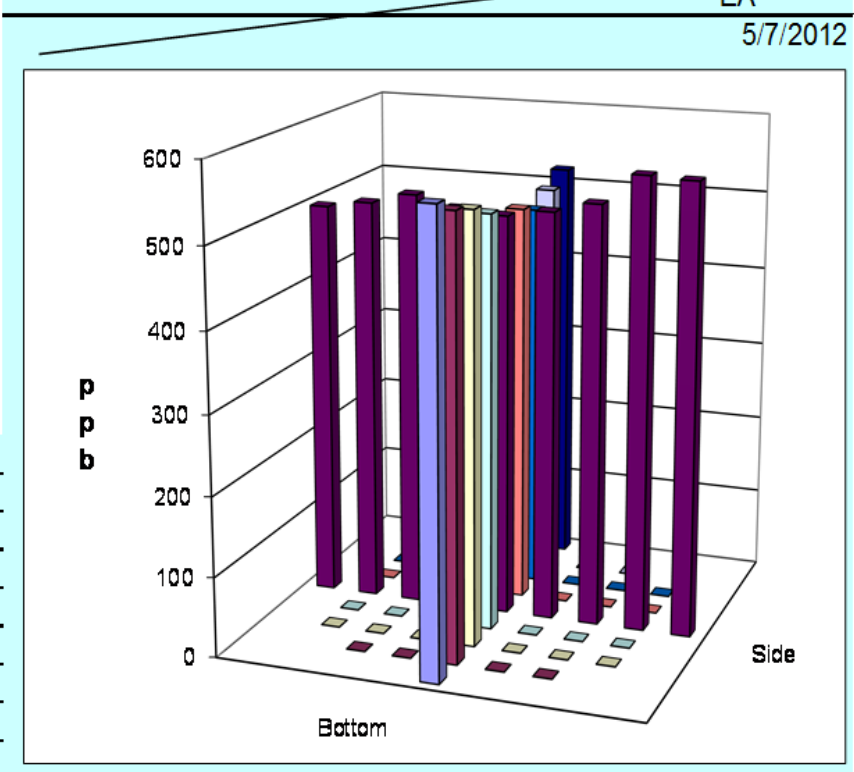

Technical Data Review performed by: Susan Sande

Signature/date

7/10/2012

Signature on file with original TT-WTPSP-078 
Rev. 0

31-JuL06

Date
Testers $\frac{6 / 20 / 2012}{\mathrm{XY}, \mathrm{CA}}$
Stack Dia.
Stack X-Area
Test Port $\frac{11.938 \text { in. }}{111.9 \text { in. }^{2}}$
Distance to disturbance
Measurement units pob SF

TRACER GAS TRAVERSE DATA FORU

\begin{tabular}{|c|c|c|c|c|c|c|c|c|c|}
\hline \multicolumn{4}{|c|}{ Measurement units pob SF 6} & \multicolumn{5}{|c|}{ Injection Point C-Bottom } & \\
\hline \multirow{3}{*}{$\begin{array}{l}\text { Order } \rightarrow \\
\text { Traverse } \rightarrow \\
\text { Trial } \longrightarrow\end{array}$} & & \multicolumn{2}{|c|}{ 2nd } & & & \multicolumn{2}{|c|}{ 1st } & & \\
\hline & & \multicolumn{4}{|c|}{ Side } & \multicolumn{4}{|c|}{ Bottom } \\
\hline & & 1 & 2 & 3 & Mean & 1 & 2 & 3 & Mean \\
\hline Point & Depth, in. & \multicolumn{4}{|c|}{ ppb } & \multicolumn{4}{|c|}{ ppb } \\
\hline 1 & 0.50 & 560 & 519 & 567 & 548.7 & 613 & 533 & 529 & 558.3 \\
\hline 2 & 1.25 & 575 & 636 & 517 & 576.0 & 557 & 523 & 563 & 547.7 \\
\hline 3 & 2.32 & 628 & 586 & 576 & 596.7 & 623 & 612 & 610 & 615.0 \\
\hline 4 & 3.86 & 618 & 552 & 638 & 602.7 & 554 & 548 & 574 & 558.7 \\
\hline Center & 5.97 & 576 & 641 & 623 & 613.3 & 528 & 679 & 585 & 597.3 \\
\hline 5 & 8.08 & 585 & 524 & 599 & 569.3 & 536 & 540 & 541 & 539.0 \\
\hline 6 & 9.62 & 548 & 623 & 591 & 587.3 & 608 & 591 & 556 & 585.0 \\
\hline 7 & 10.68 & 600 & 554 & 496 & 550.0 & 595 & 587 & 666 & 616.0 \\
\hline 8 & 11.44 & 666 & 564 & 549 & 593.0 & 580 & 572 & 636 & 596.0 \\
\hline \multicolumn{2}{|c|}{ Averages $\longrightarrow$} & 595.1 & 577.7 & 572.9 & 581.9 & 577.1 & 576.1 & 584.4 & 579.2 \\
\hline
\end{tabular}

Fan Configuration Fan B Only

Stack Temp Hz

Start/End Tme 9:30/11:12

Center $2 / 3$ from

$80 \operatorname{deg} F$

1.10 to:

Points in Center $2 / 3$ 2 to: 7

Injection Point C-Bottom \begin{tabular}{|lr}
\hline$A I I$ & $\mathrm{ppb}$ \\
Mean & 580.6 \\
Min Point & 539.0 \\
Max Point & 616.0 \\
\hline
\end{tabular}

Avg. Conc.

$577.46 \mathrm{ppb}$

\begin{tabular}{|c|c|c|}
\hline \multirow{4}{*}{$\begin{array}{l}\text { Tracer tank pressure } \\
\text { Injection flowmeter } \\
\text { Stack Temp }\end{array}$} & Start & Fnish \\
\hline & 125 & 125 \\
\hline & 30 & 30 \\
\hline & 79.4 & 79.6 \\
\hline Mean stack velocity & 2930 & 2938 \\
\hline Sampling flowmeter & 5 & 5 \\
\hline Ambient pressure & 1009 & 1008 \\
\hline Ambient humidity & $31 \%$ & $31 \%$ \\
\hline Ambient Temp & 70.7 & 74.3 \\
\hline B\&K vapor correction & $\mathrm{Y}$ & $\mathrm{Y}$ \\
\hline Back-Gd gas & $7,2,10,2,13$ & $6,12,9,5,6$ \\
\hline No. Bk-Gd samples & 5 & 5 \\
\hline
\end{tabular}

Gas analyzer checked:

$6 / 19 / 2012$

Notes: $\quad$ Re-do of GT-4 Fan B max target 2619 to $3014 \mathrm{fpm}$ at Bottom 3.

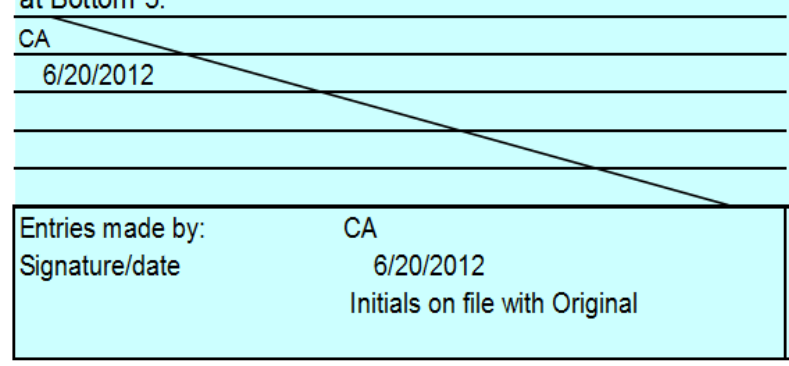

\begin{tabular}{r|l} 
Dev. from mean & $\frac{\text { Center 2/3 }}{\text { Mean }}$ \\
$-7.2 \%$ & Std. Dev. \\
$6.1 \%$ & Cov as
\end{tabular}

$6.1 \%$ COV as \%

\section{Instuments Used:}

B\&K 1302 Gas Analyzer SN 1765299

TSI VelociCalc SN T95351203001 $1 / 17 / 2013$

Fisher Scientific SN 90936818
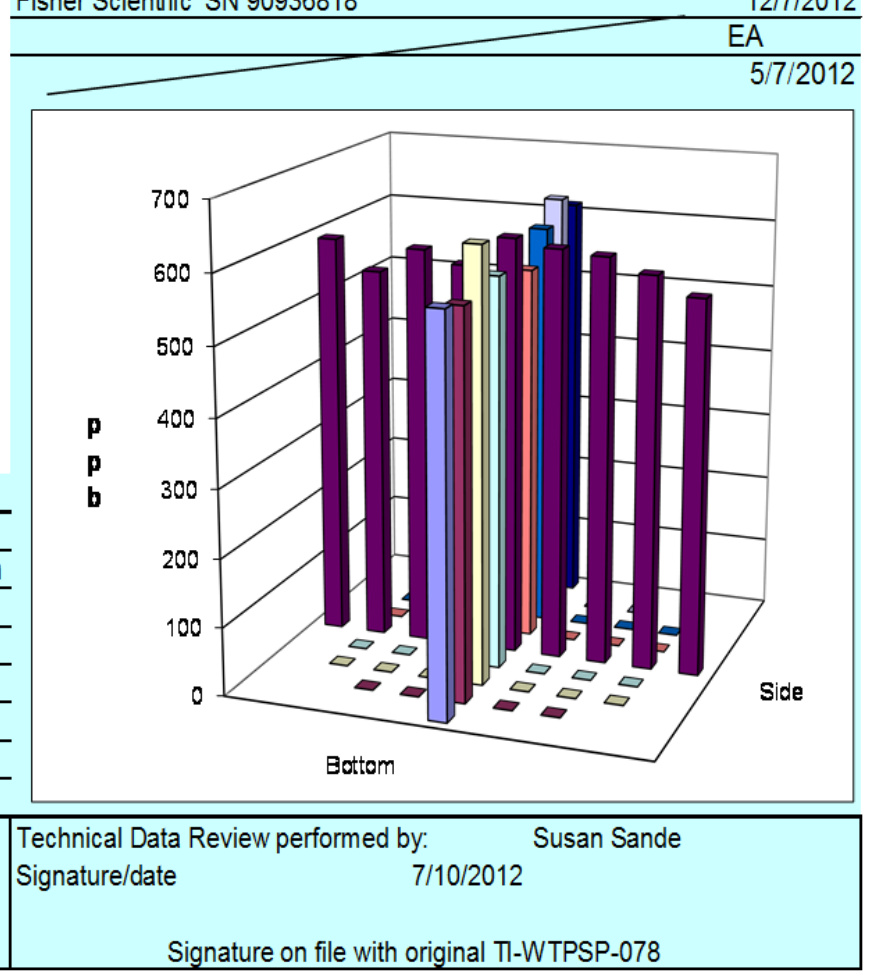
Rev. 0

31-JuL06

\begin{tabular}{|c|c|}
\hline \multicolumn{2}{|c|}{ Site HV-S2 Model } \\
\hline Date & $6 / 20 / 2012$ \\
\hline \multicolumn{2}{|c|}{ Testers $X Y, C A$} \\
\hline Stack Dia. & 11.938 in. \\
\hline Stack X-Area & 111.9 in. $^{2}$ \\
\hline Test Port & 2 \\
\hline
\end{tabular}

Distance to disturbance 96.4 inches

Measurement units pob SF6

TRACER GAS TRAVERSE DATA FORI

Order $\rightarrow$

Traverse $\rightarrow$

Tíal $\longrightarrow$

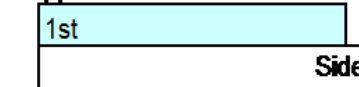

\begin{tabular}{|c|c|c|c|}
\hline Tíal $\longrightarrow$ & & 1 & 2 \\
\hline Point & Depth, in. & & \\
\hline 1 & 0.50 & 593 & \\
\hline 2 & 1.25 & 590 & \\
\hline 3 & 2.32 & 610 & 6 \\
\hline 4 & 3.86 & 597 & \\
\hline Center & 5.97 & 552 & \\
\hline 5 & 8.08 & 575 & 5 \\
\hline 6 & 9.62 & 565 & 5 \\
\hline 7 & 10.68 & 559 & 6 \\
\hline 8 & 11.44 & 591 & 5 \\
\hline Averages - & $\rightarrow$ & 581.3 & 586.3 \\
\hline
\end{tabular}

Run No. GT-14

Fan Configuration Fan B only

Fan Setting 60

Stack Temp

Hz

Start/End Tme 11:16/12:45

Center $2 / 3$ from

$83.65 \operatorname{deg} F$

Points in Center $2 / 3$ 1.10 to: 10.84

Injection Point C-Top

2 to: 7

\begin{tabular}{l|ll|}
\cline { 2 - 4 } & 2nd & \\
\hline
\end{tabular}

3

Mean

3

Mean

\begin{tabular}{|l|l|l|r|r|r|r|}
\hline ppb & & \multicolumn{5}{|c|}{ ppb } \\
\hline 595 & 575 & $\mathbf{5 8 7 . 7}$ & 607 & 604 & 583 & $\mathbf{5 9 8 . 0}$ \\
\hline 599 & 622 & $\mathbf{6 0 3 . 7}$ & 587 & 617 & 604 & $\mathbf{6 0 2 . 7}$ \\
\hline 605 & 586 & $\mathbf{6 0 0 . 3}$ & 650 & 596 & 613 & $\mathbf{6 1 9 . 7}$ \\
\hline 584 & 578 & $\mathbf{5 8 6 . 3}$ & 602 & 595 & 599 & $\mathbf{5 9 8 . 7}$ \\
\hline 563 & 614 & $\mathbf{5 7 6 . 3}$ & 571 & 576 & 566 & $\mathbf{5 7 1 . 0}$ \\
\hline 550 & 593 & $\mathbf{5 7 2 . 7}$ & 581 & 600 & 555 & $\mathbf{5 7 8 . 7}$ \\
\hline 581 & 548 & $\mathbf{5 6 4 . 7}$ & 561 & 555 & 584 & $\mathbf{5 6 6 . 7}$ \\
\hline 613 & 613 & $\mathbf{5 9 5 . 0}$ & 554 & 548 & 579 & $\mathbf{5 6 0 . 3}$ \\
\hline 587 & 569 & $\mathbf{5 8 2 . 3}$ & 573 & 569 & 540 & $\mathbf{5 6 0 . 7}$ \\
\hline & $\mathbf{5 8 8 . 7}$ & $\mathbf{5 8 5 . 4}$ & $\mathbf{5 8 7 . 3}$ & $\mathbf{5 8 4 . 4}$ & $\mathbf{5 8 0 . 3}$ & $\mathbf{5 8 4 . 0}$ \\
\hline
\end{tabular}

\begin{tabular}{|lr}
\hline All & ppb \\
Mean & 584.7 \\
Mn Point & 560.3 \\
Max Point & 619.7 \\
\hline
\end{tabular}

Avg. Conc.

$586.13 \mathrm{ppb}$

619.7

\begin{tabular}{r|l} 
Dev. from mean & Center 2/3 \\
$-4.2 \%$ & Mean \\
$6.0 \%$ & Std. Dev. \\
COV as \%
\end{tabular}

\begin{tabular}{|c|c|c|}
\hline Side & Bottom & Al \\
\hline 585.6 & 585.4 & 585.5 \\
\hline 14.9 & 21.9 & 18.0 \\
\hline 2.5 & 3.7 & 3.1 \\
\hline
\end{tabular}

\section{Instuments Used:}

B\&K 1302 Gas Analyzer SN $1765299 \quad$ Cat2 M\&TE

TSI VelociCalc SN T95351203001 $1 / 17 / 2013$

Tracer tank pressure Injection flowmeter Stack Temp

Mean stack velocity Sampling flowmeter Ambient pressure Ambient humidity Ambient Temp B\&K vapor correction Back-Gd gas

No. Bk-Gd samples

\begin{tabular}{|c|c|c|}
\hline Start & Finish & \\
\hline 250 & 250 & \\
\hline 30 & 30 & \\
\hline 81.6 & 85.7 & \\
\hline 2997 & 3000 & \\
\hline 5 & 5 & \\
\hline 1008 & 1007 & \\
\hline $31 \%$ & $26 \%$ & \\
\hline 75.2 & 79.7 & \\
\hline$Y$ & $Y$ & \\
\hline $6,12,9,5,6$ & $10,11,8,11,5$ & \\
\hline 5 & 5 & \\
\hline
\end{tabular}

Gas anahzer checked:

$6 / 19 / 2012$

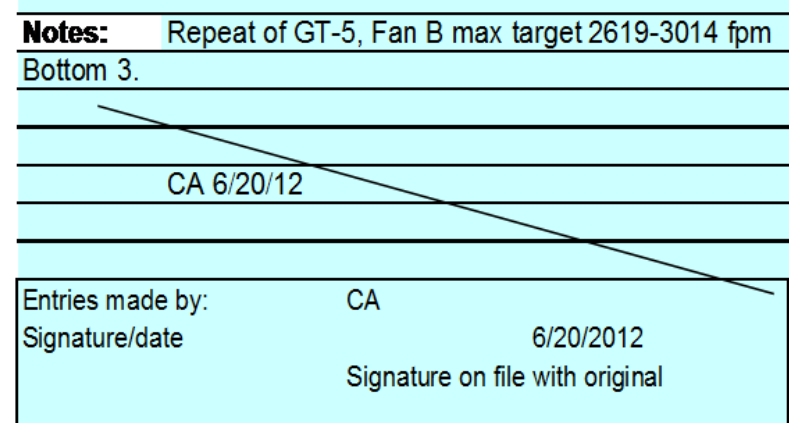
Fisher Scientific SN 90936818 $12 / 7 / 2012$

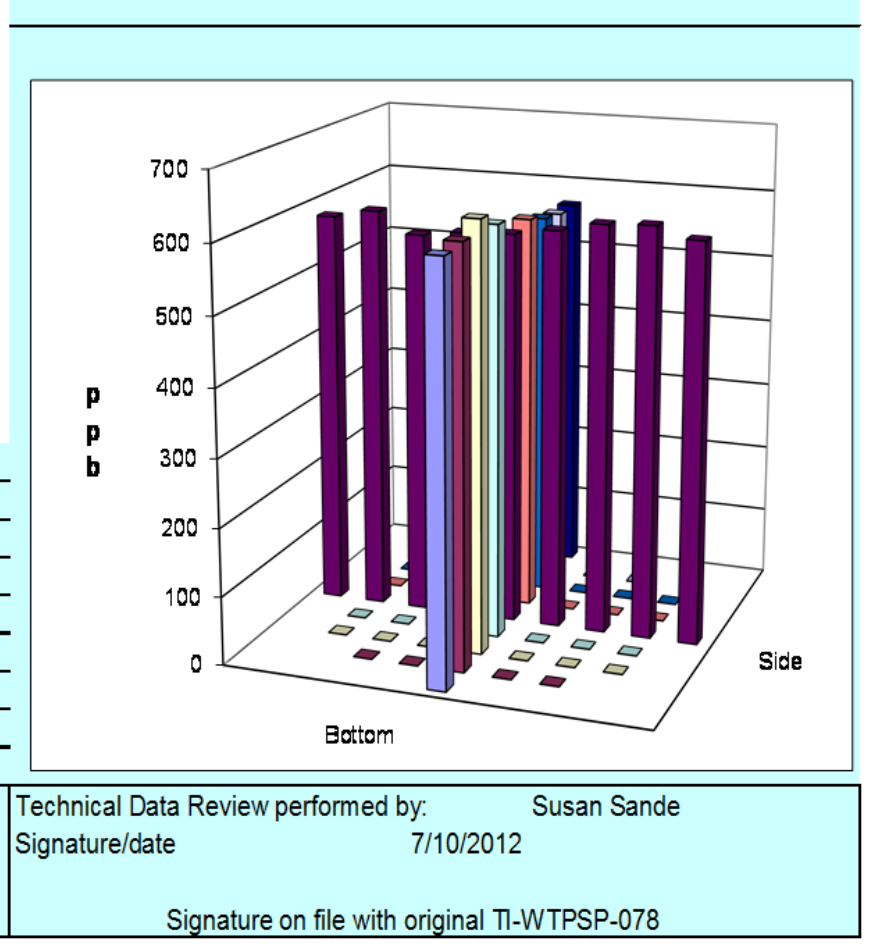


Rev. 0

31-JuL06

\begin{aligned} Site HV-S2 Model & \multicolumn{6}{c}{$\begin{array}{r}6 / 22 / 2012 \\ \text { Date }\end{array}$} \\ Testers & $\mathrm{CA}, \mathrm{XY} \\$ Stack Dia. & 11.938 in. \\ Stack X-Area & $\mathbf{1 1 1 . 9 \text { in. } ^ { 2 }} \\$ Test Port & 2\end{aligned}

Distance to disturbance 96.4 inches

Measurement units pgb SF6

TRACER GAS TRAYERSE DATA FORI

Order $\rightarrow$

Traverse $\rightarrow$

2 2nd

2nd

Run No. GT-15

Fan Configuration Fan B only

\begin{tabular}{|c|c|c|c|}
\hline \multicolumn{3}{|c|}{ Fan Configuration Fan B only } & \\
\hline Fan Setfing 57.7 & \multicolumn{2}{|c|}{ Hz } & \\
\hline Stack Temp & \multicolumn{2}{|c|}{$87 \operatorname{deg} F$} & \\
\hline \multicolumn{3}{|c|}{ Start/End Tme 915/1100 } & \\
\hline Center $2 / 3$ from & 1.10 & to: & 10.84 \\
\hline Points in Center $2 / 3$ & 2 & to: & 7 \\
\hline
\end{tabular}

Points in Center 2/3
Injection Point C- Near

\begin{tabular}{|c|c|c|c|c|c|c|}
\hline$A$ AlI & $\mathrm{ppb}$ & Dev. from mean & Center 2/3 & Side & Bottom & A] \\
\hline Mean & 600.5 & & Mean & 602.5 & 604.1 & 603.3 \\
\hline Mn Point & 569.0 & $-5.2 \%$ & Std. Dev. & 16.6 & 16.7 & 16.0 \\
\hline Max Point & 618.0 & $2.9 \%$ & COV as \% & 2.8 & 2.8 & 2.7 \\
\hline
\end{tabular}

Tíal $\longrightarrow$

\begin{tabular}{|c|c|}
\hline Point & Depth, in. \\
\hline 1 & 0. \\
\hline 2 & 1. \\
\hline 3 & 2. \\
\hline 4 & 3. \\
\hline Center & 5. \\
\hline 5 & 8. \\
\hline 6 & 9. \\
\hline 7 & 10. \\
\hline 8 & 11. \\
\hline
\end{tabular}

Avg. Conc. $\quad 598.92 \mathrm{ppb}$

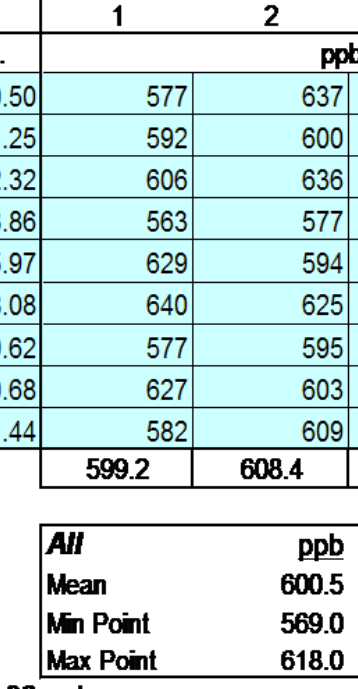

Tracer tank pressure Injection flowmeter Stack Temp

Mean stack velocity Sampling flowmeter Ambient pressure Ambient humidity Ambient Temp B\&K vapor correction Back-Gd gas

No. Bk-Gd samples

\begin{tabular}{|c|c|c|}
\hline Start & Finish & \\
\hline 250 & 250 & \\
\hline 30 & 30 & \\
\hline 85.7 & 87.9 & \\
\hline 3009 & 2937 & \\
\hline 5 & 5 & \\
\hline 991 & 991 & \\
\hline $27 \%$ & $27 \%$ & \\
\hline 90.5 & 89.6 & \\
\hline$Y$ & $Y$ & \\
\hline $\begin{array}{c}0,-6,-6,-5 \\
-9\end{array}$ & $5,3,9,5,5$ & \\
\hline 5 & 5 & \\
\hline
\end{tabular}

Gas analyzer checked: $\quad$ 6/12/2012

Notes: $\quad$ Repeat of GT-6
Entries made by: Signature/date

\section{Instuments Used:}

B\&K 1302 Gas Analyzer SN 1765299

TSI VelociCalc SN T95351203001 $\quad$ Cat2 M\&TE

Fisher Scientific SN 90936818

$12 / 7 / 2012$

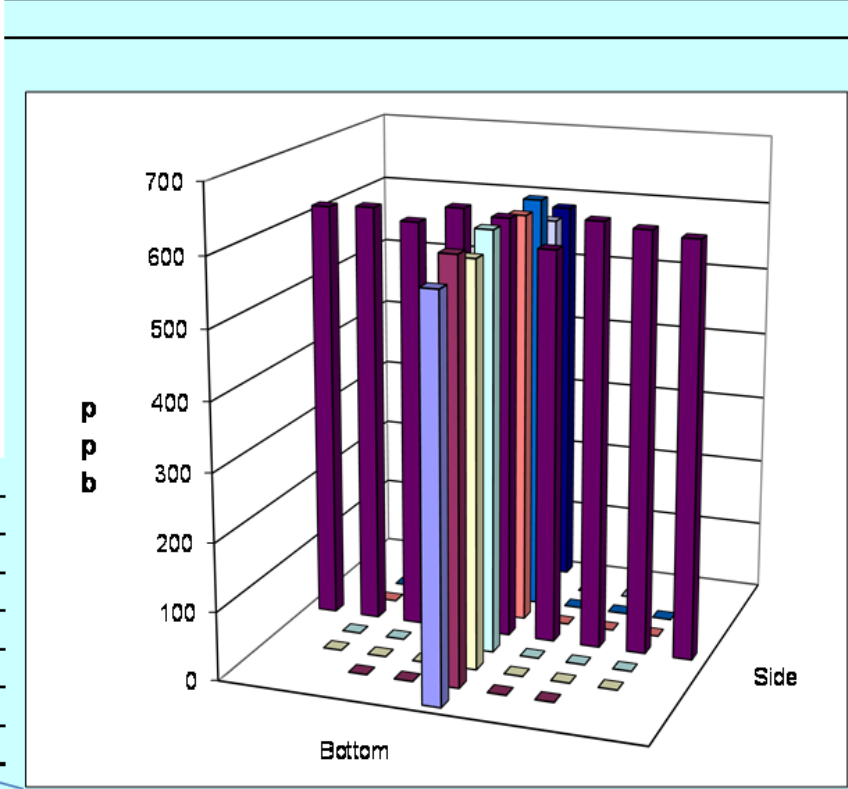

Technical Data Review performed by: Susan Sande
Signature/date
\[ / 10 / 2012 \]
Signature on file with original TI-WTPSP-078 
Rev. 0

31-JuL06

\begin{aligned} Site HV-S2 Model & \multicolumn{1}{c}{$\frac{6 / 22 / 2012}{\text { Date }}$} \\ Testers & CAXY \\ Stack Dia. & 11.938 in. \\ Stack X-Area & 111.9 in. $^{2} \\$ Test Port & 2\end{aligned}

Distance to disturbance 96.4 inches

Measurement units pob SF6

TRACER GAS TRAVERSE DATA FORI

Order $\rightarrow$

Traverse $\rightarrow$

Trial $\longrightarrow$

\begin{tabular}{|r|r|r|r|}
\multicolumn{2}{c|}{} & \multicolumn{2}{c|}{$\mathbf{1}$} \\
\hline Point & Depth, in. & \multicolumn{2}{c|}{} \\
\hline $\mathbf{1}$ & 0.50 & 587 & 57 \\
\hline $\mathbf{2}$ & 1.25 & 604 & 5 \\
\hline $\mathbf{3}$ & 2.32 & 579 & 6 \\
\hline $\mathbf{4}$ & 3.86 & 595 & 5 \\
\hline Center & 5.97 & 587 & 5 \\
\hline $\mathbf{5}$ & 8.08 & 559 & 5 \\
\hline $\mathbf{6}$ & 9.62 & 560 & 5 \\
\hline $\mathbf{7}$ & 10.68 & 579 & 5 \\
\hline $\mathbf{8}$ & 11.44 & 550 & 5 \\
\hline & & $\mathbf{5 7 7 . 8}$ & $\mathbf{5 8 1 . 3}$ \\
\hline Averages
\end{tabular}

Run No. GT-16

Fan Configuration Fan B only

Fan Setting $59.1 \quad$ Hz

Stack Temp

$89 \operatorname{deg} F$

Start/End Trme 11:11/13:00

Center $2 / 3$ from

Points in Center $2 / 3$

1.10 to: 10.84

Injection Point C - Far

2 to: 7

$2 n$

\begin{tabular}{|c|c|c|c|c|c|}
\hline 3 & Mean & 1 & 2 & 3 & Mean \\
\hline \multicolumn{2}{|l|}{ ppb } & \multicolumn{4}{|c|}{ ppb } \\
\hline 594 & 584.3 & 616 & 570 & 572 & 586.0 \\
\hline 593 & 594.7 & 606 & 545 & 626 & 592.3 \\
\hline 593 & 596.0 & 602 & 592 & 578 & 590.7 \\
\hline 564 & 578.0 & 595 & 580 & 601 & 592.0 \\
\hline 579 & 588.0 & 626 & 606 & 599 & 610.3 \\
\hline 592 & 569.7 & 587 & 602 & 567 & 585.3 \\
\hline 590 & 581.0 & 557 & 574 & 605 & 578.7 \\
\hline 593 & 587.7 & 585 & 614 & 585 & 594.7 \\
\hline 571 & 554.3 & 608 & 566 & 580 & 584.7 \\
\hline 35 & 581.5 & 98.0 & 5832 & 590.3 & 90.5 \\
\hline
\end{tabular}

\begin{tabular}{|c|c|c|c|c|c|}
\hline \multirow{2}{*}{\multicolumn{2}{|c|}{ Mean }} & \\
\hline & & 1 & 2 & 3 & Mean \\
\hline \multicolumn{2}{|l|}{$p p b$} & \multicolumn{4}{|c|}{$p p b$} \\
\hline 594 & 584.3 & 616 & 570 & 572 & $586 . C$ \\
\hline 593 & 594.7 & 606 & 545 & 626 & 592. \\
\hline 593 & 596.0 & 602 & 592 & 578 & 590.7 \\
\hline 564 & 578.0 & 595 & 580 & 601 & 592. \\
\hline 579 & 588.0 & 626 & 606 & 599 & 610. \\
\hline 592 & 569.7 & 587 & 602 & 567 & 585. \\
\hline 590 & 581.0 & 557 & 574 & 605 & 578.7 \\
\hline 593 & 587.7 & 585 & 614 & 585 & 594.7 \\
\hline 571 & 554.3 & 608 & 566 & 580 & 584.7 \\
\hline 585.4 & 581.5 & 598.0 & 5832 & 590.3 & 590.5 \\
\hline
\end{tabular}

Bottom

Avg. Conc.

\begin{tabular}{|lr}
\hline All & ppb \\
Mean & 586.0 \\
Min Point & 554.3 \\
Max Point & 610.3 \\
\hline
\end{tabular}

\section{$584.38 \mathrm{ppb}$}

\begin{tabular}{|c|c|}
\hline Dev. from mean & Center 2/3 \\
\hline $\begin{array}{r}-5.4 \% \\
4.1 \%\end{array}$ & $\begin{array}{l}\text { Mean } \\
\text { Std. Dev. } \\
\text { CON as \% }\end{array}$ \\
\hline
\end{tabular}

\begin{tabular}{rrr|} 
Side & Bottom & Al \\
585.0 & 592.0 & 588.5 \\
9.4 & 9.7 & 9.9 \\
1.6 & 1.6 & 1.7 \\
\hline
\end{tabular}

\section{Instuments Used:}

\begin{tabular}{lrr} 
B\&K 1302 Gas Analyzer SN $\quad 1765299$ & Cat2 M\&TE \\
\hline TSI VelociCalc SN T95351203001 & $1 / 17 / 2013$ \\
\hline Fisher Scientific SN 90936818 & $12 / 7 / 2012$ \\
\hline
\end{tabular}

Tracer tank pressure Injection flowmeter Stack Temp Mean stack velocity Sampling flowmeter Ambient pressure Ambient humidity Ambient Temp B\&K vapor correction Back-Gd gas

No. Bk-Gd samples

\begin{tabular}{|c|c|}
\hline Start & Finish \\
\hline 250 & 250 \\
\hline 30 & 30 \\
\hline 86.3 & 91.3 \\
\hline 2912 & 2974 \\
\hline 5 & 5 \\
\hline 991 & 990 \\
\hline $27 \%$ & $27 \%$ \\
\hline 89.6 & 88.7 \\
\hline$Y$ & $Y$ \\
\hline $7,5,8,12,8$ & $7,1,7,1,5$ \\
\hline 5 & 5 \\
\hline
\end{tabular}

Gas anahzer checked:

\section{$6 / 19 / 2012$}

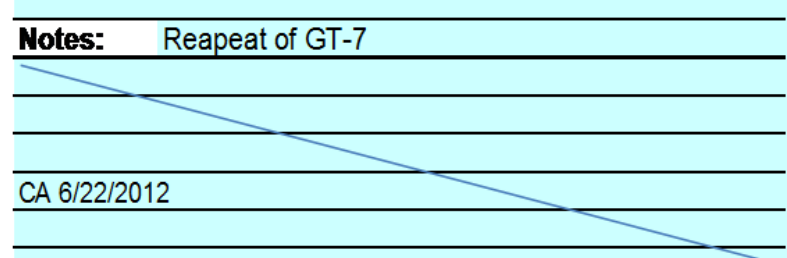

Carmen Arimescu

Signature on file with original
6/22/2012 Technical Data Review performed by: Signature/date

Signature on file with original TT-WTPSP-078 
Rev. 0

31-Jul-06

\begin{tabular}{lr}
\multicolumn{1}{c}{} & $\begin{array}{r}\text { Date } \\
\text { Testers } \\
\text { Stack Dia } \\
\text { Stack X-Area } \\
\text { Test Por }\end{array}$ \\
Distance to disturbance \\
Measurement units \\
Order --> \\
Traverse--> \\
Trial ----> \\
\hline Point & Depth, in. \\
\hline 1 & 0.50 \\
\hline 2 & 1.25 \\
\hline 3 & 2.32 \\
\hline 4 & 3.86 \\
\hline Center & 5.97 \\
\hline 5 & 8.08 \\
\hline 6 & 9.62 \\
\hline 7 & 10.68 \\
\hline 8 & 11.44 \\
\hline Averages -------->
\end{tabular}

$5 / 1 / 2012$

K X-Area

\begin{tabular}{c}
\hline 11.938 in. \\
\hline 111.9 in. $^{2}$ \\
\hline 2 \\
\hline
\end{tabular}

$$
\frac{96.4}{\text { ppb SF6 }}
$$
\begin{tabular}{|r|}
\hline 1 st \\
\hline
\end{tabular}

pp

TRACER GAS TRAVERSE DATA FORM

Run No. GT-2

Fan Configuration Fan B only

Fan Setting 26

$\mathrm{Hz}$

Points in Center $2 / 3$

Stack Temp

Start/End Time $1013 / 1130$

Center $2 / 3$ from

Injection Point E-Center

$67.2 \operatorname{deg} F$

1.10 to: 10.84

2 to: 7

Injection Point E-Center \begin{tabular}{l|ll|}
\cline { 2 - 3 } \multicolumn{1}{c|}{} & 2nd & \\
\hline
\end{tabular} \begin{tabular}{llll|llll}
1 & 2 & 3 & Mean & 1 & 2 & 3 & Mean \\
\hline
\end{tabular}

\begin{tabular}{|r|r|r|r|r|r|r|}
\hline $\mathrm{ppb}$ & & \multicolumn{5}{|c|}{$\mathrm{ppb}$} \\
\hline 740 & 732 & 724.0 & 736 & 735 & 751 & 740.7 \\
\hline 716 & 731 & 730.0 & 727 & 729 & 706 & 720.7 \\
\hline 730 & 737 & 721.3 & 747 & 713 & 737 & 732.3 \\
\hline 768 & 770 & 759.7 & 727 & 744 & 754 & 741.7 \\
\hline 711 & 729 & 720.0 & 729 & 762 & 727 & 739.3 \\
\hline 722 & 710 & 736.3 & 677 & 715 & 739 & 710.3 \\
\hline 727 & 718 & 715.0 & 725 & 750 & 706 & 727.0 \\
\hline 718 & 712 & 719.7 & 737 & 753 & 733 & 741.0 \\
\hline 719 & 725 & 712.0 & 703 & 738 & 726 & 722.3 \\
\hline & 729.3 & 726.4 & 723.1 & 737.7 & 731.0 & 730.6 \\
\hline
\end{tabular}

Avg. Conc. $728.38 \mathrm{ppb}$

\begin{tabular}{|lr}
\hline All & $\mathrm{ppb}$ \\
Mean & 728.5 \\
Min Point & 710.3 \\
Max Point & 759.7 \\
\hline
\end{tabular}

Tracer tank pressure Injection flowmeter Stack Temp

Mean stack velocity Sampling flowmeter Ambient pressure Ambient humidity Ambient Temp B\&K vapor correction Back-Gd gas

No. Bk-Gd samples

\begin{tabular}{|c|c|c|}
\hline Start & Finish & \\
\hline 125 & 125 & \\
\hline 15 & 15 & \\
\hline 65.8 & 68.6 & \\
\hline 1042 & 1087 & \\
\hline 5 & 5 & \\
\hline 1002 & 1002 & \\
\hline $28 \%$ & $24 \%$ & \\
\hline 72.5 & 76.1 & \\
\hline $\mathrm{Y}$ & $Y$ & \\
\hline $\begin{array}{c}9,11,11,10,1 \\
0\end{array}$ & $3,7,8,10,8$ & \\
\hline 5 & 5 & \\
\hline
\end{tabular}

Gas analyzer checked: $4 / 30 / 2012$

CA $5 / 1 / 12$

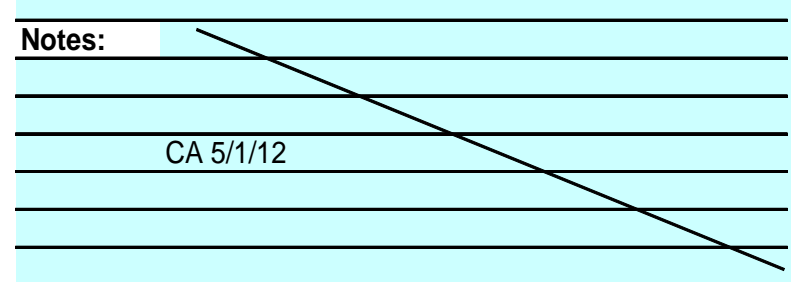
Entries made by: Signature/date

\begin{tabular}{r|l} 
Dev. from mean & Center $2 / 3$ \\
\hline & Mean \\
$-2.5 \%$ & Std. Dev. \\
$4.3 \%$ & CoV as \% \\
\hline
\end{tabular}

Instuments Used:

B\&K 1302 Gas Analyzer SN TSI VelociCalc SN T95351203001

Fisher Scientific SN 90936818

\begin{tabular}{rrr|} 
Side & Bottom & All \\
& 730.3 & 729.6 \\
15.4 & 11.8 & 13.2 \\
2.1 & 1.6 & $\mathbf{1 . 8}$ \\
\hline
\end{tabular}

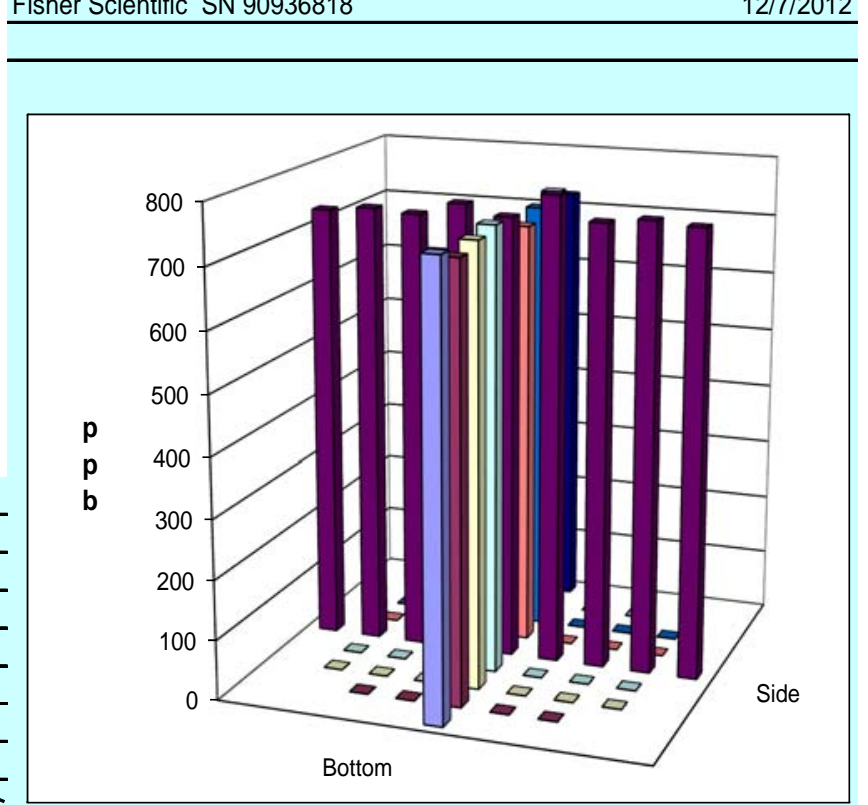

Technical Data Review performed by: Signature/date
7/10/2012

Signature on file with original 


\section{B.5 HV-S2 Particle Tracer Uniformity Data Sheets}

Rev. 0

3 Aug.

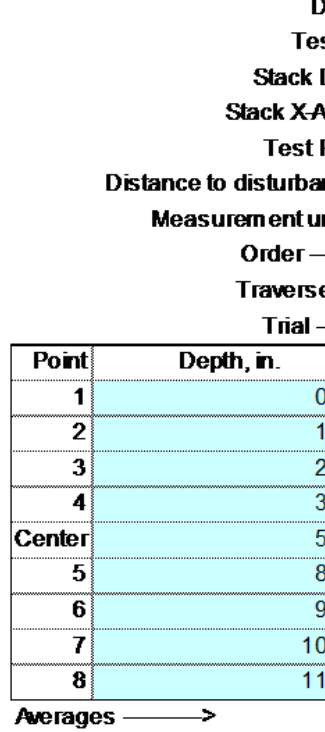

Site HV-S2 Model

Date $5 / 7 / 2012$

Tester JAG, XYY

Stack Dia. 11.938 in.

ack XArea

est Port 111.9 in. 2

2$$
96.4 \text { inches }
$$

les/43

Order $\longrightarrow$ 2nd

verse $\rightarrow$

Side

\section{PARTICL IRACER IRAVERE DATA FORU}

\author{
Run No. PT-1
}

Fan configuration $A$

Fan Setting 60

Stack Temp

Start/End Time 13:30/15:38

Center 23 from

Points in Center 2/3

Injection Point E Center

Injedion Point

$6 \operatorname{deg} F$

$\frac{10}{2}$

to: $\quad \mathbf{1 0 . 8 4}$

3 Mean $\quad \begin{array}{llll} & & & \text { Bottom }\end{array}$

\begin{tabular}{|r|r|r|r|r|r|}
\multicolumn{1}{|c|}{ Mean } & $\mathbf{1}$ & $\mathbf{2}$ & \multicolumn{1}{c|}{$\mathbf{3}$} \\
\hline 1319 & $\mathbf{1 2 5 6 . 0}$ & 1329 & 1370 & 1289 & $\mathbf{1 3 2 9 . 3}$ \\
\hline 1398 & $\mathbf{1 3 4 6 . 0}$ & 1714 & 1624 & 1663 & $\mathbf{1 6 6 7 . 0}$ \\
\hline 1392 & $\mathbf{1 3 8 0 . 3}$ & 1688 & 1741 & 1657 & $\mathbf{1 6 9 5 . 3}$ \\
\hline 1357 & $\mathbf{1 2 6 7 . 0}$ & 1680 & 1646 & 1696 & $\mathbf{1 6 7 4 . 0}$ \\
\hline 1335 & $\mathbf{1 3 8 6 . 3}$ & 1581 & 1608 & 1495 & $\mathbf{1 5 6 1 . 3}$ \\
\hline 1236 & $\mathbf{1 2 9 7 . 7}$ & 1344 & 1261 & 1415 & $\mathbf{1 3 4 0 . 0}$ \\
\hline 1149 & $\mathbf{1 1 5 0 . 7}$ & 1222 & 1306 & 1332 & $\mathbf{1 2 8 6 . 7}$ \\
\hline 943 & $\mathbf{9 9 2 . 3}$ & 1178 & 1122 & 1150 & $\mathbf{1 1 5 0 . 0}$ \\
\hline 887 & $\mathbf{8 5 4 . 3}$ & 981 & 986 & 1098 & $\mathbf{1 0 2 1 . 7}$ \\
\hline $\mathbf{1 2 2 4 . 0}$ & $\mathbf{1 2 1 4 . 5}$ & $\mathbf{1 4 1 3 . 0}$ & $\mathbf{1 4 0 7 . 1}$ & $\mathbf{1 4 2 1 . 7}$ & $\mathbf{1 4 1 3 . 9}$ \\
\hline
\end{tabular}

\begin{tabular}{|c|c|c|c|c|c|c|c|}
\hline $\mathbf{A U}$ & $\mathrm{pt} / \mathrm{ft3}$ & Dev. from mean & Center 2/3 & Side & Bottom & $\underline{\text { A! }}$ & Nombd \\
\hline Mean & 13142 & & Mean & 1260.0 & 1482.0 & 1371.0 & 1450.58 \\
\hline Min Point & 854.3 & $-35.0 \%$ & Std Dev. & 143.1 & 220.4 & 212.5 & 188.35 \\
\hline Max Point & 1695.3 & $29.0 \%$ & covas $\%$ & 11.4 & 14.9 & 15.5 & 12.98 \\
\hline
\end{tabular}

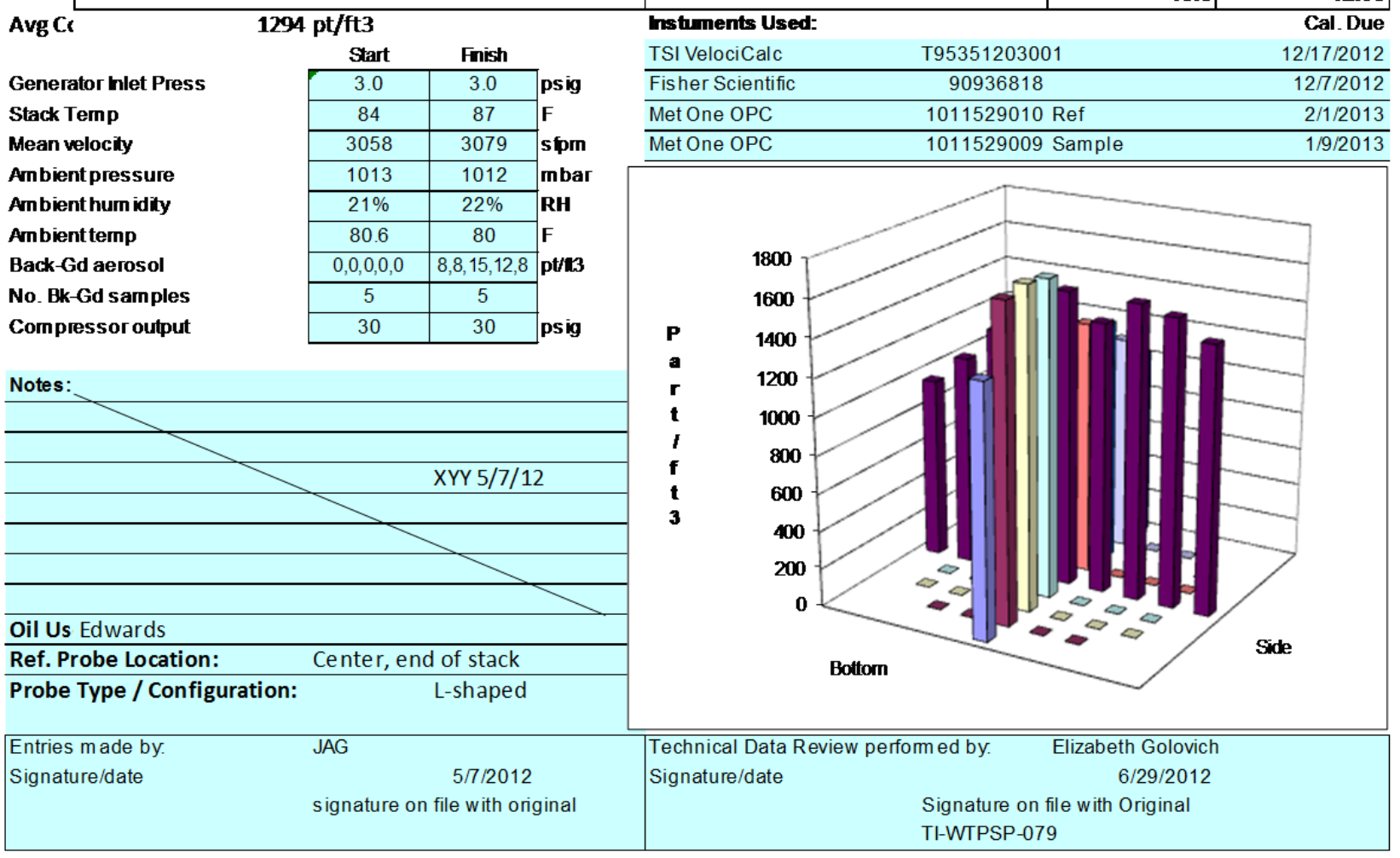


Rev. 0

3 Aug. 2006
PARTICLE TRACER TRAVERE DATA FORM

Site HV-S2 Model

Date 5/8/2012

Tester CA, JEF, XYY

Stack Dia. 11.938 in.

Stack X-Area 1119 in

Test Port

2

Distance to disturbance 96.4 inches

Measurement units parficles/13

Order $\longrightarrow$

Traverse->

Trial $\rightarrow-\rightarrow$

\begin{tabular}{|r|r|}
\hline Point & Depth, in. \\
\hline $\mathbf{1}$ & 0.50 \\
\hline $\mathbf{2}$ & 1.25 \\
\hline $\mathbf{3}$ & 2.32 \\
\hline $\mathbf{4}$ & 3.86 \\
\hline Center & 5.97 \\
\hline $\mathbf{5}$ & 8.08 \\
\hline $\mathbf{6}$ & 9.62 \\
\hline $\mathbf{7}$ & 10.68 \\
\hline $\mathbf{8}$ & 11.56 \\
\hline
\end{tabular}

Averages - - $>$
1 st
Center $2 / 3$ from

0.3

1261.7

Run No. PT-2

Fan confguration Fan B only

Fan Setting 60

Stack Temp

Start/End Time 8:30/11:01

Points in Center $2 / 3$

Injecion Point E Center
$72 \operatorname{deg} F$

Hz

2 to: $\quad \frac{10.84}{7}$

\section{2nd}

\begin{tabular}{|c|c|c|c|c|}
\hline & $\boldsymbol{A I I}$ & pt/fts & \multicolumn{2}{|c|}{ Dev. from } \\
\hline & Mean & 1471.0 & & \\
\hline & Min Point & 1111.0 & & \\
\hline & Max Point & 1873.7 & & \\
\hline \multicolumn{5}{|c|}{$1461 \mathrm{pt} / \mathrm{ft} 3$} \\
\hline & & Start & Finsh & \\
\hline \multicolumn{2}{|c|}{ Generator Inlet Press } & 5.1 & 5.1 & psig \\
\hline \multicolumn{2}{|l|}{ Stack Temp } & 65.3 & 79.6 & $F$ \\
\hline \multicolumn{2}{|l|}{ Mean } & 3092 & 3053 & sfp \\
\hline \multicolumn{2}{|c|}{ Ambient pressure } & 1007 & 1008 & mb \\
\hline \multicolumn{2}{|c|}{ Ambient humidity } & $41 \%$ & $25 \%$ & RH \\
\hline \multicolumn{2}{|c|}{ Ambient temp } & 63.5 & 79.7 & F \\
\hline \multicolumn{2}{|c|}{ Back-Gd aerosol } & $11,10,13,5$ & $4,6,9,9,6$ & $\mathrm{pt} / \mathrm{i}$ \\
\hline \multicolumn{2}{|c|}{ No. Bk-Gd samples } & 4 & 5 & \\
\hline \multicolumn{2}{|c|}{ Compressor output } & 30 & 30 & psig \\
\hline
\end{tabular}

Notes: Heat tape set at $100 \mathrm{~F}$, does not appear to be heating much.

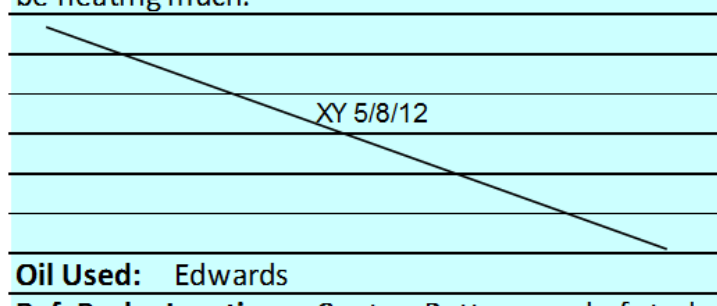

Ref. Probe Location: Center, Bottom, end of stack Probe Type / Configuration:

L-Shaped
$5 / 8 / 2012$

signature on file with original
$-24.5 \%$ Std. Dev. $27.4 \%$ covas $\%$

\begin{tabular}{|c|c|c|c|}
\hline \multicolumn{4}{|c|}{ 2nd } \\
\hline \multicolumn{4}{|c|}{ Bottom } \\
\hline 1 & 2 & 3 & Mean \\
\hline \multicolumn{4}{|c|}{ particles/fi3 } \\
\hline 1641 & 1692 & 1732 & 1688.3 \\
\hline 1766 & 1842 & 1894 & 1834.0 \\
\hline 1835 & 1879 & 1907 & 1873.7 \\
\hline 1835 & 1793 & 1921 & 1849.7 \\
\hline 1768 & 1800 & 1855 & 1807.7 \\
\hline 1808 & 1759 & 1837 & 1801.3 \\
\hline 1631 & 1675 & 1767 & 1691.0 \\
\hline 1595 & 1398 & 1407 & 1466.7 \\
\hline 1472 & 912 & 949 & 1111.0 \\
\hline 1705.7 & 1638.9 & 1696.6 & 1680.4 \\
\hline
\end{tabular}

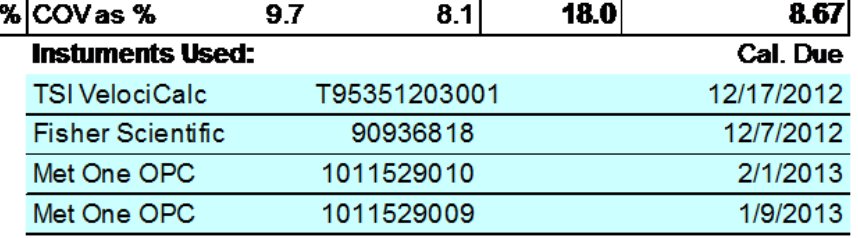

\begin{tabular}{|ll|l|}
\hline $\begin{array}{l}\text { Entries made by: } \\
\text { Signature/date }\end{array}$ & CA, XY $\begin{array}{c}5 / 8 / 2012 \\
\text { signature on file with original }\end{array}$ & $\begin{array}{l}\text { Technical Data Review performed by: Elizabeth Golovich } \\
\text { Signature/date }\end{array}$ \\
$\begin{array}{c}\text { 6/29/2012 } \\
\text { Signature on file with Original } \\
\text { TI-WTPSP-079 }\end{array}$ \\
\hline
\end{tabular}


Rev. 0

3 Aug. 2006

\begin{tabular}{|c|c|}
\hline Date & $5 / 8 / 2012$ \\
\hline Tester & $\mathrm{CA}, \mathrm{XY}$ \\
\hline Stack Dia. & 11.938 in. \\
\hline Stack X-Area & 111.9 in.2 \\
\hline Test Port & 2 \\
\hline istance to disturance & 96.4 inches \\
\hline
\end{tabular}

PARTICLE TRACER TRAVEREDATA FORM

Run No. PT-3

Fan configuration Fan B only

Fan Setting 22

Stack Temp

Start/End Time 11:0

Center $2 / 3$ fom 110

Points in Center $2 / 3 \frac{1.10}{2}$ to: $\frac{10.84}{7}$ Injection Point E Center

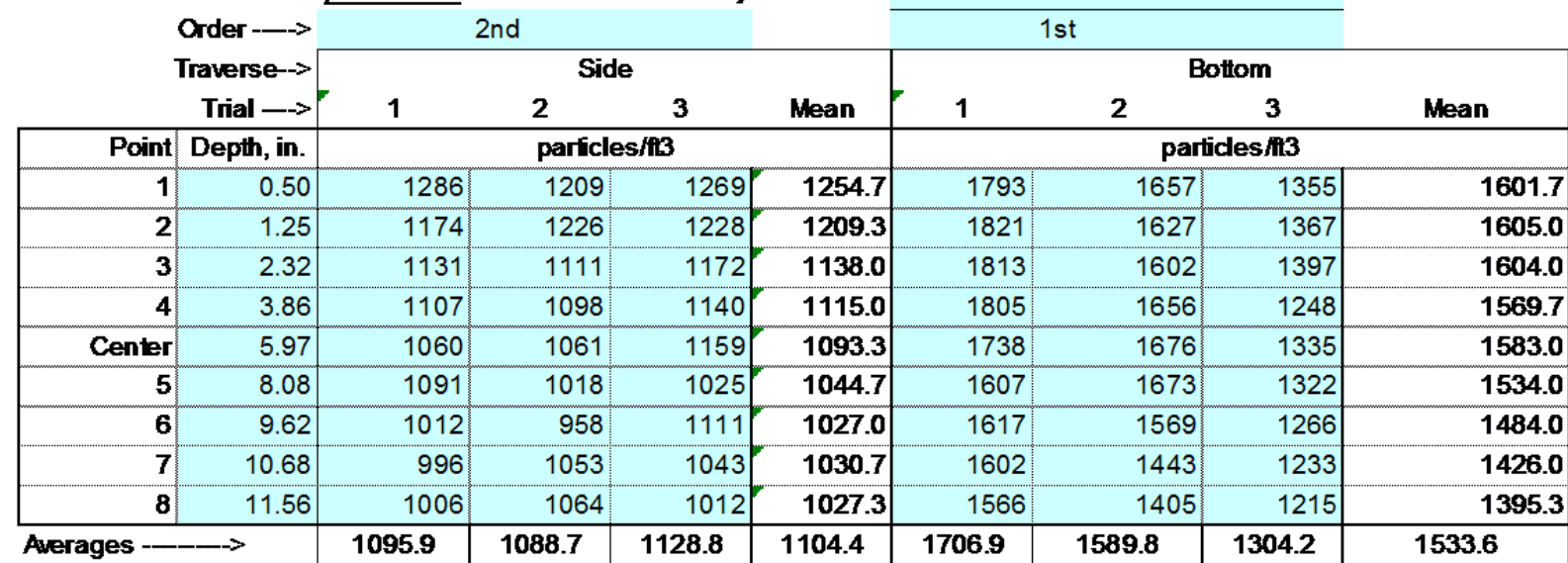

Averages

\begin{tabular}{|lr}
\hline All & pt/t/3 \\
Mean & 1319.0 \\
Min Point & 1027.0 \\
Max Point & 1605.0 \\
\hline
\end{tabular}

Avg Conc $1317 \mathrm{pt} / \mathrm{ft3}$

Generator hlet Press

Stack Temp

Mean velocity

Ambient pressure

Ambient humidity

Ambient temp

Back-Gd aerosol

No. Bk-Gd samples

Compressor output

\begin{tabular}{|c|c|}
\hline Start & Finish \\
\hline 2.5 & 2.5 \\
\hline 80.8 & 86.8 \\
\hline 1023 & 1045 \\
\hline 1008 & 1007 \\
\hline $23 \%$ & $22 \%$ \\
\hline 82.4 & 81.5 \\
\hline $6,9,2,5$ & $5,9,0,8$ \\
\hline 4 & 4 \\
\hline 30 & 30 \\
\hline
\end{tabular}

Notes:

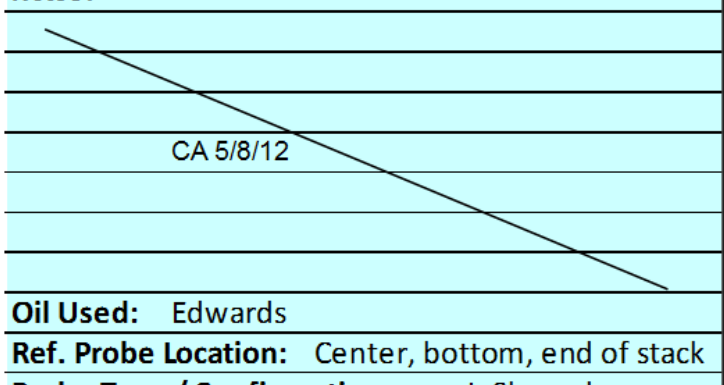

Probe Type / Configuration: L-Shaped

\begin{tabular}{r|lr} 
fom mean & Center 2/3 & Side \\
$-22.1 \%$ & Mean & 1094.0 \\
$21.7 \%$ & Std. Dev. & 66.6 \\
& COV as $\%$ & 6.1
\end{tabular}

\begin{tabular}{rrr|} 
Bottom & All & Normlzd \\
\hline 1543.7 & 1318.8 & 1563.82 \\
67.2 & 242.0 & 82.55 \\
\cline { 3 - 3 } & 18.4 & 5.28 \\
\hline
\end{tabular}

Instuments Used

\begin{tabular}{lcr} 
TSI VelociCalc & T95351203001 & $12 / 17 / 2012$ \\
\hline Fisher Scientific & 90936818 & $12 / 7 / 2012$ \\
\hline Met One OPC & 1011529010 & $2 / 1 / 2013$ \\
\hline Met One OPC & 1011529009 & $1 / 9 / 2013$ \\
\hline
\end{tabular}

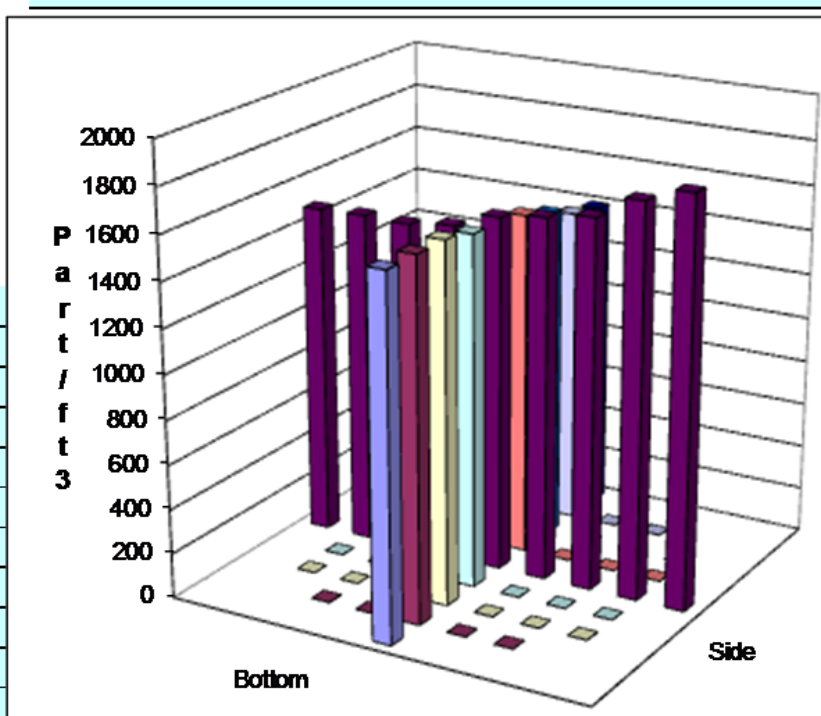

Entries made by:

$\mathrm{XY}, \mathrm{CA}$

$5 / 8 / 2012$

signature on file with original 
Rev. 0

3 Aug. 2006

\begin{tabular}{rr} 
Site HV-S2 Model \\
Date & \multicolumn{1}{|c|}{$5 / 8 / 2012$} \\
Tester & JAG, EA \\
Stack Dia. & 11.938 in. \\
\cline { 2 - 2 } Stack X-Area & $\mathbf{1 1 1 . 9}$ in $\mathbf{2}$ \\
\cline { 2 - 2 } Test Port & 2 \\
\cline { 2 - 2 } disturbance & 96.4 inches
\end{tabular}

Distance to disturbance

Measurement units particles/fis

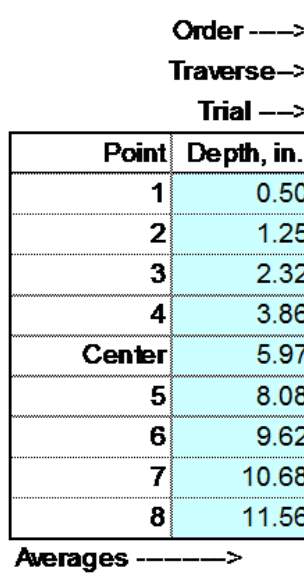

\section{PARTICLE TRACER TRAVERSE DATA FORM}

Run No. PT-4

Fan configuration $\mathrm{A}$ - min

Fan Setting 25

Stack Temp

Start/End Time 13.35/15:15

Center $2 / 3$ from

Points in Center $2 / 3$

Injection Point E Center

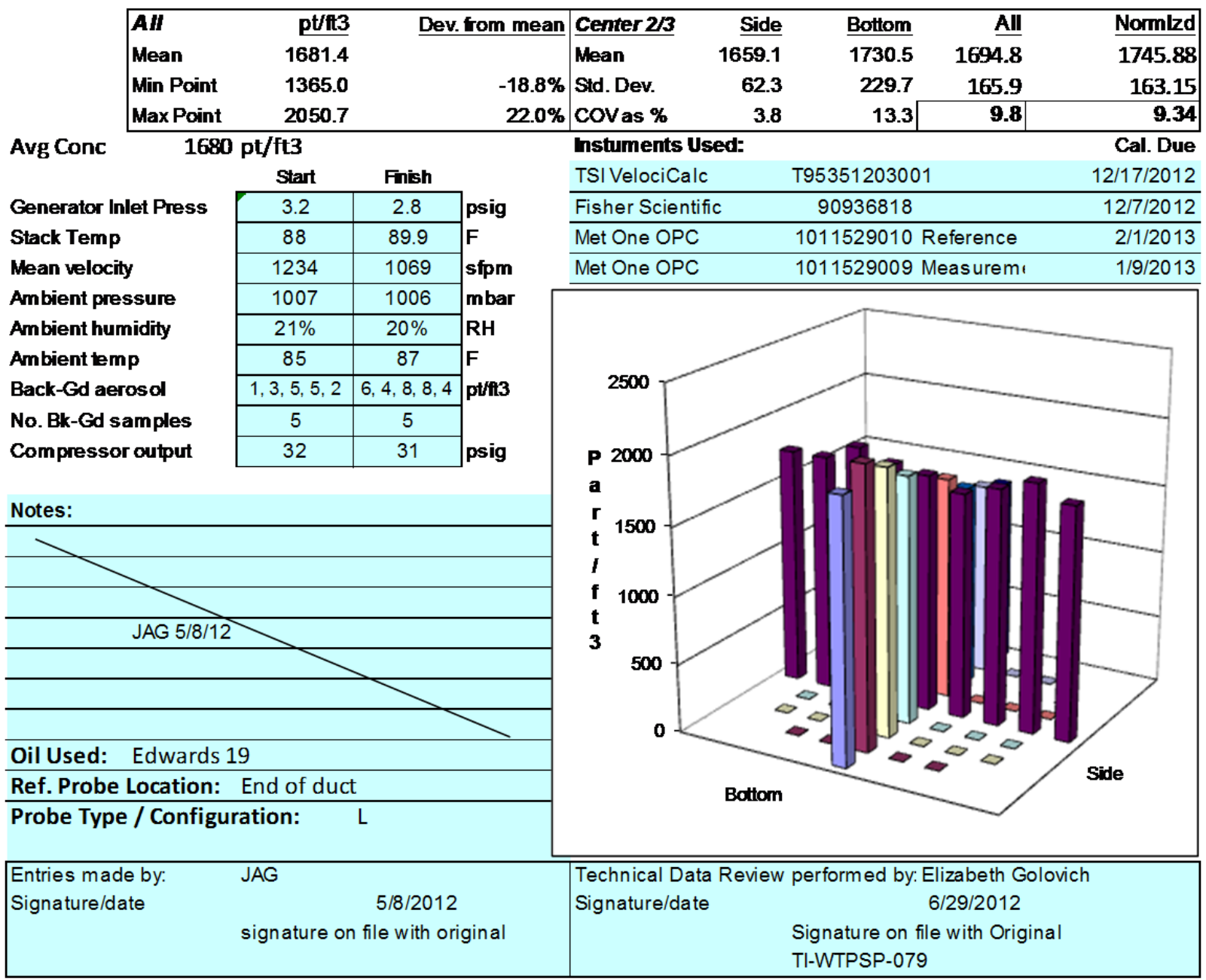


Rev. 0

3 Aug. 2006

PARTICLE TRACER TRAVERSE DATA FORM

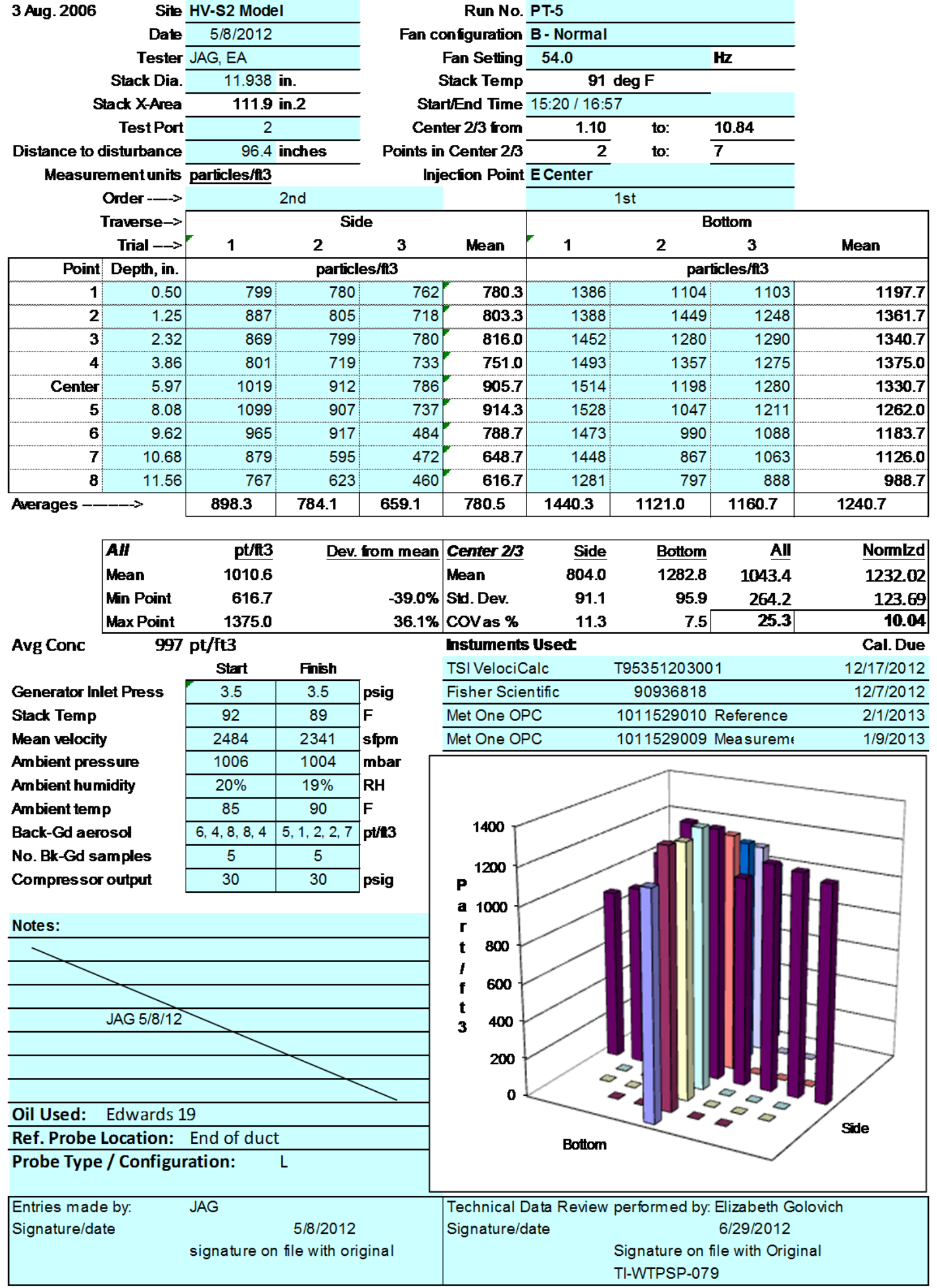


Rev. 0

3 Aug. 2006

\begin{aligned} Date & \multicolumn{1}{c}{$5 / 9 / 2012$} \\ Tester & XYY, YFS \\ Stack Dia. & $11.938 \mathrm{in} \\$. Stack X-Area & $\mathbf{1 1 1 . 9}$ in.2 \\ Test Port & 2 \\ \cline { 2 - 2 } Distance to disturbance & 96.4 inches \end{aligned}

PARTICLE TRACER TRAVERSE DATA FORM

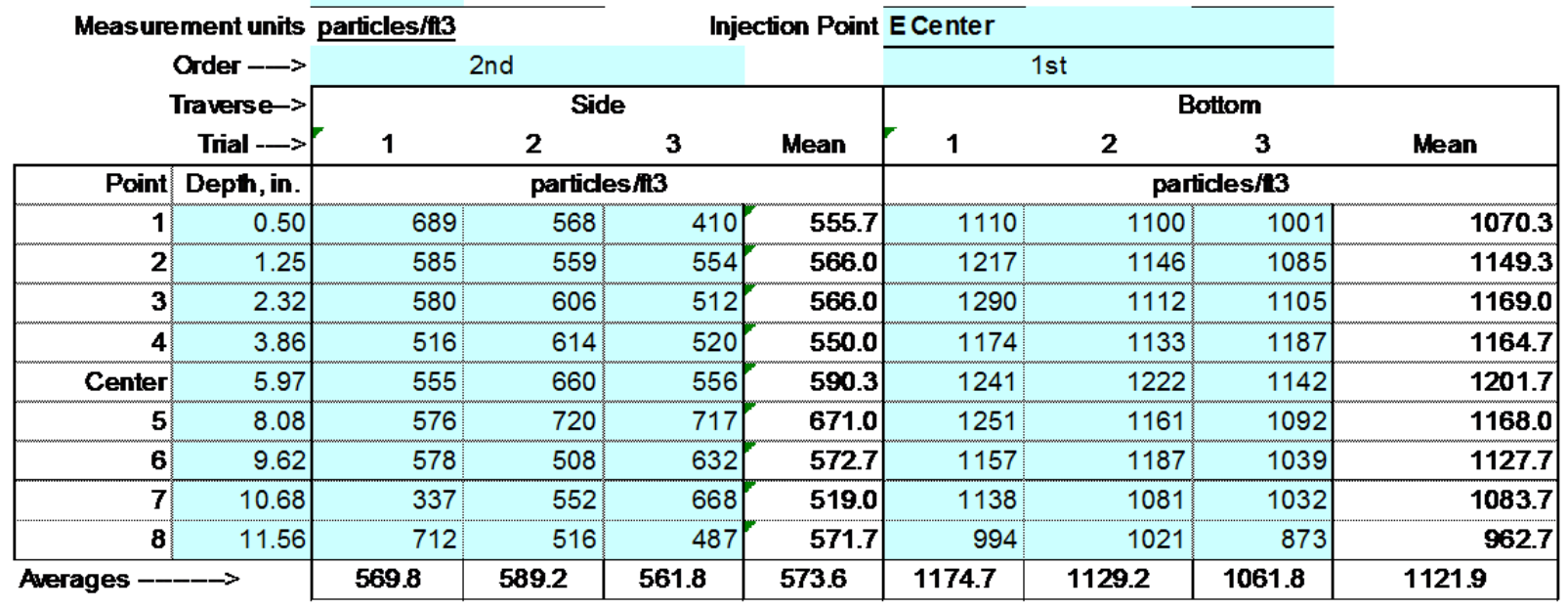

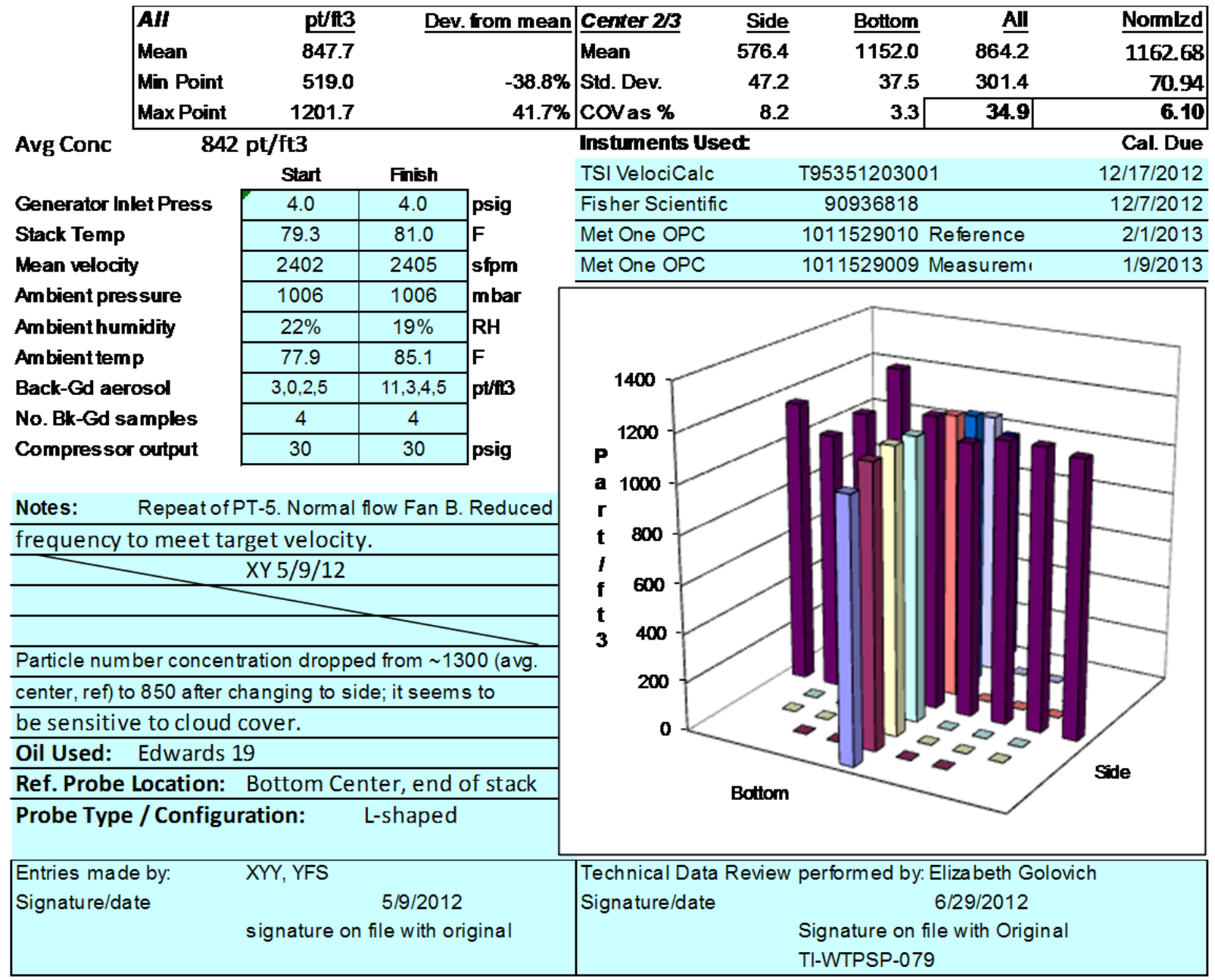

Fan configuration Fan B only

Fan Setting $48.0 \quad \mathrm{~Hz}$

Stack Temp $\mathbf{8 0} \mathbf{~ d e g ~ F}$

Start/End Time 13:10/15:10

to: 10.84

s in Center 2/3 2 to: 7

Injection Point E Center
Center 2/3 fom 110 
Rev. 0

3 Aug. 2006
PARTICLE TRACER TRAVERE DATA FORM

Site HV-S2 Model Date 5/10/2012

Tester YFS, EA

Stack Dia. 11.938 in.

Stack X-Area 111.9 in 2

Test Port

Distance to disturbance

Measurement units particles/13

Order $\rightarrow-$

Traverse-->

Trial $\rightarrow$

2

96.4 inches

1 st
Run No. PT-7

Fan configuration Fan A Max

Fan Setting

Stack Temp

$60.0 \quad \mathrm{~Hz}$

$67 \operatorname{deg} \mathrm{F}$

Start/End Time 11:00/12:34

Center $2 / 3$ from

Points in Center $2 / 3 \quad 2$

Injection Point ECenter

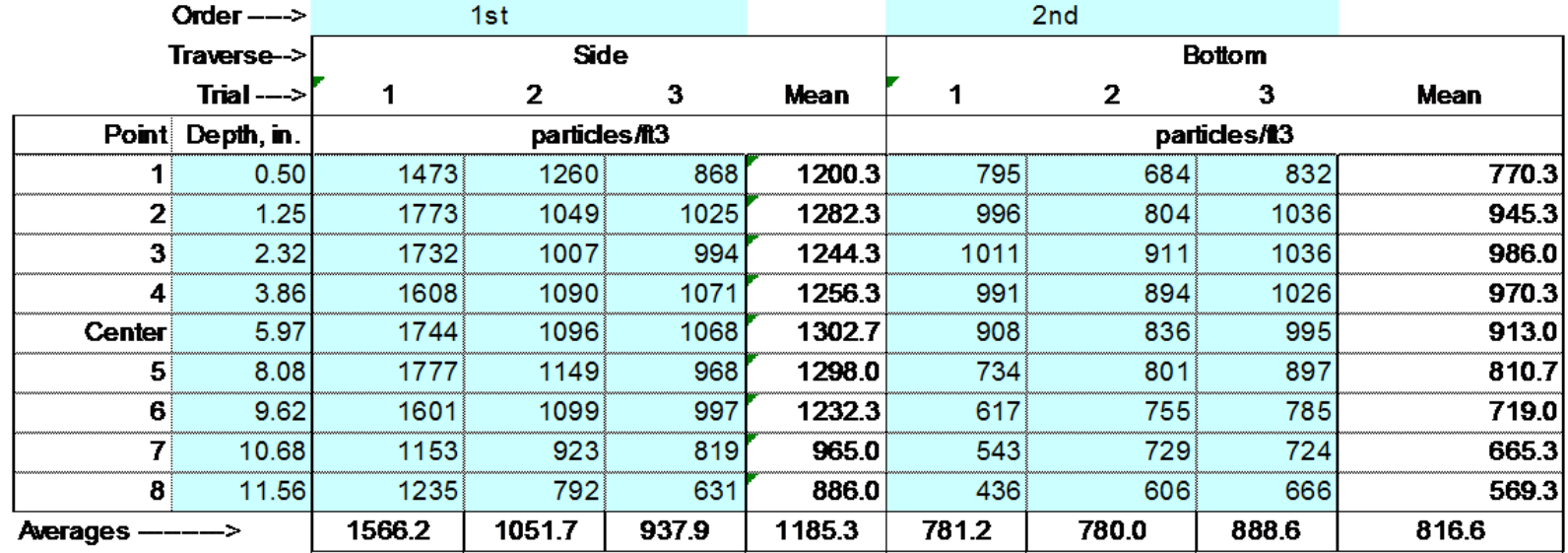

\begin{tabular}{|lr}
\hline All & pt/t3 \\
Mean & 1000.9 \\
Min Point & 569.3 \\
Max Point & 1302.7 \\
\hline
\end{tabular}

Avg Conc

$$
988 \mathrm{pt} / \mathrm{ft} 3
$$

Dev. from

Generator Inlet Press

Stack Temp

Mean velocity

Ambient pressure

Ambient humidity

Ambient temp

Back-Gd aerosol

No. Bk-Gd samples

Compressor output

\begin{tabular}{|c|c|}
\hline Start & Finish \\
\hline 7.0 & 6.9 \\
\hline 67.1 & 67.8 \\
\hline 3279 & 3107 \\
\hline 30.00 & 29.97 \\
\hline $22 \%$ & $19 \%$ \\
\hline 77.9 & 85.1 \\
\hline $3,0,2,5$ & $11,3,4,5$ \\
\hline 4 & 4 \\
\hline 30 & 30 \\
\hline
\end{tabular}

Notes: Stack velocity measu red at bottom port, point 5

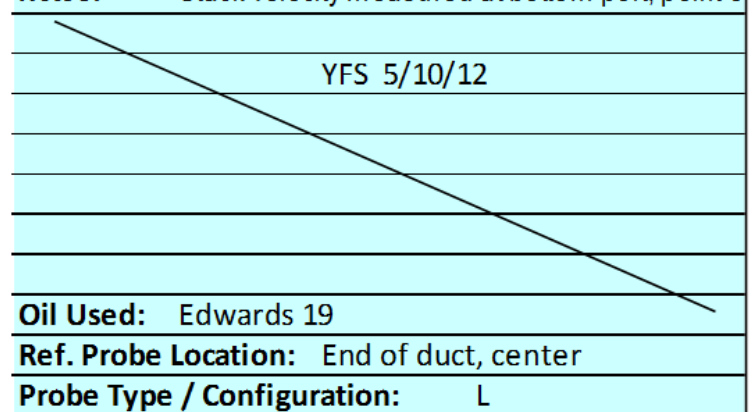

\begin{tabular}{|ll|lr|}
\hline $\begin{array}{l}\text { Entries made by: } \\
\text { Signature/date }\end{array}$ & YFS, EA $\begin{array}{c}5 / 10 / 2012 \\
\text { signature on file with original }\end{array}$ & $\begin{array}{l}\text { Technical Data Review performed by: Elizabeth Golovich } \\
\text { Signature/date }\end{array}$ & $\begin{array}{c}\text { Signature on file with Original } \\
\text { TI-WTPSP-079 }\end{array}$ \\
\hline
\end{tabular}


Rev. 0

3 Aug. 2006
PARTICLE TRACER TRAVERE DATA FORM

Site HV-S2 Model

Date 5/10/2012

Tester EA, YFS, XYY

Stack Dia. 11.938 in.

Stack X-Area 111.9 in 2

Test Port

2

Distance to disturbance

Measurement units particles/fi3

\begin{tabular}{|c|c|c|c|c|c|c|c|c|c|}
\hline \multirow{3}{*}{\multicolumn{2}{|c|}{$\begin{array}{r}\text { Order } \longrightarrow \\
\text { Traverse-> } \\
\quad \text { Trial } \longrightarrow>\end{array}$}} & \multicolumn{4}{|c|}{ 2nd } & \multicolumn{3}{|c|}{ 1st } & \multirow[b]{3}{*}{ Mngn } \\
\hline & & \multicolumn{4}{|c|}{ Side } & \multicolumn{3}{|c|}{ Bottom } & \\
\hline & & 1 & 2 & 3 & Mean & 1 & 2 & 3 & \\
\hline Point & Depth, in. & \multicolumn{4}{|c|}{ particles/13 } & \multicolumn{4}{|c|}{ parficles/ti3 } \\
\hline 1 & 0.50 & 936 & 844 & 911 & 897.0 & 1238 & 1325 & 1183 & 1248.7 \\
\hline 2 & 1.25 & 957 & 965 & 971 & 964.3 & 1473 & 1443 & 1317 & 1411.0 \\
\hline 3 & 2.32 & 981 & 924 & 900 & 935.0 & 1536 & 1453 & 1387 & 1458.7 \\
\hline 4 & 3.86 & 932 & 958 & 925 & 938.3 & 1426 & 1368 & 1356 & 1383.3 \\
\hline Center & 5.97 & 976 & 990 & 1035 & 1000.3 & 1306 & 1330 & 1220 & 1285.3 \\
\hline 5 & 8.08 & 1015 & 961 & 974 & 983.3 & 1180 & 1176 & 1065 & 1140.3 \\
\hline 6 & 9.62 & 994 & 906 & 953 & 951.0 & 1143 & 1149 & 1036 & 1109.3 \\
\hline 7 & 10.68 & 807 & 853 & 841 & 833.7 & 1008 & 985 & 963 & 985.3 \\
\hline 8 & 11.56 & 866 & 799 & 727 & 797.3 & 933 & 938 & 840 & 903.7 \\
\hline \multicolumn{2}{|l|}{ Averages - } & 940.4 & 911.1 & 915.2 & 922.3 & 1249.2 & 1240.8 & 1151.9 & 1214.0 \\
\hline
\end{tabular}

Run No. PT-8

Fan confguration Fan A only

Fan Setting 49

Stack Temp $73 \mathrm{deg} F$

Start/End Time 12:45/14:30

1.10

Points in Center $2 / 3 \quad 2$ Injection Point E Center
Center $2 / 3$ from $\begin{array}{lr}\text { to: } & \frac{10.84}{7} \\ \text { to: } & \\ & \\ 2 & 3\end{array}$ 915.2

\begin{tabular}{|c|c|c|c|c|c|c|c|}
\hline $\mathbf{A I I}$ & pt/fi3 & Dev. from mean & Center 2/3 & $\underline{\text { Side }}$ & Bottom & $\underline{\text { All }}$ & Normlzd \\
\hline Mean & 1068.1 & & Mean & 943.7 & 1253.3 & 1098.5 & 123296 \\
\hline Min Point & 797.3 & $-25.4 \%$ & Std. Dev. & 54.0 & 178.1 & 204.4 & 131.54 \\
\hline Max Point & 1458.7 & $36.6 \%$ & covas $\%$ & 5.7 & 14.2 & 18.6 & 10.67 \\
\hline
\end{tabular}

\begin{tabular}{|c|c|}
\hline Avg Conc & pt/ft3 \\
\hline Generator Inlet Press & 4.5 \\
\hline Stack Temp & 70.3 \\
\hline Mean velocity & 245 \\
\hline Ambient pressure & 29.9 \\
\hline Ambient humidity & $24 \%$ \\
\hline Ambient temp & 68 \\
\hline Back-Gd aerosol & $6,8,8$ \\
\hline No. Bk-Gd samples & 5 \\
\hline Compressor output & 30 \\
\hline
\end{tabular}

$$
p t / f t 3
$$
Instuments Used:

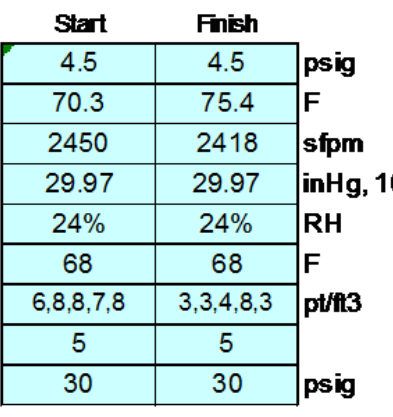

Notes: $\quad$ Stack velocity measured at bottom port, point 5

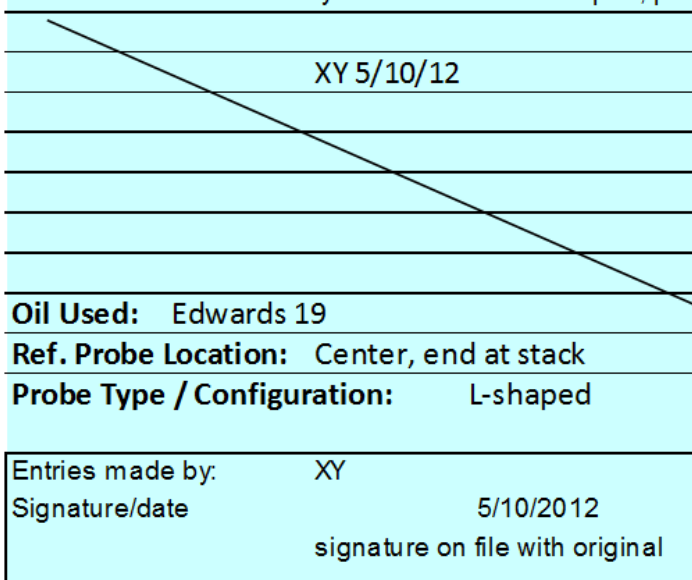

TSIVelociCalc

\begin{tabular}{lcr} 
TSI VelociCalc & T95351203001 & $12 / 17 / 2012$ \\
\hline Fisher Scientific & 90936818 & $12 / 7 / 2012$ \\
\hline Met One OPC & 1011529010 Ref & $2 / 1 / 2013$ \\
\hline Met One OPC & 1011529009 Sample & $1 / 9 / 2013$ \\
\hline
\end{tabular}

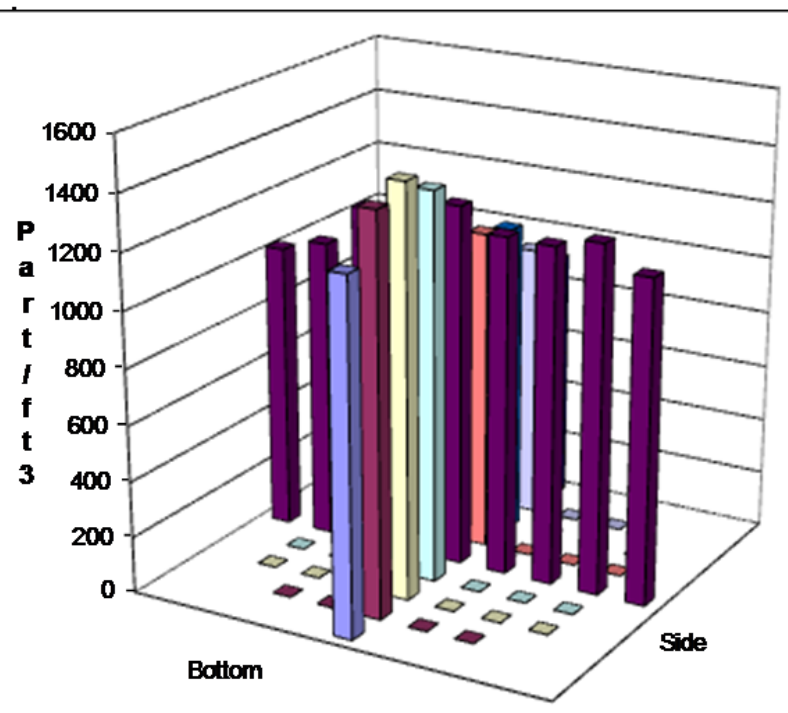

Technical Data Review performed by: Elizabeth Golovich Signature/date $6 / 29 / 2012$

Signature on file with Original TI-WTPSP-079 

Appendix C

\section{IHLW-S1 Data Sheets}





\section{C.1 IHLW-S1 Calibration of Ventilation Flow Controller}

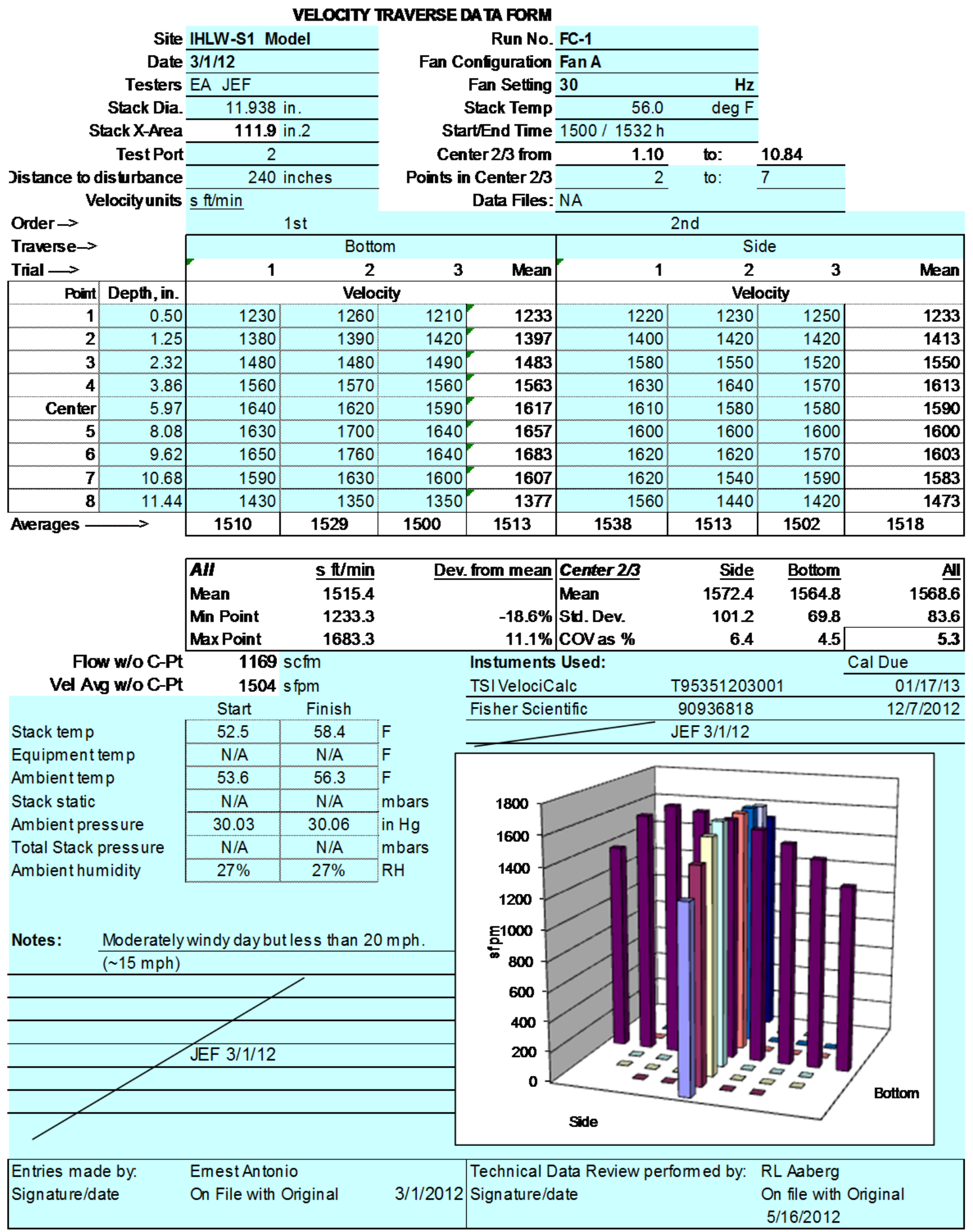




\section{VEOCITY TRAVERSE DATA FORM}

Site IHLW-S1 Mode

Date 3/1/12

Testers EA JEF

Stack Dia.

Stack X-Area

Test Port

Jistance to disturbance

Velocity units $\mathrm{s} \mathrm{ft} / \mathrm{min}$
Run No. FC-2

Fan Configuration Fan B

Fan Setting 30

Stack Temp

StartEnd Time $1542 / 1600$

Center $2 / 3$ from

Points in Center $2 / 3$

Data Files: NA

\begin{tabular}{|c|c|}
\hline $\begin{array}{l}\text { Order } \rightarrow \\
\text { Traverse } \rightarrow \\
\text { Trial } \longrightarrow\end{array}$ & \\
\hline Point & Depth, in. \\
\hline 1 & 0.5 \\
\hline 2 & 1.25 \\
\hline 3 & 2.32 \\
\hline 4 & 3.86 \\
\hline Center & 5.97 \\
\hline 5 & 8.08 \\
\hline 6 & 9.62 \\
\hline 7 & 10.68 \\
\hline 8 & 11.44 \\
\hline
\end{tabular}

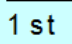

$1 \quad$ Side

\section{2nd}

\begin{tabular}{rr} 
& $\mathrm{Hz}$ \\
$56.0 \quad \operatorname{deg} \mathrm{F}$ \\
\hline 600
\end{tabular}

0

10 to:

10.84

(2) Bottom

\begin{tabular}{|c|c|c|c|c|c|c|c|}
\hline & 2 & 3 & Mean & 1 & 2 & 3 & Mean \\
\hline \multicolumn{4}{|c|}{ Velocity } & \multicolumn{4}{|c|}{ Velocity } \\
\hline 1350 & 1360 & 1390 & 1367 & 1330 & 1320 & 1320 & 1323 \\
\hline 1540 & 1530 & 1530 & 1533 & 1560 & 1530 & 1500 & 1530 \\
\hline 1660 & 1670 & 1700 & 1677 & 1740 & 1660 & 1680 & 1693 \\
\hline 1700 & 1780 & 1750 & 1743 & 1760 & 1740 & 1760 & 1753 \\
\hline 1740 & 1860 & 1810 & 1803 & 1720 & 1750 & 1750 & 1740 \\
\hline 1730 & 1790 & 1770 & 1763 & 1740 & 1720 & 1770 & 1743 \\
\hline 1690 & 1730 & 1670 & 1697 & 1670 & 1630 & 1670 & 1657 \\
\hline 1560 & 1570 & 1590 & 1573 & 1560 & 1510 & 1540 & 1537 \\
\hline 1410 & 1420 & 1410 & 1413 & 1350 & 1440 & 1420 & 1403 \\
\hline 1598 & 1634 & 1624 & 1619 & 1603 & 1589 & 1601 & 1598 \\
\hline
\end{tabular}

\begin{tabular}{|c|c|c|c|c|c|c|}
\hline $\mathbf{A} \boldsymbol{I}$ & $\mathrm{s} \mathrm{ft} / \mathrm{min}$ & Dev. from mean & Center 2/3 & Side & Bottom & All \\
\hline Mean & 1608.3 & & Mean & 1684.3 & 1664.8 & 1674.5 \\
\hline Min Point & 1323.3 & $-17.7 \%$ & Std. Dev. & 99.3 & 95.9 & 94.3 \\
\hline Max Point & 1803.3 & $12.1 \%$ & covas \% & 5.9 & 5.8 & 5.6 \\
\hline
\end{tabular}

Flow w/o C-Pt Vel Avg w/o C-Pt

Stack temp

Equipment temp Ambient tem $p$

Stack static

Ambient pressure Total Stack pressure Ambient humidity

\begin{tabular}{|c|c|c|}
\hline \multicolumn{2}{|c|}{$1588 \mathrm{sfpm}$} & \\
\hline Start & Finish & \\
\hline 56.7 & 56.3 & $F$ \\
\hline $\mathrm{N} / \mathrm{A}$ & $\mathrm{N} / \mathrm{A}$ & $F$ \\
\hline 55.4 & 50.9 & $F$ \\
\hline$N / A$ & $\mathrm{~N} / \mathrm{A}$ & mbars \\
\hline 30.06 & 30.06 & in $\mathrm{Hg}$ \\
\hline $\mathrm{N} / \mathrm{A}$ & $\mathrm{N} / \mathrm{A}$ & mbars \\
\hline $27 \%$ & $28 \%$ & $\mathrm{RH}$ \\
\hline
\end{tabular}

Notes: $\quad$ Moderately windy day but less than $20 \mathrm{mph}$.

Notes: $\quad \frac{\text { Moderately }}{(\sim 15 \mathrm{mph})}$

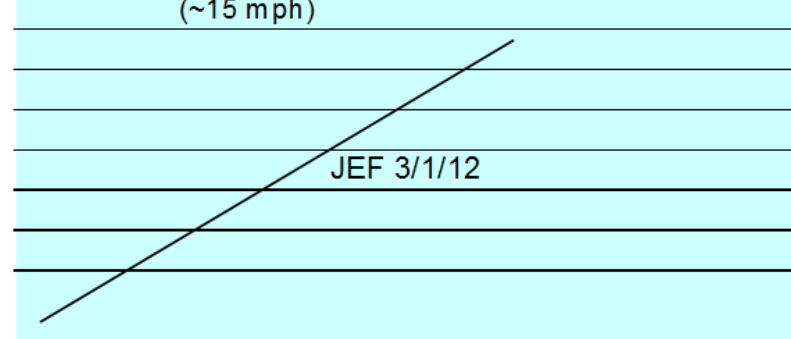

Entries made by: Julia Flaherty Signature/date

\begin{tabular}{lcc} 
Instuments Used: & & Cal Due \\
\cline { 3 - 3 } TSI VelociCalc & T95351203001 & $01 / 17 / 13$ \\
\hline Fisher Scientific & 90936818 & $12 / 7 / 2012$ \\
\hline
\end{tabular}

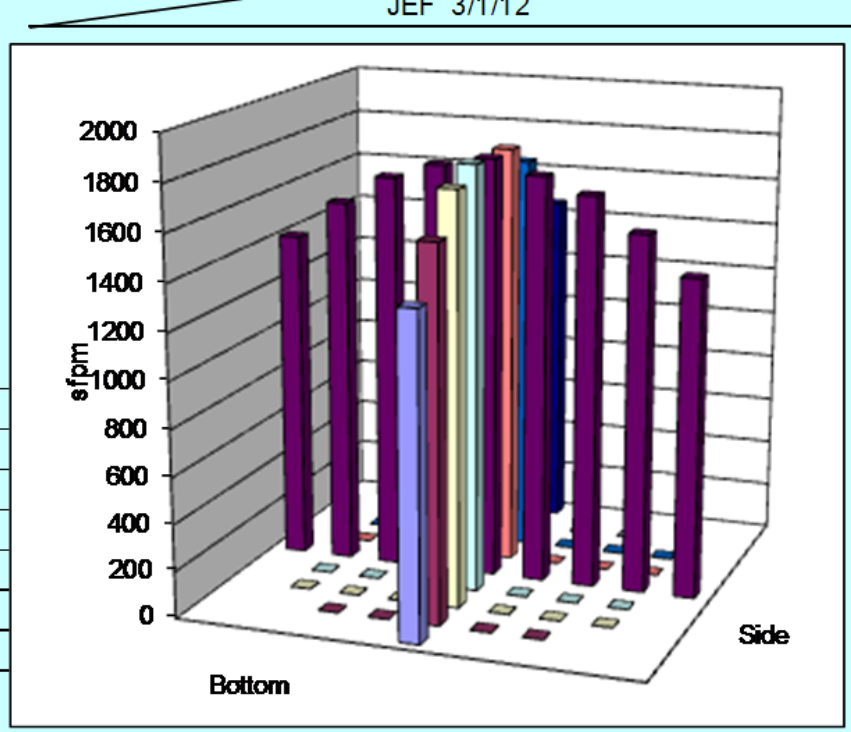

Technical Data Review performed by. RL Aaberg $3 / 1 / 2012$ Signature/date
On file with Original $5 / 16 / 2012$ 


\section{VELOCITY vS. FREQUENCY DATA FORM}

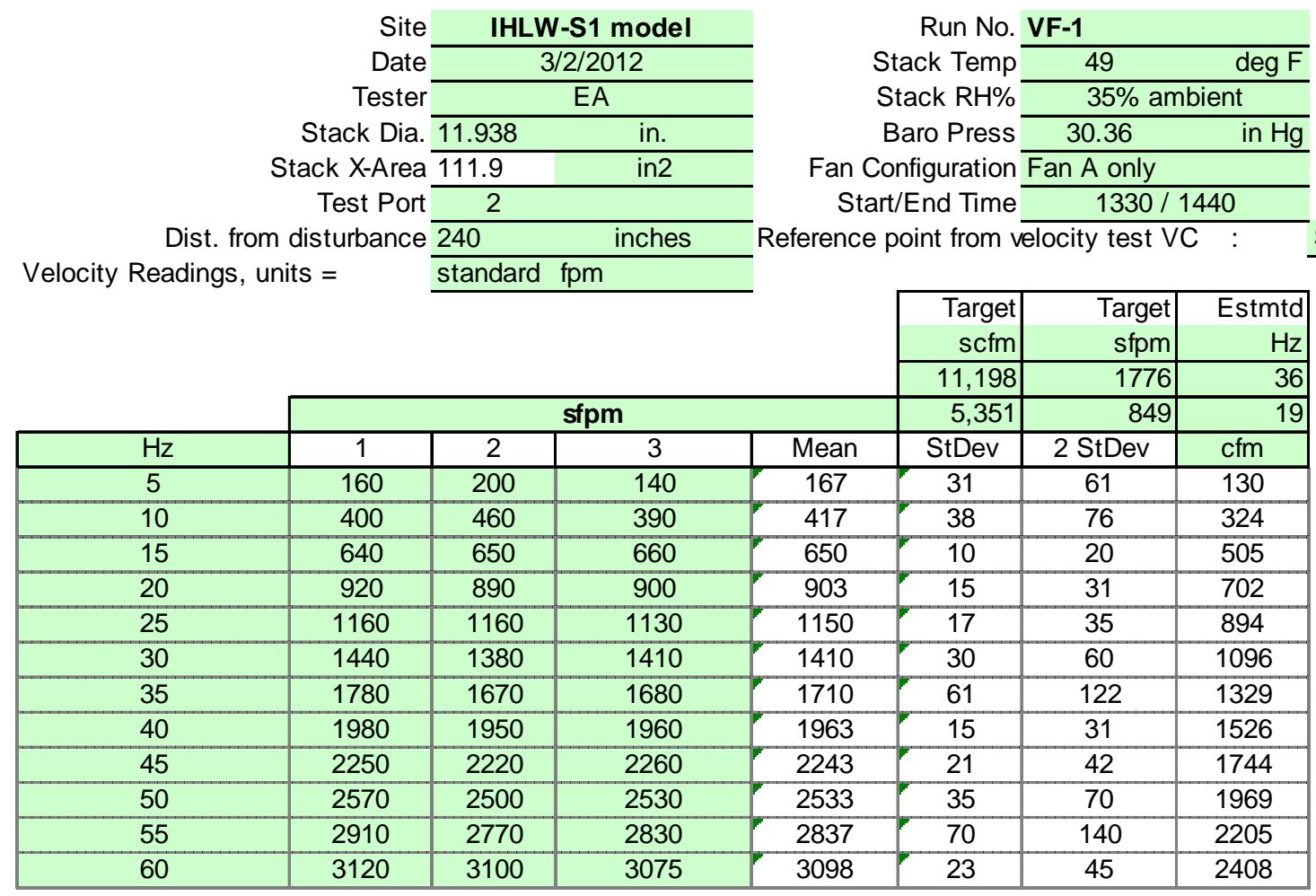

Instuments Used:

TSI VelociCalc

T95351203001

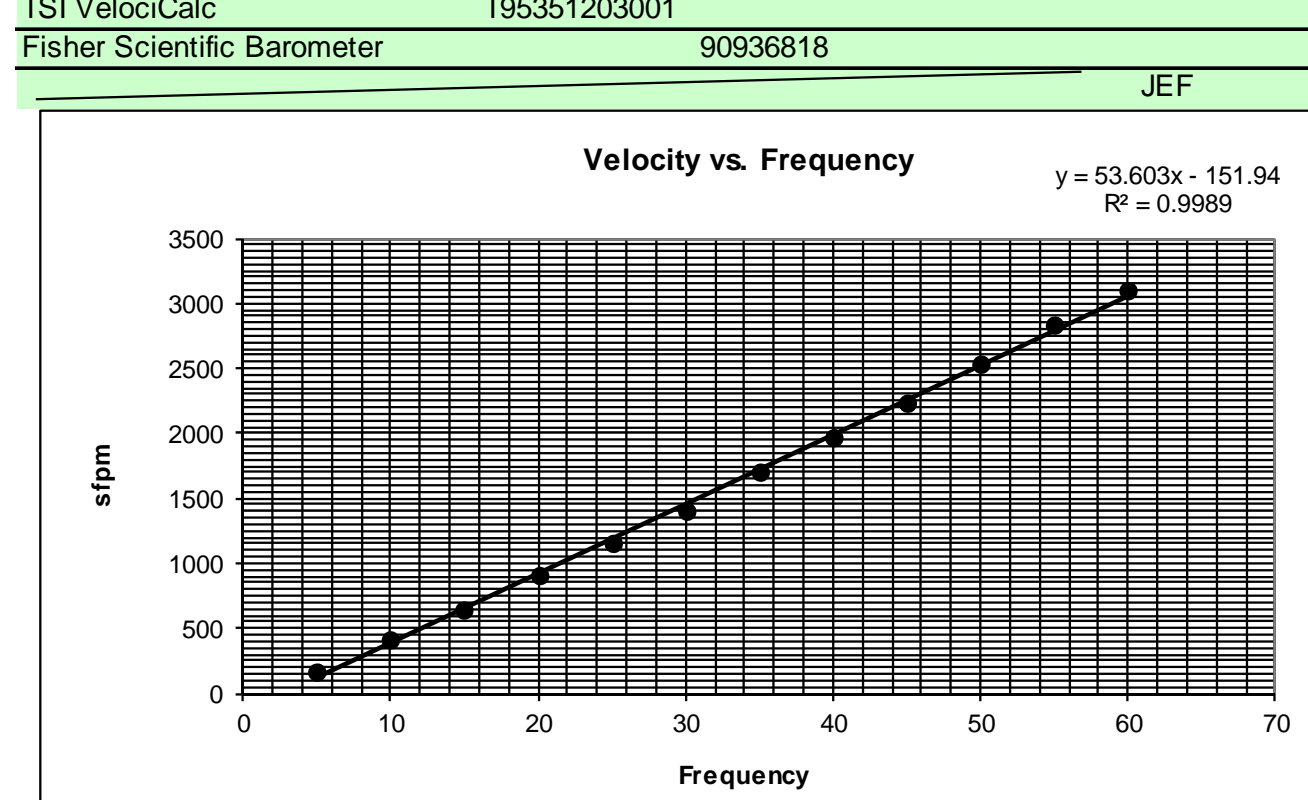

Entries made by:

Signature/date
Ernest Antonio
On File with Original
2-Mar-12
Cal Exp. Date:

$1 / 17 / 2013$

$12 / 7 / 2012$ 


\section{FREQUENCY DATA FORM}

\begin{tabular}{|c|c|c|}
\hline \multicolumn{3}{|c|}{ Site LW-S1 model } \\
\hline Date & $3 / 2 / 2012$ & \\
\hline Tester & EA & \\
\hline Stack Dia. & 11.938 & in. \\
\hline Stack X-Area & 111.9 & in2 \\
\hline Test Port & 2 & \\
\hline
\end{tabular}

Dist. from disturbance 240

Velocity Readings, units standard fpm
Run No. VF-2

\begin{tabular}{rll} 
Stack Temp & 53 & deg F \\
\cline { 2 - 3 } Stack $\mathrm{RH} \%$ & $35 \%$ ambient & \\
\cline { 2 - 3 } Baro Press & 30.36 & in $\mathrm{Hg}$ \\
\cline { 2 - 3 }
\end{tabular}

Fan Configuration Fan B Only

Start/End Time $1450 / 1550$ $\begin{array}{lll}\text { Reference point from velocity test VC : } & \text { Side } 7\end{array}$

\begin{tabular}{|c|c|c|c|c|c|c|c|}
\hline & & & & & & & \\
\hline & & & & & $\begin{array}{r}\text { Target } \\
\text { scfm }\end{array}$ & $\begin{array}{r}\text { Target } \\
\text { sfpm }\end{array}$ & $\begin{array}{r}\text { Estmtd } \\
\mathrm{Hz}\end{array}$ \\
\hline & & & & & 11,198 & 1776 & 22 \\
\hline & \multicolumn{4}{|c|}{ sfpm } & 5,351 & 849 & 17 \\
\hline $\mathrm{Hz}$ & 1 & 2 & 3 & Mean & StDev & 2 StDev & $\mathrm{cfm}$ \\
\hline 5 & 200 & 170 & 160 & 177 & 21 & 42 & 137 \\
\hline 10 & 450 & 430 & 470 & 450 & 20 & 40 & 350 \\
\hline 15 & 750 & 750 & 740 & 747 & 6 & 12 & 580 \\
\hline 20 & 1060 & 1000 & 1050 & 1037 & 32 & 64 & 806 \\
\hline 25 & 1310 & 1340 & 1320 & 1323 & 15 & 31 & 1029 \\
\hline 30 & 1640 & 1670 & 1700 & 1670 & 30 & 60 & 1298 \\
\hline 35 & 1940 & 1960 & 2010 & 1970 & 36 & 72 & 1531 \\
\hline 40 & 2260 & 2300 & 2370 & 2310 & 56 & 111 & 1796 \\
\hline 45 & 2610 & 2700 & 2670 & 2660 & 46 & 92 & 2068 \\
\hline 50 & 2920 & 3040 & 2950 & 2970 & 62 & 125 & 2309 \\
\hline 55 & 3200 & 3340 & 3350 & 3297 & 84 & 168 & 2563 \\
\hline 60 & 3650 & 3660 & 3740 & 3683 & 49 & 99 & 2863 \\
\hline
\end{tabular}

Instuments Used:

Cal Exp. Date:

TSI VelociCalc $\quad$ T95351203001

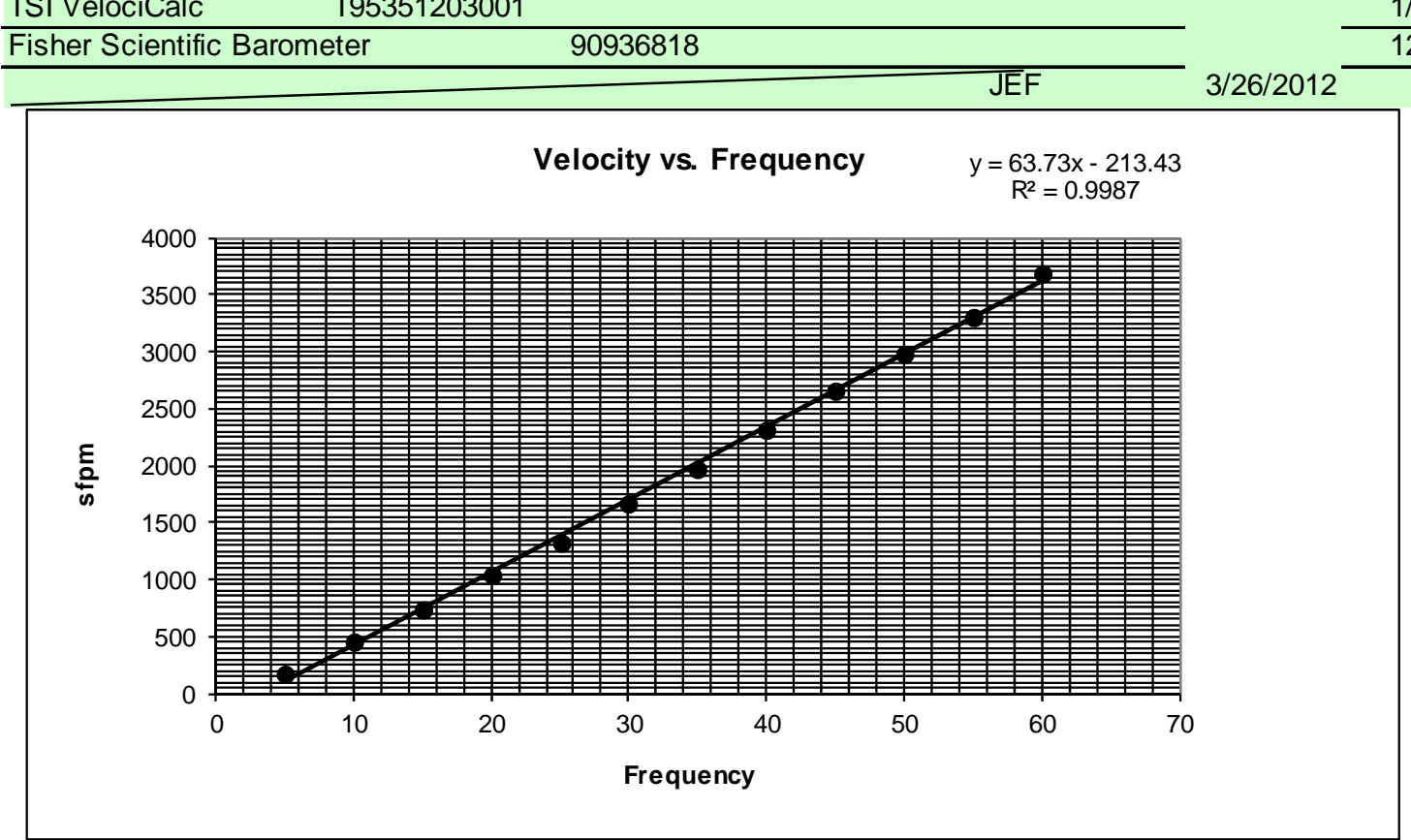

$1 / 17 / 2013$

$12 / 7 / 2012$
Entries made by:

Signature/date
Ernest Antonio

On File with Original

2-Mar-12
Technical Data Review performed by: RL Aaberg Signature/date

On file with Original $5 / 16 / 2012$ 


\section{C.2 IHLW-S1 \& Velocity Uniformity Data Sheets}

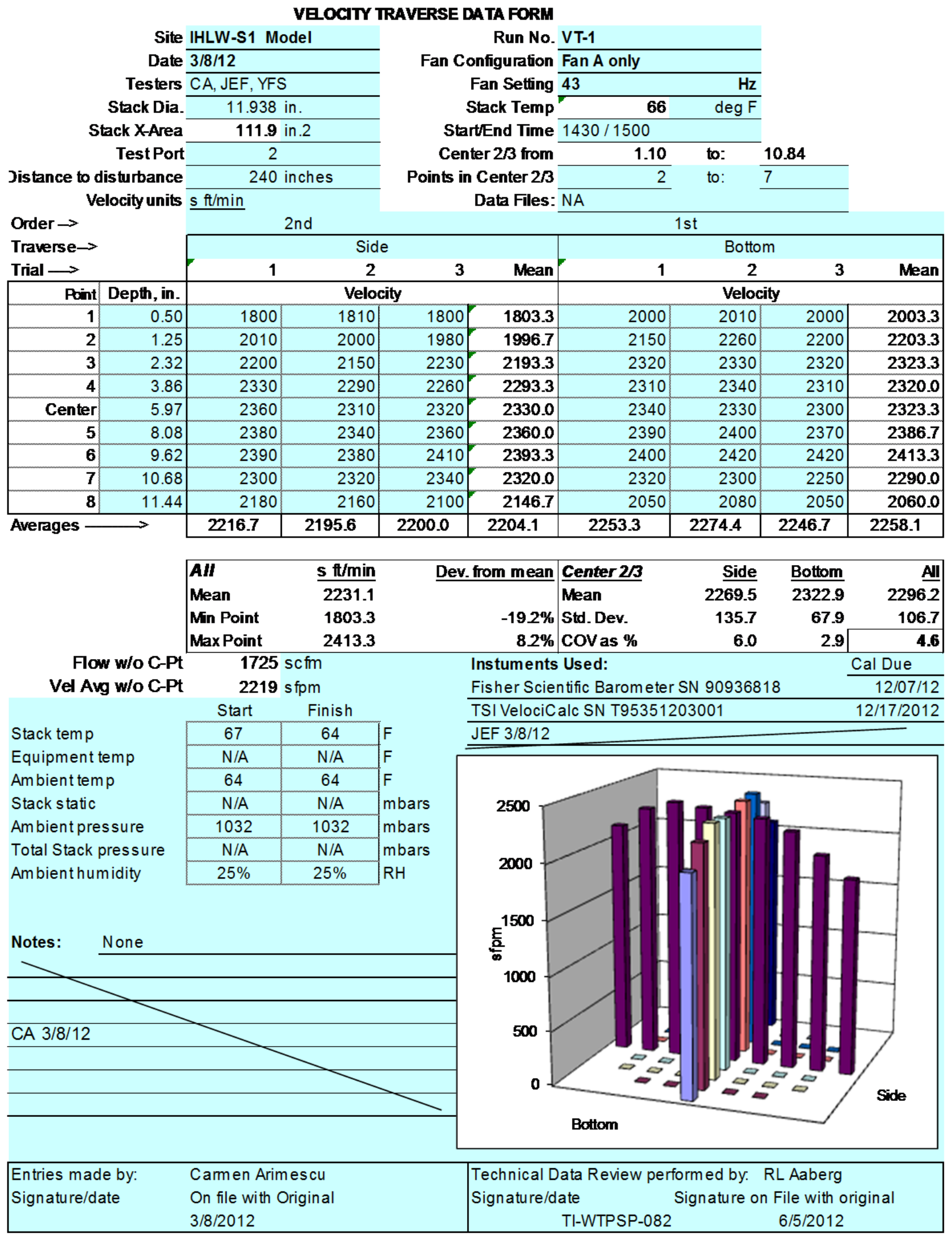




\section{VEOCITY TRAVERSE DATA FORM}

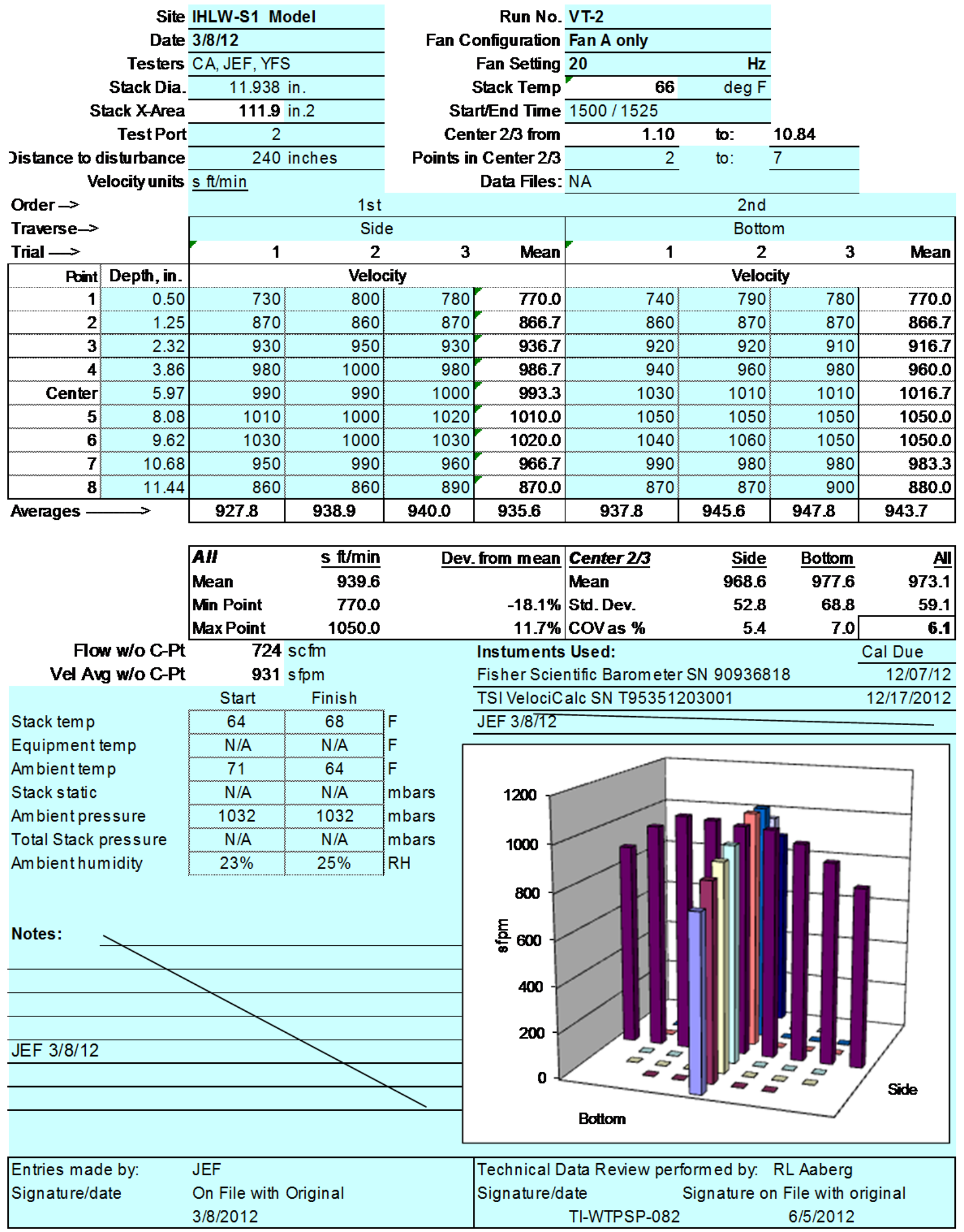




\section{VEOCITY TRAVERSE DATA FORM}

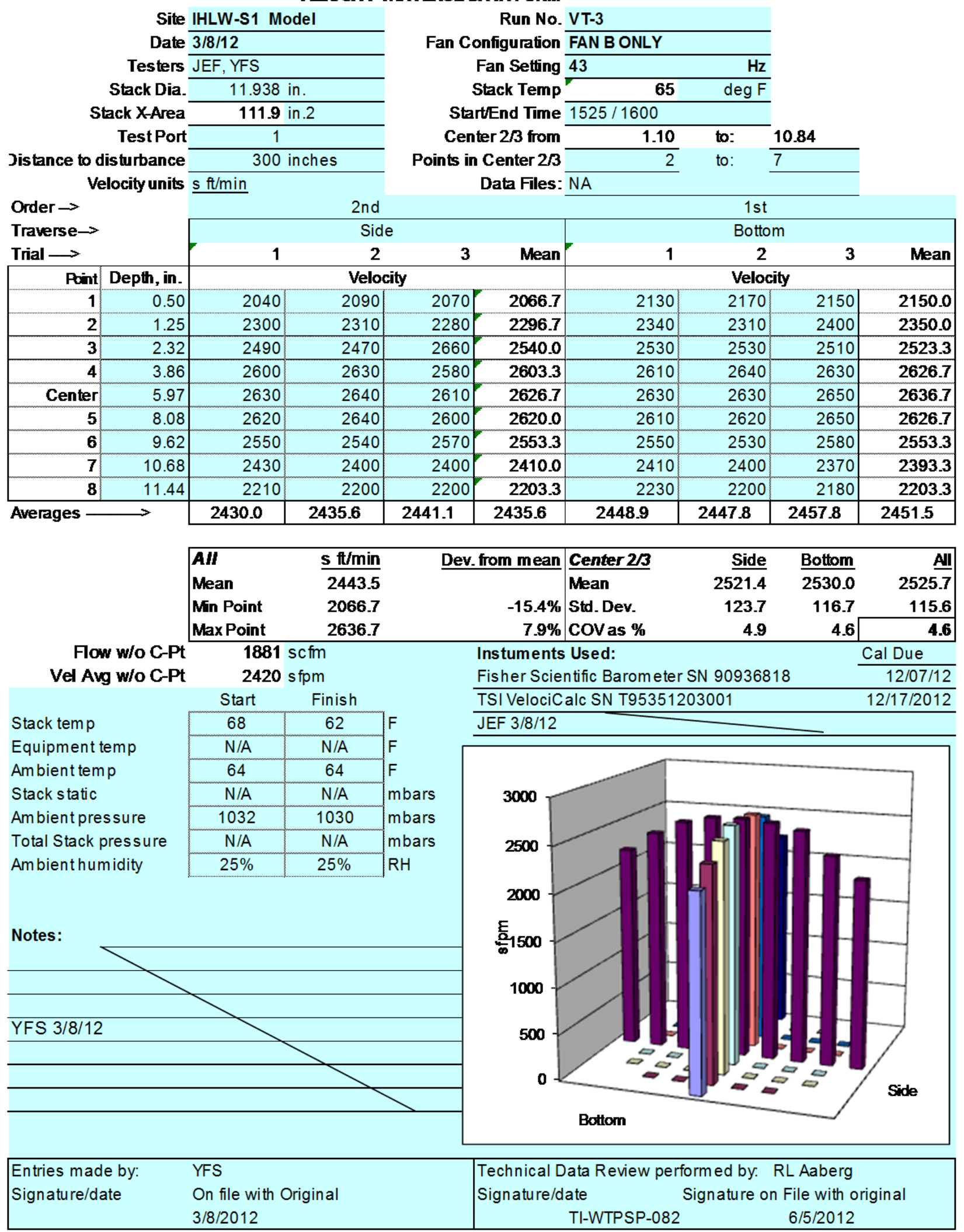




\section{VEOCITY TRAVERSE DATA FORM}

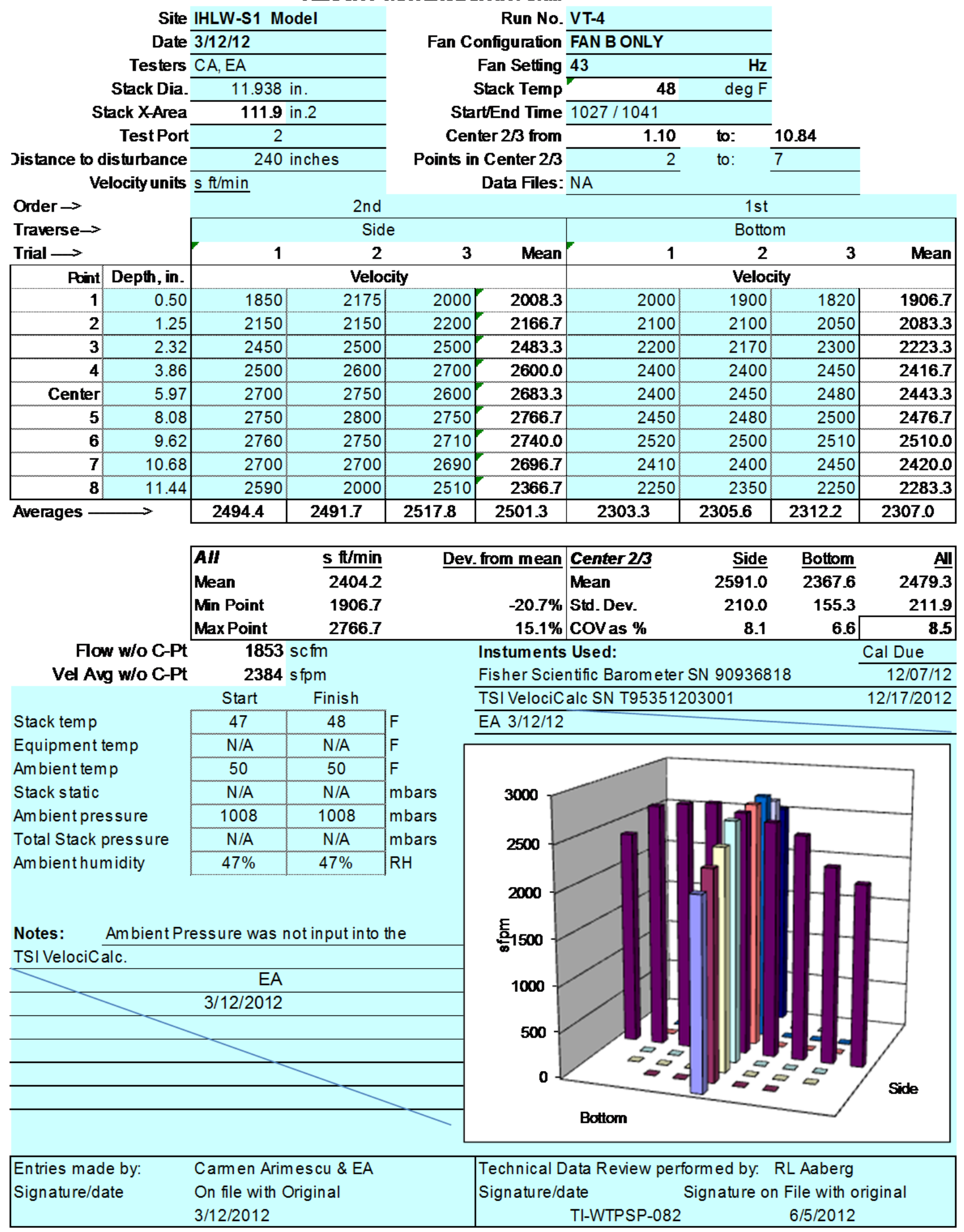


VEOCITY TRAVERSE DATA FORM

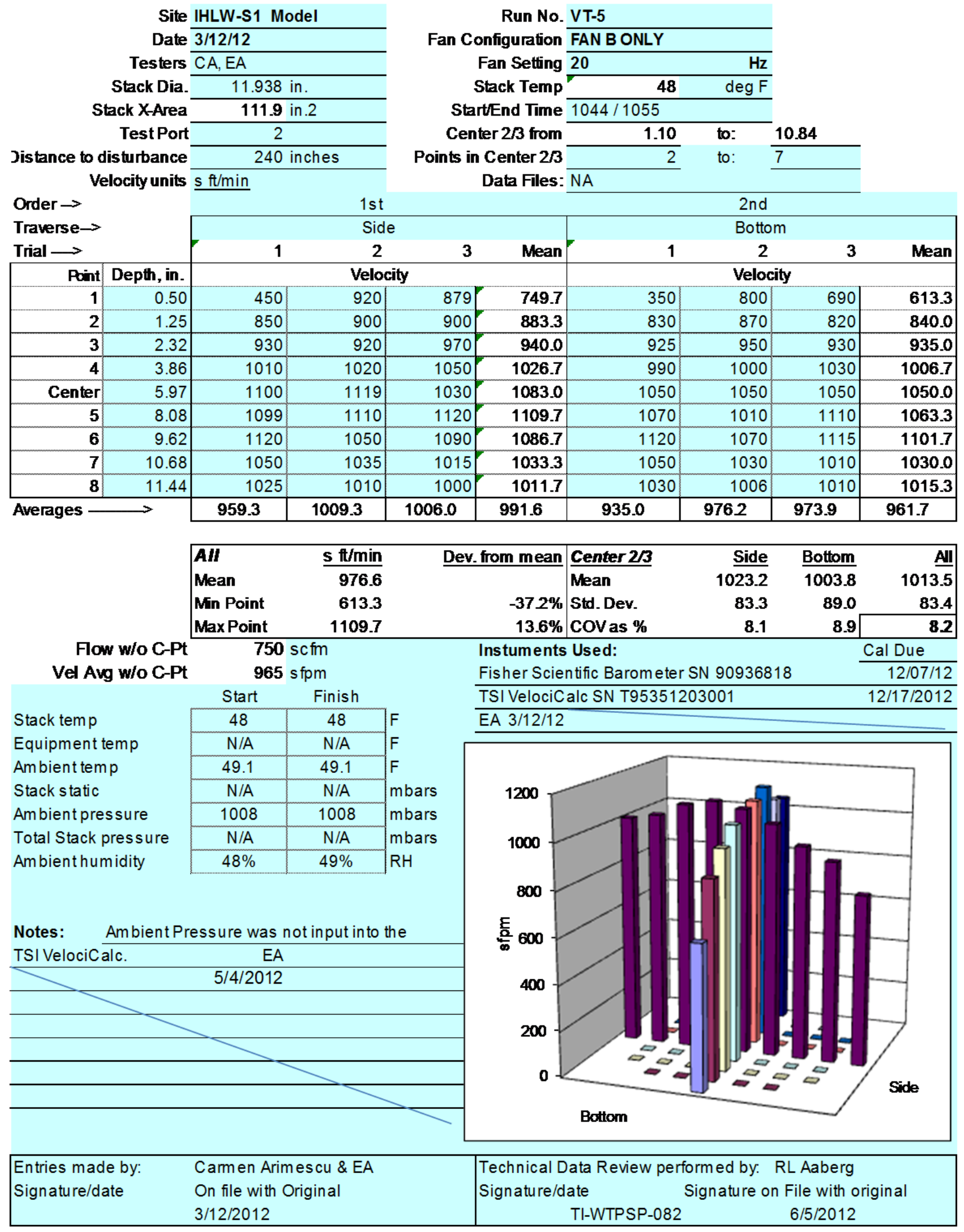


VEOCITY TRAVERSE DATA FORM

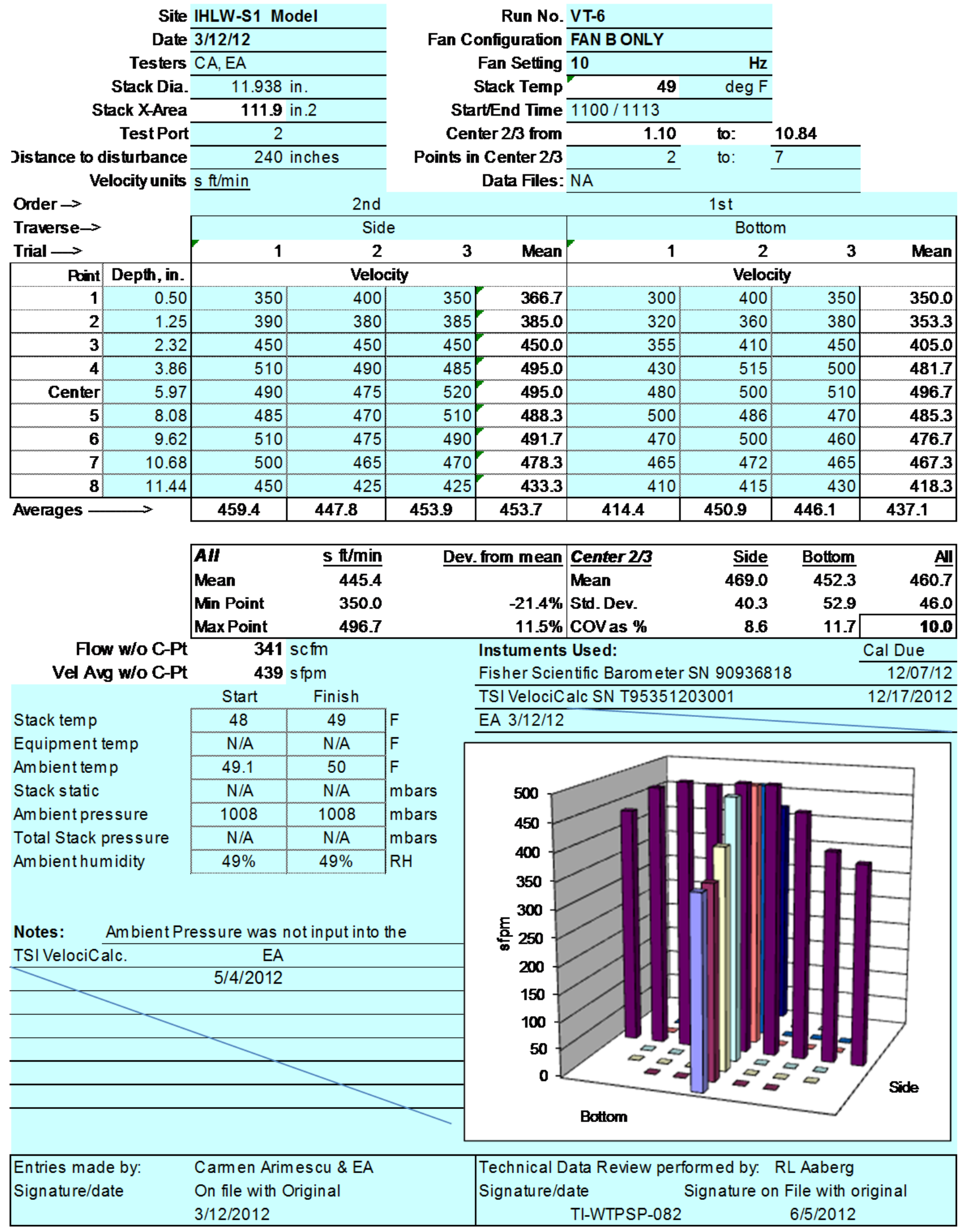




\section{VEOCITY TRAVERSE DATA FORM}

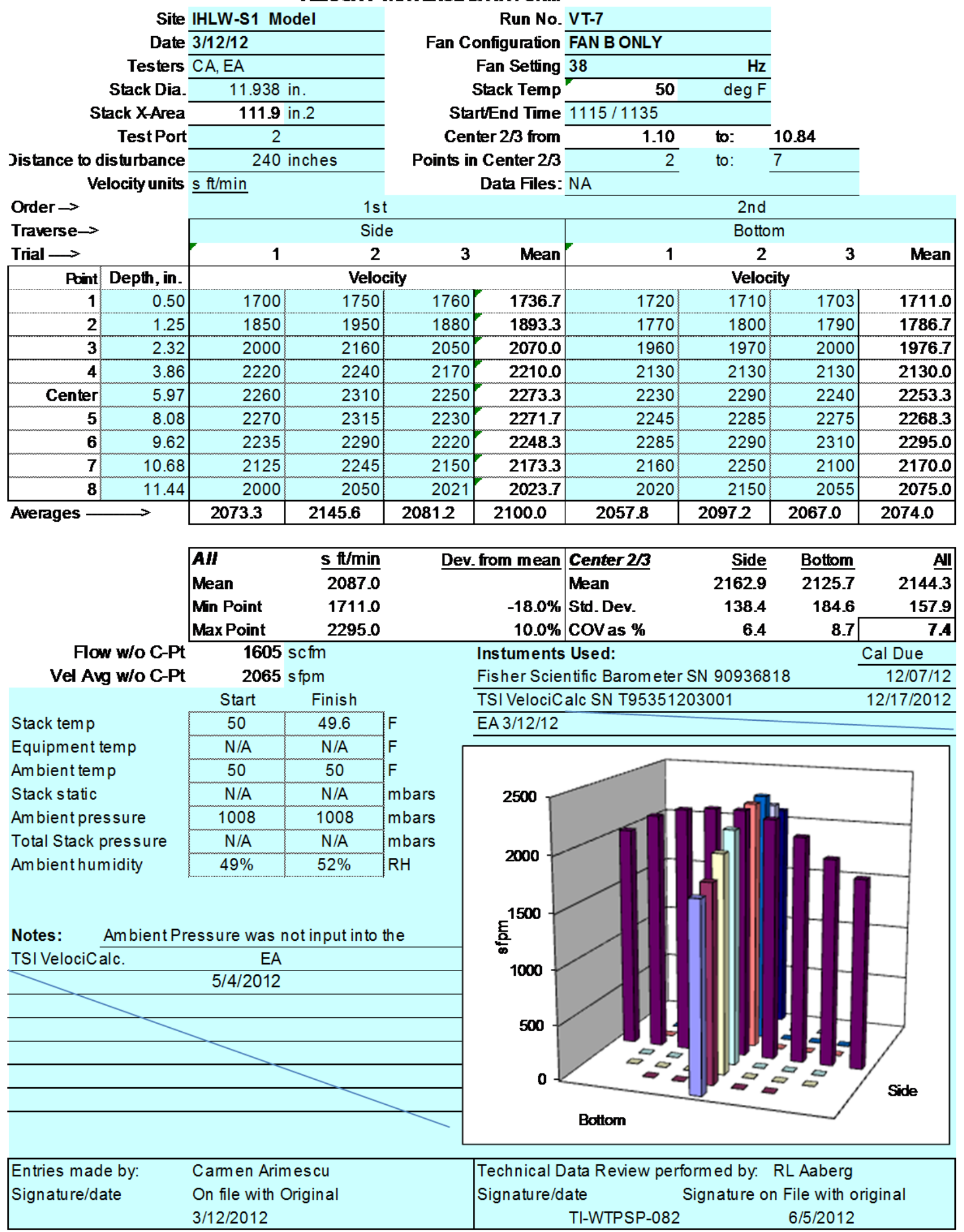




\section{VEOCITY TRAVERSE DATA FORM}

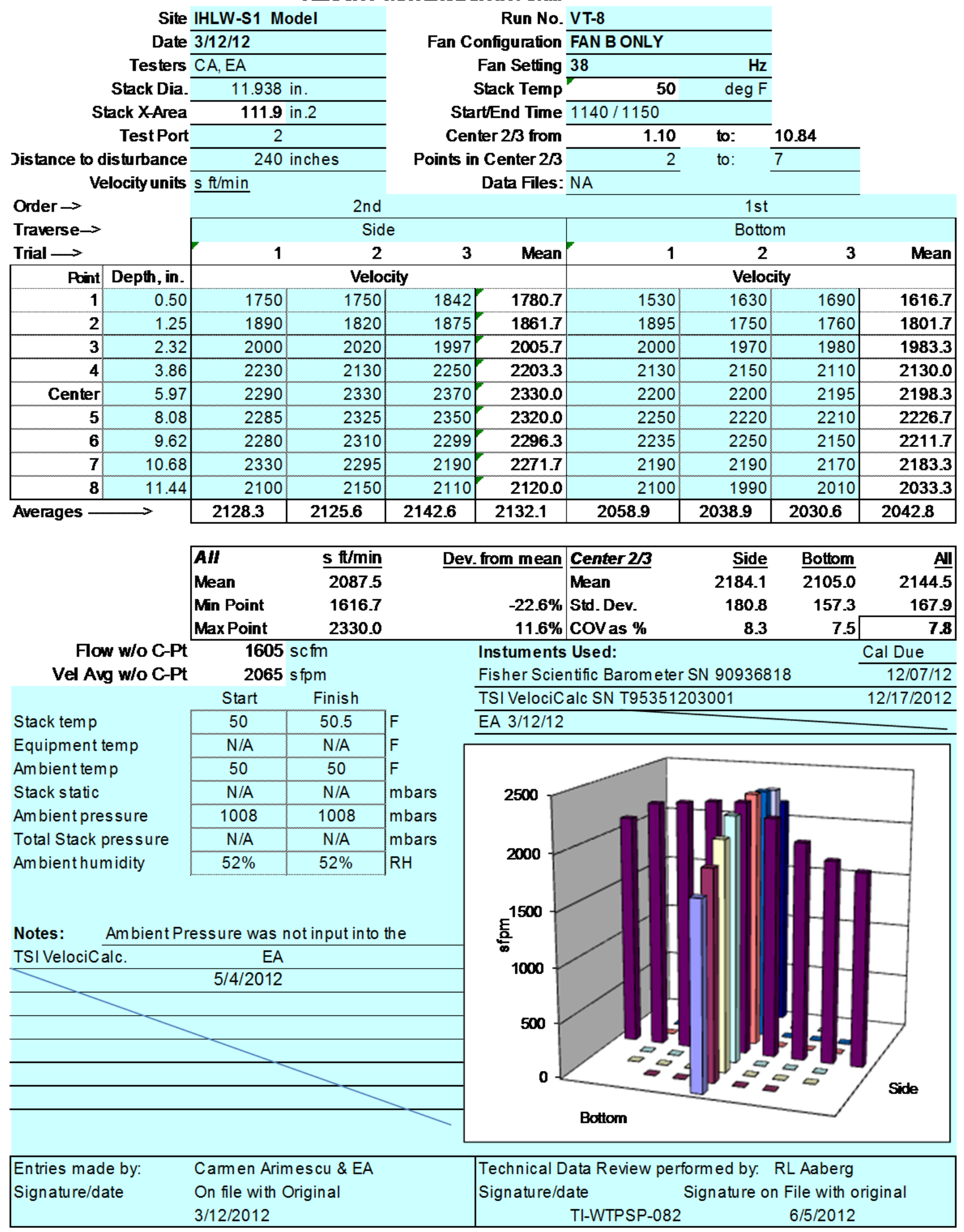




\section{VEOCITY TRAVERSE DATA FORM}

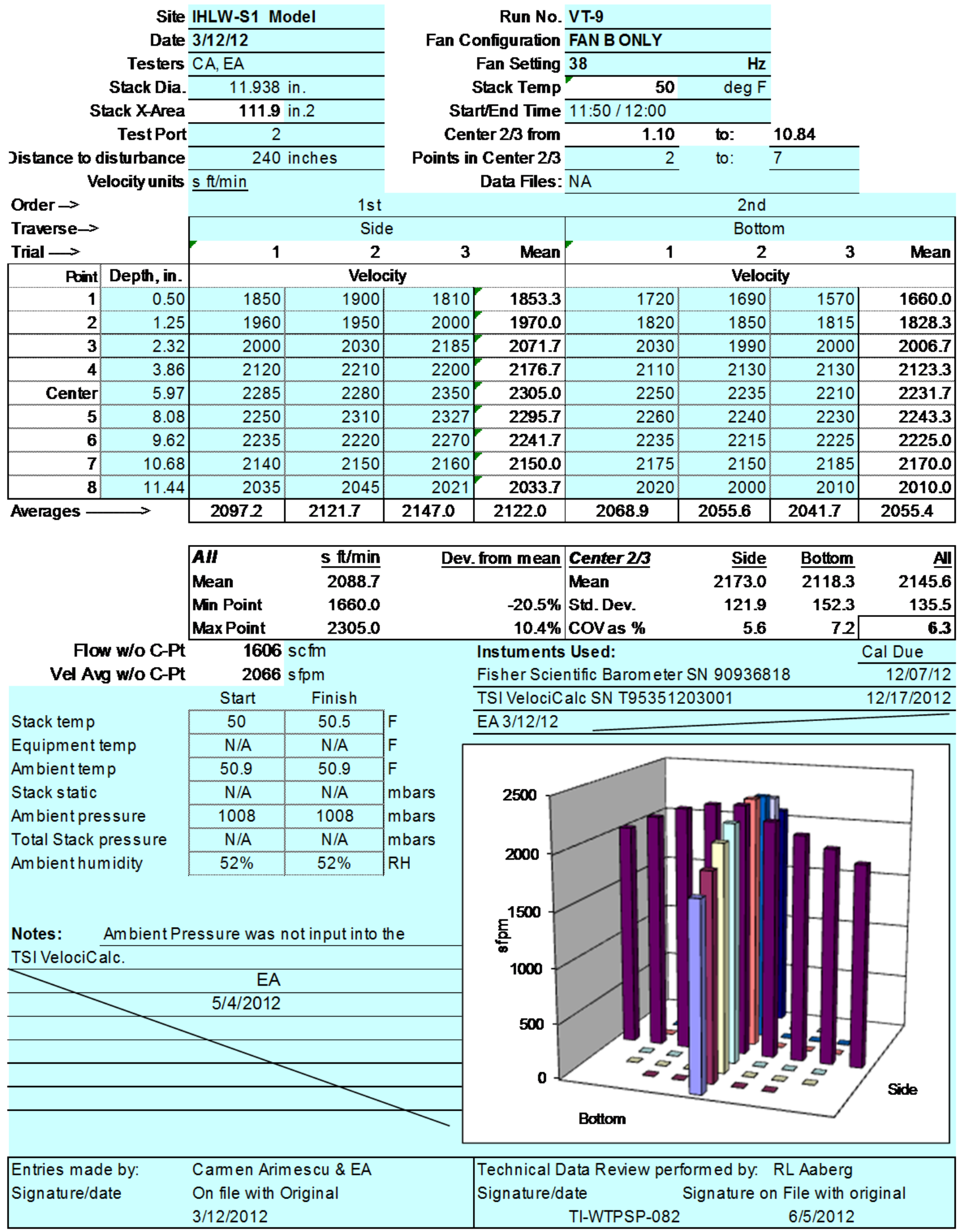




\section{VEOCITY TRAVERSE DATA FORM}

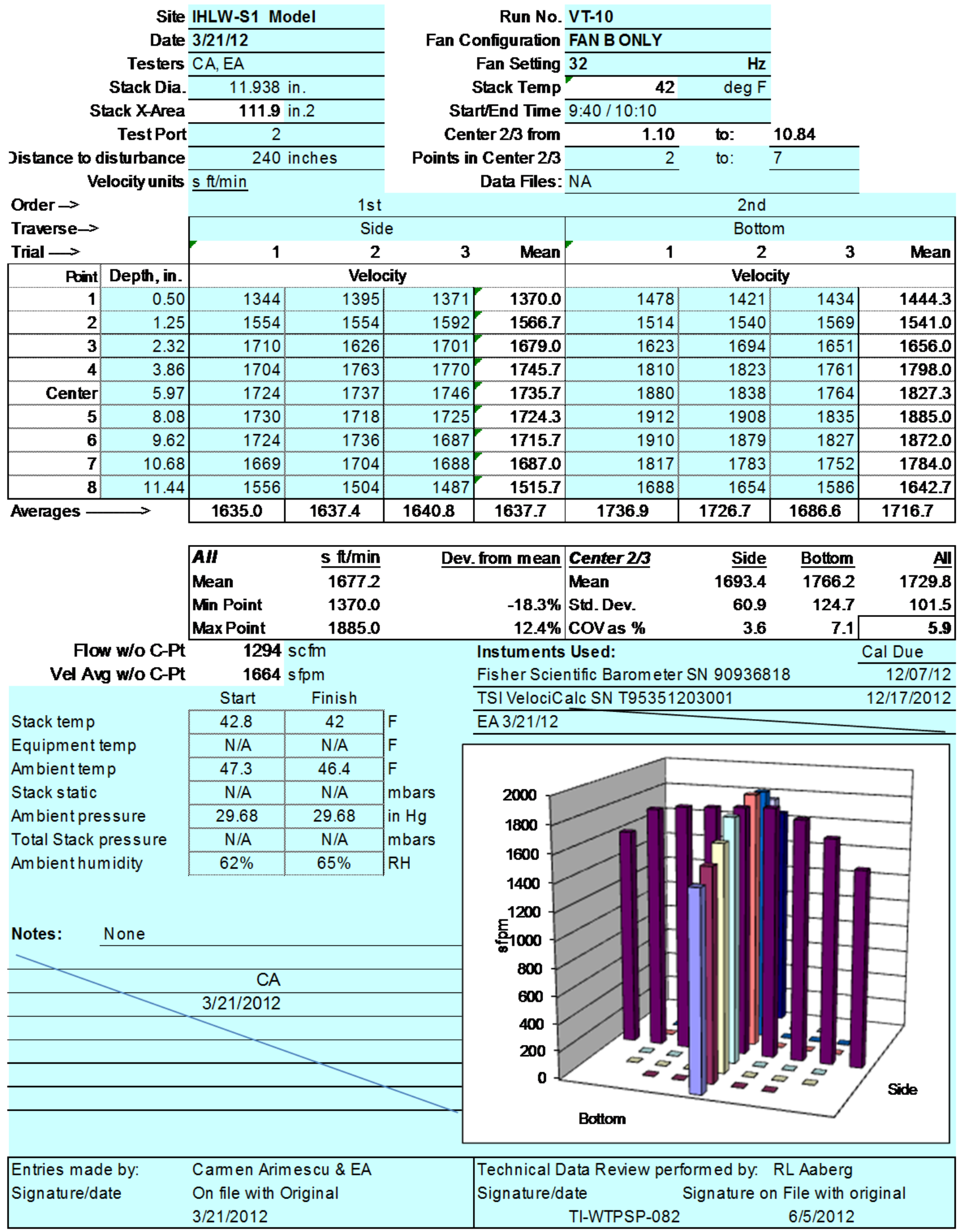




\section{VEOCITY TRAVERSE DATA FORM}

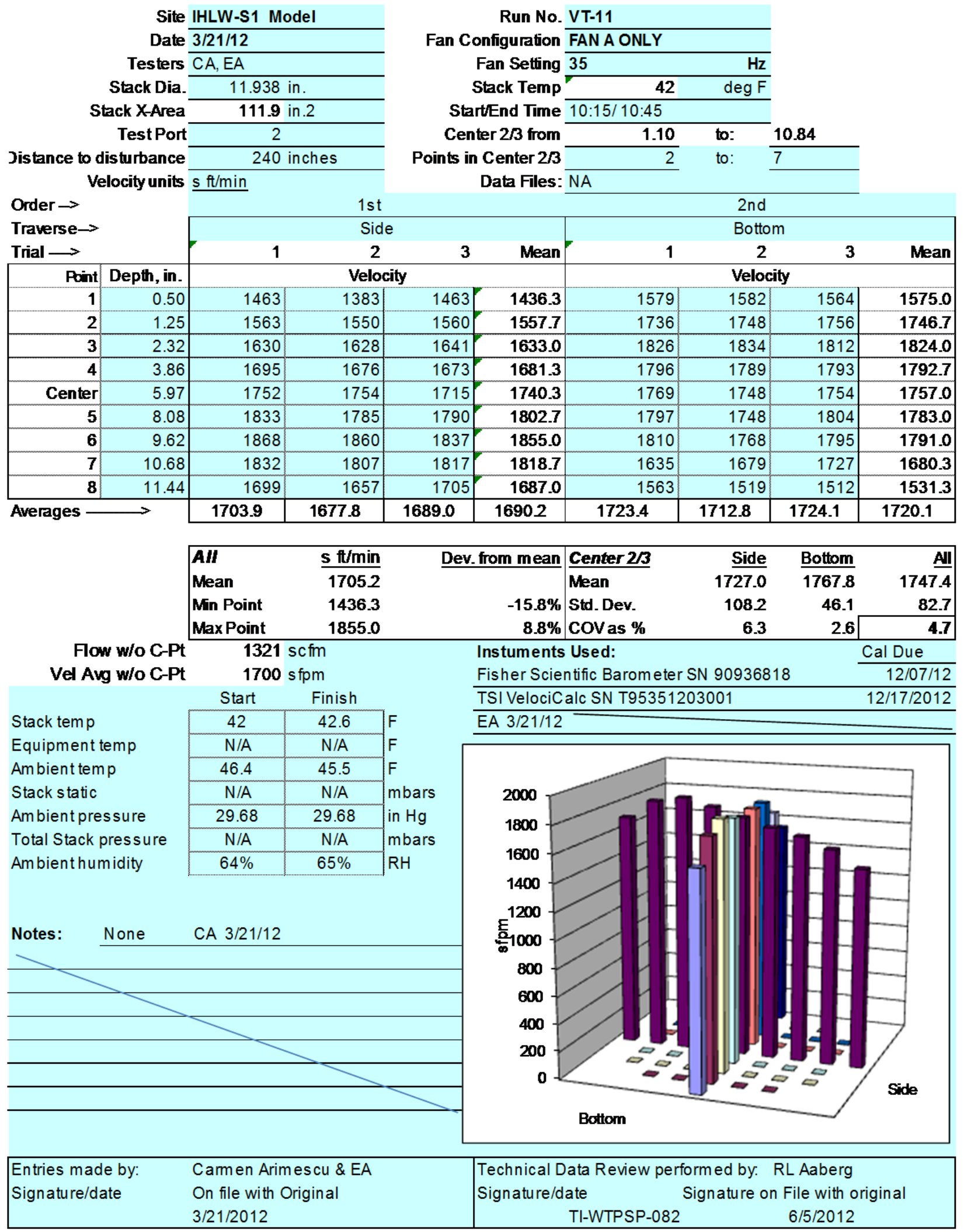




\section{VEOCITY TRAVERSE DATA FORM}

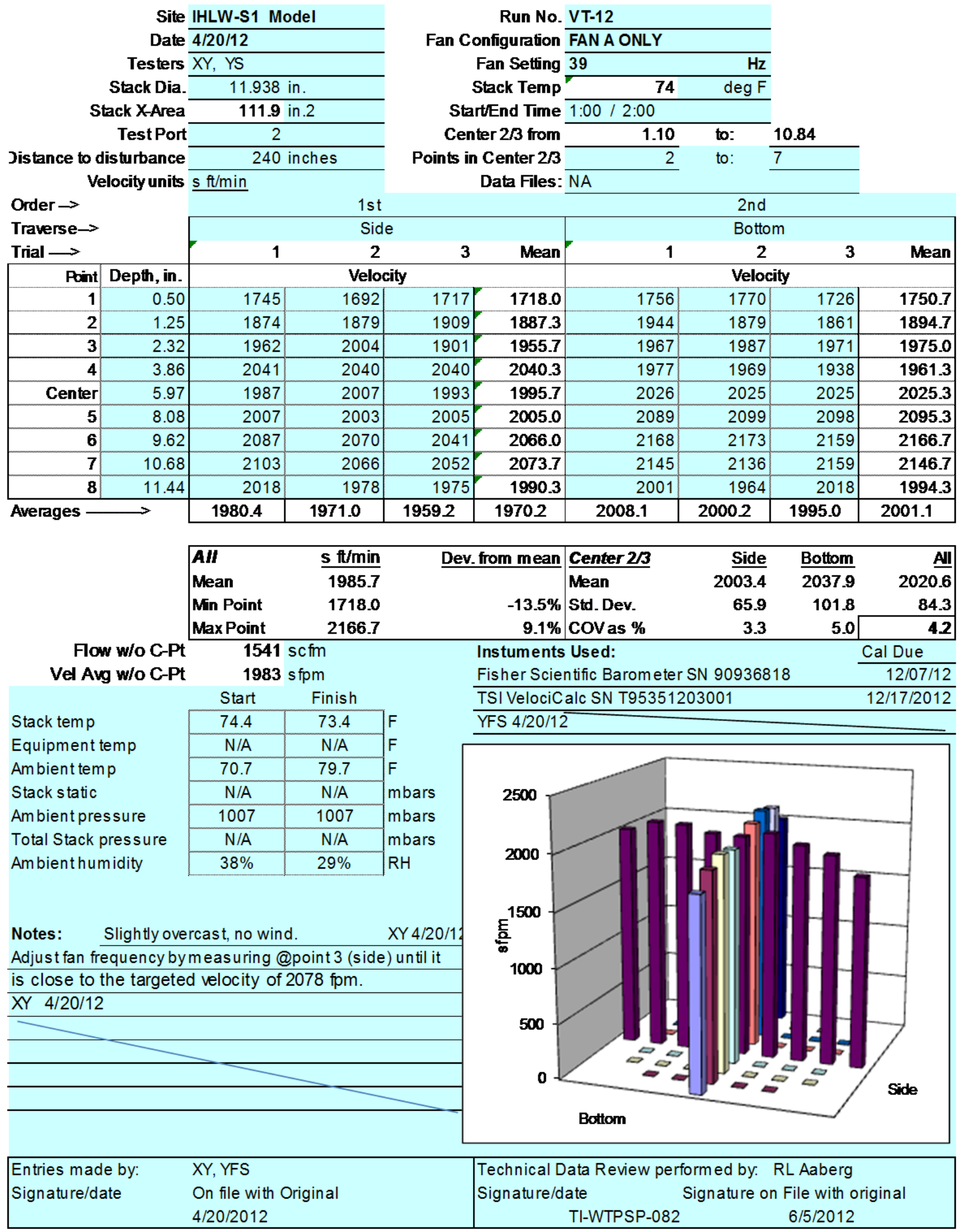




\section{VEOCITY TRAVERSE DATA FORM}

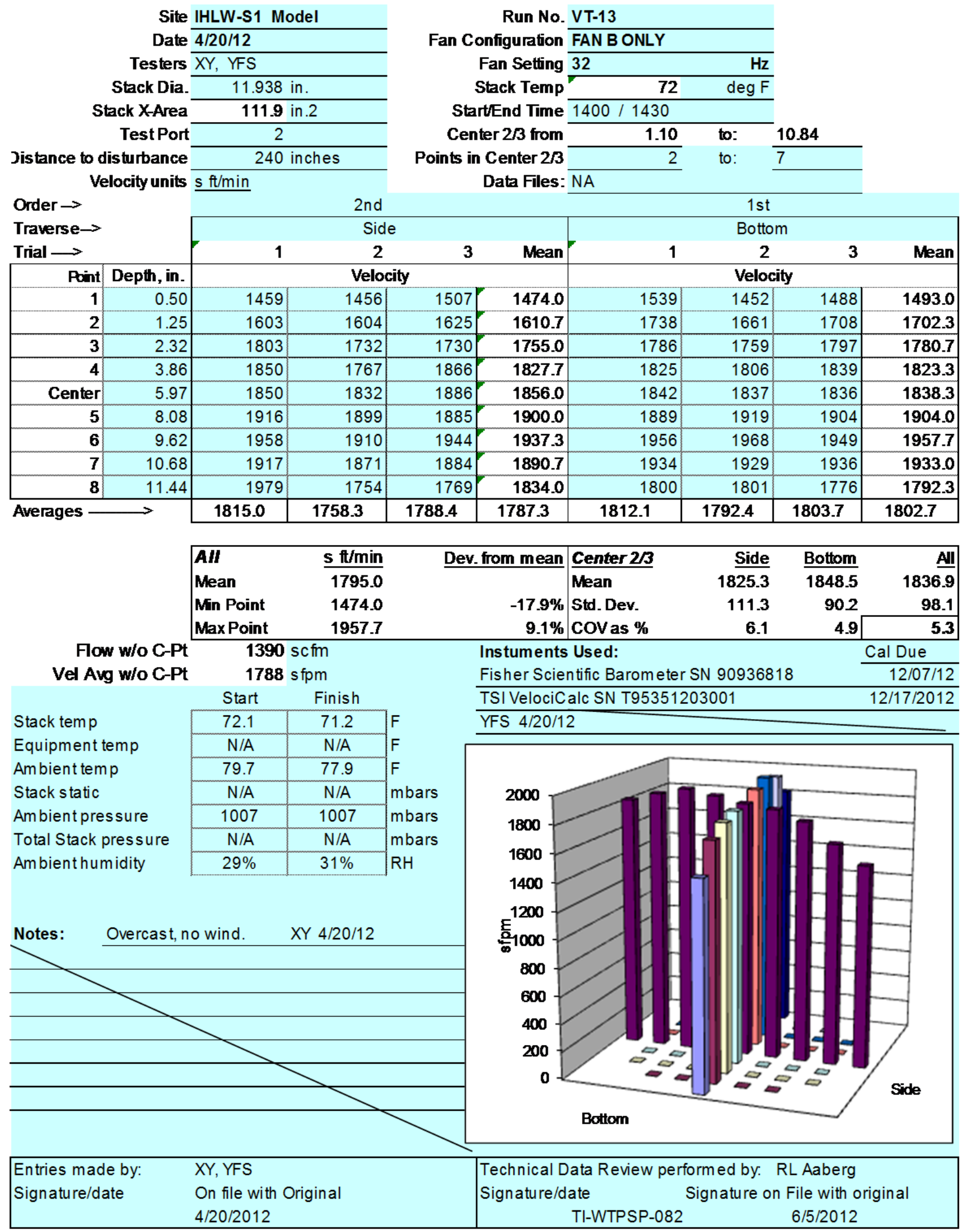




\section{VEOCITY TRAVERSE DATA FORM}

Run No. VT-14

Fan Configuration FAN B ONLY

Fan Setting 32

Stack Temp

Start/End Time

Center $2 / 3$ from

Points in Center $\mathbf{2} \boldsymbol{3}$

Data Files: NA

$\frac{72}{1430 / 1500}$

Site IHLW-S1 Model

Date 4/20/12

Stack Dia.

Stack X-Area

Test Port

Jistance to disturance

Velocity units $\mathrm{s} \mathrm{ft} / \mathrm{min}$

Order $\rightarrow$

Traverse $\rightarrow$

Trial $\longrightarrow$
Testers $X Y$, YFS
111.9 in.2

2

240 inches

1 st$$
240 \text { inches }
$$

\begin{tabular}{rr}
\hline $\mathrm{Hz}$ \\
\hline 72 & $\operatorname{deg~F}$ \\
\hline
\end{tabular}

1500

to: 10.84

to: 7 2nd Bottom \begin{tabular}{ccc}
\hline & Side \\
$\mathbf{1}$ & $\mathbf{2}$ \\
& & Velocity
\end{tabular}

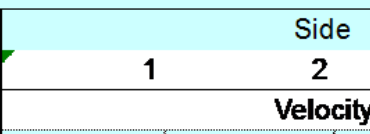

in.

$50 \quad 1510$ 0.50 1.25

\begin{tabular}{l|l}
\hline 32 & 161 \\
\hline 86 & 1782 \\
\hline 8 & 1820 \\
\hline
\end{tabular}

\begin{tabular}{|r|r|}
\hline $\mathbf{3}$ & 2.32 \\
\hline $\mathbf{4}$ & 3.86 \\
\hline Center & 5.97 \\
\hline $\mathbf{5}$ & 8.08 \\
\hline $\mathbf{6}$ & 9.62 \\
\hline $\mathbf{7}$ & 10.68 \\
\hline $\mathbf{8}$ & 11.44 \\
\hline
\end{tabular}

Averages

\begin{tabular}{|r|r|r|}
\hline 08 & 1879 & 1906 \\
\hline 62 & 1925 & 1986 \\
\hline 48 & 1985 & 1964 \\
\hline & 1929 & 1945 \\
\hline & 1758 & 1826 \\
\hline
\end{tabular}

\begin{tabular}{l}
1504 \\
1644 \\
\hline 1783 \\
185 \\
\hline 1906 \\
1986 \\
196 \\
\hline 1945 \\
1826 \\
\hline 823.6 \\
\hline
\end{tabular}

3

1.10

1773

1785.7

1832.3

\begin{tabular}{|lr}
\hline$A I I$ & $\underline{s t t / m i n}$ \\
Mean & 1822.1 \\
Min Point & 1489.3 \\
Max Point & 1986.0 \\
\hline
\end{tabular}

Flow w/o C-Pt 1410 scfm $1814 \mathrm{sfpm}$ Vel Avg w/o C-Pt

\begin{tabular}{|c|c|c|}
\hline Start & Finish & \\
\hline 71.0 & 73.6 & $F$ \\
\hline $\mathrm{N} / \mathrm{A}$ & N/A & $F$ \\
\hline 77.0 & 77.9 & $F$ \\
\hline N/A & $\mathrm{N} / \mathrm{A}$ & mbars \\
\hline 1007 & 1007 & mbars \\
\hline $\mathrm{N} / \mathrm{A}$ & $\mathrm{N} / \mathrm{A}$ & mbars \\
\hline $31 \%$ & $29 \%$ & $\mathrm{RH}$ \\
\hline
\end{tabular}

Stack tem $p$ Equipment temp Ambient tem $p$

Stack static

Ambient pressure Total Stack pressure Am bient humidity

$31 \%$

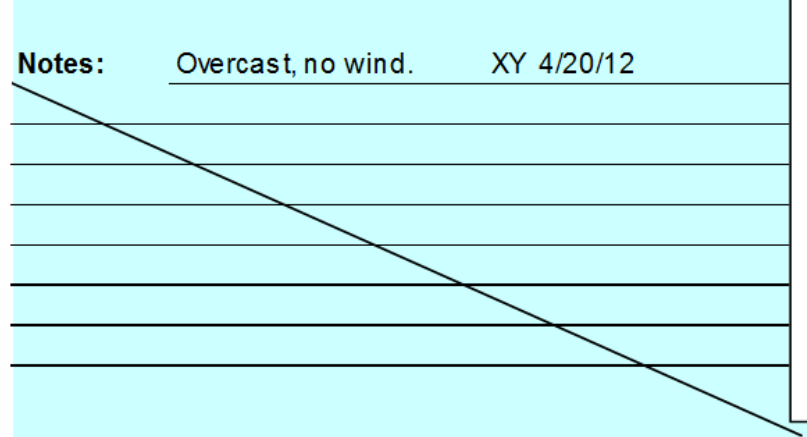
Dev. from mean Center 2/3
Mean
$-18.3 \%$ Std. Dev.
$\mathbf{9 . 0 \%}$ COV as $\%$

\begin{tabular}{|c|c|c|c|}
\hline \multicolumn{4}{|c|}{ 2nd } \\
\hline \multicolumn{4}{|c|}{ Bottom } \\
\hline 1 & 2 & 3 & Mean \\
\hline \multicolumn{4}{|c|}{ Velocity } \\
\hline 1569 & 1614 & 1616 & 1599.7 \\
\hline 1830 & 1792 & 1810 & 1810.7 \\
\hline 1899 & 1883 & 1850 & 1877.3 \\
\hline 1897 & 1882 & 1876 & 1885.0 \\
\hline 1880 & 1902 & 1908 & 1896.7 \\
\hline 1958 & 1930 & 1967 & 1951.7 \\
\hline 1996 & 1940 & 1921 & 1952.3 \\
\hline 1882 & 1830 & 1883 & 1865.0 \\
\hline 1702 & 1718 & 1678 & 1699.3 \\
\hline .9 & 1832.3 & 1834.3 & 1837.5 \\
\hline
\end{tabular}

\begin{tabular}{rrr|} 
Side & Bottom & All \\
\cline { 2 - 3 } 1855.1 & 18912 & 18732 \\
124.3 & 49.7 & 92.9 \\
6.7 & 2.6 & 5.0 \\
\hline
\end{tabular}

\begin{tabular}{lc} 
Instuments Used: & Cal Due \\
\cline { 2 - 2 } Fisher Scientific Barometer SN 90936818 & $12 / 07 / 12$ \\
\hline TSI VelociCalc SN T95351203001 & $12 / 17 / 2012$ \\
\hline YFS 4/20/12 & \\
\hline
\end{tabular}

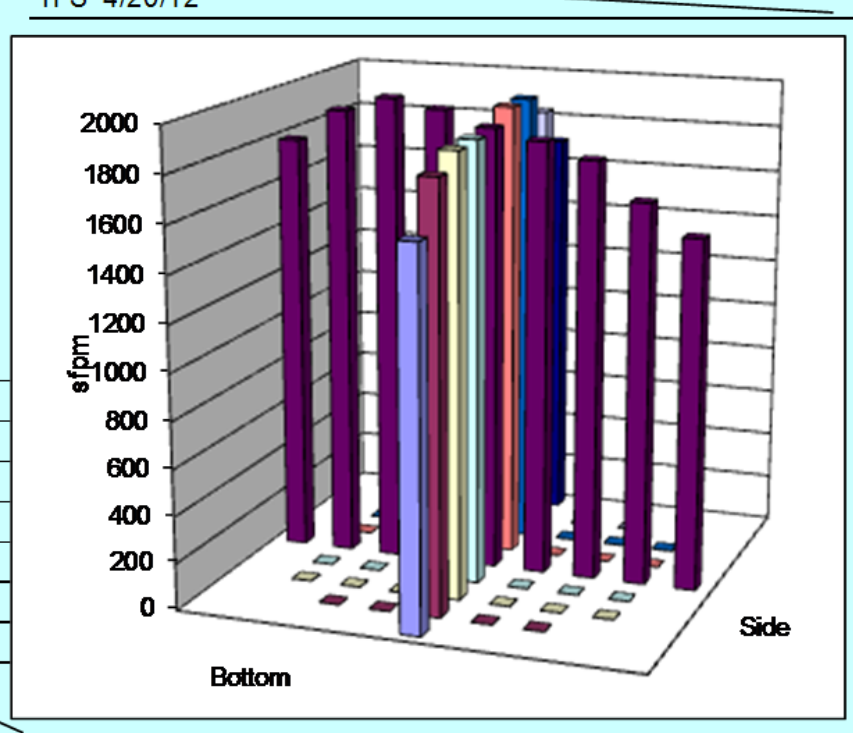

Entries made by:

$X Y, Y F S$

On file with Original $4 / 20 / 2012$
Technical Data Review performed by. RL Aaberg Signature/date TI-WTPSP-082
Signature on File with original $6 / 5 / 2012$ 


\section{VEOCITY TRAVERSE DATA FORM}

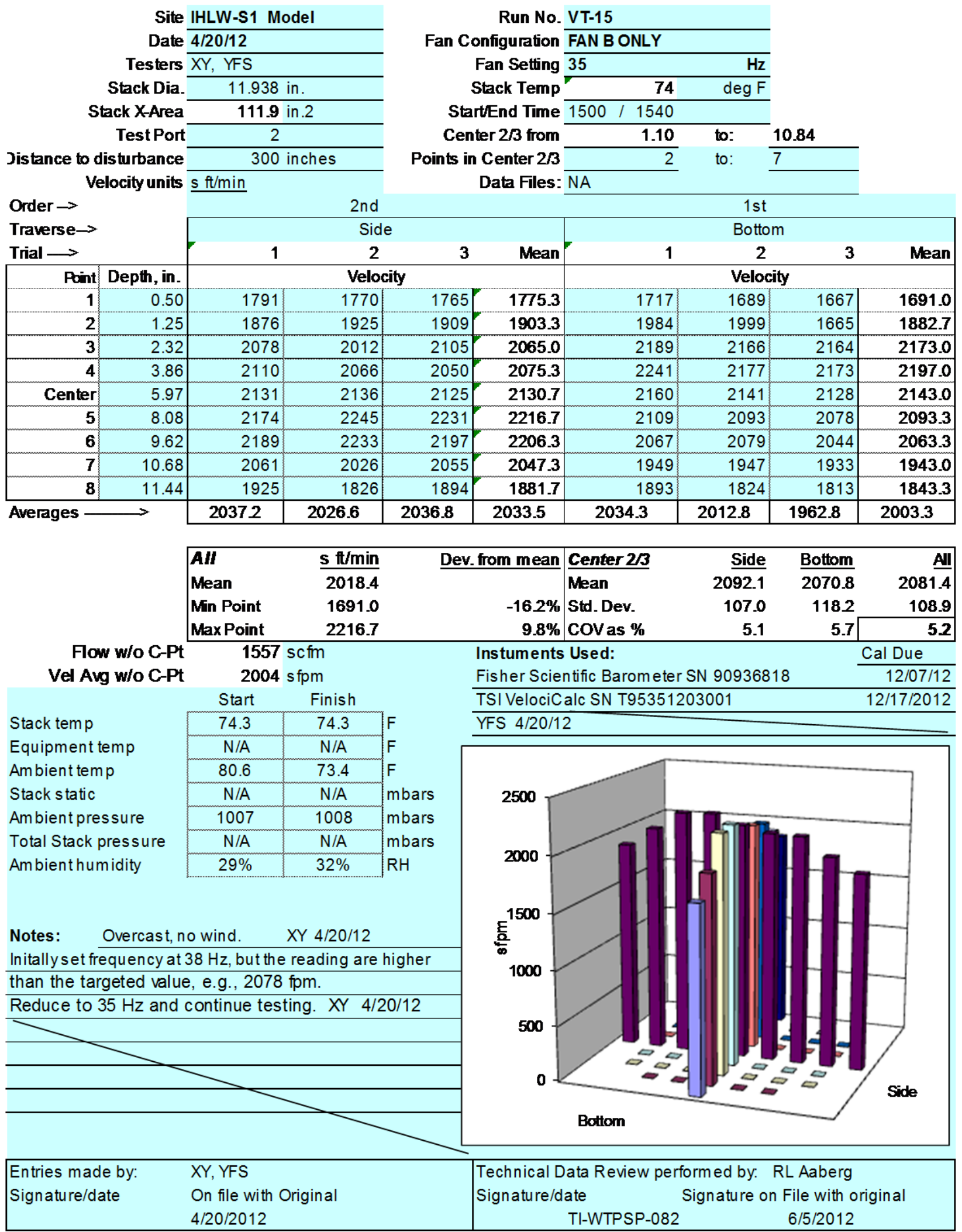




\section{C.3 IHLW-S1 Flow Angle Data Sheets}

FLOW ANGLE DATA FORM

Site IHLW-S1 scale model

Date $3 / 6 / 2012$

Tester EA, JEF, CA

Stack Dia. 11.938 in

Stack X-Area 111.9 in2

Elevation N.A. ft

Distance to disturbance 240

Start/End Time 1455h/1535h
IHLW-S1_PowAnglexts

Run No. FA-1

Fan Seting $42 \mathrm{~Hz}$

Fan configuration Fan A Only

Approx air vel. $2500 \mathrm{sfpm}$ at point $\gg>1$ bottom center Units degrees (clockwise > pos. nos.)

Port

Stack Temp 59 if

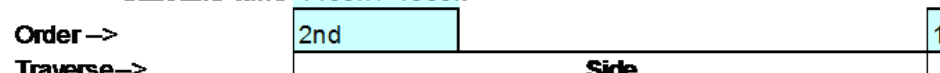
$1 \mathrm{st}$

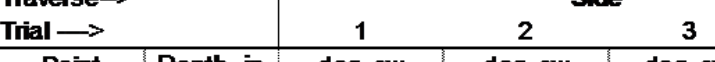

\begin{tabular}{|c|c|c|c|}
\hline Point & Depth, in. & deg.cw & deg. cw \\
\hline 1 & 0.50 & 4 & 8 \\
\hline 2 & 1.25 & 5 & 7 \\
\hline 3 & 2.32 & -1 & 5 \\
\hline 4 & 3.86 & -2 & 4 \\
\hline Center & 5.97 & -3 & -7 \\
\hline 5 & 8.08 & 2 & 0 \\
\hline 6 & 9.62 & 3 & 4 \\
\hline 7 & 10.68 & 5 & 5 \\
\hline 8 & 11.44 & 6 & 5 \\
\hline
\end{tabular}

Mean of absclute velues:

- "wo points by wall:

Instuments Used

S-type pitot

Velocity sensor

Angle indicator

Manometer

Note:

To assure similar hose connections

between the manometer and pitot tube, rotating

the pitot tube assembly clockwise drives the

meniscus to the right (to higher pos. numbers).

\section{Dwyer 24-inch S-type Pito\#10 \\ Shop built \\ Dwyer 400-5, S36N}

TSI Velocicalc SN\#T9535120300

3

\begin{tabular}{c|c|c|c} 
& 1 & 2 & 3 \\
deg. Avg. & deg. Cw & deg. cw & deg. cw
\end{tabular}

1

Bottom

3

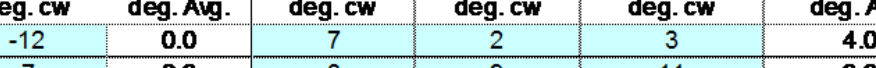

\begin{tabular}{l|r}
7 & 6.3 \\
\hline 1 & 1.7
\end{tabular}

\begin{tabular}{l|r}
1 & 1.7 \\
\hline 3 & 1.7 \\
-7 & -5.7
\end{tabular}

\begin{tabular}{l:r}
-7 & -5.7 \\
\hline 1 & 1.0
\end{tabular}

\begin{tabular}{l|r}
1 & 1.0 \\
3 & 3.3 \\
\hline
\end{tabular}

\begin{tabular}{|l|r}
3 & 3.3 \\
\hline 6 & 4.7 \\
\hline
\end{tabular}

\begin{tabular}{|l|}
\hline .3 \\
\hline 7 \\
1.7 \\
\hline .7 \\
\hline .0 \\
\hline .3 \\
4.7 \\
\hline .7 \\
\hline
\end{tabular}

$\begin{array}{r}\hline \\ \hline \\ \hline\end{array}$

\begin{tabular}{c|c}
\hline 8 & 2 \\
\hline 12 & 9 \\
\hline 5 & 7 \\
\hline 1 & 6 \\
\hline 4 & -2 \\
\hline 4 & 4 \\
\hline 5 & 3 \\
\hline 4 & 3 \\
\hline
\end{tabular}

\begin{tabular}{l|r}
11 & $\mathbf{4 . 0}$ \\
\hline 10 & 9.3 \\
\hline
\end{tabular}

\begin{tabular}{l|r}
10 & 9.7 \\
3 & 4.7
\end{tabular}

\begin{tabular}{l|l}
3 & 4.7 \\
2 & 0.3 \\
\hline & 3.3
\end{tabular}

\begin{tabular}{l|l}
3 & $\mathbf{3 . 3}$ \\
\hline
\end{tabular}

3.3

3.5

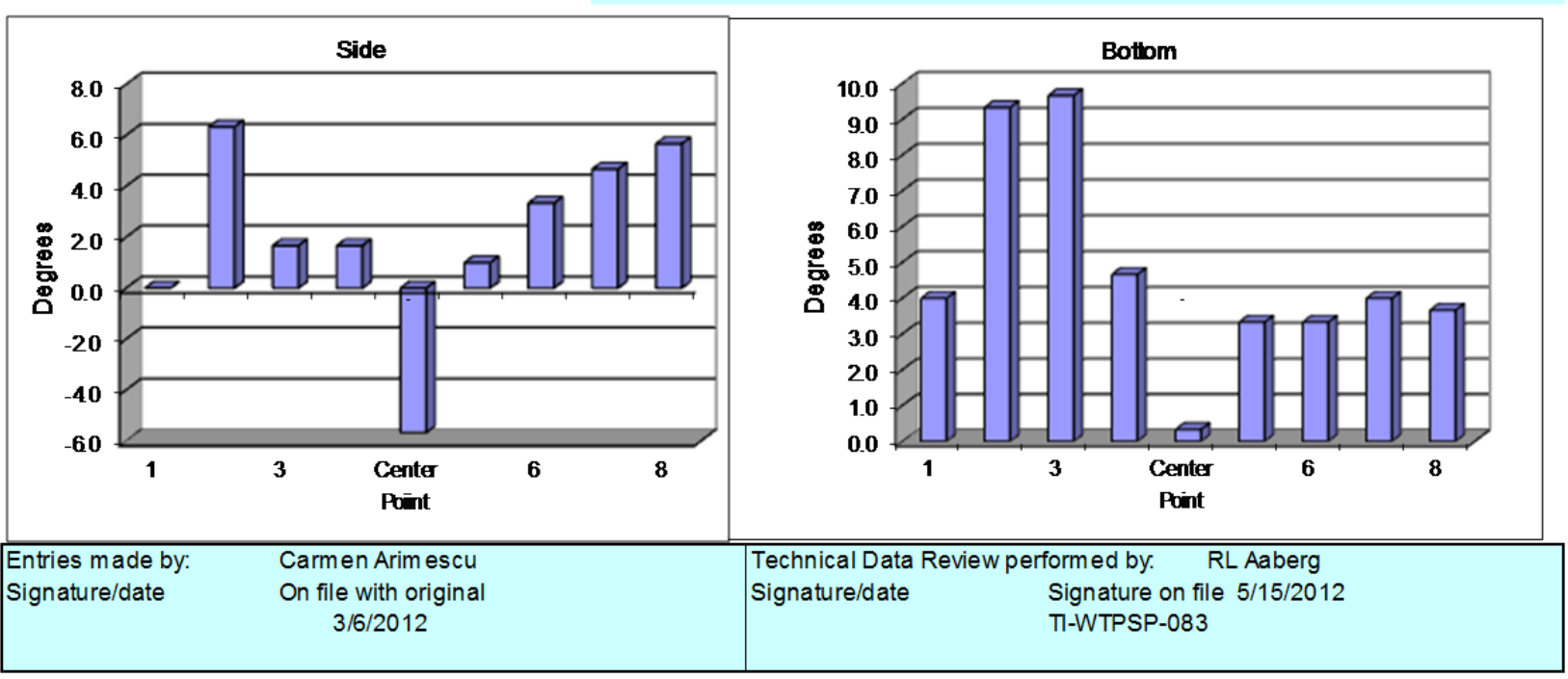


FLOW ANGLE DATA FORM

Site IHLW-S1 scale model

Date $3 / 6 / 2012$

Tester EA, JEF, CA

Stack Dia. 11.938 in

Stack X-Area 111.9 in2

Elevation N.A. $\mathrm{ft}$

Distance to disturbance $\frac{240}{15}$ in

Start/End Time $\overline{1543 \mathrm{~h} / 1627 \mathrm{~h}}$
IHLW-S1_FlowAngle.Xls

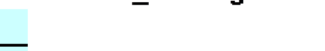
-

Run No. FA-2
Fan Setting $\frac{10}{10} \mathrm{~Hz}$
Fan configuration Fan A Only
Approx air vel. $500 \mathrm{sfpm}$ at point $\gg=1$ bottom center Units degrees (clockwise > pos. nos.)
Stack Temp Port $\frac{2}{62}{ }^{\circ} \mathrm{F}$

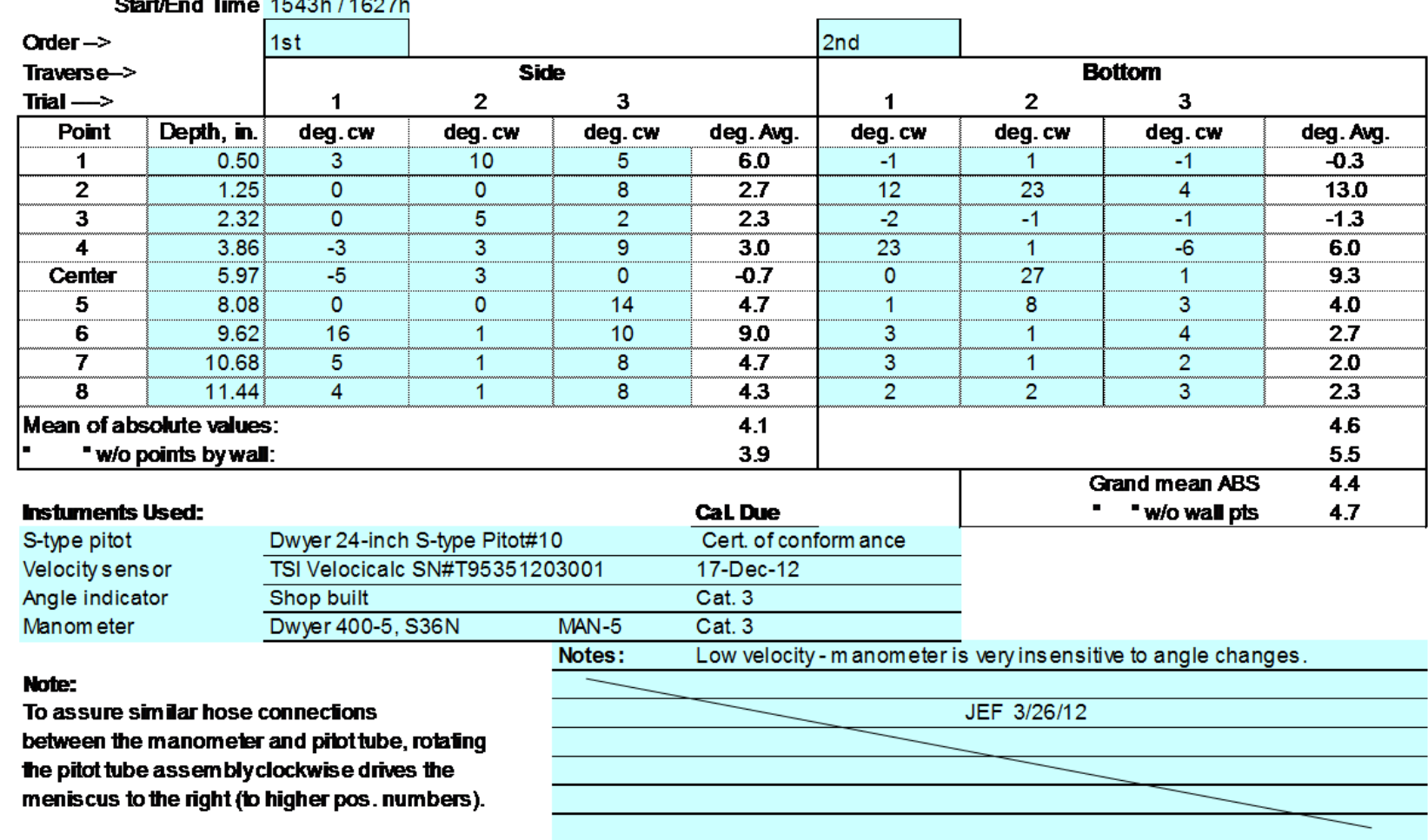

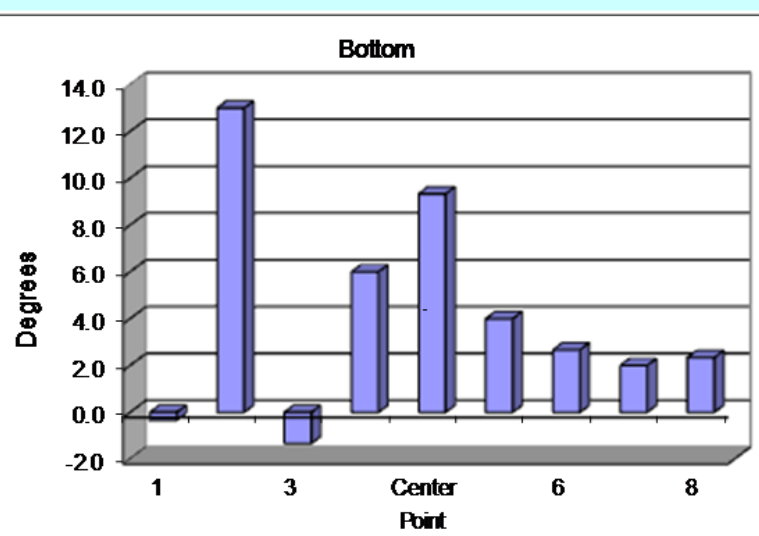

Entries $m$ ade by.

Signature/date

\author{
Carmen Arim escu \\ On file with original \\ $3 / 6 / 2012$
}

Technical Data Review performed by: $\quad$ RL Aaberg

Signature/date Signature on file 5/15/2012

TT-WTPSP-083 
FLOW ANGLE DATA FORM

Site IHLW-S1 scale model

Date $3 / 7 / 2012$

Tester EA, CA

Stack Dia. 11.938 in

Stack X-Area 111.9 in2

Bevation N.A.

Distance to disturbance 240 in

Start/End Tme $1405 \mathrm{~h} / 1507 \mathrm{~h}$
IHLW-S1_RowAnglexds

Run No. FA-3

Fan Setting $42 \mathrm{~Hz}$

Fan configuration Fan B Only

Approx air vel. 2570 sfpm at point $\gg 1$ bottom center Units degrees (clockwise > pos. nos.)

$$
\text { Port }
$$

Stack Temp

$60.4{ }^{\circ} \mathrm{F}$

1 st

Order $\rightarrow$

Traverse $\rightarrow$

Trial $\longrightarrow$

$$
\text { 2nd }
$$

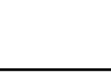

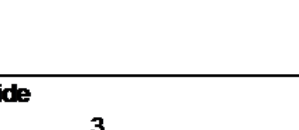

\begin{tabular}{|c|c|c|c|c|}
\hline Point & Depth, in. & deg. cw & deg. cw & deg. \\
\hline $\mathbf{1}$ & 0.50 & 6 & 9 & \\
\hline $\mathbf{2}$ & 1.25 & -4 & -5 \\
\hline $\mathbf{3}$ & 2.32 & 6 & -4 & \\
\hline $\mathbf{4}$ & 3.86 & 5 & -5 & \\
\hline Center & 5.97 & 5 & 8 & \\
\hline $\mathbf{5}$ & 8.08 & 3 & 4 & \\
\hline $\mathbf{6}$ & 9.62 & 3 & 3 \\
\hline $\mathbf{7}$ & 10.68 & 5 & 5 \\
\hline $\mathbf{8}$ & 11.44 & 7 & 5 \\
\hline
\end{tabular}

Mean of absolute values:

- - w/ points by wall:

Instuments Used:

S-type pitot

Velocity sens or

Angle indicator

Manometer

\begin{tabular}{c|c}
\hline$g . c w$ & de \\
\hline 9 \\
-5 \\
-5 \\
-4 \\
5 \\
\hline 4 \\
\hline 5 \\
\hline 5 \\
5
\end{tabular}

\begin{tabular}{l|c|cc} 
& 1 & 2 & 3 \\
deg. Avg. & deg. cw & deg. cw & deg.
\end{tabular}

\begin{tabular}{|c|c|c|c|c}
\hline 8.0 & 3 & deg. $c w$ & deg. cw & deg. Avg
\end{tabular}

\begin{tabular}{l|l|l|l|}
-4.7 & 11 & 10 & -4 \\
\hline
\end{tabular}

$-1.0$

$-1.3$

6.0

3.7

$\mathbf{3 . 7}$

5.7

4.3

3.6

11
11

1
1
1

1
2
2
3

$\mid$

\begin{tabular}{l|l}
-4 & 5.7 \\
-2 & $\mathbf{2 . 0}$
\end{tabular}

\begin{tabular}{l|l}
1 & 2.0 \\
0 & 0.7
\end{tabular}

\begin{tabular}{l|l}
0 & 0.3 \\
\hline
\end{tabular}

0
-1
1

-1
1 $\quad \begin{aligned} & 0.3 \\ & 3\end{aligned}$

\begin{tabular}{l|l}
3 & 1.0 \\
\hline & 2.3
\end{tabular}

$2 \quad 2.7$

Note:

To assure sim iar hose connections between the manometer and pibt tube, rotaing the pitot tube assembly clockwise drives the meniscus to the right (to higher pos. numbers).
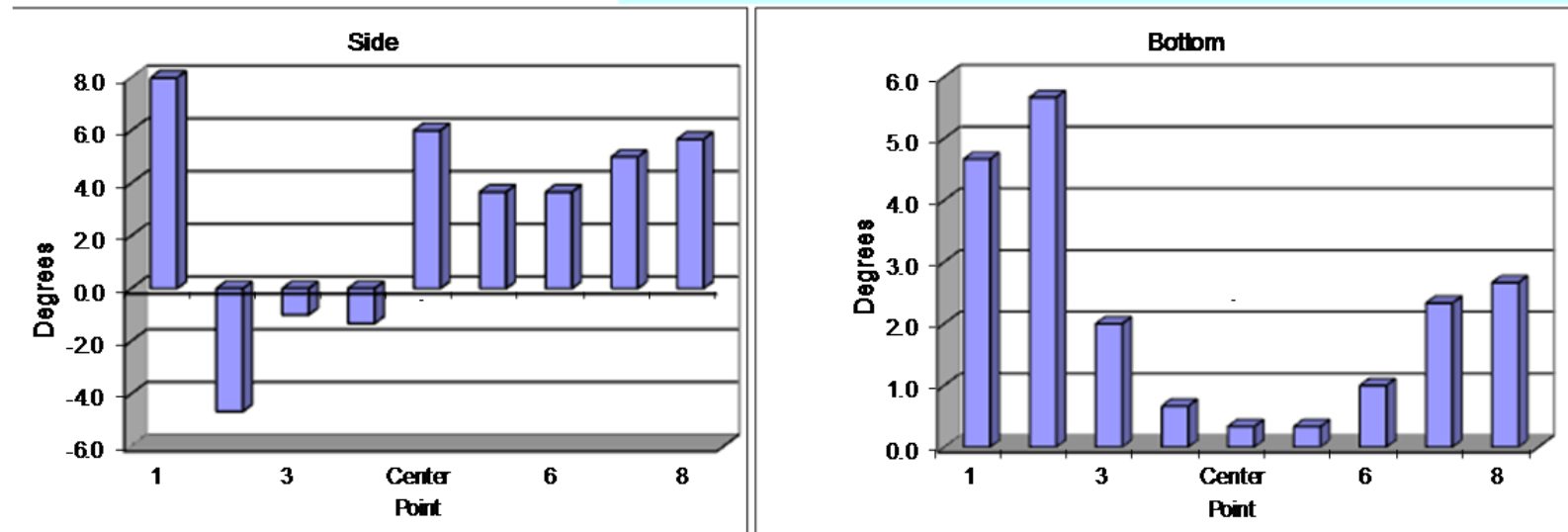

Cal. Due

Cert. of conformance

17-Dec-12

Shop built

Cat. 3

Notes:

Notes: $\longrightarrow$

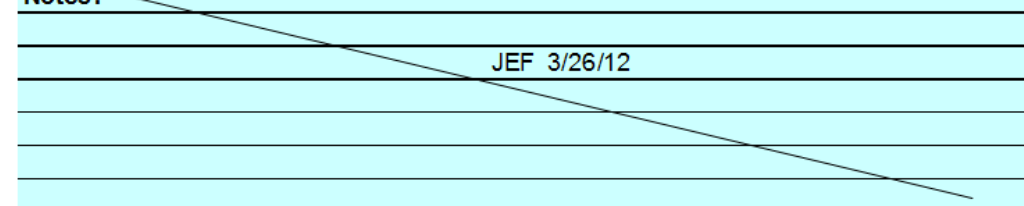

Grand mean ABS

1.8

- who wall pts $\quad 2.7$ 
FLOW ANGLE DATA FORM

Site IHLW-S1 scale model

Date 3/7/2012

Tester EA, CA

Stack Dia. 11.938 in

Stack X-Area 111.9 in2

Elevation N.A.

Distance to disturbance 300 in

Start/End Time $\overline{1515 \mathrm{~h} / 1610 \mathrm{~h}}$
IHLW-S1_RowAnglexts -

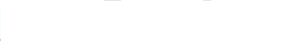

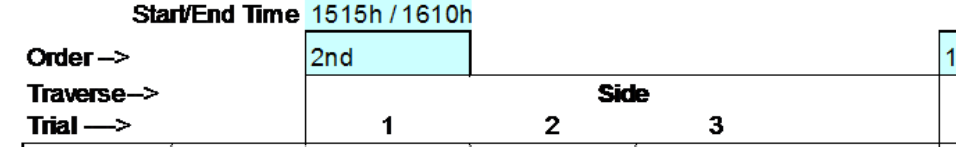

Run No. FA-4
Fan configuration Fan B only
Approx air vel. $2590 \mathrm{sfpm}$ at point $\gg>1$ bottom center Units degrees (clockwise > pos. nos.)
Stack Temp $\begin{array}{cc} & 1 \\ & 5^{\circ} \mathrm{F}\end{array}$

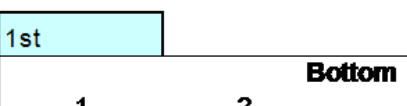

Mean of absolute velues:

- "w/o point bywall:

Instuments Used

S-type pitot

Velocity sensor

Angle indicator

Manometer

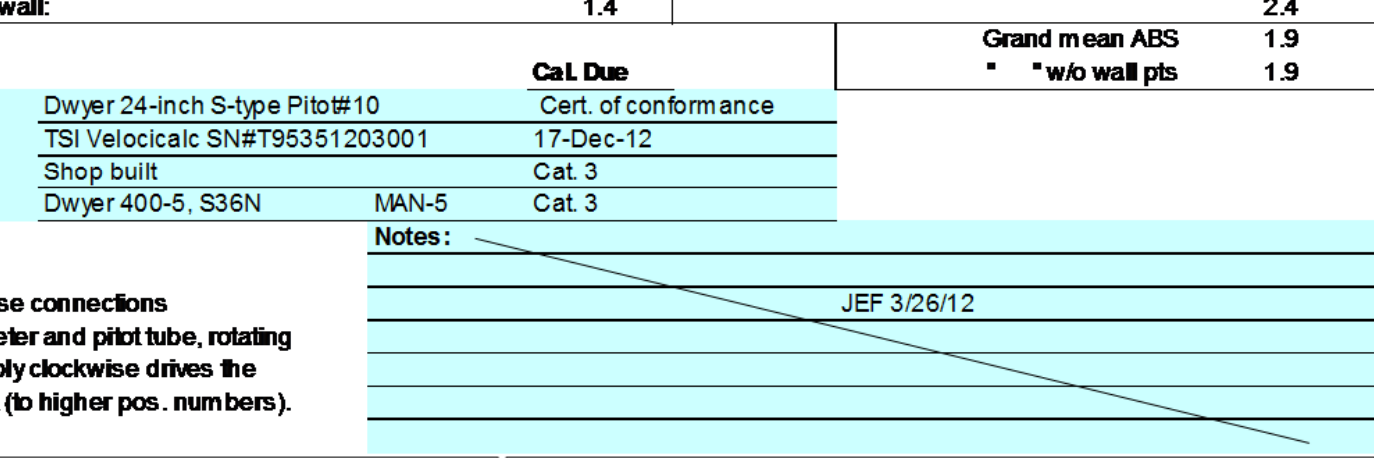

To assure similar hose connections between the manometer and pitbt tube, rotating the pitot tube assembly clockwise drives the meniscus to the right (to higher pos. numbers)

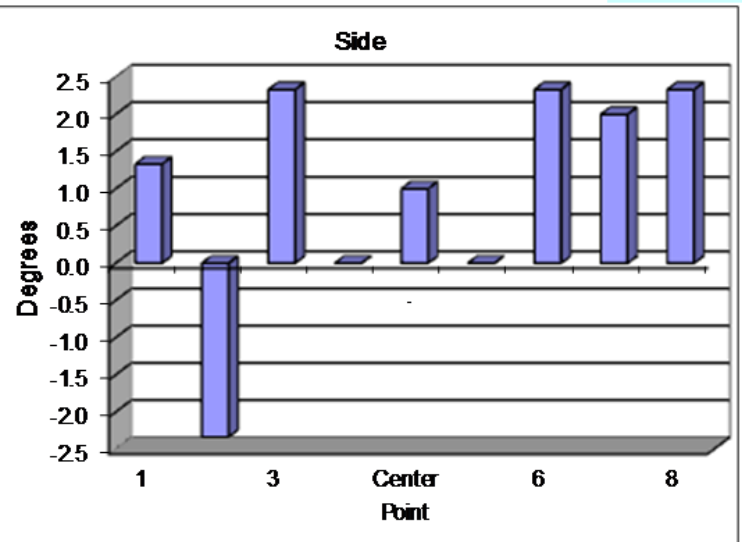

\begin{tabular}{|lc}
\hline Entries made by: & Carmen Arim escu \\
Signature/date & On file with original \\
& $3 / 7 / 2012$
\end{tabular}

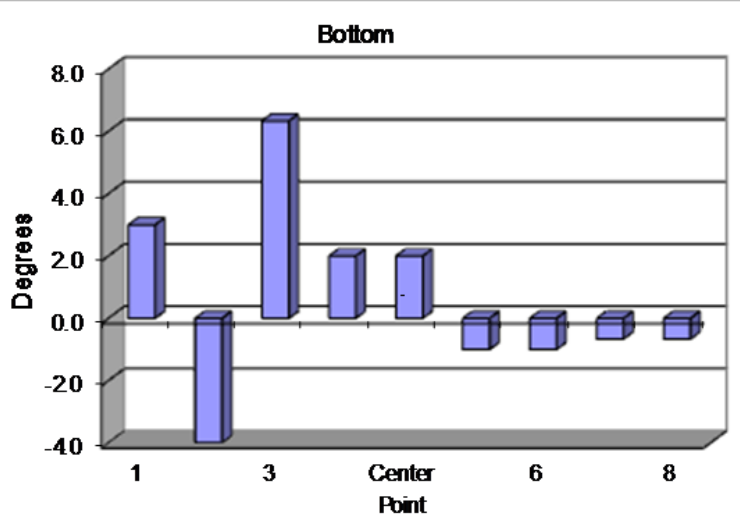

Technical Data Review performed by. RL Aaberg

Signature/date Signature on file 5/15/2012

T1-WTPSP-083 
FLOW ANGLE DATA FORM

Site IHLW-S1 scale model

Date $3 / 8 / 2012$

Tester CA, JEF

Stack Dia. 11.938 in

Stack X-Area 111.9 in2

Elevation N.A.

Distance b disturbance 240 in

Start/End Time $\overline{1300 \mathrm{~h} / 1335}$

IHLW-S1_RowAnglexts

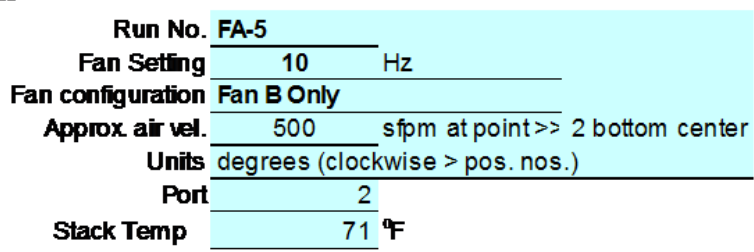

Run No. FA-5

Fan Seting

Approx air vel. $500 \mathrm{sfpm}$ at point $\gg 2$ bottom center Units degrees (clockwise > pos. nos.)

Stack Temp

1 st

Order $\rightarrow$

Traverse- $\rightarrow$

Trial $\longrightarrow$

$$
\text { 2nd }
$$

side

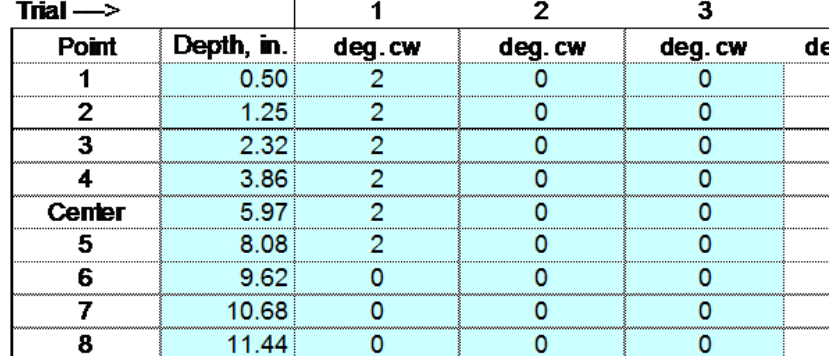

Meen of absclute velues:

- "who points bywall:

Instuments Used

S-type pitot

Velocity sensor

Angle indicator

Manometer wall: 0.5

Note:

To assure similar hose connections between the manometer and pitot tube, rotating the pitot tube assembly clockwise drives the meniscus to the right (to higher pos. numbers).

\begin{tabular}{|c|c|}
\hline & CaL Due \\
\hline Dwyer 24-inch S-type Pitot\#10 & Cert. of conform ance \\
\hline TSI Velocicalc SN\#T95351203001 & $17-$ Dec-12 \\
\hline Shop built & Cat. 3 \\
\hline Dwyer $400-5$, S36N & Cat. 3 \\
\hline
\end{tabular}

\begin{tabular}{l|l|l|l} 
& & \\
\hline
\end{tabular}

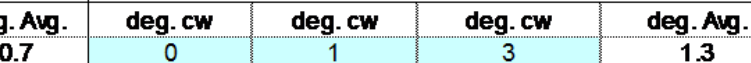

\begin{tabular}{|l|l|l|l|r|}
0.7 & 1 & 1 & 2 & 1.3 \\
\hline
\end{tabular}

\begin{tabular}{l|l|l|l|l}
0.7 & 1 & 1 & 2 & 1.3 \\
\hline
\end{tabular}

0.7

0.7

0.7

0.0

0.0

0.0

0.5

Notes: Highly insensitive to angle. Oil level remains at zero -15 to $+15 \mathrm{deg}$.

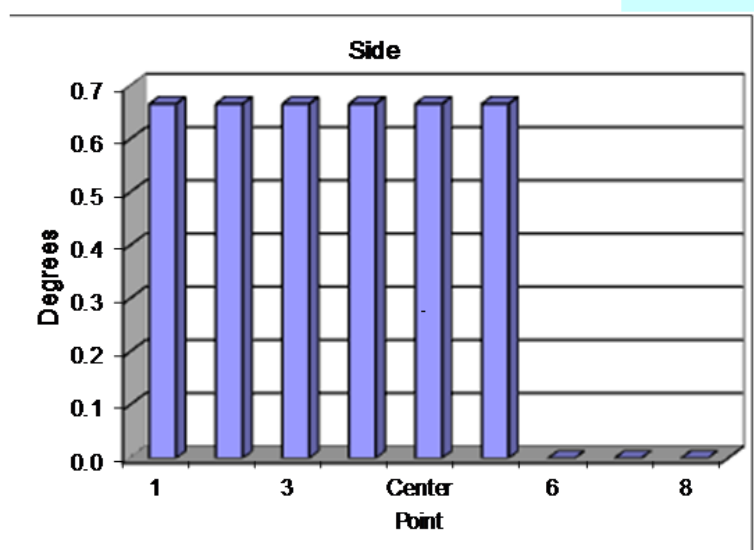

\begin{tabular}{|ll}
\hline Entries made by: & Carmen Arim escu \\
Signature/date & On file with Original \\
& $3 / 8 / 2012$
\end{tabular}

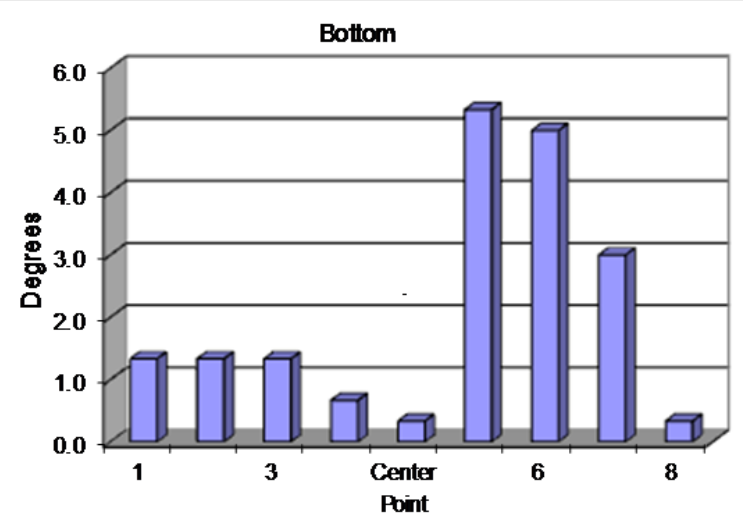

Technical Data Review performed by. RL Aaberg

Signature/date Signature on file 5/15/2012

T7-WTPSP-083 


\section{FLOW ANGLE DATA FORM}

Site IHLW-S1 scale mode

Date 3/8/2012

Tester CA, JEF, YFS

Stack Dia. 11.938 in

Stack X-Area 111.9 in 2

Elevation N.A. ft

Distance to disturbance $\frac{240}{13}$

Start/End Tme $1345 \mathrm{~h} / 1410 \mathrm{~h}$
IHLW-S1_FlowAngle.xts

Run No. FA-6

Fan Setting $33 \quad \mathrm{~Hz}$

Fan configuration Fan B Only

Approx.air vel. 1800 sfpm at point $>>1$ side center Units degrees (clockwise > pos. nos.)

Port 2

Stack Temp $69{ }^{\circ} \mathrm{F}$

\begin{tabular}{l|ll|l}
$\substack{\text { Order } \rightarrow \\
\text { Traverse } \rightarrow}$ & 1 st & Side
\end{tabular}

Trial $\longrightarrow$

\begin{tabular}{|c|r|}
\hline Point & Depth, in \\
\hline $\mathbf{1}$ & 0.50 \\
\hline $\mathbf{2}$ & 1.25 \\
\hline $\mathbf{3}$ & 2.32 \\
\hline $\mathbf{4}$ & 3.86 \\
\hline Center & 5.97 \\
\hline $\mathbf{5}$ & 8.08 \\
\hline $\mathbf{6}$ & 9.62 \\
\hline $\mathbf{7}$ & 10.68 \\
\hline $\mathbf{8}$ & 11.44 \\
\hline
\end{tabular}

Mean of absolute values:

- w/o point by wall:

Instuments Used:

S-type pitot Velocity sensor Angle indicator

Manometer

Note:

To assure similar hose connections

between the manometer and pitot tube, rotating

the pitot tube assembly clockwise drives the

meniscus to the right (to higher pos. numbers).

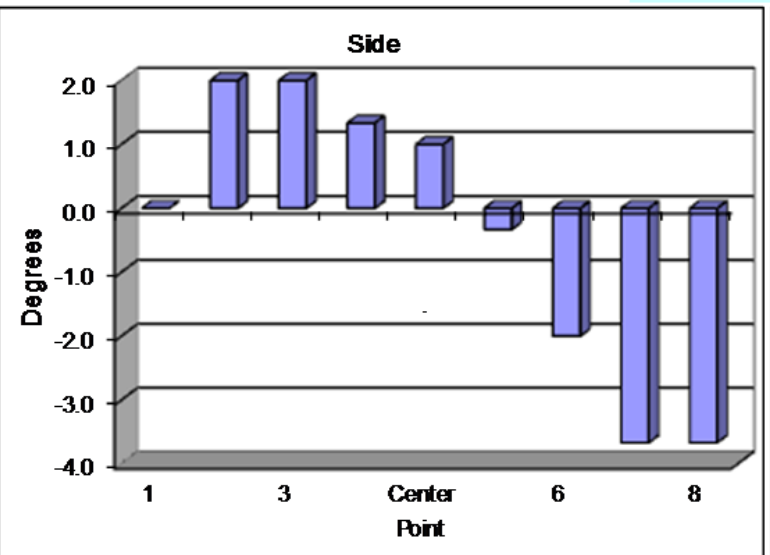

Entries made by:

Carm en Arim escu

Signature/date

On file with Original

$3 / 8 / 2012$

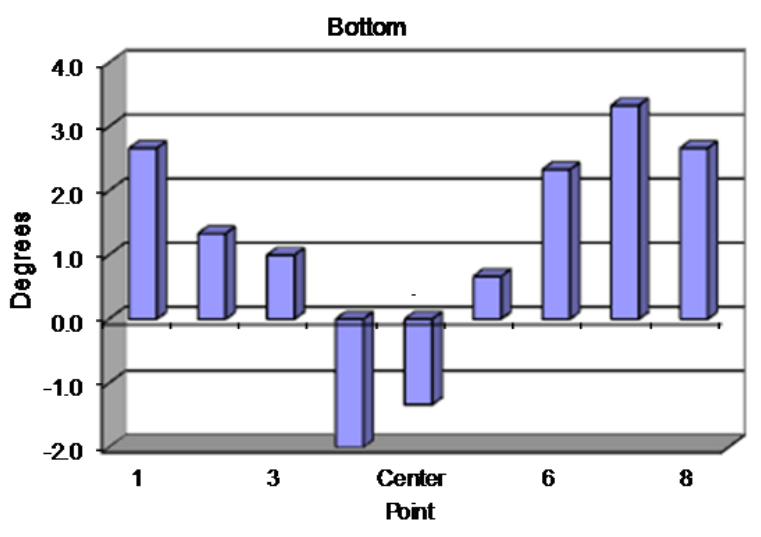

Technical Data Review perform ed by. RL Aaberg

Signature/date Signature on file 5/15/2012

TI-WTPSP-083 


\section{FLOW ANGLE DATA FORM}

Site IHLW-S1 scale mode

Date 3/21/2012

Tester CA, JAG

Stack Dia. 11.938 in

Stack X-Area 111.9 in 2

Elevation N.A. ft

Distance to disturbance $\frac{240}{14: 10 / 15: 15}$ in

Start/End Tme 14:10/15:15

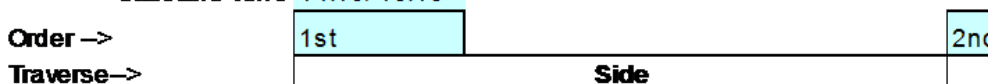

Trial $\longrightarrow$

\begin{tabular}{|c|r|}
\hline Point & Depth, in \\
\hline $\mathbf{1}$ & 0.5 \\
\hline $\mathbf{2}$ & 1.2 \\
\hline $\mathbf{3}$ & 2.3 \\
\hline $\mathbf{4}$ & 3.86 \\
\hline Center & 5.97 \\
\hline $\mathbf{5}$ & 8.08 \\
\hline $\mathbf{6}$ & 9.62 \\
\hline $\mathbf{7}$ & 10.68 \\
\hline $\mathbf{8}$ & 11.44 \\
\hline
\end{tabular}

Mean of absolute values:

- w/o points by wall:

Instuments Used:

S-type pitot

Velocity sensor

Angle indicator

Manometer

Note:

To assure similar hose connections

between the manometer and pitot tube, rotating

the pitot tube assem bly clockwise drives the

meniscus to the right (to higher pos. numbers).

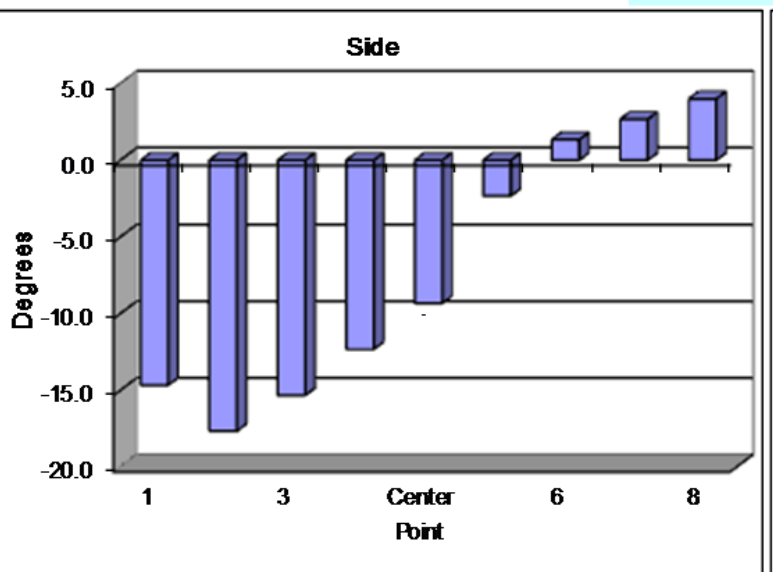

\begin{tabular}{|lc}
\hline Entries m ade by: & Carm en Arim escu \\
Signature/date & $3 / 21 / 2012$
\end{tabular}

IHLW-S1_FlowAngle.xls

3

3

deg. Avg.

\begin{tabular}{l|r}
\hline g. CW & deg. \\
\hline-14 & -17 \\
\hline-19 & -17 \\
\hline-14 & -15 \\
\hline-12 & -11 \\
\hline-8 & -10 \\
\hline-3 & -3 \\
\hline 3 & \\
\hline 3 & \\
\hline 3 &
\end{tabular}

8.9

8.7

Lue

TSI Velocicalc SN\#T95351203001 17-Dec-12

\begin{tabular}{ll}
\hline Shop built & Cat. 3
\end{tabular}

Notes:

Cat. 3

Run No. FA-7

Fan Setting $35.5 \mathrm{~Hz}$

Fan configuration FAN B ONLY

Approx. air vel. $1775 \mathrm{sfpm}$ at point $\gg>$

Units degrees (clockwise > pos. nos.)

Port

Stack Temp $45.2^{\circ} \mathrm{F}$

nd

1

Bottom

Bottom

\begin{tabular}{|c|c|c|c|}
\hline deg. cw & deg. Avg. & deg. cw & deg. \\
\hline-17 & -14.7 & -2 \\
\hline-17 & -17.7 & -10 \\
\hline-15 & -15.3 & -9 & \\
\hline-11 & -12.3 & -8 \\
\hline-10 & -9.3 & 3 & \\
\hline-3 & -2.3 & 5 \\
\hline 0 & 1.3 & 5 & \\
\hline 3 & 2.7 & 4 \\
\hline 5 & 4.0 & 6 & \\
\hline
\end{tabular}

23

\begin{tabular}{|l|l|l}
\hline eg. cw & deg.cw & deg. Avg.
\end{tabular}

\begin{tabular}{l|c|c}
-8 & -8 & -6.0 \\
-14 & -17 & -13.7
\end{tabular}

\begin{tabular}{l|l|l}
-14 & -17 & -13.7
\end{tabular}

\begin{tabular}{l|l|l}
-11 & -13 & -3.7
\end{tabular}

\begin{tabular}{l|l}
-3 & -11 \\
- & -7.3
\end{tabular}

$\left[\begin{array}{ll}-4 & -0.3\end{array}\right.$

\begin{tabular}{c|c|r}
3 & 3.7
\end{tabular}

6
7

\begin{tabular}{l|l|l}
6 & 7 & 5.7 \\
\hline 7 & 7 & 6.7
\end{tabular}

5.7

5.7

Cert. of conform ance

JAG $3 / 21 / 12$

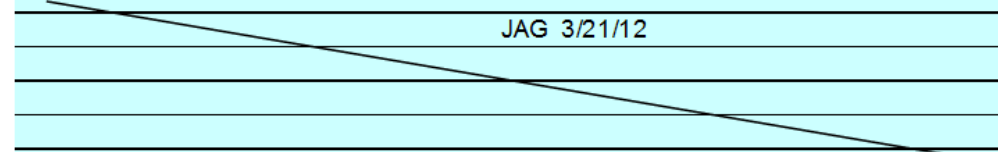

$\begin{array}{ll}\text { Grand mean ABS } & 7 \\ \text { - } \quad \text { w/o wall pts } & 7\end{array}$

7.1 
FLOW ANGLE DATA FORM

Site IHLW-S1 scale mode

Date 3/21/2012

Tester CA, JAG

Stack Dia. $\frac{11.938 \text { in }}{111.9}$

Stack X-Area 111.9 in 2

Elevation N.A.

Distance to disturbance $\frac{240}{15 \cdot 25 / 16: 15}$

Start/End Tme 15:25/16:15

\begin{tabular}{l|l|l} 
& Order $\rightarrow$ & 2nd
\end{tabular}
IHLW-S1_FlowAngle.xls

Run No. FA-8

Fan Setting $35.1 \mathrm{~Hz}$

Fan configuration FAN A ONLY

Approx. air vel. 1797 sfpm at point $\gg$

Units degrees (clockwise > pos. nos.)

Port 2

Stack Temp $42.5^{\circ} \mathrm{F}$

Bottom

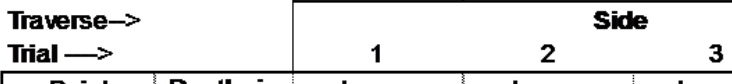

\begin{tabular}{|c|r|c|r|}
\hline Point & Depth, in. & deg. cw & deg. \\
\hline $\mathbf{1}$ & 0.50 & -13 & -1 \\
\hline $\mathbf{2}$ & 1.25 & -19 & -20 \\
\hline $\mathbf{3}$ & 2.32 & -16 & -17 \\
\hline $\mathbf{4}$ & 3.86 & -11 & \\
\hline Center & 5.97 & -5 & \\
\hline $\mathbf{5}$ & 8.08 & 0 & \\
\hline $\mathbf{6}$ & 9.62 & 2 & \\
\hline $\mathbf{7}$ & 10.68 & 7 & \\
\hline $\mathbf{8}$ & 11.44 & 7 & \\
\hline
\end{tabular}

Mean of absolute values:

- -w/o points by wall:

Instuments Used:

S-type pitot

Velocity sensor

Angle indicator

Manometer

eg.cw deg. cw

$-17$

deg. Avg.

\begin{tabular}{c|c}
1 & 2 \\
\hline eg. $c w$ & deg. cw
\end{tabular}

Bottom

\begin{tabular}{l:l}
\hline-18 & -17 \\
\hline-20 & -21 \\
\hline-17 & -15 \\
\hline-9 & -2 \\
\hline 5 & \\
\hline 6 & \\
\hline 8 &
\end{tabular}

\begin{tabular}{c|}
-21 \\
-15 \\
-7 \\
-2 \\
1 \\
4 \\
7 \\
7
\end{tabular}

$-16.0$

$-20.0$

$-16.0$

$-9.0$

$-3.0$

2.0

4.0

7.3

9.4

8.8

deg.cw, deg.cw deg.cw

\begin{tabular}{c|c|c|c}
\hline-1 & -3 & -9 & -4.3 \\
\hline-14 & -17 & -15 & $-\mathbf{1 5 . 3}$
\end{tabular}

\begin{tabular}{l|l}
-13 & -16
\end{tabular}

\begin{tabular}{l|l}
-16 & -14
\end{tabular}

$\begin{array}{l:l}-6 & -8 \\ 3 & 6\end{array}$

\begin{tabular}{c|c}
3 & -8 \\
\hline
\end{tabular}

$\begin{array}{l:r}6 & 6 \\ 9 & 10\end{array}$

\begin{tabular}{l|r}
9 & 10 \\
10 & 12 \\
\hline
\end{tabular}

\begin{tabular}{c|c|c|r}
10 & 12 & 12 & 11.3 \\
\hline 11 & 12 & 11 & 11.3
\end{tabular}

Note:

To assure similar hose connections

between the manometer and pitot wbe, rotating

the pitot tube assem bly clockwise drives the

meniscus to the right (to higher pos. numbers).

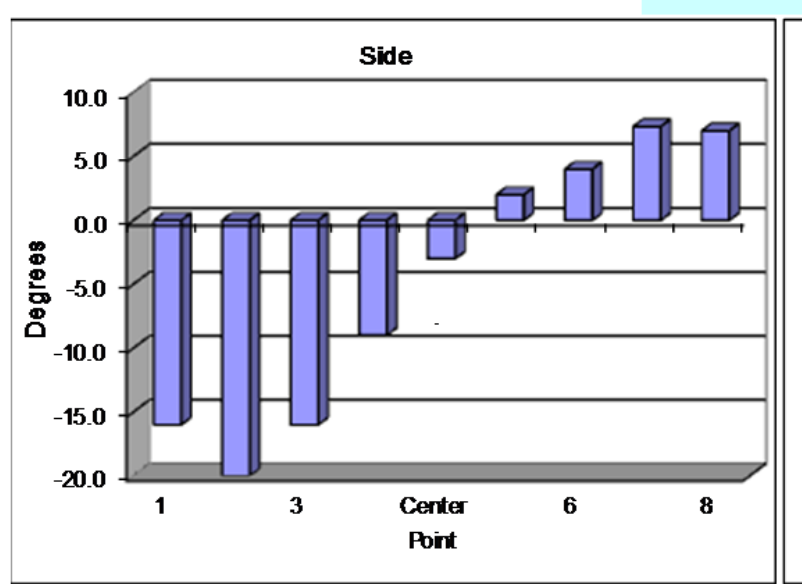

Cal. Due

Dwyer 24-inch S-type Pitot\#10

Cert. of conform ance

TSI Velocicalc SN\#T95351203001 17-Dec-12

Shop built

Cat. 3

MAN-5

Cat. 3

Notes:
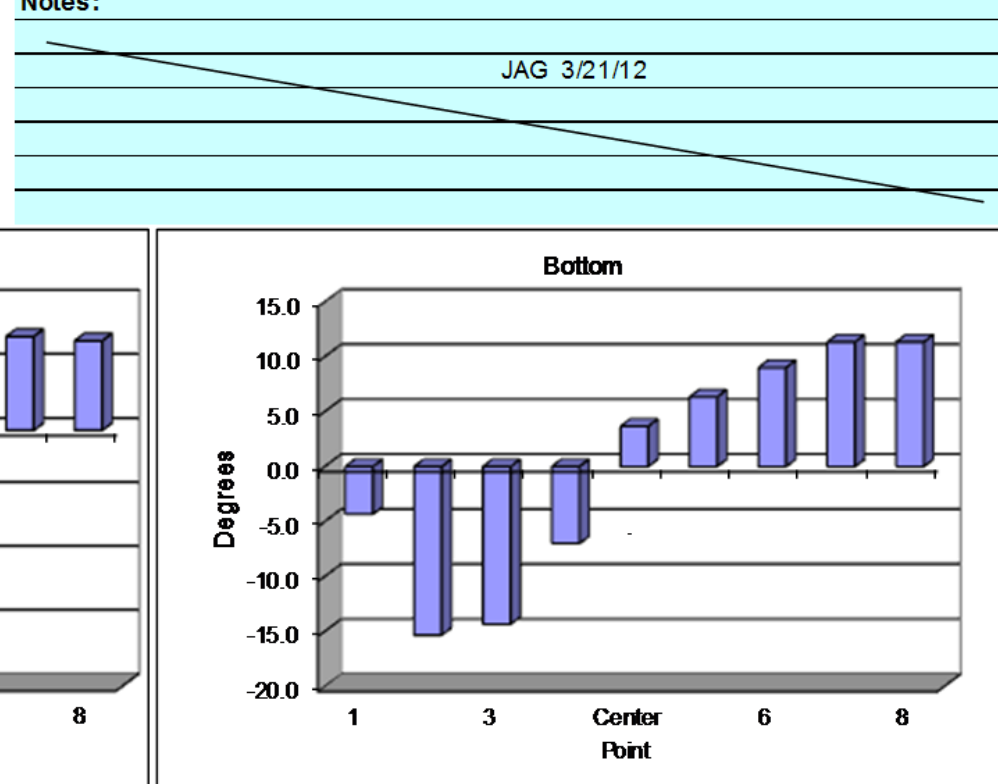


\section{C.4 IHLW-S1 Gas Tracer Calibration and Uniformity Data Sheets}

\section{SULFUR HEXAFLUORIDE GAS INSTRUMENT CALIBRATION}

\begin{tabular}{rlr} 
Site & HV-S1 Model \\
\cline { 3 - 3 } Date/Time & 4/16/2012 $12: 35$ PM \\
Testers & CA, XY, EA &
\end{tabular}

Setup

$7.7 \mathrm{ft}$

$1004 \mathrm{mbar}$

59.9 deg F ambient temp analyzer corrects to 20 deg C

$65 \%$ percent $\mathrm{RH}$ ambient humidity

$\begin{aligned} & \text { Instrument } \text { B\&K Model } 1302 \\ & \text { Serial No. } 1765299 \\ & \text { Property No. WD17210 }\end{aligned}$

Property No. WD17210
B\&K

Calibration

readings: (ppb)

Compensating for water vapor

\begin{tabular}{|r|}
\hline 108 \\
\hline 110 \\
\hline 106 \\
\hline 105 \\
\hline 104 \\
\hline
\end{tabular}

Not compensating for water vapor

\begin{tabular}{|r|}
\hline 108 \\
\hline 108 \\
\hline 110 \\
\hline 107 \\
\hline 107 \\
\hline $107=$ avg \\
$1.07=$ avg/standard
\end{tabular}

Standards Used:

Air Liquide 0.1 ppm SF6 in air, CAL11936

Air Liquide 4.97 ppm SF6 in air, FF34346

Weather Station Used:

Fisher Scientific S/N 90936818

$\mathrm{ppb}$

Cylinder CAL11936

start $P=1600$ psi

end $P=1600$ psi

$\begin{aligned} 107 & =\text { avg } \\ 1.07 & =\text { avg/standard }\end{aligned}$
Entries made by: $\quad$ EA, CA

Signature/date
$4 / 16 / 2012$

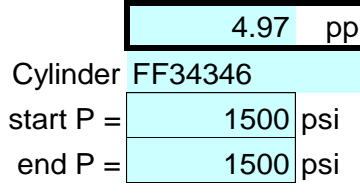

B\&K

Calibration

readings: (ppm)

Compensating for water vapor

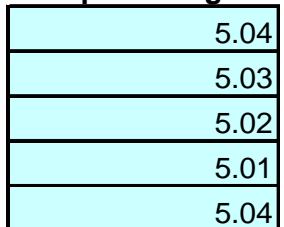

Not compensating for water vapor

5.01

5.01

5.00

5.00

5.00

$5.02=a v g$

$1.01=\mathrm{avg} / \mathrm{standard}$

Expiration date:

$3 / 19 / 2013$

$3 / 19 / 2014$

$12 / 7 / 2012$

Technical Data Review performed by: E. G.

Signature/date Signature on file with Original

TI-WTPSP-084

$7 / 10 / 2012$ 


\section{SULFUR HEXAFLUORIDE GAS INSTRUMENT CALIBRATION}

\author{
Site IHLW-S1 Model \\ Date/Time 4/23/12 7:55AM \\ Testers JEF, CA
}

\author{
Instrument B\&K Model 1302 \\ Serial No. 1765299 \\ Property No. WD17210
}

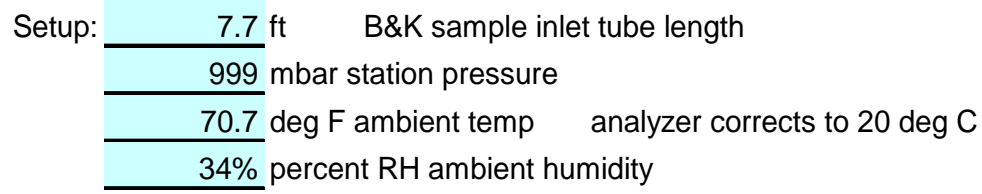

\[ \begin{array}{rr}100 \mathrm{ppb} \\ \text { Cylinder CAL11936 }\end{array} \]
start $\mathrm{P}=\frac{\mathrm{psi}}{1600} \mathrm{psi}$

$B \& K$

Calibration

readings: (ppb)

Compensating for water vapor

\begin{tabular}{|r|}
\hline 106 \\
\hline 107 \\
\hline 107 \\
\hline 104 \\
\hline 108 \\
\hline
\end{tabular}

Not compensating for water vapor

\begin{tabular}{|r|}
\hline 103 \\
\hline 108 \\
\hline 108 \\
\hline 108 \\
\hline 109 \\
\hline $107=$ avg \\
$1.07=$ avg/standard
\end{tabular}

Standards Used:

Air Liquide 0.1 ppm SF6 in air, CAL11936

Air Liquide 4.97 ppm SF6 in air, FF34346

Weather Station Used:

Fisher Scientific S/N 90936818

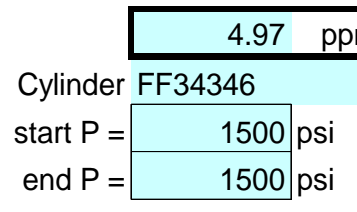

B\&K

Calibration

readings: (ppm)

Compensating for water vapor

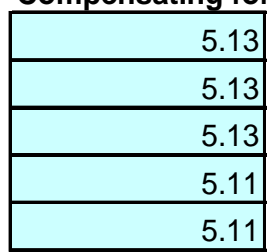

Not compensating for water vapor

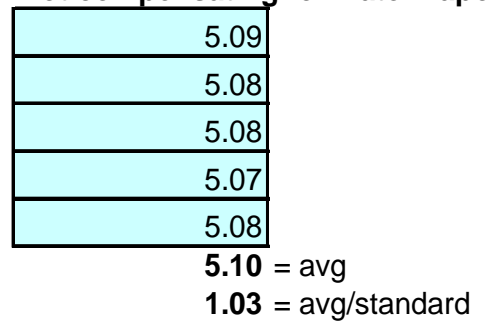

Expiration date: $3 / 19 / 2013$ $3 / 19 / 2014$

$12 / 7 / 2012$

\begin{tabular}{|l|lr}
\hline $\begin{array}{l}\text { Entries made by: } \quad \text { JEF 4/23/2012 } \\
\text { Signature/date }\end{array}$ & $\begin{array}{l}\text { Technical Data Review performed by: } \\
\text { Signature/dater G. }\end{array}$ \\
& Signature on file with Original \\
&
\end{tabular}


SULFUR HEXAFLUORIDE GAS INSTRUMENT CALIBRATION

\author{
Site IHLW-S1 Model \\ Date/Time 4/23/12 11:15AM \\ Testers JEF, CA
}

\author{
Instrument B\&K Model 1302 \\ Serial No. SN 1765299 \\ Property No. WD17210
}

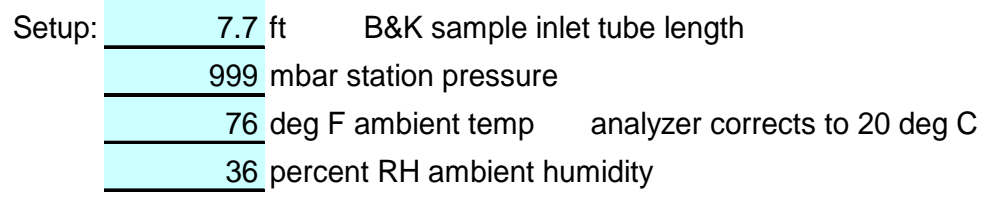

\begin{tabular}{rr|} 
& $100 \mathrm{ppb}$ \\
Cylinder CAL11936 & \\
start $P=$ & $1550 \mathrm{psi}$ \\
end $P=$ & $1550 \mathrm{psi}$
\end{tabular}

B\&K

Calibration

readings: (ppb)

Compensating for water vapor

\begin{tabular}{|r|}
\hline 104 \\
\hline 105 \\
\hline 106 \\
\hline 105 \\
\hline 106 \\
\hline
\end{tabular}

Not compensating for water vapor

\begin{tabular}{|r|}
\hline 106 \\
\hline 104 \\
\hline 103 \\
\hline 110 \\
\hline 105 \\
\hline $105=a v g$ \\
$1.05=$ avg/standard
\end{tabular}

Standards Used:

Air Liquide 0.1 ppm SF6 in air, CAL11936

Air Liquide 4.97 ppm SF6 in air, FF34346

Weather Station Used:

Fisher Scientific S/N 90936818
$12 / 7 / 2012$

\begin{tabular}{|c|c|c|}
\hline & 4.97 & \\
\hline Cylinder & FF34346 & \\
\hline & 1500 & psi \\
\hline & 1500 & ps \\
\hline
\end{tabular}

B\&K

Calibration

readings: (ppm)

Compensating for water vapor

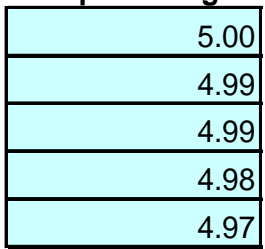

Not compensating for water vapor

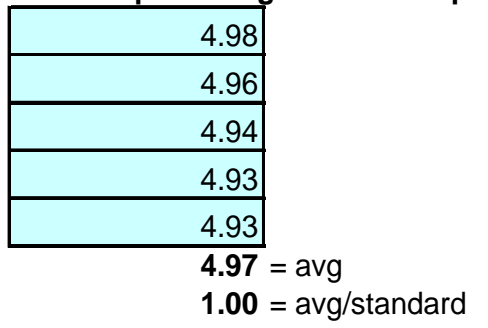

Expiration date:

$3 / 19 / 2013$

$3 / 19 / 2014$
Technical Data Review performed by: E. G.

Signature/date Signature on file with Original TI-WTPSP-084

$7 / 10 / 2012$ 
Rev. 0

31-Jul-06

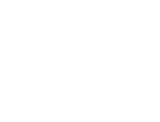

TRACER GAS TRAVERSE DATA FORM

\begin{aligned} Stack X-Area & 111.9 in. $^{2} \\ &$ Test Port \\ \cline { 2 - 2 } Distance to disturbance & 240 inches \end{aligned}

Measurement units ppb SF6

Site IHLW-S1 Model

Date 4/17/2012

Testers CA, XY

Stack Dia. 11.938 in.

ck X-Area

240 inches

Order $\rightarrow$

Traverse $\rightarrow$

Trial $\rightarrow$ 2nd
Run No. GT-1

Fan Confguration Fan B only

Fan Setting 43

Stack Temp

Start/End Time 9:30/11:40

Center $2 / 3$ from

Points in Center $2 / 3$

hjection Point 1-center

\begin{tabular}{|c|c|c|c|c|c|c|c|c|c|}
\hline Trial $\rightarrow$ & & 1 & 2 & 3 & Mean & 1 & 2 & 3 & Mean \\
\hline Point & Depth, in. & \multicolumn{4}{|c|}{ ppb } & \multicolumn{4}{|c|}{ ppb } \\
\hline 1 & 0.50 & 562 & 550 & 571 & 561.00 & 575 & 567 & 570 & 570.67 \\
\hline 2 & 1.24 & 562 & 549 & 583 & 564.67 & 577 & 576 & 567 & 573.33 \\
\hline 3 & 2.29 & 557 & 549 & 550 & 552.00 & 565 & 574 & 572 & 570.33 \\
\hline 4 & 3.82 & 587 & 582 & 582 & 583.67 & 581 & 576 & 577 & 578.00 \\
\hline Center & 5.91 & 564 & 561 & 582 & 569.00 & 567 & 575 & 578 & 573.33 \\
\hline 5 & 8.00 & 591 & 561 & 575 & 575.67 & 589 & 569 & 552 & 570.00 \\
\hline 6 & 9.52 & 574 & 572 & 568 & 571.33 & 586 & 585 & 575 & 582.00 \\
\hline 7 & 10.57 & 572 & 574 & 572 & 572.67 & 578 & 578 & 563 & 573.00 \\
\hline 8 & 11.31 & 581 & 586 & 576 & 581.00 & 577 & 606 & 556 & 579.67 \\
\hline Averages - & $\rightarrow$ & 572.22 & 564.89 & 573.22 & 570.11 & 57722 & 578.44 & 567.78 & 574.48 \\
\hline
\end{tabular}

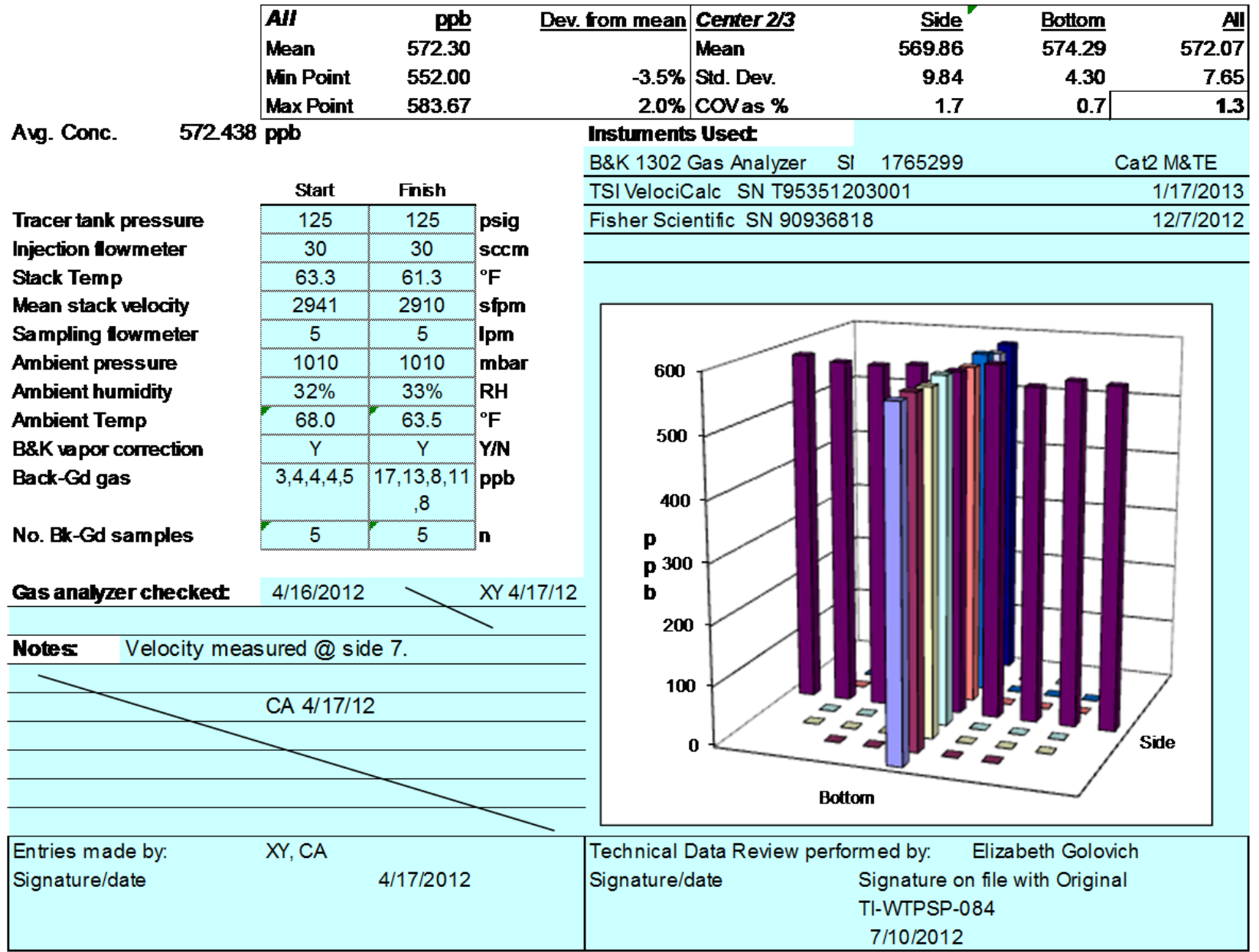


Rev. 0

31-Jul-06

Measurement units ppb SF6

TRACER GAS TRAVERSE DATA FORM

\begin{tabular}{|c|c|}
\hline \multicolumn{2}{|c|}{ Site IHLW-S1 Model } \\
\hline Date & $4 / 17 / 2012$ \\
\hline Testers & $\mathrm{XY}, \mathrm{CA}$ \\
\hline Słack Dia. & 11.938 in. \\
\hline Stack X-Area & 111.9 in. $^{2}$ \\
\hline Test Port & 2 \\
\hline disturbance & 240 inches \\
\hline
\end{tabular}

Run No. GT-2

Fan Configuration Fan A only

Fan Setting 43

Stack Temp Hz

StartEnd Time 11:50/13:02

Center 23 from

Points in Center $2 / 3$

Injection Point 1 Center

$\begin{aligned} & \text { Order } \rightarrow \\
& \text { Traverse } \rightarrow \\
& \text { Trial } \rightarrow\end{aligned}$
\begin{tabular}{|r|r|}
\hline Point & Depth, in. \\
\hline $\mathbf{1}$ & 0.50 \\
\hline $\mathbf{2}$ & 1.24 \\
\hline $\mathbf{3}$ & 2.29 \\
\hline $\mathbf{4}$ & 3.82 \\
\hline Center & 5.91 \\
\hline $\mathbf{5}$ & $\mathbf{8 . 0 0}$ \\
\hline $\mathbf{6}$ & 9.52 \\
\hline $\mathbf{7}$ & 10.57 \\
\hline $\mathbf{8}$ & 11.31 \\
\hline
\end{tabular}

1 st

2nd

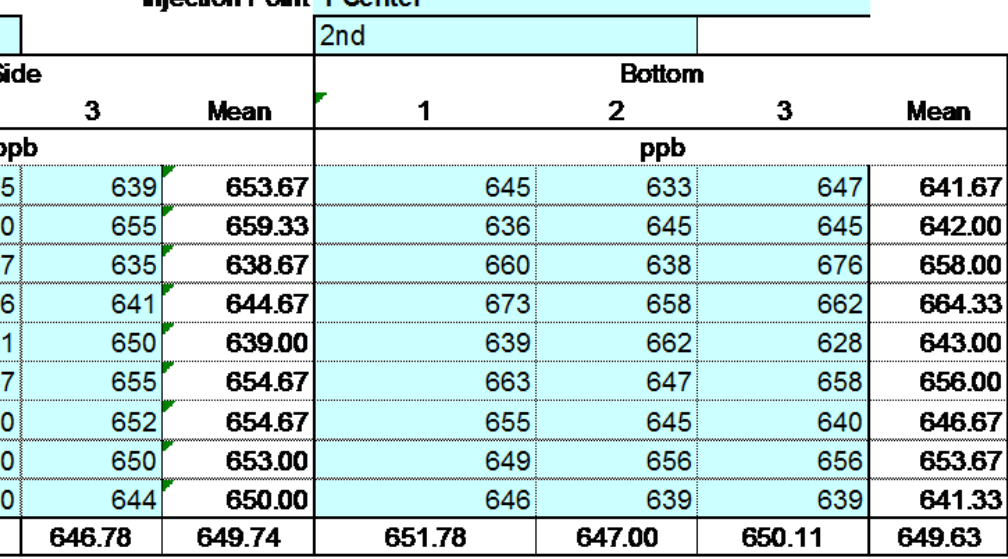

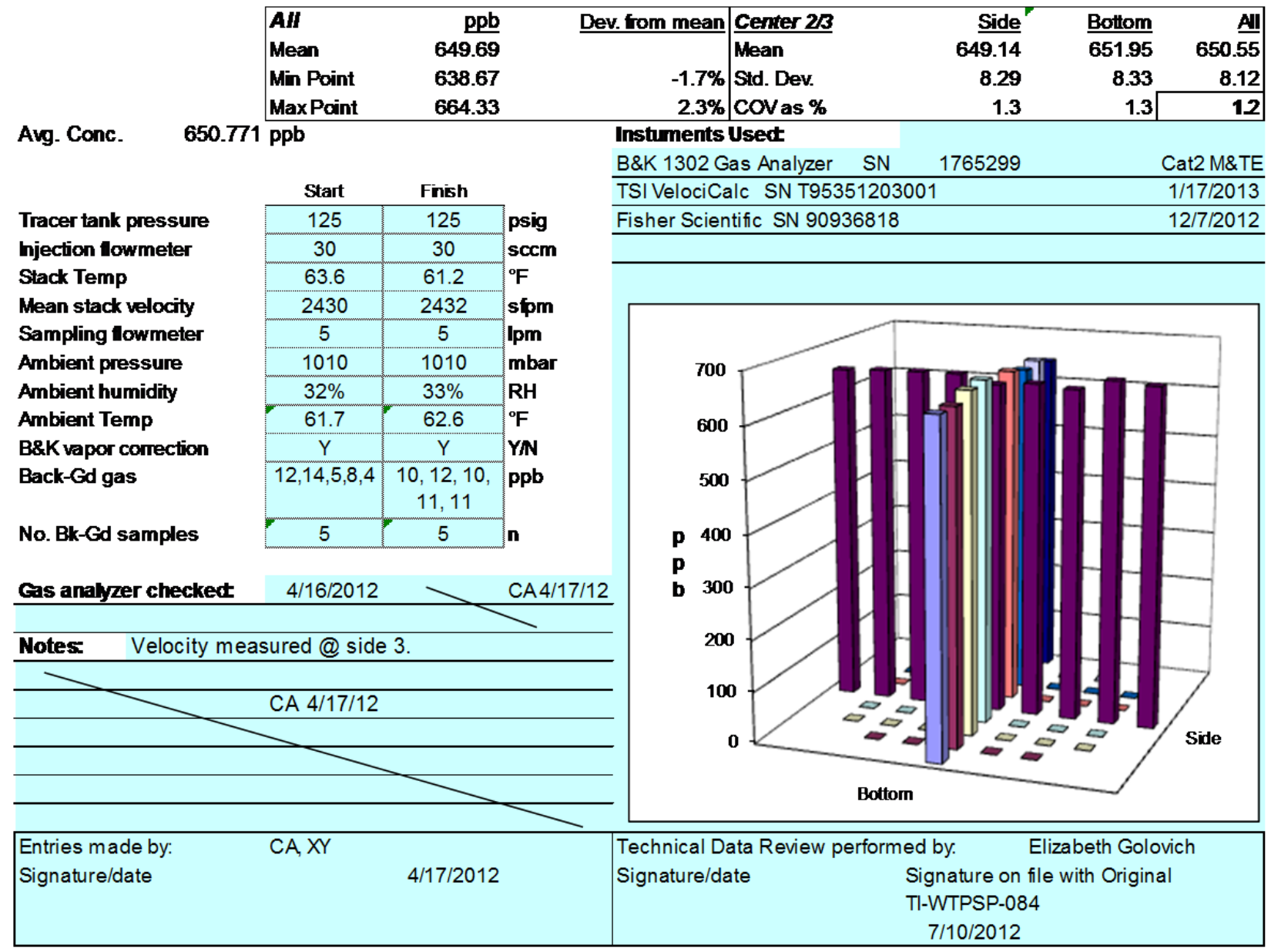


Rev. 0

31-JuL-06

IRACER GAS TRAVERSE DATA FORM

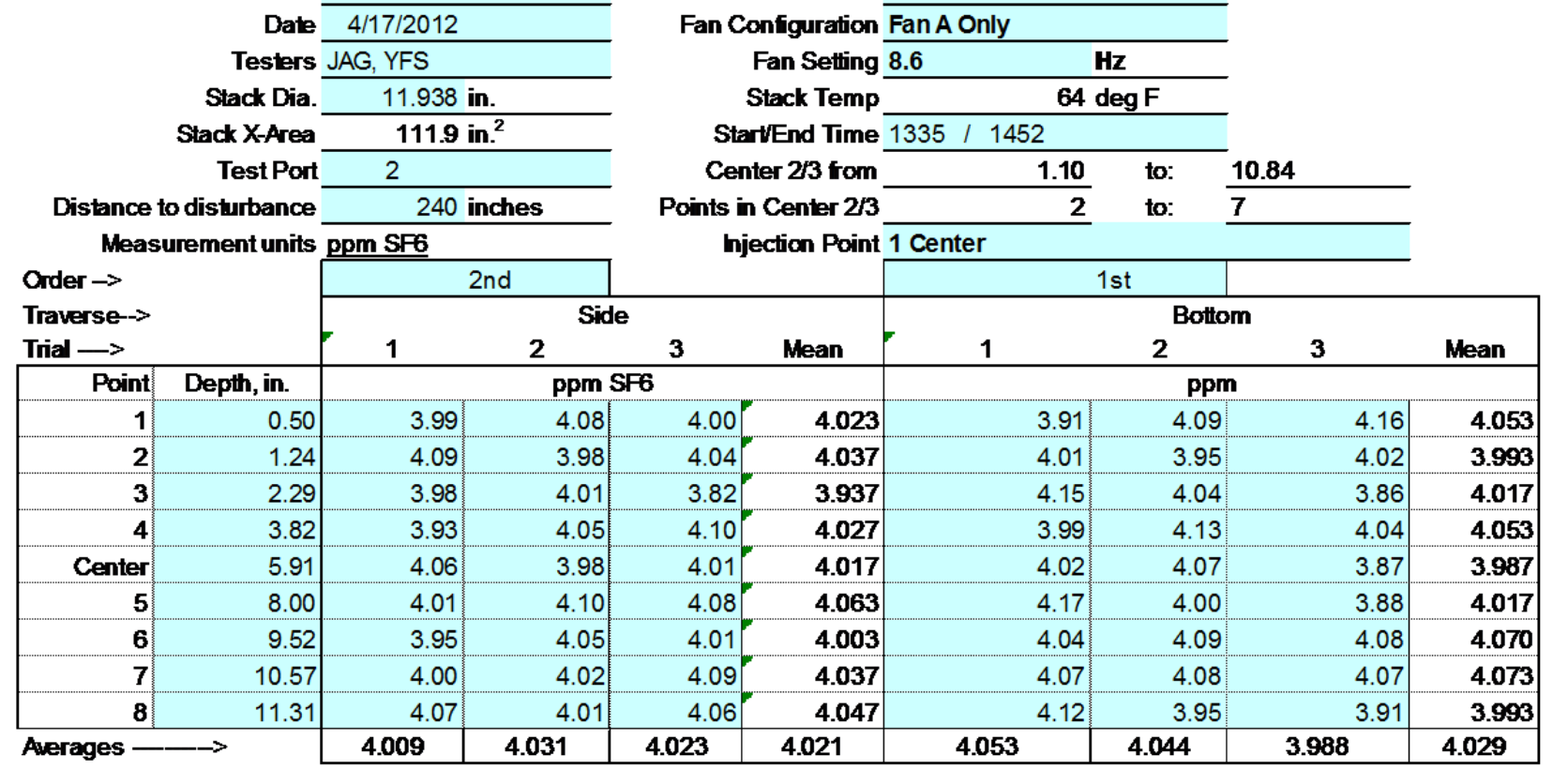

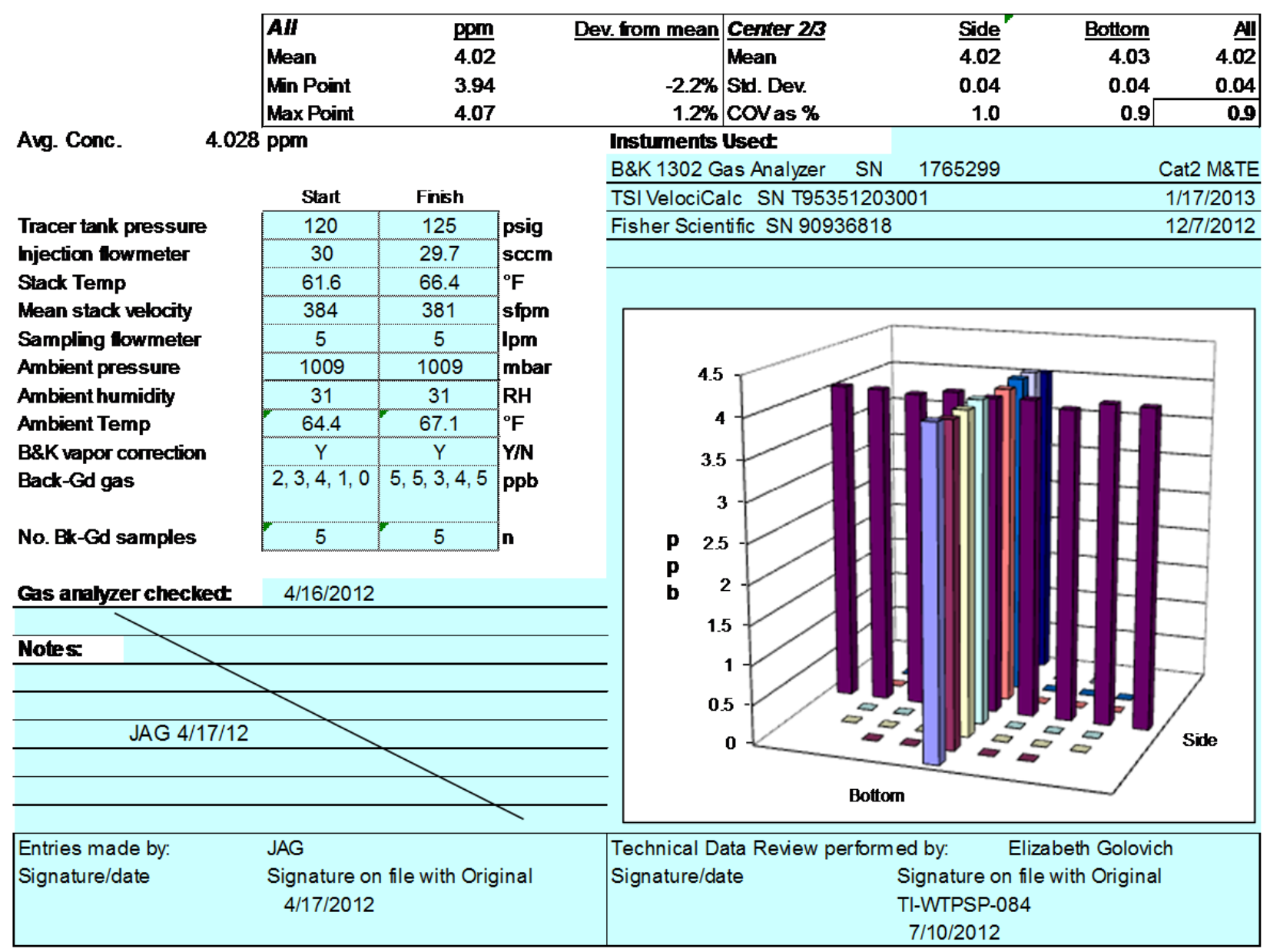


Rev. 0

31-JuL-06

IRACER GAS TRAVERSE DATA FORM

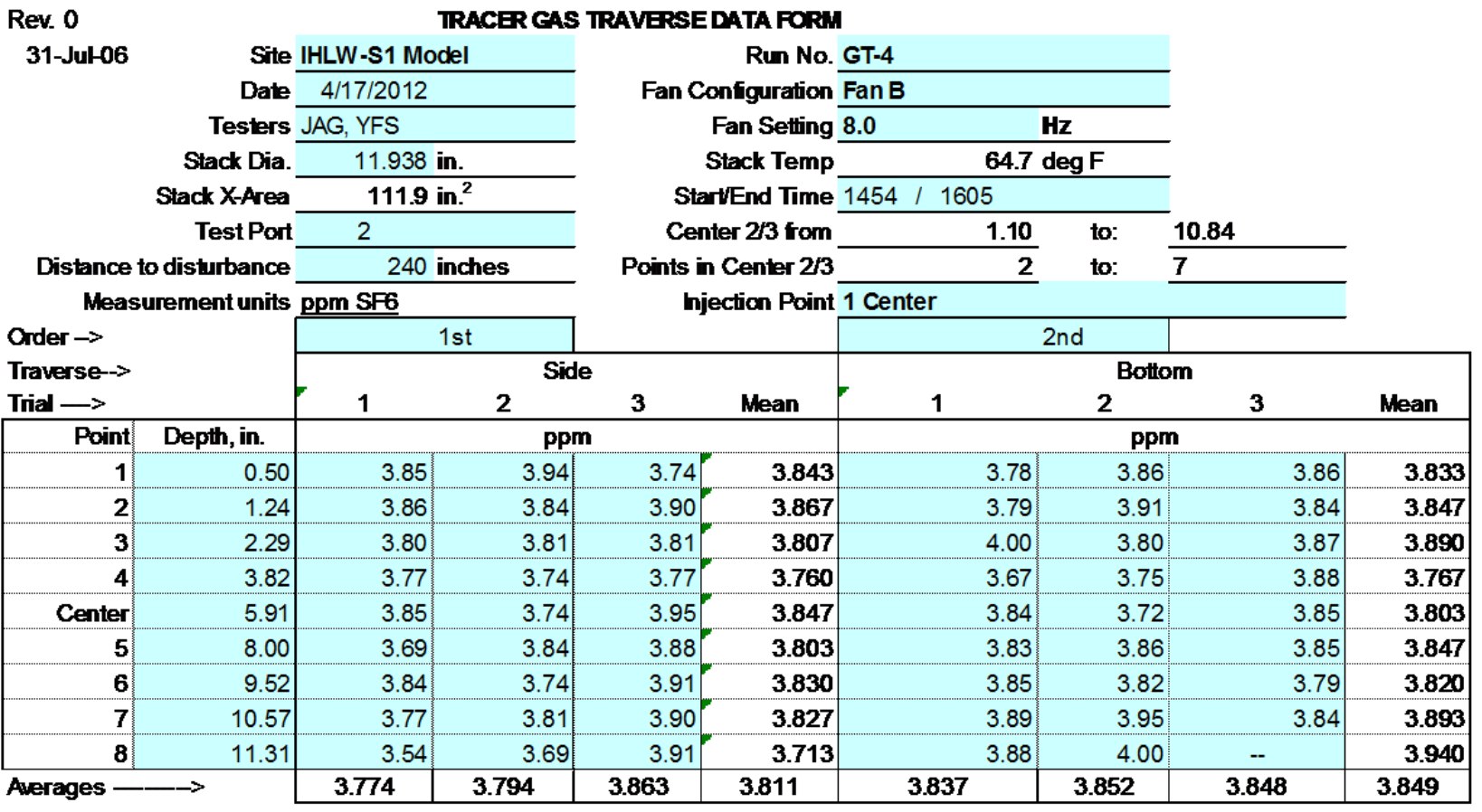

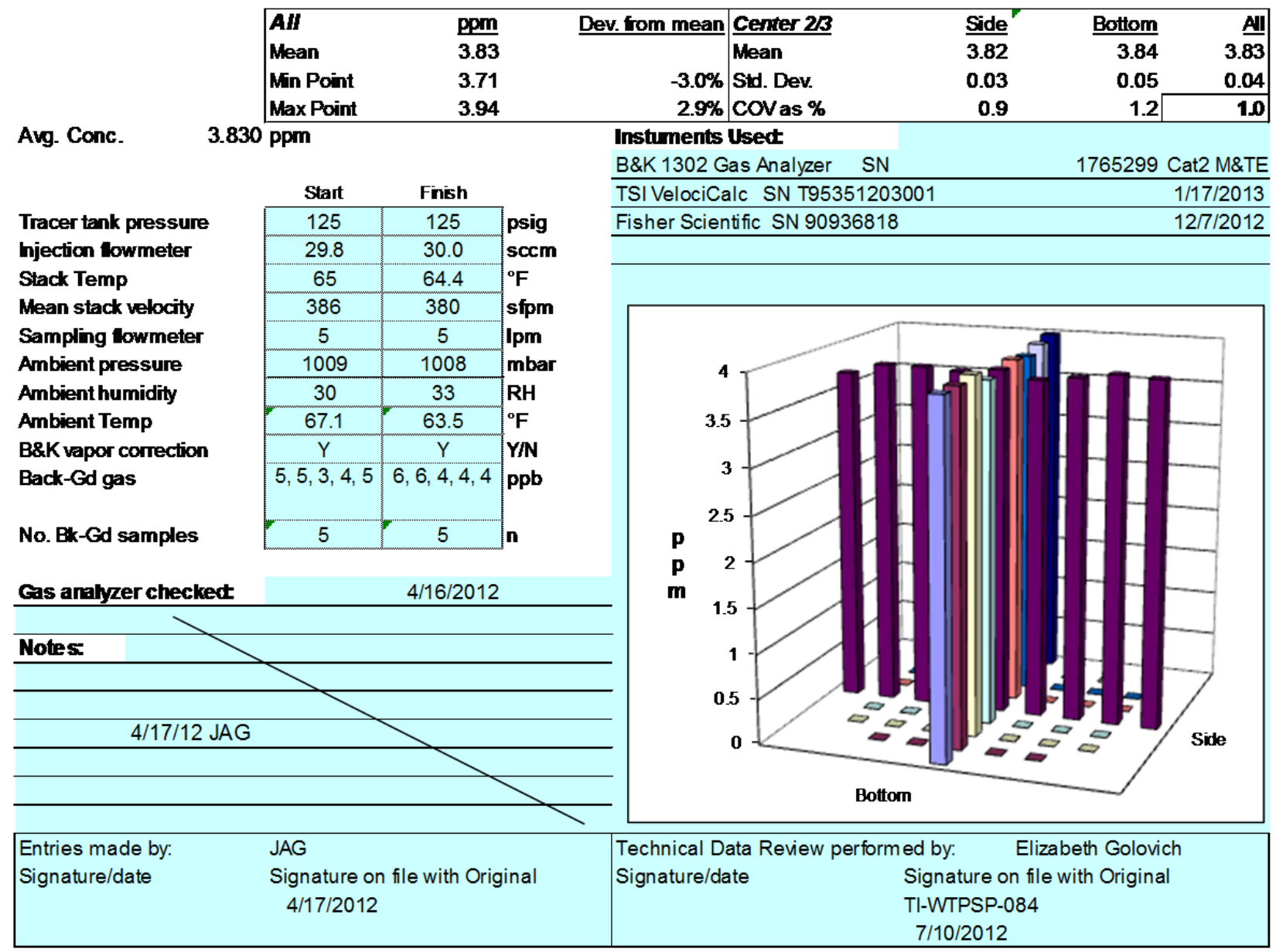


Rev. 0 31-JuL-06

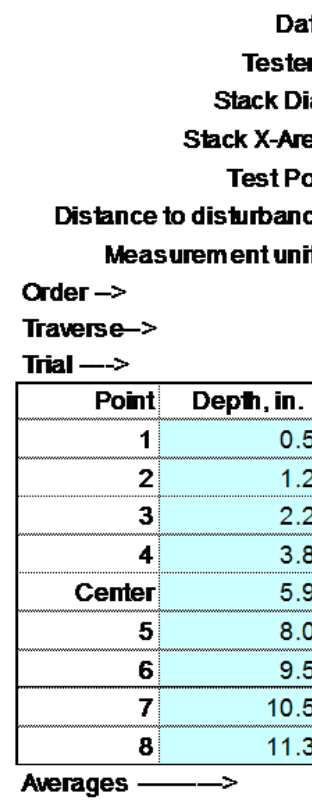

Site IHLW-S1 Model

Date 4/18/2012

Testers XY, CA

Stack Dia. 11.938 in.

bance 240 inches

\begin{tabular}{|l|}
\hline 2nd \\
\hline
\end{tabular}

TRACER GAS TRAVERE DATA FORU

Run No. GT-5

Fan Configuration Fan B only

Fan Setting 32

Stack Temp

Hz

Start/End Time $930 / 1100$

Center $2 / 3$ from

Points in Center 2/3

Injection Point 1 Center

$63.1 \operatorname{deg} F$

\begin{tabular}{l|ll|l}
\cline { 2 - 3 } & $1 \mathrm{st}$ & \\
\multicolumn{2}{c|}{ Bottom }
\end{tabular}

3 Mean $\quad 1 \quad 2^{\text {Botbm }} \quad 3$ Mean

\begin{tabular}{|r|r|r|}
\hline & & Pp \\
\hline 50 & 798 & 806 \\
\hline 24 & 795 & 823 \\
\hline 29 & 813 & 791 \\
\hline 82 & 821 & 807 \\
\hline 91 & 821 & 795 \\
\hline 00 & 791 & 808 \\
\hline 52 & 806 & 809 \\
\hline 57 & 787 & 822 \\
\hline 31 & 808 & 807 \\
\hline & $\mathbf{8 0 4 . 4 4}$ & $\mathbf{8 0 7 . 5 6}$ \\
\hline
\end{tabular}

\begin{tabular}{|r|r}
\hline 803 & 802.33 \\
\hline 809 & 809.00 \\
795 & 799.67 \\
770 & 799.33 \\
793 & $\mathbf{8 0 3 . 0 0}$ \\
\hline 816 & $\mathbf{8 0 5 . 0 0}$ \\
\hline 810 & $\mathbf{8 0 8 . 3 3}$ \\
\hline 789 & $\mathbf{7 9 9 . 3 3}$ \\
\hline 826 & $\mathbf{8 1 3 . 6 7}$ \\
\hline 22 & $\mathbf{8 0 4 . 4 1}$
\end{tabular}

\begin{tabular}{|r|r|}
\hline 33 & 81 \\
\hline 00 & 798 \\
\hline 33 & 798 \\
\hline 00 & 817 \\
\hline 00 & 796 \\
\hline 33 & 806 \\
\hline 33 & 800 \\
\hline & 81 \\
\hline
\end{tabular}

\begin{tabular}{|r|r|r|}
\hline $\mathbf{2}$ & $\mathbf{3}$ & \multicolumn{1}{l|}{ Mean } \\
\hline $\mathbf{P p b}$ & & \\
\hline 822 & 805 & $\mathbf{8 1 3 . 3 3}$ \\
\hline 808 & 798 & $\mathbf{8 0 1 . 3 3}$ \\
\hline 819 & 803 & $\mathbf{8 0 6 . 6 7}$ \\
\hline 837 & 812 & $\mathbf{8 2 2 . 0 0}$ \\
\hline 811 & 816 & $\mathbf{8 0 7 . 6 7}$ \\
\hline 815 & 812 & $\mathbf{8 1 1 . 0 0}$ \\
\hline 832 & 820 & $\mathbf{8 1 7 . 3 3}$ \\
\hline 808 & 892 & $\mathbf{8 0 4 . 3 3}$ \\
\hline 808 & 819 & $\mathbf{8 0 9 . 3 3}$ \\
\hline 17.78 & $\mathbf{8 0 8 . 5 6}$ & $\mathbf{8 1 0 . 3 3}$ \\
\hline
\end{tabular}

\begin{tabular}{|lr|}
\hline All & ppb \\
Mean & 807.37 \\
Min Point & 799.33 \\
Max Point & 822.00 \\
\hline
\end{tabular}

Avg. Conc.

$807.625 \mathrm{ppb}$

\begin{tabular}{|c|c|}
\hline Dev. from mean & Center 2/3 \\
\hline & Mean \\
\hline $\begin{array}{r}-1.0 \% \\
1.8 \% \\
\end{array}$ & $\begin{array}{l}\text { Std. Dev. } \\
\text { covas \% }\end{array}$ \\
\hline
\end{tabular}

Side
803.38
4.19
0.5

\begin{tabular}{rr|}
\hline Bottom & All \\
\hline 810.05 & 806.71 \\
7.33 & 6.70 \\
& $\mathbf{0 . 8}$ \\
\hline
\end{tabular}

\begin{tabular}{|c|c|c|c|}
\hline \multirow{2}{*}{\multicolumn{4}{|c|}{ Instuments Used }} \\
\hline & & & \\
\hline B\&K 1302 Gas Analyzer & $\mathrm{SN}$ & 1765299 & Cat2 M\&TE \\
\hline \multicolumn{3}{|c|}{ TSI VelociCalc SN T95351203001 } & $1 / 17 / 2013$ \\
\hline \multicolumn{3}{|c|}{ Fisher Scientific SN 90936818} & $12 / 7 / 2012$ \\
\hline
\end{tabular}

hiossure Stack Temp

Mean stack velocity

Sampling lowmeter Ambient pressure Ambient humidity Ambient Temp B\&K vapor correction Back-Gd gas

No. Bk-Gd samples

\begin{tabular}{|c|c|c|}
\hline Start & Fnish & \\
\hline 100 & 125 & Jpsig \\
\hline 30 & 30 & $\mathrm{sccm}$ \\
\hline 58.5 & 67.7 & ${ }^{\circ} \mathrm{F}$ \\
\hline 1754 & 1744 & sfpm \\
\hline 5 & 5 & Ipm \\
\hline 1005 & 1005 & mbar \\
\hline $46 \%$ & $36 \%$ & RH \\
\hline 57.2 & 66.2 & ${ }^{\circ} \mathrm{F}$ \\
\hline $\mathrm{Y}$ & $\mathrm{Y}$ & Y/N \\
\hline & & ppb \\
\hline $7,9,5,7,7$ & $13,18,15,9,12$ & \\
\hline 5 & 5 & $n$ \\
\hline
\end{tabular}

Gas analyzer checked:

$4 / 16 / 2012$

CA $4 / 18 / 12$
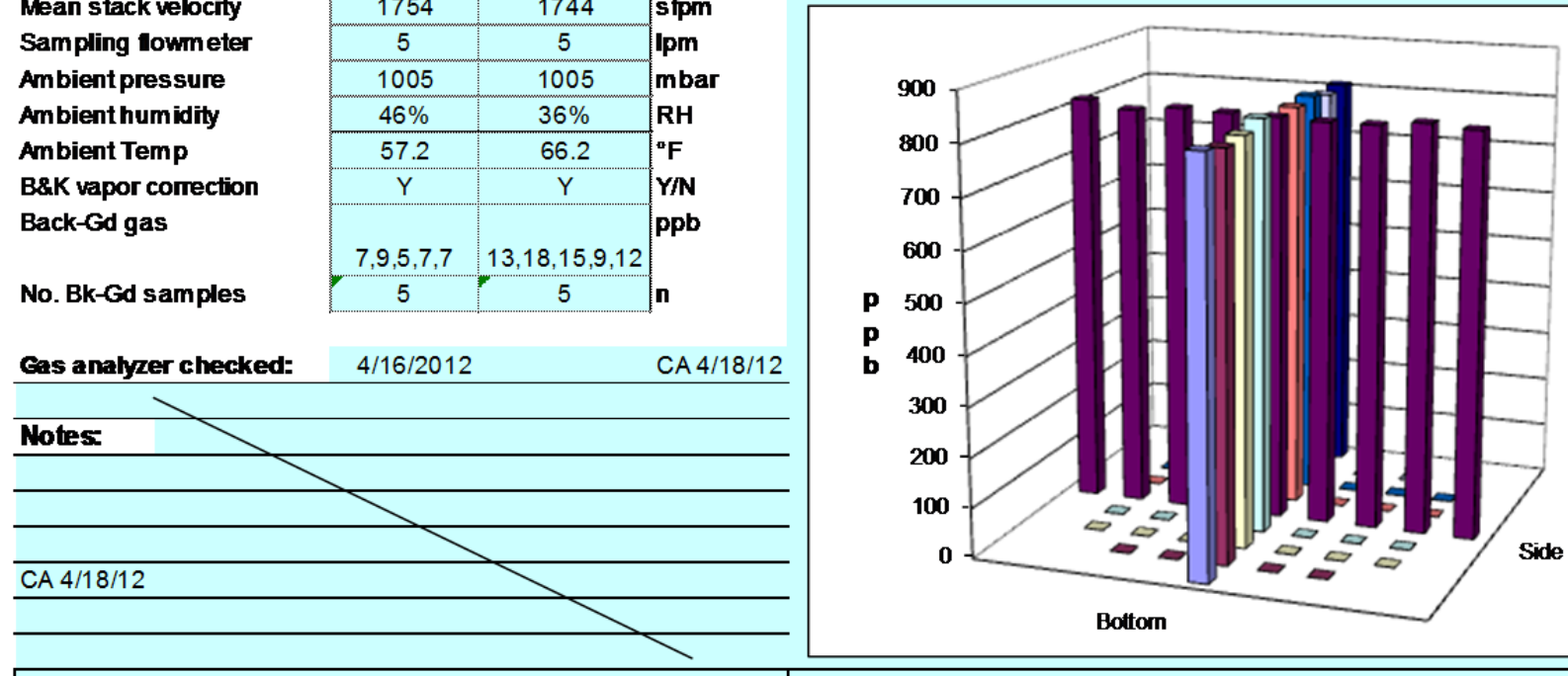

Technical Data Review perform ed by:

Elizabeth Golovich Signature/date Signature/date Signature on file with Original TI-WTPSP-084 $7 / 10 / 2012$ 
Rev. 0

31-JuL-06

TRACER GAS TRAVERE DATA FORM

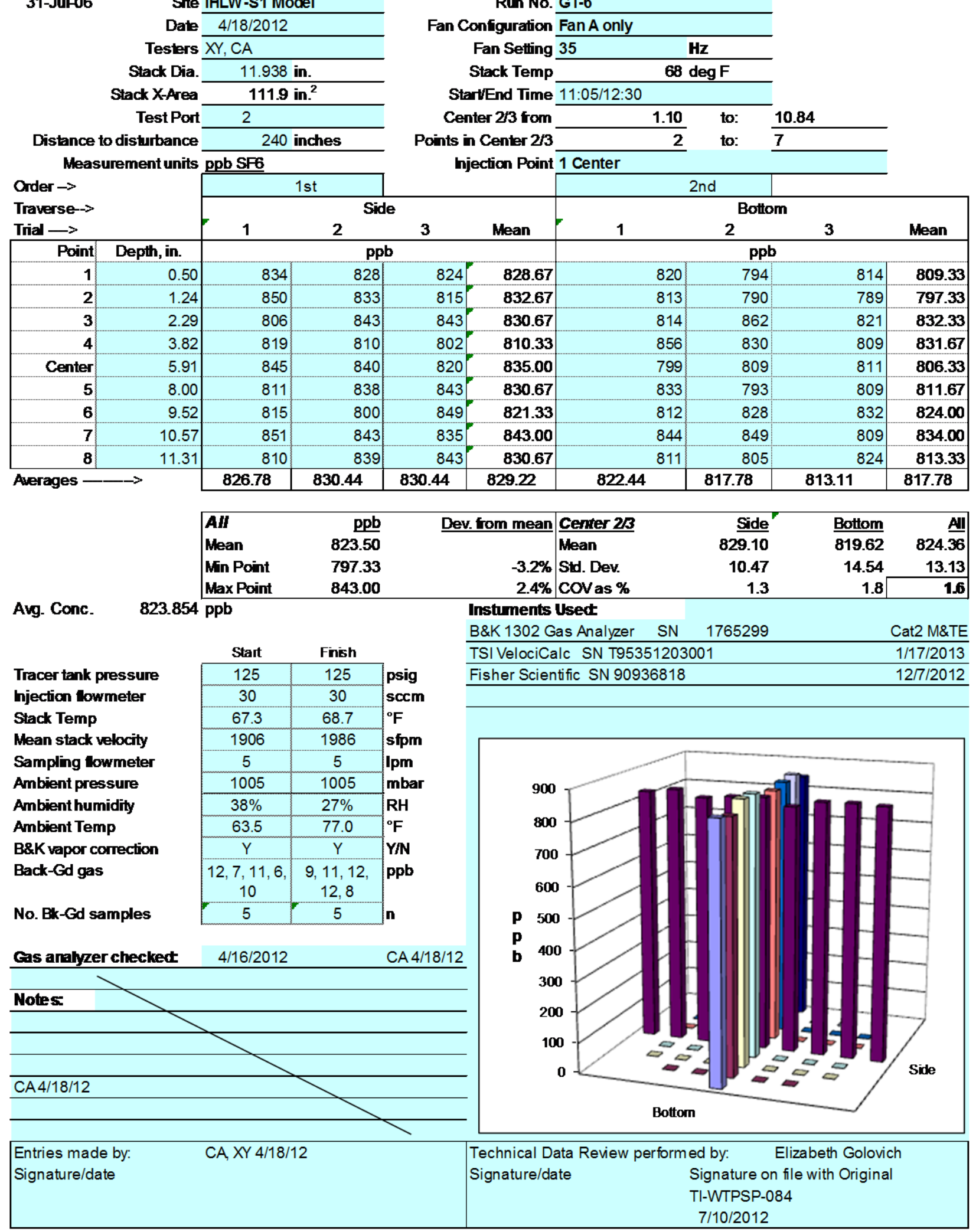


Rev. 0

31-JuL-06

\section{TRACER CAS TRAVERSE DATA FORM}

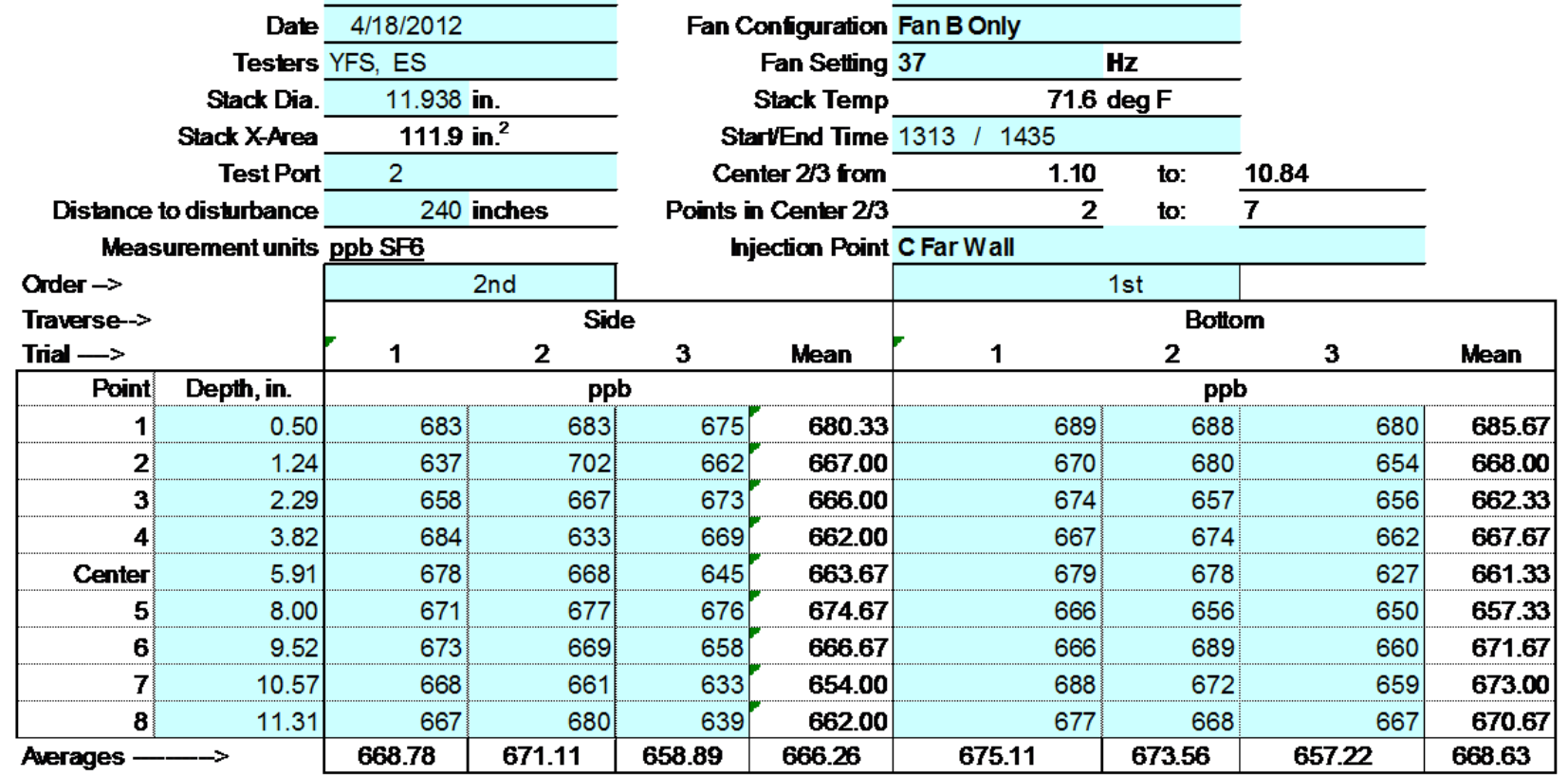

Avg. Conc

\begin{tabular}{|lr|}
\hline AII & ppb \\
Mean & 667.44 \\
Min Point & 654.00 \\
Max Point & 685.67 \\
\hline
\end{tabular}
$668.063 \mathrm{ppb}$

Tracer tank pressure
hjection towmeter
Stack Temp
Mean stack velocity
Sampling lowmeter
Ambient pressure
Ambient humidity
Ambient Temp
B\&K vapor correction
Back-Gd gas

No. Bk-Gd samples

\begin{tabular}{|c|c|c|}
\hline Start & Finsh & \\
\hline 200 & 200 & \\
\hline 30 & 30 & \\
\hline 69.5 & 73.7 & \\
\hline 2210 & 2207 & \\
\hline 5 & 5 & \\
\hline 1005 & 1005 & \\
\hline $29 \%$ & $27 \%$ & \\
\hline 75 & 75 & \\
\hline Y & $\mathrm{Y}$ & \\
\hline $3,6,4,4,2$ & $7,5,3,2,-1$ & \\
\hline 5 & 5 & \\
\hline
\end{tabular}

Gs anahyzer checked $\quad$ 4/16/2012

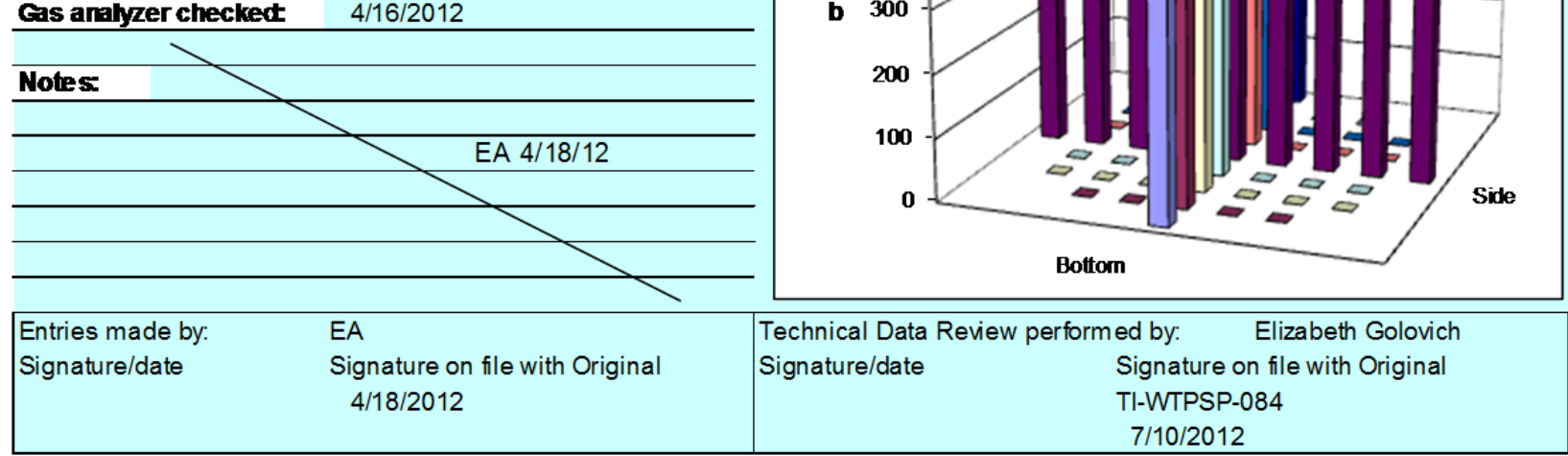


Rev. 0

31-JuL-06

\section{TRACER CAS TRAVERSE DATA FORY}

\begin{tabular}{|c|c|c|c|c|c|c|c|c|c|}
\hline & Date & $4 / 18 / 2012$ & & Fan C & nfiguration & Fan B Only & & & \\
\hline & Testers & YFS, ES & & & Fan Setting & 37 & Iz & & \\
\hline & Stack Dia. & 11.938 & & & tack Temp & 72.05 & $\operatorname{leg} F$ & & \\
\hline & Stack X-Area & 111.9 & & Sta & VEnd Time & $1440 / 1540$ & & & \\
\hline & Test Port & 2 & & Cen & er $2 / 3$ from & 1.10 & to: & 10.84 & \\
\hline Distance $t$ & o disturbance & 240 & ches & Points i & Center 233 & 2 & to: & 7 & \\
\hline Meas & urement units & ppb SF6 & & & ction Point & C Near Wall & & & \\
\hline Order $\rightarrow$ & & & st & & & & nd & & \\
\hline Traverse-> & & & Sid & & & & Botto & $\mathrm{om}$ & \\
\hline Trial $\longrightarrow$ & & 1 & 2 & 3 & Mean & 1 & 2 & 3 & Mean \\
\hline Point & Depth, in. & & ppl & & & & $\mathrm{ppb}$ & & \\
\hline 1 & 0.50 & 684 & 701 & 691 & 692.00 & 655 & 692 & 694 & 680.33 \\
\hline 2 & 1.24 & 658 & 698 & 677 & 677.67 & 675 & 701 & 705 & 693.67 \\
\hline 3 & 2.29 & 671 & 708 & 689 & 689.33 & 679 & 689 & 681 & 683.00 \\
\hline 4 & 3.82 & 681 & 668 & 674 & 674.33 & 686 & 693 & 663 & 680.67 \\
\hline Center & 5.91 & 682 & 669 & 703 & 684.67 & 676 & 711 & 688 & 691.67 \\
\hline 5 & 8.00 & 680 & 687 & 665 & 677.33 & 670 & 683 & 662 & 671.67 \\
\hline 6 & 9.52 & 698 & 685 & 661 & 681.33 & 681 & 679 & 652 & 670.67 \\
\hline 7 & 10.57 & 682 & 686 & 681 & 683.00 & 659 & 678 & 684 & 673.67 \\
\hline 8 & 11.31 & 682 & 672 & 684 & 679.33 & 672 & 650 & 639 & 653.67 \\
\hline Averages - & $\rightarrow$ & 679.78 & 686.00 & 680.56 & 682.11 & 672.56 & 686.22 & 674.22 & 677.67 \\
\hline
\end{tabular}

Avg. Conc.

\begin{tabular}{|lr}
\hline$A I I$ & ppb \\
Mean & 679.89 \\
Min Point & 653.67 \\
Max Point & 693.67 \\
\hline
\end{tabular}

\section{Tracer tank pressure hjection lowmeter Stack Temp \\ Mean stack velocity \\ Sampling lowmeter Ambient pressure Ambient humidity Ambient Temp B\&K vapor correction Back-Gd gas}

No. Bk-Gd samples

\begin{tabular}{|c|c|}
\hline Start & Finish \\
\hline 200 & 200 \\
\hline 30 & 30 \\
\hline 73.7 & 70.4 \\
\hline 2267 & 2254 \\
\hline 5 & 5 \\
\hline 1005 & 1004 \\
\hline $28 \%$ & $28 \%$ \\
\hline 73 & 71 \\
\hline$Y$ & $Y$ \\
\hline $\begin{array}{c}3,4,3,-0.7 \\
-0.4\end{array}$ & $\begin{array}{c}3,-1,3,3 \\
0.4\end{array}$ \\
\hline 5 & 5 \\
\hline
\end{tabular}

Gs anabyer checked $\quad 4 / 16 / 2012$

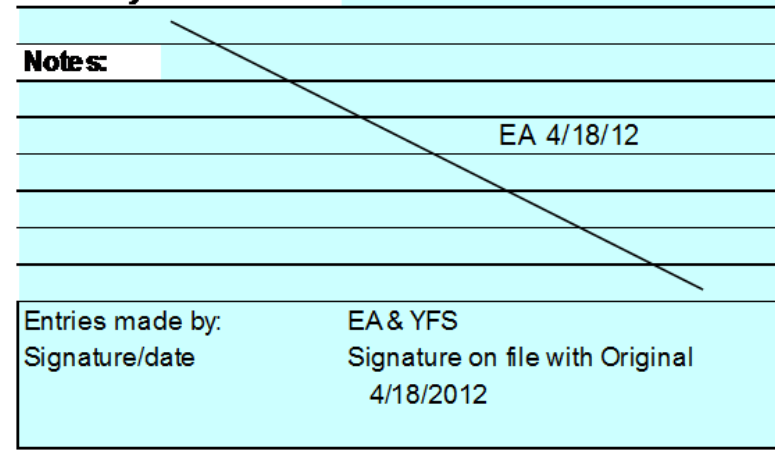

Dev. from mean Center 23

$$
\text { Mean }
$$

$-3.9 \%$ Std. Dev. $2.0 \%$ COVas \%

Instuments Usect

B\&K 1302 Gas Analyzer SN

\begin{tabular}{rrr|} 
Side & Bottom & All \\
& 680.71 & 680.90 \\
5.10 & 9.36 & 7.24 \\
0.7 & 1.4 & 1.1 \\
\hline
\end{tabular}
TSIVelociCalc SN T95351203001

Fisher Scientific SN 90936818

1765299 Cat2 M\&TE

$12 / 7 / 2012$

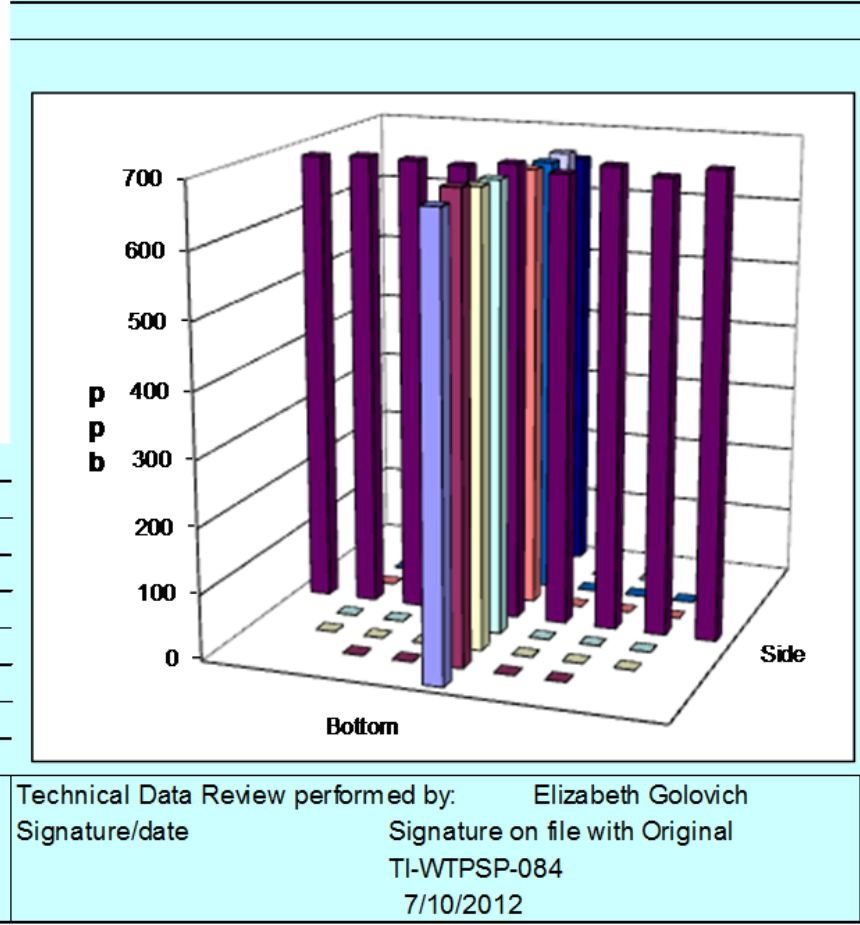


Rev. 0

31-JuL-06

\section{TRACER CAS TRAVERSE DATA FORY}

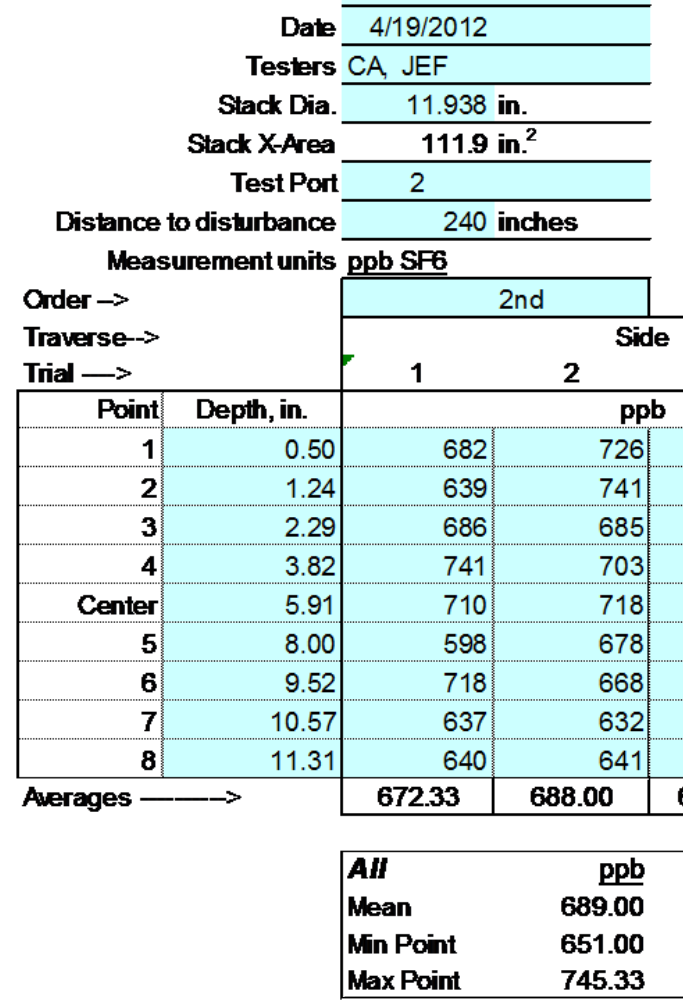

Avg. Conc. $\quad 688.167 \mathrm{ppb}$

Tracer tank pressure Injection lowmeter Stack Temp

Mean stack velocity

Sampling lowmeter Ambient pressure

Ambient humidity

Ambient Temp

B\&K vapor correction

Back-Gd gas

No. Bk-Gd samples

\begin{tabular}{|c|c|}
\hline Start & Finish \\
\hline 10 & 10 \\
\hline 30 & 30 \\
\hline 53.8 & 60.8 \\
\hline 2169 & 2230 \\
\hline 5 & 5 \\
\hline 1006 & 1007 \\
\hline $48 \%$ & $39 \%$ \\
\hline 50.9 & 59.9 \\
\hline$Y$ & $Y$ \\
\hline $\begin{array}{c}0.5,2,4,6 \\
4\end{array}$ & $\begin{array}{c}14,14,10 \\
11,12\end{array}$ \\
\hline 5 & 5 \\
\hline
\end{tabular}

Gs anahyer checkedt $4 / 16 / 2012>$ CA 4/19/12

Notes Tracer tank pressure appears very low, just barely of the zero bar, perhaps due to low air temperature.

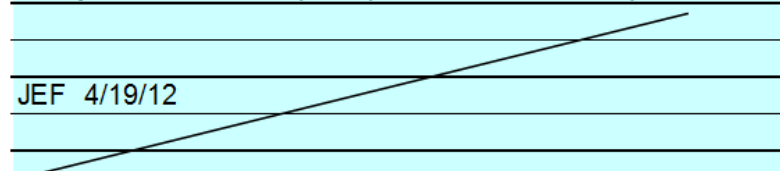

\begin{tabular}{|ll}
\hline Entries made by: & JEF, CA \\
Signature/date & Signatures on file with Original \\
& $4 / 19 / 2012$
\end{tabular}

Run No. GT-9

Fan Configuration Fan B Only

Fan Setting 37

Stack Temp

StartEnd Time $0820 / 0939$

Center $2 / 3$ from

Points in Center $2 / 3$

Injection Point Center Bottom

\begin{tabular}{|c|c|c|}
\hline & & \\
\hline 57.3 & & \\
\hline 939 & & \\
\hline 1.10 & to: & 10.84 \\
\hline 2 & to: & 7 \\
\hline
\end{tabular}

3 Mean $\quad$\begin{tabular}{lllll} 
& \multicolumn{3}{|c|}{ Bottom } \\
3 & 1 & 2 & 3 & Mean
\end{tabular}

\begin{tabular}{|c|c|c|c|c|c|}
\hline & \multicolumn{5}{|c|}{$\mathbf{2}$} \\
\hline 653 & $\mathbf{6 8 7 . 0 0}$ & 751 & 658 & 660 & $\mathbf{6 8 9 . 6 7}$ \\
\hline 645 & $\mathbf{6 7 5 . 0 0}$ & 697 & 602 & 709 & $\mathbf{6 6 9 . 3 3}$ \\
\hline 676 & $\mathbf{6 8 2 . 3 3}$ & 690 & 717 & 698 & 701.67 \\
\hline 648 & 697.33 & 713 & 686 & 648 & $\mathbf{6 8 2 . 3 3}$ \\
\hline 695 & 707.67 & 717 & 693 & 641 & $\mathbf{6 8 3 . 6 7}$ \\
\hline 677 & $\mathbf{6 5 1 . 0 0}$ & 689 & 651 & 739 & $\mathbf{6 9 3 . 0 0}$ \\
\hline 774 & $\mathbf{7 2 0 . 0 0}$ & 700 & 725 & 726 & $\mathbf{7 1 7 . 0 0}$ \\
\hline 686 & $\mathbf{6 5 1 . 6 7}$ & 664 & 722 & 678 & $\mathbf{6 8 8 . 0 0}$ \\
\hline 699 & $\mathbf{6 6 0 . 0 0}$ & 757 & 691 & 788 & $\mathbf{7 4 5 . 3 3}$ \\
\hline 683.67 & $\mathbf{6 8 1 . 3 3}$ & $\mathbf{7 0 8 . 6 7}$ & $\mathbf{6 8 2 7 8}$ & $\mathbf{6 9 8 . 5 6}$ & $\mathbf{6 9 6 . 6 7}$ \\
\hline
\end{tabular}

\begin{tabular}{|c|c|c|c|c|}
\hline Dev. from mean & Center 23 & Side & Bottom & All \\
\hline & Mean & 683.57 & 690.71 & 687.14 \\
\hline$-5.5 \%$ & Std. Dev. & 26.62 & 15.28 & 21.18 \\
\hline $8.2 \%$ & COVas $\%$ & 3.9 & 2.2 & 3.1 \\
\hline
\end{tabular}

\begin{tabular}{lr} 
Instuments Usect & \\
B\&K 1302 Gas Analyzer SN & 1765299 Cat2 M\&TE \\
\hline TSI VelociCalc SN T95351203001 & $1 / 17 / 2013$ \\
\hline Fisher Scientific SN 90936818 & $12 / 7 / 2012$ \\
\hline
\end{tabular}

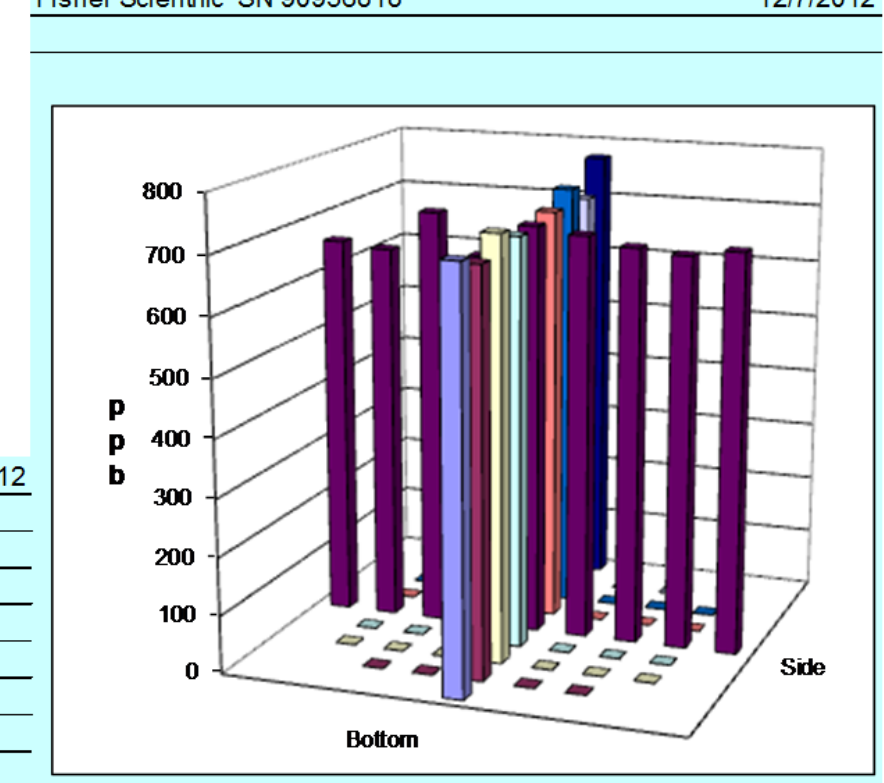

Technical Data Review performed by: Elizabeth Golovich

Signature/date Signature on file with Original TI-WTPSP-084 $7 / 10 / 2012$ 
Rev. 0

31-JuL-06

IRACER GAS TRAVERSE DATA FORM

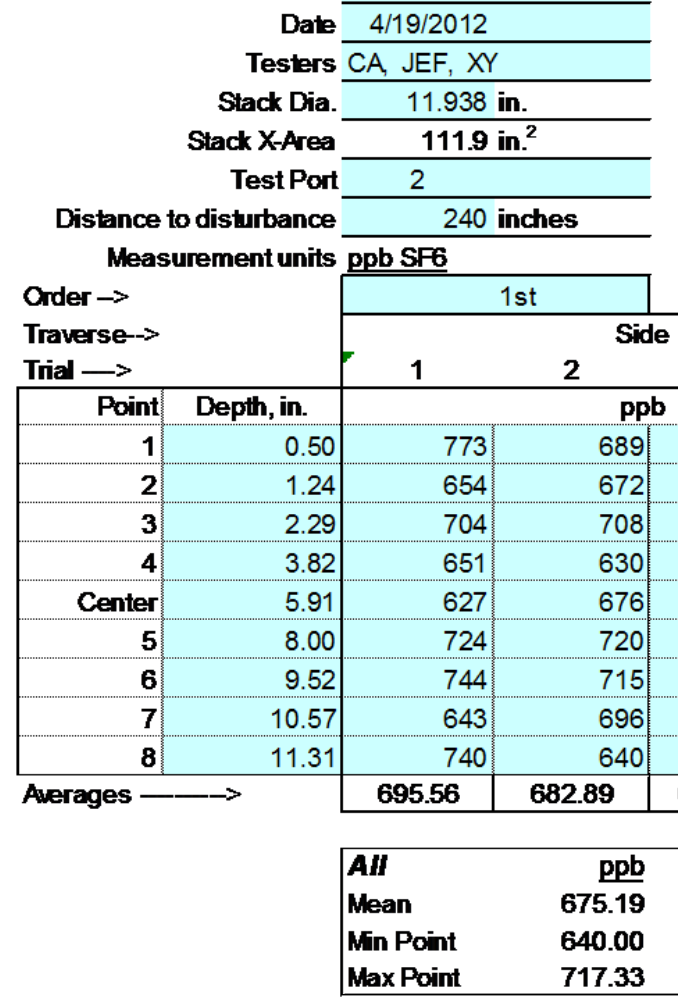

Avg. Conc.

$677.563 \mathrm{ppb}$

\begin{tabular}{|c|c|c|c|}
\hline \multirow{3}{*}{$\begin{array}{l}\text { Tracer tank pressure } \\
\text { hjection lowmeter }\end{array}$} & Start & Finsh & \multirow[b]{2}{*}{ psig } \\
\hline & 100 & 125 & \\
\hline & 30 & 30 & sccm \\
\hline ack Temp & 58.9 & 64.0 & ${ }^{\circ} \mathrm{F}$ \\
\hline ean stack velocity & 2212 & 2218 & sfpm \\
\hline ampling towmeter & 5 & 5 & Ipm \\
\hline nbient pressure & 1007 & 1007 & mbar \\
\hline nbient humidity & $35 \%$ & $32 \%$ & RH \\
\hline mbient Temp & 63.5 & 67.1 & ${ }^{\circ} \mathrm{F}$ \\
\hline B\&K vapor correction & Y & $\mathrm{Y}$ & YIN \\
\hline Back-Gd gas & $\begin{array}{c}14,10,11 \\
12,8\end{array}$ & $\begin{array}{c}7,6,14,10 \\
10\end{array}$ & ppb \\
\hline . Bk-Gd samples & 5 & 5 & $n$ \\
\hline
\end{tabular}

Gs analyzer checked $\quad 4 / 16 / 2012$

CA $4 / 19 / 12$

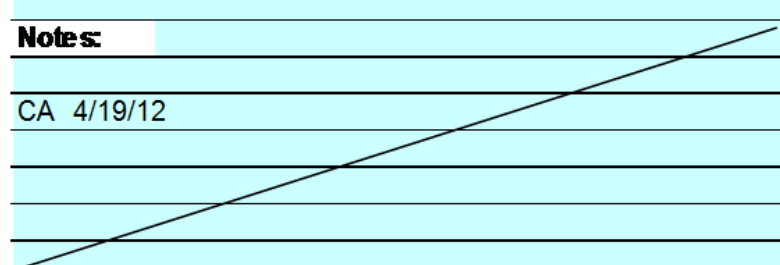

\begin{tabular}{|ll}
\hline Entries made by: & CA, XY, JEF \\
Signature/date & Signatures on file with Original \\
& $4 / 19 / 2012$
\end{tabular}

Run No. GT-10

Fan Configuration Fan B Only

Fan Setting 37

Stack Temp

StartEnd Time $0955 / 1108$

Center 233 from

Points in Center $2 / 3$

Injection Point $\mathrm{C}$ TOP

\begin{tabular}{|c|c|c|}
\hline \multicolumn{2}{|c|}{ Hz } & \\
\hline \multicolumn{2}{|c|}{$61.45 \mathrm{deg} F$} & \\
\hline 1108 & & \\
\hline 1.10 & to: & 10.84 \\
\hline 2 & to: & 7 \\
\hline
\end{tabular}

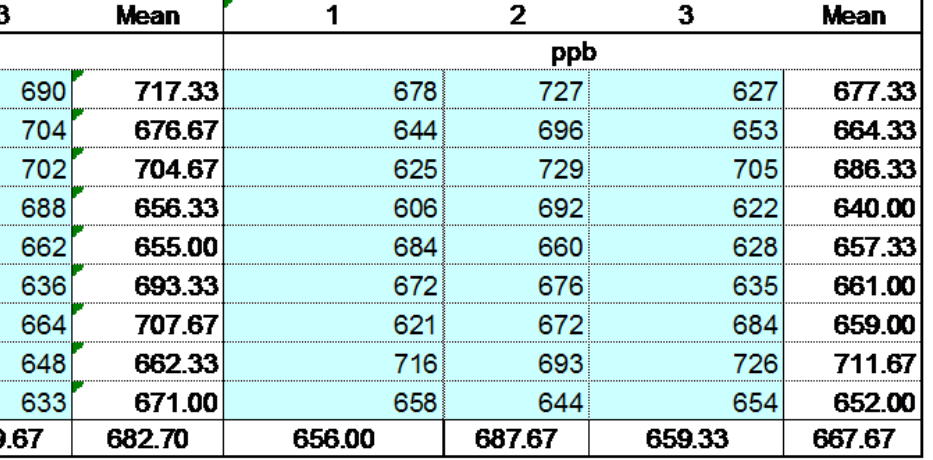

\begin{tabular}{r|lrrr|} 
Dev. fom mean & Center 23 & Side & Bottom & 세 \\
\cline { 2 - 5 } & Mean & 679.43 & 668.52 & 673.98 \\
$-5.2 \%$ & Std. Dev. & 22.57 & 23.39 & 22.80 \\
$6.2 \%$ & COV as \% & 3.3 & 3.5 & $\mathbf{3 . 4}$ \\
\hline
\end{tabular}

Instuments Usect

\begin{tabular}{lr} 
B\&K 1302 Gas Analyzer SN & 1765299 Cat2 M\&TE \\
\hline TSI VelociCalc SN T95351203001 & $1 / 17 / 2013$ \\
\hline Fisher Scientific SN 90936818 & $12 / 7 / 2012$ \\
\hline
\end{tabular}

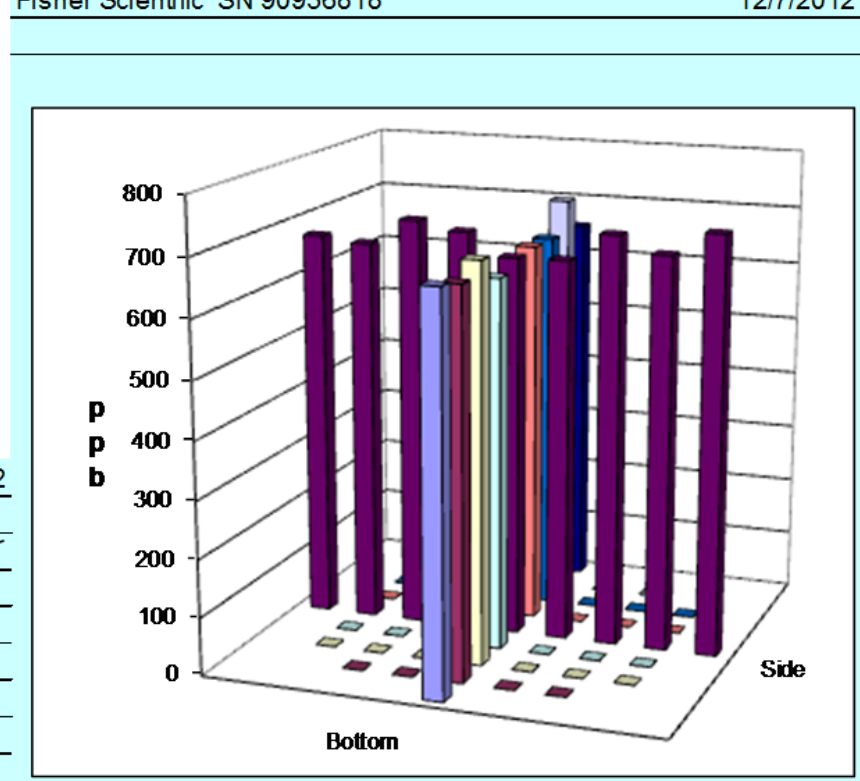

Technical Data Review performed by: Elizabeth Golovich Signature/date Signature on file with Original TI-WTPSP-084 $7 / 10 / 2012$ 
Rev. 0

31-JuL-06

TRACER CAS TRAVERSE DATA FORM

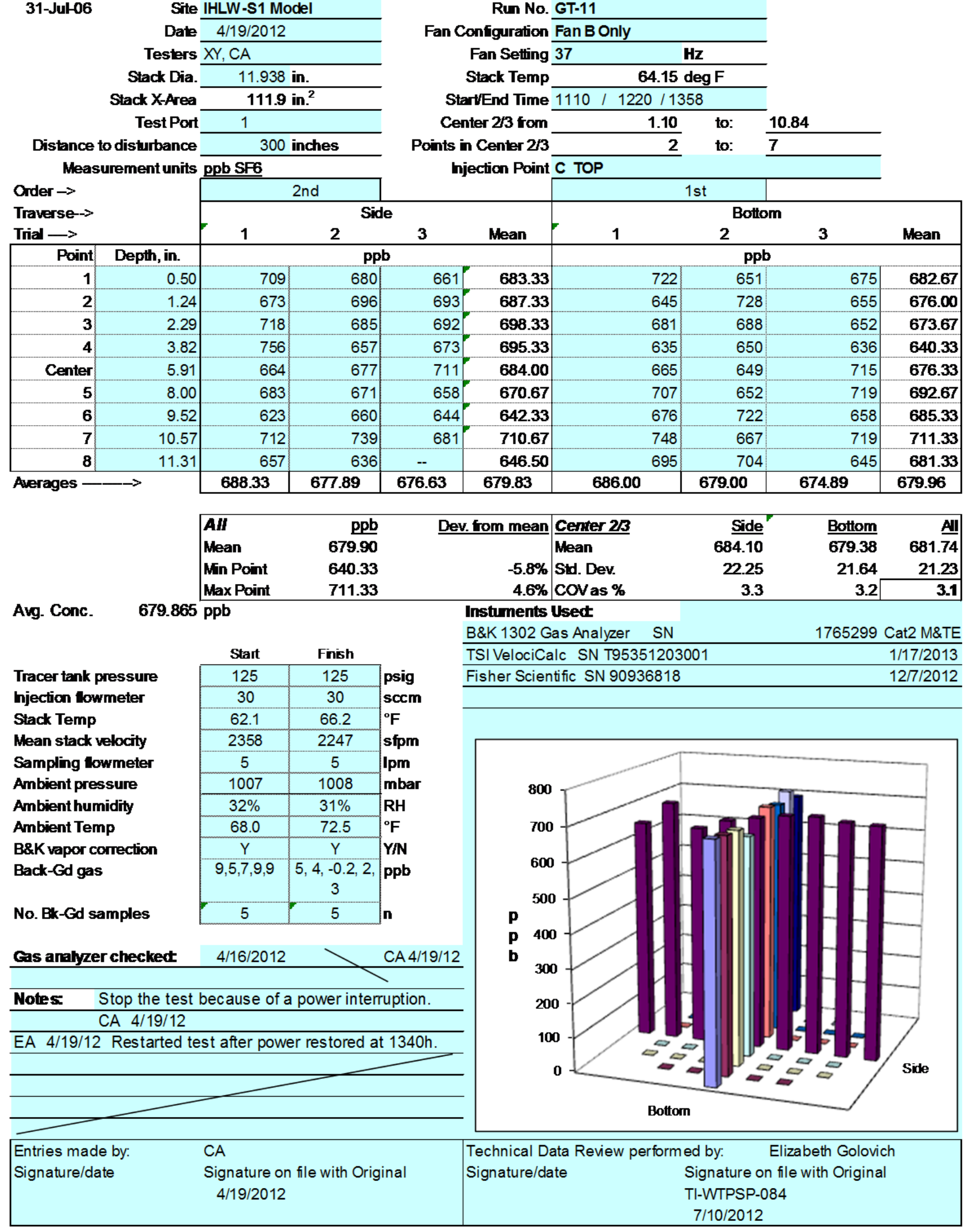


Rev. 0

31-JuL-06

TRACER CAS TRAVERE DATA FORM

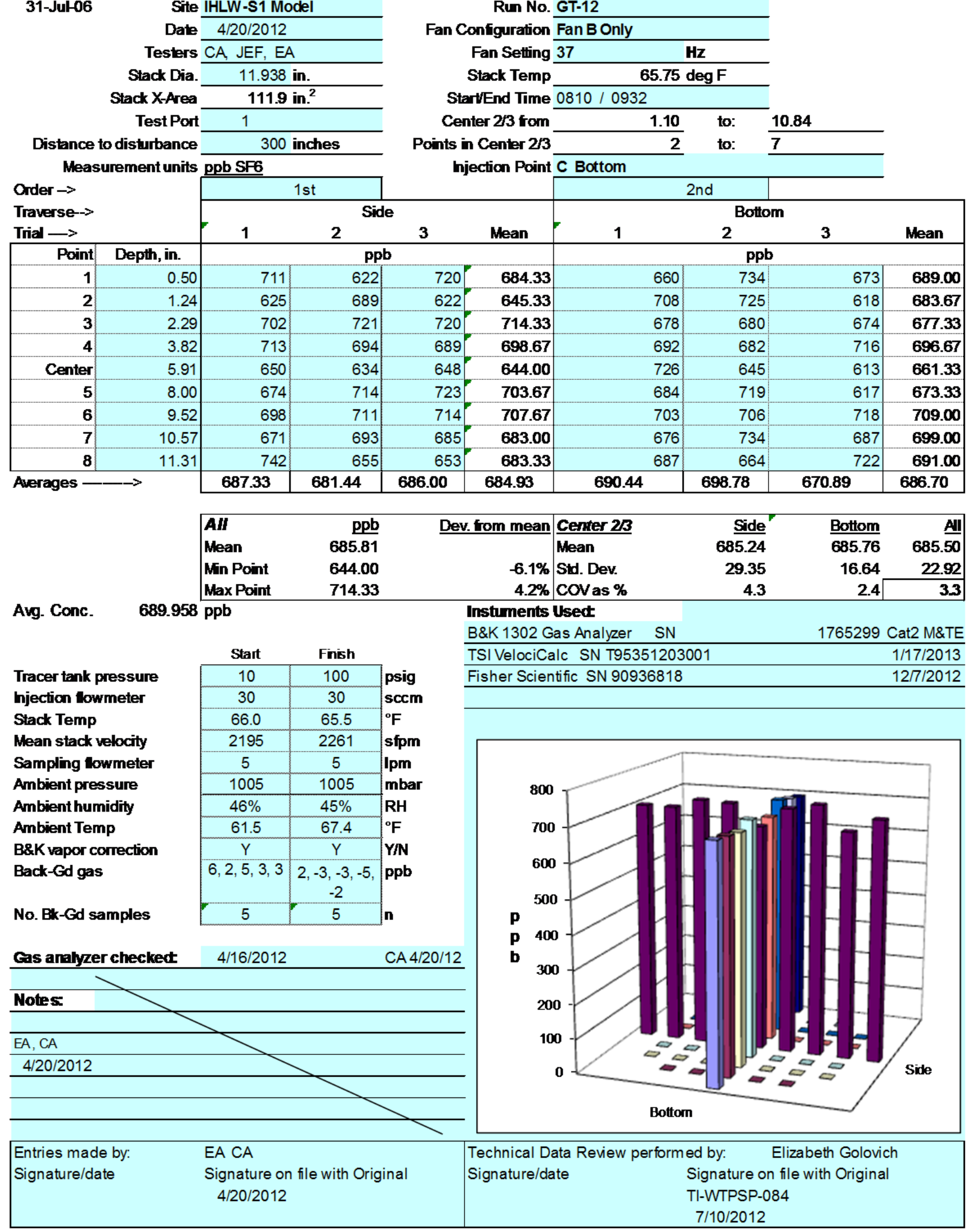


Rev. 0

31-JuL-06

TRACER CAS TRAVERSE DATA FORY

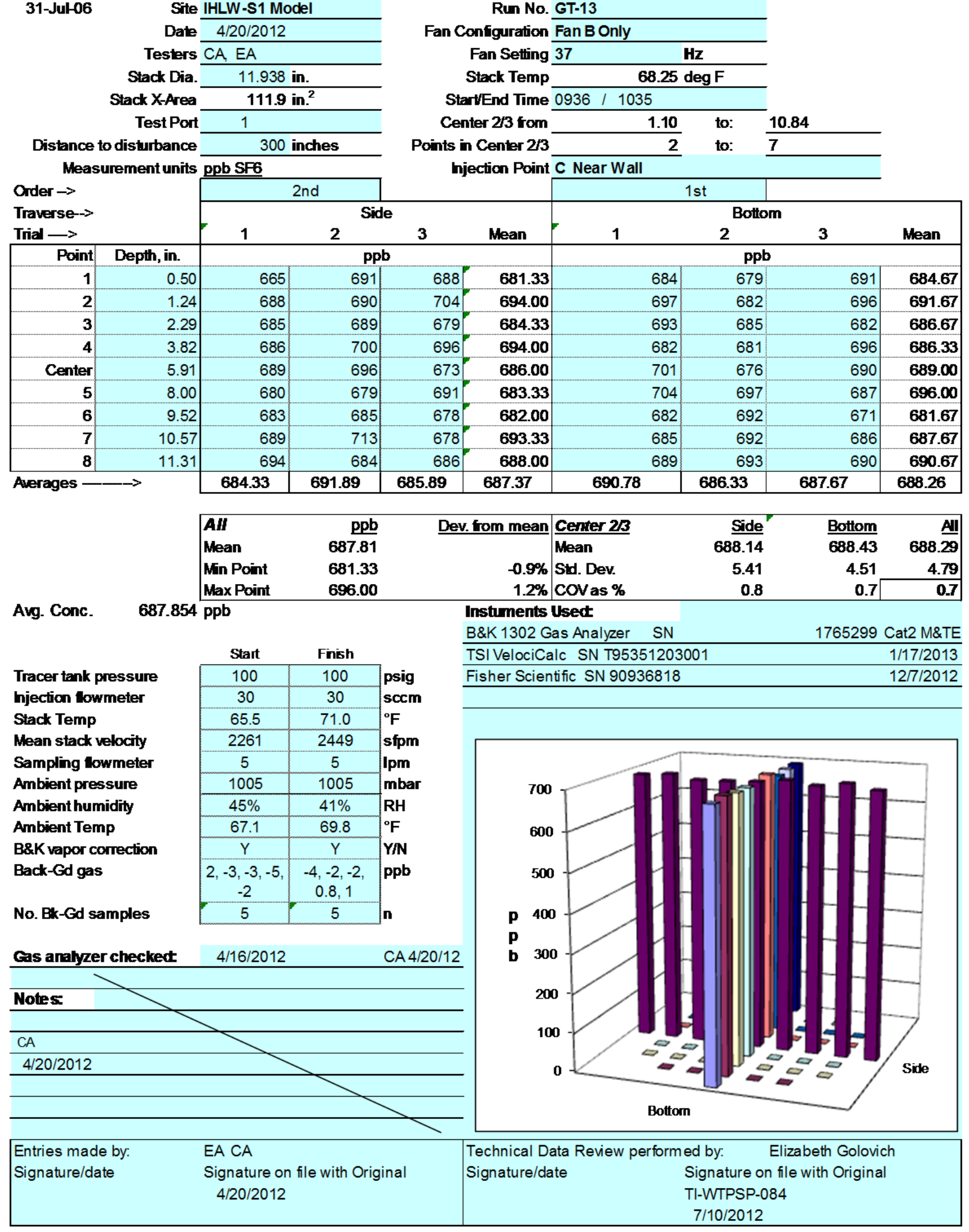


Rev. 0

31-JuL-06

TRACER CAS TRAVERSE DATA FORM

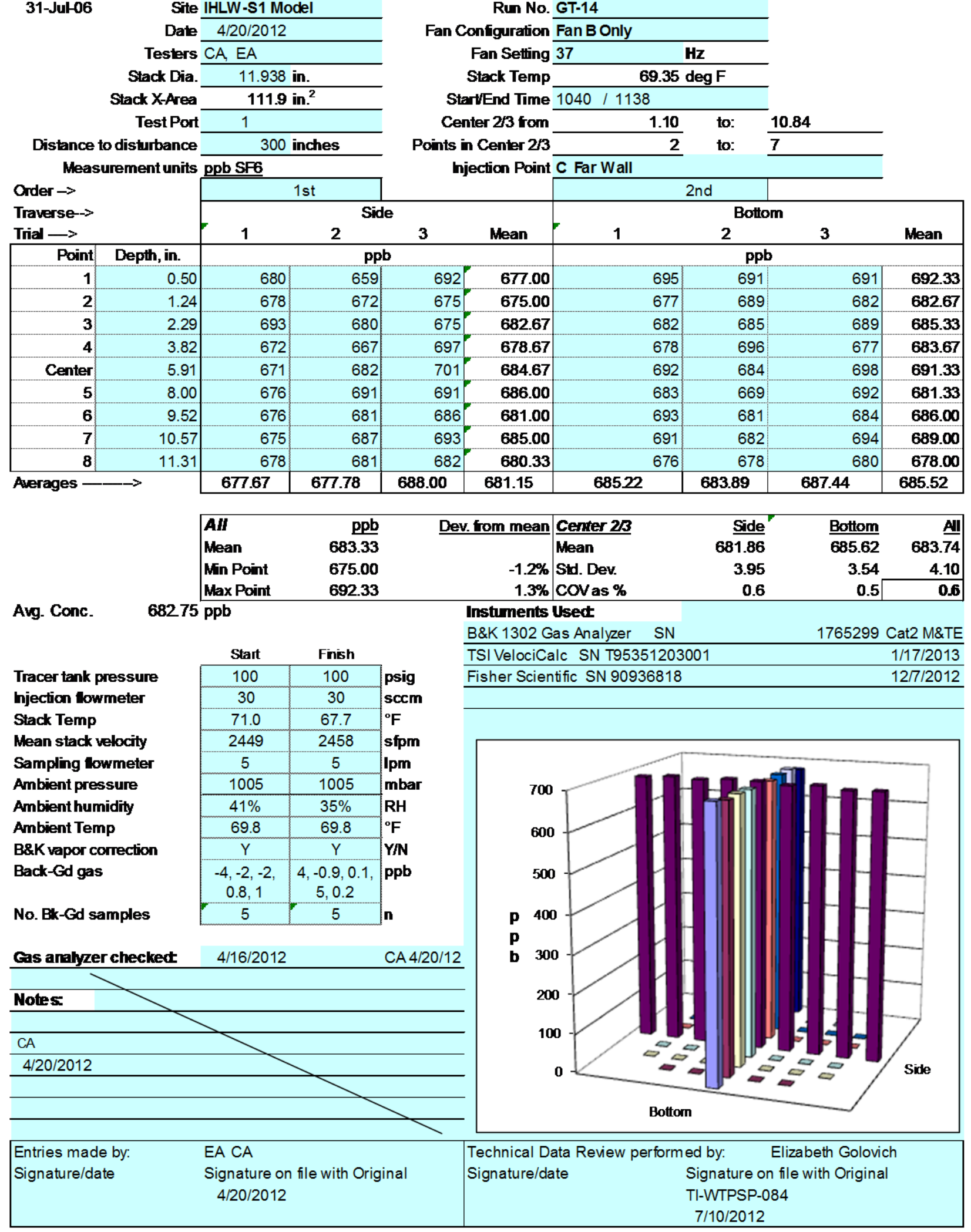


Rev. 0

31-JuL-06

TRACER GAS TRAVERE DATA FORM

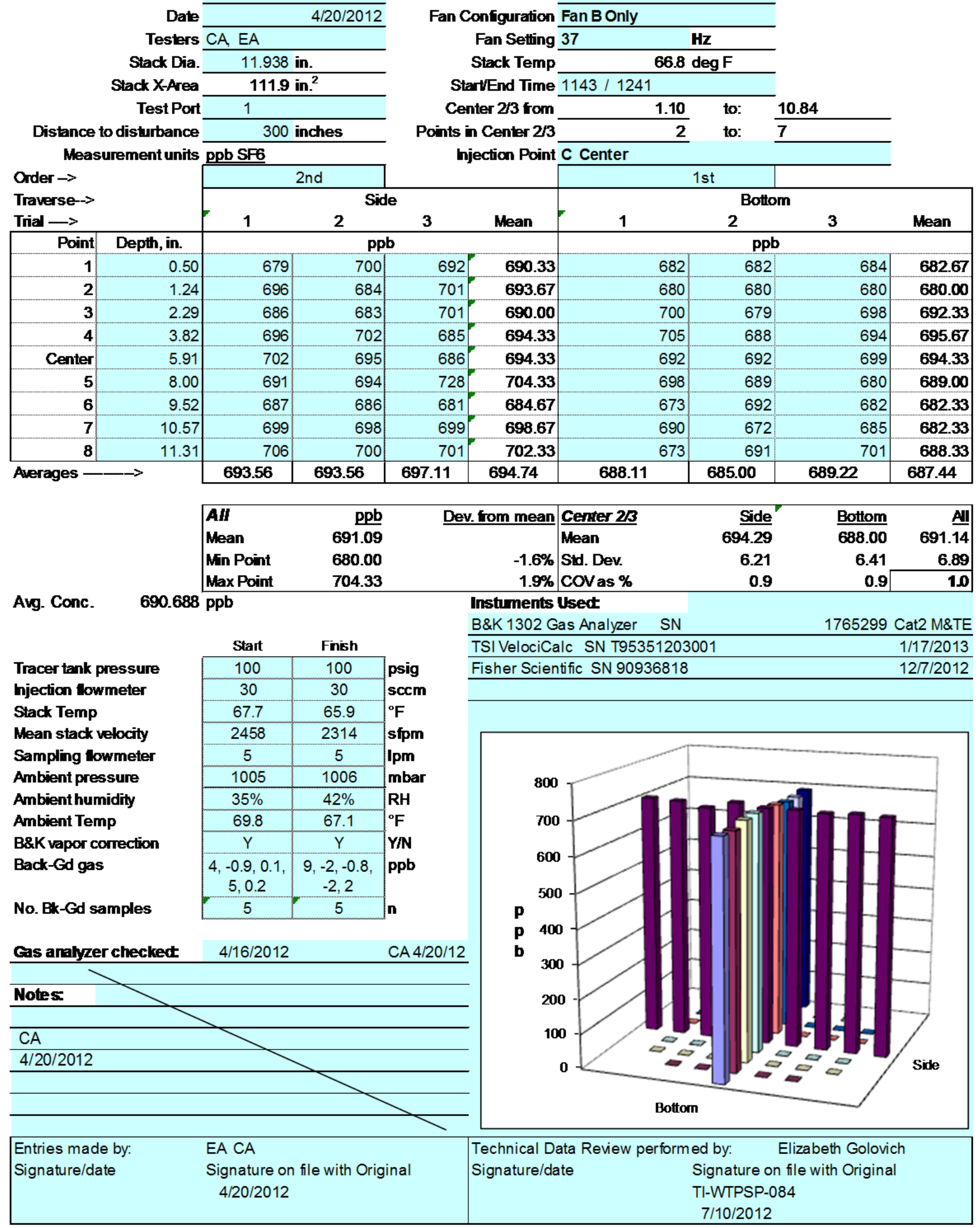


Rev. 0

31-JuL-06

TRACER CAS TRAVERSE DATA FORM

$\begin{aligned} & \text { Site IHLW-S1 Model } \\ & \text { Date } \frac{4 / 20 / 2012}{\text { Testers }} \\ & \text { XY, YFS } \\ & \text { Stack Dia. } \frac{11.938 \text { in. }}{111.9 \text { in. }^{2}} \\ & \text { Stack X-Area } \\ & \text { Test Port }\end{aligned}$

Distance to disturbance $240 \quad$ inches

Measurement units ppb SF6
Run No. GT-16

Fan Configuration Fan B Only

Fan Setting 35

Stack Temp

StartEnd Time $1545 / 1705$

Center $2 / 3$ from

Points in Center $2 / 3$

mjection Point $\mathrm{C}$ Bottom

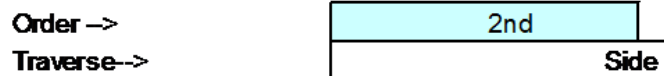

\begin{tabular}{|c|c|c|c|c|c|c|c|c|c|}
\hline \multirow{2}{*}{\multicolumn{2}{|c|}{$\begin{array}{l}\text { Traverse--> } \\
\text { Trial } \longrightarrow\end{array}$}} & \multicolumn{4}{|c|}{ Side } & \multicolumn{4}{|c|}{ Bottom } \\
\hline & & 1 & 2 & 3 & Mean & 1 & 2 & 3 & Mean \\
\hline Point & Depth, in. & \multicolumn{4}{|c|}{ ppb } & \multicolumn{4}{|c|}{ ppb } \\
\hline 1 & 0.50 & 646 & 661 & 710 & 672.33 & 709 & 739 & 708 & 718.67 \\
\hline 2 & 1.24 & 611 & 698 & 825 & 711.33 & 863 & 622 & 730 & 738.33 \\
\hline 3 & 2.29 & 569 & 737 & 701 & 669.00 & 793 & 687 & 690 & 723.33 \\
\hline 4 & 3.82 & 756 & 707 & 787 & 750.00 & 713 & 703 & 883 & 766.33 \\
\hline Center & 5.91 & 575 & 697 & 737 & 669.67 & 650 & 699 & 713 & 687.33 \\
\hline 5 & 8.00 & 592 & 855 & 741 & 729.33 & 645 & 782 & 740 & 722.33 \\
\hline 6 & 9.52 & 695 & 665 & 678 & 679.33 & 689 & 656 & 545 & 630.00 \\
\hline 7 & 10.57 & 678 & 838 & 808 & 774.67 & 746 & 683 & 770 & 733.00 \\
\hline 8 & 11.31 & 814 & 693 & 687 & 731.33 & 615 & 710 & 688 & 671.00 \\
\hline \multicolumn{2}{|c|}{ Averages $\longrightarrow \longrightarrow$} & 659.56 & 727.89 & 741.56 & 709.67 & 713.67 & 697.89 & 718.56 & 710.04 \\
\hline
\end{tabular}

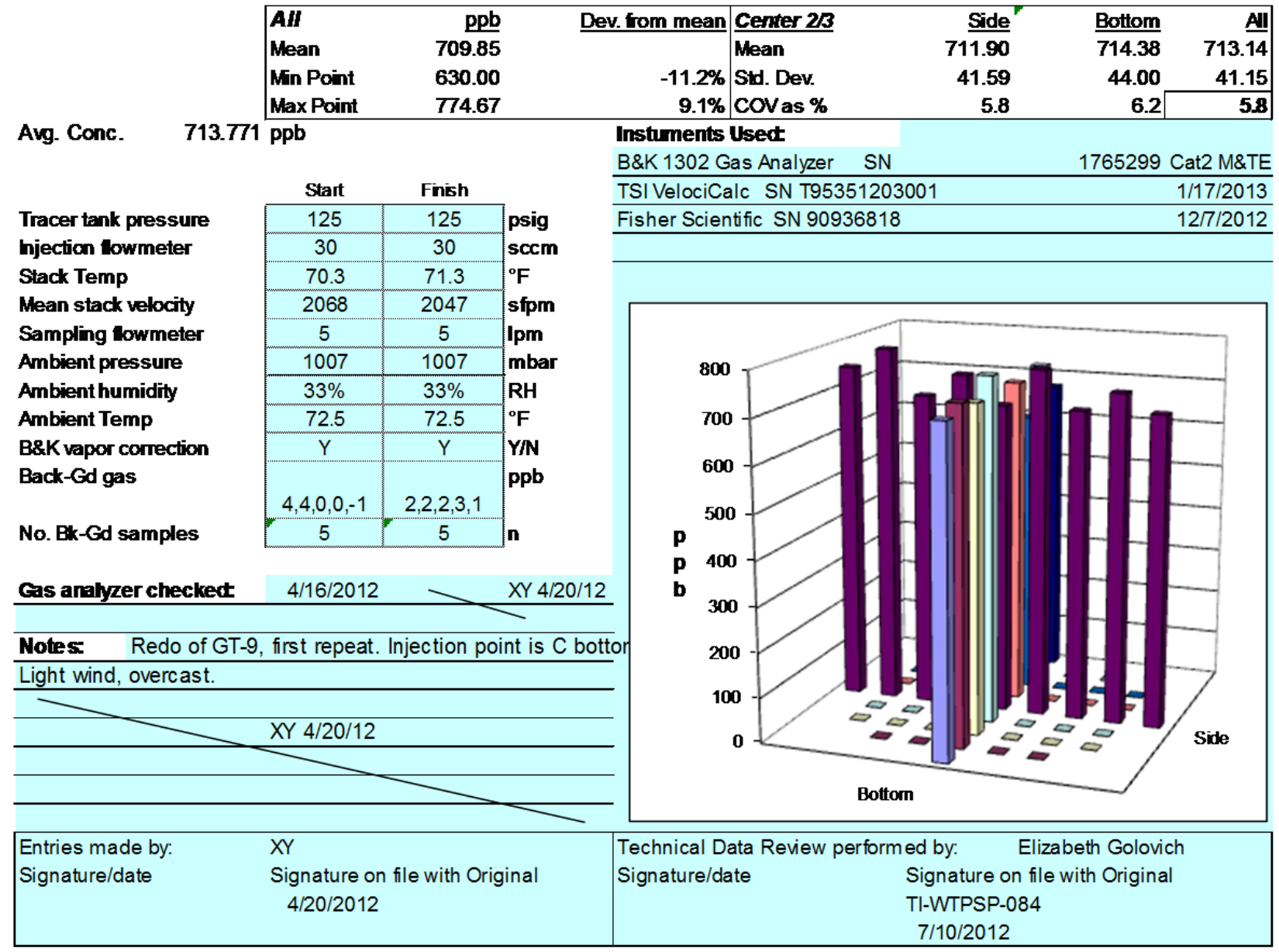


Rev. 0

31-JuL-06

TRACER GAS TRAVERSE DATA FORM

\begin{tabular}{|c|c|c|c|c|c|c|c|c|}
\hline & & ACER CAS & RAVERSE & ATA FORM & & & & \\
\hline Site & IHLW-S1 Mo & & & Run No. & -17 & & & \\
\hline Date & $4 / 23 / 20012$ & & Fan C & nfiguration & B only & & & \\
\hline Testers & CA, EA, JEF & & & Fan Setting & & $\mathbf{z}$ & & \\
\hline Stack Dia. & 11.938 & & & tack Temp & 77.25 & $\operatorname{leg} F$ & & \\
\hline Stack X-Area & 111.9 & & & VEnd Time & $5 / 958$ & & & \\
\hline Test Port & 2 & & Cen & er 233 from & 1.10 & to: & 10.84 & \\
\hline o disturbance & 240 & ches & Points i & Center 2/3 & 2 & to: & 7 & \\
\hline urement units & ppb SF6 & & & ction Point & 3ottom & & & \\
\hline & & nd & & & & st & & \\
\hline & & Sid & & & & Bottc & & \\
\hline & 1 & 2 & 3 & Mean & 1 & 2 & 3 & Mean \\
\hline Depth, in. & & ppt & & & & ppl & & \\
\hline 0.50 & 726 & 781 & 697 & 734.67 & 646 & 771 & 625 & 680.67 \\
\hline 1.24 & 679 & 667 & 621 & 655.67 & 655 & 664 & 597 & 638.67 \\
\hline 2.29 & 788 & 681 & 631 & 700.00 & 717 & 774 & 623 & 704.67 \\
\hline 3.82 & 730 & 800 & 665 & 731.67 & 639 & 640 & 727 & 668.67 \\
\hline 5.91 & 697 & 666 & 660 & 674.33 & 743 & 676 & 669 & 696.00 \\
\hline 8.00 & 703 & 629 & 661 & 664.33 & 728 & 684 & 666 & 692.67 \\
\hline 9.52 & 660 & 724 & 627 & 670.33 & 699 & 619 & 693 & 670.33 \\
\hline 10.57 & 661 & 742 & 663 & 688.67 & 696 & 673 & 641 & 670.00 \\
\hline 11.31 & 636 & 683 & 621 & 646.67 & 676 & 640 & 625 & 647.00 \\
\hline & 697.78 & 708.11 & 649.56 & 685.15 & 688.78 & 68233 & 651.78 & 674.30 \\
\hline
\end{tabular}

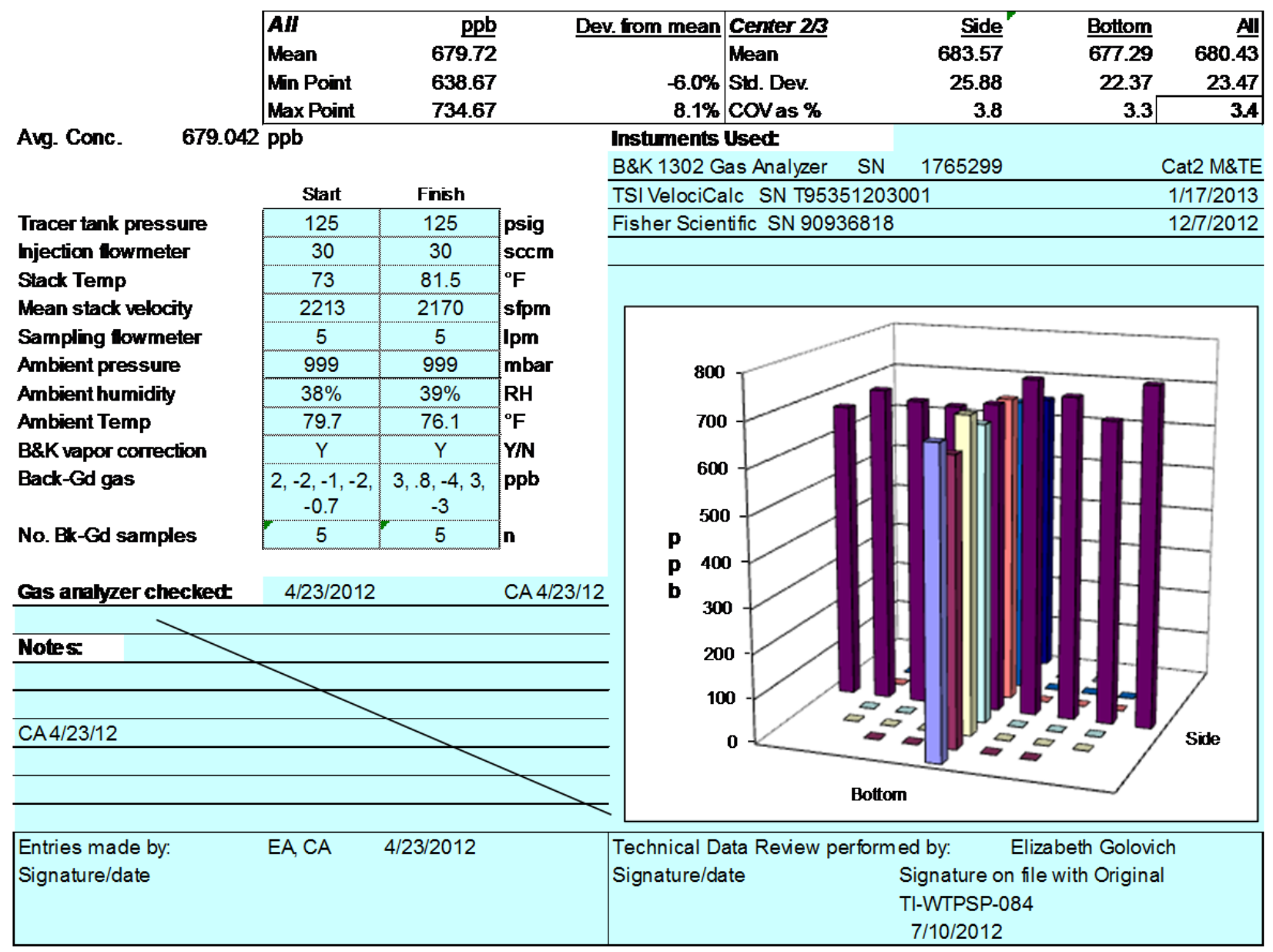


Rev. 0

31-JuL-06

\section{TRACER CAS TRAVERSE DATA FORY}

\begin{aligned} & Date \multicolumn{1}{c}{$4 / 3 / 2012$} \\ & Testers CA, EA, JEF \\ & Stack Dia. 11.938 in. \\ & Stack X-Area 111.9 in. $^{2} \\ &$ Test Port 2 \\ & Distance to disturbance \\ & Measurement units ppb SF6 \end{aligned}

Run No. GT-18

Fan Configuration Fan B only

Fan Setting 37

Stack Temp

Start/End Time $1000 / 1100$

Center $2 / 3$ from

Points in Center $2 / 3$

Injection Point C Bottom

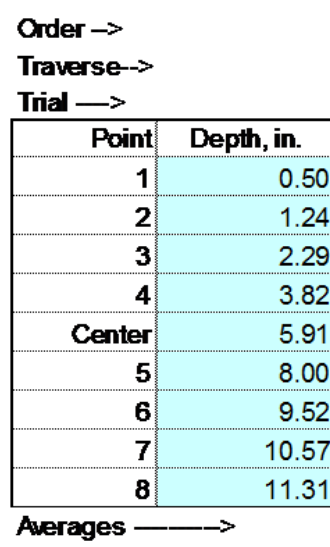

\begin{tabular}{|r|r|}
\hline 1 st \\
\hline
\end{tabular}

2nd

3 Mean 1

$\mathbf{H 3 ~ d e g}$

$3 \operatorname{deg} F$

$\frac{1.10}{2}$ to: $\frac{10.84}{7}$

2 to: 7

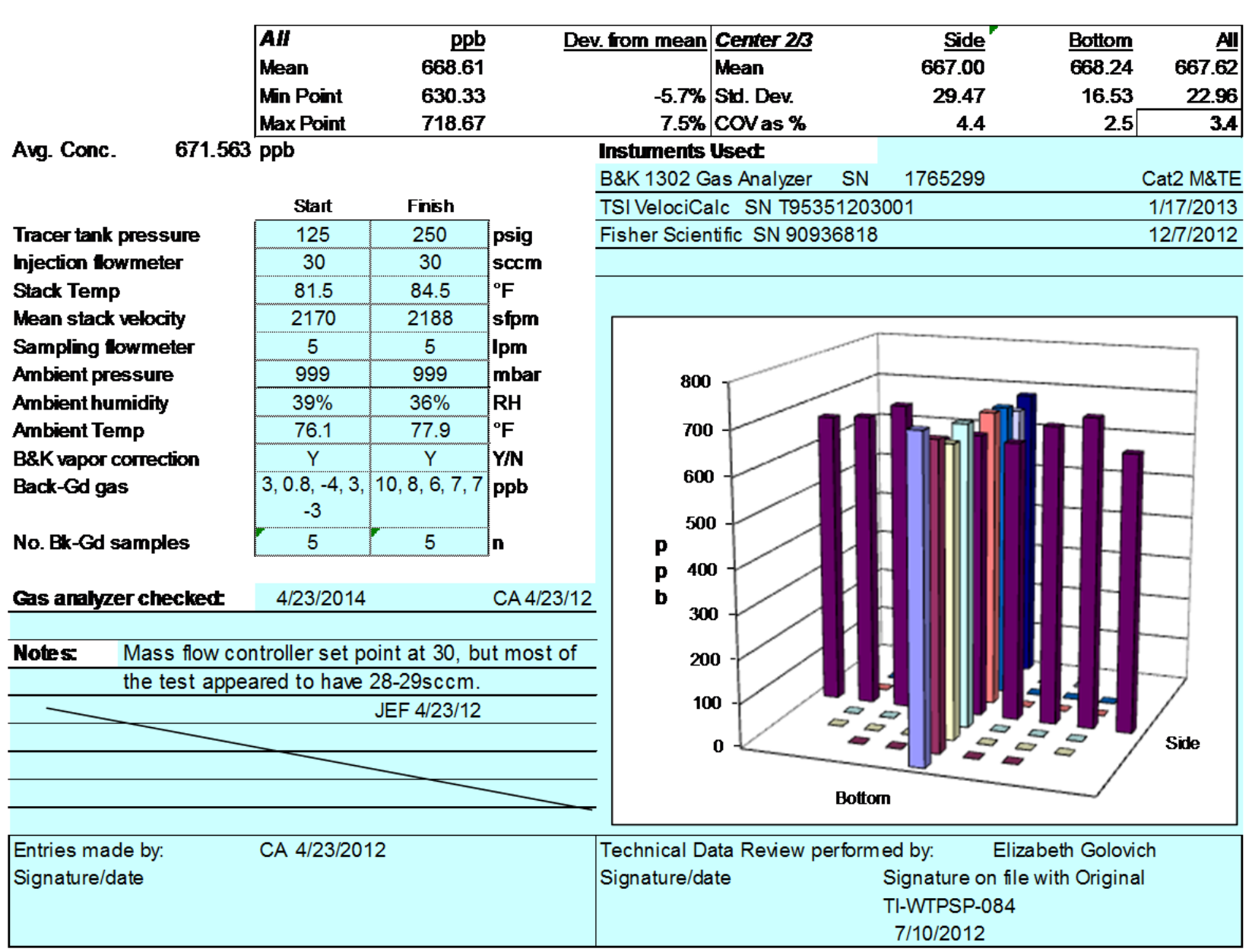


Rev. 0

31-JuL-06

IRACER CAS TRAVERE DATA FORM

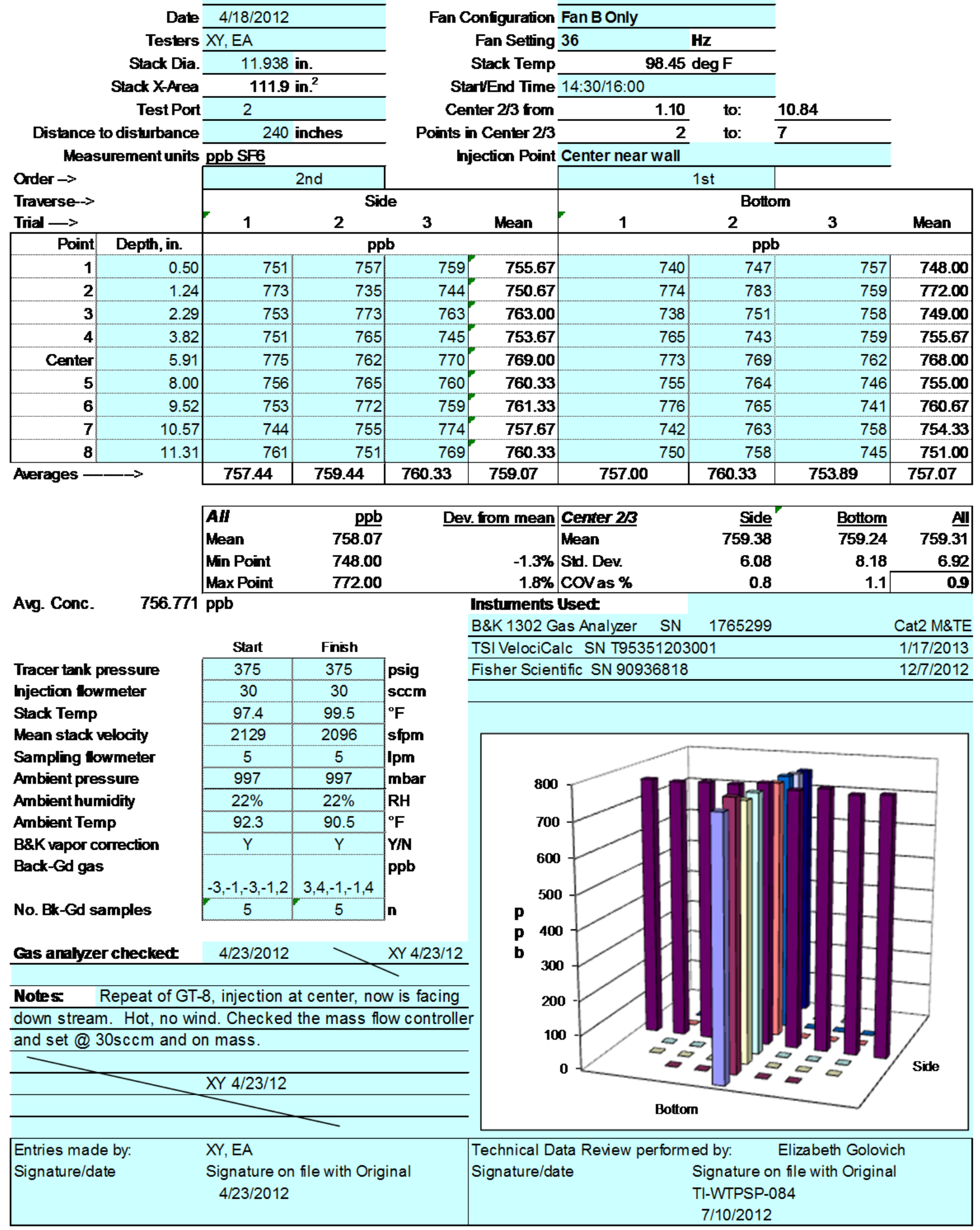


Rev. 0

31-JuL-06

TRACER CAS TRAVERSE DATA FORM

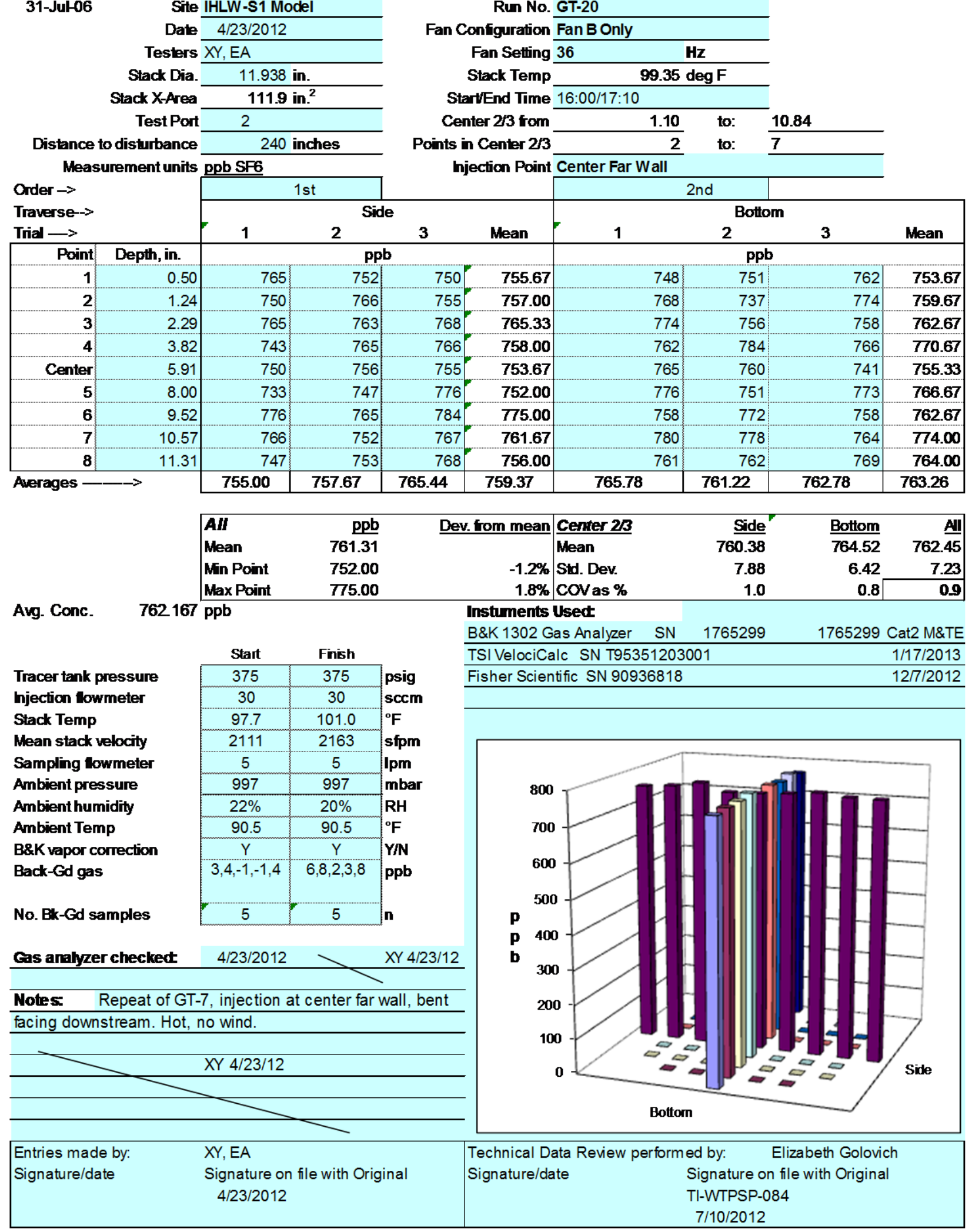


Rev. 0

31-JuL-06

TRACER CAS IRAVERSE DATA FORY

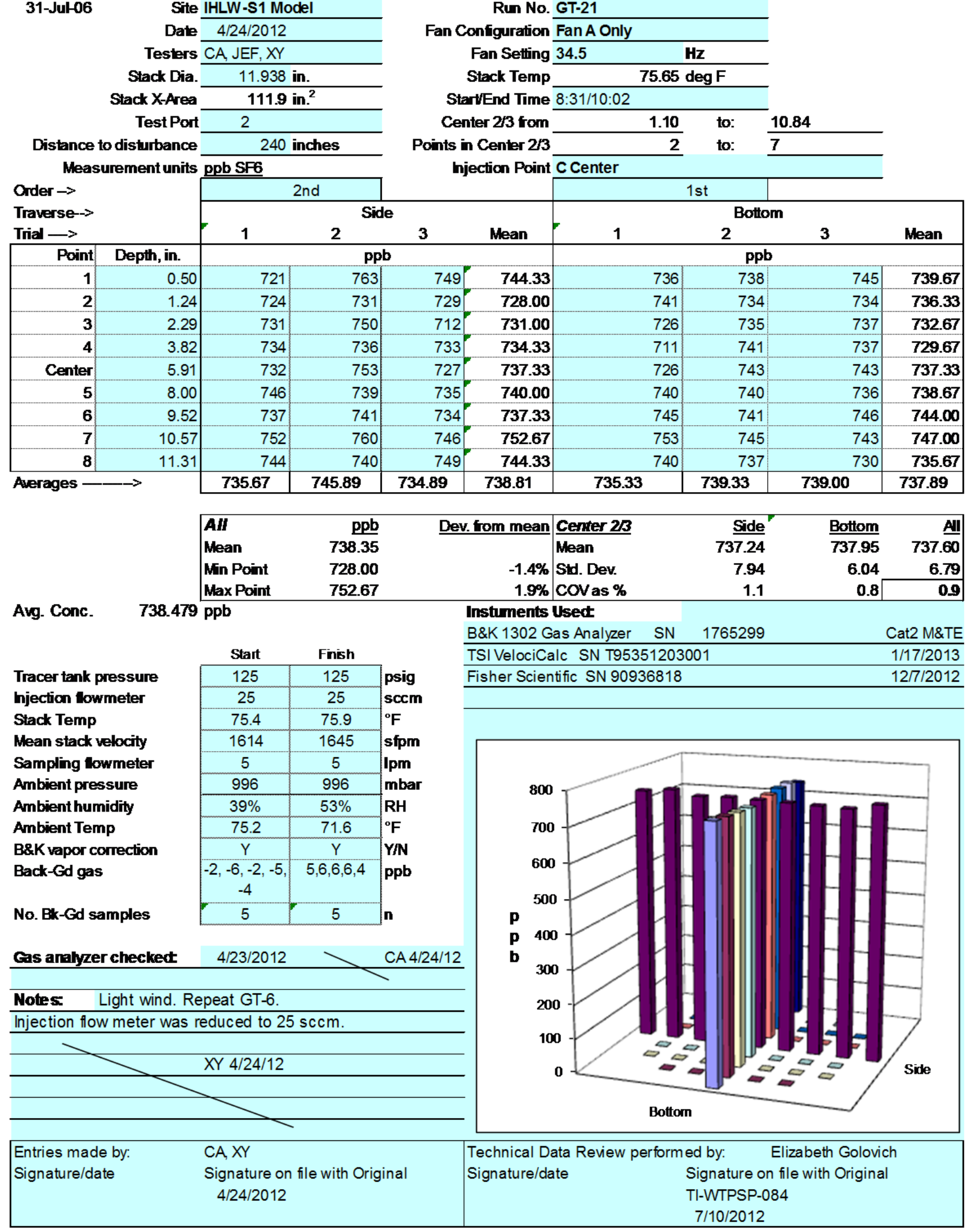


Rev. 0

31-JuL-06

TRACER GAS TRAVERSE DATA FORM

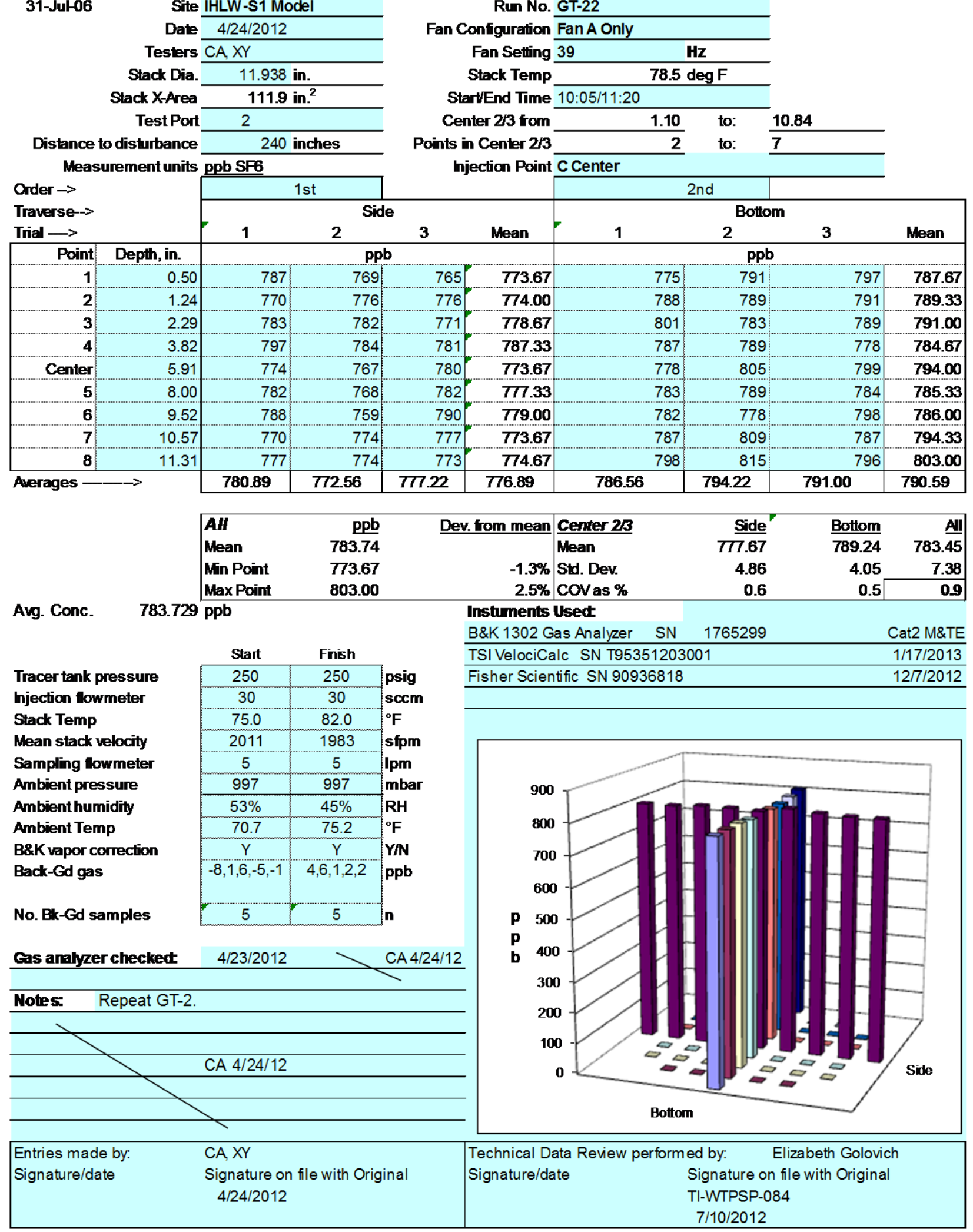


Rev. 0

31-JuL-06

TRACER GAS TRAVERSE DATA FORM

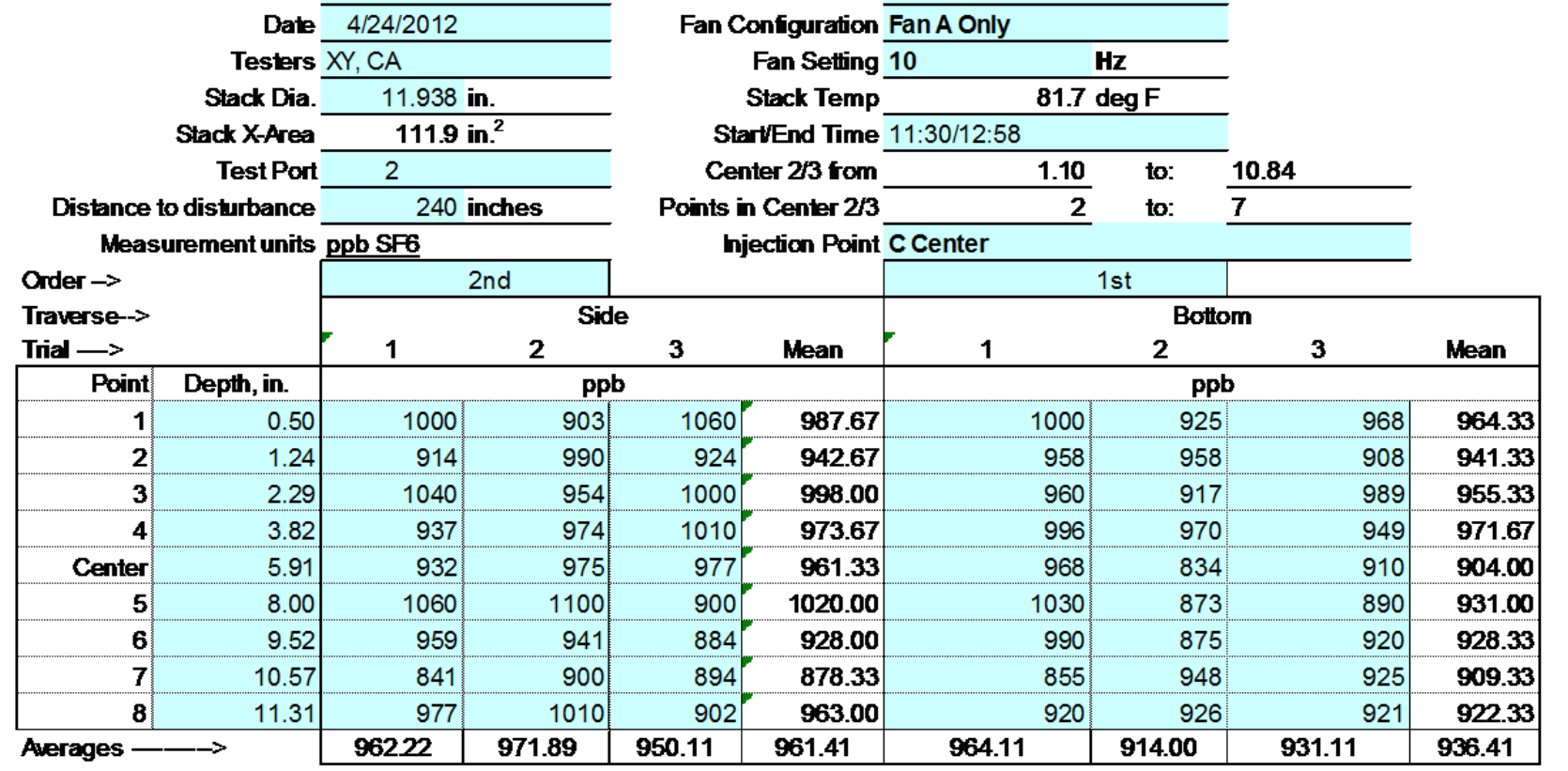

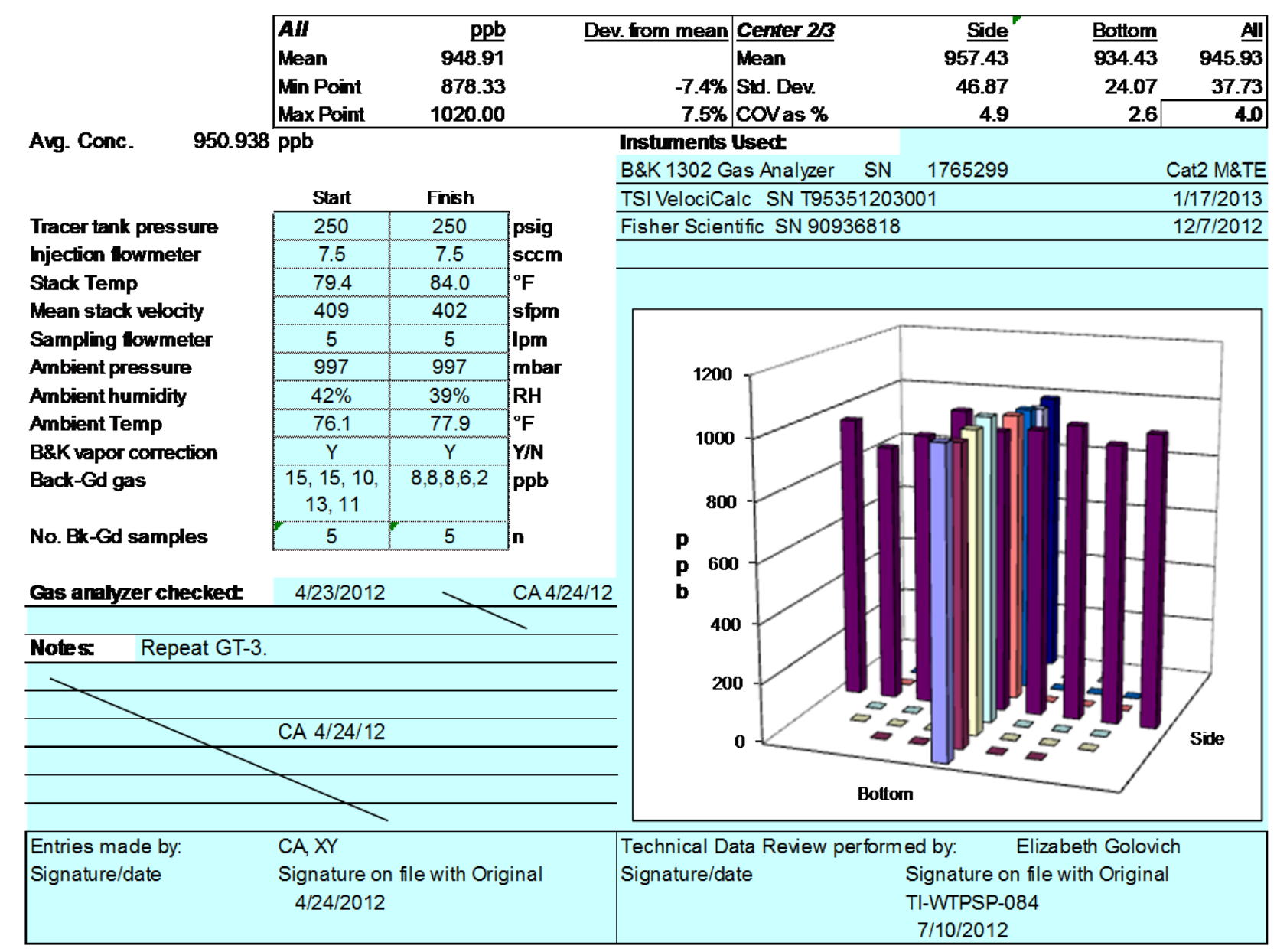


Rev. 0

31-JuL-06

TRACER CAS TRAVERSE DATA FORM

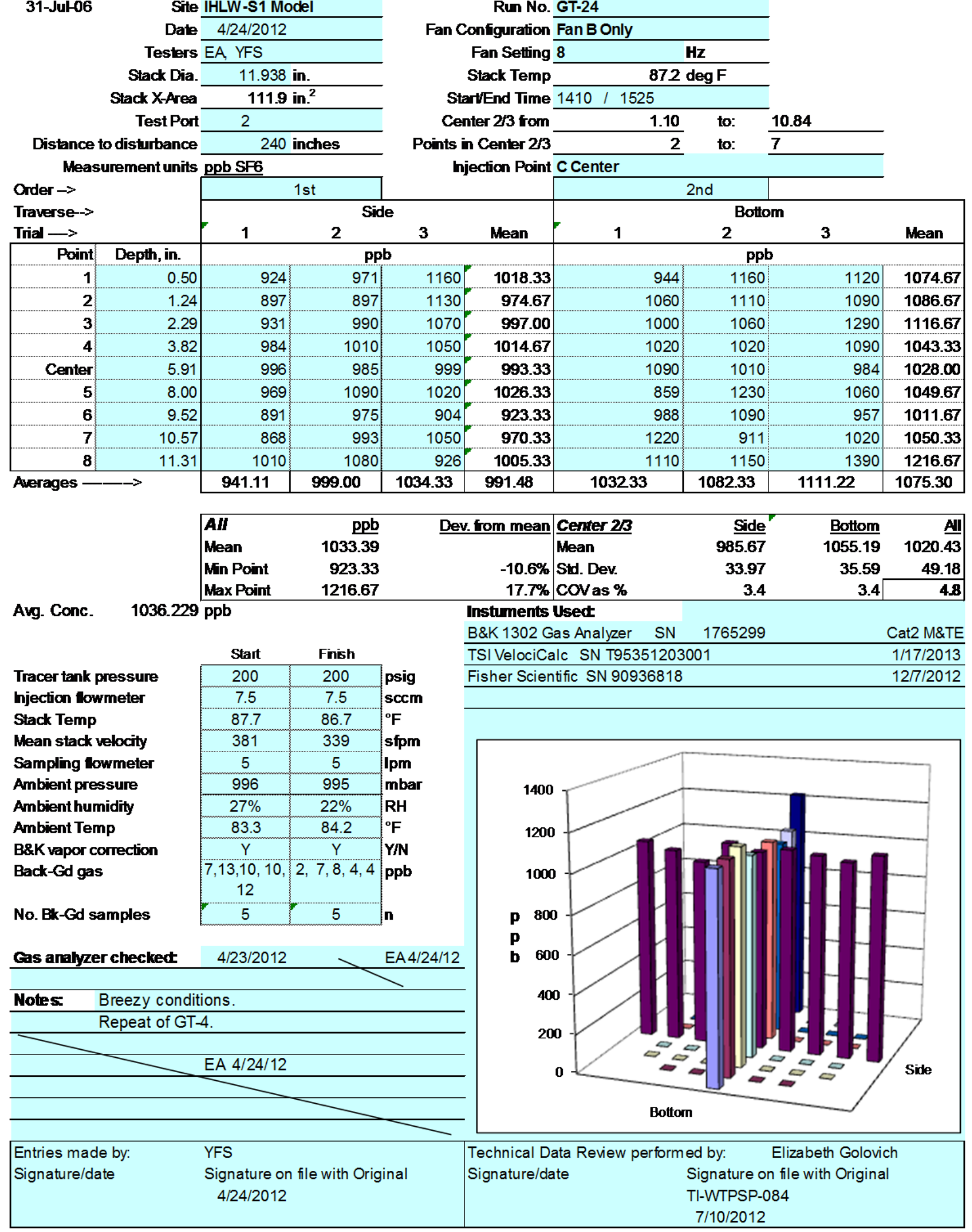


Rev. 0

31-JuL-06

TRACER GAS TRAVERSE DATA FORM

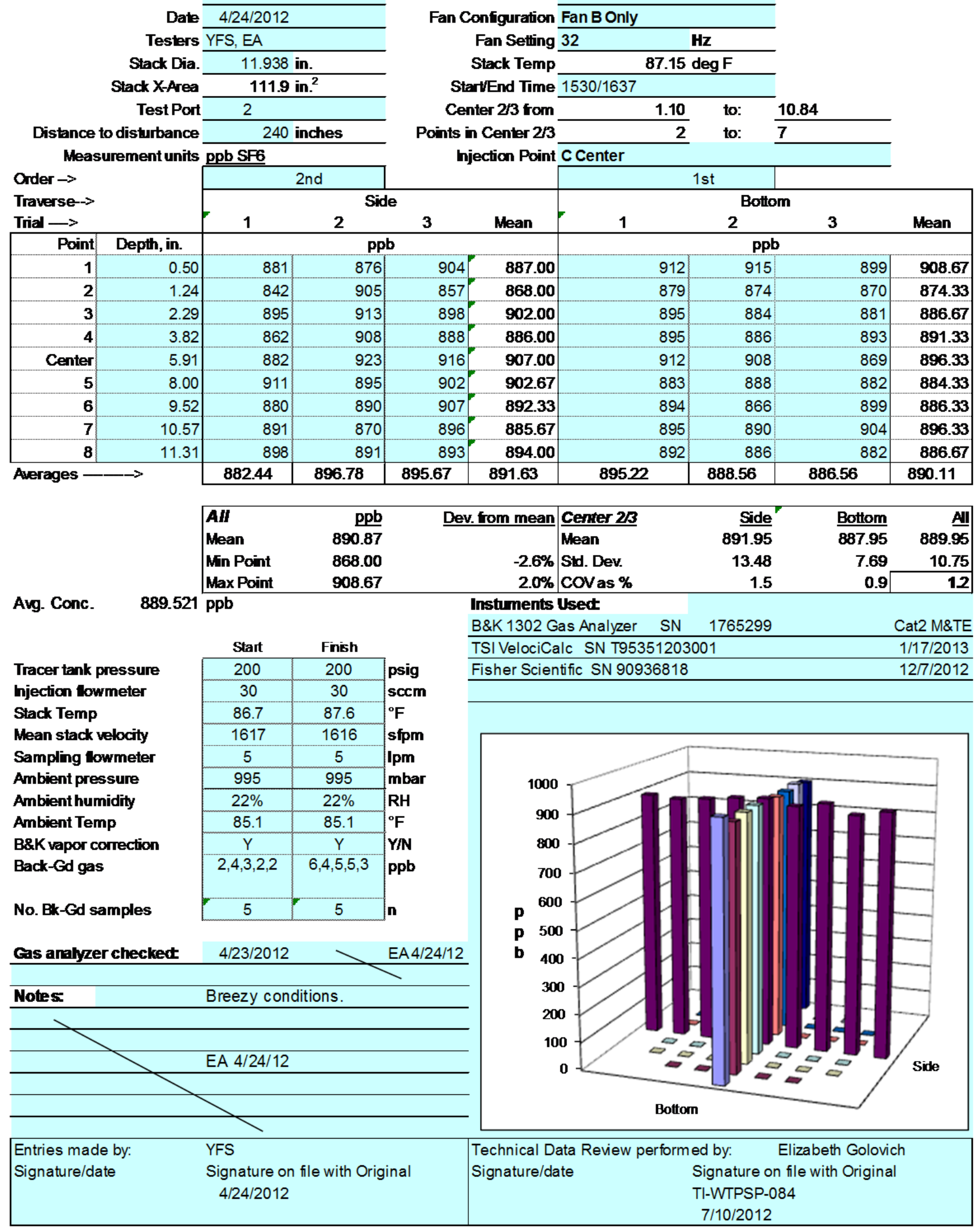


Rev. 0

31-JuL-06

TRACER CAS TRAVERE DATA FORM

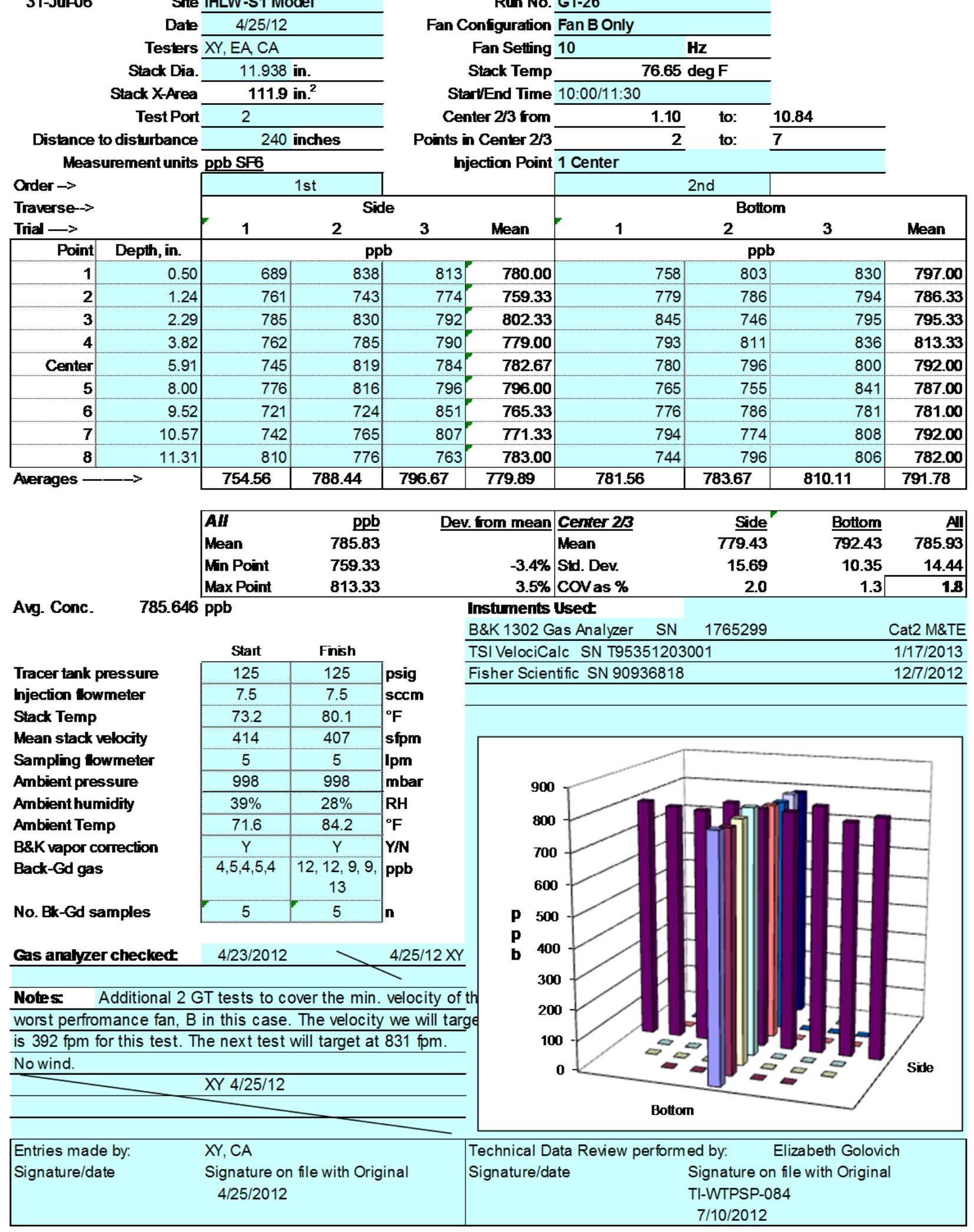


Rev. 0

31-JuL-06

IRACER CAS IRAVERSE DATA FORM

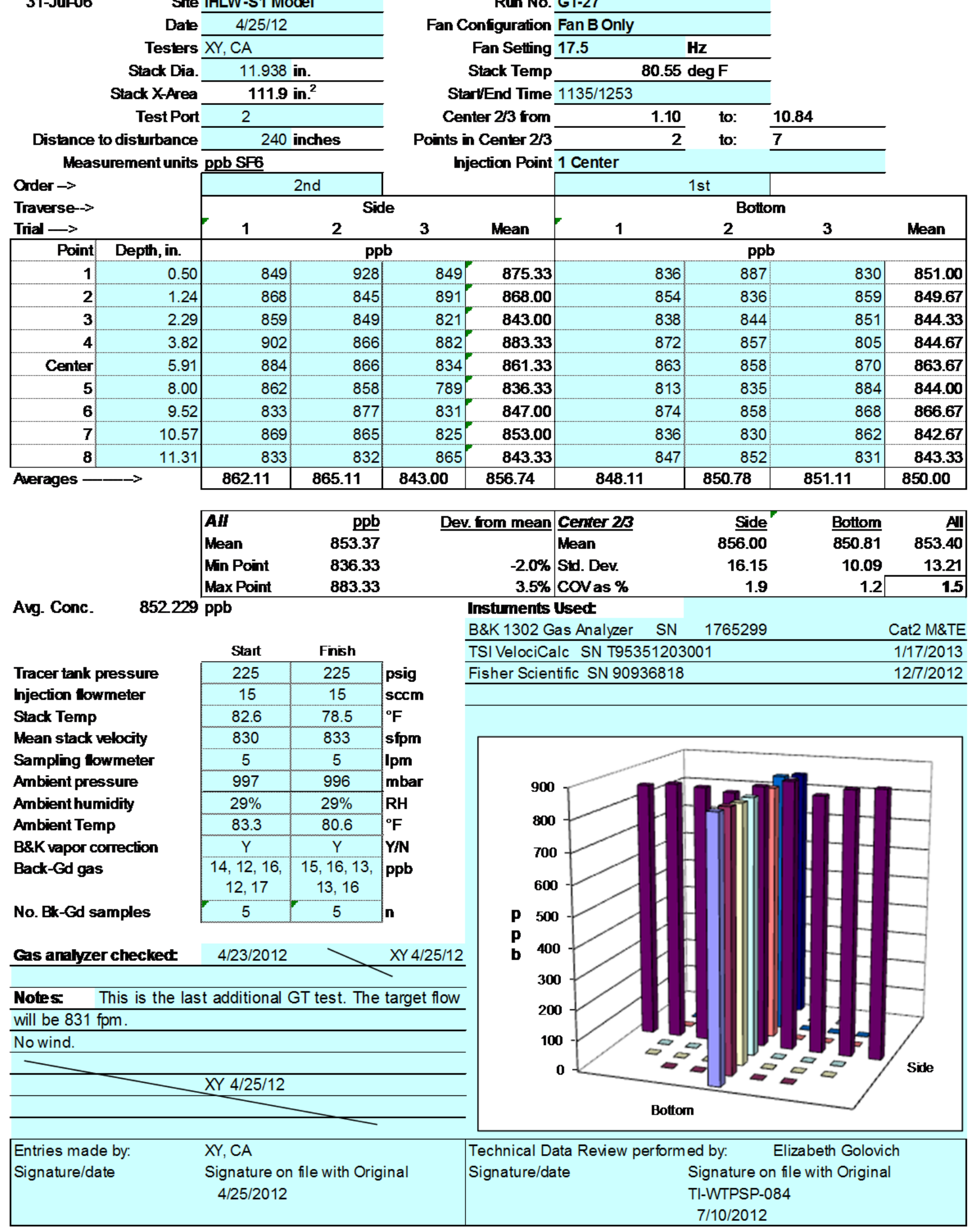




\section{C.5 IHLW-S1 Particle Tracer Uniformity Data Sheets}

Rev. 0

3 Aug. 2006

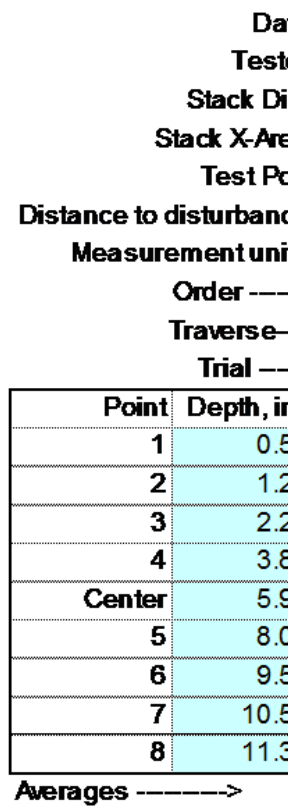

Site IHLW-S1 Model

Date 3/14/2012

Tester CA, XY, JEF

Stack Dia. 11.938 in.

$\mathrm{X}$-Area 111.9 in 2

est Port particles/tis
240 inches

1 st
Run No.

Fan configuration Fan A only

Fan Setting 42

Stack Temp $\mathbf{4 9 . 4 5 ~ d e g ~ F ~}$

Start/End Time 10:00/12:00

Center $2 \beta$ from $\quad 1.10$

Points in Center $2 / 3 \longdiv { 2 }$ Injection Point Prt C Center

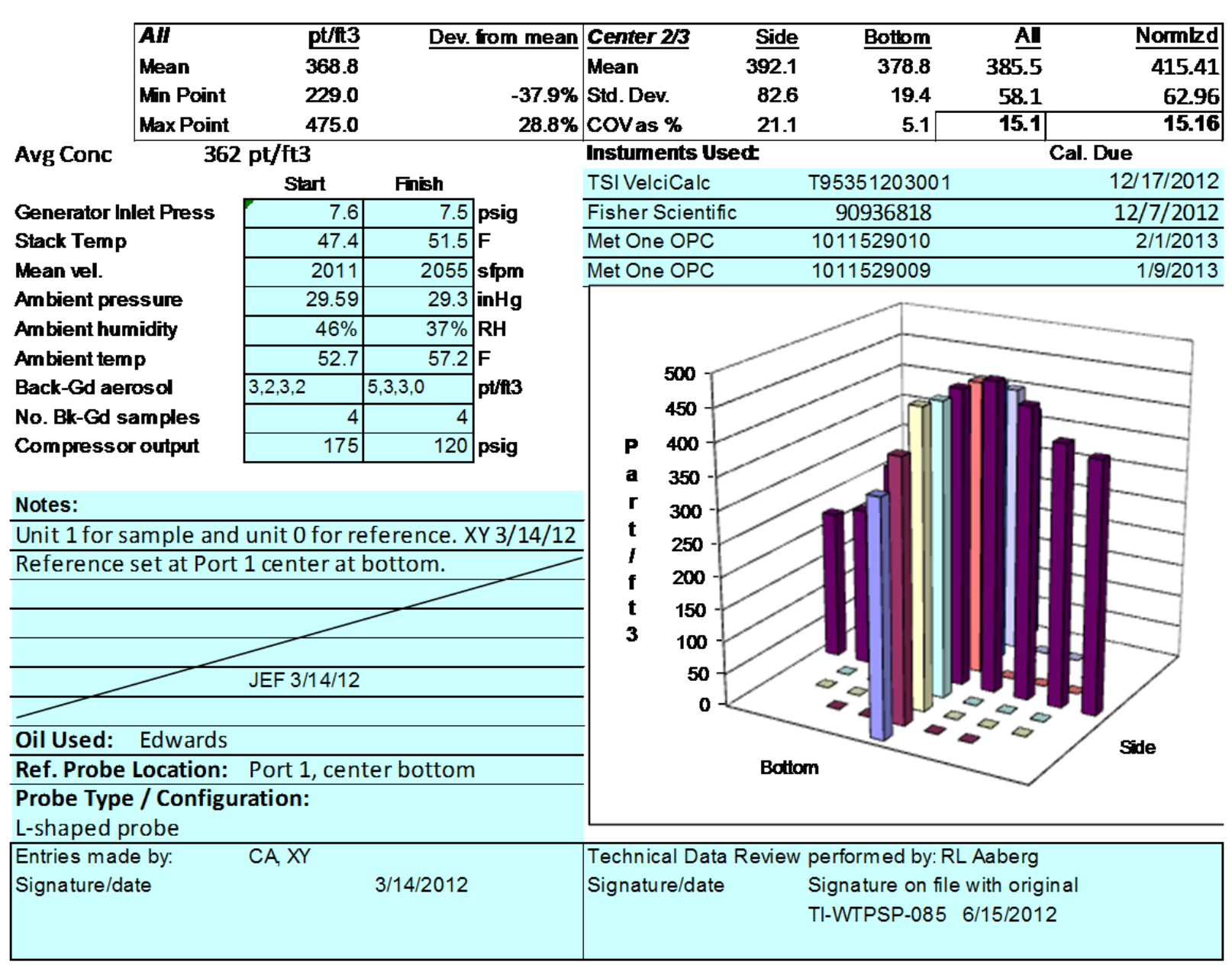


Rev. 0

3 Aug. 2006

\begin{tabular}{|c|c|}
\hline 3 Aug. 2006 & IHLW-s1 Model \\
\hline Date & $3 / 15 / 2012$ \\
\hline \multicolumn{2}{|c|}{ Tester $\overline{C A}, X Y$} \\
\hline Stack Dia. & 11.938 in. \\
\hline Stack X-Area & 111.9 in 2 \\
\hline Test Port & 2 \\
\hline Distance to disturbance & 240 inches \\
\hline
\end{tabular}

\begin{tabular}{|c|c|c|c|c|c|c|c|c|c|}
\hline \multirow{4}{*}{\multicolumn{2}{|c|}{$\begin{array}{r}\text { Measurement units } \\
\text { Order } \longrightarrow \\
\text { Traverse } \rightarrow \\
\text { Trial } \longrightarrow\end{array}$}} & \multicolumn{7}{|c|}{ Injection Point Prt C Center } & \\
\hline & & \multicolumn{4}{|c|}{ 2nd } & \multicolumn{3}{|c|}{$1 \mathrm{st}$} & \\
\hline & & \multicolumn{4}{|c|}{ Side } & \multicolumn{3}{|c|}{ Bottom } & \multirow{3}{*}{ Mean } \\
\hline & & 1 & 2 & \multirow[b]{2}{*}{$s \mathrm{ft3}$} & \multirow[t]{2}{*}{ Mean } & \multicolumn{3}{|c|}{2} & \\
\hline Point & Depth, in. & \multicolumn{2}{|c|}{ particles/ft3 } & & & \multicolumn{3}{|c|}{ particles/ft3 } & \\
\hline 1 & 0.50 & 890 & 1020 & 987 & 965.7 & 868 & 787 & 959 & 8713 \\
\hline 2 & 1.24 & 877 & 1145 & 957 & 993.0 & 976 & 948 & 1042 & 988.7 \\
\hline 3 & 2.29 & 770 & 1135 & 1215 & 1040.0 & 1116 & 1045 & 1195 & 1118.7 \\
\hline 4 & 3.82 & 992 & 1414 & 1258 & 1221.3 & 1192 & 1069 & 1206 & 1155.7 \\
\hline Center & 5.91 & 1085 & 1273 & 1263 & 1207.0 & 1245 & 1158 & 1317 & 1240.0 \\
\hline 5 & 8.00 & 1081 & 1303 & 1237 & 1207.0 & 1142 & 1076 & 1290 & 1169.3 \\
\hline 6 & 9.52 & 1108 & 1223 & 1164 & 1165.0 & 1028 & 1110 & 1213 & 1117.0 \\
\hline 7 & 10.57 & 1016 & 1187 & 1020 & 1074.3 & 913 & 897 & 1091 & 9670 \\
\hline 8 & 11.31 & 992 & 930 & 871 & 931.0 & 866 & 794 & 935 & 8650 \\
\hline Averages - & $\rightarrow$ & 979.0 & 1181.1 & 1108.0 & 1089.4 & 1038.4 & 987.1 & 1138.7 & 1054.7 \\
\hline
\end{tabular}

Run No. PT-2

Fan confguration Fan B only

Fan Setting 36

Stack Temp

Start/End Time 9:55/12:00

Center $2 / 3$ from 1.10

Points in Center $2 \beta$ 2 to: $(2$ 10.84 to: 7 Injection Point Prt C Center

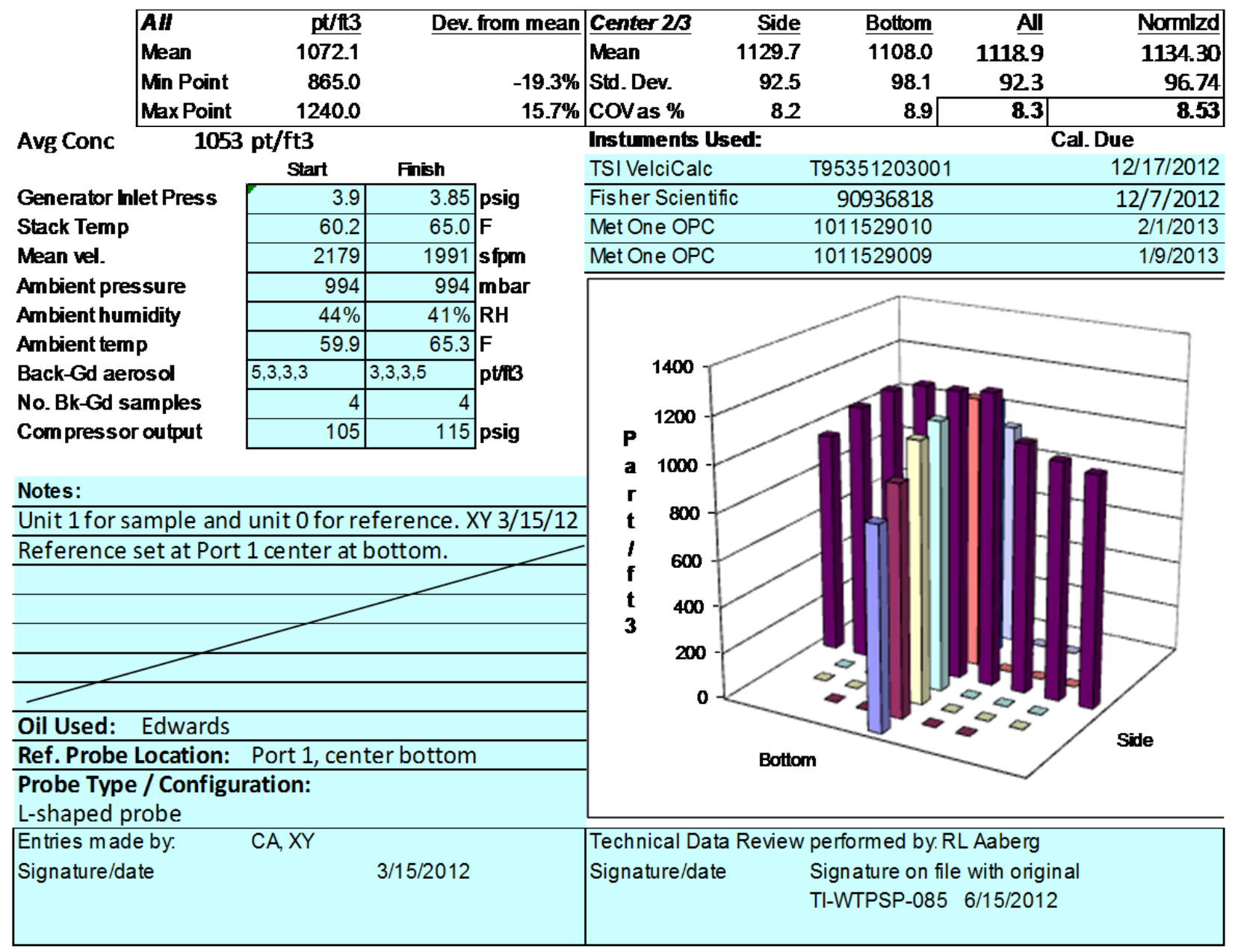


Rev. 0

3 Aug. 2006
PARTICLE TRACER TRAVERSE DATA FORM

Site IHLW-S1 Model

Date $3 / 16 / 2012$

Tester CA, EA, XY

Stack Dia. 11.938 in

Stack XArea 111.9 in 2

Test Port

2

Distance to disturbance 240 inches

Measurement units particles/113

Order $\longrightarrow$

Traverse-

Trial $\rightarrow$

\begin{tabular}{|r|r|}
\hline Point & Depth, in \\
\hline $\mathbf{1}$ & 0.5 \\
\hline $\mathbf{2}$ & 1.2 \\
\hline $\mathbf{3}$ & 2.2 \\
\hline $\mathbf{4}$ & 3.8 \\
\hline $\mathbf{C e n t e r}$ & 5.9 \\
\hline $\mathbf{5}$ & 8.00 \\
\hline $\mathbf{6}$ & 9.5 \\
\hline $\mathbf{7}$ & 10.5 \\
\hline $\mathbf{8}$ & 11.3 \\
\hline
\end{tabular}

Averages

$1 \mathrm{st}$

\begin{tabular}{l|l}
\hline & \\
\hline & \\
\hline & \\
\hline & 3 \\
\hline 9 & 3 \\
\hline 2 & 3 \\
\hline 0 & 5 \\
\hline 2 & 5 \\
\hline 7 & 3 \\
\hline 1 & 57 \\
\hline & 431.8 \\
\hline
\end{tabular}

Run No. PT-3

Fan configuration FAN A ONLY

Fan Setting 8

Stack Temp

$62.9 \operatorname{deg} \mathrm{F}$

Start/End Time 11:00/1:31

Center $2 / 3$ from 1.10

Points in Center $2 / 3$ Ijection Point Port C Center

$\begin{array}{ll}\text { to: } & 10.84 \\ \text { to: } & \end{array}$
2nd

\begin{tabular}{|c|c|c|}
\hline \multicolumn{2}{|c|}{ Bottom } & \multirow[b]{2}{*}{ Mean } \\
\hline 2 & 3 & \\
\hline \multicolumn{2}{|c|}{ particles $/ \mathrm{t} 3$} & \\
\hline 1292 & 735 & 8540 \\
\hline 603 & 542 & 5990 \\
\hline 588 & 624 & 551.3 \\
\hline 586 & 716 & 630.0 \\
\hline 492 & 320 & 475.0 \\
\hline 417 & 263 & 334.3 \\
\hline 85 & 369 & 206.0 \\
\hline 273 & 51 & 172.7 \\
\hline 94 & 127 & 178.7 \\
\hline 2 & 416.3 & \\
\hline
\end{tabular}

\begin{tabular}{|c|c|c|c|c|c|c|c|}
\hline $\mathbf{A H}$ & $\mathrm{pt} / \mathrm{ft} 3$ & Dev. from mean & Center 2/3 & Side & Bottom & All & Normlzd \\
\hline Mean & 542.0 & & Mean & 676.6 & 424.0 & 550.3 & 70284 \\
\hline Min Point & 172.7 & $-68.1 \%$ & Std. Dev. & 165.8 & 187.4 & 214.6 & 247.61 \\
\hline Max Point & 938.0 & $73.1 \%$ & Covas \% & 24.5 & 442 & 39.0 & 35.23 \\
\hline
\end{tabular}

\section{Avg Conc $\quad 529 \mathrm{pt} / \mathrm{ft} 3$}

\begin{tabular}{|c|c|c|c|}
\hline & Start & Finish & \\
\hline Generator hlet Press & 0.85 & 0.9 & psig \\
\hline tack Temp & 61 & 64.8 & $F$ \\
\hline ean vel. & 279 & 140 & sfpm \\
\hline nbient pressure & 995 & 995 & mbar \\
\hline nbient humidity & $30 \%$ & $28 \%$ & $\mathrm{RH}$ \\
\hline nbient temp & 64.4 & 65.3 & $F$ \\
\hline ack-Gd aerosol & $45,48,24,30$ & $30,48,39,8$ & ptifi3 \\
\hline Bk-Gal cam & 4 & 4 & \\
\hline ompressor output & 110 & 110 & psig \\
\hline
\end{tabular}

Notes: Generator pressure is increased 1.2

\section{Instuments Used:}

Cal. Due

Probe Type / Configuration:

L-shaped probe

Entries made by.

Signature/date

Carmen Arimescu
$3 / 16 / 2012$

\begin{abstract}
Techign
\end{abstract}
TSI VelciCalc

Fisher Scientific

Met One OPC

Met One OPC 1011529009

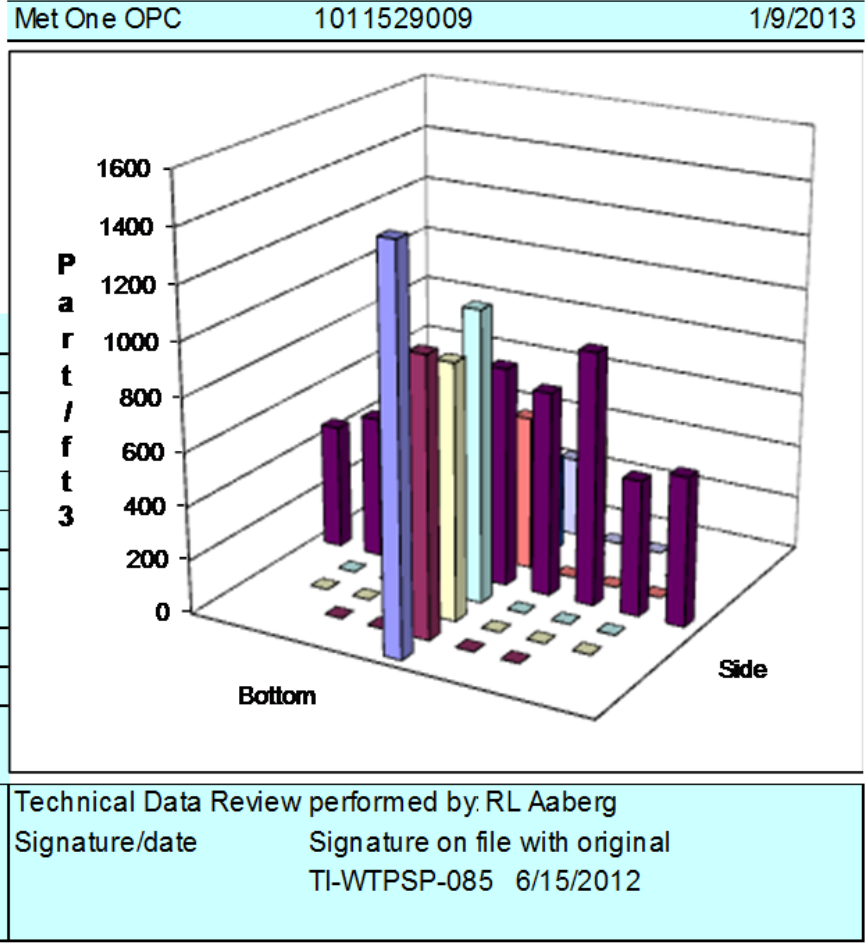

$12 / 17 / 2012$

$12 / 7 / 2012$ $2 / 1 / 2013$ $1 / 9 / 2013$ 
Rev. 0

3 Aug. 2006

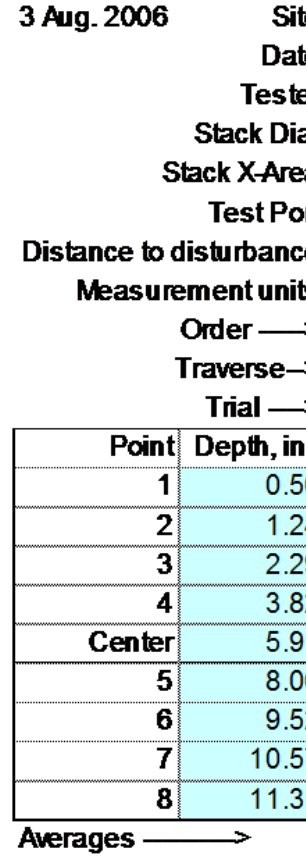

PARTICLE TRACER TRAVERSE DATA FORM

Site IHLW-S1 Model

Date $3 / 16 / 2012$

Tester CA, XY

Stack Dia. 11.938 in

TestPort

111.9 in 2

$\frac{2}{240 \text { inches }}$

240 inches

particles $/ \mathrm{it} 3$
Run No. PT-4

Fan configuration FAN B ONLY

Fan Setting 9

Stack Temp

$68.15 \operatorname{deg} \mathrm{F}$

Start/End Time 1:40/3:10

Center $2 \beta$ from

Points in Center $2 / 3$

njection Point $\overline{\text { Port C Center }}$

$\frac{1.10}{2}$ to: $\quad$ to: $\frac{10.84}{7}$

\section{2nd}

1st

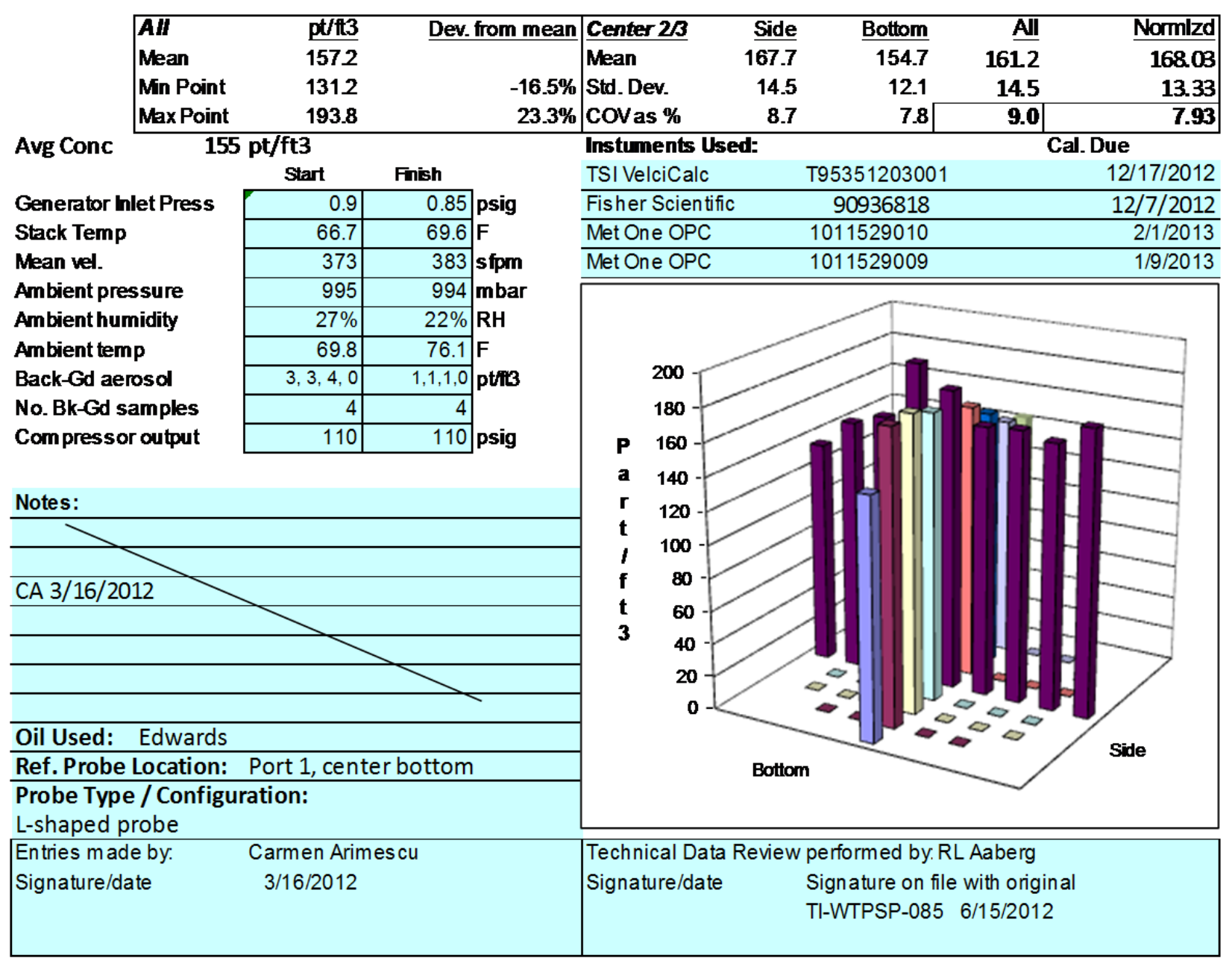

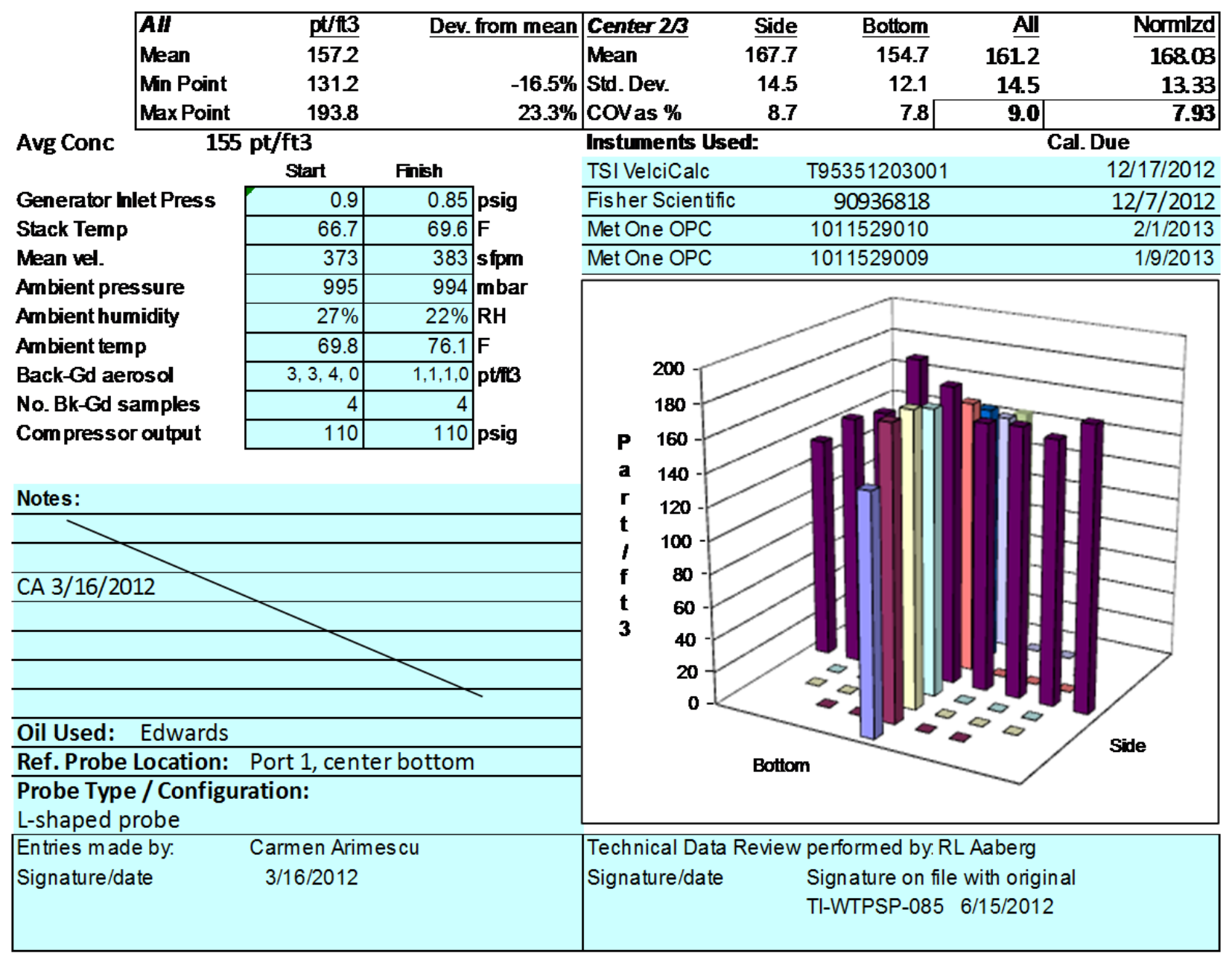

Notes:

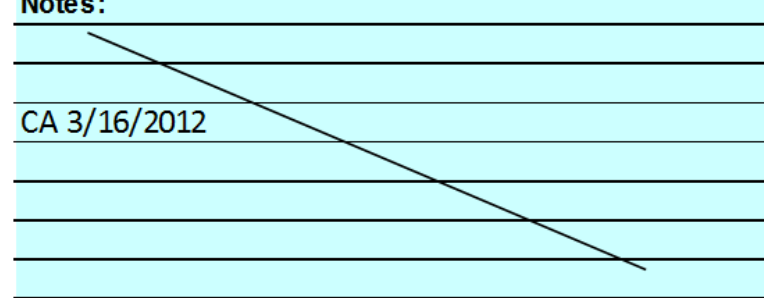

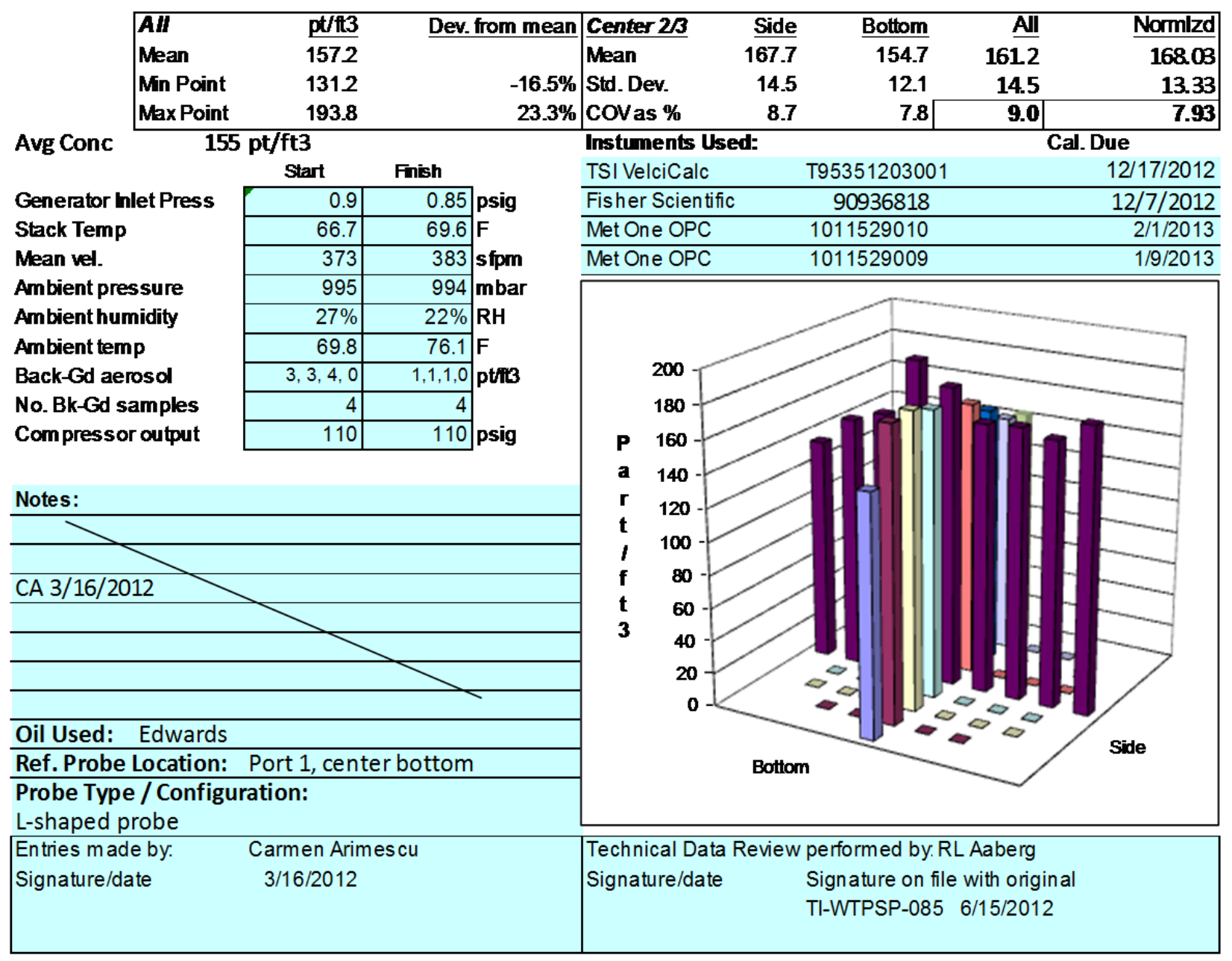

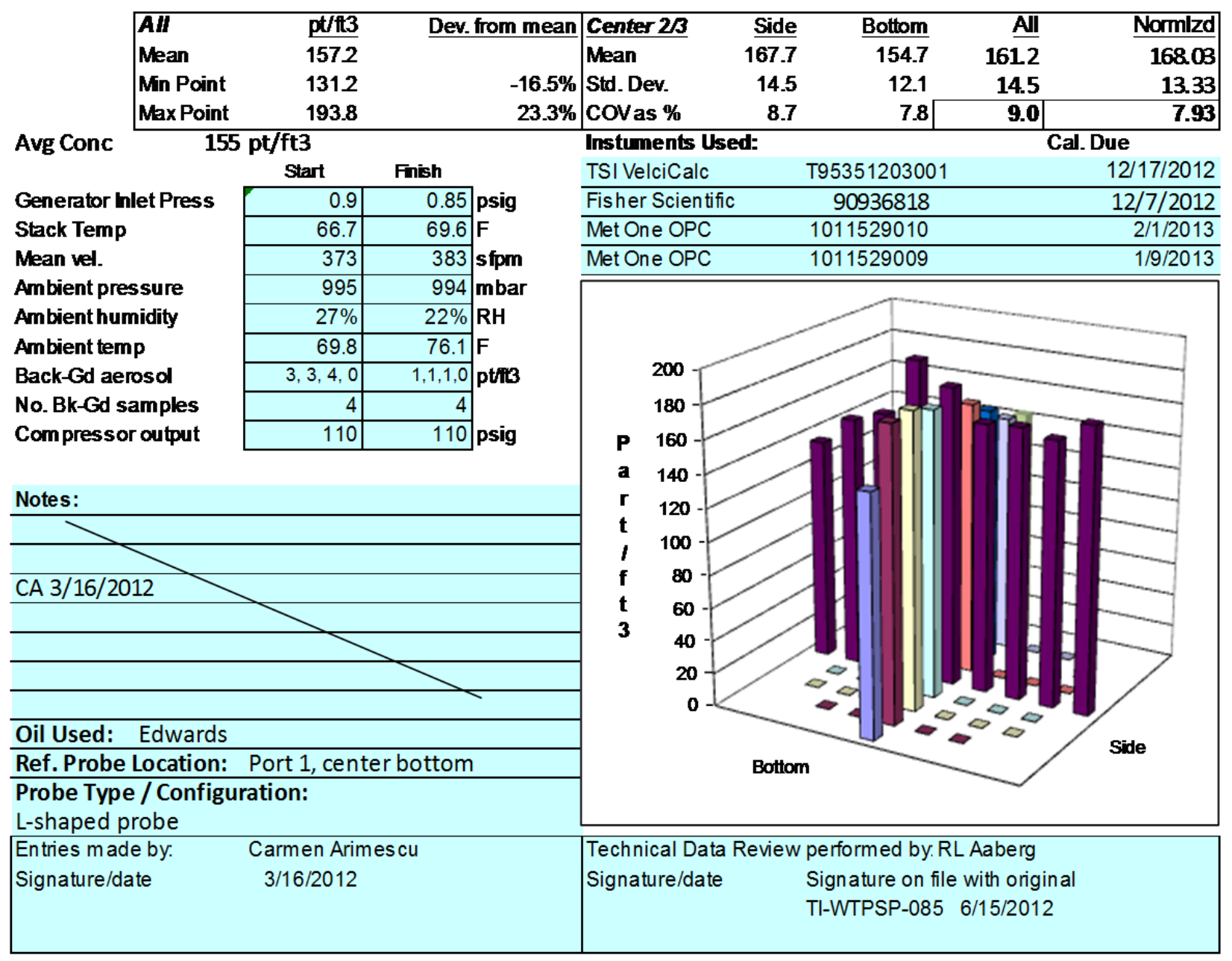

\begin{tabular}{|r|r|r|r|r|}
\hline & \multicolumn{4}{|c|}{ Bottom } \\
\hline & $\mathbf{1}$ & \multicolumn{2}{|c|}{$\mathbf{2}$} & $\mathbf{3}$ \\
\hline $\mathbf{1 7 2 . 4}$ & 121.5 & 118.5 & 160.6 & $\mathbf{1 3 3 . 5}$ \\
\hline $\mathbf{1 6 0 . 3}$ & 146.6 & 189.8 & 152.6 & $\mathbf{1 6 3 .}$ \\
\hline $\mathbf{1 6 4 . 3}$ & 160.6 & 186.7 & 145.6 & $\mathbf{1 6 4 . 3}$ \\
\hline $\mathbf{1 6 3 . 0}$ & 160.6 & 169.7 & 148.6 & $\mathbf{1 5 9 . 6}$ \\
\hline $\mathbf{1 8 1 . 0}$ & 170.7 & 171.7 & 156.6 & $\mathbf{1 6 6 . 3}$ \\
\hline $\mathbf{1 9 3 . 8}$ & 161.6 & 153.6 & 141.6 & $\mathbf{1 5 2 . 3}$ \\
\hline $\mathbf{1 5 8 . 9}$ & 147.6 & 141.6 & 141.6 & $\mathbf{1 4 3 . 6}$ \\
\hline $\mathbf{1 5 2 . 2}$ & 148.6 & 115.5 & 137.6 & $\mathbf{1 3 3 . 9}$ \\
\hline $\mathbf{1 3 5 . 3}$ & 135.5 & 129.5 & 128.5 & $\mathbf{1 3 1 2}$ \\
\hline $\mathbf{1 6 4 . 6}$ & $\mathbf{1 5 0 . 4}$ & $\mathbf{1 5 3 . 0}$ & $\mathbf{1 4 5 . 9}$ & $\mathbf{1 4 9 . 7}$ \\
\hline
\end{tabular}


Rev. 0

3 Aug. 2006
PARTICLE TRACER TRAVERSEDATA FORM

Site IHLW-S1 Model

Date 3/19/2012

Tester CA, XY

Stack Dia. 11.938 in.

Stack X-Area 111.9 in.2

Test Port

Distance to disturbance

Measurement units parficles/ft

300 inches

Order $-\rightarrow$

Traverse-->

Trial $-\rightarrow$

Point Depth, in

\begin{tabular}{|r|r|}
\hline Point & Depth, in \\
\hline $\mathbf{1}$ & 0.5 \\
\hline $\mathbf{2}$ & 1.2 \\
\hline $\mathbf{3}$ & 2.2 \\
\hline $\mathbf{4}$ & 3.8 \\
\hline Center & 5.9 \\
\hline $\mathbf{5}$ & 8.00 \\
\hline $\mathbf{6}$ & 9.5 \\
\hline $\mathbf{7}$ & 10.5 \\
\hline $\mathbf{8}$ & 11.31 \\
\hline
\end{tabular}

Averages --- ->
1 st
Run No. PT-5

Side

Fan configuration FAN BONLY

Fan Setting

Stack Temp

51.4 deg $F$

Start/End Time 9:45/13:00

Center $2 / 3$ from 1.10

Points in Center $2 / 3$

Injection Point $\overline{\text { Port C Center }}$ $\begin{array}{ll}\text { b: } & \mathbf{1 0 . 8 4} \\ \text { to: } & \end{array}$

2nd

$3 \quad$ Mean 1

Bottom

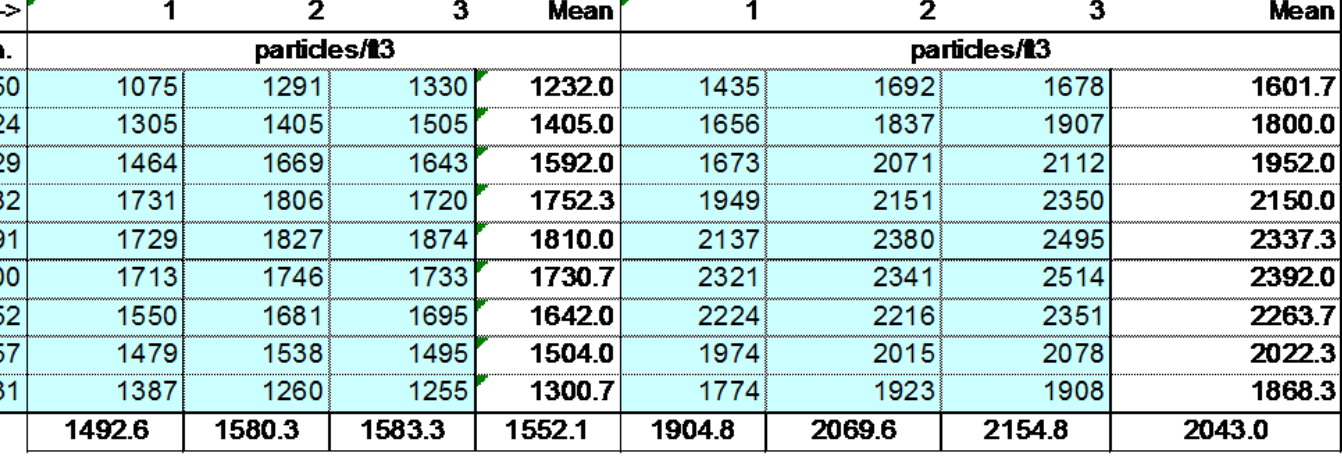

\begin{tabular}{|lr|}
\hline All & $\mathrm{pt} / \mathrm{ft} 3$ \\
Mean & 1797.6 \\
Min Point & 1232.0 \\
Max Point & 2392.0 \\
\hline \multicolumn{2}{|c|}{$1763 \mathrm{pt} / \mathrm{ft3}$} \\
\hline
\end{tabular}

Avg Conc $1763 \mathrm{pt} / \mathrm{ft} 3$

Generator Inlet Press

Stack Temp

Mean vel.

Ambient pressure

Ambient humidity

Ambient temp

Back-Gd aerosol

No. Bk-Gd samples

Compressor output

\begin{tabular}{|c|c|c|}
\hline $\mathrm{pt/f3}$ & Dev. & from $n$ \\
\hline 1797.6 & & \\
\hline 1232.0 & & \\
\hline 2392.0 & & \\
\hline $\mathrm{pt} / \mathrm{ft} 3$ & & \\
\hline Start & Finish & \\
\hline 6.2 & 6.15 & psig \\
\hline 49.4 & 53.4 & $F$ \\
\hline 2113 & 2095 & sfpm \\
\hline 29.56 & 29.56 & $\mathrm{inHg}$ \\
\hline $27 \%$ & $28 \%$ & RH \\
\hline 53.5 & 51.8 & $F$ \\
\hline $0,1,1,1$ & $3,5,4,2$ & $\mathrm{pt} / \mathrm{ti3}$ \\
\hline 4 & 4 & \\
\hline 155 & 170 & psig \\
\hline
\end{tabular}

Notes:

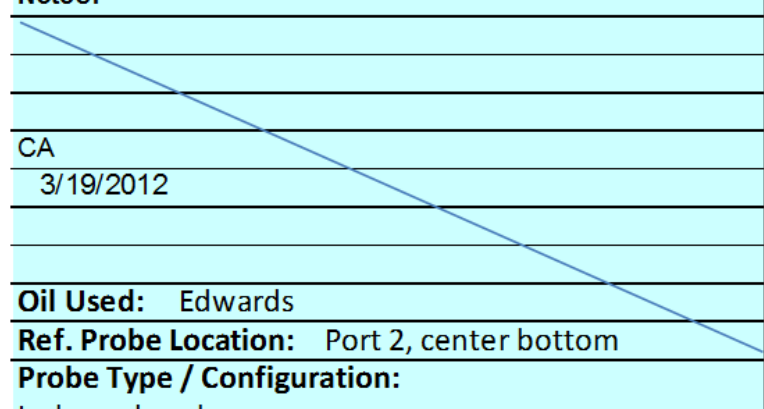

L-shaped probe

\begin{tabular}{|lc}
\hline Entries made by: & Carmen Arimescu \\
Signature/date & $3 / 19 / 2012$
\end{tabular}

Signature/date

$3 / 19 / 2012$

33

\begin{tabular}{l|lr} 
mean & Center 2/3 & Side \\
Mean & 1633.7 \\
$-31.5 \%$ & Std. Dev. & 144.6 \\
$33.1 \%$ & COVas \% & 8.9 \\
\hline \multicolumn{3}{|l}{ Instuments Usect }
\end{tabular}

\begin{tabular}{rrrr|}
\hline Bottom & \multicolumn{1}{c}{$\underline{\text { II }}$} & Normlzd \\
\hline 2131.0 & 1882.4 & 2120.37 \\
216.8 & 313.0 & 194.72 \\
10.2 & 16.6 & 9.18 \\
\hline
\end{tabular}

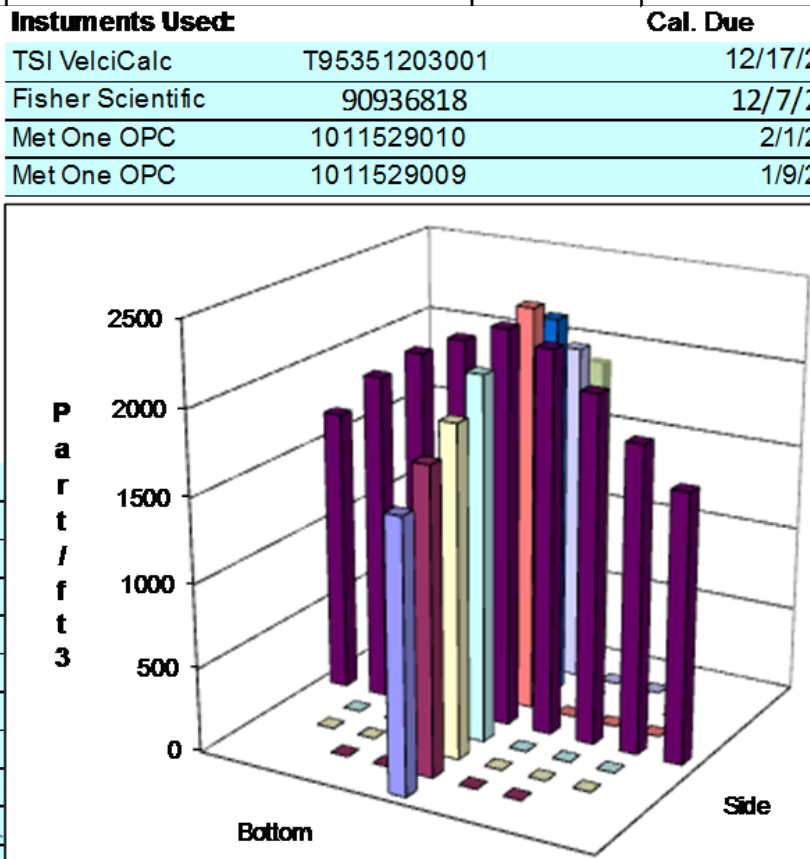

Technical Data Review performed by: RL Aaberg

Signature/date

Signature on file with original

TI-WTPSP-085 6/15/2012 
Rev. 0

3 Aug. 2006

PARTICLE TRACER TRAVERSEDATA FORM

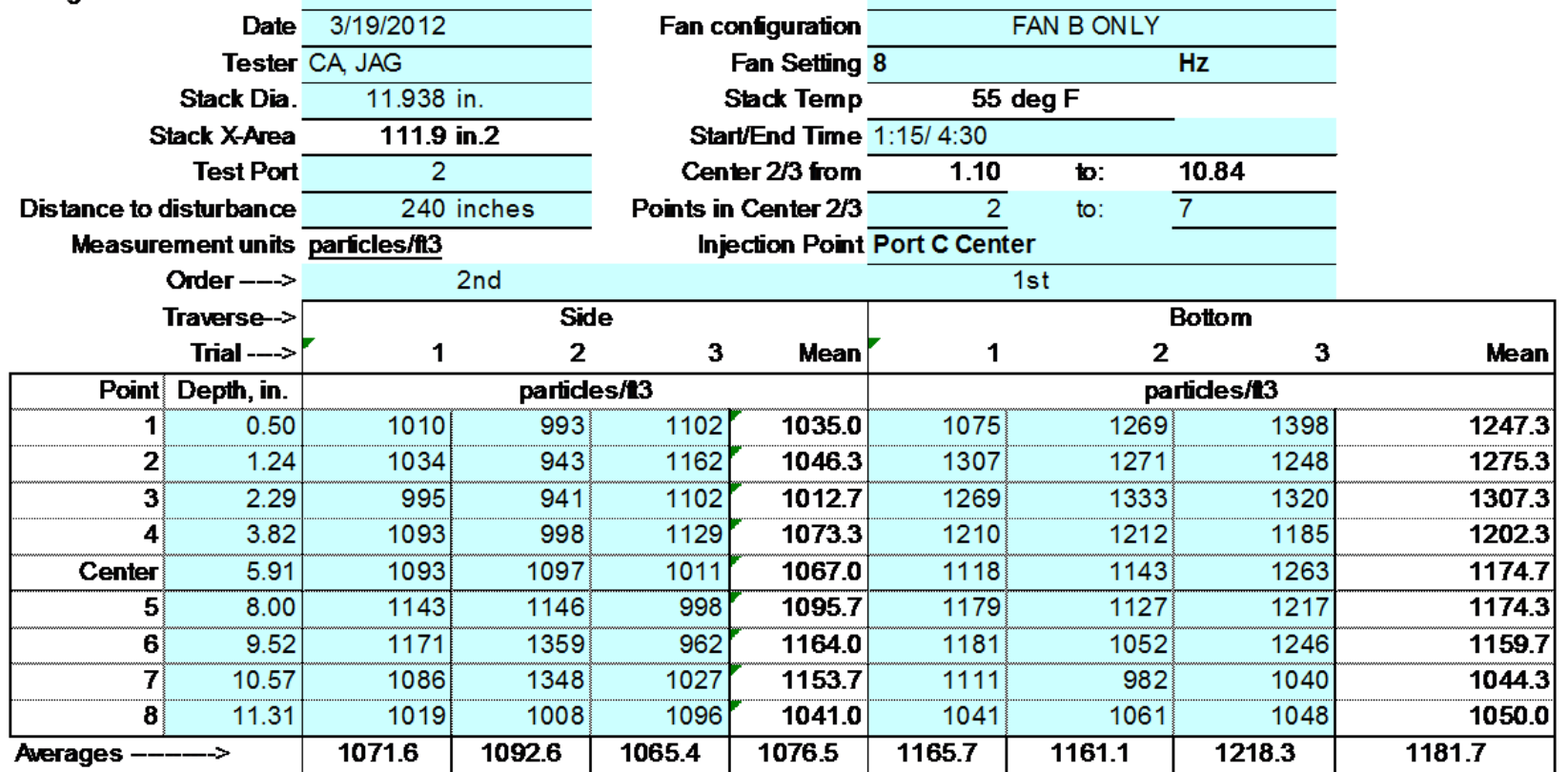

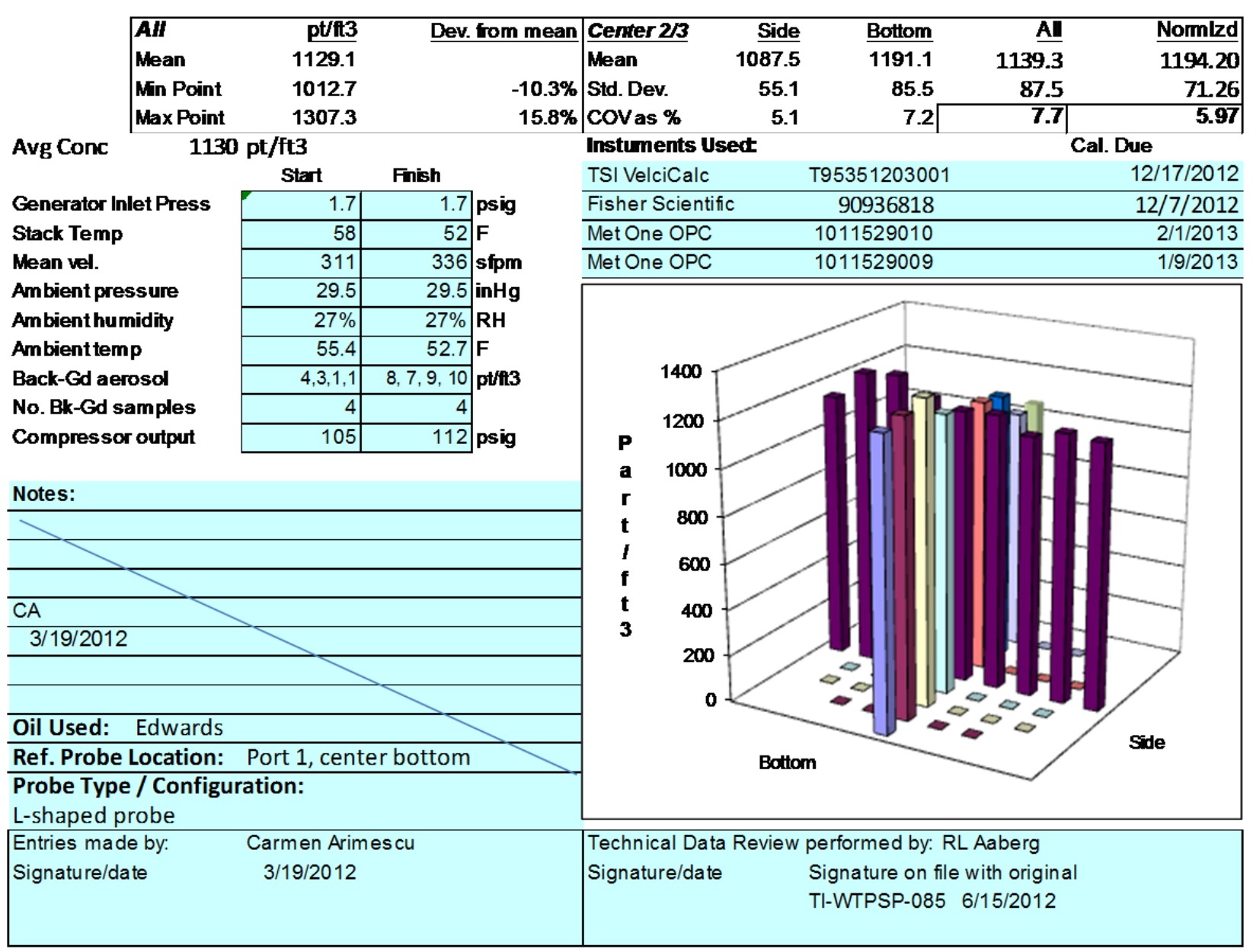


Rev. 0

3 Aug. 2006

\begin{tabular}{|c|c|c|}
\hline & Date & $3 / 22 / 2012$ \\
\hline & Tester & $\mathrm{CA}, \mathrm{YFS}$ \\
\hline & Stack Dia. & 11.938 \\
\hline & tack X-Area & 111.9 \\
\hline & Test Port & 2 \\
\hline istance to 0 & disturbance & 240 \\
\hline Measure & ement units & parficles/fi3 \\
\hline & Onder --> & $1 \mathrm{st}$ \\
\hline & Traverse--> & \\
\hline & Trial --> & 1 \\
\hline Point & Depth, in. & \\
\hline 1 & 0.50 & 524 \\
\hline 2 & 1.24 & 557 \\
\hline 3 & 2.29 & 576 \\
\hline 4 & 3.82 & 632 \\
\hline Center & 5.91 & 570 \\
\hline 5 & 8.00 & 576 \\
\hline 6 & 9.52 & 593 \\
\hline 7 & 10.57 & 546 \\
\hline 8 & 11.31 & 505 \\
\hline Averages -- & $\rightarrow$ & 564.3 \\
\hline & AII & $\mathrm{pt} / \mathrm{tt3}$ \\
\hline & Mean & 663.9 \\
\hline & Min Point & 538.3 \\
\hline & Max Point & 819.3 \\
\hline
\end{tabular}

Avg Conc $661 \mathrm{pt} / \mathrm{ft} 3$

Generator Inlet Press Stack Temp

Mean vel.

Ambient pressure

Ambienthumidity

Ambient temp

Back-Gd aerosol

No. Bk-Gd samples

Compressor output

\begin{tabular}{|r|r|l}
\multicolumn{1}{|c|}{ Start } & Fnish & \\
\hline 1.7 & 1.7 & psig \\
\hline 56.8 & 60.7 & F \\
\hline 408 & 388 & sfpm \\
\hline 29.71 & 29.71 & inH \\
\hline $48 \%$ & $35 \%$ & RH \\
\hline 49.1 & 59.9 & F \\
\hline $1,1,2,2$ & $0,0,0,2$ & pt/f33 \\
\hline 4 & 4 & \\
\hline 110 & 120 & psig \\
\hline & &
\end{tabular}

Notes:
Run No. PT-7

Fan configuration FAN A ONLY

Fan Setting 9.5

Stack Temp $\quad 58.75 \mathrm{deg} F$

Start/End Time 1130/1300

Center 2/3 from

Points in Center 2/3

Injection Point $\overline{\text { Port C Center }}$ $\begin{array}{ll}\text { to: } & \mathbf{1 0 . 8 4} \\ \text { to: } & \end{array}$

2nd

\begin{tabular}{|c|c|c|c|c|c|c|}
\hline \multirow{2}{*}{\multicolumn{3}{|c|}{ Side }} & \multicolumn{3}{|c|}{ 2nd } & \multirow[b]{3}{*}{ Mean } \\
\hline & & & \multicolumn{3}{|c|}{ Bottom } & \\
\hline 2 & 3 & Mean & 1 & 2 & 3 & \\
\hline \multicolumn{3}{|c|}{ particles/13 } & \multicolumn{4}{|c|}{ particles/13 } \\
\hline 543 & 559 & 542.0 & 686 & 727 & 794 & 735.7 \\
\hline 586 & 577 & 573.3 & 755 & 626 & 775 & 718.7 \\
\hline 535 & 571 & 560.7 & 752 & 668 & 870 & 763.3 \\
\hline 536 & 546 & 571.3 & 793 & 680 & 812 & 761.7 \\
\hline 560 & 533 & 554.3 & 783 & 743 & 932 & 819.3 \\
\hline 551 & 587 & 571.3 & 747 & 761 & 908 & 805.3 \\
\hline 544 & 577 & 571.3 & 734 & 794 & 910 & 812.7 \\
\hline 523 & 590 & 553.0 & 639 & 743 & 824 & 735.3 \\
\hline 513 & 597 & 538.3 & 658 & 825 & 803 & 762.0 \\
\hline 3.4 & 570.8 & 559.5 & 727.4 & 729.7 & 847.6 & 768.2 \\
\hline
\end{tabular}

\begin{tabular}{rrrr|} 
Side & Bottom & $\underline{\text { AI }}$ & Nomlzd \\
\cline { 2 - 4 } 8.0 & 773.8 & 669.4 & 804.47 \\
1.6 & 39.5 & 111.7 & 42.58 \\
\cline { 3 - 5 } & 5.1 & 16.7 & 5.29
\end{tabular}

$23.4 \%$ COV as \% $\quad 1.6$

Instuments Usedt

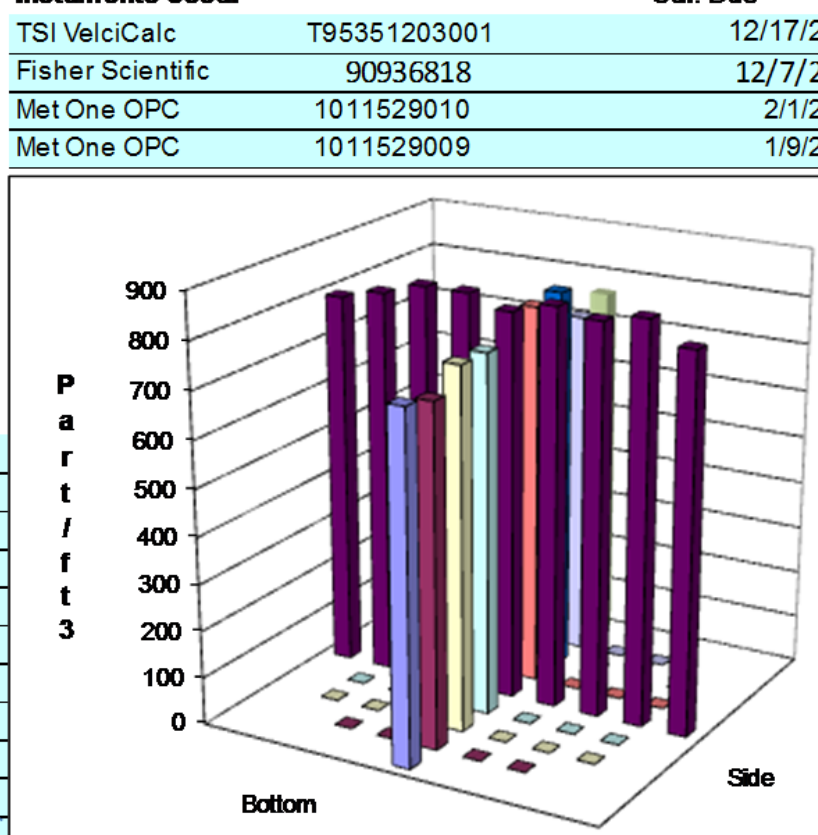

Probe Type / Configuration

L-shaped probe

Entries made by: Carmen Arimescu

Signature/date

$3 / 22 / 2012$ 
Rev. 0

3 Aug. 2006

PARTICLE TRACER TRAVERSE DATA FORM

\section{Site IHLW-S1 Mode \\ Date 5/24/2012}

Tester YFS, EA

\begin{aligned} Stack Dia. & 11.938 in. \\ \cline { 2 - 2 } Stack X-Area & $\mathbf{1 1 1 . 9}$ in.2 \\ \cline { 2 - 2 } Test Port & 2 \\ \cline { 2 - 2 } disturbance & 240 inches \end{aligned}

Distance to disturbance 240 inches

Measurement units parficles/th3

Order $\rightarrow 1$ 1st
Run No. PT-8

Fan configuration Fan B Only

Fan Setting 14

Stack Temp \#DIVO! $\operatorname{deg} F$

Start/End Time $1500 / 1745$

Center 2/3 fom

Points in Center 2/3

Injection Point $\overline{\text { Port C Center }}$

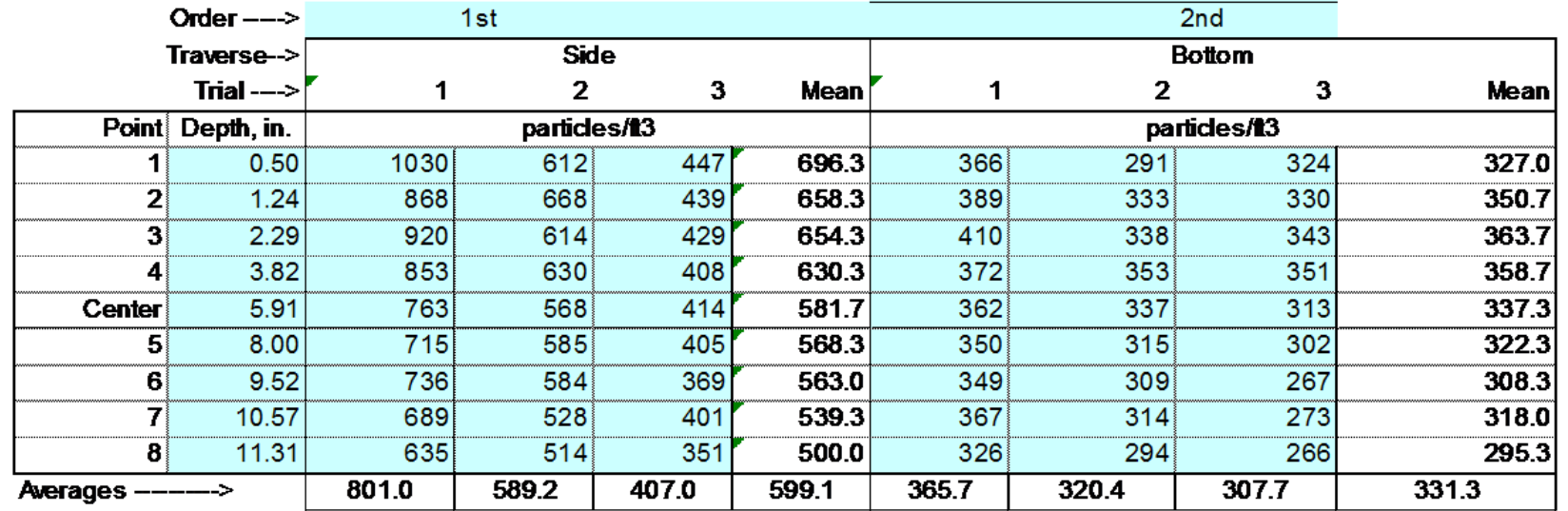

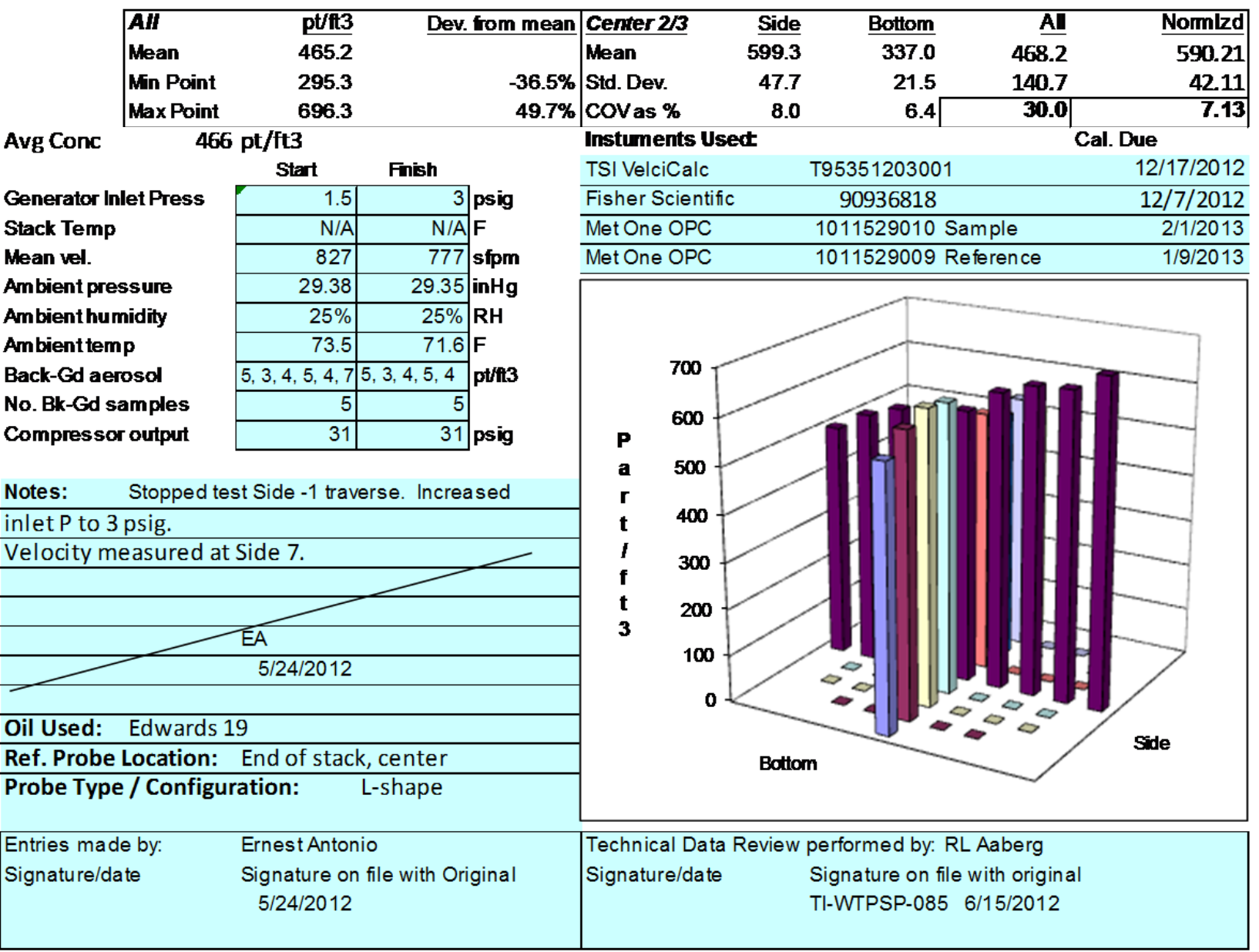


Rev. 0

3 Aug. 2006

PARTICLE TRACER TRAVERSEDATA FORM

Site IHLW-S1 Mode
Date

Stack Dia. 11.938 in.

Stack X-Area 111.9 in.2

Test Port

Distance to disturbance

Measurement units parficles/ti3

240 inches

Order $\rightarrow->$

Traverse-->

Trial $\rightarrow$

\begin{tabular}{|l|l|}
\hline Point & Depth, in. \\
\hline
\end{tabular}

10.50

\begin{tabular}{|r|r|}
\hline $\mathbf{1}$ & 0.5 \\
\hline $\mathbf{2}$ & 1.2 \\
\hline $\mathbf{3}$ & 2.2 \\
$\mathbf{4}$ & 3.8 \\
\hline Center & 5.9 \\
\hline $\mathbf{5}$ & 8.00 \\
\hline $\mathbf{6}$ & 9.5 \\
\hline $\mathbf{7}$ & 10.5 \\
\hline $\mathbf{8}$ & 11.3 \\
\hline
\end{tabular} 2nd

$\begin{array}{lc}1 & \text { Side } \\ 1 & 2\end{array}$

\begin{tabular}{|r|r|}
\hline 50 & 1788 \\
\hline 24 & 1837 \\
\hline 2 & 1766 \\
\hline 1 & 1842 \\
\hline 50 & 1734 \\
\hline 52 & 1766 \\
\hline 31 & 1678 \\
\hline
\end{tabular}

1750.9
Run No. PT-9

Fan configuration Fan B Only

Fan Setting $\mathbf{1 4 . 9}$

Stack Temp

$84.8 \mathrm{deg} F$

Start/End Time 9:40/11:40

Center $2 / 3$ from

Points in Center $2 / 3$

Injection Point $\overline{\text { Port C, center }}$ to:
to: $\quad \underline{10.84}$

\begin{tabular}{c|c}
\cline { 2 - 3 } & $1 \mathrm{st}$ \\
Bottom
\end{tabular}

3

\begin{tabular}{|c|c|c|c|c|c|c|c|}
\hline & 2 & 3 & Mean & 1 & 2 & 3 & Mean \\
\hline & particle & $\sqrt{113}$ & & & & $\operatorname{des} / 13$ & \\
\hline & 1610 & 1922 & 1773.3 & 2100 & 1936 & 1758 & 1931.3 \\
\hline & 1822 & 1936 & 1865.0 & 2062 & 2002 & 1779 & 1947.7 \\
\hline & 1844 & 1929 & 1846.3 & 2177 & 2110 & 1876 & 2054.3 \\
\hline & 1879 & 1903 & 1874.7 & 2032 & 2088 & 1922 & 2014.0 \\
\hline & 1911 & 1978 & 1874.3 & 2076 & 2183 & 1921 & 2060.0 \\
\hline & 1847 & 1830 & 1814.3 & 2032 & 1953 & 2013 & 1999.3 \\
\hline & 1900 & 1824 & 1800.7 & 2084 & 1987 & 2049 & 2040.0 \\
\hline & 1860 & 1701 & 1752.7 & 1880 & 1722 & 1884 & 1828.7 \\
\hline & 1724 & 1729 & 1701.0 & 1804 & 1718 & 1934 & 1818.7 \\
\hline & 21.9 & 1861.3 & 1811.4 & 2027.4 & 1966.6 & 1904.0 & 1966.0 \\
\hline
\end{tabular}

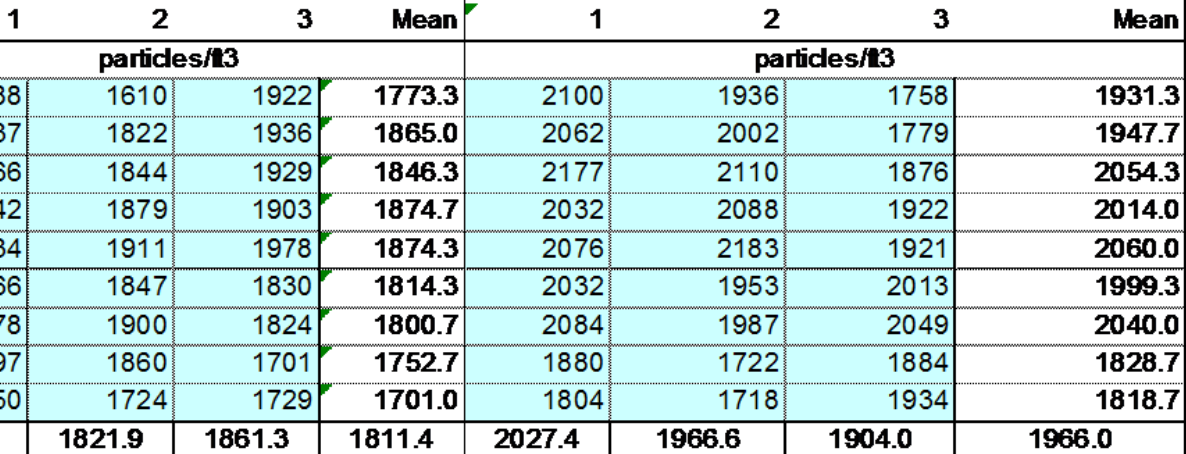

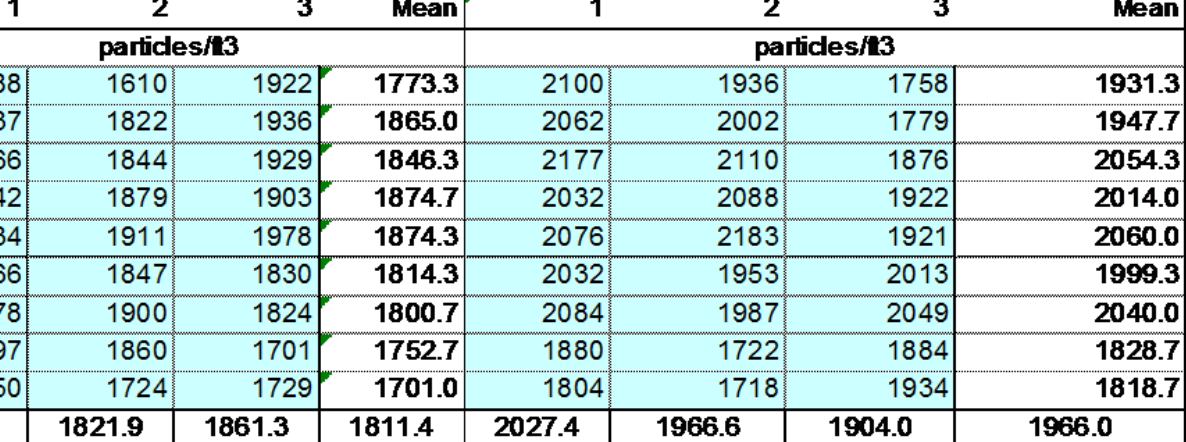

particles/13

\begin{tabular}{|lr|}
\hline All & $\mathrm{pt} / \mathrm{ft} 3$ \\
Mean & 1888.7 \\
Min Point & 1701.0 \\
Max Point & 2060.0 \\
\hline \multicolumn{2}{|c|}{$1879 \mathrm{pt} / \mathrm{ft3}$} \\
\hline
\end{tabular}

Avg Conc $1879 \mathrm{pt} / \mathrm{ft} 3$

Generator Inlet Press

Stack Temp

Mean vel.

Ambient pressure

Ambienthumidity

Ambient temp

Back-Gd aerosol

No. Bk-Gd samples

Compressor output

\begin{tabular}{|c|c|c|}
\hline $\mathrm{pt} / \mathrm{ft3}$ & Dev. & from \\
\hline 1888.7 & & \\
\hline 1701.0 & & \\
\hline 2060.0 & & \\
\hline $9 \mathrm{pt} / \mathrm{ft} 3$ & & \\
\hline Start & Finish & \\
\hline 0.6 & 0.6 & psig \\
\hline 81.7 & 87.9 & $F$ \\
\hline 880 & 817 & sfpm \\
\hline 29.59 & 29.39 & in $\mathrm{Hg}$ \\
\hline $41 \%$ & $27 \%$ & $\mathbf{R H}$ \\
\hline 77.6 & 92.3 & $\mathrm{~F}$ \\
\hline $7,7,5,4$ & $4,2,5,5$ & pt/f3 \\
\hline 4 & 4 & \\
\hline 30 & 30 & psig \\
\hline
\end{tabular}

Notes:

Oil Used: Edwards 19

Ref. Probe Location: End of stack, center Probe Type / Configuration: L-shape

\begin{tabular}{|ll}
\hline Entries made by: & CA, XY \\
Signature/date & Signature on file with Original \\
& $6 / 1 / 2012$
\end{tabular}

\begin{tabular}{r|lr} 
mean & Center 2/3 & Side \\
$-9.9 \%$ & 1832.6 \\
$9.1 \%$ & Std. Dean & 45.6 \\
COV as \% & 2.5
\end{tabular}

\begin{tabular}{lcr} 
Instuments Usect & \multicolumn{1}{c}{ Cal. Due } \\
TSI VelciCalc & T95351203001 & $12 / 17 / 2012$ \\
\hline Fisher Scientific & 90936818 & $12 / 7 / 2012$ \\
\hline Met One OPC & 1011529010 Sample & $2 / 1 / 2013$ \\
\hline Met One OPC & 1011529009 Reference & $1 / 9 / 2013$ \\
\hline
\end{tabular}

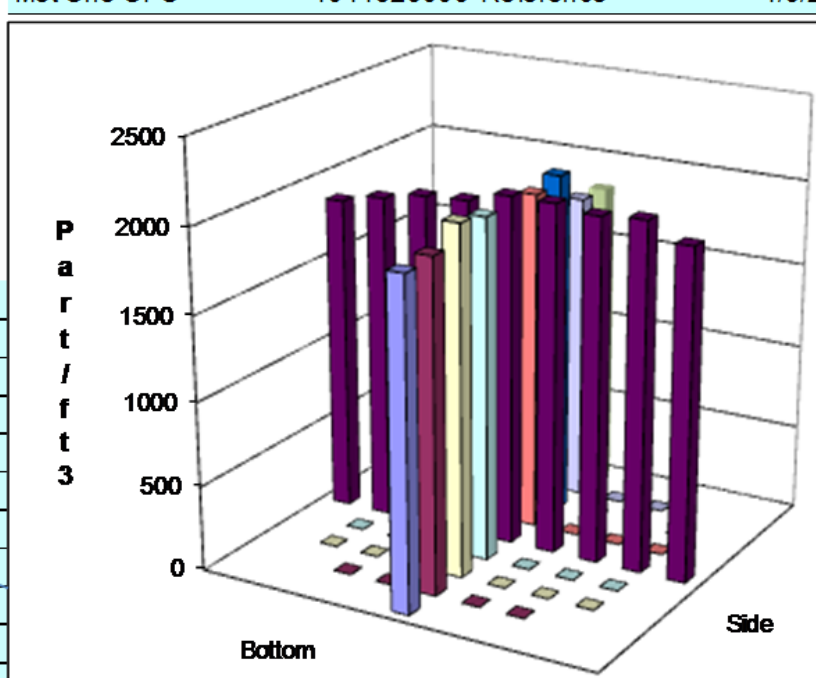

Technical Data Review performed by: RL Aaberg

Signature/date

Signature on file with original

TI-WTPSP-085 6/15/2012 
Appendix D

Flow Unit Conversions 



\section{Appendix D}

\section{Flow Unit Conversions}

Flow units provided by BNI were in actual flow units. For consistency with the measurement equipment used for velocity and flow rate measurements, these were converted to standard flow units. Converting from acfm to scfm was done by simple ratio of the standard temperature (in absolute units, Rankine) to the actual temperature of the stack provided by BNI, as follows:

$$
Q_{s c f m}=Q_{a c f m}\left(\frac{T_{s t d}}{T_{a c t}}\right)
$$

The table below lists the flow and velocity values provided by BNI as well as the calculated values in standard units.

\begin{tabular}{lccccc}
\hline & $\begin{array}{c}\text { Temperature } \\
(\mathrm{F})\end{array}$ & $\begin{array}{c}\text { Air Flow } \\
(\mathrm{acfm})\end{array}$ & $\begin{array}{c}\text { Air Flow } \\
(\mathrm{scfm})\end{array}$ & $\begin{array}{c}\text { Air Velocity } \\
(\mathrm{afpm})\end{array}$ & $\begin{array}{c}\text { Air Velocity } \\
\text { (sfpm) }\end{array}$ \\
\hline HV-S1 & & & & & \\
\hline Single fan-max flow & 105 & 59,300 & 55,627 & 3,030 & 2,833 \\
Single fan -norm flow & 93 & 47,500 & 45,524 & 2,419 & 2,319 \\
Single fan-min flow & 59 & 23,700 & 24,202 & 1,207 & 1,233 \\
\hline HV-S2 & & & & & \\
\hline Single fan-max flow & 170 & 63,750 & 53,631 & 3,041 & 2,558 \\
Single fan-norm flow & 102 & 51,000 & 48,096 & 2,433 & 2,294 \\
Single fan-min flow & 59 & 25,500 & 26,040 & 1,216 & 1,242 \\
\hline IHLW-S1 & & & & & \\
\hline Single fan-max flow & 160 & 13,100 & 11,198 & 2,078 & 1,776 \\
Single fan-norm flow & 102 & 10,480 & 9,883 & 1,662 & 1,567 \\
Single fan-min flow & 59 & 5,240 & 5,351 & 831 & 849 \\
\hline
\end{tabular}



Appendix E Document List 



\section{Appendix E}

\section{Document List}

\begin{tabular}{lll}
\hline Project Plan & PP-WTPSP-045 & $\begin{array}{l}\text { Air Sampling Probe Location Tests for Waste Treatment Plant } \\
\text { HV-S1, HV-S2 and IHLW-S1 (Group 3-4) Air Exhaust Systems }\end{array}$ \\
\hline Test Plan & TP-WTPSP-032 & $\begin{array}{l}\text { Air Sampling Probe Location Tests for Waste Treatment Plant } \\
\text { HV-S1, HV-S2 and IHLW-S1 (Group 3-4) Air Exhaust Systems }\end{array}$ \\
\hline Test Instructions & TI-WTPSP-066 & Measurements HV-S1 Scale Model \\
& TI-WTPSP-067 & Calibration of Ventilation Flow Controller for HV-S1 Scale Model \\
& Stack \\
& TI-WTPSP-068 & Velocity Uniformity Measurements of HV-S1 Scale Model \\
TI-WTPSP-069 & Determine Flow Angle in HV-S1 Scale Model Stack \\
TI-WTPSP-070 & Tests of Gas Tracer Mixing in HV-S1 Scale Model Stack \\
TI-WTPSP-071 & Tests of Particle Tracer Mixing in HV-S1 Scale Model Stack \\
& TI-WTPSP-072 & Measurements HV-S2 Scale Model \\
TI-WTPSP-073 & Calibration of Ventilation Flow Controller for HV-S2 Scale Model \\
& Stack \\
TI-WTPSP-074 & Velocity Uniformity Measurements of HV-S3 Scale Model \\
TI-WTPSP-075 & Determine Flow Angle in HV-S2 Scale Model Stack \\
TI-WTPSP-078 & Tests of Gas Tracer Mixing in HV-S2 Scale Model Stack \\
TI-WTPSP-079 & Tests of Particle Tracer Mixing in HV-S2 Scale Model Stack \\
TI-WTPSP-080 & Measurements of IHLW-S1 Scale Model \\
TI-WTPSP-083 & Determine Flow Angle in IHLW-S1 Scale Model Stack \\
TI-WTPSP-082 & Velocity Uniformity Measurements of IHLW-S1 Scale Model \\
TI-WTPSP-084 & Tests of Gas Tracer Mixing in IHLW-S1 Scale Model Stack \\
TI-WTPSP-085 & Tests of Particle Tracer Mixing in IHLW-S1 Scale Model Stack \\
TI-WTPSP-081 & Calibration of Ventilation Controller for IHLW-S1 Scale Model \\
& Stack \\
\hline & \\
\hline
\end{tabular}

Calculation

Packages

CCP-WTPSP-1227

CCP-WTPSP-1228

CCP-WTPSP-1229

CCP-WTPSP-1230

CCP-WTPSP-1231

CCP-WTPSP-1232

CCP-WTPSP-1233

CCP-WTPSP-1234

CCP-WTPSP-1235

CCP-WTPSP-1236

CCP-WTPSP-1237

CCP-WTPSP-1208
Calibration of Ventilation Flow Controller for IHLW-S1

Determine Flow Angle in IHLW-S1 Scale Model Stack

Determine Air Velocity Uniformity of IHLW-S1 Scale Stack Model

Determine Particle Tracer Uniformity of IHLW-S1 Scale Model Stack

Gas Tracer Mixing in the IHLW-S1 Scale Model Stack

Calibration of Ventilation Flow Controller for HV-S1 Scale Model

Determine Flow Angle in HV-S1 Scale Model Stack

Determine Air Velocity Uniformity of HV-S1 Scale Stack Model

Determine Particle Tracer Uniformity of HV-S1 Scale Model Stack

Gas Tracer Mixing in the HV-S1 Scale Model Stack

Calibration of Ventilation Flow Controller for HV-S2 Scale Model

Determine Flow Angle in HV-S2 Scale Model Stack 
CCP-WTPSP-1209 Determine Air Velocity Uniformity of HV-S2 Scale Stack Model

CCP-WTPSP-1210 Determine Particle Tracer Uniformity of HV-S2 Scale Model Stack

CCP-WTPSP-1211 Gas Tracer Mixing in the HV-S2 Scale Model Stack 
PNNL-21998

WTP-RPT-224 Rev 0

\section{Distribution}

No. of

Copies

ONSITE*

4 Bechtel National, Inc.

FW Damerow

JM Meyer

MM Mwembeshi

WTP Docs
H4-02

H4-02

H4-02

H4-02
No. of

Copies

7 Pacific Northwest National Laboratory

EJ Antonio

K3-54

JE Flaherty

JA Glissmeyer

K9-30

DE Kurath

LM Peurrung

Information Release

Project File
K3-54

K3-52

K9-09

K3-52

K3-52

*All distribution will be made electronically.

Distr. 1 


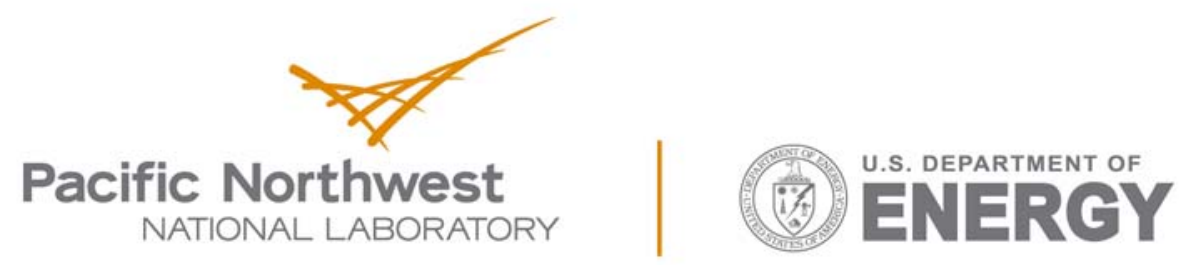

Proudly Operated by Battelle Since 1965

902 Battelle Boulevard

P.O. Box 999

Richland, WA 99352

1-888-375-PNNL (7665)

www.pnnl.gov 